\title{
Antipsychotics for the Prevention and Treatment of Delirium
}




\title{
Comparative Effectiveness Review
}

Number 219

\section{Antipsychotics for the Prevention and Treatment of Delirium}

\author{
Prepared for: \\ Agency for Healthcare Research and Quality \\ U.S. Department of Health and Human Services \\ 5600 Fishers Lane \\ Rockville, MD 20857 \\ www.ahrq.gov
}

Contract No. 290-2015-00006-I, 290-32008-T

Prepared by:

Johns Hopkins University Evidence-based Practice Center

Baltimore, MD

Investigators:

Karin J. Neufeld, M.D., M.P.H.

Dale M. Needham, M.D., Ph.D.

Esther S. Oh, M.D., Ph.D.

Lisa M. Wilson, Sc.M.

Roozbeh Nikooie, M.D.

Allen Zhang, B.S.

Mounica Koneru, M.B.B.S.

Amulya Balagani, M.B.B.S.

Sriharsha Singu, M.B.B.S.

Louay Aldabain, M.D.

Karen A. Robinson, Ph.D.

AHRQ Publication No. 19-EHC019-EF

September 2019 


\section{Key Messages}

\section{Purpose of Review}

To assess the benefits and harms of antipsychotics for the prevention and treatment of delirium among adult patients.

\section{Key Messages}

- Haloperidol or second-generation antipsychotics used to prevent or treat delirium did not decrease length of stay in hospital.

- There was little or no evidence to determine the effect of antipsychotics on cognitive function, delirium severity, or caregiver burden, or for sedation when used for prevention.

- Second-generation antipsychotics may lower the occurrence of delirium in postoperative patients.

- Haloperidol or second-generation antipsychotics used to prevent or treat delirium may lead to little or no difference in sedation or extrapyramidal side effects (problems with muscles such as spasms or restlessness). Heart-related side effects tended to occur more frequently with the use of antipsychotics, in particular QT interval prolongation (a type of heart rhythm problem) in second-generation antipsychotics.

- Future studies are needed to assess the effects of using antipsychotics on patient agitation and distress, subsequent memories of delirium, caregiver burden and distress, inappropriate continuation of antipsychotic therapy, and long-term cognitive and functional outcomes. 
This report is based on research conducted by the Johns Hopkins University Evidence-based Practice Center (EPC) under contract to the Agency for Healthcare Research and Quality (AHRQ), Rockville, MD (Contract No. 290-2015-00006-I-2). The findings and conclusions in this document are those of the authors, who are responsible for its contents; the findings and conclusions do not necessarily represent the views of AHRQ. Therefore, no statement in this report should be construed as an official position of AHRQ or of the U.S. Department of Health and Human Services.

Two investigators were members of the guideline panel for a 2018 Society for Clinical Care Medicine guideline for the management of pain, sedation, delirium, rehabilitation, and sleep for critically ill patients. Two investigators co-authored the American Geriatric Society guidelines for post-operative delirium in older adults. None of the other investigators have any affiliations or financial involvement that conflicts with the material presented in this report.

The information in this report is intended to help healthcare decision makers-patients and clinicians, health system leaders, and policymakers, among others-make well-informed decisions and thereby improve the quality of healthcare services. This report is not intended to be a substitute for the application of clinical judgment. Anyone who makes decisions concerning the provision of clinical care should consider this report in the same way as any medical reference and in conjunction with all other pertinent information, i.e., in the context of available resources and circumstances presented by individual patients.

This report is made available to the public under the terms of a licensing agreement between the author and the Agency for Healthcare Research and Quality. This report may be used and reprinted without permission except those copyrighted materials that are clearly noted in the report. Further reproduction of those copyrighted materials is prohibited without the express permission of copyright holders.

AHRQ or U.S. Department of Health and Human Services endorsement of any derivative products that may be developed from this report, such as clinical practice guidelines, other quality enhancement tools, or reimbursement or coverage policies, may not be stated or implied.

This report may periodically be assessed for the currency of conclusions. If an assessment is done, the resulting surveillance report describing the methodology and findings will be found on the Effective Health Care Program website at www.effectivehealthcare.ahrq.gov. Search on the title of the report.

People using assistive technology may not be able to fully access information in this report. For assistance contact EPC@ahrq.hhs.gov.

Suggested citation: Neufeld KJ, Needham DM, Oh ES, Wilson LM, Nikooie R, Zhang A, Koneru M, Balagani A, Singu S, Aldabain L, Robinson KA. Antipsychotics for the Prevention and Treatment of Delirium. Comparative Effectiveness Review No. 219. (Prepared by the Johns Hopkins University Evidence-based Practice Center under Contract No. 290-2015-00006-I-2.) AHRQ Publication No. 19-EHC019-EF. Rockville, MD: Agency for Healthcare Research and Quality; September 2019. Posted final reports are located on the Effective Health Care Program search page. DOI: https://doi.org/10.23970/AHRQEPCCER219. 


\section{Preface}

The Agency for Healthcare Research and Quality (AHRQ), through its Evidence-based Practice Centers (EPCs), sponsors the development of systematic reviews to assist public- and private-sector organizations in their efforts to improve the quality of healthcare in the United States. These reviews provide comprehensive, science-based information on common, costly medical conditions, and new healthcare technologies and strategies.

Systematic reviews are the building blocks underlying evidence-based practice; they focus attention on the strength and limits of evidence from research studies about the effectiveness and safety of a clinical intervention. In the context of developing recommendations for practice, systematic reviews can help clarify whether assertions about the value of the intervention are based on strong evidence from clinical studies. For more information about AHRQ EPC systematic reviews, see www.effectivehealthcare.ahrq.gov/reference/purpose.cfm.

AHRQ expects that these systematic reviews will be helpful to health plans, providers, purchasers, government programs, and the healthcare system as a whole. Transparency and stakeholder input are essential to the Effective Health Care Program. Please visit the website (www.effectivehealthcare.ahrq.gov) to see draft research questions and reports or to join an email list to learn about new program products and opportunities for input.

If you have comments on this systematic review, they may be sent by mail to the Task Order Officer named below at: Agency for Healthcare Research and Quality, 5600 Fishers Lane, Rockville, MD 20857, or by email to epc@ahrq.hhs.gov.

Gopal Khanna, M.B.A.

Director

Agency for Healthcare Research and Quality

Stephanie Chang, M.D., M.P.H.

Director

Evidence-based Practice Center Program

Center for Evidence and Practice Improvement Agency for Healthcare Research and Quality
Arlene S. Bierman, M.D., M.S.

Director

Center for Evidence and Practice Improvement

Agency for Healthcare Research and Quality

Laura L. Pincock, Pharm.D., M.P.H.

Task Order Officer

Center for Evidence and Practice

Improvement

Agency for Healthcare Research and Quality 


\section{Acknowledgments}

The authors gratefully acknowledge the following individuals for their contributions to this project: Sumana Vasishta, M.B.B.S., for her help with reviewing articles and Jeanette Edelstein, M.A., for her services copyediting the report.

\section{Key Informants}

In designing the study questions, the EPC consulted several Key Informants who represent the end-users of research. The EPC sought the Key Informant input on the priority areas for research and synthesis. Key Informants are not involved in the analysis of the evidence or the writing of the report. Therefore, in the end, study questions, design, methodological approaches, and/or conclusions do not necessarily represent the views of individual Key Informants.

Key Informants must disclose any financial conflicts of interest greater than $\$ 5,000$ and any other relevant business or professional conflicts of interest. Because of their role as end-users, individuals with potential conflicts may be retained. The TOO and the EPC work to balance, manage, or mitigate any conflicts of interest.

The list of Key Informants who provided input to this report follows:

Donna Fick, Ph.D., R.N.

College of Nursing, Pennsylvania State

James L. Rudolph, M.D., S.M.

University

University Park, PA

Warren Alpert Medical School of Brown

University

John James, Ph.D.

Brown University School of Public Health

Patient Advocate

Providence, RI

Houston, TX

Marianne Shaughnessy, Ph.D., C.N.R.P.

Veterans Health Administration

Ann M. Kolanowski, Ph.D., R.N.

College of Nursing, Pennsylvania State

Alexandria, VA

University

University Park, PA

Eric Weakly, M.S.W., M.B.A.

Substance Abuse and Mental Health

Julie A. Lanphere, D.O.

Services Administration

University of Pittsburgh

Rockville, MD

Pittsburgh, PA

Michelle T. Weckmann, M.S., M.D.

Javier Muniz, M.D.

University of Iowa Health Care

Iowa City, IA

Food and Drug Administration

Silver Spring, MD

Teisha Robertson, Pharm.D., M.B.A.

Defense Health Agency

Falls Church, VA 


\section{Technical Expert Panel}

In designing the study questions and methodology at the outset of this report, the EPC consulted several technical and content experts. Broad expertise and perspectives were sought. Divergent and conflicted opinions are common and perceived as healthy scientific discourse that results in a thoughtful, relevant systematic review. Therefore, in the end, study questions, design, methodologic approaches, and/or conclusions do not necessarily represent the views of individual technical and content experts.

Technical Experts must disclose any financial conflicts of interest greater than \$5,000 and any other relevant business or professional conflicts of interest. Because of their unique clinical or content expertise, individuals with potential conflicts may be retained. The TOO and the EPC work to balance, manage, or mitigate any potential conflicts of interest identified.

The list of Technical Experts who provided input to this report follows:

Noll Campbell, Pharm.D., M.S.*

Purdue University

Indianapolis, IN

E. Wesley Ely, M.D., M.P.H.

Vanderbilt University School of Medicine

Nashville, TN

James C. Jackson, Psy.D.

Vanderbilt University School of Medicine

Nashville, TN

James L. Rudolph, M.D., S.M.*

Warren Alpert Medical School of Brown University

Brown University School of Public Health

Providence, RI

Marianne Shaughnessy, Ph.D., C.N.R.P.*

Veterans Health Administration

Alexandria, VA

Christine Soong, M.D., M.Sc.

Mount Sinai Hospital

Toronto, Ontario, Canada

*Provided input on Draft Report. 


\section{Peer Reviewers}

Prior to publication of the final evidence report, EPCs sought input from independent Peer Reviewers without financial conflicts of interest. However, the conclusions and synthesis of the scientific literature presented in this report do not necessarily represent the views of individual reviewers.

Peer Reviewers must disclose any financial conflicts of interest greater than $\$ 5,000$ and any other relevant business or professional conflicts of interest. Because of their unique clinical or content expertise, individuals with potential nonfinancial conflicts may be retained. The TOO and the EPC work to balance, manage, or mitigate any potential nonfinancial conflicts of interest identified.

The list of Peer Reviewers follows:

Nicole Brandt, Pharm.D., M.B.A.

University of Maryland School of Pharmacy

Baltimore, MD

Biren Kamdar, M.D., M.B.A., M.H.S.

UC San Diego School of Medicine

Division of Pulmonary, Critical Care and Sleep Medicine

La Jolla, CA 


\section{Antipsychotics for the Prevention and Treatment of Delirium}

\section{Structured Abstract}

Objectives. To assess benefits and harms of antipsychotics for the prevention and treatment of delirium in adult patient populations.

Data sources. We searched PubMed ${ }^{\circledR}$, Embase ${ }^{\circledR}$, the Cochrane Central Register of Controlled Trials (CENTRAL), Cumulative Index to Nursing and Allied Health Literature (CINAHL ${ }^{\circledR}$ ), and PsycINFO $^{\circledR}$ through March 2019. We also hand-searched the reference lists of included articles, relevant reviews, and delirium-specific bibliographic repositories.

Review methods. We included randomized controlled trials (RCTs) of antipsychotics that evaluated benefits or harms, and also observational studies that reported harms. Two reviewers independently screened search results for eligibility, serially abstracted data, and independently assessed the risk of bias of the studies and graded the strength of evidence (SOE) for prespecified critical outcomes: delirium severity, cognitive functioning, length of stay in hospital, inappropriate continuation of antipsychotic drugs, falls, sedation, and caregiver burden/strain.

Results. We identified 14 RCTs and 1 observational study evaluating the use of antipsychotics in prevention of delirium. For the treatment of delirium, we identified 19 RCTs and 25 observational studies. Two RCTs were classified as both a prevention and treatment trial. In trials of the prevention of delirium across all populations, there was no difference in delirium incidence for haloperidol versus placebo (relative risk [RR], 0.94; 95\% confidence interval [CI], 0.77 to 1.16). Second-generation antipsychotics, compared with placebo, may decrease delirium incidence in postoperative patients at risk for delirium (RR, 0.36; 95\% CI, 0.26 to 0.50). Antipsychotics (both haloperidol and second-generation), compared to placebo, demonstrated no differences for length of stay in hospital (low SOE for second-generation antipsychotics and high SOE for haloperidol). We were unable to draw conclusions regarding the effect of antipsychotics on sedation, falls, and delirium severity (insufficient SOE). We found no studies evaluating cognitive functioning, inappropriate continuation of antipsychotic drugs, or caregiver burden/strain. For treatment of delirium, there was little to no difference in effect of haloperidol and second-generation antipsychotics compared with placebo for length of stay in hospital (moderate SOE) and sedation (low and moderate SOE, respectively) with insufficient or no evidence for cognitive functioning or delirium severity. Also, effects of second-generation antipsychotics were not significantly different compared with haloperidol for delirium severity (moderate SOE), cognitive functioning (low SOE), length of stay in hospital (moderate SOE), and sedation (moderate SOE). We found no studies reporting inappropriate continuation of antipsychotic drugs, falls, or caregiver burden/strain. We did not find statistically significant differences for haloperidol or second-generation antipsychotics in neurological harms, including extrapyramidal side effects and neuroleptic malignant syndrome. However, cardiac harms tended to occur more frequently with antipsychotics, specifically prolongation of QT interval with second-generation antipsychotics. 
Conclusions. Haloperidol or second-generation antipsychotics, compared to placebo, used for the prevention or treatment of delirium did not improve length of stay in hospital. We found little or no evidence to determine the effect of antipsychotics on cognitive function, delirium severity, or caregiver burden. Second-generation antipsychotics may decrease delirium incidence in postoperative patients, but this evidence is limited and requires more study. We did not detect neurological harms associated with haloperidol or second-generation antipsychotics used for the prevention or treatment of delirium. However, cardiac effects tended to occur more frequently in those receiving antipsychotics. Future studies should include standardized, clinically meaningful measures of patient distress, subsequent memories of delirium, caregiver burden and distress, inappropriate continuation of antipsychotic therapy, and long-term cognitive and functional outcomes. 


\section{Contents}

Evidence Summary ............................................................................................................................. ES-1

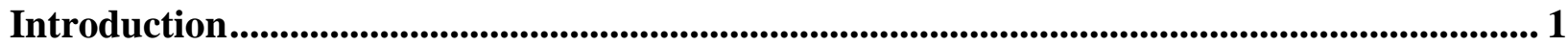

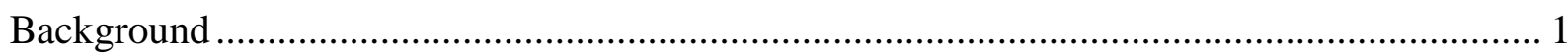

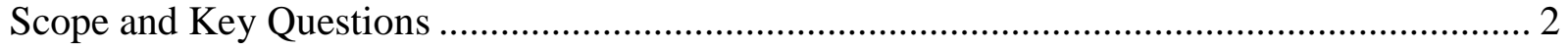

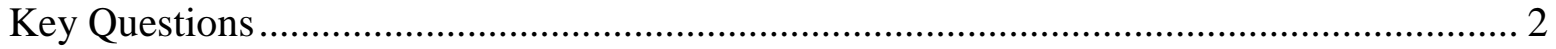

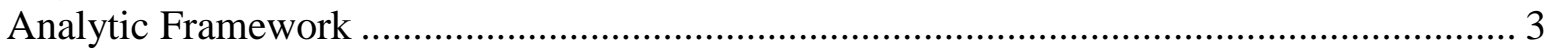

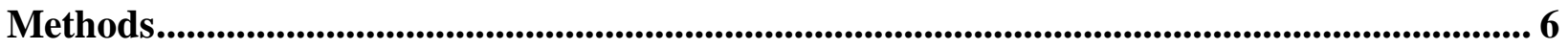

Topic Refinement and Protocol Development............................................................................... 6

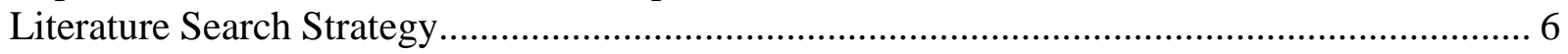

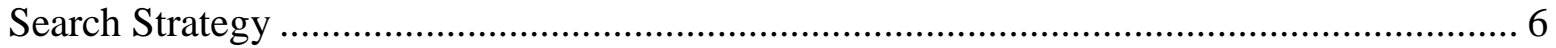

Inclusion and Exclusion Criteria............................................................................................... 7

Process for Study Selection ................................................................................................... 8

Data Extraction and Data Management .............................................................................. 8

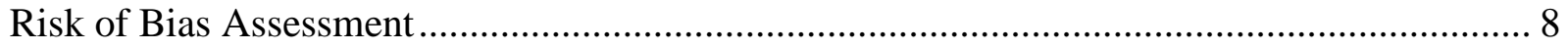

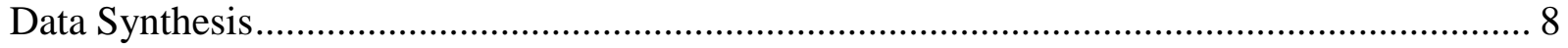

Grading the Body of Evidence for Each Key Question.............................................................. 9

Peer Review and Public Commentary ................................................................................. 10

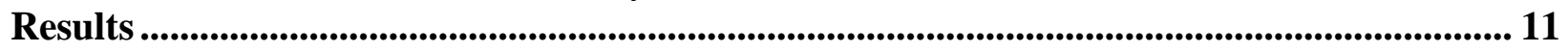

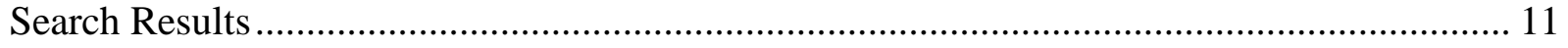

Key Question 1. Benefits and Harms of Antipsychotics To Prevent Delirium ......................... 14

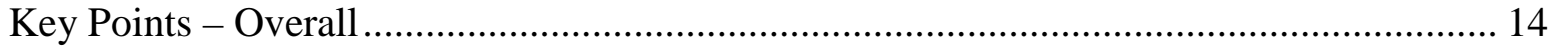

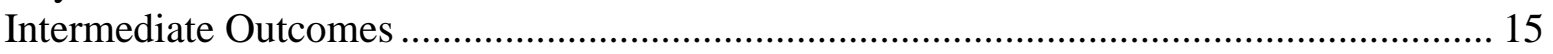

Final Health or Patient-Centered Outcomes .......................................................................... 22

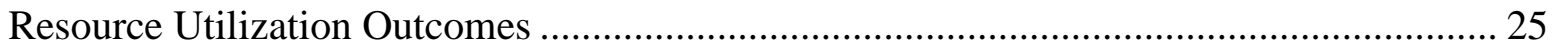

Adverse Effects .............................................................................................................. 29

Key Question 1a. Benefits and Harms of Antipsychotics To Prevent Delirium in Persons Aged

65 Years and Older ........................................................................................................ 36

Key Points - Aged 65 Years and Older .......................................................................... 36

Intermediate Outcomes ................................................................................................... 37

Final Health or Patient-Centered Outcomes ............................................................................ 43

Resource Utilization Outcomes ...................................................................................... 46

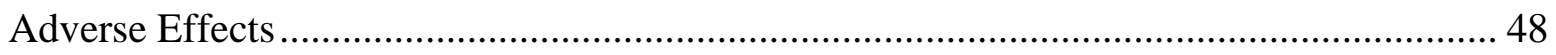

Key Question 1b. Benefits and Harms of Antipsychotics To Prevent Delirium in Persons with

Dementia ..................................................................................................................... 52

Key Question 1c. Benefits and Harms of Antipsychotics To Prevent Delirium in Patients in an

Intensive Care Unit .............................................................................................................. 52

Key Points - Intensive Care Unit................................................................................... 52

Intermediate Outcomes ..................................................................................................... 53

Final Health or Patient-Centered Outcomes …………………………………………......... 59

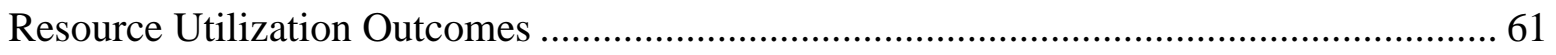

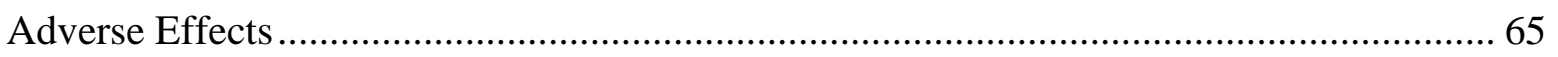

Key Question 1d. Benefits and Harms of Antipsychotics To Prevent Delirium in Patients in a

Post-Acute Care Facility ............................................................................................................ 69

Key Question 1e. Benefits and Harms of Antipsychotics To Prevent Delirium in Patients in

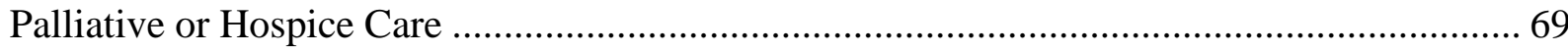


Key Question 1f. Benefits and Harms of Antipsychotics To Prevent Delirium in Patients in

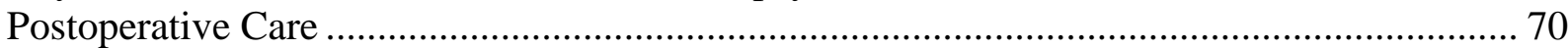

Key Points - Postoperative Care....................................................................................... 70

Intermediate Outcomes ............................................................................................. 70

Final Health or Patient-Centered Outcomes ..................................................................... 75

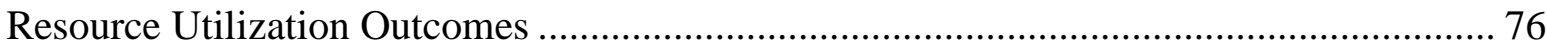

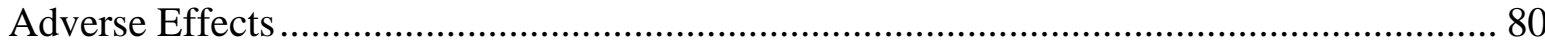

Key Question 2. Benefits and Harms of Antipsychotics To Treat Delirium ............................. 82

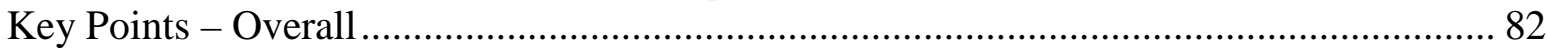

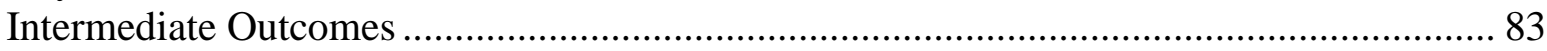

Final Health or Patient-Centered Outcomes ....................................................................... 94

Resource Utilization Outcomes ....................................................................................... 100

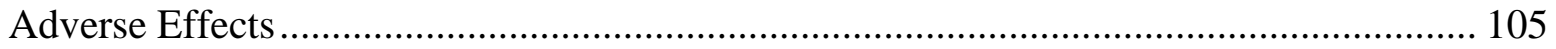

Key Question 2a. Benefits and Harms of Antipsychotics To Treat Delirium in Persons Aged

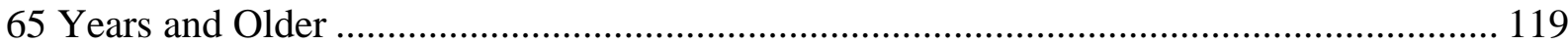

Key Points - Aged 65 Years and Older …………………............................................... 119

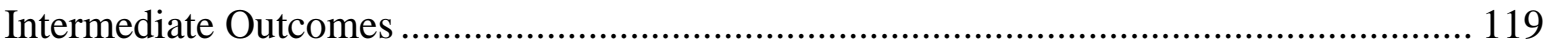

Final Health or Patient-Centered Outcomes - Aged 65 Years and Older ........................... 122

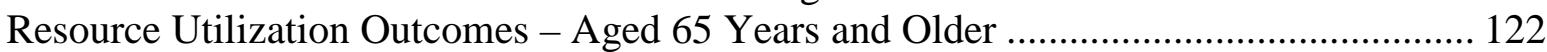

Adverse Effects - Aged 65 Years and Older ..................................................................... 122

Key Question 2b. Benefits and Harms of Antipsychotics To Treat Delirium in Persons With

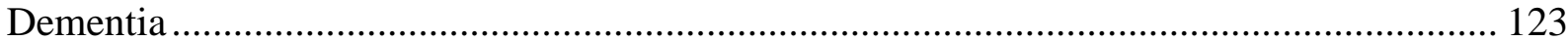

Key Question 2c. Benefits and Harms of Antipsychotics To Treat Delirium in Patients in an

Intensive Care Unit ........................................................................................................ 123

Key Points - Intensive Care Unit..................................................................................... 123

Intermediate Outcomes .................................................................................................. 124

Final Health or Patient-Centered Outcomes ....................................................................... 126

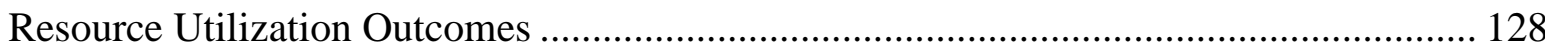

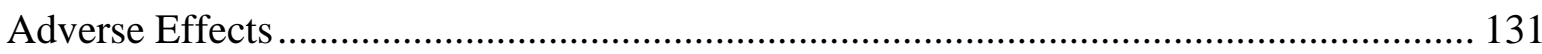

Key Question 2d. Benefits and Harms of Antipsychotics To Treat Delirium in Patients in a Post-Acute Care Facility ................................................................................................... 137

Key Question 2e. Benefits and Harms of Antipsychotics To Treat Delirium in Patients in

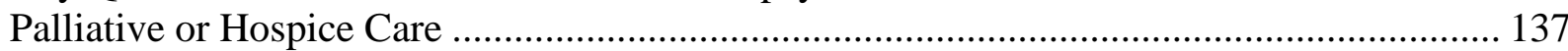

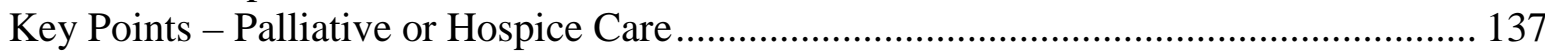

Intermediate Outcomes ................................................................................................... 138

Final Health or Patient-Centered Outcomes .................................................................... 140

Resource Utilization Outcomes - Palliative or Hospice Care ............................................... 141

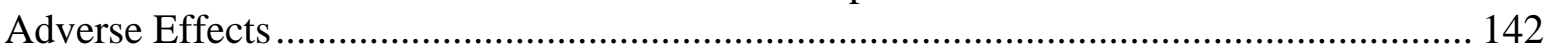

Key Question 2f. Benefits and Harms of Antipsychotics To Treat Delirium in Patients in

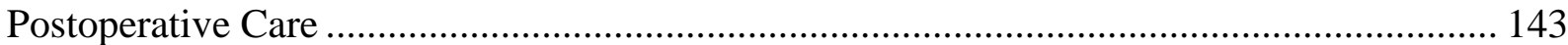

Key Points - Postoperative Care.......................................................................................... 143

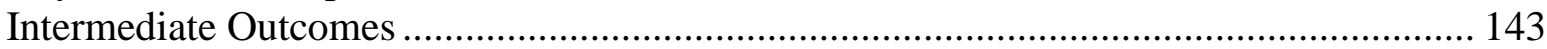

Final Health or Patient-Centered Outcomes .................................................................... 144

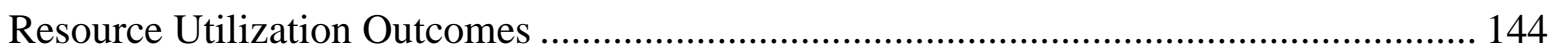

Adverse Effects - Postoperative ……………………....................................................... 147

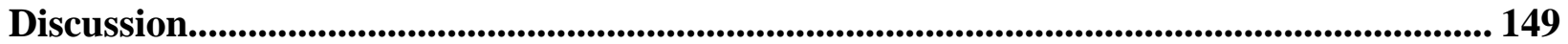


Key Findings and Strength of Evidence ……………..................................................... 149

Findings in Relationship to What Is Already Known....................................................... 150

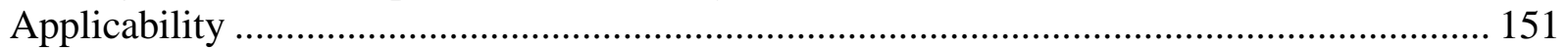

Implications for Clinical and Policy Decisionmaking ............................................................ 151

Limitations of the Systematic Review Process ....................................................................... 152

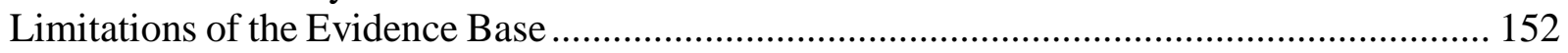

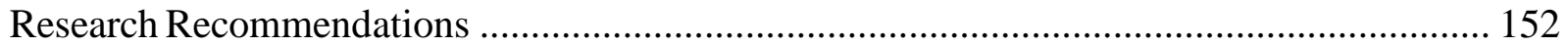

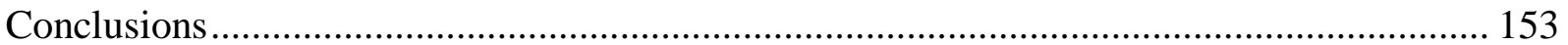

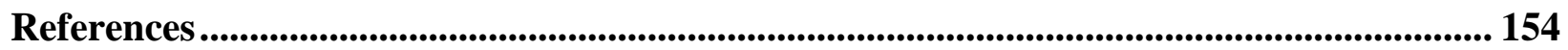

Abbreviations and Acronyms ............................................................................................................... 160

\section{Tables}

Table A. Summary of the strength of evidence and conclusion for the effects of antipsychotics used for the prevention of delirium on critical outcomes among adults at risk for delirium.......... 4 Table B. Summary of the strength of evidence and conclusion for the effects of antipsychotics used for the treatment of delirium on critical outcomes among adults with delirium ....................... 6

Table 1. Antipsychotic agents ....................................................................................... 2

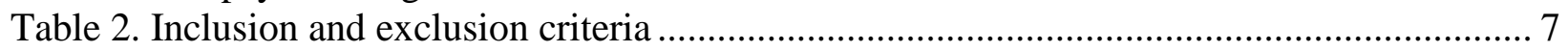

Table 3. Critical outcomes for each subpopulation .................................................................. 10

Table 4. Number of studies evaluating antipsychotics for the prevention of delirium for overall

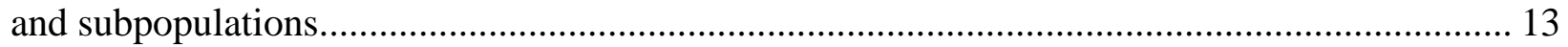

Table 5. Number of studies evaluating antipsychotics for the treatment of delirium for overall

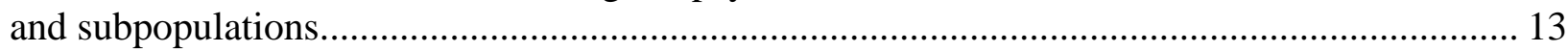

Table 6. Strength of evidence domains for trials evaluating antipsychotics for the prevention of delirium in terms of delirium severity ……………………................................................... 18

Table 7. Strength of evidence domains for trials evaluating antipsychotics for the prevention of

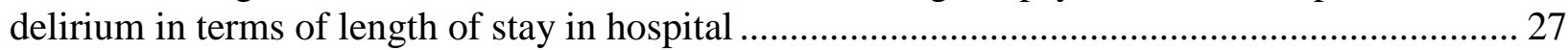

Table 8. Strength of evidence domains for studies reporting sedation in the evaluation of antipsychotics for delirium prevention ...................................................................................... 31

Table 9. Strength of evidence domains for trials evaluating antipsychotics for the prevention of delirium in terms of delirium severity in patients aged 65 years and older .................................. 40 Table 10. Strength of evidence domains for trials reporting falls in the evaluation of antipsychotics for the prevention of delirium in patients 65 years of age and older ..... .45

Table 11. Strength of evidence domains for studies reporting sedation in the evaluation of antipsychotics for delirium prevention ........................................................................................ 49 Table 12. Strength of evidence domains for trials evaluating antipsychotics for the prevention of delirium in terms of delirium severity for patients in an intensive care unit ................................ 56 Table 13. Strength of evidence domains for trials evaluating antipsychotics for the prevention of delirium in terms of length of stay in hospital for patients in an intensive care unit..................... 63 Table 14. Strength of evidence domains for studies reporting sedation in the evaluation of antipsychotics for delirium prevention in patients in an intensive care unit................................... 66 Table 15. Strength of evidence domains for trials evaluating antipsychotics for the prevention of delirium in terms of delirium severity in postoperative patients ................................................... 73 Table 16. Strength of evidence domains for trials evaluating antipsychotics for the prevention of delirium in terms of length of stay in hospital in postoperative patients. 78 
Table 17. Strength of evidence domains for trials evaluating antipsychotics for the treatment of delirium in terms of delirium severity ........................................................................ 89

Table 18. Strength of evidence domains for trials evaluating antipsychotics for the treatment of delirium in terms of cognitive functioning

Table 19. Strength of evidence domains for trials evaluating antipsychotics for the treatment of delirium in terms of length of stay in hospital ................................................................... 103

Table 20. Strength of evidence domains for trials evaluating antipsychotics for the treatment of delirium in terms of sedation.... 108

Table 21. Strength of evidence domains for trials evaluating antipsychotics for the treatment of delirium in terms of delirium severity in patients aged 65 years and older. 121 Table 22. Strength of evidence domains for trials evaluating antipsychotics for the treatment of delirium in terms of length of stay in hospital for patients in an intensive care unit................. 130 Table 23. Strength of evidence domains for studies evaluating antipsychotics for the treatment of delirium in terms of sedation in patients in the intensive care unit .... 133 Table 24. Strength of evidence domains for trials evaluating antipsychotics for the treatment of delirium in terms of delirium severity in patients in palliative or hospice care. 139 Table 25. Strength of evidence domains for trials evaluating antipsychotics for the treatment of delirium in terms of length of stay in hospital for postoperative patients

\section{Figures}

Figure 1. Analytic framework for antipsychotics for delirium prevention .................................. 4

Figure 2. Analytic framework for antipsychotics for delirium treatment................................... 5

Figure 3. Summary of the literature search.................................................................... 12

Figure 4. Meta-analysis of delirium incidence in trials comparing either haloperidol or secondgeneration antipsychotics with placebo among patients at risk for delirium............................. 16 Figure 5. Meta-analysis of use of rescue therapy in trials comparing haloperidol with placebo in patients at risk for delirium....

Figure 6. Meta-analysis of mortality in trials comparing haloperidol with placebo in patients at risk for delirium ........................................................................................................... 23 Figure 7. Meta-analysis of sedation in trials comparing haloperidol with placebo in patients at

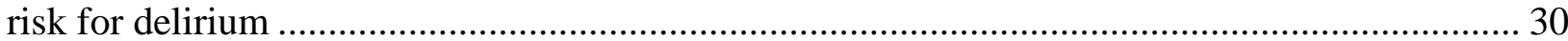
Figure 8. Meta-analysis of cardiac effects in trials comparing haloperidol with placebo in patients

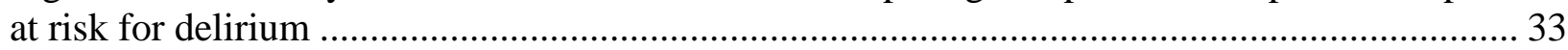

Figure 9. Meta-analysis of extrapyramidal side effects in trials comparing haloperidol with

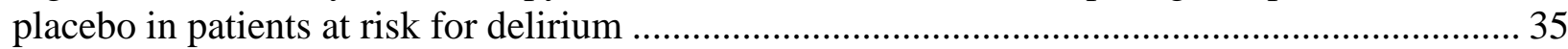
Figure 10. Meta-analysis of delirium incidence in trials comparing haloperidol with placebo in patients aged 65 years and older at risk for delirium...

Figure 11. Meta-analysis of delirium incidence in trials comparing haloperidol with placebo in critically ill patients at risk for delirium ....................................................................... 54

Figure 12. Meta-analysis of the use of rescue therapy in trials comparing haloperidol with placebo in critically ill patients at risk for delirium ......................................................... 58 Figure 13. Meta-analysis of mortality in trials comparing haloperidol with placebo in critically ill

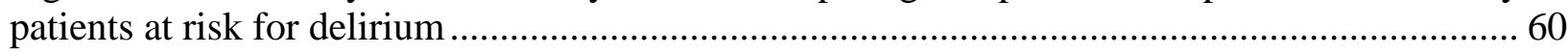
Figure 14. Meta-analysis of neurologic effects in trials comparing haloperidol with placebo in critically ill patients at risk for delirium 
Figure 15. Meta-analysis of delirium incidence in trials comparing haloperidol with placebo in postoperative patients at risk for delirium

Figure 16. Meta-analysis of delirium severity scores as measured by the DRS-R-98* in trials comparing haloperidol with second-generation antipsychotics in patients with delirium 87 Figure 17. Meta-analysis of patients with delirium severity scores below identified threshold in trials comparing haloperidol with second-generation antipsychotics in patients with delirium... 88 Figure 18. Meta-analysis of duration of delirium in trials comparing haloperidol with secondgeneration antipsychotics in patients with delirium. 92

Figure 19. Meta-analysis of the use of rescue therapy in trials comparing haloperidol with placebo in patients with delirium.... 93

Figure 20. Meta-analysis of mortality in trials comparing haloperidol with placebo in patients with delirium.... 95 Figure 21. Meta-analysis of mortality in trials comparing second-generation antipsychotics with placebo in patients with delirium.... 96

Figure 22. Meta-analysis of mortality in trials comparing haloperidol with second-generation antipsychotics in patients with delirium 97 Figure 23. Meta-analysis of sedation in trials comparing second-generation antipsychotics with placebo in patients with delirium 106

Figure 24. Meta-analysis of sedation outcomes in trials comparing second-generation antipsychotics with haloperidol 106

Figure 25. Meta-analysis of cardiac outcomes in trials comparing haloperidol or secondgeneration antipsychotics with placebo in patients with delirium...

Figure 26. Meta-analysis of neurological outcomes in trials comparing haloperidol with placebo in patients with delirium

Figure 27. Meta-analysis of neurologic outcomes in trials comparing second-generation antipsychotics with placebo...

Figure 28. Meta-analysis of neurologic outcomes in trials comparing haloperidol with secondgeneration antipsychotics in patients with delirium.

Figure 29. Meta-analysis of mortality in trials with an intensive care unit population comparing haloperidol or second-generation antipsychotics with placebo 127 Figure 30. Meta-analysis of neurological extrapyramidal symptoms outcomes in trials with an intensive care unit population comparing haloperidol with placebo 136

\section{Appendixes}

Appendix A. Detailed Search Strategy

Appendix B. Screening and Data Abstraction Forms

Appendix C. Included and Excluded Articles

Appendix D. Evidence Tables 


\section{Evidence Summary}

\section{Introduction}

\section{Background}

Delirium is a syndrome characterized by an abrupt impairment in cognition, with a specific deficit in attention, that is associated with an underlying medical cause or causes. ${ }^{1}$ Delirium is a common and important condition in all healthcare settings, but is particularly prevalent in older adults and patients with critical illness. Delirium is strongly associated with increased mortality and longer hospital stay, with an estimated cost of \$38 to \$152 billion annually for patients aged 70 years or older. ${ }^{2}$ Additionally, delirium experienced during a hospitalization is strongly associated with new or worsening long-term cognitive impairment. ${ }^{3}$

Preventive and therapeutic interventions are needed to reduce the burden of delirium and associated long-term cognitive impairments. Currently, there are no medications approved by the U.S. Food and Drug Administration for the prevention and treatment of delirium. Antipsychotics, medications approved for use in psychotic conditions such as schizophrenia, are frequently used in patients with delirium or at risk of delirium despite the lack of clear evidence to support their use. ${ }^{4}$ Previous reviews of antipsychotics for delirium were inconclusive about benefit or harm owing to few studies, particularly in older adults and other susceptible patient populations, and heterogeneity of interventions. ${ }^{5,6}$ Chronic use of antipsychotics in management of conditions other than delirium has been shown to increase the risk of stroke and sudden death in older adults, and particularly those with dementia. ${ }^{7-9}$ Recently, increasing numbers of randomized controlled trials (RCTs) of antipsychotic medications have been conducted for the treatment or prevention of delirium. We conducted a systematic review of the benefits and harms of antipsychotics for the prevention and treatment of delirium.

\section{Key Questions}

We sought to address two Key Questions:

1. What are the benefits and harms of antipsychotics compared with each other, placebo, or non-drug approaches to prevent delirium?

2. What are the benefits and harms of antipsychotics compared with each other, placebo, or non-drug approaches to treat delirium?

For each Key Question, we first considered evidence about the overall population and then considered the following populations or settings:

- Persons aged 65 years or older

- Persons with dementia

- Patients in an intensive care unit

- Patients in a post-acute care facility

- Patients in palliative or hospice care

- Patients in postoperative care 


\section{Methods}

We followed the methods outlined in the Agency for Healthcare Research and Quality's (AHRQ's) Methods Guide for Effectiveness and Comparative Effectiveness Reviews. ${ }^{10}$ Our protocol is posted on the AHRQ Effective Health Care Program's website (www.effectivehealthcare.ahrq.gov) and registered on PROSPERO (CRD42018109552). The searches were conducted in March 2019. Details of the methodology can be found in the full report.

\section{Results}

After screening abstracts and full-text, we included 57 studies (published in 62 articles). Of the eligible studies, 15 studies, including 14 RCTs and 1 observational study, addressed the prevention of delirium. Forty-four studies, including 19 RCTs and 25 observational studies or non-randomized trials, addressed the treatment of delirium. Two trials enrolled patients with and without delirium; we classified these as both a prevention and treatment trial for the purposes of this report. ${ }^{11,12}$ The trials for prevention of delirium evaluated delirium incidence, length of hospital stay, sedation and severity, with most of the studies focused on postoperative or intensive care unit-based populations. The treatment trials primarily evaluated hospital length of stay and sedation effects, with most of the studies focused on inpatients, particularly those with critical illness. Evidence tables with details about the included studies are in the appendixes of the full report.

\section{Antipsychotics for the Prevention of Delirium}

Table A summarizes the evidence for the use of antipsychotics for the prevention of delirium. The critical outcomes for the prevention of delirium in adults at risk for delirium included: cognitive functioning, delirium severity, length of stay in the hospital, inappropriate continuation of antipsychotic medication once initiated, and sedation. Critical outcomes varied by patient group and are listed in Table A.

In the prevention of delirium across all populations, haloperidol made little to no difference on delirium incidence compared with placebo (relative risk [RR], 0.94; 95\% confidence interval [CI], 0.77 to 1.16) or length of hospital stay (high strength of evidence); there was insufficient evidence to determine the effect of haloperidol versus placebo on delirium severity, sedation, or cognitive outcomes. Second-generation antipsychotics decreased the incidence of delirium compared with placebo in patients at risk of delirium (RR, 0.36; 95\% CI, 0.26 to 0.50); the studies included in this meta-analysis included postoperative patients only. However there was no effect on the length of hospital stay (low strength of evidence) and insufficient evidence to determine the impact on the severity of delirium for second-generation antipsychotics versus placebo. We were unable to draw conclusions for any type of drug-drug comparisons between second-generation antipsychotics or comparisons to any other types of therapies (i.e., other than antipsychotics) due to the absence or insufficiency of evidence.

We also examined different populations at risk of delirium. There was insufficient evidence to determine the impact of haloperidol or second-generation antipsychotics on delirium severity, sedation, or falls in patients 65 years of age and older. Haloperidol used in patients at risk for delirium in the intensive care unit had little to no effect on length of stay in hospital (high strength of evidence). We found insufficient or no evidence for haloperidol or second-generation antipsychotics on cognitive functioning, delirium severity, inappropriate continuation, or 
sedation for patients at risk of delirium in the intensive care unit. Haloperidol or secondgeneration antipsychotics for the prevention of delirium in postoperative patients had little to no effect on the hospital length of stay for second-generation agents (low strength of evidence) and insufficient or no evidence for the other critical outcomes. We found no evidence for antipsychotics in patients at risk for delirium with dementia, those in a post-acute care facility, and among patients in palliative or hospice care.

\section{Antipsychotics for the Treatment of Delirium}

Table B summarizes the effects of antipsychotics used for the treatment of delirium. The critical outcomes for the treatment of delirium in adults with delirium included: cognitive functioning, delirium severity, length of stay in the hospital, inappropriate continuation of antipsychotic medication once initiated, and sedation. Critical outcomes by patient group are listed in Table B.

Haloperidol or second-generation antipsychotics for the treatment of delirium had little or similar effect on hospital length of stay (moderate strength of evidence) or sedation (low strength of evidence for haloperidol and moderate strength of evidence for second-generation antipsychotics; RR, 1.10; 95\% CI, 0.78 to 1.53) compared with placebo. In comparing haloperidol with second-generation antipsychotics, there were no clinically meaningful differences for cognitive functioning (low strength of evidence), and delirium severity (e.g., mean between-group difference in Delirium Rating Scale-R-98 scores, -0.03; 95\% CI, -2.04 to 1.98), length of stay in hospital, and sedation (all moderate strength of evidence; RR, 1.26; 95\% CI, 0.92 to 1.72). We were unable to draw conclusions for any type of drug-drug comparisons between second-generation antipsychotics or comparisons with any other types of therapies (i.e., other than antipsychotics) due to the absence of studies or insufficient evidence.

Antipsychotics compared with placebo or head-to-head trials in patients in intensive care unit settings showed no or similar effect on length of stay in hospital (moderate strength of evidence) or sedation (moderate strength of evidence). Patients being treated with haloperidol or secondgeneration antipsychotics compared with those who received placebo in palliative care or hospice settings may have slightly less improvement in delirium severity over time (low strength of evidence). We found no or insufficient evidence for the effects of antipsychotics to treat delirium among patients aged 65 years or older, those with dementia, among patients in a postacute care facility, and among patients in postoperative care. 
Table A. Summary of the strength of evidence and conclusion for the effects of antipsychotics used for the prevention of delirium on critical outcomes among adults at risk for delirium

\begin{tabular}{|c|c|c|c|c|c|c|c|}
\hline $\begin{array}{l}\text { Critical } \\
\text { Outcome }\end{array}$ & $\begin{array}{l}\text { Populations } \\
\text { Assessed* }\end{array}$ & $\begin{array}{c}\text { Haloperidol } \\
\text { Versus Placebo }\end{array}$ & $\begin{array}{c}\text { Second- } \\
\text { Generation } \\
\text { Antipsychotics } \\
\text { Versus Placebo }\end{array}$ & $\begin{array}{l}\text { Haloperidol } \\
\text { Versus Second- } \\
\text { Generation } \\
\text { Antipsychotics }\end{array}$ & $\begin{array}{c}\text { Second- } \\
\text { Generation } \\
\text { Antipsychotics } \\
\text { Versus Second- } \\
\text { Generation } \\
\text { Antipsychotics }\end{array}$ & $\begin{array}{l}\text { Haloperidol } \\
\text { Versus Other } \\
\text { Therapies }\end{array}$ & $\begin{array}{c}\text { Second- } \\
\text { Generation } \\
\text { Antipsychotics } \\
\text { Versus Other } \\
\text { Therapies }\end{array}$ \\
\hline \multirow{4}{*}{$\begin{array}{l}\text { Cognitive } \\
\text { functioning }\end{array}$} & Overall & & & & & & \\
\hline & Aged $\geq 65$ yrs & & & & & & \\
\hline & Critically ill & & & & & & \\
\hline & Postoperative & & & & & & \\
\hline \multirow{4}{*}{$\begin{array}{l}\text { Delirium } \\
\text { severity }\end{array}$} & Overall & Insufficient & Insufficient & & & & \\
\hline & Aged $\geq 65$ yrs & Insufficient & Insufficient & & & & \\
\hline & Critically ill & Insufficient & & & & & \\
\hline & Postoperative & Insufficient & Insufficient & & & & \\
\hline Falls & Aged $\geq 65$ yrs & Insufficient & & & & & \\
\hline \multirow{3}{*}{$\begin{array}{l}\text { Length of stay } \\
\text { in hospital }\end{array}$} & Overall & High; = & Low; = & Insufficient & & Insufficient & \\
\hline & Critically ill & High; = & Insufficient & Insufficient & & Insufficient & \\
\hline & Postoperative & Insufficient & Low; = & & & & \\
\hline \multirow{4}{*}{$\begin{array}{l}\text { Inappropriate } \\
\text { continuation of } \\
\text { antipsychotic } \\
\text { medication }\end{array}$} & Overall & & & & & & \\
\hline & Aged $\geq 65$ yrs & & & & & & \\
\hline & Critically ill & & & & & & \\
\hline & Postoperative & & & & & & \\
\hline \multirow{3}{*}{ Sedation } & Overall & Insufficient & & & & & \\
\hline & Aged $\geq 65$ yrs & Insufficient & & & & & \\
\hline & Critically ill & Insufficient & & & & & \\
\hline
\end{tabular}

Blank cells indicate no evidence.

Conclusion: = we concluded that there was little to no difference between interventions; + favors the intervention over control; - favors the control over the intervention

Strength of evidence: Insufficient = the body of evidence has unacceptable deficiencies, precluding a conclusion; Low = low confidence that the evidence reflects the true effect and further research is likely to change our confidence in the estimate of the effect and is likely to change the estimate; Moderate = indicating moderate confidence that the evidence reflects the true effect but further research could change our confidence in the estimate of the effect and may change the estimate; High = high confidence that the evidence reflects the true effect and further research is very unlikely to change our confidence in the estimate of the effect 
* We did not find any evidence for the critical outcomes among the following populations at risk for delirium: persons with dementia, patients in a postacute care facility, and patients in palliative or hospice care. 
Table B. Summary of the strength of evidence and conclusion for the effects of antipsychotics used for the treatment of delirium on critical outcomes among adults with delirium

\begin{tabular}{|c|c|c|c|c|c|c|c|}
\hline $\begin{array}{l}\text { Critical } \\
\text { Outcome }\end{array}$ & $\begin{array}{l}\text { Populations } \\
\text { Assessed* }\end{array}$ & $\begin{array}{c}\text { Haloperidol } \\
\text { Versus Placebo }\end{array}$ & $\begin{array}{c}\text { Second- } \\
\text { Generation } \\
\text { Antipsychotics } \\
\text { Versus Placebo }\end{array}$ & $\begin{array}{c}\text { Haloperidol } \\
\text { Versus Second- } \\
\text { Generation } \\
\text { Antipsychotics }\end{array}$ & $\begin{array}{c}\text { Second- } \\
\text { Generation } \\
\text { Antipsychotics } \\
\text { Versus Second- } \\
\text { Generation } \\
\text { Antipsychotics }\end{array}$ & $\begin{array}{l}\text { Haloperidol } \\
\text { Versus Other } \\
\text { Therapies }\end{array}$ & $\begin{array}{c}\text { Second- } \\
\text { Generation } \\
\text { Antipsychotics } \\
\text { Versus Other } \\
\text { Therapies }\end{array}$ \\
\hline \multirow{2}{*}{$\begin{array}{l}\text { Cognitive } \\
\text { functioning }\end{array}$} & Overall & & Insufficient & Low; = & Insufficient & Insufficient & \\
\hline & Critically ill & & & & & & \\
\hline \multirow{3}{*}{$\begin{array}{l}\text { Delirium } \\
\text { severity }\end{array}$} & Overall & Insufficient & Insufficient & Moderate; = & Insufficient & Insufficient & \\
\hline & Critically ill & & & & & & \\
\hline & Palliative & Low; - & Low; - & Insufficient & & & \\
\hline $\begin{array}{l}\text { Caregiver } \\
\text { burden/strain }\end{array}$ & Palliative & & & & & & \\
\hline \multirow{2}{*}{$\begin{array}{l}\text { Length of stay } \\
\text { in hospital }\end{array}$} & Overall & Moderate; = & Moderate; = & Moderate; = & & Insufficient & \\
\hline & Critically ill & Moderate; = & Moderate; = & Moderate; = & & & \\
\hline \multirow{2}{*}{$\begin{array}{l}\text { Inappropriate } \\
\text { continuation of } \\
\text { antipsychotic } \\
\text { medication } \\
\end{array}$} & Overall & & & & & & \\
\hline & Critically ill & & & & & & \\
\hline \multirow{3}{*}{ Sedation } & Overall & Low; = & Moderate; = & Moderate; = & Insufficient & & \\
\hline & Critically ill & Moderate; = & Moderate; = & Moderate; = & & & \\
\hline & Palliative & & & & & & \\
\hline
\end{tabular}

Blank cells indicate no evidence.

Conclusion: = we concluded that there was little to no difference between interventions; + favors the intervention over control; - favors the control over the intervention

Strength of evidence: Insufficient = the body of evidence has unacceptable deficiencies, precluding a conclusion; Low = low confidence that the evidence reflects the true effect and further research is likely to change our confidence in the estimate of the effect and is likely to change the estimate; Moderate = indicating moderate confidence that the evidence reflects the true effect but further research could change our confidence in the estimate of the effect and may change the estimate; High = high confidence that the evidence reflects the true effect and further research is very unlikely to change our confidence in the estimate of the effect

* We did not find any evidence for the critical outcomes among the following populations with delirium: persons aged 65 years and older, persons with dementia, patients in a post-acute care facility, patients in palliative or hospice care, and patients in post-operative care. 


\section{Adverse Effects}

We evaluated cardiac and neurological harms. Most or all of the studies assessing cardiac effects included critically ill patients who may be at a higher risk of cardiac events compared to other patient populations. In all RCTs and observational studies evaluating haloperidol versus placebo, second-generation antipsychotics versus placebo, haloperidol versus second-generation antipsychotics, and second-generation antipsychotics versus second-generation antipsychotics, there were no statistically significant differences in the occurrence of any of types of cardiac effects reported. However, potentially important cardiac effects cannot be excluded since these tended to occur more frequently with antipsychotics.

Studies that reported neurological harms included critically ill patients who may be at a higher risk of neurological events compared to other patient populations. Extrapyramidal symptoms were the most commonly monitored neurological adverse effect. Apart from a single RCT in patients receiving palliative care which reported a statistically significant increase in extrapyramidal symptoms for both haloperidol compared with placebo and for second-generation antipsychotics compared with placebo, the larger body of evidence in all other patient populations, found no statistically significant increase in any neurological effect for any first- or second-generation antipsychotic compared with placebo or in other head-to-head trials.

\section{Discussion}

Second-generation antipsychotics may lower the incidence of delirium in postoperative patients at risk of delirium, but the evidence is limited and requires further study. For those being treated for delirium in palliative care or hospice settings, haloperidol or second-general antipsychotics may have slightly less improvement in delirium severity than those treated with placebo. However, for all other antipsychotics and outcomes, we found little to no effect or there was not enough evidence to determine the effect.

The greatest challenge to the applicability of this body of evidence is related to the populations and outcomes studied. Trials were often conducted in medical and surgical critically ill patients. The overall results of this report may not be directly applicable to other populations, including postoperative patients, older inpatients, and patients with dementia. Critically ill patients may have differing pathophysiological etiologies of delirium compared with other populations, as well as more severe physiological and metabolic derangements. Any benefit and risk of antipsychotics for prevention or treatment of delirium within the context of critical illness may not be generalizable to other populations, such as those with dementia, in postacute care, or in palliative care.

For the vast majority of outcomes predetermined to be of critical importance by our panel of experts and key informants, studies did not exist or were inadequate in design or number to answer the key questions. There was insufficient or no evidence for many comparisons and outcomes due to the paucity of studies. For instance, there was insufficient evidence comparing haloperidol with second-generation antipsychotics and for drug-drug comparisons within the class of second-generation antipsychotics. Poor reporting meant that the risk of bias was frequently unclear, especially regarding sequence generation and allocation concealment for the RCTs.

Moreover, there was also frequent unclear risk of bias related to missing outcome data and selective outcome reporting. There was inconsistency in measurement instruments used and approaches to statistical analysis and reporting, even in evaluating the same outcome domain, 
such as delirium severity. Many studies were underpowered, with insufficient duration to adequately assess longer-term clinical outcomes, particularly related to cognitive impairment - a well-known sequela of delirium. Finally, study designs were fairly heterogeneous, using different dosing routes, and a range of doses and frequencies of different antipsychotic agents. Combining heterogeneous treatment and prevention approaches may bias the findings toward the null hypothesis. So too, combining a range of dosing exposures may obscure adverse outcomes, associated with higher doses of medications.

Future studies evaluating pharmacologic prevention and treatment strategies should examine patient groups that are anticipated to have similarity with respect to their delirium risk factor(s) and associated pathophysiology given that these factors may affect response to therapy. Research focused on more homogeneous patient groups is needed. This applies to postoperative patient populations where larger well-controlled trials of second-generation antipsychotics in the prevention of delirium are needed to clarify whether there is any beneficial role for the perioperative setting.

Heterogeneity of outcome domains and measurement instruments emphasize the need for greater standardization. Such standardization would assist with comparison, synthesis and metaanalysis of studies and reduce omission of critical outcomes in future trials in an effort to reduce research waste. In particular, careful identification of the outcomes of greatest importance to clinicians, patients/caregivers and researchers will advance future research. The field would benefit from the development of standardized, clinically meaningful measures of the following outcomes: patient agitation and distress, subsequent memories of delirium, caregiver burden and distress, inappropriate continuation of antipsychotic therapy, and long-term cognitive and functional outcomes.

A striking finding resulting from this review was the lack of investigation of many important patient and care-giver centered outcomes in the study of delirium prevention and treatment. Much more research is needed to study questions such as the comparison between the pharmacologic and non-pharmacologic approaches, quality of life outcomes and best treatment approaches in populations of patients such as those with pre-existing dementia. Studies answering these questions will have important implications for policy and treatment recommendations for patients within our healthcare system.

\section{Conclusions}

Haloperidol or second-generation antipsychotics, compared with placebo, used for the prevention or treatment of delirium did not improve clinically important outcomes. In postoperative patients, second-generation antipsychotics may decrease delirium incidence. We did not detect neurological harms associated with haloperidol or second-generation antipsychotics used for the prevention or treatment of delirium, but cardiac effects tended to occur more frequently in antipsychotics compared with placebo. Future studies should include standardized, clinically meaningful measures of patient agitation and distress, subsequent memories of delirium, caregiver burden and distress, inappropriate continuation of antipsychotic therapy, and long-term cognitive and functional outcomes. 


\section{References}

1. American Psychiatric Association. DSM-5 Task Force. Diagnostic and Statistical Manual of Mental Disorders. DSM-5. 5th ed. Arlington, VA: American Psychiatric Association; 2013.

2. Leslie DL, Marcantonio ER, Zhang Y, et al. Oneyear health care costs associated with delirium in the elderly population. Arch Intern Med. 2008 Jan 14;168(1):27-32. doi:

10.1001/archinternmed.2007.4. PMID: 18195192.

3. Sakusic A, O'Horo JC, Dziadzko M, et al. Potentially Modifiable Risk Factors for LongTerm Cognitive Impairment After Critical Illness: A Systematic Review. Mayo Clin Proc. 2018 Jan;93(1):68-82. doi: 10.1016/j.mayocp.2017.11.005. PMID: 29304923.

4. Devlin JW, Bhat S, Roberts RJ, et al. Current perceptions and practices surrounding the recognition and treatment of delirium in the intensive care unit: a survey of 250 critical care pharmacists from eight states. Ann Pharmacother. 2011 Oct;45(10):1217-29. doi: 10.1345/aph.1Q332. PMID: 21934036.

5. Neufeld KJ, Yue J, Robinson TN, et al. Antipsychotic Medication for Prevention and Treatment of Delirium in Hospitalized Adults: A Systematic Review and Meta-Analysis. J Am Geriatr Soc. 2016 Apr;64(4):705-14. doi: 10.1111/jgs.14076. PMID: 27004732.

6. Kishi T, Hirota T, Matsunaga S, et al. Antipsychotic medications for the treatment of delirium: a systematic review and meta-analysis of randomised controlled trials. J Neurol Neurosurg Psychiatry. 2016 Jul;87(7):767-74. doi: 10.1136/jnnp-2015-311049. PMID: 26341326.

7. Yang C, Hao Z, Tian J, et al. Does antipsychotic drug use increase the risk of long term mortality? A systematic review and meta-analysis of observational studies. Oncotarget. 2018 Mar 13;9(19):15101-10. doi: 10.18632/oncotarget.24120. PMID: 29599930.

8. Chen WY, Chen LY, Liu HC, et al. Antipsychotic medications and stroke in schizophrenia: A casecrossover study. PLoS One.

2017;12(6):e0179424. doi:

10.1371/journal.pone.0179424. PMID: 28614417.
9. Wu CS, Wu KY, Lo YR, et al. Psychotropic use and risk of stroke among patients with bipolar disorders: 10-year nationwide population based study. J Affect Disord. 2018 Jan 15;226:77-84. doi: 10.1016/j.jad.2017.09.020. PMID: 28964996.

10. Methods Guide for Effectiveness and Comparative Effectiveness Reviews. AHRQ Publication No. 10(14)-EHC063-EF. Agency for Healthcare Research and Quality. Rockville, MD. January 2014. 2014.

www.effectivehealthcare.ahrq.gov.

11. Girard TD, Pandharipande PP, Carson SS, et al. Feasibility, efficacy, and safety of antipsychotics for intensive care unit delirium: the MIND randomized, placebo-controlled trial. Crit Care Med. 2010 Feb;38(2):428-37. PMID: 20095068.

12. Page VJ, Ely EW, Gates S, et al. Effect of intravenous haloperidol on the duration of delirium and coma in critically ill patients (HopeICU): a randomised, double-blind, placebocontrolled trial. Lancet Respir Med. 2013 Sep;1(7):515-23. doi: 10.1016/s22132600(13)70166-8. PMID: 24461612. 


\section{Introduction}

\section{Background}

Delirium is a syndrome characterized by an abrupt impairment in cognition, with a specific deficit in attention, that is associated with an underlying medical cause or causes. ${ }^{1}$ Delirium is a common condition in all healthcare settings, but it is particularly prevalent in older adults and in patients with critical illness. Advanced age is an independent risk factor for delirium, ${ }^{2}$ with a prevalence of over 70 percent in critically ill patients aged 60 years and older. ${ }^{3,4}$ Delirium is strongly associated with increased mortality and longer hospital stays, with an estimated cost of $\$ 38$ to $\$ 152$ billion U.S. dollars annually for patients aged 70 years and older. ${ }^{5}$

Delirium experienced during a hospitalization is associated with new or worsening long-term cognitive impairment. A large, multi-site prospective study of critically ill patients in the United States found that, among 12-month survivors, about 25 percent had cognitive test scores similar to patients with mild Alzheimer's disease. ${ }^{6}$ A 2018 systematic review reported that delirium duration is the modifiable factor most strongly associated with long-term cognitive impairment after critical illness. ${ }^{7}$ These findings are consistent with community-based epidemiological studies of older adults, demonstrating that delirium is significantly associated with incident dementia (odds ratio [OR], 8.7) in those without pre-existing cognitive impairment, ${ }^{8}$ with accelerated cognitive decline ${ }^{9}$ in those with pre-existing dementia, and with worsening dementia severity (OR, 3.1) and global function (OR, 2.8).

Preventive and therapeutic interventions are needed to reduce the burden of delirium and associated long-term cognitive impairments. Currently, there are no medications approved by the U.S. Food and Drug Administration for the prevention and treatment of delirium. Antipsychotics, medications approved for use in patients with psychotic disorders such as schizophrenia, are frequently used in patients with delirium or those at risk for delirium despite the lack of clear evidence to support their use. ${ }^{10}$ First-generation antipsychotic medications, including haloperidol, were developed as early as the 1950s and are associated with more neurologic (also referred to as extrapyramidal) symptoms. Second-generation antipsychotics developed in the 1980s are thought to have fewer neurologic symptoms because of novel mechanisms of actions in the brain. ${ }^{11}$ Table 1 lists the antipsychotic agents by drug class. Previous reviews of antipsychotics for delirium were inconclusive about benefit or harm owing to few studies, particularly in older adults and other susceptible patient populations, and heterogeneity of interventions. ${ }^{12,13}$ Chronic use of antipsychotics in management of conditions other than delirium is associated with increased risk of stroke and sudden death in older adults - particularly those with dementia. ${ }^{14-16}$ Recently completed randomized controlled trials of antipsychotic medications for the treatment and prevention of delirium are not included in these previous reviews. 
Table 1. Antipsychotic agents

\begin{tabular}{|c|c|}
\hline Class & Antipsychotic \\
\hline First-generation antipsychotic & $\begin{array}{ll}\text { - } & \text { Chlorpromazine } \\
\text { - } & \text { Droperidol } \\
\text { - } & \text { Fluphenazine } \\
\text { - } & \text { Haloperidol } \\
\text { - } & \text { Molindone } \\
\text { - } & \text { Perphenazine } \\
\text { - } & \text { Pimozide } \\
\text { - } & \text { Thiothixene } \\
\text { - } & \text { Thioridazine } \\
\end{array}$ \\
\hline Second-generation antipsychotic & $\begin{array}{ll}\text { - } & \text { Aripiprazole } \\
\text { - } & \text { Asenapine } \\
\text { - } & \text { Brexpiprazole } \\
\text { - } & \text { Cariprazine } \\
\text { - } & \text { lloperidone } \\
\text { - } & \text { Lurasidone } \\
\text { - } & \text { Olanzapine } \\
\text { - } & \text { Paliperidone } \\
\text { - } & \text { Risperiapine } \\
\text { - } & \text { Ziprasidone } \\
\end{array}$ \\
\hline
\end{tabular}

We conducted a systematic review of the benefits and harms of antipsychotics for the prevention and treatment of delirium.

\section{Scope and Key Questions}

\section{Key Questions}

Key Question 1. What are the benefits and harms of antipsychotics compared with each other, placebo, or non-drug approaches to prevent delirium?

a. What are the benefits and harms of antipsychotics compared with each other, placebo, or non-drug approaches to prevent delirium in persons aged 65 years and older?

b. What are the benefits and harms of antipsychotics compared with each other, placebo, or non-drug approaches to prevent delirium in persons with dementia?

c. What are the benefits and harms of antipsychotics compared with each other, placebo, or non-drug approaches to prevent delirium in patients in an intensive care unit? 
d. What are the benefits and harms of antipsychotics compared with each other, placebo, or non-drug approaches to prevent delirium in patients in a post-acute care facility?

e. What are the benefits and harms of antipsychotics compared with each other, placebo, or non-drug approaches to prevent delirium in patients in palliative or hospice care?

f. What are the benefits and harms of antipsychotics compared with each other, placebo, or non-drug approaches to prevent delirium in patients in postoperative care?

Key Question 2. What are the benefits and harms of antipsychotics compared with each other, placebo, or non-drug approaches to treat delirium?

a. What are the benefits and harms of antipsychotics compared with each other, placebo, or non-drug approaches to treat delirium in persons aged 65 years and older?

b. What are the benefits and harms of antipsychotics compared with each other, placebo, or non-drug approaches to treat delirium in persons with dementia?

c. What are the benefits and harms of antipsychotics compared with each other, placebo, or non-drug approaches to treat delirium in patients in an intensive care unit?

d. What are the benefits and harms of antipsychotics compared with each other, placebo, or non-drug approaches to treat delirium in patients in a post-acute care facility?

e. What are the benefits and harms of antipsychotics compared with each other, placebo, or non-drug approaches to treat delirium in patients in palliative or hospice care?

f. What are the benefits and harms of antipsychotics compared with each other, placebo, or non-drug approaches to treat delirium in patients in postoperative care?

\section{Analytic Framework}

Figures 1 and 2 graphically display the Key Questions. 
Figure 1. Analytic framework for antipsychotics for delirium prevention

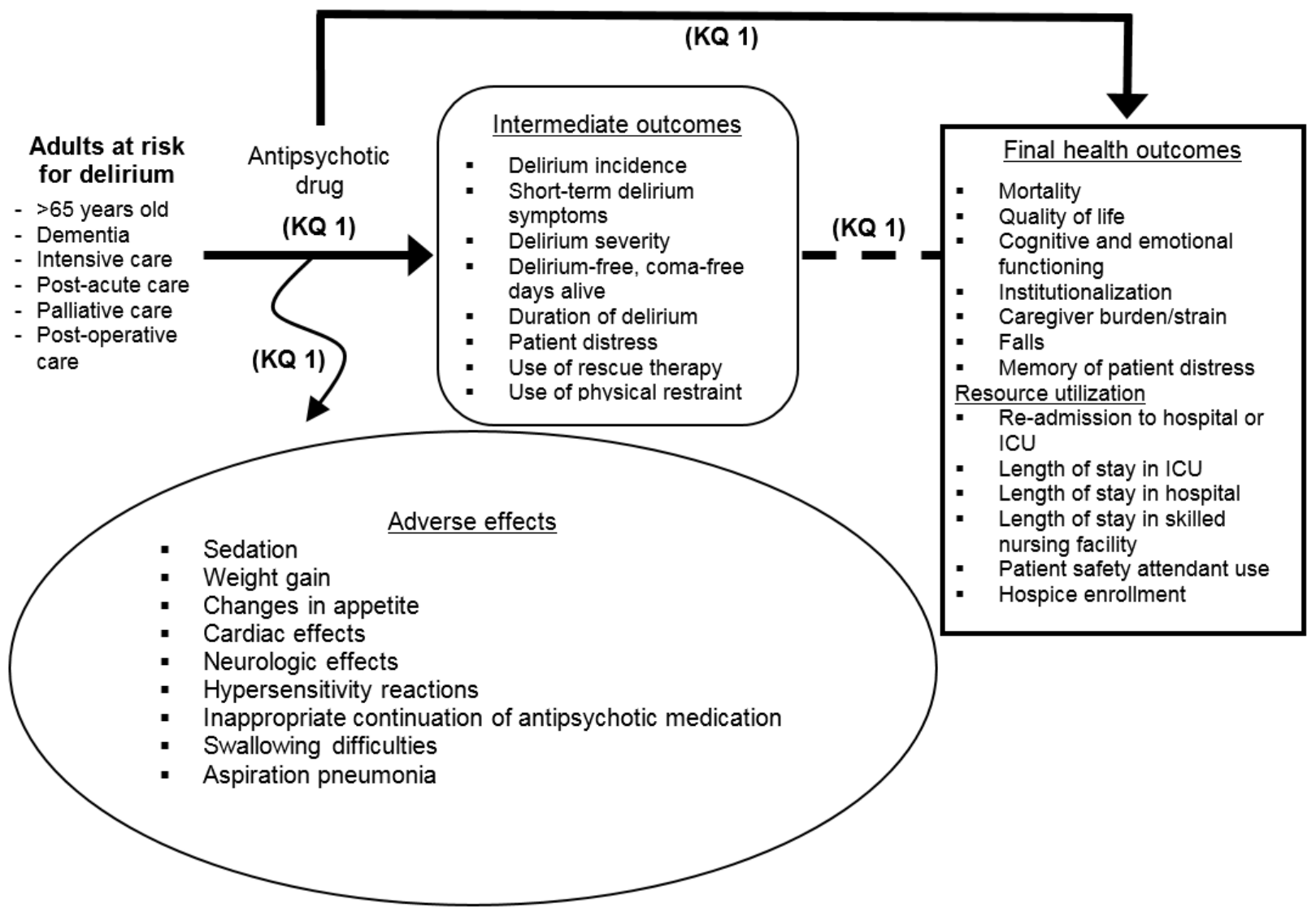

ICU = intensive care unit; KQ = Key Question 
Figure 2. Analytic framework for antipsychotics for delirium treatment

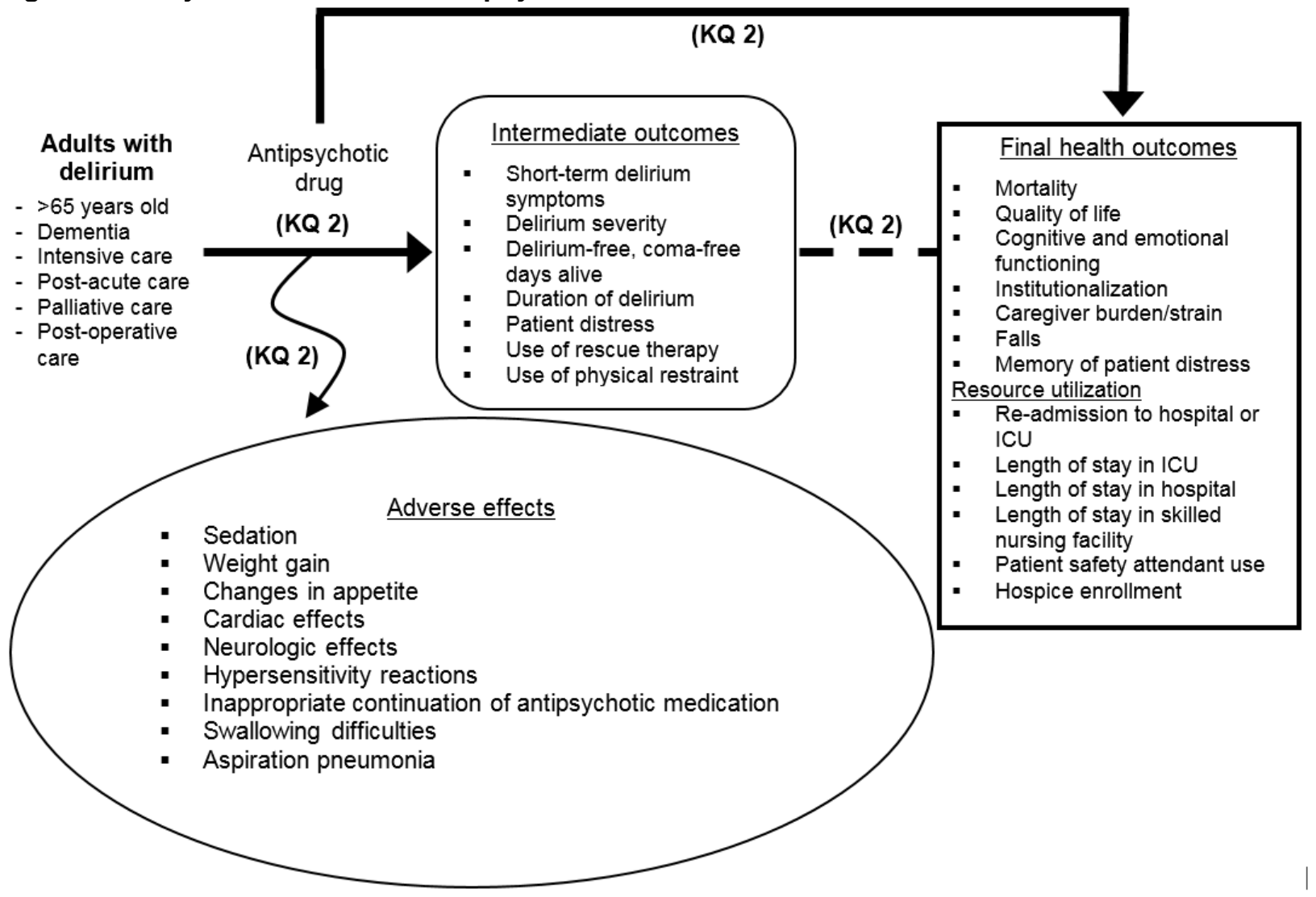

ICU = intensive care unit; KQ = Key Question 


\section{Methods}

We followed the methods outlined in the Agency for Healthcare Research and Quality (AHRQ) Methods Guide for Effectiveness and Comparative Effectiveness Reviews. ${ }^{17}$

\section{Topic Refinement and Protocol Development}

A professional society nominated the topic for the review and the scope was initially developed by the Scientific Resource Center for AHRQ’s Effective Health Care Program. Preliminary Key Questions were posted on AHRQ’s website for public comment.

We further refined the Key Questions with input from Key Informants, representatives from AHRQ, representatives from the nominating partner, and from public comments. Key Informants included patient advocates; representatives from rehabilitation, nursing, geriatrics and palliative care, critical care, pharmacy; and representatives from government agencies.

We recruited a Technical Expert Panel (TEP) to review a draft of the protocol. The TEP included representatives from critical care, geriatrics and palliative care, internal medicine, pharmacy, psychology, and nursing. With the feedback from the TEP and the AHRQ and professional society representatives, we finalized the protocol and posted it on the AHRQ Effective Health Care Program's website (www.effectivehealthcare.ahrq.gov). We also registered the protocol on PROSPERO (CRD42018109552).

\section{Literature Search Strategy}

\section{Search Strategy}

We searched the following databases for primary studies through March 2019: PubMed ${ }^{\circledR}$, Embase $^{\circledR}$, the Cochrane Central Register of Controlled Trials (CENTRAL), Cumulative Index to Nursing and Allied Health Literature (CINAHL ${ }^{\circledR}$ ), and PsycINFO ${ }^{\circledR}$. We developed a search strategy for PubMed based on an analysis of medical subject headings $\left(\mathrm{MeSH}^{\circledR}\right)$ and text words from eligible primary studies identified a priori (Appendix A). Our search was peer reviewed by a medical librarian with experience in developing literature searches in the field of delirium. We handsearched the reference lists of included articles and relevant reviews. We also handsearched the references included in several delirium-specific bibliographic repositories. ${ }^{18-20}$ We will update the search during the peer review process.

We conducted grey literature searches of ClinicalTrials.gov to identify data and studies not reported in the published literature, to assess for publication and reporting bias, and to inform future research needs. Studies identified through grey literature searches were considered for inclusion in the review under two conditions: 1) they were a source of a unique study that met inclusion criteria and provided enough methodologic detail to assess risk of bias, or 2) they were matched to an original publication that had been included in the review when the abstract or presentation reported data on an outcome that was not reported in the original publication. The team searched ClinicalTrials.gov to identify any relevant registered trials. We will update the ClinicalTrials.gov literature search during the peer review process. We reviewed any material that was submitted through the Supplemental Evidence and Data for Systematic Reviews (SEADS) portal and the Federal Register. 


\section{Inclusion and Exclusion Criteria}

Table 2 lists our inclusion and exclusion criteria using the PICOTS (Population, Intervention, Comparison, Outcomes, Timing, Setting and Study) design framework.

Table 2. Inclusion and exclusion criteria

\begin{tabular}{|c|c|c|}
\hline PICOTS & Inclusion Criteria & Exclusion Criteria \\
\hline Population & $\begin{array}{ll}\text { - } & \text { KQ 1: adults who are at risk of delirium } \\
\text { - } & \text { KQ 2: adults with delirium }\end{array}$ & $\begin{array}{l}\text { KQ 2: studies that did not use a } \\
\text { validated instrument to diagnose } \\
\text { delirium }{ }^{21} \\
\text { - } \quad \text { Studies of children }\end{array}$ \\
\hline Intervention & $\begin{array}{l}\text { Studies that evaluated an antipsychotic drug, } \\
\text { including: } \\
\text { o Any first-generation agent (chlorpromazine, } \\
\text { droperidol, fluphenazine, haloperidol, loxapine, } \\
\text { molindone, perphenazine, pimozide, } \\
\text { prochlorperazine, thiothixene, thioridazine, } \\
\text { trifluoperazine) } \\
\text { o Any second-generation agent (aripiprazole, } \\
\text { asenapine, brexpiprazole, cariprazine, } \\
\text { clozapine, iloperidone, lurasidone, olanzapine, } \\
\text { paliperidone, quetiapine, risperidone, } \\
\text { ziprasidone) }\end{array}$ & $\begin{array}{l}\text { - Studies in which the effects of the } \\
\text { antipsychotic drugs cannot be } \\
\text { isolated }\end{array}$ \\
\hline Comparison & $\begin{array}{l}\text { KQ 1: non-drug approaches to preventing delirium, } \\
\text { including placebo, active control, and usual care } \\
\text { KQ 2: non-drug approaches to treating delirium, } \\
\text { including placebo, active control, and usual care }\end{array}$ & $\begin{array}{l}\text { - Studies that do not have a } \\
\text { comparison group for outcomes } \\
\text { other than adverse events }\end{array}$ \\
\hline Outcomes & $\begin{array}{l}\text { - Studies addressing the following outcomes: } \\
\text { Intermediate outcomes (delirium incidence, } \\
\text { short-term delirium symptoms*; delirium } \\
\text { severity; delirium-free, coma-free days alive; } \\
\text { duration of delirium; patient distress; use of } \\
\text { rescue therapy; use of physical restraint) } \\
\text { - } \text { Final health or patient-centered outcomes } \\
\text { (mortality; quality of life; cognitive and } \\
\text { emotional functioning; long-term cognitive } \\
\text { impairment; institutionalization; caregiver } \\
\text { burden/strain; falls; memory of patient distress) } \\
\text { Resource utilization (readmission to the hospital } \\
\text { or ICU; length of stay in ICU; length of stay in } \\
\text { hospital; length of stay in skilled nursing facility; } \\
\text { patient safety attendant use; hospice } \\
\text { enrollment) } \\
\text { Adverse effects (sedation; weight gain; } \\
\text { changes in appetite; cardiac effects; neurologic } \\
\text { effects; paradoxical reactions; hypersensitivity } \\
\text { reactions; inappropriate continuation of } \\
\text { antipsychotics; swallowing difficulties; aspiration } \\
\text { pneumonia) }\end{array}$ & \\
\hline $\begin{array}{c}\text { Timing and } \\
\text { setting }\end{array}$ & - $\quad$ Studies of any length of followup & \\
\hline $\begin{array}{l}\text { Type of } \\
\text { study }\end{array}$ & $\begin{array}{ll}\text { - } & \text { RCTs for all outcomes except adverse events } \\
\text { - } & \text { RCTs, non-RCTs, and prospective cohort studies } \\
\text { with and without a comparison group for adverse } \\
\text { events } \\
\text { - } \quad \text { Studies in any language } \\
\end{array}$ & $\begin{array}{ll}\text { - } & \text { Studies with no original data } \\
\text { - } & \text { Meeting abstracts }\end{array}$ \\
\hline
\end{tabular}

ICU = intensive care unit; KQ = Key Question; PICOTS = population, intervention, comparison, outcomes, timing and setting, and type of study; RCT = randomized controlled trial

* For short-term delirium symptoms, we considered agitation and/or increased psychomotor activity, sleep-wake cycle disturbances, and delusions. 


\section{Process for Study Selection}

Two reviewers independently screened each abstract. Both reviewers needed to agree that an article met at least one of the exclusion criteria to be excluded (see Table 2 for inclusion/exclusion criteria). We tracked and resolved differences between reviewers through consensus adjudication. Articles promoted on the basis of the abstract screen underwent another screen using the same process.

\section{Data Extraction and Data Management}

We created and pilot tested standardized forms for data extraction (Appendix B). Each article underwent double review by the study investigators for data abstraction. The second reviewer confirmed the first reviewer's abstracted data for completeness and accuracy. Reviewer pairs were formed to include personnel with both clinical and methodological expertise.

For all articles, the reviewers extracted information on general study characteristics (e.g., study design, study period, and followup); study participants (e.g., population, age, sex, presence of dementia, setting, presence of substance use, presence of hypertension); interventions (e.g., dose, administration, frequency of use, and duration of use); comparisons; the method of ascertainment of outcomes; and the outcome results, including measures of variability. Non-drug interventions were categorized following the scheme developed by $\mathrm{Oh}$ and colleagues. ${ }^{22} \mathrm{We}$ contacted the authors of the included studies for additional data, if necessary.

All information from the article review process was entered into a DistillerSR database (Evidence Partners Inc., Ottawa, Canada) by the first reviewer. We used the DistillerSR database to maintain the data and to create detailed evidence tables and summary tables.

\section{Risk of Bias Assessment}

Two reviewers independently assessed risk of bias for each study. For randomized controlled trials (RCTs), we used the Cochrane Risk of Bias Tool. ${ }^{23}$ For observational studies, we used the Cochrane Risk of Bias in Non-Randomized Studies of Interventions (ROBINS-I) tool. ${ }^{24}$ We resolved differences between reviewers through consensus. We judged the overall risk of bias for each study based on the adjudicated ratings for the individual risk of bias items. RCTs had three overall ratings for risk of bias (low, high, and unclear) and observational studies had five overall ratings (low, moderate, serious, critical, and no information).

\section{Data Synthesis}

We organized the report by Key Question and, then, by outcome. We conducted qualitative synthesis for each Key Question. We created detailed evidence tables containing all information extracted from eligible studies. We conducted meta-analyses when there were sufficient data (i.e., at least three studies) and studies were sufficiently homogenous with respect to key variables (e.g., population characteristics, study duration, treatment, and outcome definition). We decided as a team what could be pooled in a meta-analysis. For the subquestion regarding persons aged 65 years and older, we included any study in which at least 50 percent of the population was aged 65 years and older.

Although the drugs may have different mechanisms of action, we anticipated that most drugs within a class would have similar clinical effects. Therefore, we combined studies of unique medications within classes when reporting outcomes. If we saw substantial statistical heterogeneity (I-squared $>50 \%$ ) in pooled estimates for any outcome, we explored whether this 
was a result of pooling studies of unique medications. We then stratified studies by medication and repeated the pooled analyses and measures of heterogeneity. We also conducted sensitivity analyses by omitting one study at a time to assess the influence of any single study on the pooled estimates.

For continuous outcomes, we calculated a mean difference by using a random-effects model with the DerSimonian and Laird formula in settings of low heterogeneity ${ }^{25}$ or with appropriate analyses when there is higher heterogeneity. ${ }^{26}$ Because methods to transform medians where there are skewed data can under- and over-estimate the true effect in a meta-analysis, we decided not conduct meta-analysis in these circumstances (e.g., for length of stay in hospital). ${ }^{27}$ For dichotomous outcomes, we calculated a pooled effect estimate of the relative risk between the trial arms of RCTs, with each study weighted by the inverse variance, by using a random-effects model with the DerSimonian and Laird formula for calculating between-study variance in settings of low heterogeneity ${ }^{25}$ or with appropriate analyses when there is higher heterogeneity. ${ }^{26}$

For the outcome of delirium severity, we used a crosswalk ${ }^{28,29}$ to convert the scores for the Memorial Delirium Assessment Scale (MDAS) or the Confusion Assessment Method-Severity (CAM-S) to the Delirium Rating Scale-Revised-98 (DRS-R-98).

We planned to examine publication bias using Begg's test and Egger's test, including evaluation of the asymmetry of funnel plots for each comparison of interest for the outcomes for which meta-analyses were conducted and there were at least 10 studies. ${ }^{30,31}$ Publication bias was also qualitatively considered as part of the strength of evidence determination.

We used STATA statistical software (Intercooled, version 12.1, StataCorp, College Station, TX) for all meta-analyses.

We qualitatively summarized studies that were not amenable to pooling.

\section{Grading the Body of Evidence for Each Key Question}

We graded the strength of evidence using the grading scheme recommended by the Guide for Conducting Comparative Effectiveness Reviews. ${ }^{32}$ We applied evidence grades to the bodies of evidence about each comparison for each critical outcome.

Critical outcomes were determined a priori and separately for each subpopulation. We asked the Key Informants and the Technical Experts to select the five most important outcomes for each of the patient subpopulations, with at least one outcome being a potential adverse effect. We defined importance as those outcomes that have the greatest relevance to decision-making about the use of antipsychotics for the prevention or treatment of delirium in the specific patient group. Table 3 lists the outcomes that were considered critical for each subpopulation.

We assessed the study limitations, consistency, directness, precision, and reporting bias. When scoring study limitations, we considered how well the study design and the study methods (using risk of bias assessments) protected against bias. We classified evidence into four categories: (1) "high" grade (indicating high confidence that the evidence reflects the true effect and further research is very unlikely to change our confidence in the estimate of the effect); (2) "moderate" grade (indicating moderate confidence that the evidence reflects the true effect but further research could change our confidence in the estimate of the effect and may change the estimate); (3) "low" grade (indicating low confidence that the evidence reflects the true effect and further research is likely to change our confidence in the estimate of the effect and is likely to change the estimate); and (4) "insufficient" grade (indicating evidence is unavailable or the body of evidence has unacceptable deficiencies, precluding a conclusion). 
Table 3. Critical outcomes for each subpopulation

\begin{tabular}{|c|c|}
\hline Subpopulation & Critical Outcomes \\
\hline Overall & $\begin{array}{ll}\text { - } & \text { Cognitive functioning } \\
\text { - } & \text { Delirium severity } \\
\text { - } & \text { Length of stay in hospital } \\
\text { - } & \text { Inappropriate continuation of antipsychotics } \\
\text { - } & \text { Sedation }\end{array}$ \\
\hline Persons aged 65 years and older & $\begin{array}{ll}\text { - } & \text { Cognitive functioning } \\
\text { - } & \text { Delirium severity } \\
\text { - } & \text { Falls } \\
\text { - } & \text { Inappropriate continuation of antipsychotics } \\
\text { - } & \text { Sedation }\end{array}$ \\
\hline Persons with dementia & $\begin{array}{ll} & \text { Cognitive functioning } \\
\text { - } & \text { Caregiver burden/strain } \\
\text { - } & \text { Length of stay in hospital } \\
\text { - } & \text { Inappropriate continuation of antipsychotics } \\
\end{array}$ \\
\hline Patients in an intensive care unit & $\begin{array}{ll}\text { - } & \text { Cognitive functioning } \\
\text { - } & \text { Delirium severity } \\
\text { - } & \text { Length of stay in hospital } \\
\text { - } & \text { Inappropriate continuation of antipsychotics } \\
\text { - } & \text { Sedation }\end{array}$ \\
\hline Patients in a post-acute care facility & $\begin{array}{ll}\text { - } & \text { Cognitive functioning } \\
\text { - } & \text { Falls } \\
\text { - } & \text { Length of stay in hospital } \\
\text { - } & \text { Sedation } \\
\end{array}$ \\
\hline Patients in palliative or hospice care & $\begin{array}{ll} & \text { Caregiver burden/strain } \\
\text { - } & \text { Delirium severity } \\
\text { - } & \text { Sedation } \\
\end{array}$ \\
\hline Patients in post-operative care & $\begin{array}{ll} & \text { Cognitive functioning } \\
\text { - } & \text { Delirium severity } \\
\text { - } & \text { Length of stay in hospital } \\
\text { - } & \text { Inappropriate continuation of antipsychotics }\end{array}$ \\
\hline
\end{tabular}

\section{Peer Review and Public Commentary}

Experts in the fields of critical care, geriatrics, hospital medicine, nursing, palliative care, pharmacy, psychology, and surgery and individuals representing stakeholder and user communities were invited to provide external peer review of this systematic review; AHRQ and an associate editor also provided comments. The draft report was posted on the AHRQ website for 4 weeks to elicit public comment. We addressed all reviewer comments, revising the text as appropriate, and documented everything in a disposition of comments report that will be made available 3 months after the Agency posts the final systematic review on the Effective Health Care website. 


\section{Results}

\section{Search Results}

We retrieved 9,427 unique citations (Figure 3). After screening abstracts and full-text, we included 57 studies (published in 62 articles). Appendix C provides a list of the included articles and the articles excluded at full-text screen.

Of the eligible studies, 15 studies, including 14 randomized controlled trials (RCTs) ${ }^{33-46}$ and 1 observational study, ${ }^{47}$ addressed the prevention of delirium. Forty-four studies, including 19 RCTs $^{44,45,48-64}$ and 25 observational studies or non-randomized trials, ${ }^{65-89}$ addressed the treatment of delirium. Two trials enrolled patients with and without delirium; we classified these as both a prevention and treatment trial for the purposes of this report. ${ }^{44,45}$

Tables 4 and 5 list the included studies by population by comparison. We found one study that compared haloperidol with another first-generation antipsychotic. ${ }^{51}$ We decided not to discuss this comparison further because of the infrequent use of chlorpromazine in current clinical practice.

Details of the included studies are provided in the Evidence Tables in Appendix D. 
Figure 3. Summary of the literature search

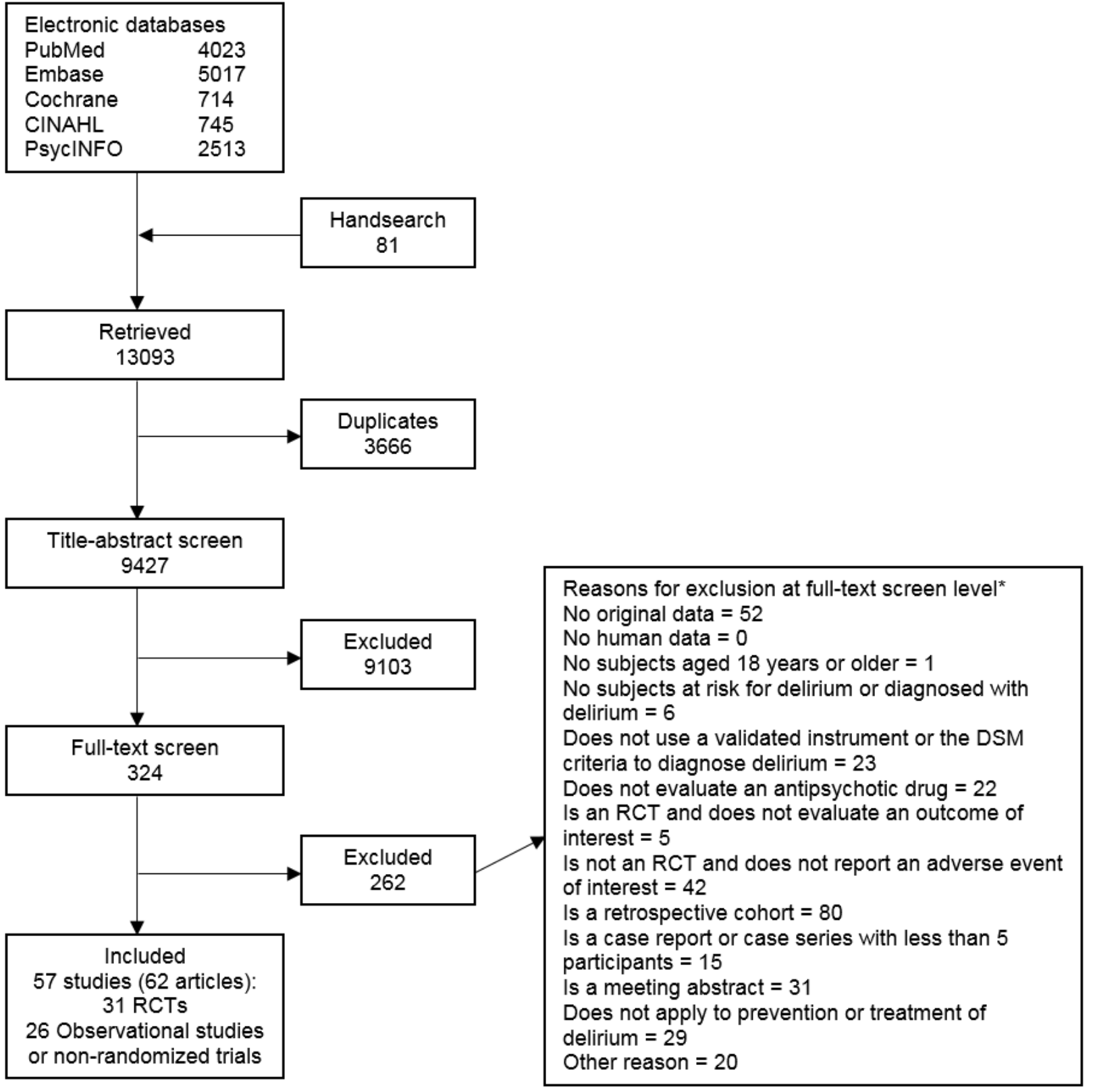

Abbreviations: CINAHL = Cumulative Index to Nursing and Allied Health Literature; DSM = Diagnostic and Statistical Manual; RCT $=$ randomized controlled trial 
Table 4. Number of studies evaluating antipsychotics for the prevention of delirium for overall and subpopulations

\begin{tabular}{|c|c|c|c|c|c|c|c|}
\hline & $\begin{array}{c}\text { Overall } \\
\text { Patient } \\
\text { Population }\end{array}$ & $\begin{array}{l}\text { Patients, } \\
\text { Aged } 65 \\
\text { Years Or } \\
\text { Older }\end{array}$ & $\begin{array}{l}\text { Patients } \\
\text { With } \\
\text { Dementia }\end{array}$ & $\begin{array}{l}\text { Patients } \\
\text { In } \\
\text { Intensive } \\
\text { Care Unit }\end{array}$ & $\begin{array}{l}\text { Patients } \\
\text { In Post- } \\
\text { Acute } \\
\text { Care } \\
\text { Facility } \\
\end{array}$ & $\begin{array}{l}\text { Patients In } \\
\text { Palliative } \\
\text { Or } \\
\text { Hospice } \\
\text { Care } \\
\end{array}$ & $\begin{array}{l}\text { Patients } \\
\text { In Post- } \\
\text { Operative } \\
\text { Care }\end{array}$ \\
\hline $\begin{array}{l}\text { Haloperidol vs. } \\
\text { placebo }\end{array}$ & $\begin{array}{l}11^{33-35,37,38,} \\
41-46\end{array}$ & $4^{35,37,41,43}$ & 0 & $7^{33,34,42-46}$ & 0 & 0 & $\begin{array}{l}5^{35,37,38,43,} \\
46\end{array}$ \\
\hline $\begin{array}{l}\text { Second-generation } \\
\text { antipsychotic vs. } \\
\text { placebo }\end{array}$ & $3^{36,39,40}$ & $2^{36,39}$ & 0 & 0 & 0 & 0 & $3^{36,39,40}$ \\
\hline $\begin{array}{l}\text { Haloperidol vs. } \\
\text { second-generation } \\
\text { antipsychotic }\end{array}$ & $1^{44}$ & 0 & 0 & $1^{44}$ & 0 & 0 & 0 \\
\hline $\begin{array}{l}\text { Second-generation } \\
\text { antipsychotic vs. } \\
\text { second-generation } \\
\text { antipsychotic }\end{array}$ & 0 & 0 & 0 & 0 & 0 & 0 & 0 \\
\hline $\begin{array}{l}\text { Haloperidol vs. } \\
\text { other treatment }\end{array}$ & $1^{33}$ & 0 & 0 & $1^{33}$ & 0 & 0 & 0 \\
\hline $\begin{array}{l}\text { Second-generation } \\
\text { antipsychotic vs. } \\
\text { other treatment }\end{array}$ & 0 & 0 & 0 & 0 & 0 & 0 & 0 \\
\hline No comparison & $1^{47}$ & 0 & 0 & $1^{47}$ & 0 & 0 & 0 \\
\hline
\end{tabular}

Table 5. Number of studies evaluating antipsychotics for the treatment of delirium for overall and subpopulations

\begin{tabular}{|c|c|c|c|c|c|c|c|}
\hline & $\begin{array}{c}\text { Overall } \\
\text { Patient } \\
\text { Population }\end{array}$ & $\begin{array}{l}\text { Patients, } \\
\text { Aged } 65 \\
\text { Years Or } \\
\text { Older }\end{array}$ & $\begin{array}{l}\text { Patients } \\
\text { With } \\
\text { Dementia }\end{array}$ & $\begin{array}{l}\text { Patients } \\
\text { In } \\
\text { Intensive } \\
\text { Care Unit }\end{array}$ & $\begin{array}{l}\text { Patients } \\
\text { In Post- } \\
\text { Acute } \\
\text { Care } \\
\text { Facility }\end{array}$ & $\begin{array}{c}\text { Patients In } \\
\text { Palliative } \\
\text { Or } \\
\text { Hospice } \\
\text { Care }\end{array}$ & $\begin{array}{l}\text { Patients } \\
\text { In Post- } \\
\text { Operative } \\
\text { Care }\end{array}$ \\
\hline $\begin{array}{l}\text { Haloperidol vs. } \\
\text { placebo }\end{array}$ & $5^{44,45,48,57,79}$ & $1^{57}$ & 0 & $3^{44,45,79}$ & 0 & $1^{48}$ & 0 \\
\hline $\begin{array}{l}\text { Second-generation } \\
\text { vs. placebo }\end{array}$ & $\begin{array}{l}6^{44,48,52,57} \\
63,79\end{array}$ & $1^{57}$ & 0 & $3^{44,52,79}$ & 0 & $1^{48}$ & 0 \\
\hline $\begin{array}{l}\text { Haloperidol vs. } \\
\text { second-generation } \\
\text { antipsychotic }\end{array}$ & $\begin{array}{l}2044,48,53-58 \\
61,62,64-66,68 \\
70,75,79,84,85 \\
89\end{array}$ & $1^{57}$ & 0 & $4^{44,53,79,85}$ & 0 & $2^{48,61}$ & 0 \\
\hline $\begin{array}{l}\text { Second-generation } \\
\text { antipsychotic vs. } \\
\text { second-generation } \\
\text { antipsychotic }\end{array}$ & $\begin{array}{l}7^{54,59,60,66,} \\
68,69,89\end{array}$ & 0 & 0 & $1^{54}$ & 0 & 0 & 0 \\
\hline $\begin{array}{l}\text { Haloperidol vs. } \\
\text { other treatment }\end{array}$ & $3^{49-51}$ & 0 & 0 & 0 & 0 & 0 & $1^{49}$ \\
\hline $\begin{array}{l}\text { Second-generation } \\
\text { antipsychotic vs. } \\
\text { other treatment }\end{array}$ & 0 & 0 & 0 & 0 & 0 & 0 & 0 \\
\hline No comparison & $\begin{array}{l}15^{67,71-74,76-} \\
78,80-83,86-88\end{array}$ & & & & & & \\
\hline
\end{tabular}




\section{Key Question 1. Benefits and Harms of Antipsychotics To Prevent Delirium}

\section{Key Points - Overall}

\section{Delirium Severity}

- We were unable to draw a conclusion regarding the effects of antipsychotics on delirium severity in patients at risk for delirium for the following comparisons:

o Haloperidol versus placebo (insufficient evidence)

o Second-generation antipsychotics versus placebo (insufficient evidence)

o Haloperidol versus second-generation antipsychotics (no trials)

o Second-generation antipsychotics versus second-generation antipsychotics (no trials)

o Haloperidol versus other therapies (no trials)

o Second-generation antipsychotics versus other therapies (no trials)

\section{Cognitive Functioning}

- We found no trials evaluating cognitive functioning in patients at risk for delirium that compared an antipsychotic with a placebo, another antipsychotic, or another treatment.

\section{Length of Stay in Hospital}

- We concluded that there was little to no difference in length of stay in hospital for patients at risk of delirium for the following comparisons:

o Haloperidol versus placebo (Strength of evidence [SOE]: High)

o Second-generation antipsychotics versus placebo (SOE: Low)

- We are unable to draw a conclusion regarding the effects of antipsychotics on length of stay in hospital for patients at risk for delirium for the following comparisons:

o Haloperidol versus second-generation antipsychotics (insufficient evidence)

o Second-generation antipsychotics versus second-generation antipsychotics (no trials)

o Haloperidol versus other therapies (insufficient evidence)

o Second-generation antipsychotics versus other therapies (no trials)

\section{Inappropriate Continuation of Antipsychotics}

- We found no studies evaluating inappropriate continuation of antipsychotics in patients at risk for delirium that compared an antipsychotic with a placebo, another antipsychotic, or another treatment.

\section{Sedation}

- We were unable to draw a conclusion regarding the effects of antipsychotics on sedation in patients at risk for delirium for the following comparisons:

o Haloperidol versus placebo (insufficient evidence)

o Second-generation antipsychotics versus placebo (no studies)

o Haloperidol versus second-generation antipsychotics (no studies)

o Second-generation antipsychotics versus second-generation antipsychotics (no studies)

o Haloperidol versus other therapies (no studies) 
o Second-generation antipsychotics versus other therapies (no studies)

\section{Intermediate Outcomes}

\section{Delirium Incidence - Overall}

\section{Haloperidol Versus Placebo}

Nine randomized controlled trials (RCTs) directly compared delirium incidence between haloperidol and placebo groups. These RCTs enrolled 3,408 patients in both surgical and medical intensive care and non-intensive care unit settings and used a variety of validated delirium detection instruments. Five of the trials were low risk of bias, ${ }^{34,37,41,42,46}$ three had unclear risk of bias, ${ }^{33,38,43}$ and one had high risk of bias owing to lack of blinding and allocation concealment. ${ }^{35}$ Intravenous haloperidol was administered in all except two trials; in those two exceptions, oral doses were given. ${ }^{37,41}$ These nine trials were pooled, as they each identified new onset of delirium (incidence) within the week after exposure to prophylactic haloperidol or placebo. We found no clinically meaningful difference in the relative risk of delirium in the comparison of haloperidol with placebo (relative risk [RR], 0.94; 95\% confidence interval [CI], 0.77 to 1.16, I-squared, 44\%; Figure 4). Exclusion of any one trial did not change the inference of the meta-analysis. We considered the results to be consistent and precise, but this is limited due to some trials with high or unclear risk of bias.

\section{Second-Generation Antipsychotics Versus Placebo}

Three RCTs, two with low risk of bias ${ }^{36,39}$ and one with high risk of bias due to lack of blinding, ${ }^{40}$ enrolled 627 patients without delirium prior to surgery and compared delirium occurrence in the postoperative setting following randomization with postoperative risperidone or placebo ${ }^{36,40}$ or peri-operative olanzapine or placebo. ${ }^{39}$ Two of these trials included patients aged 65 years and older prior to either on-pump cardiac or joint-replacement surgery. We found a statistically significant and clinically meaningful difference in the relative risk of delirium favoring second-generation antipsychotics compared with placebo for the prevention of delirium (RR, 0.36; 95\% CI, 0.26 to 0.50, I-squared, 0\%) (Figure 4). Exclusion of any one trial did not change the inference of the meta-analysis. Although the results were consistent and precise, all of the studies were conducted in the postoperative setting and these results may not apply to all adult patients at risk for delirium.

\section{Haloperidol Versus Other Therapies}

We found one three-arm RCT, with unclear risk of bias that evaluated delirium incidence and, in addition to a placebo arm, compared intravenous haloperidol with intravenous dexmedetomidine, administered for treatment of agitation. ${ }^{33}$ This RCT enrolled patients with severe systemic illness in the intensive care unit. The results of this trial favored the use of dexmedetomidine over haloperidol for the prevention of delirium.

\section{Other Comparisons}

We found no delirium prevention trials evaluating delirium incidence for the following comparisons:

- Haloperidol versus second-generation antipsychotics

- Second-generation antipsychotics versus second-generation antipsychotics 
- Second-generation antipsychotics versus other therapies.

Figure 4. Meta-analysis of delirium incidence in trials comparing either haloperidol or secondgeneration antipsychotics with placebo among patients at risk for delirium

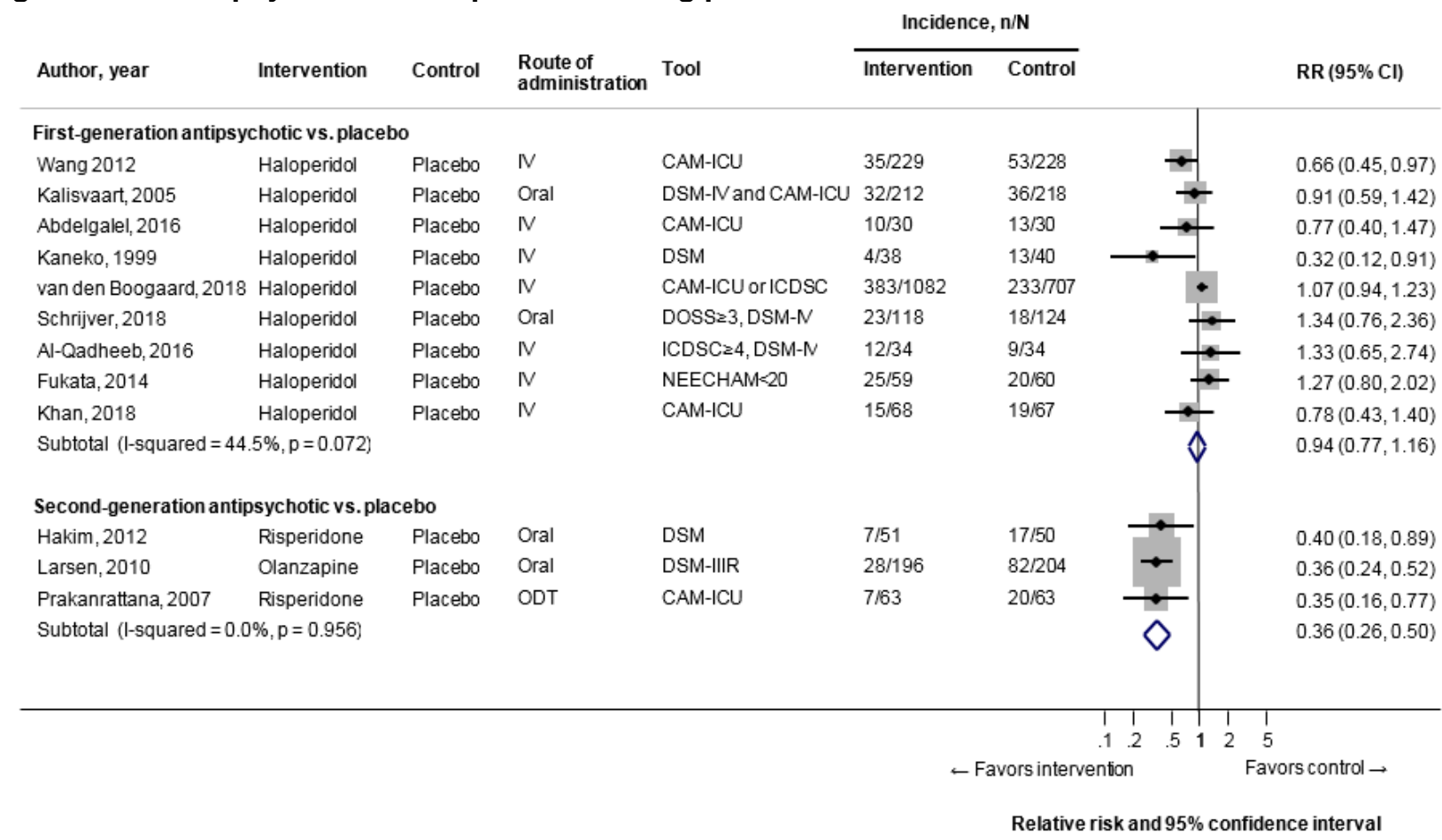

CAM-ICU = Confusion Assessment Method for the ICU; CI = confidence interval; DOSS = Delirium Observation Screening Scale; DSM = Diagnostic and Statistical Manual of Mental Disorders; DSM-IIIR = Diagnostic and Statistical Manual of Mental Disorders, 3rd ed. Revision; DSM-IV = Diagnostic and Statistical Manual of Mental Disorders, $4^{\text {th }}$ ed.; ICDSC = Intensive Care Delirium Screening Checklist; IV = intravenous; $\mathrm{n}=$ incidence within arm; $\mathrm{N}=$ total arm population; NEECHAM = NeelonChampagne Confusion Scale; $\mathrm{p}=\mathrm{p}$-value; $\mathrm{RR}$ = relative risk

\section{Short-Term Delirium Symptoms - Overall}

\section{Haloperidol Versus Placebo}

We found two RCTs, one with low risk of bias and one with unclear risk of bias, enrolling critically ill mechanically ventilated patients or patients undergoing elective gastrointestinal surgery. ${ }^{38,45}$ These trials compared the effect of haloperidol and placebo on two different delirium symptoms: agitation and sleep duration. Haloperidol compared to placebo did not change sleep duration. ${ }^{38}$ Haloperidol compared to placebo was statistically significantly associated with decreased agitation. ${ }^{45}$ We concluded that these single studies provided insufficient evidence to make a determination about the impact of haloperidol on short-term delirium symptoms when used to prevent delirium.

\section{Other Comparisons}

We found no delirium prevention trials evaluating delirium symptoms for the following comparisons:

- Second-generation antipsychotics versus placebo

- Haloperidol versus second-generation antipsychotics

- Second-generation antipsychotics versus second-generation antipsychotics 
- Haloperidol versus other therapies

- Second-generation antipsychotics versus other therapies.

\section{Delirium Severity - Overall}

\section{Haloperidol Versus Placebo}

Three RCTs, with low risk of bias, compared haloperidol with placebo and assessed delirium severity using the same instrument, the Delirium Rating Scale-Revised-98, in different populations with inconsistent results. ${ }^{37,41,46}$ We conducted a meta-analysis, but there was substantial statistical heterogeneity (I-squared, $82 \%$ ). When we conducted a profile likelihood analysis, the results would change the inference and remained limited by substantial heterogeneity (I-squared, 70\%). Also, one trial was missing the Delirium Rating Scale-Revised98 results for over half of the patients (54\%). ${ }^{41}$ We were unable to draw conclusions based on the inconsistency and methodological limitations. (SOE: Insufficient)

\section{Second-Generation Antipsychotics Versus Placebo}

Two RCTs, with low risk of bias, compared second-generation antipsychotics with placebo and assessed delirium severity using two instruments, the Delirium Rating Scale-Revised-98 and the Intensive Care Delirium Screening Checklist, in surgical populations with inconsistent results. ${ }^{36,39}$ We were unable to draw conclusions based on the inconsistent results and nonrepresentativeness in these two trials. (SOE: Insufficient)

\section{Other Comparisons}

We found no delirium prevention trials evaluating delirium severity for the following comparisons:

- Haloperidol versus second-generation antipsychotics

- Second-generation antipsychotics versus second-generation antipsychotics

- Haloperidol versus other therapies

- Second-generation antipsychotics versus other therapies.

\section{Strength of Evidence}

Table 6 summarizes the strength of evidence findings for antipsychotics for the prevention of delirium in terms of delirium severity. 
Table 6. Strength of evidence domains for trials evaluating antipsychotics for the prevention of delirium in terms of delirium severity

\begin{tabular}{|c|c|c|c|c|c|c|c|c|}
\hline Comparison & $\begin{array}{l}\text { Number Of } \\
\text { Trials (N) }\end{array}$ & $\begin{array}{c}\text { Study } \\
\text { Limitations }\end{array}$ & Directness & Consistency & Precision & $\begin{array}{l}\text { Reporting } \\
\text { Bias }\end{array}$ & $\begin{array}{l}\text { Strength Of } \\
\text { Evidence }\end{array}$ & Summary \\
\hline $\begin{array}{l}\text { Haloperidol vs. } \\
\text { placebo }\end{array}$ & $\begin{array}{l}3^{37,41,46} \\
(807)\end{array}$ & Moderate* & Indirect $^{\dagger}$ & Inconsistent & Precise & Undetected & Insufficient & $\begin{array}{l}\text { We are unable to draw a } \\
\text { conclusion. }\end{array}$ \\
\hline $\begin{array}{l}\text { Second- } \\
\text { generation } \\
\text { antipsychotics } \\
\text { vs. placebo }\end{array}$ & $2^{36,39}(501)$ & Low & Indirect $^{\ddagger}$ & Inconsistent & Precise & Undetected & Insufficient & $\begin{array}{l}\text { We are unable to draw a } \\
\text { conclusion. }\end{array}$ \\
\hline $\begin{array}{l}\text { Haloperidol vs. } \\
\text { second- } \\
\text { generation } \\
\text { antipsychotics }\end{array}$ & No trials & & & & & & & \\
\hline $\begin{array}{l}\text { Second- } \\
\text { generation } \\
\text { antipsychotics } \\
\text { vs. second- } \\
\text { generation } \\
\text { antipsychotics }\end{array}$ & No trials & & & & & & & \\
\hline $\begin{array}{l}\text { Haloperidol vs. } \\
\text { other therapies }\end{array}$ & No trials & & & & & & & \\
\hline $\begin{array}{l}\text { Second- } \\
\text { generation } \\
\text { antipsychotics } \\
\text { vs. other } \\
\text { therapies }\end{array}$ & No trials & & & & & & & \\
\hline
\end{tabular}

* Moderate rating for study limitations is primarily due to missing Delirium Rating Scale-Revised-98 scores in $53.7 \%$ of the patients in one trial ${ }^{41}$

† Indirect rating due to a limited patient population (medical and/or surgical, but not including critical illness); results may not be generalizable to other patients with delirium.

¥ Indirect rating due to a single patient population; results may not be generalizable to other patients with delirium. 


\section{Delirium-Free, Coma-Free Days Alive - Overall}

\section{Haloperidol Versus Placebo}

We identified four RCTs, comparing haloperidol with placebo that assessed delirium- and coma-free days alive at different followup time points in populations of medical and surgical patients in intensive care units. ${ }^{42-45}$ Three trials, with low risk of bias, reported no statistically significant effect on delirium- and coma-free days alive for haloperidol compared with placebo. ${ }^{42,44,45}$ However, one trial, with unclear risk of bias, reported a statistically greater number of delirium- and coma-free days for haloperidol compared with placebo. ${ }^{43}$ Based on our calculations of the same data we found no statistically or clinically significant difference in this study. We concluded that haloperidol had no effect on delirium-free or coma-free days alive; we considered the evidence from these trials to be consistent and precise, although limited to patients in intensive care units.

\section{Second-Generation Antipsychotics Versus Placebo}

We identified one RCT, with low risk of bias and enrolling patients receiving mechanical ventilation, that compared second-generation antipsychotics with placebo for delirium- and coma-free days. ${ }^{44}$ This trial, with 21-day followup, reported no statistically significant effect on delirium- and coma-free days alive for ziprasidone compared with placebo.

\section{Haloperidol Versus Second-Generation Antipsychotics}

We identified one RCT, with low risk of bias and enrolling patients receiving mechanical ventilation, that compared haloperidol with second-generation antipsychotics for delirium- and coma-free days. ${ }^{44}$ This trial, with 21-day followup, reported no statistically significant effect on delirium- and coma-free days alive for ziprasidone compared with haloperidol.

\section{Other Comparisons}

We found no delirium prevention trials evaluating delirium- and coma-free days alive that compared for the following comparisons:

- Second-generation antipsychotics versus second-generation antipsychotics

- Haloperidol versus other therapies

- Second-generation antipsychotics versus other therapies.

\section{Duration of Delirium - Overall}

\section{Haloperidol Versus Placebo}

Seven RCTs, comparing haloperidol with placebo for the prevention of delirium in patients admitted to medical or surgical intensive care units or acute inpatient medical or surgical wards, reported the duration of delirium. Six of these RCTs had low risk of bias, ${ }^{34,37,41,44-46}$ and one had high risk of bias owing to lack of blinding and allocation concealment. ${ }^{35}$ Six of the seven trials reported no statistically significant difference in delirium duration between haloperidol and placebo. ${ }^{34,35,41,44-46}$ One perioperative study found a significant difference favoring haloperidol. ${ }^{37}$ We did not conduct a meta-analysis because these trials generally provided results as median values only. The skewed nature of length of stay data precluded conversion or transformation of the data. Based on the consistent findings in six of seven trials we concluded 
that haloperidol has no effect on delirium duration when used as a preventive agent in all populations at risk of delirium.

\section{Second-Generation Antipsychotics Versus Placebo}

Three RCTs, with low risk of bias, compared a second-generation antipsychotic with placebo for the prevention of delirium and assessed delirium duration in medical and surgical patients in intensive care units and post-surgical acute inpatient wards. ${ }^{36,39,44}$ Two of the trials demonstrated no statistically significant effect on delirium duration. ${ }^{36,44}$ A third study demonstrated a statistically significant shorter duration of delirium in the placebo group in a postoperative population. ${ }^{39}$ We did not conduct a meta-analysis because two trials provided results as median values. The skewed nature of length of stay data precluded conversion or transformation of the data. These studies highlight the heterogeneity of patient populations and design of interventions. There is insufficient evidence regarding the impact of perioperative use of second-generation antipsychotics versus placebo on delirium duration.

\section{Haloperidol Versus Second-Generation Antipsychotics}

One three-arm RCT, with low risk of bias, enrolled patients receiving mechanical ventilation in mixed medical and surgical intensive care units. ${ }^{44}$ This RCT reported no statistically significant effect on delirium duration for patients randomized to treatment with a median daily dose of $15 \mathrm{mg}$ oral haloperidol compared with patients randomized to a median daily dose of 113 mg of oral ziprasidone.

\section{Other Comparisons}

We found no delirium prevention trials evaluating delirium duration for the following comparisons:

- Second-generation antipsychotics versus second-generation antipsychotics

- Haloperidol versus other therapies

- Second-generation antipsychotics versus other therapies.

\section{Patient Distress - Overall}

We found no trials evaluating patient distress in patients at risk for delirium that compared an antipsychotic with a placebo, another antipsychotic, or another treatment.

\section{Use of Rescue Therapy - Overall}

\section{Haloperidol Versus Placebo}

Four RCTs, three with low risk of bias ${ }^{41,44,45}$ and one with uncertain risk of bias, ${ }^{43}$ compared haloperidol with placebo for the prevention of delirium in 941 patients and reported on the use of rescue therapy, defined as the use of any open-label antipsychotic. Three of the RCTs, of which one was a three-arm trial, ${ }^{44}$ enrolled medical and/or surgical patients in the intensive care unit setting; one RCT enrolled patients on acute inpatient wards. ${ }^{41}$ We found no statistically significant between-group difference in the use of rescue therapy when comparing haloperidol with placebo (pooled RR, 0.63; 95\% CI, 0.34 to 1.15; I-squared, 62\%; Figure 5). This is a possibly relevant decrease in use of open-label antipsychotic but the potential impact is difficult to assess. Exclusion of any one study did not change the inference from the meta-analysis. 


\section{Second-Generation Antipsychotics Versus Placebo}

One three-arm RCT, with low risk of bias, enrolled medical and surgical patients requiring mechanical ventilation in the intensive care unit and included the comparison of ziprasidone with placebo for the prevention of delirium. There was no between-group difference in the use of rescue therapy, defined as the use of any open-label antipsychotic. ${ }^{44}$

\section{Haloperidol Versus Second-Generation Antipsychotics}

One three-arm RCT, with low risk of bias, enrolled medical and surgical patients requiring mechanical ventilation in the intensive care unit and included the comparison of ziprasidone with haloperidol for the prevention of delirium. We found no between-group difference in the use of rescue therapy, defined as the use of any open-label antipsychotic. ${ }^{44}$

\section{Other Comparisons}

We found no delirium prevention trials evaluating the use of rescue therapy for the following comparisons:

- Second-generation antipsychotics versus second-generation antipsychotics

- Haloperidol versus other therapies

- Second-generation antipsychotics versus other therapies.

Figure 5. Meta-analysis of use of rescue therapy in trials comparing haloperidol with placebo in patients at risk for delirium

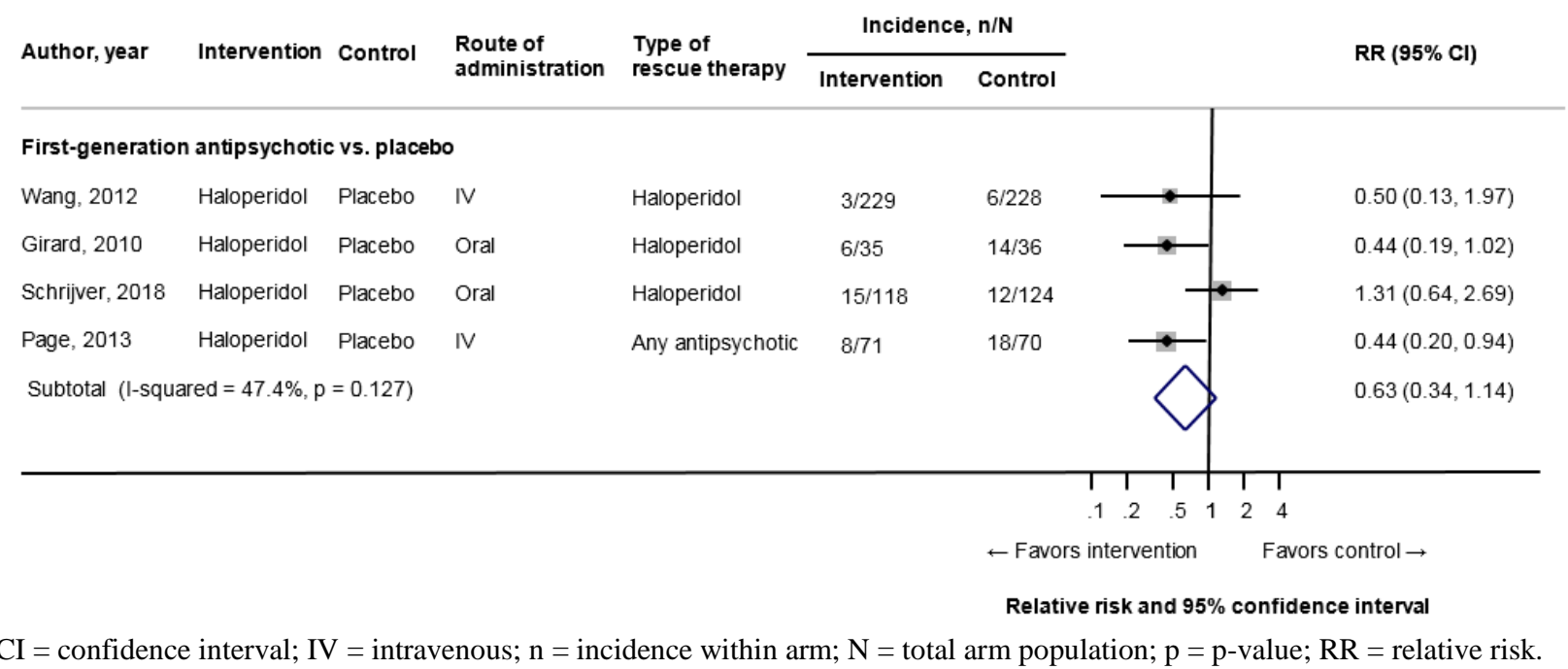

\section{Use of Physical Restraint - Overall}

\section{Haloperidol Versus Placebo}

One three-arm RCT with low risk of bias, enrolled critically ill patients without delirium admitted to surgical and medical intensive care units and compared restraint use between placebo and two haloperidol arms ( $1 \mathrm{mg}$ or $2 \mathrm{mg}$, 3 times per day). ${ }^{42}$ This trial reported a small statistically non-significant difference in the number of patients requiring restraint, favoring placebo. Another two-arm RCT, enrolling patients undergoing major non-cardiac thoracic surgery compared haloperidol (0.5 mg, 3 times per day for 4 days) with placebo, reported no 
difference in use of physical restraint. ${ }^{46}$ There is inconsistent evidence on the use of physical restraint.

\section{Second-Generation Antipsychotics Versus Placebo}

One RCT, with low risk of bias, enrolled patients undergoing joint replacement surgery. This RCT randomized patients to prophylactic use of $5 \mathrm{mg}$ olanzapine or placebo before and after surgery and reported restraint use. ${ }^{39}$ This trial reported no statistical difference in restraint use in patients randomized to olanzapine versus placebo. There were few events, so the results were imprecise.

\section{Other Comparisons}

We found no delirium prevention trials evaluating restraint use for the following comparisons:

- Haloperidol versus second-generation antipsychotics

- Second-generation antipsychotics versus second-generation antipsychotics

- Haloperidol versus other therapies

- Second-generation antipsychotics versus other therapies.

\section{Final Health or Patient-Centered Outcomes}

\section{Mortality - Overall}

\section{Haloperidol Versus Placebo}

We conducted a meta-analysis of eight RCTs ${ }^{33,34,41-46}$ that compared haloperidol with placebo; one of these studies was excluded from the analysis because there were no deaths. ${ }^{46}$ Six of these RCTs had low risk of bias, ${ }^{34,41,42,44-46}$ and two RCTs had unclear risk of bias. ${ }^{33,43}$ Our analysis showed no between-group differences in short-term mortality, defined as mortality while in hospital or up to 30 days after randomization, for haloperidol compared with placebo (pooled RR, 0.98; 95\% CI, 0.82 to 1.17) (Figure 6). We tested the effect of each individual trial on the combined point estimate. No single trial influenced the pooled results. No substantial statistical heterogeneity was identified. All but one ${ }^{41}$ of the RCTs enrolled critically ill patients.

Of these eight RCTs, two trials, with low risk of bias, ${ }^{41,} 42$ also examined 90 -day mortality, including the largest trial with 1,789 critically ill patients. ${ }^{42}$ The results of a meta-analysis of these trials were similar to the results for short-term mortality, with no between-group differences when comparing haloperidol with placebo (pooled RR, 0.97; 95\% CI, 0.81 to 1.16; Figure 6). One RCT also examined 180-day mortality, with no between-group differences when comparing haloperidol with placebo (RR, 0.95; 95\% CI, 0.53 to 1.70$).{ }^{41}$

One RCT, with low risk of bias and enrolling 1,789 critically ill patients, reported no statistically significant effect on survival at 28 days for haloperidol (median, 28 days; interquartile range [IQR], 28 to 28 days) compared with placebo (median, 28 days; IQR, 28 to 28 days) (differences in median, 0 days; $95 \%$ CI, 0 to 0 days). ${ }^{42}$ This trial reported a similar effect on survival at 90 days for haloperidol (median, 90 days; IQR, 90 to 90 days) compared with placebo (median, 90 days; IQR, 90 to 90 days) (differences in median, 0 days; 95\% CI, 0 to 0 days). 


\section{Second-Generation Antipsychotics Versus Placebo}

Two RCTs, with low risk of bias, compared second-generation antipsychotics (risperidone and ziprasidone) with placebo and reported no between-group differences in short-term mortality, defined as mortality while in hospital or up to 30 days after randomization. ${ }^{36,44}$

Figure 6. Meta-analysis of mortality in trials comparing haloperidol with placebo in patients at risk for delirium

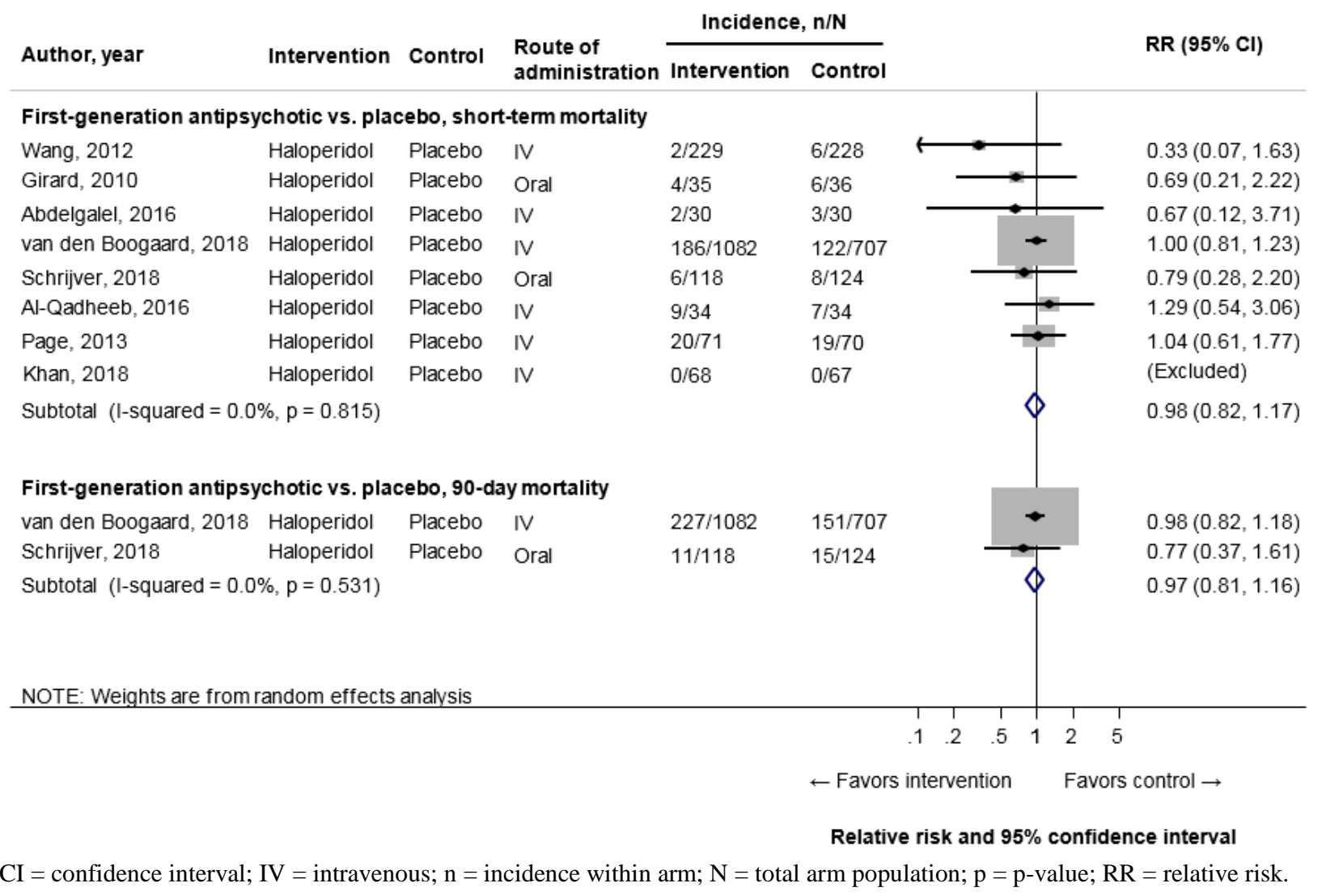

\section{Haloperidol Versus Second-Generation Antipsychotics}

One RCT, with low risk of bias, compared the second-generation antipsychotic, ziprasidone, with haloperidol and reported no between-group differences in short-term mortality, defined as mortality while in hospital or up to 30 days after randomization. ${ }^{44}$

\section{Haloperidol Versus Other Therapies}

One RCT, with unclear risk of bias, compared haloperidol with dexmedetomidine and reported no between-group differences in short-term mortality, defined as mortality while in hospital or up to 30 days after randomization. ${ }^{33}$

\section{Other Comparisons}

We found no delirium prevention trials evaluating mortality for the following comparisons:

- Second-generation antipsychotics versus second-generation antipsychotics

- Second-generation antipsychotics versus other therapies. 


\section{Institutionalization - Overall}

\section{Haloperidol Versus Placebo}

One RCT, with low risk of bias, compared oral haloperidol with placebo for the prevention of delirium and reported on new institutionalization. The RCT enrolled patients aged 70 years and older admitted to medical or surgical acute inpatient wards. ${ }^{41}$ This trial reported no statistically significant between-group difference in new institutionalization at the time of discharge, at 3 months followup, or 6 months followup.

\section{Second-Generation Antipsychotics Versus Placebo}

One RCT, with low risk of bias and enrolling orthopedic surgery patients aged 65 years and older and treated with oral olanzapine or placebo, reported a statistically significant betweengroup difference in institutionalization (defined as discharge to a rehabilitation or skilled nursing facility) favoring the second-generation antipsychotic. ${ }^{39}$

\section{Other Comparisons}

We found no delirium prevention trials evaluating institutionalization for the following comparisons:

- Haloperidol versus second-generation antipsychotics

- Second-generation antipsychotics versus second-generation antipsychotics

- Haloperidol versus other therapies

- Second-generation antipsychotics versus other therapies.

\section{Falls - Overall}

\section{Haloperidol Versus Placebo}

One RCT, with high risk of bias owing to lack of blinding and allocation concealment, compared haloperidol with no intervention for the prevention of delirium in patients undergoing abdominal or orthopedic surgery and reported on falls as an adverse outcome. ${ }^{35} \mathrm{~A}$ single fall occurred in one patient on day seven of the trial, four days after the last dose of haloperidol was administered and was determined by the authors as unlikely to be related to the study intervention.

\section{Other Comparisons}

We found no delirium prevention trials evaluating falls for the following comparisons:

- Second-generation antipsychotics versus placebo

- Haloperidol versus second-generation antipsychotics

- Second-generation antipsychotics versus second-generation antipsychotics

- Haloperidol versus other therapies

- Second-generation antipsychotics versus other therapies.

\section{Other Final Health or Patient-Centered Outcomes - Overall}

We found no trials in patients at risk for delirium that compared an antipsychotic with a placebo, another antipsychotic, or another treatment and evaluated the following final health or patient-centered outcomes: 
- Quality of life

- Cognitive functioning

- Caregiver burden/strain

- Memory of patient distress

\section{Resource Utilization Outcomes}

\section{Readmission to Hospital - Overall}

\section{Haloperidol Versus Placebo}

One RCT, with low risk of bias, compared haloperidol with placebo for the prevention of delirium in hospitalized medical and surgical patients aged 65 years and older. ${ }^{41}$ This trial demonstrated no statistically significant between-group differences on readmission to the acute care hospital of treatment group at 3 months followup or 6 months followup.

\section{Other Comparisons}

We found no delirium prevention trials evaluating readmission to the acute care hospital for the following comparisons:

- Second-generation antipsychotics versus placebo

- Haloperidol versus second-generation antipsychotics

- Second-generation antipsychotics versus second-generation antipsychotics

- Haloperidol versus other therapies

- Second-generation antipsychotics versus other therapies.

\section{Readmission to Intensive Care Unit - Overall}

\section{Haloperidol Versus Placebo}

Two RCTs, with low risk of bias, compared haloperidol with placebo in critically ill medical and surgical patients. ${ }^{42,45}$ Both RCTs demonstrated no effect on readmission to the intensive care unit, but these results were imprecise.

\section{Other Comparisons}

We found no delirium prevention trials evaluating readmission to the intensive care unit for the following comparisons:

- Second-generation antipsychotics versus placebo

- Haloperidol versus second-generation antipsychotics

- Second-generation antipsychotics versus second-generation antipsychotics

- Haloperidol versus other therapies

- Second-generation antipsychotics versus other therapies.

\section{Length of Stay in Hospital - Overall}

\section{Haloperidol Versus Placebo}

Eight RCTs comparing haloperidol with placebo assessed length of stay in hospital in medical and surgical patients in both intensive and non-intensive care units. ${ }^{33,37,41-46}$ Six RCTs 
had low risk of bias, ${ }^{37,41,42,44-46}$ and two RCTs had unclear risk of bias. ${ }^{33,43}$ We did not conduct a meta-analysis because these trials generally provided results as median values and the length of stay in hospital data are skewed, precluding conversion or transformation of the data.

Seven of the eight RCTs reported no statistically significant difference in length of stay in hospital for the overall trial population when comparing haloperidol versus placebo. ${ }^{37,41-46}$ One of the eight RCTs reported a statistically significant difference in length of stay in hospital, with fewer days in hospital for patients receiving haloperidol compared with patients receiving placebo. ${ }^{33}$ Considering the consistent findings in seven of eight trials, we concluded that there was no effect of haloperidol compared with placebo on the length of stay in hospital. (SOE: High)

\section{Second-Generation Antipsychotics Versus Placebo}

Three RCTs comparing second-generation antipsychotics with placebo assessed length of stay in hospital. ${ }^{36,40,44}$ Two RCTs had low risk of bias, ${ }^{36,44}$ and one RCT had high risk of bias, owing to lack of blinding of participants and unclear risk of bias in selective outcome reporting. ${ }^{40}$ We did not conduct a meta-analysis because these trials generally provided results as median values and the length of stay in hospital data are skewed, precluding conversion or transformation of the data. Across all these trials, second-generation antipsychotics compared with placebo had no effect on length of stay in hospital. (SOE: Low)

\section{Haloperidol Versus Second-Generation Antipsychotics}

One RCT, with low risk of bias, compared haloperidol with ziprasidone and assessed length of stay in hospital in medical and surgical patients in intensive care units. ${ }^{44}$ This three-arm trial, enrolling patients receiving mechanical ventilation, reported no statistically significant effect on length of stay in hospital for ziprasidone compared with haloperidol. We were unable to draw a conclusion on the effect of haloperidol compared with second-generation antipsychotics due to lack of evidence. (SOE: Insufficient)

\section{Haloperidol Versus Other Therapies}

One RCT, with unclear risk of bias and enrolling critically ill patients, compared haloperidol with dexmedetomidine and reported significantly shorter length of stay in hospital in the dexmedetomidine group compared with haloperidol. ${ }^{33}$ We are unable to draw a conclusion. (SOE: Insufficient)

\section{Other Comparisons}

We found no delirium prevention trials evaluating length of stay in hospital for the following comparisons:

- Second-generation antipsychotics versus second-generation antipsychotics

- Second-generation antipsychotics versus other therapies.

\section{Strength of Evidence}

Table 7 summarizes the SOE of evidence findings for antipsychotics for the prevention of delirium in terms of length of stay in hospital. 
Table 7. Strength of evidence domains for trials evaluating antipsychotics for the prevention of delirium in terms of length of stay in hospital

\begin{tabular}{|c|c|c|c|c|c|c|c|c|}
\hline Comparison & $\begin{array}{c}\text { Number Of Trials } \\
(\mathrm{N})\end{array}$ & $\begin{array}{c}\text { Study } \\
\text { Limitations }\end{array}$ & Directness & Consistency & Precision & $\begin{array}{c}\text { Reporting } \\
\text { Bias }\end{array}$ & $\begin{array}{c}\text { Strength of } \\
\text { Evidence }\end{array}$ & Summary \\
\hline $\begin{array}{l}\text { Haloperidol vs. } \\
\text { placebo }\end{array}$ & $8^{33,37,41-46}(3385)$ & Low & Direct & Consistent & Precise & Undetected & High & $\begin{array}{l}\text { All RCTs were } \\
\text { conducted in medical } \\
\text { and surgical patients in } \\
\text { both intensive and non- } \\
\text { intensive care units. } \\
\text { Most trials ( } n=7 \text { ) } \\
\text { reported no statistically } \\
\text { significant difference } \\
\text { when comparing } \\
\text { haloperidol to placebo. }\end{array}$ \\
\hline $\begin{array}{l}\text { Second- } \\
\text { generation } \\
\text { antipsychotics vs. } \\
\text { placebo }\end{array}$ & $3^{36,40,44}(328)$ & Medium & Indirect* & Consistent & Imprecise & Undetected & Low & $\begin{array}{l}\text { All RCTs reported no } \\
\text { statistically significant } \\
\text { difference. }\end{array}$ \\
\hline $\begin{array}{l}\text { Haloperidol vs. } \\
\text { second-generation } \\
\text { antipsychotics }\end{array}$ & $1^{44}(101)$ & Low & Indirect $^{\dagger}$ & $\begin{array}{l}\text { Consistency } \\
\text { unknown, } \\
\text { (single study) }\end{array}$ & Imprecise & Undetected & Insufficient & $\begin{array}{l}\text { We were unable to draw } \\
\text { a conclusion. }\end{array}$ \\
\hline $\begin{array}{l}\text { Second- } \\
\text { generation } \\
\text { antipsychotics vs. } \\
\text { second-generation } \\
\text { antipsychotics }\end{array}$ & No trials & & & & & & & \\
\hline $\begin{array}{l}\text { Haloperidol vs. } \\
\text { other therapies }\end{array}$ & $1^{33}(90)$ & Medium & Indirect $^{\dagger}$ & $\begin{array}{l}\text { Consistency } \\
\text { unknown, } \\
\text { (single study) } \\
\end{array}$ & Imprecise & Undetected & Insufficient & $\begin{array}{l}\text { We were unable to draw } \\
\text { a conclusion. }\end{array}$ \\
\hline $\begin{array}{l}\text { Second- } \\
\text { generation } \\
\text { antipsychotics vs. } \\
\text { other therapies }\end{array}$ & No trials & & & & & & & \\
\hline
\end{tabular}

RCT = randomized controlled trial

* Indirect rating due to a limited patient population (critical care and postoperative); results may not be generalizable to other patients with delirium.

$\dagger$ Indirect rating due to a single patient population in one RCT; results may not be generalizable to other patients with delirium. 


\section{Length of Stay in Intensive Care Unit - Overall}

\section{Haloperidol Versus Placebo}

Seven RCTs, five with low risk of bias ${ }^{34,42,44-46}$ and two with uncertain risk of bias, ${ }^{33,43}$ comparing haloperidol with placebo for the prevention of delirium in patients admitted to medical or surgical intensive care units, reported length of stay in the intensive care unit. We did not conduct a meta-analysis because these trials generally provided results as median values. The skewed nature of length of stay data precluded conversion or transformation of the data. Because all of the studies reported either no statistical difference, or no meaningful clinical difference we concluded that there is no effect on length of stay in the intensive care unit for all patients at risk of delirium for haloperidol compared with placebo.

\section{Second-Generation Antipsychotics Versus Placebo}

Three RCTs, two with low risk of bias ${ }^{36,44}$ and one with high risk of bias owing to a lack of blinding, ${ }^{40}$ compared oral second-generation antipsychotics with placebo for the prevention of delirium in critically ill and post-surgical patients and evaluated the length of stay in the intensive care unit. We did not conduct a meta-analysis because these trials generally provided results as median values. The skewed nature of length of stay data precluded conversion or transformation of the data. All three RCTs consistently reported no statistically significant effect on length of stay in the intensive care unit.

\section{Haloperidol Versus Second-Generation Antipsychotics}

One RCT, with low risk of bias, enrolled patients with and without delirium who were receiving mechanical ventilation. ${ }^{44}$ This three-arm trial reported no statistically significant effect on length of stay in the intensive care unit for those patients randomized to receive a median daily dose of $40 \mathrm{mg}$ of oral ziprasidone compared with placebo.

\section{Haloperidol Versus Other Therapies}

One three-arm RCT, with unclear risk of bias, included adult patients with severe systemic illness in an intensive care unit and demonstrated a statistically significant difference in length of stay in the intensive care unit in 30 patients randomized to receive a 2.5 loading dose of haloperidol followed by 0.5 to $2.0 \mathrm{mg}$ per hour, based on sedation level versus 30 patients randomized to receive dexmedetomidine. ${ }^{33}$

\section{Other Comparisons}

We found no delirium prevention trials evaluating length of stay in the intensive care unit for the following comparisons:

- Second-generation antipsychotics versus second-generation antipsychotics

- Second-generation antipsychotics versus other therapies.

\section{Patient Safety Attendant Use - Overall}

\section{Second-Generation Antipsychotics Versus Placebo}

One RCT, with low risk of bias and enrolling 400 orthopedic surgery patients aged 65 years and older and treated with $10 \mathrm{mg}$ of oral olanzapine in the perioperative setting or placebo, 
reported no statistically significant between-group difference in patient safety attendant use (RR, 2.34; $95 \%$ CI, 0.73 to 7.48$).^{39}$

\section{Other Comparisons}

We found no delirium prevention trials evaluating patient safety attendant use for the following comparisons:

- Haloperidol versus placebo

- Haloperidol versus second-generation antipsychotics

- Second-generation antipsychotics versus second-generation antipsychotics

- Haloperidol versus other therapies

- Second-generation antipsychotics versus other therapies.

\section{Other Resource Utilization Outcomes - Overall}

We found no trials in patients at risk for delirium that compared an antipsychotic with a placebo, another antipsychotic, or another treatment and evaluated the following resource utilization outcomes:

- Length of stay in skilled nursing facility

- Hospice enrollment

\section{Adverse Effects}

\section{Sedation - Overall}

\section{Haloperidol Versus Placebo}

Four RCTs, all with low risk of bias and enrolling a total of 881 patients, compared haloperidol with placebo for the prevention of delirium and reported on sedation (described as excessive or oversedation, or daytime somnolence). ${ }^{34,37,41,45}$ Two RCTs, enrolled medical and surgical patients in the intensive care unit and administered multiple doses of intravenous haloperidol or placebo for treatment and/or prevention of delirium (maximum daily dose range: 4 to $7.5 \mathrm{mg}$ ). ${ }^{34,45}$ Two RCTs enrolled medical and surgical patients admitted to the acute inpatient wards and administered multiple doses of oral haloperidol for the prevention of delirium (maximum daily dose range: 1.5 to $2 \mathrm{mg}$ ). ${ }^{37,41}$ One trial found no sedation in either arm and was not included in the meta-analysis. ${ }^{37}$ The remaining trials were combined and although we found no statistically significant between-group difference in sedation (pooled RR, 2.05; 95\% CI, 0.86 to 4.85; I-squared, $0 \%$ ) (Figure 7), given the few events in these trials, we were unable to draw a conclusion about the effects of haloperidol on sedation. Exclusion of any one trial did not change the inference from the meta-analysis. (SOE: Insufficient)

We did not find any observational studies addressing this outcome for this comparison in patients at risk for delirium. 
Figure 7. Meta-analysis of sedation in trials comparing haloperidol with placebo in patients at risk for delirium

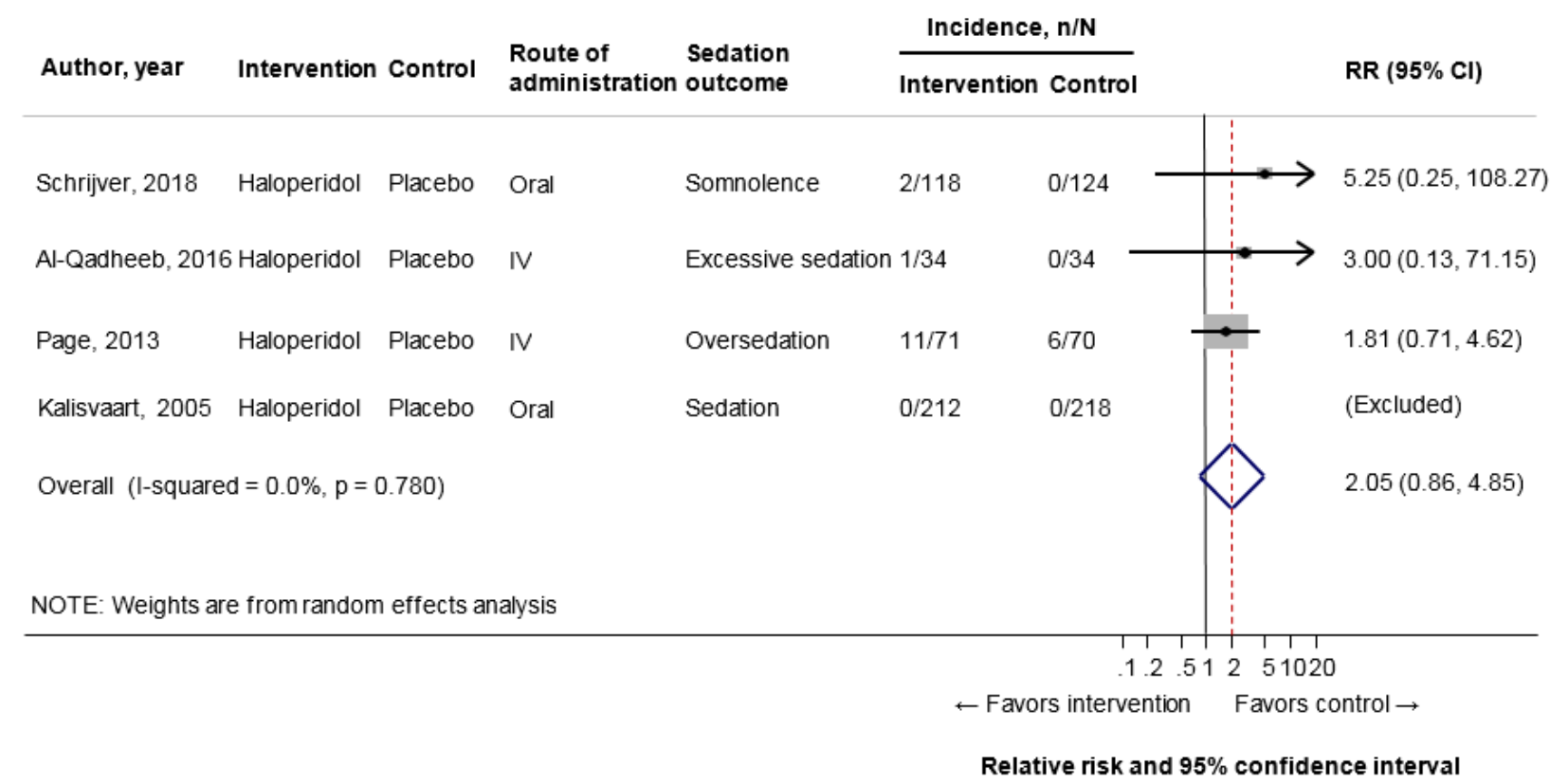

$\mathrm{CI}$ = confidence interval; IV = intravenous; $\mathrm{n}$ = incidence within arm; $\mathrm{N}$ = total arm population; $\mathrm{p}=\mathrm{p}$-value; $\mathrm{RR}=$ relative risk.

\section{Other Comparisons}

We found no studies of delirium prevention evaluating sedation for the following comparisons:

- Second-generation antipsychotics versus placebo

- Haloperidol versus second-generation antipsychotics

- Second-generation antipsychotics versus second-generation antipsychotics

- Haloperidol versus other therapies

- Second-generation antipsychotics versus other therapies.

\section{Strength of Evidence}

Table 8 summarizes the strength of evidence findings for antipsychotics used for the prevention of delirium in terms of sedation. 
Table 8. Strength of evidence domains for studies reporting sedation in the evaluation of antipsychotics for delirium prevention

\begin{tabular}{|c|c|c|c|c|c|c|c|c|}
\hline Comparison & $\begin{array}{l}\text { Number Of } \\
\text { Studies (N) }\end{array}$ & $\begin{array}{c}\text { Study } \\
\text { Limitations }\end{array}$ & Directness & Consistency & Precision & $\begin{array}{c}\text { Reporting } \\
\text { Bias }\end{array}$ & $\begin{array}{c}\text { Strength of } \\
\text { Evidence }\end{array}$ & Summary \\
\hline $\begin{array}{l}\text { Haloperidol vs. } \\
\text { placebo }\end{array}$ & $\begin{array}{l}4^{34,37,41,45} \\
(881)\end{array}$ & Low & Direct & Consistent & Imprecise* & Undetected & Insufficient & $\begin{array}{l}\text { We are unable to draw a } \\
\text { conclusion. }\end{array}$ \\
\hline $\begin{array}{l}\text { Second- } \\
\text { generation } \\
\text { antipsychotics } \\
\text { vs. placebo }\end{array}$ & No studies & & & & & & & \\
\hline $\begin{array}{l}\text { Haloperidol vs. } \\
\text { second- } \\
\text { generation } \\
\text { antipsychotics }\end{array}$ & No studies & & & & & & & \\
\hline $\begin{array}{l}\text { Second- } \\
\text { generation } \\
\text { antipsychotics } \\
\text { vs. second- } \\
\text { generation } \\
\text { antipsychotics }\end{array}$ & No studies & & & & & & & \\
\hline $\begin{array}{l}\text { Haloperidol vs. } \\
\text { other therapies }\end{array}$ & No studies & & & & & & & \\
\hline $\begin{array}{l}\text { Second- } \\
\text { generation } \\
\text { antipsychotics } \\
\text { vs. other } \\
\text { therapies }\end{array}$ & No studies & & & & & & & \\
\hline
\end{tabular}

* Numbers of events were few. 


\section{Cardiac Effects - Overall}

\section{Haloperidol Versus Placebo}

Seven RCTs, five with low risk of bias ${ }^{34,42,44-46}$ and two with uncertain risk of bias, ${ }^{33,43}$ compared haloperidol with placebo for the prevention of delirium in patients admitted to medical or surgical intensive care units and reported a range of cardiac effects. All seven trials administered haloperidol intravenously (maximum fixed daily dose range: 1.5 to $7.5 \mathrm{mg}$ ) except one that administered oral doses (maximum fixed daily dose: $20 \mathrm{mg}$ ). ${ }^{44}$ Two of the RCTs were three-arm trials. ${ }^{33,44}$ We found no statistical difference in the occurrence of each of three types of cardiac effects across all six RCTs. There were few cardiac events in these trials, so the results are imprecise.

Six RCTs reported arrhythmias (including atrial fibrillation, supraventricular tachycardia, and monomorphic ventricular tachycardia) in 2,713 patients in intervention and placebo arms. ${ }^{33}$, ${ }^{42-46}$ One trial reported no ventricular arrhythmias in either group and was not included in the meta-analysis. ${ }^{44}$ Although we found no statistically significant between-group difference in arrhythmias (pooled RR, 1.27; 95\% CI, 0.72 to 2.21; I-squared, $0 \%$ ) more arrhythmias were reported in the intervention arms (Figure 8).

Seven RCTs reported corrected QT interval prolongation in 2,781 patients randomized to intervention and placebo arms. ${ }^{33,34,42-46}$ We found no statistically significant between-group difference in corrected QT interval prolongation (pooled RR, 1.11; 95\% CI, 0.80 to 1.55; Isquared, 0\%) (Figure 8).

Three RCTs reported hypotension ${ }^{33,34,45}$ in 299 critically ill patients in both the intervention and placebo arms. We found no statistically significant between-group difference in hypotension (pooled RR, 1.15; 95\% CI, 0.40 to 3.32; I-squared, 0\%) (Figure 8).

In addition to the trials included in the meta-analysis above, one RCT with low risk of bias, randomized 242 patients aged 70 years and older, at risk of delirium, and admitted from the Emergency Department to surgical or medical units. This trial reported no statistically significant difference in acute coronary syndrome in those patients randomized to $2 \mathrm{mg}$ per day of oral haloperidol versus placebo (RR, 1.05; 95\% CI, 0.15 to 7.34 ). ${ }^{41}$

We did not find any observational studies addressing this outcome for this comparison in patients at risk for delirium.

\section{Second-Generation Antipsychotics Versus Placebo}

Four RCTs, three with low risk of bias ${ }^{36,39,44}$ and one with high risk of bias owing to a lack of blinding, ${ }^{40}$ compared oral second-generation antipsychotics with placebo for the prevention of delirium in critically ill and post-surgical patients and evaluated a variety of cardiac effects. There were no statistically significant between-group differences in the occurrence of cardiac effects across all four RCTs. The results were consistent, but imprecise.

Three RCTs reported no statistically significant between-group differences in the occurrence of arrhythmias.

Two RCTs reported corrected QT interval prolongation. One three-arm trial, of mechanically ventilated, critically ill patients, compared oral ziprasidone with placebo and reported no statistically significant between group differences in corrected QT interval prolongation. ${ }^{44}$ The other RCT included cardiac surgery patients receiving oral risperidone versus placebo and reported no occurrences of corrected QT interval prolongation. ${ }^{36}$ 
We did not find any observational studies addressing cardiac effects for second-generation anti-psychotics compared with placebo in patients at risk for delirium.

Figure 8. Meta-analysis of cardiac effects in trials comparing haloperidol with placebo in patients at risk for delirium

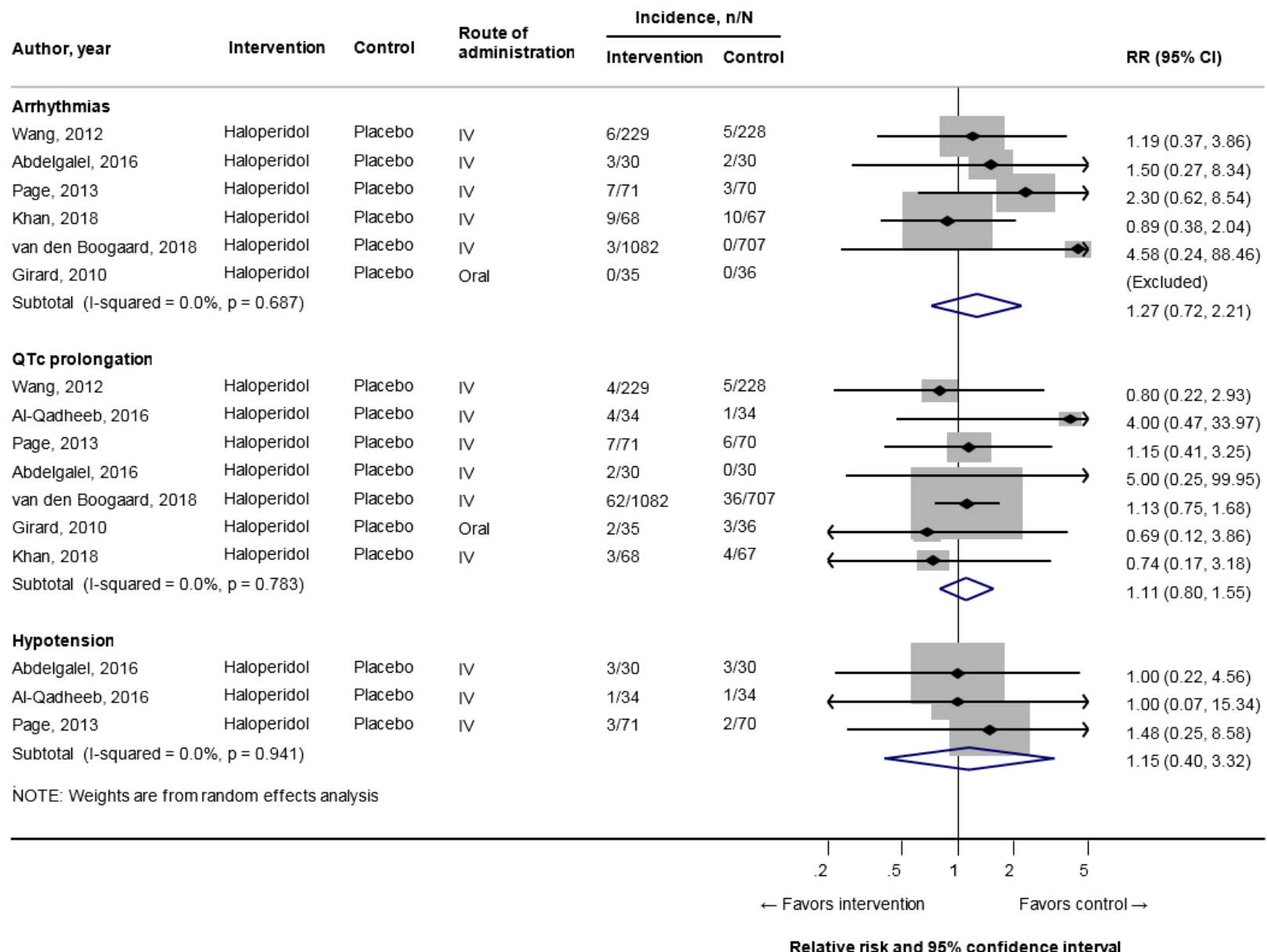

$\mathrm{CI}$ = confidence interval; $\mathrm{IV}$ = intravenous; $\mathrm{n}$ = incidence within arm; monomorphic $\mathrm{MT}$ = monomorphic ventricular tachycardia; $\mathrm{N}$ = total arm population; $\mathrm{p}=\mathrm{p}$-value; $\mathrm{QTc}=\mathrm{QT}$ interval; $\mathrm{RR}=$ relative risk.

\section{Haloperidol Versus Second-Generation Antipsychotics}

One RCT, with low risk of bias and enrolling patients with and without delirium, evaluated cardiac effects. ${ }^{44}$ This three-arm trial included mechanically ventilated, critically ill patients treated with oral haloperidol or ziprasidone and reported no statistically significant betweengroup differences in the prolongation of the corrected QT interval; no ventricular arrhythmias occurred in either group. ${ }^{44}$

We did not find any observational studies addressing cardiac effects for haloperidol compared with second-generation antipsychotics in patients at risk for delirium.

\section{Haloperidol Versus Other Therapies}

One three-arm RCT, with unclear risk of bias, compared haloperidol with dexmedetomidine in the prevention of delirium in critically ill adults and reported no statistically significant between-group differences for the following cardiac outcomes: arrhythmias, prolongation of the corrected QT interval, hypotension, or bradycardia. ${ }^{33}$ 
We did not find any observational studies addressing cardiac effects for haloperidol compared with other therapies in patients at risk for delirium.

\section{Other Comparisons}

We found no studies of delirium prevention evaluating cardiac effects for the following comparisons:

- Second-generation antipsychotics versus second-generation antipsychotics

- Second-generation antipsychotics versus other therapies.

\section{Studies With No Comparison Group}

In one observational study with 177 critically ill patients at high risk of delirium who received haloperidol prophylaxis, nine patients (5\%) had to stop the use of haloperidol because of QTc prolongation. ${ }^{47}$

\section{Neurologic Effects - Overall}

\section{Haloperidol Versus Placebo}

Six RCTs, ${ }^{34,41,42,44-46}$ with low risk of bias, compared haloperidol with placebo for the prevention of delirium in 2,480 patients admitted to medical or surgical intensive care units or acute inpatient wards and reported a range of neurologic effects. Four trials administered haloperidol intravenously (maximum fixed daily dose range: 1.5 to $7.5 \mathrm{mg}$ ) $34,42,45,46$ and two administered oral doses of haloperidol (maximum fixed daily dose range: 2 to $20 \mathrm{mg}$ ). ${ }^{41,44}$ One RCT was a three-arm study. ${ }^{44}$ We found no statistically significant difference in between-group occurrence of extrapyramidal side effects (including akathisia, muscle stiffness, and dystonic movements such as torticollis) in a meta-analysis of five trials (pooled RR, 1.02; 95\% CI, 0.58 to 1.79; I-squared, 0\%) (Figure 9). Exclusion of any one trial did not change the inference from the meta-analysis. We excluded one study from the analysis because they reported individual symptoms of extrapyramidal side effects. ${ }^{46}$ This trial reported no statistically significant differences in elbow rigidity, wrist rigidity, or resting tremor and no events of dystonia, tardive dyskinesia, or pseudoparkinsonism.

The three RCTs described above monitored the occurrence of neuroleptic malignant syndrome. One RCT reported no statistically significant between-group difference in patients randomized to intervention and placebo for $3 \mathrm{mg}$ and $6 \mathrm{mg}$ daily intravenous dosage arms, respectively, finding that 2 cases of suspected neuroleptic malignant syndrome occurred in patients randomized to placebo while no cases were detected in the haloperidol group. ${ }^{42}$ The other two RCTs reported no occurrence of neuroleptic malignant syndrome in either the haloperidol or placebo arms in critically ill, mechanically ventilated patients or thoracic surgery patients. $^{44,46}$

We did not find any observational studies addressing neurologic effects that compared haloperidol with placebo in patients at risk for delirium. 
Figure 9. Meta-analysis of extrapyramidal side effects in trials comparing haloperidol with placebo in patients at risk for delirium

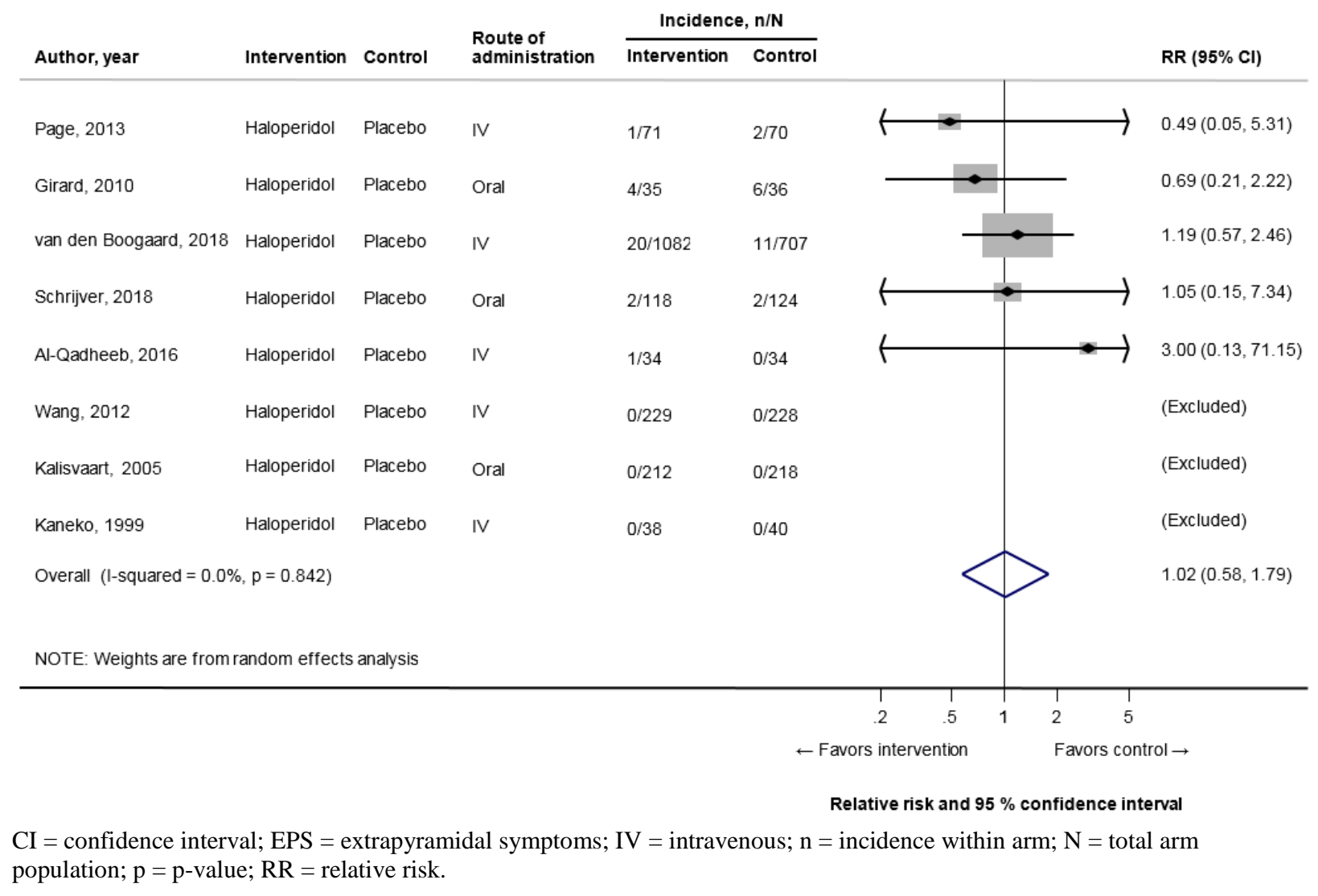

\section{Second-Generation Antipsychotics Versus Placebo}

Two RCTs, with low risk of bias, compared oral second-generation antipsychotics with placebo for the prevention of delirium in critically ill and post-surgical patients and reported neurologic effects. ${ }^{36,44}$ This three-arm trial, enrolling mechanically ventilated, critically ill patients, compared oral ziprasidone versus placebo and reported no statistically significant between-group differences in akathisia or extrapyramidal symptoms not including akathisia. Neuroleptic malignant syndrome did not occur in either group in this study. The other RCT included cardiac surgery patients receiving oral risperidone versus placebo and reported no statistically significant between-group differences in extrapyramidal side effects. ${ }^{36}$ There were few events in both trials, so the results are imprecise.

We did not find any observational studies addressing neurologic effects that compared second-generation antipsychotics with placebo in patients at risk for delirium.

\section{Haloperidol Versus Second-Generation Antipsychotics}

One three-arm RCT, with low risk of bias, enrolling mechanically ventilated, critically ill patients, compared oral haloperidol with ziprasidone for the treatment and prevention of delirium and reported no statistically significant between-group differences in akathisia or extrapyramidal symptoms not including akathisia, and reported no instances of neuroleptic malignant syndrome. $^{44}$

We did not find any observational studies addressing neurologic effects that compared haloperidol with second-generation antipsychotics in patients at risk for delirium. 


\section{Other Comparisons}

We found no studies of delirium prevention evaluating neurologic effects for the following comparisons:

- Second-generation antipsychotics versus second-generation antipsychotics

- Haloperidol versus other therapies

- Second-generation antipsychotics versus other therapies.

\section{Studies With No Comparison Group}

In one observational study with 177 critically ill patients at high risk of delirium who received intravenous haloperidol prophylaxis, one patient (1\%) had to stop haloperidol because of an extrapyramidal side effect described as "signs of Parkinsonism," and one patient (1\%) had to stop haloperidol because of suspected neuroleptic malignant syndrome. ${ }^{47}$

\section{Other Adverse Effects - Overall}

We found no studies in patients at risk for delirium that compared an antipsychotic with a placebo, another antipsychotic, or another treatment and evaluated the following adverse events:

- Weight gain

- Changes in appetite

- Hypersensitivity reactions

- Inappropriate continuation of antipsychotics

- Swallowing difficulties

- Aspiration pneumonia

\section{Key Question 1a. Benefits and Harms of Antipsychotics To Prevent Delirium in Persons Aged 65 Years and Older}

\section{Key Points - Aged 65 Years and Older}

\section{Delirium Severity}

- We were unable to draw a conclusion regarding the effects of antipsychotics on delirium severity in patients aged 65 years and older at risk for delirium for the following comparisons:

o Haloperidol versus placebo (insufficient evidence)

o Second-generation antipsychotics versus placebo (insufficient evidence)

o Haloperidol versus second-generation antipsychotics (no trials)

o Second-generation antipsychotics versus second-generation antipsychotics (no trials)

o Haloperidol versus other therapies (no trials)

o Second-generation antipsychotics versus other therapies (no trials)

\section{Falls}

- We were unable to draw a conclusion regarding the effects of antipsychotics on falls in patients aged 65 years and older at risk for delirium for the following comparisons:

o Haloperidol versus placebo (insufficient evidence)

o Second-generation antipsychotics versus placebo (no trials)

o Haloperidol versus second-generation antipsychotics (no trials) 
o Second-generation antipsychotics versus second-generation antipsychotics (no trials)

o Haloperidol versus other therapies (no trials)

o Second-generation antipsychotics versus other therapies (no trials)

\section{Sedation}

- We were unable to draw a conclusion regarding the effects of antipsychotics on sedation for the following comparisons:

o Haloperidol versus placebo (insufficient evidence)

o Second-generation antipsychotics versus placebo (no studies)

o Haloperidol versus second-generation antipsychotics (no studies)

o Second-generation antipsychotics versus second-generation antipsychotics (no studies)

o Haloperidol versus other therapies (no studies)

o Second-generation antipsychotics versus other therapies (no studies)

\section{Cognitive Functioning, Inappropriate Continuation of Antipsychotics}

- We found no studies evaluating cognitive functioning or inappropriate continuation of antipsychotics in patients aged 65 years and older with delirium that compared an antipsychotic with a placebo, another antipsychotic, or another treatment.

\section{Intermediate Outcomes}

\section{Delirium Incidence - Aged 65 Years and Older}

\section{Haloperidol Versus Placebo}

Three RCTs, one at low risk of bias, ${ }^{41}$ one at unclear risk of bias, ${ }^{43}$ and one at high risk of bias owing to lack of blinding and allocation concealment, ${ }^{35}$ enrolled 820 patients, aged 65 years and older, in surgical intensive care and non-intensive medical and surgical acute hospital ward settings. Three different validated delirium detection instruments were used to directly compare delirium occurrence between haloperidol and placebo groups. Intravenous haloperidol was administered in the surgical intensive care unit setting ${ }^{43}$ and oral doses were administered to patients on inpatient ward settings. ${ }^{37,41}$ These trials were pooled and included in a meta-analysis because they identified new onset of delirium (incidence) over time after exposure to prophylactic haloperidol or placebo. There was little to no difference in the relative risk of delirium for haloperidol compared with placebo (pooled RR, 1.01; 95\% CI, 0.62 to 1.64, Isquared, 69\%) (Figure 10). Exclusion of any one study did not change the inference of the metaanalysis. These results were inconsistent and imprecise. 
Figure 10. Meta-analysis of delirium incidence in trials comparing haloperidol with placebo in patients aged 65 years and older at risk for delirium

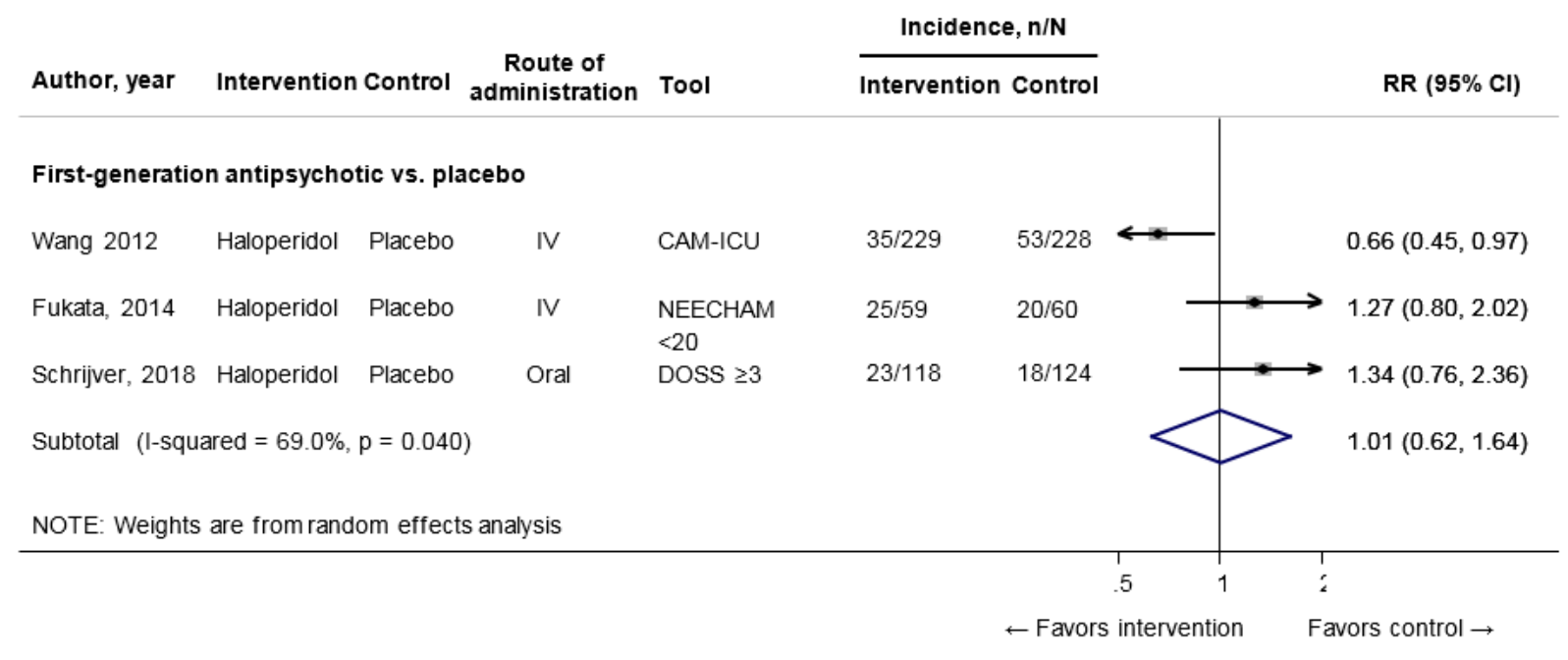

Relative risk and $95 \%$ confidence interval

CAM-ICU = Confusion Assessment Method for the Intensive Care Unit; CI = confidence interval; DOSS = Delirium Observation Screening Scale; IV = intravenous; MMSE = Mini-mental state examination; $\mathrm{n}=$ incidence within arm; $\mathrm{N}=$ total arm population; $\mathrm{p}=\mathrm{p}$-value; $\mathrm{RR}$ = relative risk.

\section{Second-Generation Antipsychotics Versus Placebo}

Two RCTs, with low risk of bias, enrolled patients aged 65 years and older without delirium prior to surgery and compared delirium occurrence in the postoperative setting and both reported a decreased incidence of delirium in the intervention group. ${ }^{36,37}$ These results were consistent, but imprecise.

\section{Other Comparisons}

We found no delirium prevention trials evaluating delirium incidence for the following comparisons:

- Haloperidol versus second-generation antipsychotics

- Second-generation antipsychotics versus second-generation antipsychotics

- Haloperidol versus other therapies

- Second-generation antipsychotics versus other therapies.

\section{Delirium Severity - Aged 65 Years and Older}

\section{Haloperidol Versus Placebo}

One RCT, with low risk of bias, enrolled patients aged 70 years and older in medical or surgical specialty and reported no statistically significant effect in terms of the Delirium Rating Scale-Revised-98 scores for haloperidol compared with placebo. ${ }^{41}$ However, Delirium Rating Scale-Revised-98 scores were missing in 53.7 percent of the subjects and, therefore, the results should be interpreted with caution. We were unable to draw conclusions. (SOE in persons aged 65 years and older: Insufficient) 


\section{Second-Generation Antipsychotics Versus Placebo}

The findings presented in the "Delirium Severity - Overall” results section are wholly represented by this subgroup. Please refer to the "Delirium Severity - Overall” results section. (SOE in persons aged 65 years and older: Insufficient)

\section{Other Comparisons}

We found no delirium prevention trials evaluating delirium severity in persons aged 65 years and older for the following comparisons:

- Haloperidol versus second-generation antipsychotics

- Second-generation antipsychotics versus second-generation antipsychotics

- Haloperidol versus other therapies

- Second-generation antipsychotics versus other therapies.

\section{Strength of Evidence}

Table 9 summarizes the strength of evidence findings for the effects of antipsychotics on delirium severity in persons aged 65 years and older at risk for delirium. 
Table 9. Strength of evidence domains for trials evaluating antipsychotics for the prevention of delirium in terms of delirium severity in patients aged 65 years and older

\begin{tabular}{|c|c|c|c|c|c|c|c|c|}
\hline Comparison & $\begin{array}{c}\text { Number Of } \\
\text { Trials (N) }\end{array}$ & $\begin{array}{c}\text { Study } \\
\text { Limitations }\end{array}$ & Directness & Consistency & Precision & $\begin{array}{l}\text { Reporting } \\
\text { Bias }\end{array}$ & $\begin{array}{l}\text { Strength Of } \\
\text { Evidence }\end{array}$ & Summary \\
\hline $\begin{array}{l}\text { Haloperidol vs. } \\
\text { placebo }\end{array}$ & $1^{41}(242)$ & Low & Direct & $\begin{array}{l}\text { Consistency } \\
\text { unknown, } \\
\text { (single study) }\end{array}$ & Imprecise & Undetected & Insufficient & $\begin{array}{l}\text { We were unable to draw a } \\
\text { conclusion. }\end{array}$ \\
\hline $\begin{array}{l}\text { Second-generation } \\
\text { antipsychotics vs. } \\
\text { placebo }\end{array}$ & $2^{36,39}(501)$ & Low & Direct & Inconsistent & Precise & Undetected & Insufficient & $\begin{array}{l}\text { We were unable to draw a } \\
\text { conclusion. }\end{array}$ \\
\hline $\begin{array}{l}\text { Haloperidol vs. } \\
\text { second-generation } \\
\text { antipsychotics }\end{array}$ & No trials & & & & & & & \\
\hline $\begin{array}{l}\text { Second-generation } \\
\text { antipsychotics vs. } \\
\text { second-generation } \\
\text { antipsychotics }\end{array}$ & No trials & & & & & & & \\
\hline $\begin{array}{l}\text { Haloperidol vs. other } \\
\text { therapies }\end{array}$ & No trials & & & & & & & \\
\hline $\begin{array}{l}\text { Second-generation } \\
\text { antipsychotics vs. } \\
\text { other therapies }\end{array}$ & No trials & & & & & & & \\
\hline
\end{tabular}




\section{Delirium-Free, Coma-Free Days Alive - Aged 65 Years and Older}

\section{Haloperidol Versus Placebo}

One RCT, with unclear risk of bias, enrolled patients aged 65 years and older admitted to the intensive care unit after noncardiac surgery and reported longer delirium-free and coma-free days for haloperidol (mean, 6.8 days; standard deviation [SD], 0.7) compared with placebo (mean, 6.7 days; SD, 0.9) $(P=0.03)$ for the overall study population. ${ }^{43}$ Our own calculation shows a nonstatistically significant difference between the two means for the overall study population with a mean difference of 0.1 ( $95 \%$ CI, -0.05 to 0.25 ). This difference in delirium-free, coma-free days alive was not considered to be a clinically significant difference.

\section{Other Comparisons}

We found no delirium prevention trials evaluating delirium- and coma-free days in persons aged 65 years and older for the following comparisons:

- Second-generation antipsychotics versus placebo

- Haloperidol versus second-generation antipsychotics

- Second-generation antipsychotics versus second-generation antipsychotics

- Haloperidol versus other therapies

- Second-generation antipsychotics versus other therapies.

\section{Duration of Delirium - Aged 65 Years and Older}

\section{Haloperidol Versus Placebo}

Two RCTs that compared haloperidol with placebo or no medication for the prevention of delirium in patients aged 65 years and older did not demonstrate a statistically significant difference in delirium duration. ${ }^{35,41}$

\section{Second-Generation Antipsychotics Versus Placebo}

Two RCTs, with low risk of bias, compared a second-generation antipsychotic with placebo for the prevention of delirium and reported inconsistent results.

\section{Haloperidol Versus Second-Generation Antipsychotics}

One RCT, with low risk of bias, enrolled patients with and without delirium who received mechanical ventilation. ${ }^{44}$ This three-arm trial reported no statistically significant effect on delirium duration for a median daily dose of $20 \mathrm{mg}$ oral ziprasidone compared with a median daily dose of $15 \mathrm{mg}$ oral haloperidol.

\section{Other Comparisons}

We found no delirium prevention trials evaluating delirium duration in patients aged 65 years and older for the following comparisons:

- Second-generation antipsychotics versus second-generation antipsychotics

- Haloperidol versus other therapies

- Second-generation antipsychotics versus other therapies. 


\section{Use of Rescue Therapy - Aged 65 Years and Older}

\section{Haloperidol Versus Placebo}

Two RCTs compared haloperidol with placebo for the prevention of delirium in patients aged 65 years and older and reported on the use of rescue therapy, defined as the use of any open-label antipsychotic. ${ }^{41,43}$ One RCT, with low risk of bias, randomized patients aged 70 years and older and at risk of delirium and admitted from the Emergency Department to surgical or medical units. ${ }^{41}$ The other RCT, with unclear risk of bias, included adult patients aged 65 years and older undergoing non-cardiac surgery and recovery in an intensive care unit. ${ }^{43}$ Both trials reported no statistically significant difference in the use of rescue medications in those patients randomized to haloperidol compared with placebo. However, these results were inconsistent and imprecise.

\section{Other Comparisons}

We found no delirium prevention trials evaluating the use of rescue therapy in patients aged 65 years and older for the following comparisons:

- Haloperidol versus second-generation antipsychotics

- Second-generation antipsychotics versus second-generation antipsychotics

- Haloperidol versus other therapies

- Second-generation antipsychotics versus other therapies.

\section{Use of Physical Restraint - Aged 65 Years and Older}

\section{Second-Generation Antipsychotics Versus Placebo}

The findings presented in the "Use of Physical Restraint - Overall" results section are wholly represented by this subgroup. Please refer to the "Use of Physical Restraint - Overall" results section.

\section{Other Comparisons}

We found no delirium prevention trials evaluating the use of physical restraint for patients aged 65 years and older for the following comparisons:

- Haloperidol versus placebo

- Haloperidol versus second-generation antipsychotics

- Second-generation antipsychotics versus second-generation antipsychotics

- Haloperidol versus other therapies

- Second-generation antipsychotics versus other therapies.

\section{Other Intermediate Outcomes - Aged 65 Years and Older}

We found no trials enrolling patients aged 65 years and older at risk for delirium that compared an antipsychotic with a placebo, another antipsychotic, or another treatment and evaluated the following intermediate outcomes:

- Short-term delirium symptoms

- Patient distress 


\section{Final Health or Patient-Centered Outcomes}

\section{Mortality - Aged 65 Years and Older}

\section{Haloperidol Versus Placebo}

Two RCTs compared haloperidol with placebo and reported no between-group differences in short-term mortality, defined as mortality while in hospital or up to 30 days after randomization. ${ }^{41,43}$ One RCT, with unclear risk of bias, enrolled patients aged 65 years and older in an intensive care unit. The other RCT, with low risk of bias, enrolled medical and surgical patients aged 70 years and older. ${ }^{\text {a41 }}$ Both trials reported no statistically significant difference in short-term mortality when comparing haloperidol with placebo. One of the trials trial also examined 90-day and 180-day mortality. ${ }^{41}$ The results were similar to the short-term mortality, with no between-group differences when comparing haloperidol with placebo at 90 days ${ }^{\mathrm{a}}$ and at 180 days. ${ }^{a}$ There were few events in both trials, so the results are imprecise.

\section{Second-Generation Antipsychotics Versus Placebo}

One RCT, with low risk of bias, enrolled patients aged 65 years and older and compared risperidone with placebo. This trial reported no between-group differences in short-term mortality, defined as mortality while in hospital or up to 30 days after randomization. ${ }^{36}$

\section{Other Comparisons}

We found no delirium prevention trials evaluating mortality in patients aged 65 years and older for the following comparisons:

- Haloperidol versus second-generation antipsychotics

- Second-generation antipsychotics versus second-generation antipsychotics

- Haloperidol versus other therapies

- Second-generation antipsychotics versus other therapies.

\section{Institutionalization - Aged 65 Years and Older}

\section{Haloperidol Versus Placebo}

The findings presented in the "Institutionalization - Overall" results section are wholly represented by this subgroup. Please refer to the "Institutionalization - Overall" results section.

\section{Second-Generation Antipsychotics Versus Placebo}

The findings presented in the "Institutionalization - Overall" results section are wholly represented by this subgroup. Please refer to the "Institutionalization - Overall" results section.

\section{Other Comparisons}

We found no delirium prevention trials evaluating institutionalization in patients aged 65 years and older for the following comparisons:

- Haloperidol versus second-generation antipsychotics

a Schrijver et al. reported mortality outcomes as odds ratios: i) 30-day mortality (OR, 0.78; 95\% CI, 0.26 to 2.33; $P$ $=0.65$ ); ii) 90 -day mortality (OR, 0.77; $95 \%$ CI, 0.34 to 1.75; $P=0.53$ ); iii) 180 -day mortality (OR, 0.98 ; $95 \%$ CI, 0.49 to $2.00 ; P=0.96$ ) 
- Second-generation antipsychotics versus second-generation antipsychotics

- Haloperidol versus other therapies

- Second-generation antipsychotics versus other therapies.

\section{Falls - Aged 65 Years and Older}

\section{Haloperidol Versus Placebo}

One RCT, with high risk of bias owing to a lack of blinding and allocation concealment, compared haloperidol with no intervention for the prevention of delirium and reported on falls as an adverse outcome. ${ }^{35}$ The trial enrolled patients aged 75 years and older undergoing abdominal or orthopedic surgery. A single fall occurred in one patient on day seven of the trial, four days after the last dose of haloperidol was administered. The study authors determined that the fall was unlikely to be related to the study intervention. We were unable to draw a conclusion. (SOE: Insufficient)

\section{Other Comparisons}

We found no delirium prevention trials evaluating falls in patients aged 65 years and older for the following comparisons:

- Second-generation antipsychotics versus placebo

- Haloperidol versus second-generation antipsychotics

- Second-generation antipsychotics versus second-generation antipsychotics

- Haloperidol versus other therapies

- Second-generation antipsychotics versus other therapies.

\section{Strength of Evidence}

Table 10 summarizes the strength of evidence findings for the effects of antipsychotics in terms of falls in patients aged 65 years and older. 
Table 10. Strength of evidence domains for trials reporting falls in the evaluation of antipsychotics for the prevention of delirium in patients 65 years of age and older

\begin{tabular}{|c|c|c|c|c|c|c|c|c|}
\hline Comparison & $\begin{array}{l}\text { Number Of } \\
\text { Trials (N) }\end{array}$ & $\begin{array}{c}\text { Study } \\
\text { Limitations }\end{array}$ & Directness & Consistency & Precision & $\begin{array}{c}\text { Reporting } \\
\text { Bias }\end{array}$ & $\begin{array}{c}\text { Strength Of } \\
\text { Evidence }\end{array}$ & Summary \\
\hline $\begin{array}{l}\text { Haloperidol vs. } \\
\text { placebo }\end{array}$ & $1^{35}(119)$ & High* & Direct & Unknown & Imprecise $^{\dagger}$ & Undetected & Insufficient & $\begin{array}{l}\text { We were unable to draw a } \\
\text { conclusion. }\end{array}$ \\
\hline $\begin{array}{l}\text { Second-generation } \\
\text { antipsychotics vs. } \\
\text { placebo }\end{array}$ & No trials & & & & & & & \\
\hline $\begin{array}{l}\text { Haloperidol vs. } \\
\text { second-generation } \\
\text { antipsychotics }\end{array}$ & No trials & & & & & & & \\
\hline $\begin{array}{l}\text { Second-generation } \\
\text { antipsychotics vs. } \\
\text { second-generation } \\
\text { antipsychotics }\end{array}$ & No trials & & & & & & & \\
\hline $\begin{array}{l}\text { Haloperidol vs. } \\
\text { other therapies }\end{array}$ & No trials & & & & & & & \\
\hline $\begin{array}{l}\text { Second-generation } \\
\text { antipsychotics vs. } \\
\text { other therapies }\end{array}$ & No trials & & & & & & & \\
\hline
\end{tabular}

other therapies

* RCT was open-label and lacking in allocation concealment.

† Only one fall was reported during the study. 


\section{Other Final Health or Patient-Centered Outcomes - Aged 65 Years and Older}

We found no trials in patients aged 65 years and older at risk for delirium that compared an antipsychotic with a placebo, another antipsychotic, or another treatment and evaluated the following final health or patient-centered outcomes:

- Quality of life

- Cognitive functioning

- Caregiver burden/strain

- Memory of patient distress

\section{Resource Utilization Outcomes}

\section{Readmission to Hospital - Aged 65 Years and Older}

\section{Haloperidol Versus Placebo}

One RCT, with low risk of bias, compared haloperidol with placebo for the prevention of delirium in hospitalized medical and surgical patients aged 65 years of age and older. ${ }^{41}$ This RCT reported no statistically significant effect on readmission to the acute care hospital by treatment group at 3 months or 6 months followup.

\section{Other Comparisons}

We found no delirium prevention trials evaluating readmission to the acute care hospital in patients aged 65 years and older for the following comparisons:

- Second-generation antipsychotics versus placebo

- Haloperidol versus second-generation antipsychotics

- Second-generation antipsychotics versus second-generation antipsychotics

- Haloperidol versus other therapies

- Second-generation antipsychotics versus other therapies.

\section{Length of Stay in Hospital - Aged 65 Years and Older}

\section{Haloperidol Versus Placebo}

Two RCTs compared haloperidol with placebo and assessed length of stay in hospital for patients aged 65 or older. ${ }^{41,43}$ One RCT, with low risk of bias, enrolled patients aged 70 years and older in medical or surgical specialty. ${ }^{41}$ The other RCT, with unclear risk of bias, enrolled patients aged 65 years and older admitted to the intensive care unit after noncardiac surgery. ${ }^{43}$ Both trials consistently reported no statistically significant effect on length of stay in hospital for haloperidol compared with placebo for the overall study population. One of the trials also reported no statistically significant effect on length of stay in hospital for haloperidol compared with placebo in patients who had developed postoperative delirium. ${ }^{43}$

\section{Second-Generation Antipsychotics Versus Placebo}

One RCT, with low risk of bias, enrolled patients aged 65 years and older who experienced subsyndromal delirium after on-pump cardiac surgery examined the effect of risperidone 
compared with placebo on length of stay in hospital. ${ }^{36}$ This trial reported no statistically significant effect for risperidone compared with placebo.

\section{Other Comparisons}

We found no delirium prevention trials evaluating length of stay in hospital for patients aged 65 years and older for the following comparisons:

- Haloperidol versus second-generation antipsychotics

- Second-generation antipsychotics versus second-generation antipsychotics

- Haloperidol versus other therapies

- Second-generation antipsychotics versus other therapies.

\section{Length of Stay in Intensive Care Unit - Aged 65 Years and Older}

\section{Haloperidol Versus Placebo}

One RCT, with unclear risk of bias, enrolled patients aged 65 years and older undergoing non-cardiac surgery and recovery in an intensive care unit. ${ }^{43}$ This trial reported a statistically significant difference for length of stay in the intensive care unit for patients randomized to a 0.5 mg loading dose of haloperidol followed by $0.1 \mathrm{mg}$ per hour infusion compared with placebo. However, this difference of less than 2 hours in the intensive care unit is not clinically important.

\section{Second-Generation Antipsychotics Versus Placebo}

One RCT, with low risk of bias, enrolled cardiac surgery patients aged 65 years and older with subsyndromal delirium in the postoperative setting and reported no statistically significant difference in length of stay in the intensive care unit for those randomized to receive $1 \mathrm{mg}$ per day of oral risperidone compared with placebo. ${ }^{36}$

\section{Other Comparisons}

We found no delirium prevention trials evaluating length of stay in the intensive care unit among patients aged 65 years and older for the following comparisons:

- Haloperidol versus second-generation antipsychotics

- Second-generation antipsychotics versus second-generation antipsychotics

- Haloperidol versus other therapies

- Second-generation antipsychotics versus other therapies.

\section{Patient Safety Attendant Use - Aged 65 Years and Older}

\section{Second-Generation Antipsychotics Versus Placebo}

One RCT, with low risk of bias and enrolling orthopedic surgery patients aged 65 years and older, compared treatment with $10 \mathrm{mg}$ of oral olanzapine in the perioperative setting with placebo and reported no statistically significant between-group difference in patient safety attendant. $^{39}$

\section{Other Comparisons}

We found no delirium prevention trials evaluating patient safety attendant use for patients aged 65 years and older for the following comparisons:

- Haloperidol versus placebo 
- Haloperidol versus second-generation antipsychotics

- Second-generation antipsychotics versus second-generation antipsychotics

- Haloperidol versus other therapies

- Second-generation antipsychotics versus other therapies.

\section{Other Resource Utilization Outcomes - Aged 65 years and older}

We found no trials in patients aged 65 years and older at risk for delirium that compared an antipsychotic with a placebo, another antipsychotic, or another treatment and evaluated the following resource utilization outcomes:

- Readmission to intensive care unit

- Length of stay in skilled nursing facility

- Hospice enrollment.

\section{Adverse Effects}

\section{Sedation - Aged 65 Years and Older}

\section{Haloperidol Versus Placebo}

One RCT, with low risk of bias, enrolled patients aged 70 years and older admitted to medical or surgical acute inpatient wards from the Emergency Department and reported on sedation (described as somnolence) in patients randomized to oral haloperidol (maximum fixed daily dose: $2 \mathrm{mg}$ ) compared with placebo for the prevention of delirium. ${ }^{41}$ There was no statistically significant between-group difference in the relative risk of developing somnolence. However, there were too few events in this trial to draw a conclusion about the effects of haloperidol on sedation. (SOE: Insufficient)

We did not find any observational studies addressing sedation that compared haloperidol with placebo in patients aged 65 years and older at risk for delirium.

\section{Other Comparisons}

We found no studies of delirium prevention evaluating sedation in patients aged 65 years and older for the following comparisons:

- Second-generation antipsychotics versus placebo

- Haloperidol versus second-generation antipsychotics

- Second-generation antipsychotics versus second-generation antipsychotics

- Haloperidol versus other therapies

- Second-generation antipsychotics versus other therapies.

\section{Strength of Evidence}

Table 11 summarizes the strength of evidence findings for the effects of antipsychotics in terms of sedation in patients aged 65 years and older. 
Table 11. Strength of evidence domains for studies reporting sedation in the evaluation of antipsychotics for delirium prevention

\begin{tabular}{|c|c|c|c|c|c|c|c|c|}
\hline Comparison & $\begin{array}{l}\text { Number Of } \\
\text { Studies (N) }\end{array}$ & $\begin{array}{c}\text { Study } \\
\text { Limitations }\end{array}$ & Directness & Consistency & Precision & $\begin{array}{c}\text { Reporting } \\
\text { Bias }\end{array}$ & $\begin{array}{c}\text { Strength of } \\
\text { Evidence }\end{array}$ & Summary \\
\hline $\begin{array}{l}\text { taloperidol vs. } \\
\text { lacebo }\end{array}$ & $1^{41}(242)$ & Low & Direct & $\begin{array}{l}\text { Unknown } \\
\text { (single study) }\end{array}$ & Imprecise* $^{*}$ & Undetected & Insufficient & $\begin{array}{l}\text { We are unable to draw a } \\
\text { conclusion. }\end{array}$ \\
\hline $\begin{array}{l}\text { Second-generation } \\
\text { antipsychotics vs. } \\
\text { lacebo }\end{array}$ & No studies & & & & & & & \\
\hline $\begin{array}{l}\text { Haloperidol vs. } \\
\text { econd-generation } \\
\text { antipsychotics }\end{array}$ & No studies & & & & & & & \\
\hline $\begin{array}{l}\text { econd-generation } \\
\text { antipsychotics vs. } \\
\text { econd-generation } \\
\text { antipsychotics }\end{array}$ & No studies & & & & & & & \\
\hline $\begin{array}{l}\text { Jaloperidol vs. } \\
\text { ther therapies }\end{array}$ & No studies & & & & & & & \\
\hline $\begin{array}{l}\text { Second-generation } \\
\text { antipsychotics vs. } \\
\text { ther therapies }\end{array}$ & No studies & & & & & & & \\
\hline
\end{tabular}

other therapies

* Numbers of events were few. 


\section{Cardiac Effects - Aged 65 Years and Older}

\section{Haloperidol Versus Placebo}

Two RCTs compared haloperidol with placebo for the prevention of delirium in patients aged 65 years and older and reported no statistically significant between-group difference in cardiac effects. ${ }^{41,43}$ There were few events in the trials, so the results are imprecise.

One RCT, with low risk of bias and enrolling patients aged 70 years and older admitted from the Emergency Department to medical or surgical acute inpatient wards, compared oral haloperidol (maximum fixed daily dose: $2 \mathrm{mg}$ ) with placebo for the prevention of delirium and reported on adverse cardiac events. ${ }^{41}$ This trial reported no statistically significant between-group difference in acute coronary syndrome.

Another RCT, with unclear risk of bias, enrolled patients aged 65 years and older undergoing non-cardiac surgery and recovery in an intensive care unit. ${ }^{43}$ Patients randomized to a $0.5 \mathrm{mg}$ loading dose of haloperidol, followed by $0.1 \mathrm{mg}$ per hour infusion did not have any statistically significant between-group differences when compared with placebo in corrected QT interval prolongation or the development of arrhythmia during study drug infusion.

We did not find any observational studies addressing cardiac effects that compared haloperidol with placebo in patients aged 65 years and older at risk for delirium.

\section{Second-Generation Antipsychotics Versus Placebo}

Two RCTs compared olanzapine with placebo and reported on cardiac effects. ${ }^{36,39}$ One RCT, with low risk of bias and enrolling orthopedic surgery patients aged 65 years and older treated with $10 \mathrm{mg}$ of oral olanzapine versus placebo in the perioperative period, reported no statistically significant between-group differences in arrhythmias or atrial fibrillation. ${ }^{39}$ Another RCT, with low risk of bias, enrolled cardiac surgery patients aged 65 years and older with subsyndromal delirium in the postoperative period and reported no prolongation of the corrected QT interval patients randomized to $1 \mathrm{mg}$ per day of risperidone compared with placebo. ${ }^{36}$

We did not find any observational studies addressing cardiac effects that compared secondgeneration antipsychotics with placebo in patients aged 65 years and older at risk for delirium.

\section{Other Comparisons}

We found no studies of delirium prevention evaluating cardiac effects in patients aged 65 years and older for the following comparisons:

- Haloperidol versus second-generation antipsychotics

- Second-generation antipsychotics versus second-generation antipsychotics

- Haloperidol versus other therapies

- Second-generation antipsychotics versus other therapies.

\section{Neurologic Effects - Aged 65 Years and Older}

\section{Haloperidol Versus Placebo}

Three RCTs compared haloperidol with placebo or no medication for the prevention of delirium in patients aged 65 years and older and reported no statistically significant betweengroup difference in neurologic symptoms. ${ }^{35,41,43}$ There were few events in the trials, so the results are imprecise. 
One RCT, with low risk of bias, randomized patients aged 70 years and older at risk of delirium and admitted from the Emergency Department to surgical or medical units. ${ }^{41}$ There was no statistically significant between-group difference in neurologic symptoms in those patients randomized to $2 \mathrm{mg}$ per day of oral haloperidol compared with placebo for occurrence of extrapyramidal side effects or reports of adverse events including transient ischemic attacks.

One RCT, with high risk of bias owing to a lack of blinding and allocation concealment, enrolled patients aged 75 years and older undergoing elective digestive or orthopedic surgeries in an open-label study. ${ }^{35}$ This trial reported no between-group differences in adverse events including confusions and loss of consciousness in patients randomized to $2.5 \mathrm{mg}$ per day of oral haloperidol compared with those patients randomized to no drug.

One RCT, with unclear risk of bias, enrolled patients aged 65 years and older undergoing non-cardiac surgery and recovery in an intensive care unit. ${ }^{43}$ This trial reported the absence of the occurrence of neuroleptic malignant syndrome in patients in either arm.

We did not find any observational studies addressing neurologic effects that compared haloperidol with placebo in patients aged 65 years and older at risk for delirium.

\section{Second-Generation Antipsychotics Versus Placebo}

One RCT, with low risk of bias, compared oral second-generation antipsychotics with placebo for the prevention of delirium in post-surgical patients and reported neurologic effects. ${ }^{36}$ This trial enrolled 101 cardiac surgery patients receiving oral risperidone versus placebo and reported no statistically significant between-group differences in extrapyramidal side. ${ }^{36}$

We did not find any observational studies addressing neurologic effects that compared second-generation antipsychotics with placebo in patients aged 65 years and older at risk for delirium.

\section{Haloperidol Versus Second-Generation Antipsychotics}

One RCT, with low risk of bias, enrolled cardiac surgery patients aged 65 years and older with subsyndromal delirium in the postoperative setting and reported no statistically significant between-group difference in neurologic effects, including extrapyramidal symptoms for those randomized to receive $1 \mathrm{mg}$ per day of oral risperidone compared with. ${ }^{36}$

We did not find any observational studies addressing neurologic effects that compared haloperidol with second-generation antipsychotics in patients aged 65 years and older at risk for delirium.

\section{Other Comparisons}

We found no studies of delirium prevention that evaluated neurologic effects in patients aged 65 years and older for the following comparisons:

- Second-generation antipsychotics versus second-generation antipsychotics

- Haloperidol versus other therapies

- Second-generation antipsychotics versus other therapies.

\section{Other Adverse Effects - Aged 65 Years and Older}

We found no studies in patients aged 65 years and older at risk for delirium that compared an antipsychotic with a placebo, another antipsychotic, or another treatment and evaluated the following adverse events:

- Weight gain 
- Changes in appetite

- Hypersensitivity reactions

- Inappropriate continuation of antipsychotics

- Swallowing difficulties

- Aspiration pneumonia

\section{Key Question 1b. Benefits and Harms of Antipsychotics To Prevent Delirium in Persons with Dementia}

We found no trials evaluating antipsychotics for the prevention of delirium in persons with dementia.

\section{Key Question 1c. Benefits and Harms of Antipsychotics To Prevent Delirium in Patients in an Intensive Care Unit}

\section{Key Points - Intensive Care Unit}

\section{Delirium Severity}

- We were unable to draw a conclusion regarding the effects of antipsychotics on delirium severity for the following comparisons:

o Haloperidol versus placebo (insufficient evidence)

o Second-generation antipsychotics versus placebo (no trials)

o Haloperidol versus second-generation antipsychotics (no trials)

o Second-generation antipsychotics versus second-generation antipsychotics (no trials)

o Haloperidol versus other therapies (no trials)

o Second-generation antipsychotics versus other therapies (no trials)

\section{Length of Stay in Hospital}

- In critically ill medical and surgical patients, there was little to no difference in length of stay in hospital for haloperidol compared with placebo. (SOE: High)

- We were unable to draw a conclusion regarding the effects of antipsychotics on length of stay in hospital for the following comparisons:

o Second-generation antipsychotics versus placebo (insufficient evidence)

o Haloperidol versus second-generation antipsychotics (insufficient evidence)

o Second-generation antipsychotics versus second-generation antipsychotics (no trials)

o Haloperidol versus other therapies (insufficient evidence)

o Second-generation antipsychotics versus other therapies (no trials)

\section{Cognitive Functioning, Inappropriate Continuation of Antipsychotics}

- We found no studies evaluating cognitive functioning or inappropriate continuation of antipsychotics in patients in an intensive care unit at risk for delirium that compared an antipsychotic with a placebo, another antipsychotic, or another treatment. 


\section{Sedation}

- We were unable to draw a conclusion regarding the effects of antipsychotics on sedation for the following comparisons:

o Haloperidol versus placebo (insufficient evidence)

o Second-generation antipsychotics versus placebo (no studies)

o Haloperidol versus second-generation antipsychotics (no studies)

o Second-generation antipsychotics versus second-generation antipsychotics (no studies)

o Haloperidol versus other therapies (no studies)

o Second-generation antipsychotics versus other therapies (no studies)

\section{Intermediate Outcomes}

\section{Delirium Incidence - Intensive Care Unit}

\section{Haloperidol Versus Placebo}

Five RCTs, three with low risk of bias ${ }^{34,42,46}$ and two with unclear risk of bias, ${ }^{33,43}$ directly compared delirium occurrence in patients randomized to haloperidol and placebo groups. The five RCTs enrolled 2,557patients in both surgical and medical intensive care unit settings and used a variety of validated delirium detection instruments. Intravenous haloperidol was administered in all trials. We conducted a meta-analysis of these trials because they identified new onset of delirium (incidence) after exposure to prophylactic haloperidol or placebo in surgical and medical intensive care unit patients. We found no difference in the relative risk of delirium in the pooled analysis (RR, 0.90; 95\% CI, 0.69 to 1.17, I-squared, 47\%) (Figure 11). Exclusion of any one study did not change the inference of the meta-analysis. We considered these results to be consistent, but imprecise. 
Figure 11. Meta-analysis of delirium incidence in trials comparing haloperidol with placebo in critically ill patients at risk for delirium

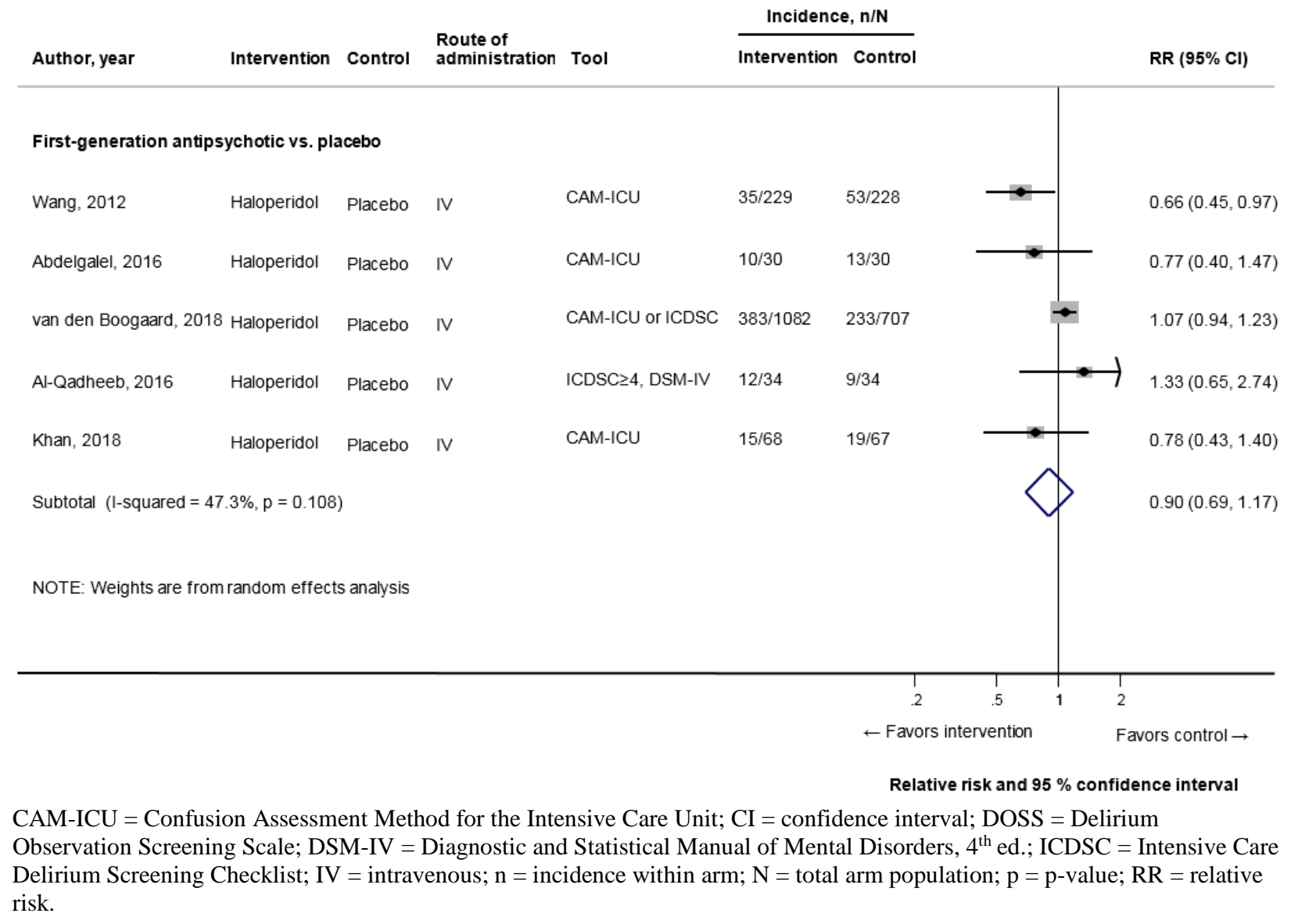

\section{Second-Generation Antipsychotics Versus Placebo}

One RCT, ${ }^{36}$ with low risk of bias, and one RCT, with high risk of bias owing to a lack of blinding, ${ }^{40}$ compared delirium occurrence in the postoperative setting following randomization to risperidone or placebo. These two RCTs enrolled patients without delirium prior to cardiac surgery and recovery in the intensive care unit. Both of these trials demonstrated statistically significant between-group differences in the incidence of delirium.

\section{Haloperidol Versus Other Therapies}

The findings presented in the "Delirium Incidence - Overall” results section are wholly represented by this subgroup. Please refer to the "Delirium Incidence - Overall” results section.

\section{Other Comparisons}

We found no delirium prevention trials evaluating delirium incidence among critically ill patients for the following comparisons:

- Haloperidol versus second-generation antipsychotics

- Second-generation antipsychotics versus second-generation antipsychotics

- Second-generation antipsychotics versus other therapies. 


\section{Short-Term Delirium Symptoms - Intensive Care Unit}

\section{Haloperidol Versus Placebo}

One RCT, with low risk of bias, included patients receiving mechanical ventilation enrolled within 72 hours of intensive care unit admission. ${ }^{45}$ This study showed a statistically significant effect of haloperidol on the proportion of patients with a Richmond Agitation-Sedation Scale (RASS) score of +2 or more compared with the placebo.

\section{Other Comparisons}

We found no delirium prevention trials evaluating short-term delirium symptoms in critically ill patients for the following comparisons:

- Second-generation antipsychotics versus placebo

- Haloperidol versus second-generation antipsychotics

- Second-generation antipsychotics versus second-generation antipsychotics

- Haloperidol versus other therapies

- Second-generation antipsychotics versus other therapies.

\section{Delirium Severity - Intensive Care Unit}

\section{Haloperidol Versus Placebo}

One low risk of bias RCT evaluated delirium severity in 135 patients at risk of delirium undergoing major non-cardiac thoracic surgery randomized to intravenous haloperidol or placebo. ${ }^{46}$ No statistically significant between-group differences were found in delirium severity between haloperidol and placebo among patients who developed delirium (results provided by author). Because the results were imprecise, we are unable to draw any conclusions about the effects of haloperidol versus placebo on delirium severity among patients in the intensive care unit at risk for delirium. (SOE: Insufficient)

\section{Other Comparisons}

We found no delirium prevention trials evaluating delirium severity for critically ill patients for the following comparisons:

- Second-generation antipsychotics versus placebo

- Haloperidol versus second-generation antipsychotics

- Second-generation antipsychotics versus second-generation antipsychotics

- Haloperidol versus other therapies

- Second-generation antipsychotics versus other therapies.

\section{Strength of Evidence}

Table 12 summarizes the strength of evidence findings for antipsychotics for the prevention of delirium in terms of delirium severity for critically ill patients. 
Table 12. Strength of evidence domains for trials evaluating antipsychotics for the prevention of delirium in terms of delirium severity for patients in an intensive care unit

\begin{tabular}{|c|c|c|c|c|c|c|c|c|}
\hline Comparison & $\begin{array}{c}\text { Number Of } \\
\text { Trials (N) }\end{array}$ & $\begin{array}{c}\text { Study } \\
\text { Limitations } \\
\end{array}$ & Directness & Consistency & Precision & $\begin{array}{c}\text { Reporting } \\
\text { Bias }\end{array}$ & $\begin{array}{c}\text { Strength Of } \\
\text { Evidence }\end{array}$ & Summary \\
\hline $\begin{array}{l}\text { Haloperidol vs. } \\
\text { placebo }\end{array}$ & $1^{46}(135)$ & Low & Direct & $\begin{array}{l}\text { Consistency } \\
\text { unknown, } \\
\text { (single study) }\end{array}$ & Imprecise & Undetected & Insufficient & $\begin{array}{l}\text { We are unable to draw } \\
\text { any conclusions. }\end{array}$ \\
\hline $\begin{array}{l}\text { Second-generation } \\
\text { antipsychotics vs. } \\
\text { placebo }\end{array}$ & No trials & & & & & & & \\
\hline $\begin{array}{l}\text { Haloperidol vs. } \\
\text { second-generation } \\
\text { antipsychotics }\end{array}$ & No trials & & & & & & & \\
\hline $\begin{array}{l}\text { Second-generation } \\
\text { antipsychotics vs. } \\
\text { second-generation } \\
\text { antipsychotics }\end{array}$ & No trials & & & & & & & \\
\hline $\begin{array}{l}\text { Haloperidol vs. other } \\
\text { therapies }\end{array}$ & No trials & & & & & & & \\
\hline $\begin{array}{l}\text { Second-generation } \\
\text { antipsychotics vs. } \\
\text { other therapies }\end{array}$ & No trials & & & & & & & \\
\hline
\end{tabular}




\section{Delirium-Free, Coma-Free Days Alive - Intensive Care Unit}

\section{Haloperidol Versus Placebo}

The findings presented in the "Delirium-Free, Coma-Free Days Alive - Overall” results section are wholly represented by this subgroup. Please refer to the "Delirium-Free, Coma-Free Days Alive - Overall” results section.

\section{Second-Generation Antipsychotics Versus Placebo}

The findings presented in the "Delirium-Free, Coma-Free Days Alive - Overall” results section are wholly represented by this subgroup. Please refer to the "Delirium-Free, Coma-Free Days Alive - Overall” results section.

\section{Haloperidol Versus Second-Generation Antipsychotics}

The findings presented in the "Delirium-Free, Coma-Free Days Alive - Overall” results section are wholly represented by this subgroup. Please refer to the "Delirium-Free, Coma-Free Days Alive - Overall” results section.

\section{Other Comparisons}

We found no delirium prevention trials evaluating delirium- and coma-free days in critically ill patients for the following comparisons:

- Second-generation antipsychotics versus second-generation antipsychotics

- Haloperidol versus other therapies

- Second-generation antipsychotics versus other therapies.

\section{Duration of Delirium - Intensive Care Unit}

\section{Haloperidol Versus Placebo}

Four RCTs, ${ }^{34,44-46}$ with low risk of bias, compared haloperidol with placebo for the prevention of delirium in patients admitted to medical or surgical intensive care units and requiring mechanical ventilation. All four of the trials consistently reported no statistically significant difference in delirium duration in haloperidol compared with placebo. We did not conduct a meta-analysis because these trials generally provided results as median values. The skewed nature of length of stay data precluded conversion or transformation of the data.

\section{Second-Generation Antipsychotics Versus Placebo}

One RCT, with low risk of bias, enrolled patients with and without delirium who received mechanical ventilation in mixed medical and surgical intensive care units. ${ }^{44}$ This three-arm trial reported no statistically significant effect on delirium duration for 30 patients randomized to a median dose of $113 \mathrm{mg}$ per day of oral ziprasidone compared with 36 patients randomized to placebo.

\section{Haloperidol Versus Second-Generation Antipsychotics}

The findings presented in the "Duration of Delirium - Overall" results section are wholly represented by this subgroup. Please refer to the "Duration of Delirium - Overall" results section. 


\section{Other Comparisons}

We found no delirium prevention trials evaluating delirium duration in critically ill patients for the following comparisons:

- Second-generation antipsychotics versus second-generation antipsychotics

- Haloperidol versus other therapies

- Second-generation antipsychotics versus other therapies.

\section{Use of Rescue Therapy - Intensive Care Unit}

\section{Haloperidol Versus Placebo}

Three RCTs, two with low risk of bias ${ }^{44,45}$ and one with uncertain risk of bias, ${ }^{43}$ compared haloperidol with placebo for the prevention of delirium and reported on the use of rescue therapy, defined as the use of any open-label antipsychotic. All three RCTs, of which one was a three-arm trial, ${ }^{44}$ enrolled medical and/or surgical patients in the intensive care unit setting. We found a statistically significant between-group difference in the use of rescue therapy favoring the haloperidol group (pooled RR, 0.45; 95\% CI, 0.27 to 0.75; I-squared, 0\%) (Figure 12). This is a possibly relevant decrease but the potential impact is difficult to assess. Exclusion of any one study did not change the inference from the meta-analysis.

Figure 12. Meta-analysis of the use of rescue therapy in trials comparing haloperidol with placebo in critically ill patients at risk for delirium

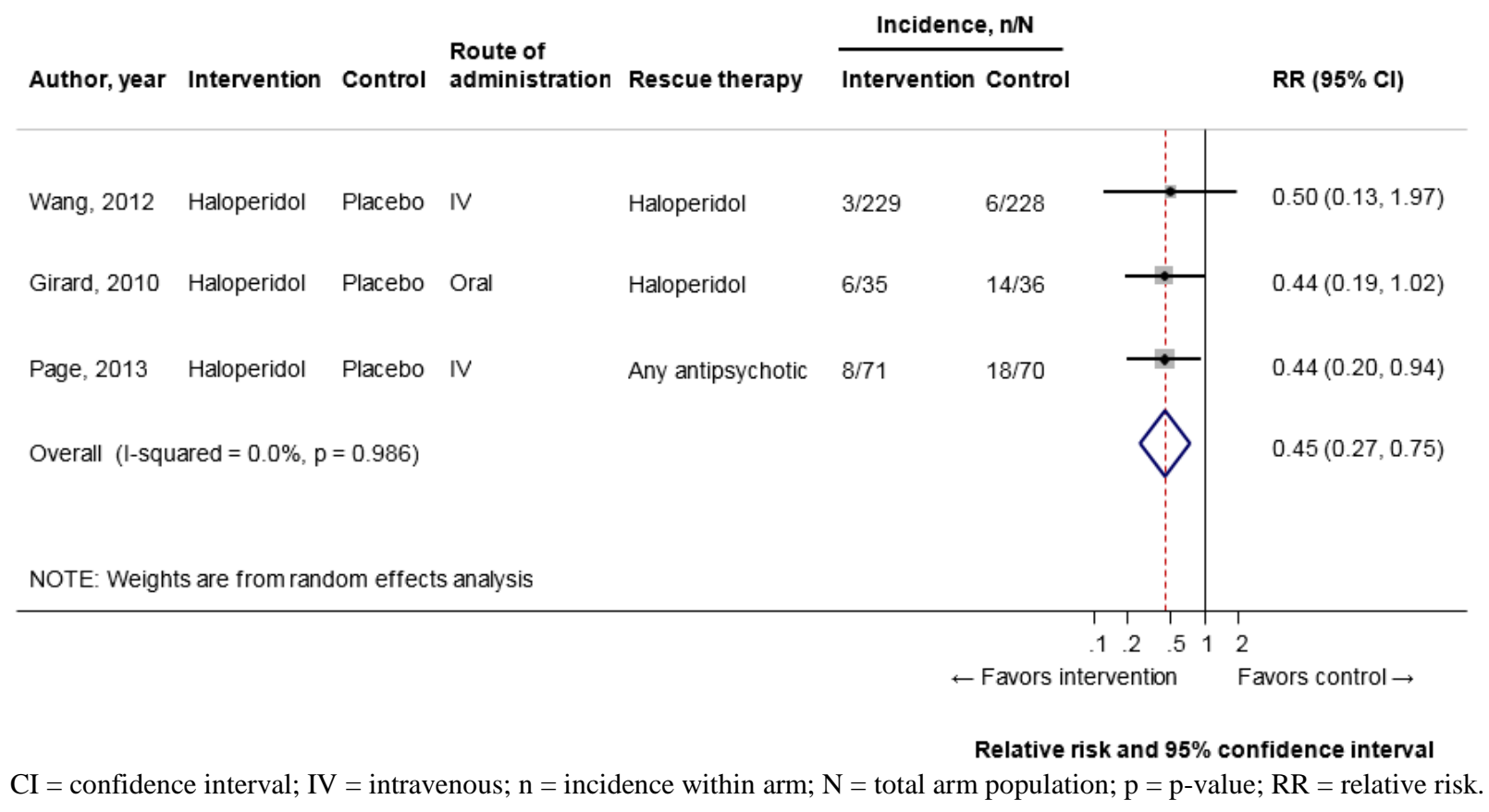

\section{Second-Generation Antipsychotics Versus Placebo}

The findings presented in the "Use of Rescue Therapy - Overall" results section are wholly represented by this subgroup. Please refer to the "Use of Rescue Therapy - Overall” results section. 


\section{Haloperidol Versus Second-Generation Antipsychotics}

The findings presented in the "Use of Rescue Therapy - Overall" results section are wholly represented by this subgroup. Please refer to the "Use of Rescue Therapy - Overall” results section.

\section{Other Comparisons}

We found no delirium prevention trials evaluating the use of rescue therapy in critically ill patients for the following comparisons:

- Second-generation antipsychotics versus second-generation antipsychotics

- Haloperidol versus other therapies

- Second-generation antipsychotics versus other therapies.

\section{Use of Physical Restraint - Intensive Care Unit}

\section{Haloperidol Versus Placebo}

The findings presented in the "Use of Physical Restraint - Overall" results section are wholly represented by this subgroup. Please refer to the "Use of Physical Restraint - Overall" results section.

\section{Other Comparisons}

We found no delirium prevention trials evaluating the use of physical restraint for critically ill patients for the following comparisons:

- Second-generation antipsychotics versus placebo

- Haloperidol versus second-generation antipsychotics

- Second-generation antipsychotics versus second-generation antipsychotics

- Haloperidol versus other therapies

- Second-generation antipsychotics versus other therapies.

\section{Other Intermediate Outcomes - Intensive Care Unit}

We found no trials in patients in an intensive care unit at risk for delirium that compared an antipsychotic with a placebo, another antipsychotic, or another treatment and evaluated the following intermediate outcomes:

- $\quad$ Patient distress

\section{Final Health or Patient-Centered Outcomes}

\section{Mortality - Intensive Care Unit}

\section{Haloperidol Versus Placebo}

We conducted a meta-analysis of seven RCTs that compared haloperidol with placebo in patients in intensive care units; one study was excluded from the analysis because there were no events. We found no between-group differences in short-term mortality, defined as mortality while in hospital or up to 30 days after randomization (pooled RR, 0.98; 95\% CI, 0.83 to 1.15; $P$ $=0.74$; Figure 13)..$^{33,34,42-46}$ Five of the RCTs were low risk of bias ${ }^{34,42,44-46}$ and two of the RCTs had unclear risk of bias. ${ }^{33,43}$ The evidence was consistent and precise. We tested the effect 
of each individual trial on the combined point estimate. No single trial influenced the pooled results. No substantial statistical heterogeneity was identified.

The largest trial, with low risk of bias enrolling a total of 1,789 critically ill patients, also examined 90-day mortality. ${ }^{42}$ The result was similar to the short-term mortality, with no between-group differences in comparing $3 \mathrm{mg}$ or $6 \mathrm{mg}$ haloperidol with placebo (RR, 0.99; 95\% CI, 0.77 to 1.27$)$.

Figure 13. Meta-analysis of mortality in trials comparing haloperidol with placebo in critically ill patients at risk for delirium

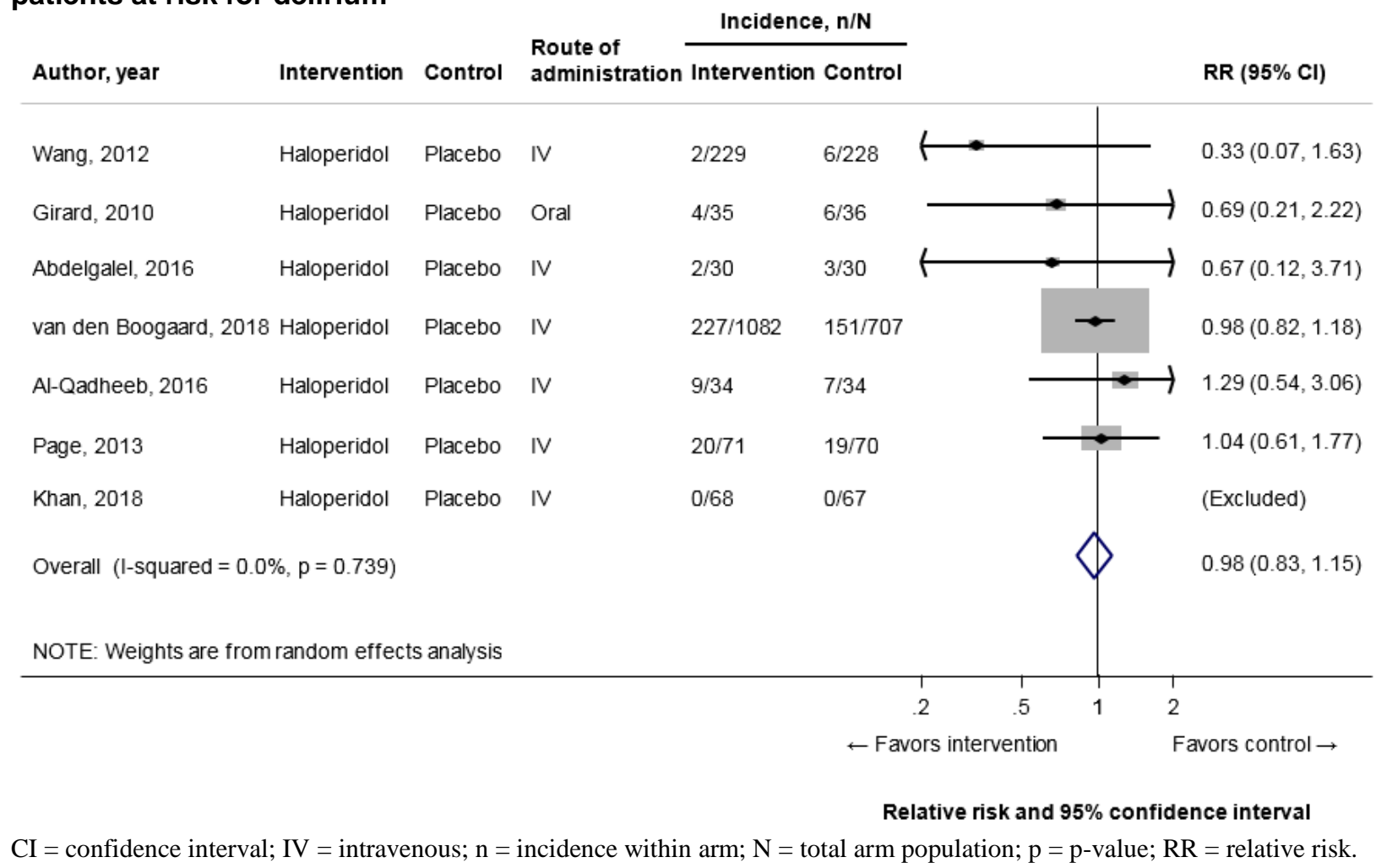

\section{Second-Generation Antipsychotics Versus Placebo}

One RCT, with low risk of bias, compared ziprasidone with placebo and reported no between-group differences in short-term mortality, defined as mortality while in hospital or up to 30 days after randomization. ${ }^{44}$

\section{Haloperidol Versus Second-Generation Antipsychotics}

The findings presented in the "Mortality - Overall" results section are wholly represented by this subgroup. Please refer to the "Mortality - Overall” results section.

\section{Haloperidol Versus Other Therapies}

The findings presented in the "Mortality - Overall” results section are wholly represented by this subgroup. Please refer to the "Mortality - Overall" results section.

\section{Other Comparisons}

We found no delirium prevention trials evaluating mortality in critically ill patients for the following comparisons: 
- Second-generation antipsychotics versus second-generation antipsychotics

- Second-generation antipsychotics versus other therapies.

\section{Other Final Health or Patient-Centered Outcomes - Intensive Care Unit}

We found no trials in patients in an intensive care unit at risk for delirium that compared an antipsychotic with a placebo, another antipsychotic, or another treatment and evaluated the following final health or patient-centered outcomes:

- Quality of life

- Cognitive functioning

- Institutionalization

- Caregiver burden/strain

- Falls

- Memory of patient distress

\section{Resource Utilization Outcomes}

\section{Readmission to Intensive Care Unit - Intensive Care Unit}

\section{Haloperidol Versus Placebo}

The findings presented in the "Readmission to Intensive Care Unit - Overall" results section are wholly represented by this subgroup. Please refer to the "Readmission to Intensive Care Unit - Overall” results section.

\section{Other Comparisons}

We found no delirium prevention trials evaluating readmission to intensive care unit in critically ill patients for the following comparisons:

- Second-generation antipsychotics versus placebo

- Haloperidol versus second-generation antipsychotics

- Second-generation antipsychotics versus second-generation antipsychotics

- Haloperidol versus other therapies

- Second-generation antipsychotics versus other therapies.

\section{Length of Stay in Hospital - Intensive Care Unit}

\section{Haloperidol Versus Placebo}

Six RCTs compared haloperidol with placebo and assessed length of stay in hospital for medical and surgical patients in intensive care units. ${ }^{33,42-46}$ Four of the RCTs had low risk of bias, ${ }^{42,44-46}$ and two of the RCTs had unclear risk of bias. ${ }^{33,43}$ We did not conduct a metaanalysis because these trials generally provided results as median values and the length of stay data are skewed, precluding conversion or transformation of the data. Five of the RCTs reported no statistically significant difference in length of stay in hospital when comparing haloperidol with placebo. ${ }^{42-46}$ However, one three-arm RCT reported significantly shorter length of stay in hospital in the haloperidol group compared with placebo. ${ }^{33}$ 
In summary, we concluded that haloperidol compared to placebo did not affect the length of stay in hospital when used to prevent delirium. (SOE for patients in the intensive care unit: High)

\section{Second-Generation Antipsychotics Versus Placebo}

One RCT, with low risk of bias and enrolling patients receiving mechanical ventilation, reported no statistically significant effect on length of stay in hospital for ziprasidone compared with placebo. ${ }^{44}$ We were unable to draw conclusions based upon this one study. (SOE for patients in the intensive care unit: Insufficient)

\section{Haloperidol Versus Second-Generation Antipsychotics}

The findings presented in the "Length of Stay in Hospital - Overall" results section are wholly represented by this subgroup. Please refer to the "Length of Stay in Hospital - Overall" results section. We were unable to draw a conclusion. (SOE for patients in the intensive care unit: Insufficient)

\section{Haloperidol Versus Other Therapies}

The findings presented in the "Length of Stay in Hospital - Overall" results section are wholly represented by this subgroup. Please refer to the "Length of Stay in Hospital - Overall" results section. We were unable to draw a conclusion. (SOE for patients in the intensive care unit: Insufficient)

\section{Other Comparisons}

We found no delirium prevention trials evaluating length of stay in hospital for critically ill patients for the following comparisons:

- Second-generation antipsychotics versus second-generation antipsychotics

- Second-generation antipsychotics versus other therapies.

\section{Strength of Evidence}

Table 13 summarizes the strength of evidence findings for antipsychotics for the prevention of delirium in terms of length of stay in hospital for critically ill patients. 
Table 13. Strength of evidence domains for trials evaluating antipsychotics for the prevention of delirium in terms of length of stay in hospital for patients in an intensive care unit

\begin{tabular}{|c|c|c|c|c|c|c|c|c|}
\hline Comparison & $\begin{array}{l}\text { Number Of } \\
\text { Trials (N) }\end{array}$ & $\begin{array}{c}\text { Study } \\
\text { Limitations }\end{array}$ & Directness & Consistency & Precision & $\begin{array}{l}\text { Reporting } \\
\text { Bias }\end{array}$ & $\begin{array}{c}\text { Strength Of } \\
\text { Evidence }\end{array}$ & Summary \\
\hline $\begin{array}{l}\text { Haloperidol vs. } \\
\text { placebo }\end{array}$ & $\begin{array}{l}6^{33,42-46} \\
(2,623)\end{array}$ & Low & Direct & Consistent & Precise & Undetected & High & $\begin{array}{l}\text { All RCTs were } \\
\text { conducted in medical } \\
\text { and surgical patients in } \\
\text { intensive care units. } \\
\text { There was no statistically } \\
\text { significant difference in } \\
\text { length of stay in hospital } \\
\text { comparing haloperidol to } \\
\text { placebo. }\end{array}$ \\
\hline $\begin{array}{l}\text { Second-generation } \\
\text { antipsychotics vs. } \\
\text { placebo }\end{array}$ & $1^{44}(101)$ & Low & Direct & $\begin{array}{l}\text { Consistency } \\
\text { unknown, } \\
\text { (single study) }\end{array}$ & Imprecise & Undetected & Insufficient & $\begin{array}{l}\text { We were unable to draw } \\
\text { a conclusion. }\end{array}$ \\
\hline $\begin{array}{l}\text { Haloperidol vs. } \\
\text { second-generation } \\
\text { antipsychotics }\end{array}$ & $1^{44}(101)$ & Low & Direct & $\begin{array}{l}\text { Consistency } \\
\text { unknown, } \\
\text { (single study) }\end{array}$ & Imprecise & Undetected & Insufficient & $\begin{array}{l}\text { We were unable to draw } \\
\text { a conclusion. }\end{array}$ \\
\hline $\begin{array}{l}\text { Second-generation } \\
\text { antipsychotics vs. } \\
\text { second-generation } \\
\text { antipsychotics }\end{array}$ & No trials & & & & & & & \\
\hline $\begin{array}{l}\text { Haloperidol vs. other } \\
\text { therapies }\end{array}$ & $1^{33}(90)$ & Medium & Direct & $\begin{array}{l}\text { Consistency } \\
\text { unknown, } \\
\text { (single study) }\end{array}$ & Imprecise & Undetected & Insufficient & $\begin{array}{l}\text { We were unable to draw } \\
\text { a conclusion. }\end{array}$ \\
\hline $\begin{array}{l}\text { Second-generation } \\
\text { antipsychotics vs. } \\
\text { other therapies }\end{array}$ & No trials & & & & & & & \\
\hline
\end{tabular}

RCT = randomized controlled trial. 


\section{Length of Stay in Intensive Care Unit - Intensive Care Unit}

\section{Haloperidol Versus Placebo}

The findings presented in the "Length of Stay in Intensive Care Unit - Overall” results section are wholly represented by this subgroup. Please refer to the "Length of Stay in Intensive Care Unit - Overall” results section.

\section{Second-Generation Antipsychotics Versus Placebo}

One RCT, with low risk of bias, enrolled patients with and without delirium who were receiving mechanical ventilation in mixed medical and surgical intensive care units. ${ }^{44}$ This threearm RCT reported no statistically significant effect on length of stay in the intensive care unit for patients randomized to receive a median dose of $113 \mathrm{mg}$ per day of oral ziprasidone compared with patients receiving placebo.

\section{Haloperidol Versus Second-Generation Antipsychotics}

One RCT, with low risk of bias, enrolled patients with and without delirium who were receiving mechanical ventilation in mixed medical and surgical intensive care units. ${ }^{44}$ This threearm RCT reported no statistically significant effect on length of stay in the intensive care unit for patients randomized to receive a median dose of $15 \mathrm{mg}$ per day of oral haloperidol compared with a median dose of $113 \mathrm{mg}$ per day of oral ziprasidone. ${ }^{44}$

\section{Haloperidol Versus Other Therapies}

One three-arm RCT, with unclear risk of bias, enrolled adult patients with severe systemic illness in an intensive care unit and demonstrated a statistically significant difference in length of stay in the intensive care unit for 30 patients randomized to receive a $2.5 \mathrm{mg}$ loading dose of haloperidol followed by 0.5 to $2.0 \mathrm{mg}$ per hour, based on sedation level compared with 30 patients randomized to receive $1.0 \mathrm{mcg} / \mathrm{kg}$ loading dose of dexmedetomidine followed by 0.2 to $0.7 \mathrm{mcg} / \mathrm{kg}$ per hour. ${ }^{33}$

\section{Other Comparisons}

We found no delirium prevention trials evaluating length of stay in the intensive care unit for the following comparisons:

- Second-generation antipsychotics versus second-generation antipsychotics

- Second-generation antipsychotics versus other therapies.

\section{Other Resource Utilization Outcomes - Intensive Care Unit}

We found no trials in critically ill patients at risk for delirium that compared an antipsychotic with a placebo, another antipsychotic, or another treatment and evaluated the following resource utilization outcomes:

- Readmission to hospital

- Length of stay in skilled nursing facility

- Patient safety attendant use

- Hospice enrollment 


\section{Adverse Effects}

\section{Sedation - Intensive Care Unit}

\section{Haloperidol Versus Placebo}

Two RCTs, both with low risk of bias, compared haloperidol with placebo in patients requiring mechanical ventilation in a medical or surgical intensive care unit and reported on sedation (described as excessive or oversedation). ${ }^{34,45}$ There were too few events in these trials to draw a conclusion about the effects of haloperidol on sedation. (SOE: Insufficient)

We did not find any observational studies addressing sedation that compared haloperidol with placebo in critically ill patients at risk for delirium.

\section{Other Comparisons}

We found no studies of delirium prevention evaluating sedation in critically ill patients for the following comparisons:

- Second-generation antipsychotics versus placebo

- Haloperidol versus second-generation antipsychotics

- Second-generation antipsychotics versus second-generation antipsychotics

- Haloperidol versus other therapies

- Second-generation antipsychotics versus other therapies.

\section{Strength of Evidence}

Table 14 summarizes the strength of evidence findings for antipsychotics for the prevention of delirium in terms of sedation in critically ill patients. 
Table 14. Strength of evidence domains for studies reporting sedation in the evaluation of antipsychotics for delirium prevention in patients in an intensive care unit

\begin{tabular}{|c|c|c|c|c|c|c|c|c|}
\hline Comparison & $\begin{array}{l}\text { Number Of } \\
\text { Studies (N) }\end{array}$ & $\begin{array}{c}\text { Study } \\
\text { Limitations }\end{array}$ & Directness & Consistency & Precision & $\begin{array}{c}\text { Reporting } \\
\text { Bias }\end{array}$ & $\begin{array}{l}\text { Strength of } \\
\text { Evidence }\end{array}$ & Summary \\
\hline $\begin{array}{l}\text { Haloperidol vs. } \\
\text { placebo }\end{array}$ & $2^{34,45}(209)$ & Low & Direct & Consistent & Imprecise* & Undetected & Insufficient & $\begin{array}{l}\text { We are unable to draw a } \\
\text { conclusion. }\end{array}$ \\
\hline $\begin{array}{l}\text { Second- } \\
\text { generation } \\
\text { antipsychotics vs. } \\
\text { placebo }\end{array}$ & No studies & & & & & & & \\
\hline $\begin{array}{l}\text { Haloperidol vs. } \\
\text { second- } \\
\text { generation } \\
\text { antipsychotics }\end{array}$ & No studies & & & & & & & \\
\hline $\begin{array}{l}\text { Second- } \\
\text { generation } \\
\text { antipsychotics vs. } \\
\text { second- } \\
\text { generation } \\
\text { antipsychotics }\end{array}$ & No studies & & & & & & & \\
\hline $\begin{array}{l}\text { Haloperidol vs. } \\
\text { other therapies }\end{array}$ & No studies & & & & & & & \\
\hline $\begin{array}{l}\text { Second- } \\
\text { generation } \\
\text { antipsychotics vs. } \\
\text { other therapies }\end{array}$ & No studies & & & & & & & \\
\hline
\end{tabular}

* Numbers of events were few and confidence intervals wide with only two single center studies contributing data. 


\section{Cardiac Effects - Intensive Care Unit}

\section{Haloperidol Versus Placebo}

The findings presented in the "Cardiac Effects - Overall" results section are wholly represented by this subgroup. Please refer to the "Cardiac Effects - Overall” results section.

\section{Second-Generation Antipsychotics Versus Placebo}

One RCT, with low risk of bias, compared ziprasidone with placebo and reported on cardiac effects. ${ }^{44}$ This three-arm trial included patients included 66 mechanically ventilated, critically ill patients treated with oral ziprasidone versus placebo, and reported no statistically significant between-group differences in corrected QT interval; no ventricular arrhythmias occurred in either arm.

We did not find any observational studies addressing cardiac effects that compared secondgeneration antipsychotics with placebo in critically ill patients at risk for delirium.

\section{Haloperidol Versus Second-Generation Antipsychotics}

The findings presented in the "Cardiac Effects - Overall" results section are wholly represented by this subgroup. Please refer to the "Cardiac Effects - Overall" results section.

\section{Haloperidol Versus Other Therapies}

The findings presented in the "Cardiac Effects - Overall" results section are wholly represented by this subgroup. Please refer to the "Cardiac Effects - Overall" results section.

\section{Other Comparisons}

We found no studies of delirium prevention evaluating cardiac effects in critically ill patients for the following comparisons:

- Second-generation antipsychotics versus second-generation antipsychotics

- Second-generation antipsychotics versus other therapies.

\section{Studies With No Comparison Group}

The findings presented in the "Cardiac Effects - Overall" results section are wholly represented by this subgroup. Please refer to the "Cardiac Effects - Overall" results section.

\section{Neurologic Effects - Intensive Care Unit}

\section{Haloperidol Versus Placebo}

Five RCTs, ${ }^{34,42,44-46}$ with low risk of bias, compared haloperidol with placebo for the prevention of delirium in 2,235 patients admitted to medical or surgical intensive care units and reported a range of neurologic effects. Four of the trials administered haloperidol intravenously (maximum daily dose range, 1.5 to $7.5 \mathrm{mg}$ ), ${ }^{34,42,45,46}$ and one of the trials administered oral doses (maximum daily dose, $20 \mathrm{mg}$ ). ${ }^{44}$ One of the RCTs was a three-arm study. ${ }^{44}$ There was no difference in between-group occurrence of extrapyramidal side effects (including akathisia, muscle stiffness, and dystonic movements such as torticollis) (pooled RR, 1.01; 95\% CI, 0.56 to 1.83; I-squared, 0\%) (Figure 14). Exclusion of any one trial did not change the inference from the meta-analysis. We excluded one study from the analysis because they reported individual 
symptoms of extrapyramidal side effects. ${ }^{46}$ This trial reported no statistically significant differences in elbow rigidity, wrist rigidity, or resting tremor and no events of dystonia, tardive dyskinesia, or pseudoparkinsonism.

Three RCTs reported the occurrence of neuroleptic malignant syndrome. ${ }^{42,44,46}$ One RCT reported no statistically significant between-group difference in patients randomized to intervention and placebo for $3 \mathrm{mg}$ and $6 \mathrm{mg}$ daily intravenous dosage arms. ${ }^{42}$ The other two RCTs reported no occurrence of neuroleptic malignant syndrome in either the haloperidol or the placebo arms in 206 critically ill, mechanically ventilated patients. ${ }^{44,46}$

We did not find any observational studies addressing neurologic effects that compared haloperidol with placebo in critically ill patients at risk for delirium.

Figure 14. Meta-analysis of neurologic effects in trials comparing haloperidol with placebo in critically ill patients at risk for delirium

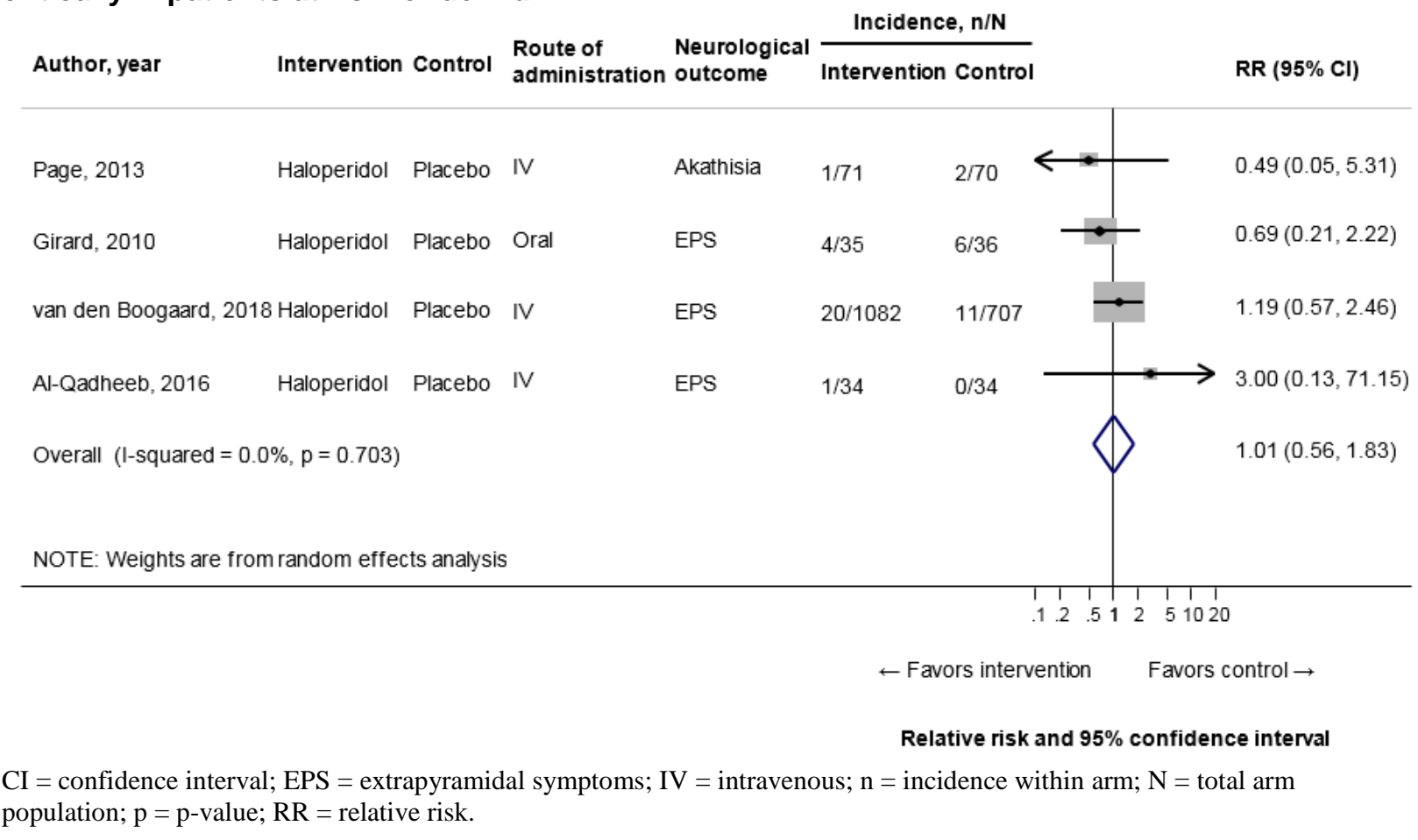

\section{Second-Generation Antipsychotics Versus Placebo}

One RCT, with low risk of bias, compared oral second-generation antipsychotics with placebo for the prevention of delirium in critically ill and post-surgical patients and evaluated neurologic effects. ${ }^{44}$ This three-arm study included a total of 101 mechanically ventilated, critically ill patients treated with oral ziprasidone versus placebo and reported no statistically significant between-group differences in akathisia or extrapyramidal symptoms not including akathisia. Neuroleptic malignant syndrome did not occur in either group in this study.

We did not find any observational studies addressing neurologic effects that compared second-generation antipsychotics with placebo in critically ill patients at risk for delirium.

\section{Haloperidol Versus Second-Generation Antipsychotics}

One three-arm RCT, with low risk of bias, compared oral haloperidol with ziprasidone in mechanically ventilated, critically ill patients and reported no statistically significant between- 
group differences in akathisia or extrapyramidal symptoms not including akathisia. This trial also reported no instances of neuroleptic malignant syndrome. ${ }^{44}$

We did not find any observational studies addressing neurologic effects that compared haloperidol with second-generation antipsychotics in critically ill patients at risk for delirium.

\section{Other Comparisons}

We found no studies of delirium prevention evaluating neurologic effects in critically ill patients for the following comparisons:

- Second-generation antipsychotics versus second-generation antipsychotics

- Haloperidol versus other therapies

- Second-generation antipsychotics versus other therapies.

\section{Studies With No Comparison Group}

In one observational study with 177 critically ill patients at high risk of delirium who received intravenous haloperidol prophylaxis, one patient (1\%) had to stop haloperidol because of an extrapyramidal side effect described as "signs of Parkinsonism," and one patient (1\%) had to stop haloperidol because of suspected neuroleptic malignant syndrome. ${ }^{47}$

\section{Other Adverse Effects - Intensive Care Unit}

We found no studies in critically ill patients at risk for delirium that compared an antipsychotic with a placebo, another antipsychotic, or another treatment and evaluated the following adverse events:

- Weight gain

- Changes in appetite

- Hypersensitivity reactions

- Inappropriate continuation of antipsychotics

- Swallowing difficulties

- Aspiration pneumonia

\section{Key Question 1d. Benefits and Harms of Antipsychotics To Prevent Delirium in Patients in a Post-Acute Care Facility}

We found no trials evaluating antipsychotics for the prevention of delirium in patients in a post-acute care facility.

\section{Key Question 1e. Benefits and Harms of Antipsychotics To Prevent Delirium in Patients in Palliative or Hospice Care}

We found no trials evaluating antipsychotics for the prevention of delirium in patients in palliative or hospice care. 


\section{Key Question 1f. Benefits and Harms of Antipsychotics To Prevent Delirium in Patients in Postoperative Care}

\section{Key Points - Postoperative Care}

\section{Delirium Severity}

- We were unable to draw a conclusion regarding the effects of antipsychotics on delirium severity in patients in postoperative care for the following comparisons:

o Haloperidol versus placebo (insufficient evidence)

o Second-generation antipsychotics versus placebo (insufficient evidence)

o Haloperidol versus second-generation antipsychotics (no trials)

o Second-generation antipsychotics versus second-generation antipsychotics (no trials)

o Haloperidol versus other therapies (no trials)

o Second-generation antipsychotics versus other therapies (no trials)

\section{Length of Stay in Hospital}

- We concluded that there was little to no difference in length of stay in hospital for postoperative patients treated with second-generation antipsychotics versus those treated with placebo. (SOE: Low)

- We were unable to draw a conclusion regarding the effects of antipsychotics on length of stay in hospital for patients in postoperative care for the following comparisons:

o Haloperidol versus placebo (insufficient evidence)

o Haloperidol versus second-generation antipsychotics (no trials)

o Second-generation antipsychotics versus second-generation antipsychotics (no trials)

o Haloperidol versus other therapies (no trials)

o Second-generation antipsychotics versus other therapies (no trials)

\section{Cognitive Functioning and Inappropriate Continuation of Antipsychotics}

- We found no studies evaluating cognitive functioning or inappropriate continuation of antipsychotics in postoperative patients at risk for delirium that compared an antipsychotic with a placebo, another antipsychotic, or another treatment.

\section{Intermediate Outcomes}

\section{Delirium Incidence - Postoperative Care}

\section{Haloperidol Versus Placebo}

Four RCTs, two with low risk of bias, ${ }^{37,46}$ one with unclear risk of bias, ${ }^{38}$ and one with high risk of bias owing to a lack of blinding and allocation concealment, ${ }^{35}$ enrolling postoperative patients and employing different validated delirium detection instruments, directly compared delirium incidence between haloperidol and placebo groups. Three trials administered intravenous haloperidol, ${ }^{35,38,46}$ and one trial ${ }^{37}$ administered oral doses. We included these trials in a meta-analysis because they identified new onset of delirium (incidence) in postoperative patients after exposure to prophylactic haloperidol or placebo. We found no difference in the 
relative risk of delirium in the meta-analysis (RR, 0.86; 95\% CI, 0.57 to 1.29; I-squared, 51\%) (Figure 15). Exclusion of any one trial did not change the inference of the meta-analysis. Owing to the substantial statistical heterogeneity, we also conducted a profile likelihood method of meta-analysis, and found a similar non-statistically significant effect on delirium incidence comparing haloperidol with placebo (pooled RR, 0.92 95\% CI, 0.52 to 1.26; I-squared, 0\%).

Figure 15. Meta-analysis of delirium incidence in trials comparing haloperidol with placebo in postoperative patients at risk for delirium

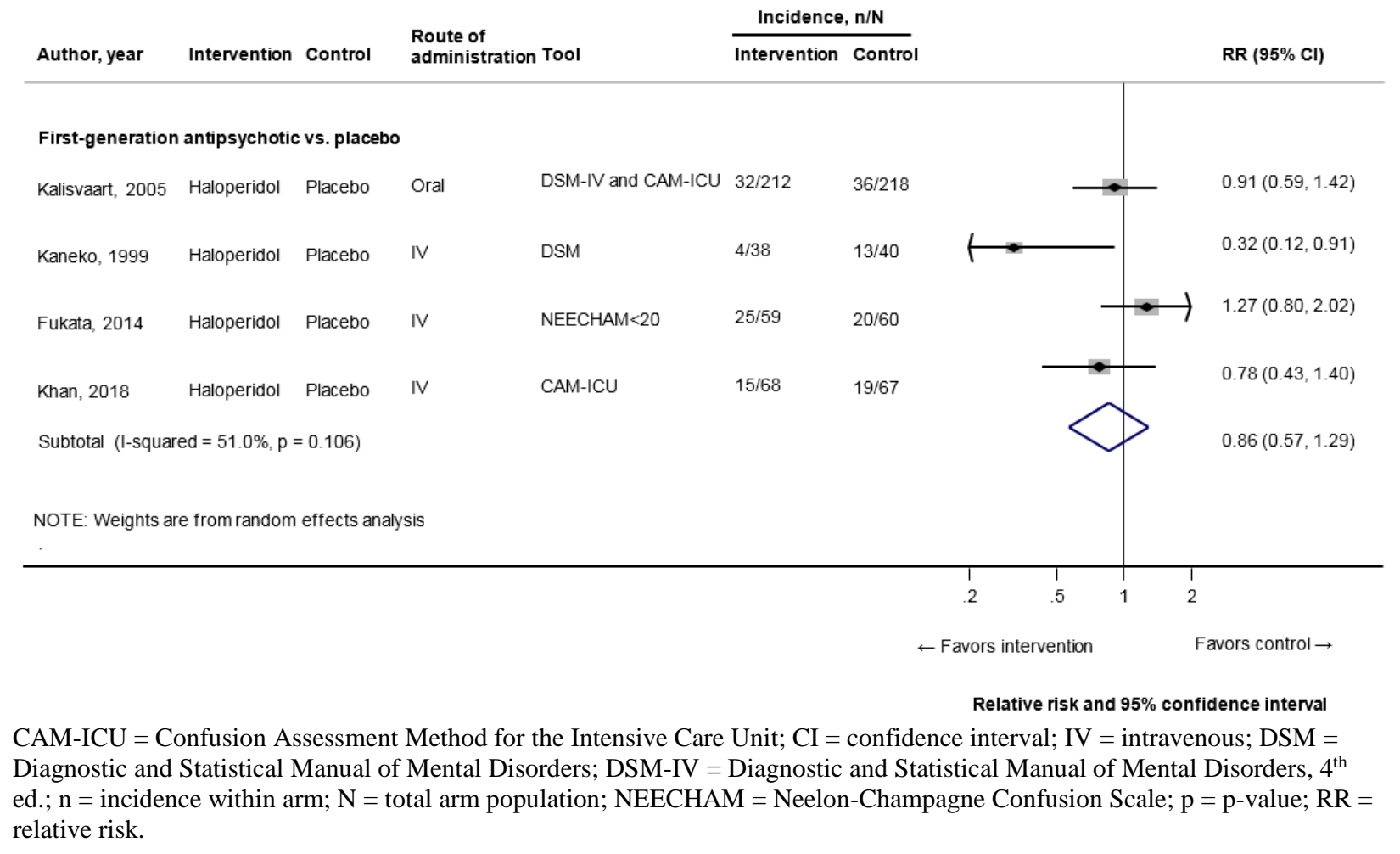

\section{Second-Generation Antipsychotics Versus Placebo}

The findings presented in the "Delirium Incidence - Overall" results section are wholly represented by this subgroup. Please refer to the "Delirium Incidence - Overall” results section.

\section{Other Comparisons}

We found no delirium prevention trials evaluating delirium incidence in postoperative patients for the following comparisons:

- Haloperidol versus second-generation antipsychotics

- Second-generation antipsychotics versus second-generation antipsychotics

- Haloperidol versus other therapies

- Second-generation antipsychotics versus other therapies.

\section{Short-Term Delirium Symptoms - Postoperative Care}

\section{Haloperidol Versus Placebo}

One RCT, with unclear risk of bias, compared haloperidol with placebo and assessed the delirium symptom of sleep duration during day and night. ${ }^{38}$ This trial, enrolling patients 
undergoing elective gastrointestinal surgery, reported no statistically significant difference between the haloperidol group and the placebo group in the average and total time of sleep.

\section{Other Comparisons}

We found no delirium prevention trials evaluating postoperative short-term delirium symptoms for the following comparisons:

- Second-generation antipsychotics versus placebo

- Haloperidol versus second-generation antipsychotics

- Second-generation antipsychotics versus second-generation antipsychotics

- Haloperidol versus other therapies

- Second-generation antipsychotics versus other therapies.

\section{Delirium Severity - Postoperative Care}

\section{Haloperidol Versus Placebo}

Two RCTs, with low risk of bias, enrolled acute or elective hip surgery patients or patients undergoing non-cardiac thoracic surgery and assessed delirium severity by the highest value of the Delirium Rating Scale-Revised-98. ${ }^{37,46}$ One RCT reported significantly lower delirium severity during the first three days after the onset of delirium in the haloperidol group compared with the placebo ${ }^{37}$ and the second reported no statistical difference in severity among those who developed delirium (results provided by author). ${ }^{46}$ We were unable to draw conclusions. (SOE in patients in postoperative care: Insufficient)

\section{Second-Generation Antipsychotics Versus Placebo}

The findings presented in the "Delirium Severity - Overall” results section are wholly represented by this subgroup. Please refer to the "Delirium Severity - Overall” results section.

\section{Other Comparisons}

We found no delirium prevention trials evaluating postoperative delirium severity for the following comparisons:

- Haloperidol versus second-generation antipsychotics

- Second-generation antipsychotics versus second-generation antipsychotics

- Haloperidol versus other therapies

- Second-generation antipsychotics versus other therapies.

\section{Strength of Evidence}

Table 15 summarizes the strength of evidence findings for antipsychotics for the prevention of delirium in terms of delirium severity in postoperative patients. 
Table 15. Strength of evidence domains for trials evaluating antipsychotics for the prevention of delirium in terms of delirium severity in postoperative patients

\begin{tabular}{|c|c|c|c|c|c|c|c|c|}
\hline Comparison & $\begin{array}{c}\text { Number Of } \\
\text { Trials (N) }\end{array}$ & $\begin{array}{c}\text { Study } \\
\text { Limitations }\end{array}$ & Directness & Consistency & Precision & $\begin{array}{c}\text { Reporting } \\
\text { Bias }\end{array}$ & $\begin{array}{c}\text { Strength Of } \\
\text { Evidence }\end{array}$ & Summary \\
\hline $\begin{array}{l}\text { Haloperidol vs. } \\
\text { placebo }\end{array}$ & $2^{37,46}(565)$ & Low & Direct & Inconsistent & Precise & Undetected & Insufficient & $\begin{array}{l}\text { We were unable to } \\
\text { draw a conclusion. }\end{array}$ \\
\hline $\begin{array}{l}\text { Second-generation } \\
\text { antipsychotics vs. } \\
\text { placebo }\end{array}$ & $2^{36,39}(501)$ & Low & Direct & Inconsistent & Precise & Undetected & Insufficient & $\begin{array}{l}\text { We were unable to } \\
\text { draw a conclusion }\end{array}$ \\
\hline $\begin{array}{l}\text { Haloperidol vs. } \\
\text { second-generation } \\
\text { antipsychotics }\end{array}$ & No trials & & & & & & & \\
\hline $\begin{array}{l}\text { Second-generation } \\
\text { antipsychotics vs. } \\
\text { second-generation } \\
\text { antipsychotics }\end{array}$ & No trials & & & & & & & \\
\hline $\begin{array}{l}\text { Haloperidol vs. } \\
\text { other therapies }\end{array}$ & No trials & & & & & & & \\
\hline $\begin{array}{l}\text { Second-generation } \\
\text { antipsychotics vs. } \\
\text { other therapies }\end{array}$ & No trials & & & & & & & \\
\hline
\end{tabular}




\section{Duration of Delirium - Postoperative Care}

\section{Haloperidol Versus Placebo}

Three RCTs, two with low risk of bias ${ }^{37,46}$ and one at high risk of bias owing to lack of blinding and allocation concealment, ${ }^{35}$ compared haloperidol with placebo for the prevention of delirium in postoperative patients and reported different findings.

\section{Second-Generation Antipsychotics Versus Placebo}

Two RCTs, with low risk of bias, compared a second-generation antipsychotic with placebo for the prevention of delirium and assessed delirium duration in postoperative patients. ${ }^{36,39}$ One trial demonstrated no statistically significant within group difference for delirium duration, ${ }^{36}$ and the other trial demonstrated a statistically significant shorter duration of delirium favoring the placebo group. ${ }^{39}$

\section{Other Comparisons}

We found no delirium prevention trials evaluating delirium duration among patients in postoperative care for the following comparisons:

- Haloperidol versus second-generation antipsychotics

- Second-generation antipsychotics versus second-generation antipsychotics

- Haloperidol versus other therapies

- Second-generation antipsychotics versus other therapies.

\section{Use of Rescue Therapy - Postoperative Care}

\section{Haloperidol Versus Placebo}

One RCT, with unclear risk of bias, enrolled patients aged 65 years and older undergoing non-cardiac surgery and recovery in an intensive care unit. ${ }^{43}$ There was no statistically significant difference in the use of rescue medications (defined as the use of any open-label antipsychotic agent) in those patients randomized to a $0.5 \mathrm{mg}$ loading dose of haloperidol followed by $0.1 \mathrm{mg}$ per hour infusion compared with placebo.

\section{Other Comparisons}

We found no delirium prevention trials evaluating the use of rescue therapy among patients in postoperative care for the following comparisons:

- Second-generation antipsychotics versus placebo

- Haloperidol versus second-generation antipsychotics

- Second-generation antipsychotics versus second-generation antipsychotics

- Haloperidol versus other therapies

- Second-generation antipsychotics versus other therapies. 


\section{Use of Physical Restraint - Postoperative Care}

\section{Haloperidol Versus Placebo}

One RCT with low risk of bias enrolled patients undergoing major thoracic non-cardiac surgery in an intensive care unit. ${ }^{46}$ This trial reported no difference in restraint use between the patients randomized to intravenous haloperidol compared with placebo.

\section{Second-Generation Antipsychotics Versus Placebo}

The findings presented in the "Use of Physical Restraint - Overall" results section are wholly represented by this subgroup. Please refer to the "Use of Physical Restraint - Overall" results section.

\section{Other Comparisons}

We found no delirium prevention trials evaluating the use of physical restraint in patients in postoperative care for the following comparisons:

- Haloperidol versus second-generation antipsychotics

- Second-generation antipsychotics versus second-generation antipsychotics

- Haloperidol versus other therapies

- Second-generation antipsychotics versus other therapies

\section{Other Intermediate Outcomes - Postoperative Care}

We found no trials in patients in postoperative care with delirium that compared an antipsychotic with a placebo, another antipsychotic, or another treatment and evaluated the following intermediate outcomes:

- Delirium- and coma-free days alive

- Patient distress

\section{Final Health or Patient-Centered Outcomes}

\section{Mortality - Postoperative Care}

\section{Haloperidol Versus Placebo}

One RCT, with low risk of bias and enrolling patients after non-cardiac thoracic surgery, compared intravenous haloperidol with placebo and reported no in-hospital mortality. ${ }^{46}$

\section{Second-Generation Antipsychotics Versus Placebo}

One RCT, with low risk of bias and enrolling patients after cardiac surgery, compared risperidone with placebo and reported no between-group differences in short-term mortality, defined as mortality while in hospital or up to 30 days after randomization. ${ }^{36}$

\section{Other Comparisons}

We found no delirium prevention trials evaluating mortality in postoperative settings for the following comparisons:

- Haloperidol versus second-generation antipsychotics

- Second-generation antipsychotics versus second-generation antipsychotics 
- Haloperidol versus other therapies

- Second-generation antipsychotics versus other therapies.

\section{Institutionalization - Postoperative Care}

\section{Second-Generation Antipsychotics Versus Placebo}

The findings presented in the "Institutionalization - Overall" results section are wholly represented by this subgroup. Please refer to the "Institutionalization - Overall" results section.

\section{Other Comparisons}

We found no delirium prevention trials evaluating institutionalization in postoperative settings for the following comparisons:

- Haloperidol versus placebo

- Haloperidol versus second-generation antipsychotics

- Second-generation antipsychotics versus second-generation antipsychotics

- Haloperidol versus other therapies

- Second-generation antipsychotics versus other therapies.

\section{Other Final Health or Patient-Centered Outcomes - Postoperative Care}

We found no trials in postoperative patients at risk for delirium that compared an antipsychotic with a placebo, another antipsychotic, or another treatment and evaluated the following final health or patient-centered outcomes:

- Quality of life

- Cognitive functioning

- Caregiver burden/strain

- Falls

- Memory of patient distress

\section{Resource Utilization Outcomes}

\section{Length of Stay in Hospital - Postoperative Care}

\section{Haloperidol Versus Placebo}

Two RCTs, both with low risk of bias, enrolled acute or elective hip surgery patients or patients undergoing non-cardiac thoracic surgery and reported different findings. ${ }^{37,46}$ One trial reported significantly shorter length of stay in hospital in the haloperidol group compared with placebo, ${ }^{37}$ and the other reported no statistically significant difference. ${ }^{46}$ We were unable to draw conclusions. (SOE in patients in postoperative care: Insufficient)

\section{Second-Generation Antipsychotics Versus Placebo}

Two RCTs comparing second-generation antipsychotics with placebo assessed length of stay in hospital. ${ }^{36,40}$ One trial had low risk of bias, ${ }^{36}$ and the other trial had high risk of bias primarily owing to a lack of blinding and unclear potential for selective outcome reporting. ${ }^{40}$ Both trials 
reported no effect on length of stay in hospital when comparing second-generation antipsychotics with placebo in postoperative settings. (SOE in patients in postoperative care: Low)

\section{Other Comparisons}

We found no delirium prevention trials evaluating length of stay in hospital in postoperative settings for the following comparisons:

- Haloperidol versus second-generation antipsychotics

- Second-generation antipsychotics versus second-generation antipsychotics

- Haloperidol versus other therapies

- Second-generation antipsychotics versus other therapies.

\section{Strength of Evidence}

Table 16 summarizes the strength of evidence findings for antipsychotics for the prevention of delirium in terms of length of stay in hospital in postoperative patients. 
Table 16. Strength of evidence domains for trials evaluating antipsychotics for the prevention of delirium in terms of length of stay in hospital in postoperative patients

\begin{tabular}{|c|c|c|c|c|c|c|c|c|}
\hline Comparison & $\begin{array}{l}\text { Number Of } \\
\text { Trials (N) }\end{array}$ & $\begin{array}{c}\text { Study } \\
\text { Limitations }\end{array}$ & Directness & Consistency & Precision & $\begin{array}{c}\text { Reporting } \\
\text { Bias }\end{array}$ & $\begin{array}{c}\text { Strength Of } \\
\text { Evidence }\end{array}$ & Summary \\
\hline $\begin{array}{l}\text { Haloperidol vs. } \\
\text { placebo }\end{array}$ & $2^{37,46}(565)$ & Low & Direct & Inconsistent & Precise & Undetected & Insufficient & $\begin{array}{l}\text { We were unable to draw a } \\
\text { conclusion. }\end{array}$ \\
\hline $\begin{array}{l}\text { Second-generation } \\
\text { antipsychotics vs. } \\
\text { placebo }\end{array}$ & $2^{36,40}(227)$ & Medium & Direct & Consistent & Imprecise & Undetected & Low & $\begin{array}{l}\text { All RCTs reported no } \\
\text { statistically significant } \\
\text { difference. }\end{array}$ \\
\hline $\begin{array}{l}\text { Haloperidol vs. } \\
\text { second-generation } \\
\text { antipsychotics }\end{array}$ & No trials & & & & & & & \\
\hline $\begin{array}{l}\text { Second-generation } \\
\text { antipsychotics vs. } \\
\text { second-generation } \\
\text { antipsychotics }\end{array}$ & No trials & & & & & & & \\
\hline $\begin{array}{l}\text { Haloperidol vs. other } \\
\text { therapies }\end{array}$ & No trials & & & & & & & \\
\hline $\begin{array}{l}\text { Second-generation } \\
\text { antipsychotics vs. } \\
\text { other therapies }\end{array}$ & No trials & & & & & & & \\
\hline
\end{tabular}

RCT $=$ randomized controlled trial. 


\section{Length of Stay in Intensive Care Unit - Postoperative Care}

\section{Haloperidol Versus Placebo}

Two RCTs, one with low risk of bias ${ }^{46}$ and a second with unclear risk of bias, ${ }^{43}$ enrolled patients undergoing major thoracic non-cardiac surgery and recovery in an intensive care unit. One trial reported no difference in intensive care length of stay ${ }^{46}$ and the second reported a statistically significant difference for length of stay in the intensive care unit for patients randomized to intravenous haloperidol compared with placebo. ${ }^{43}$ However, this difference of less than 2 hours in the intensive care unit is not considered clinically important.

\section{Second-Generation Antipsychotics Versus Placebo}

Two trials each compared risperidone with placebo and reported no statistically significant difference in length of stay in the intensive care unit. One RCT, with low risk of bias, enrolled cardiac surgery patients with postoperative subsyndromal delirium. ${ }^{36}$ The other RCT, with high risk of bias owing to a lack of blinding, enrolled cardiac surgery patients randomized to treatment with a single dose of $1 \mathrm{mg}$ sublingual risperidone versus placebo. ${ }^{40}$ There were no statisically significant between-group differences in length of stay in the intensive care unit in either trial.

\section{Other Comparisons}

We found no delirium prevention trials evaluating length of stay in the intensive care unit among postoperative patients for the following comparisons:

- Haloperidol versus second-generation antipsychotics

- Second-generation antipsychotics versus second-generation antipsychotics

- Haloperidol versus other therapies

- Second-generation antipsychotics versus other therapies.

\section{Patient Safety Attendant Use - Postoperative Care}

\section{Second-Generation Antipsychotics Versus Placebo}

The findings presented in the "Patient Safety Attendant Use - Overall" results section are wholly represented by this subgroup. Please refer to the "Patient Safety Attendant Use - Overall" results section.

\section{Other Comparisons}

We found no delirium prevention trials evaluating patient safety attendant use in postoperative settings for the following comparisons:

- Haloperidol versus placebo

- Haloperidol versus second-generation antipsychotics

- Second-generation antipsychotics versus second-generation antipsychotics

- Haloperidol versus other therapies

- Second-generation antipsychotics versus other therapies. 


\section{Other Resource Utilization Outcomes - Postoperative Care}

We found no trials in postoperative patients at risk of delirium that compared an antipsychotic with a placebo, another antipsychotic, or another treatment and evaluated the following resource utilization outcomes:

- Readmission to hospital

- Readmission to intensive care unit

- Length of stay in skilled nursing facility

- Hospice enrollment

\section{Adverse Effects}

\section{Sedation - Postoperative Care}

\section{Haloperidol Versus Placebo}

One RCT, with low risk of bias and enrolling patients undergoing elective and emergent hip surgeries at risk of delirium, reported no evidence of sedation in those randomized to $1.5 \mathrm{mg}$ per day of oral perioperative haloperidol or placebo. ${ }^{37}$

We did not find any observational studies addressing sedation that compared haloperidol with placebo in postoperative patients at risk for delirium.

\section{Other Comparisons}

We found no studies of delirium prevention evaluating sedation in postoperative patients for the following comparisons:

- Second-generation antipsychotics versus placebo

- Haloperidol versus second-generation antipsychotics

- Second-generation antipsychotics versus second-generation antipsychotics

- Haloperidol versus other therapies

- Second-generation antipsychotics versus other therapies.

\section{Cardiac Effects - Postoperative Care}

\section{Haloperidol Versus Placebo}

One RCT, with low risk of bias and enrolling patients undergoing major thoracic non-cardiac surgery at risk of delirium, reported no evidence of cardiac side effects including arrhythmias or corrected QT interval prolongation in those randomized to intravenous haloperidol versus placebo. $^{46}$

\section{Second-Generation Antipsychotics Versus Placebo}

We identified three trials assessing different cardiac effects. ${ }^{36,39,40}$ One RCT, with low risk of bias, enrolled cardiac surgery patients with postoperative subsyndromal delirium, randomized to receive $1 \mathrm{mg}$ per day oral risperidone versus placebo, and reported no corrected QT interval prolongation in either arm. ${ }^{36}$ Two of the RCTs reported no statistically significant between-group differences in the occurrence of arrhythmias. One RCT, with low risk of bias and enrolling orthopedic surgery patients treated with $10 \mathrm{mg}$ oral perioperative olanzapine versus placebo, reported no statistically significant difference in arrhythmias or atrial fibrillation. ${ }^{39}$ One RCT, 
with high risk of bias, owing to a lack of blinding, and enrolling 126 cardiac surgery patients treated with a $1 \mathrm{mg}$ sublingual dose risperidone versus placebo, reported no statistically significant between-group differences in arrhythmias. ${ }^{40}$

We did not find any observational studies addressing cardiac effects that compared secondgeneration antipsychotics with placebo in postoperative patients at risk for delirium.

\section{Other Comparisons}

We found no studies of delirium prevention evaluating cardiac effects in a postoperative setting for the following comparisons:

- Haloperidol versus second-generation antipsychotics

- Second-generation antipsychotics versus second-generation antipsychotics

- Haloperidol versus other therapies

- Second-generation antipsychotics versus other therapies.

\section{Neurologic Effects - Postoperative Care}

\section{Haloperidol Versus Placebo}

Four RCTs compared haloperidol with placebo for the prevention of delirium in postoperative patients and reported neurologic outcomes. ${ }^{35,37,38,46}$ Two RCTs, with low risk of bias and enrolling patients undergoing elective and emergent hip surgeries and major non-cardiac thoracic surgery at risk of delirium, reported no statistically significant differences in extrapyramidal effects in patients randomized to haloperidol versus placebo. ${ }^{37,46}$

One RCT, with unclear risk of bias, randomized patients undergoing gastrointestinal surgery to an intervention arm receiving $5 \mathrm{mg}$ per day of intravenous haloperidol or a placebo group. This trial reported no evidence of extrapyramidal symptoms in patients in either arm of the study. ${ }^{38}$

One RCT, with high risk of bias owing to a lack of blinding and allocation concealment, enrolling patients aged 75 years and older undergoing elective digestive or orthopedic surgeries in an open-label study, randomized patients to $2.5 \mathrm{mg}$ per day of oral haloperidol or no drug. ${ }^{35}$ This study reported no significant between-group difference in the occurrence of convulsions.

We did not find any observational studies addressing neurologic effects that compared haloperidol with placebo in postoperative patients at risk for delirium.

\section{Second-Generation Antipsychotics Versus Placebo}

One RCT, with low risk of bias, enrolled cardiac surgery patients with postoperative subsyndromal delirium and randomized to receive $1 \mathrm{mg}$ per day of oral risperidone or placebo. This trial reported no statistically significant between-group differences in extrapyramidal side effects. $^{36}$

We did not find any observational studies addressing neurologic effects that compared second-generation antipsychotics with placebo in postoperative patients at risk for delirium.

\section{Other Comparisons}

We found no studies of delirium prevention evaluating neurologic effects in a postoperative setting for the following comparisons:

- Haloperidol versus second-generation antipsychotics

- Second-generation antipsychotics versus second-generation antipsychotics 
- Haloperidol versus other therapies

- Second-generation antipsychotics versus other therapies.

\section{Other Adverse Effects - Postoperative Care}

We found no studies in postoperative patients at risk for delirium that compared an antipsychotic with a placebo, another antipsychotic, or another treatment and evaluated the following adverse events:

- Weight gain

- Changes in appetite

- Hypersensitivity reactions

- Inappropriate continuation of antipsychotics

- Swallowing difficulties

- Aspiration pneumonia

\section{Key Question 2. Benefits and Harms of Antipsychotics To Treat Delirium}

\section{Key Points - Overall}

\section{Delirium Severity}

- There was little to no effect for haloperidol versus second-generation antipsychotics on delirium severity as evaluated by the Delirium Severity Rating Scale-Revised-98 (pooled mean between-group differences, 0.0; 95\% CI, -2.0 to 2.0). (SOE: Moderate)

- We were unable to draw a conclusion regarding the effects of antipsychotics on delirium severity for the following comparisons:

o Haloperidol versus placebo (insufficient evidence)

o Second-generation antipsychotics versus placebo (insufficient evidence)

o Second-generation antipsychotics versus second-generation antipsychotics (insufficient evidence)

o Haloperidol versus other therapies (insufficient evidence)

o Second-generation antipsychotics versus other therapies (no trials)

\section{Cognitive Functioning}

- There was no clinically important or statistically significant difference in Mini-Mental State Exam scores comparing haloperidol with second-generation antipsychotics (range in mean differences, 0.14 to 0.15). (SOE: Low)

- We were unable to draw a conclusion regarding the effects of antipsychotics on cognitive functioning for the following comparisons:

o Haloperidol versus placebo (no trials)

o Second-generation antipsychotics versus placebo (insufficient evidence)

o Second-generation antipsychotics versus second-generation antipsychotics (insufficient evidence)

o Haloperidol versus other therapies (insufficient evidence)

o Second-generation antipsychotics versus other therapies (no trials) 


\section{Length of Stay in Hospital}

- RCTs, conducted exclusively in critically ill patients, reported length of stay in hospital:

o Little to no difference comparing haloperidol versus placebo, with the largest trial reporting no clinically important difference (SOE: Moderate)

o Similar effects for second-generation antipsychotics versus placebo, with the largest trial reporting no clinically important difference (SOE: Moderate)

o No clinically important or statistically significant difference for haloperidol versus second-generation antipsychotics (SOE: Moderate)

- We were unable to draw a conclusion regarding the effects of antipsychotics on length of stay in hospital for the following comparisons:

o Second-generation antipsychotics versus second-generation antipsychotics (no trials)

o Haloperidol versus other therapies (insufficient evidence)

o Second-generation antipsychotics versus other therapies (no trials)

\section{Sedation}

- In critically ill patients, there was no clinically important or statistically significant difference in rates of sedation when comparing haloperidol with placebo. (SOE: Low)

- In trials that mostly enrolled patients with critical illness, there was no clinically important or statistically significant difference in rates of sedation for the following comparisons:

o Second-generation antipsychotics versus placebo (pooled RR, 1.10; 95\% CI, 0.78 to 1.53) (SOE: Moderate)

o Haloperidol versus second-generation antipsychotics (pooled RR, 1.26; 95\% CI, 0.92 to 1.72) (SOE: Moderate)

- We were unable to draw a conclusion regarding the effects of antipsychotics on sedation for the following comparisons:

o Second-generation antipsychotics versus second-generation antipsychotics (insufficient evidence)

o Haloperidol versus other therapies (no studies)

o Second-generation antipsychotics versus other therapies (no studies)

\section{Inappropriate Continuation of Antipsychotics}

- We found no trials evaluating inappropriate continuation of antipsychotics in patients with delirium that compared an antipsychotic with a placebo, another antipsychotic, or another treatment.

\section{Intermediate Outcomes}

\section{Delirium Incidence - Overall}

\section{Second-Generation Antipsychotics Versus Placebo}

One RCT, with low risk of bias and enrolling patients admitted to a medical or surgical intensive care unit, compared quetiapine with placebo. This trial reported no statistically significant effect on delirium incidence within 14 days. ${ }^{52}$ 


\section{Other Comparisons}

We found no delirium treatment trials evaluating delirium incidence for the following comparisons:

- Haloperidol versus placebo

- Haloperidol versus second-generation antipsychotics

- Second-generation antipsychotics versus second-generation antipsychotics

- Haloperidol versus other therapies

- Second-generation antipsychotics versus other therapies

\section{Short-Term Delirium Symptoms - Overall}

\section{Haloperidol Versus Placebo}

One RCT, with low risk of bias, compared haloperidol with placebo in critically ill patients receiving mechanical ventilation and enrolled within 72 hours of intensive care unit admission. ${ }^{45}$ The trial evaluated short-term delirium symptom of agitation, defined as a Richmond AgitationSedation Scale score of greater than or equal to +2 , and reported no statistically significant effect on agitation.

\section{Second-Generation Antipsychotics Versus Placebo}

Two RCTs, ${ }^{52,63}$ with small sample sizes and low risk of bias, compared quetiapine with placebo and provided imprecise and inconsistent evidence. Each trial used different measures of short-term delirium symptoms. One of these trials enrolled critically ill patients and reported a potentially clinically important fewer hours of Sedation-Agitation Scale score of equal to or greater than five for the quetiapine group; ${ }^{52}$ the other trial enrolled non-critically ill patients ${ }^{63}$ and reported no statistically significant difference using the Brief Psychiatric Rating Scale.

\section{Haloperidol Versus Second-Generation Antipsychotics}

Two RCTs compared second-generation antipsychotics with haloperidol using different measures of sleep duration. ${ }^{54,62}$ One trial, with low risk of bias, enrolled inpatients with hyperactive delirium. ${ }^{62}$ The other trial, a three-arm RCT with unclear risk of bias, compared olanzapine, risperidone, and haloperidol in non-critically ill inpatients. ${ }^{54}$ These two trials, each with a small sample size, provided consistent but imprecise evidence of no significant difference in sleep duration for second-generation antipsychotics compared with haloperidol.

\section{Second-Generation Antipsychotics Versus Second-Generation Antipsychotics}

One three-arm RCT, with unclear risk of bias, compared olanzapine with risperidone in noncritically ill inpatients. ${ }^{54}$ This trial evaluated the short-term delirium symptom of increased duration of sleep and reported no statistically significant effect on this outcome for risperidone compared with olanzapine.

\section{Other Comparisons}

We found no delirium treatment trials evaluating short-term delirium symptoms in patients with delirium for the following comparisons:

- Haloperidol versus other therapies

- Second-generation antipsychotics versus other therapies 


\section{Delirium Severity - Overall}

\section{Haloperidol Versus Placebo}

Two three-arm RCTs comparing haloperidol with placebo assessed delirium severity using different instruments in different patient populations with inconsistent results. ${ }^{48,57}$ (SOE: Insufficient)

\section{Second-Generation Antipsychotics Versus Placebo}

Three RCTs compared three different second-generation antipsychotics with placebo, assessing delirium severity using different instruments in different patient populations, with inconsistent results. ${ }^{48,57,63}$ (SOE: Insufficient)

\section{Haloperidol Versus Second-Generation Antipsychotics}

Eight RCTs comparing haloperidol with three different second-generation antipsychotics (olanzapine, risperidone, and quetiapine) assessed delirium severity using different instruments in different patient populations. ${ }^{54-58,61,62,64}$ Six of the eight RCTs enrolled medical and surgical inpatients with and without critical illness, ${ }^{54-58,64}$ one enrolled patients receiving palliative care, ${ }^{61}$ and one enrolled inpatients with hyperactive delirium. ${ }^{62}$ Two of the eight RCTs had high risk of bias, primarily owing to a lack of blinding and missing outcome data, ${ }^{58,61}$ and three had low risk of bias. ${ }^{55,62,64}$ The three remaining RCTs had unclear risk of bias. ${ }^{54,56,57}$ Across these trials comparing haloperidol with second-generation antipsychotics, there was no clinically important or statistically significant difference of delirium severity comparing second-generation antipsychotics with haloperidol. (SOE: Moderate)

We conducted a meta-analysis of the five trials that evaluated delirium severity using Delirium Rating Scale-Revised-98 or the Memorial Delirium Assessment Scale that we converted to a Delirium Rating Scale-Revised-98 score (range, 0 to 48). ${ }^{54-56,62,64}$ One of the four RCTs was a three-arm trial with unclear risk of bias that compared both olanzapine and risperidone with haloperidol in a total of 64 inpatients without critical illness; we used the olanzapine with haloperidol comparison in our meta-analysis. ${ }^{54}$ The meta-analysis showed no statistically significant difference in delirium severity (pooled mean between-group difference, 0.0; 95\% CI, -2.0 to 2.0; I-squared, 25\%) (Figure 16). We tested the effect of each individual trial on the combined point estimate; no single trial influenced the pooled results. No substantial statistical heterogeneity was identified. We repeated the meta-analysis using the data for a comparison of risperidone with haloperidol for the one three-arm RCT, ${ }^{54}$ and the results were similar to the comparison of olanzapine with haloperidol, with a very small non-statistically significant between-group difference in the reduction of delirium severity (pooled mean between-group difference, -0.3; 95\% CI, -2.4 to 1.7; I-squared, 11\%) (Figure 16).

Three RCTs with a total of 257 patients evaluated change in delirium severity using the Clinical Global Impression scale (range, 1 to 7). ${ }^{53,57,58}$ We could not conduct a meta-analysis because these trials did not use the same measure from the Clinical Global Impression scale. Across the three RCTs, there was no clinically important or statistically significant difference in delirium severity or improvement.

We were unable to combine the following two RCTs owing to the use of heterogeneous delirium severity rating scales. The Delirium Rating Scale is a different instrument than the revised version (Delirium Rating Scale-Revised-98) and there is no crosswalk to convert the scores. Two RCTs evaluating delirium severity using the Delirium Rating Scale compared 
olanzapine with haloperidol and reported no statistically significant difference in delirium severity reduction from baseline..$^{57,61}$

One RCT, with high risk of bias, evaluated delirium severity using the Memorial Delirium Assessment Scale. ${ }^{58}$ We were unable to include this trial in the meta-analysis because it did not report sufficiently detailed information. This RCT compared olanzapine with haloperidol in inpatients and reported similar delirium severity at the end of the study for olanzapine compared with haloperidol. Using the same scale, the RCT also reported a reduction in delirium severity for patients receiving olanzapine compared with those receiving haloperidol over the study period.

Three RCTs evaluated the percentage of patients with Delirium Rating Scale-Revised-98 scores of less than a threshold of either 10 or 12 (indicating lower delirium symptoms), comparing a second-generation antipsychotic (e.g., quetiapine, olanzapine, and risperidone) with haloperidol. ${ }^{54,55,62}$ These RCTs demonstrated no statistically significant difference in percentage of patients with Delirium Rating Scale-Revised-98 scores of less than this threshold (pooled RR, 0.96; $95 \%$ CI, 0.80 to 1.16; I-squared, $0.0 \%$ ) (Figure 17). We tested the effect of each individual study on the combined point estimate. No single study influenced the pooled results. No substantial statistical heterogeneity was identified.

One three-arm RCT, with unclear risk of bias, compared olanzapine and haloperidol in a total of 175 inpatients aged 65 years and older and reported no statistically significant difference in the percentage of patients with "significant improvement" or "complete alleviation" on the Clinical Global Impression scale for olanzapine (82\%) compared with haloperidol (88\%) (RR, 0.94; $95 \%$ CI, 0.82 to 1.08$).{ }^{57}$ 
Figure 16. Meta-analysis of delirium severity scores as measured by the DRS-R-98* in trials comparing haloperidol with second-generation antipsychotics in patients with delirium

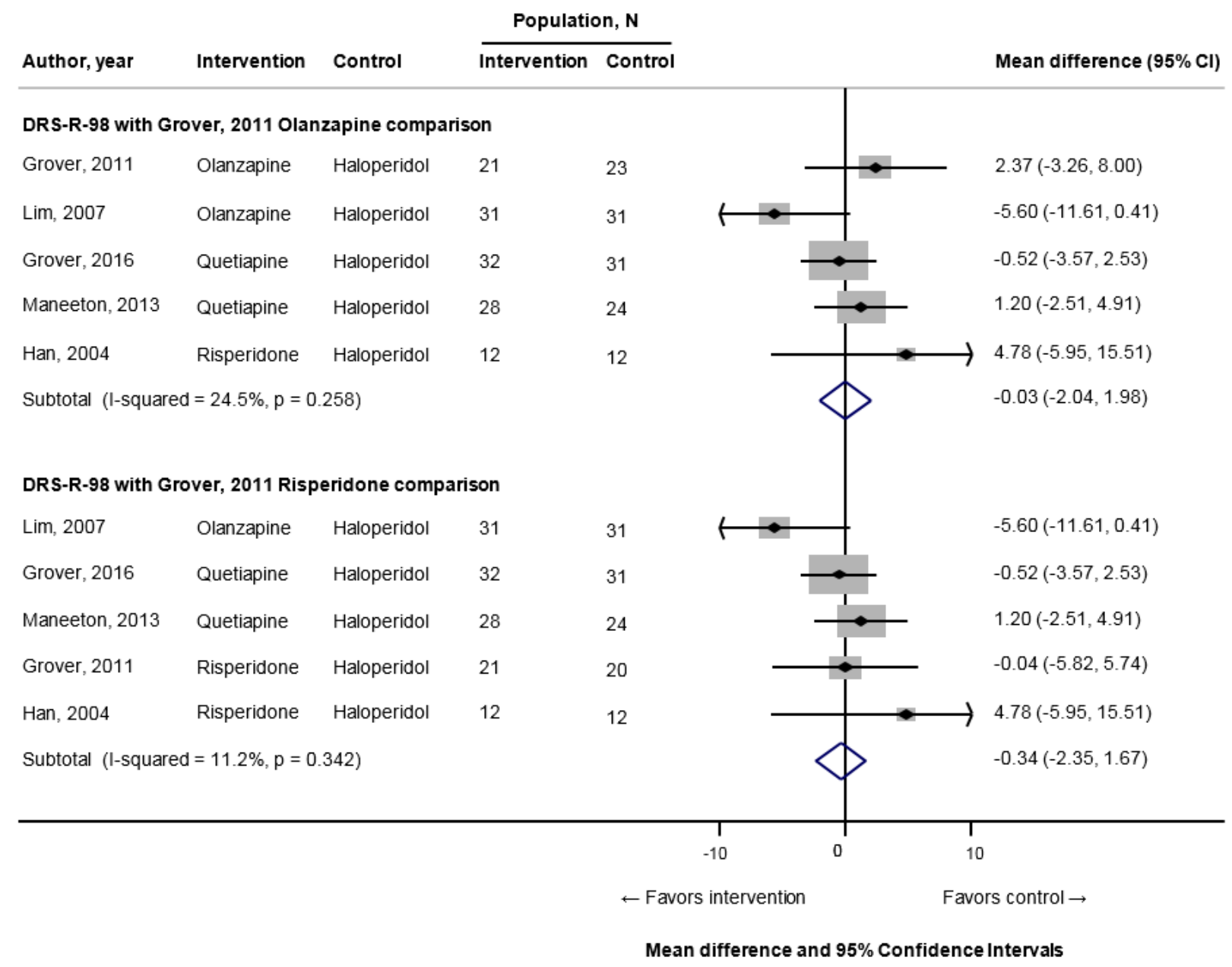

* Han, 2004 assessed delirium severity with the Memorial Delirium Assessment Scale. We converted these scores to the DRS-R98.

CI = confidence interval; DRS-R-98 = Delirium Rating Scale-Revised-98; N = sample size; p = p-value. 
Figure 17. Meta-analysis of patients with delirium severity scores below identified threshold in trials comparing haloperidol with second-generation antipsychotics in patients with delirium

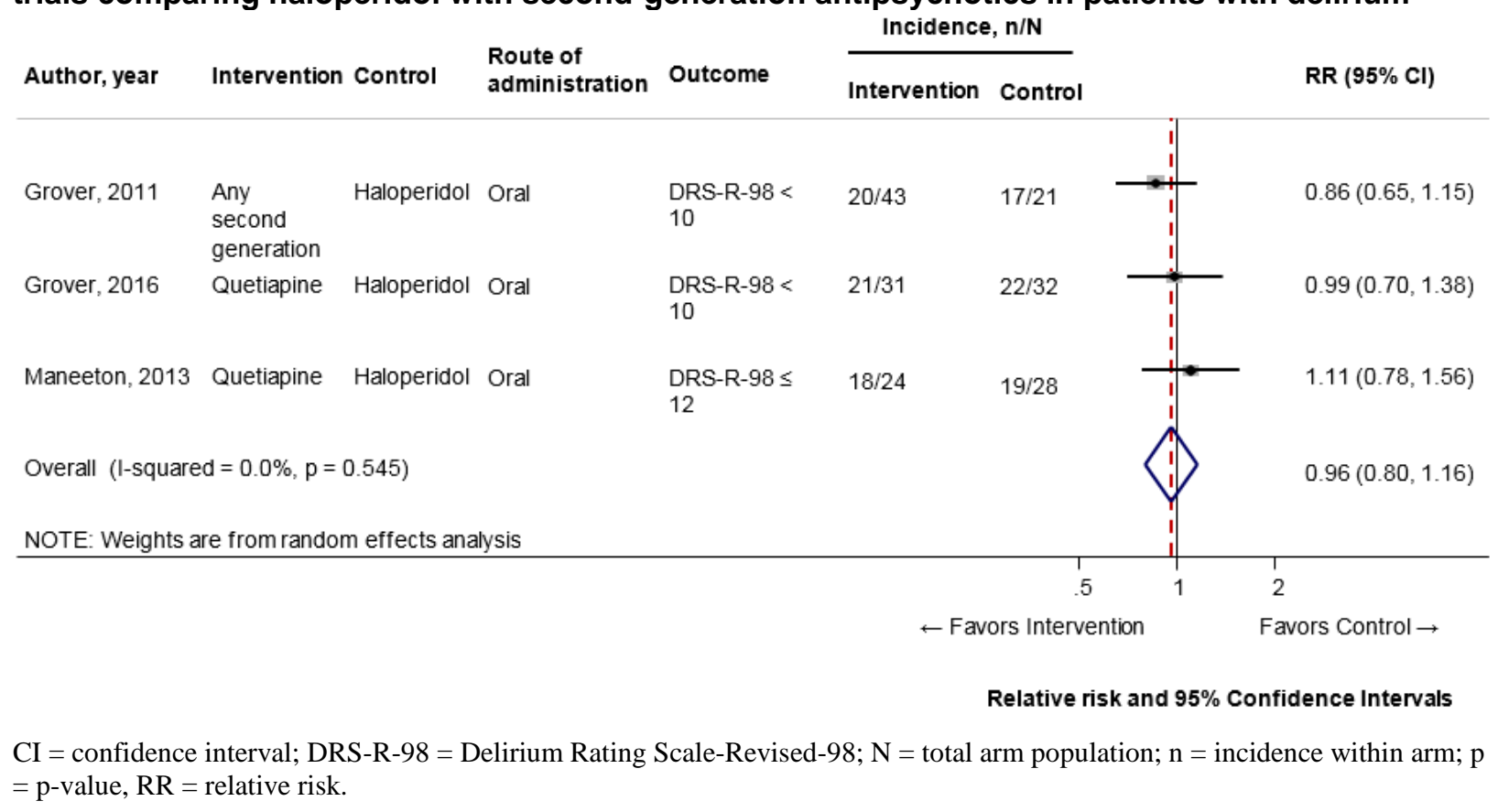

\section{Second-Generation Antipsychotics Versus Second-Generation Antipsychotics}

Three RCTs compared different second-generation antipsychotics and evaluated delirium severity using the Delirium Rating Scale-Revised-98. ${ }^{54,59,60}$ We were unable to conduct a metaanalysis because one of the trials did not report sufficient data. Across all three RCTs, with a total of 127 patients, there was no statistically significant difference in second-generation antipsychotics compared with other second-generation antipsychotics. However, the first RCT had unclear risk of bias, ${ }^{54}$ and the other two RCTs, with a total of 72 inpatients, had high risk of bias owing to a lack of blinding or missing outcome data; ${ }^{59,60}$ we were unable to draw a conclusion. (SOE: Insufficient)

\section{Haloperidol Versus Other Therapies}

Two RCTs, with a total of 126 patients, each evaluating a different patient population and using a different delirium severity instrument, compared haloperidol with three different therapies. ${ }^{50,51}$ We were unable to draw a conclusion owing to the small sample size and consequent lack of precision, comparisons with different types of therapies, and the use of different delirium severity measurement instruments. (SOE: Insufficient)

\section{Second-Generation Antipsychotics Versus Other Therapies}

We found no trials evaluating delirium severity in patients with delirium that compared second-generation antipsychotics with other therapies.

\section{Strength of Evidence}

Table 17 summarizes the strength of evidence for antipsychotics for the treatment of delirium in terms of delirium severity. 
Table 17. Strength of evidence domains for trials evaluating antipsychotics for the treatment of delirium in terms of delirium severity

\begin{tabular}{|c|c|c|c|c|c|c|c|c|}
\hline Comparison & $\begin{array}{l}\text { Number Of } \\
\text { Trials (N) }\end{array}$ & $\begin{array}{c}\text { Study } \\
\text { Limitations }\end{array}$ & Directness & Consistency & Precision & $\begin{array}{c}\text { Reporting } \\
\text { Bias }\end{array}$ & $\begin{array}{l}\text { Strength Of } \\
\text { Evidence }\end{array}$ & Summary \\
\hline $\begin{array}{l}\text { Haloperidol vs. } \\
\text { placebo }\end{array}$ & $2^{48,57}(424)$ & Medium & Indirect & Inconsistent & Imprecise & Undetected & Insufficient & $\begin{array}{l}\text { We were unable to draw a } \\
\text { conclusion. }\end{array}$ \\
\hline $\begin{array}{l}\text { Second- } \\
\text { generation } \\
\text { antipsychotics } \\
\text { vs. placebo }\end{array}$ & $\begin{array}{l}3^{48,57,63} \\
(466)\end{array}$ & Medium & Direct & Inconsistent & Imprecise & Undetected & Insufficient & $\begin{array}{l}\text { We were unable to draw a } \\
\text { conclusion. }\end{array}$ \\
\hline $\begin{array}{l}\text { Haloperidol vs. } \\
\text { second- } \\
\text { generation } \\
\text { antipsychotics }\end{array}$ & $\begin{array}{l}8^{54-58,61,62,64} \\
(570)\end{array}$ & Medium & Direct & Consistent & Precise & Undetected & Moderate & $\begin{array}{l}\text { Haloperidol vs. second- } \\
\text { generation antipsychotics had } \\
\text { no clinically important or } \\
\text { statistically significant } \\
\text { differences (pooled mean } \\
\text { between-group differences for } \\
\text { DRS-R-98 (5 trials): } 0.0 ; 95 \% \\
\text { Cl, }-2.0 \text { to } 2.0 \text { ). }\end{array}$ \\
\hline $\begin{array}{l}\text { Second- } \\
\text { generation } \\
\text { antipsychotics } \\
\text { vs. second- } \\
\text { generation } \\
\text { antipsychotics }\end{array}$ & $\begin{array}{l}3^{54,59,60} \\
(127)\end{array}$ & High & Direct & Consistent & Imprecise & Undetected & Insufficient & $\begin{array}{l}\text { We were unable to draw a } \\
\text { conclusion. }\end{array}$ \\
\hline $\begin{array}{l}\text { Haloperidol vs. } \\
\text { other therapies }\end{array}$ & $2^{50,51}(126)$ & Medium & Indirect & Unknown* & Imprecise & Undetected & Insufficient & $\begin{array}{l}\text { We were unable to draw a } \\
\text { conclusion. }\end{array}$ \\
\hline $\begin{array}{l}\text { Second- } \\
\text { generation } \\
\text { antipsychotics } \\
\text { vs. other } \\
\text { therapies }\end{array}$ & No trials & & & & & & & \\
\hline
\end{tabular}

* Consistency is unknown since each "other” therapy comparator group (i.e., lorazepam, ondansetron, and dexmedetomidine) was not replicated in a second RCT. Abbreviations: CGI = Clinical Global Impression; CI = confidence interval; DRS-R-98 = Delirium Rating Scale-Revised-98; RCT = randomized controlled trial. 


\section{Delirium- and Coma-Free Days Alive - Overall}

\section{Haloperidol Versus Placebo}

Three RCTs, with low risk of bias, compared haloperidol with placebo and assessed delirium- and coma-free days alive at different followup times among populations of medical and surgical patients in intensive care units. ${ }^{44,45,53}$ All three trials provided consistent evidence of no difference in delirium- and coma-free days alive comparing haloperidol with placebo. We did not conduct a meta-analysis because these trials generally provided results as median values, and the data for delirium- and coma-free days alive may be skewed, precluding transformation of the data.

\section{Second-Generation Antipsychotics Versus Placebo}

Two RCTs, with low risk of bias, compared ziprasidone with placebo at different followup times among populations of medical and surgical patients in intensive care units. Both welldesigned RCTs demonstrated consistent evidence of no statistically significant effect of ziprasidone on delirium- and coma-free days alive. ${ }^{44,53}$

\section{Haloperidol Versus Second-Generation Antipsychotics}

Two RCTs, with low risk of bias, compared ziprasidone with haloperidol at different followup times among populations of medical and surgical patients in intensive care units. ${ }^{44,53}$ There was consistent and precise evidence of no difference in delirium- and coma-free days alive.

\section{Other Comparisons}

We found no delirium treatment trials evaluating delirium- and coma-free days alive for the following comparisons:

- Second-generation antipsychotics versus second-generation antipsychotics

- Haloperidol versus other therapies

- Second-generation antipsychotics versus other therapies

\section{Duration of Delirium - Overall}

\section{Haloperidol Versus Placebo}

Three RCTs, with low risk of bias, compared haloperidol with placebo and assessed delirium duration in medical and surgical patients in intensive care units. ${ }^{44,45}, 53$ There was consistent and precise evidence of no statistically significant effect on delirium duration for haloperidol compared with placebo. We did not conduct a meta-analysis because these trials generally provided results as median values and delirium duration data may be skewed, precluding conversion or transformation of the data.

\section{Second-Generation Antipsychotics Versus Placebo}

Three RCTs, with low risk of bias, compared a second-generation antipsychotic with placebo and assessed delirium duration in medical and surgical patients in intensive care units. ${ }^{44,52,53}$ There was consistent and precise evidence of no statistically significant effect of secondgeneration antipsychotics on delirium duration. We did not conduct a meta-analysis because 
these trials generally provided results as median values and delirium duration data may be skewed, precluding conversion or transformation of the data.

\section{Haloperidol Versus Second-Generation Antipsychotics}

Six RCTs, with various risk of bias, assessed the effect of haloperidol on duration of delirium compared with quetiapine, ziprasidone, risperidone, or olanzapine in medical and surgical inpatients both with and without critical illness. ${ }^{44,53,56,58,62,64}$ Two of the RCTs, with low risk of bias and enrolling critically ill patients ( $\mathrm{N}=566$ and $\mathrm{N}=101)$, reported no statistically significant effect on delirium duration; ${ }^{44,53}$ these trials were not included in our meta-analysis because results were provided only as median values and delirium duration data may be skewed, precluding conversion or transformation. However, our meta-analysis of the four RCTs reporting mean values ${ }^{56,58,62,64}$ showed a non-clinically important increase in number of days with delirium for second-generation antipsychotics compared with haloperidol (pooled mean between-group difference, 0.2; 95\% CI, 0.0 to 0.4; I-squared, 38\%) (Figure 18). There was consistent and precise evidence of little to no difference in delirium duration for haloperidol compared with second-generation antipsychotics.

\section{Second-Generation Antipsychotics Versus Second-Generation Antipsychotics}

One RCT, with high risk of bias primarily owing to a lack of blinding, compared amisulpride with quetiapine and assessed delirium duration in 31 inpatients. This RCT reported no statistically significant effect for amisulpride compared with quetiapine. ${ }^{60}$

\section{Haloperidol Versus Other Therapies}

One RCT, with unclear risk of bias, compared haloperidol with morphine and assessed delirium duration in post-cardiac surgery patients with hyperactive delirium and reported no statistically significant effect for haloperidol compared with morphine. ${ }^{49}$

\section{Second-Generation Antipsychotics Versus Other Therapies}

We found no trials evaluating delirium duration that compared second-generation antipsychotics with other therapies. 
Figure 18. Meta-analysis of duration of delirium in trials comparing haloperidol with secondgeneration antipsychotics in patients with delirium

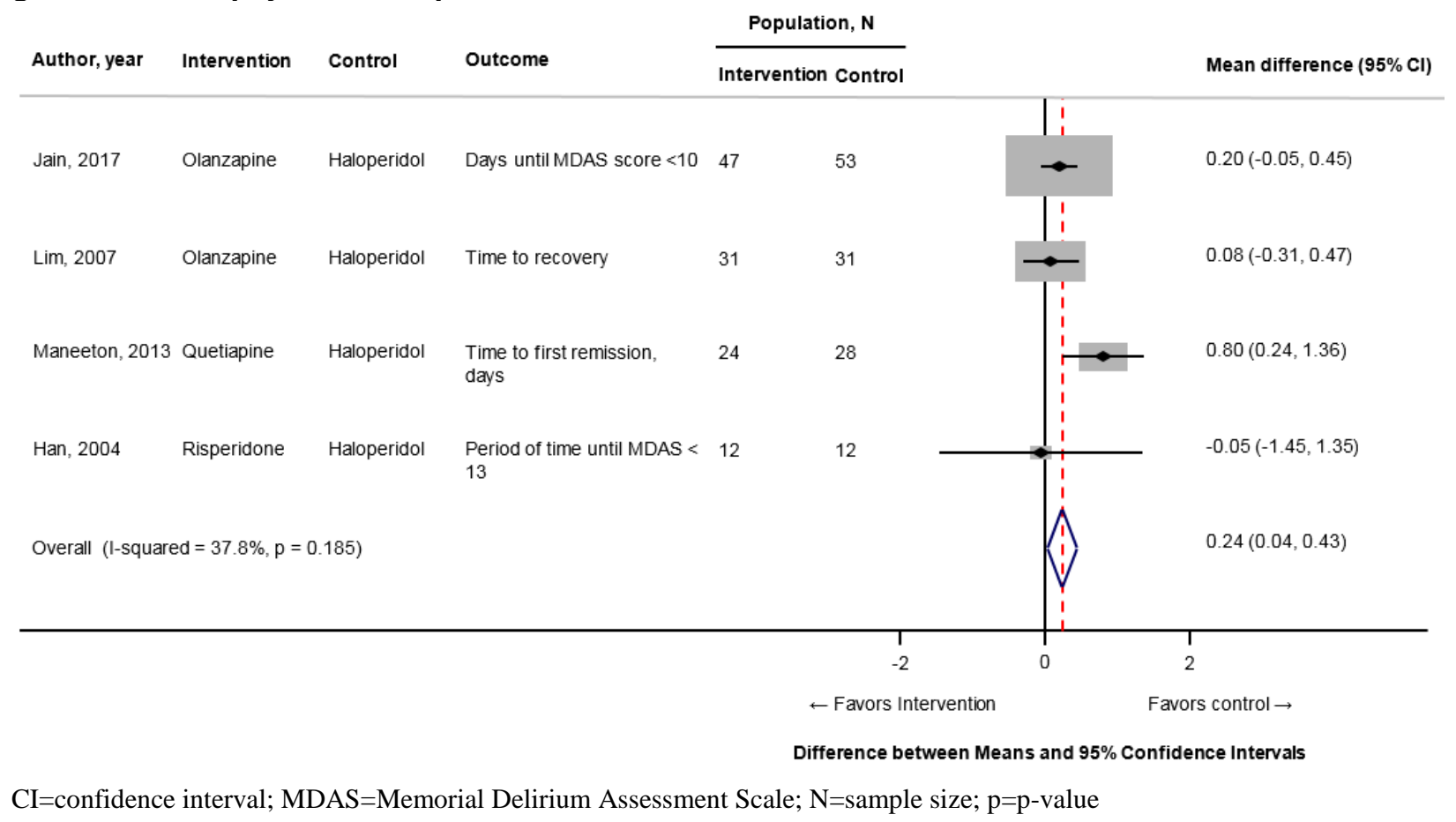

\section{Use of Rescue Therapy - Overall}

\section{Haloperidol Versus Placebo}

Three RCTs, with low risk of bias, compared haloperidol with placebo and reported on rescue therapy, defined as the use of any open-label antipsychotic. ${ }^{44,45,53}$ All three RCTs, two of which were three-arm trials, ${ }^{44,53}$ enrolled medical and surgical critically ill patients. We found a non-statistically significant decrease in the use of rescue therapy in the haloperidol group (pooled RR, 0.63; 95\% CI, 0.34 to 1.15; I-squared, 62\%) (Figure 19). This is a possibly relevant decrease but the potential impact is difficult to assess. Excluding the largest RCT ${ }^{53}$ changed the inference of the meta-analysis, suggesting a statistically significant decrease in the use of rescue therapy in patients treated with haloperidol (pooled RR with this RCT removed, 0.44; 95\% CI, 0.25 to 0.77). This trial was not qualitatively different from the other two smaller RCTs, so we retained it in the overall meta-analysis. Owing to the substantial statistical heterogeneity, we also conducted a profile likelihood method of meta-analysis and found a similar non-statistically significant decrease in the use of rescue therapy in the haloperidol group (pooled RR, 0.66 95\% CI, 0.30 to 1.19; I-squared, 42\%). There was consistent but imprecise evidence of little decrease in the use of rescue therapy for haloperidol. 
Figure 19. Meta-analysis of the use of rescue therapy in trials comparing haloperidol with placebo in patients with delirium

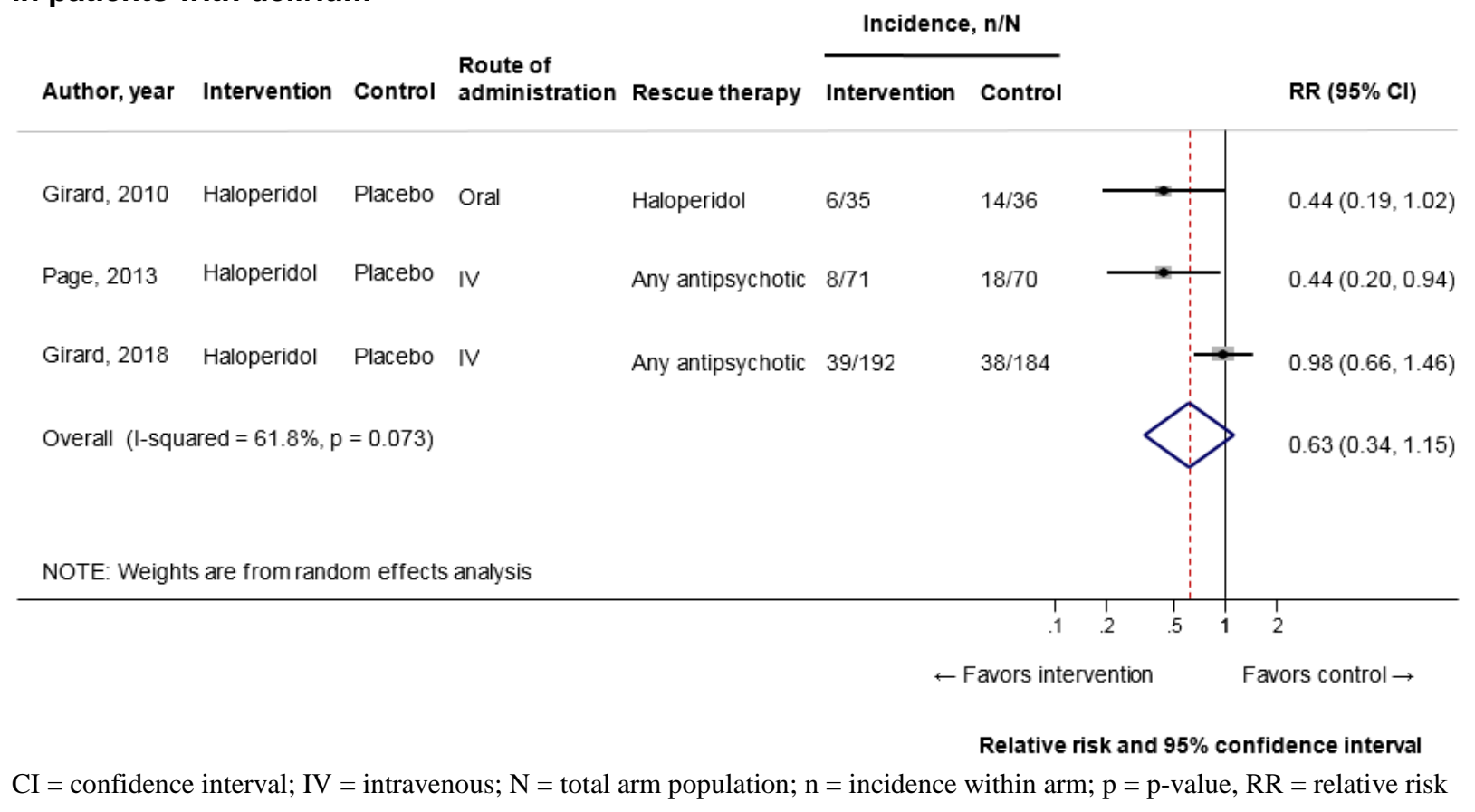

\section{Second-Generation Antipsychotics Versus Placebo}

Two three-arm RCTs, with low risk of bias, compared ziprasidone with placebo and reported on rescue therapy, defined as the use of any open-label antipsychotic. ${ }^{44,53}$ Both RCTs, enrolling medical and surgical critically ill patients, reported no effect of ziprasidone on the use of rescue therapy. The evidence from these trials was consistent but imprecise.

\section{Haloperidol and Second-Generation Antipsychotics Versus Placebo}

One three-arm RCT, with low risk of bias, compared haloperidol and risperidone with placebo and reported on rescue therapy, defined as per-protocol use of midazolam. ${ }^{48}$ This trial, enrolling patients receiving palliative care, reported a statistically significant decrease in the use of rescue therapy in the placebo group compared with the combined haloperidol and risperidone groups on all three study days. ${ }^{48}$ Because data was only reported for the combination of risperidone and haloperidol, we were unable to determine if results would be similar if data were collected for each of these two antipsychotic medications separately. However, for the use of rescue therapy, findings from this one RCT appear to favor avoidance of antipsychotics in patients receiving palliative care.

\section{Haloperidol Versus Second-Generation Antipsychotics}

Three RCTs, enrolling critically ill patients and patients receiving palliative care, compared haloperidol with two second-generation antipsychotics (ziprasidone and olanzapine). Two ${ }^{44,53}$ of the RCTs had low risk of bias and one had potential for a high risk of bias. ${ }^{61}$ Across all three RCTs, there was no evidence of statistically significant differences between haloperidol and second-generation antipsychotics in the use of rescue therapies. ${ }^{44,53,61}$ There was consistent but imprecise evidence of no statistically significant difference in the use of rescue therapy for haloperidol compared with second-generation antipsychotics. 


\section{Second-Generation Antipsychotics Versus Second-Generation Antipsychotics}

One RCT, with high risk of bias, compared risperidone with olanzapine in inpatients and reported non-statistically significant increases in the use of two separate rescue therapies (haloperidol and any benzodiazepine) for risperidone compared with olanzapine. ${ }^{59}$

\section{Haloperidol Versus Other Therapies}

One three-arm RCT, with low risk of bias, compared haloperidol with ondansetron and dexmedetomidine in trauma patients in an intensive care unit and reported on rescue therapy, defined as the use of open-label haloperidol. ${ }^{50}$ When comparing haloperidol with ondansetron, the RCT reported a statistically significant decrease in the use of rescue therapy. When comparing haloperidol with dexmedetomidine, the RCT reported a non-statistically significant decrease in the use of rescue therapy.

\section{Second-Generation Antipsychotics Versus Other Therapies}

We found no trials evaluating the use of rescue therapy in patients with delirium that compared second-generation antipsychotics with other therapies.

\section{Other Intermediate Outcomes - Overall}

We found no trials enrolling patients with delirium that compared an antipsychotic with a placebo, another antipsychotic, or another treatment and evaluated the following intermediate outcomes:

- $\quad$ Patient distress

- Use of physical restraint

\section{Final Health or Patient-Centered Outcomes}

\section{Mortality - Overall}

\section{Haloperidol Versus Placebo}

Four RCTs, with low risk of bias, compared haloperidol with placebo and showed no between-group difference in short-term mortality, defined as mortality while in hospital or up to 30 days after randomization (pooled RR, 0.98; 95\% CI, 0.75 to 1.27; I-squared, 0\%) (Figure 20). ${ }^{44,45,48,53}$ We tested the effect of each individual trial on the combined point estimate. No single trial influenced the pooled results. No substantial statistical heterogeneity was identified. Three of the four RCTs enrolled critically ill patients, ${ }^{44,45,53}$ and the fourth RCT enrolled a total of 249 patients receiving palliative care. ${ }^{48}$ Excluding the RCT enrolling patients receiving palliative care did not result in any material change to the pooled results. There was consistent but imprecise evidence of no statistically significant difference in short-term mortality for haloperidol compared with placebo.

Notably, the one RCT with low risk of bias and enrolling patients receiving palliative care was a three-arm trial and performed a time-to-event analysis that included comparison of haloperidol versus placebo. This comparison showed a decreased survival rate for the haloperidol group (median survival time, 16 vs. 26 days; hazard ratio [HR], 1.73; 95\% CI, 1.20 to $2.50 ; P=0.003) .{ }^{48}$ However, in an analysis of short-term mortality associated with the 72 hour treatment duration, there was no statistically significant effect when comparing haloperidol 
with placebo (RR, 1.17; 95\% CI, 0.47 to 2.88). Thus, it is unclear how much of the longer-term mortality risk is attributable to haloperidol use during the trial period.

Of these four RCTs, the largest trial was a three-arm trial with low risk of bias that also evaluated 90-day mortality in a total of 566 critically ill patients. ${ }^{53}$ The results of this evaluation were consistent with our meta-analysis results for short-term mortality with no between-group difference in comparing haloperidol with placebo.

Figure 20. Meta-analysis of mortality in trials comparing haloperidol with placebo in patients with delirium

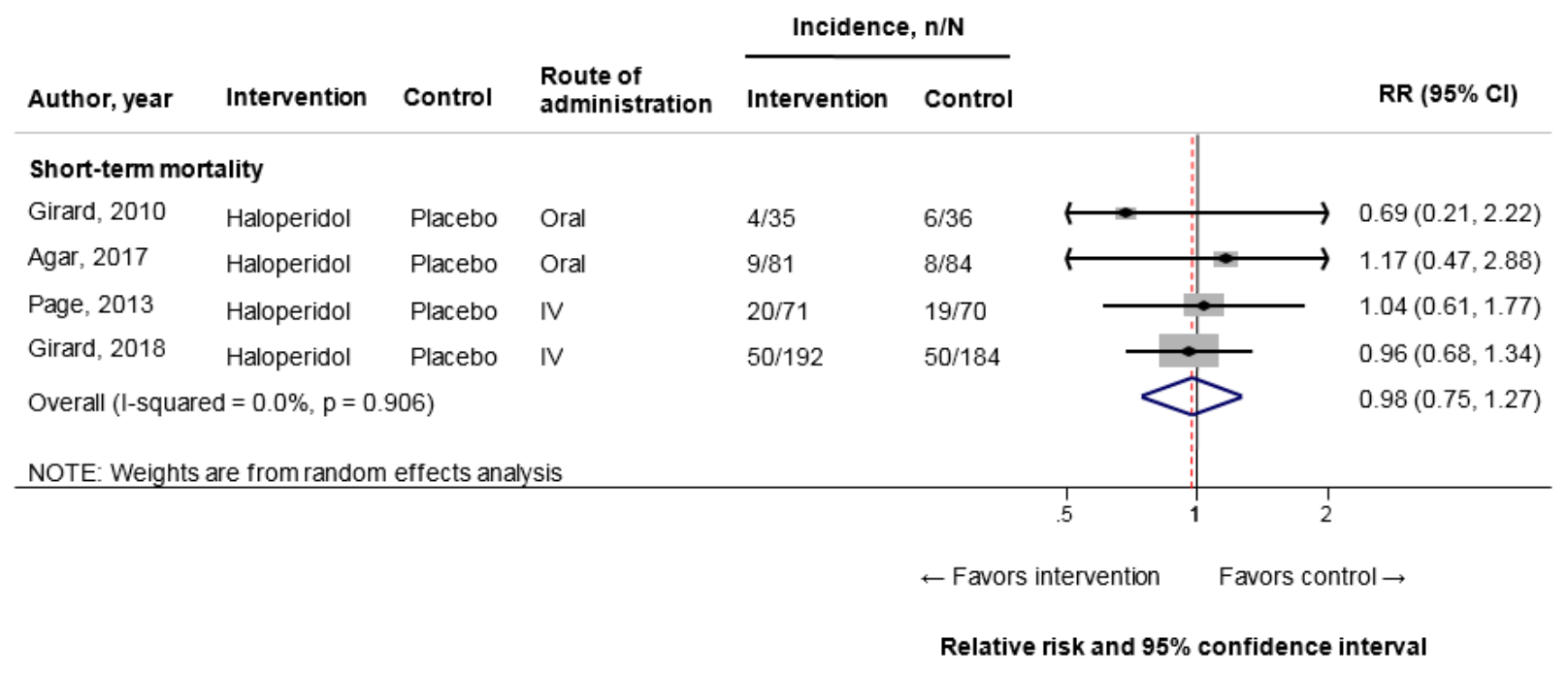

$\mathrm{CI}$ = confidence interval; $\mathrm{IV}=$ intravenous; $\mathrm{N}$ = total arm population; $\mathrm{n}=$ incidence within arm; $\mathrm{p}=\mathrm{p}$-value, $\mathrm{RR}=$ relative risk.

\section{Second-Generation Antipsychotics Versus Placebo}

Our meta-analysis of five RCTs, with low risk of bias, found no between-group difference in short-term mortality, defined as mortality while in hospital or up to 30 days after randomization, for a second-generation antipsychotic compared with placebo (pooled RR, 1.09; 95\% CI, 0.83 to 1.45; I-squared, 0\%) (Figure 21). ${ }^{44,48,52,53,63}$ Two of the RCTs evaluated quetiapine and ziprasidone; three of the RCTs evaluated risperidone. We tested the effect of each individual trial on the combined point estimate. No single trial influenced the pooled results. No substantial statistical heterogeneity was identified. Three of the five RCTs enrolled medical and surgical critically ill patients; ${ }^{44,52,53}$ the others enrolled medical and surgical inpatients without critical illness ${ }^{63}$ and patients receiving palliative care. ${ }^{48}$ Excluding the RCT that enrolled patients receiving palliative care ${ }^{48}$ did not result in any material change to the pooled results. There was consistent but imprecise evidence of no statistically significant difference in short-term mortality for second-generation antipsychotics compared with placebo.

The one RCT that enrolled patients receiving palliative care performed a time-to-event analysis comparing risperidone with placebo. This three-arm RCT reported a non-statistically significant decrease in survival for the risperidone group (median survival time, 17 vs. 26 days; HR, 1.29; $95 \%$ CI, 0.91 to $1.84 ; P=0.14) .{ }^{48}$ In an analysis of short-term mortality associated with the 72-hour treatment duration there also was no statistically significant effect when comparing risperidone with placebo (RR, 1.82; 95\% CI, 0.85 to 3.89).

Of these five RCTs, the largest was a three-arm, low risk of bias trial that also evaluated 90day mortality in a total of 566 critically ill patients. ${ }^{53}$ The results of this evaluation were 
consistent with the meta-analysis results for short-term mortality, with no between-group difference reported when comparing risperidone with placebo.

Figure 21. Meta-analysis of mortality in trials comparing second-generation antipsychotics with placebo in patients with delirium

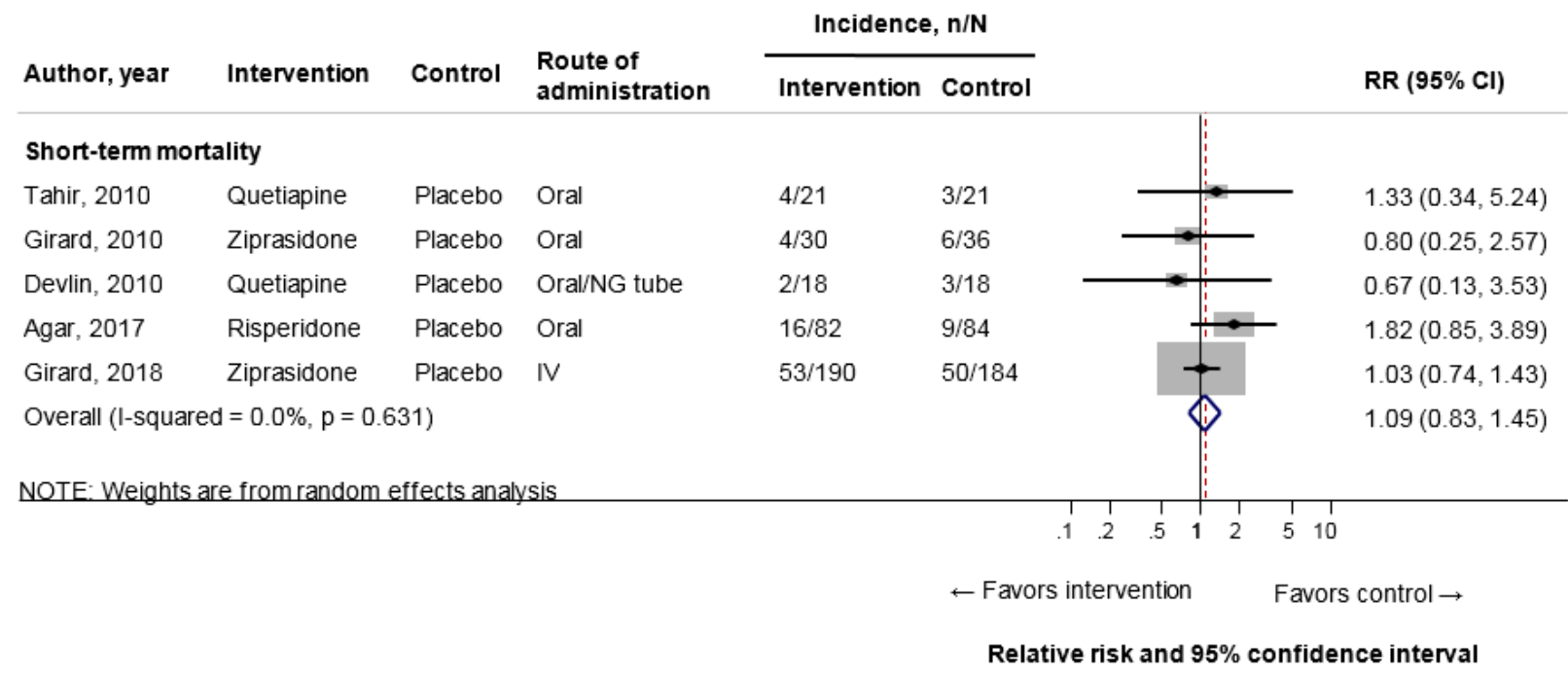

$\mathrm{CI}=$ confidence interval; IV = intravenous; $\mathrm{N}=$ total arm population; $\mathrm{n}=$ incidence within $\mathrm{arm}$; $\mathrm{NG}=$ nasogastric tube; $\mathrm{p}=\mathrm{p}-$ value, $\mathrm{RR}=$ relative risk.

\section{Haloperidol Versus Second-Generation Antipsychotics}

Our meta-analysis of six RCTs, with seven comparisons of haloperidol with a secondgeneration antipsychotic, showed no between-group difference in short-term mortality, defined as mortality while in hospital or up to 30 days after randomization (pooled RR, 1.17; 95\% CI, 0.89 to 1.55; I-squared, $0 \%$ ) (Figure 22). ${ }^{44,48,53,54,58,62}$ One of these seven comparisons evaluated quetiapine and each of the following second-generation antipsychotics were evaluated twice: risperidone, olanzapine, and ziprasidone. Four of the six RCTs were low risk of bias; ${ }^{44,48,}$ 53, 62 one RCT had unclear risk of bias; ${ }^{54}$ and one RCT was high risk of bias, primarily owing to a lack of blinding and missing outcome data. ${ }^{58}$ We tested the effect of each individual trial on the combined point estimate. No single trial influenced the pooled results. No substantial statistical heterogeneity was identified. Five of the six RCTs enrolled medical and surgical inpatients, ${ }^{44,53,}$ 54, 58, 62 with two of these five RCTs specifically focused on critically ill patients. ${ }^{44,53}$ The remaining RCT enrolled patients receiving palliative care. ${ }^{48}$ Excluding the RCT that enrolled patients receiving palliative care did not result in any material change to the pooled results. There was consistent but imprecise evidence of no statistically significant difference in short-term mortality for second-generation antipsychotics compared with haloperidol.

Of these six RCTs, the largest was a three-arm, low risk of bias trial that also evaluated 90day mortality. This RCT compared haloperidol with ziprasidone in a total of 566 critically ill patients. The results of this RCT were consistent with the results of our meta-analysis, reporting no between-group difference for short-term mortality. ${ }^{53}$ 
Figure 22. Meta-analysis of mortality in trials comparing haloperidol with second-generation antipsychotics in patients with delirium

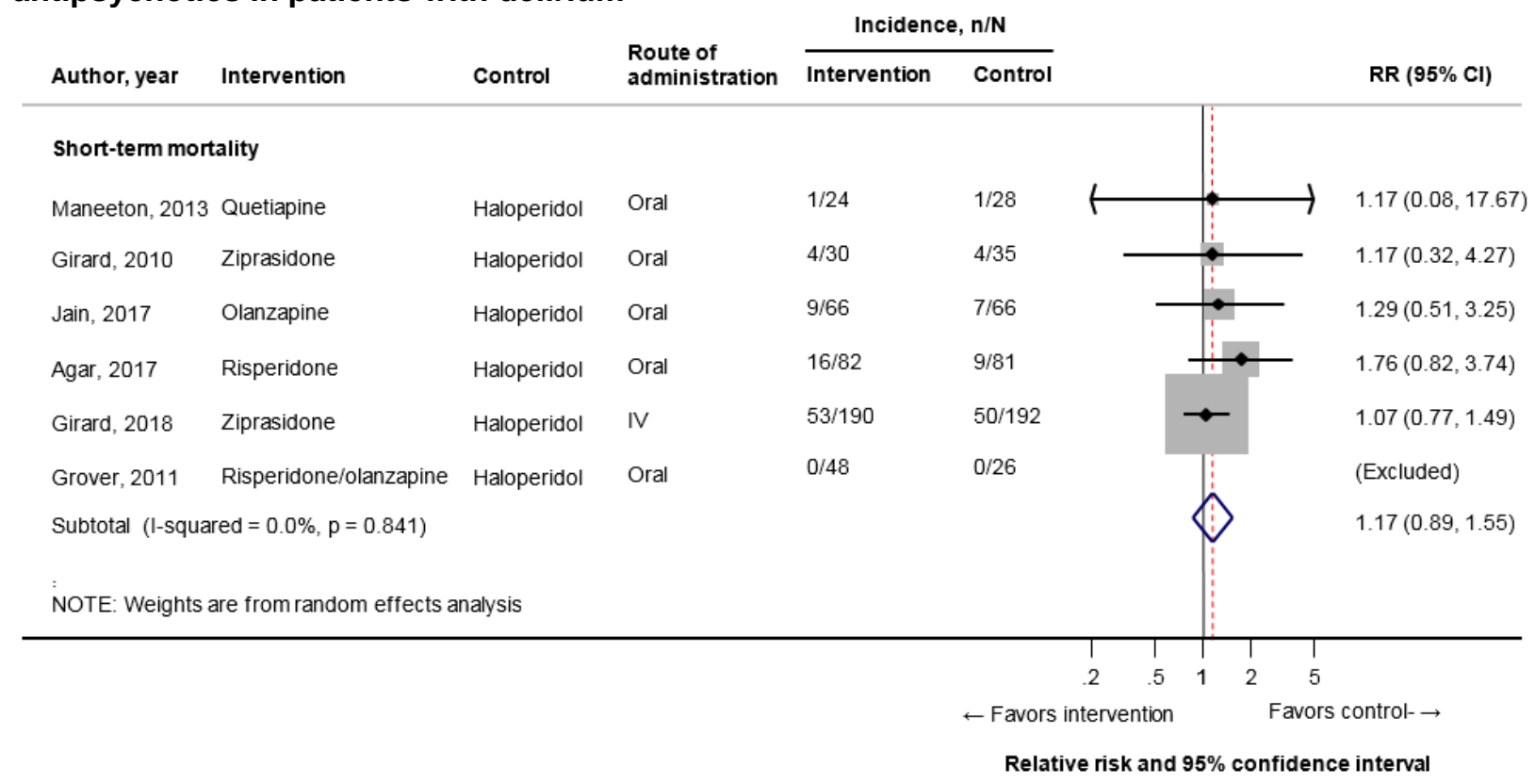

$\mathrm{CI}=$ confidence interval; $\mathrm{IV}=$ intravenous; $\mathrm{N}=$ total arm population; $\mathrm{n}=$ incidence within arm; $\mathrm{p}=\mathrm{p}$-value, $\mathrm{RR}=$ relative risk.

Second-Generation Antipsychotics Versus Second-Generation Antipsychotics

One three-arm RCT, with unclear risk of bias, compared risperidone with olanzapine in noncritically ill inpatients and reported no deaths in either treatment group. ${ }^{54}$

\section{Haloperidol Versus Other Therapies}

Two RCTs, with unclear risk of bias, compared haloperidol with another therapy, and both reported no statistically significant differences. ${ }^{49,} 51$

\section{Second-Generation Antipsychotics Versus Other Therapies}

We found no trials evaluating mortality in patients with delirium that compared secondgeneration antipsychotics with other therapies.

\section{Cognitive Functioning - Overall}

\section{Second-Generation Antipsychotics Versus Placebo}

One RCT, with low risk of bias, compared quetiapine with placebo in medical and surgical inpatients without critical illness. ${ }^{63}$ This RCT reported no clinically important or statistically significant effect on the Mini-Mental State Exam score for quetiapine compared with placebo at day 10. We were unable to draw a conclusion based upon on this small single study. (SOE: Insufficient)

\section{Haloperidol Versus Second-Generation Antipsychotics}

Two small RCTs compared haloperidol with three different second-generation antipsychotics. Overall, there were no clinically important or statistically significant differences in cognitive function, measured with the Mini-Mental State Exam score (mean differences of 
0.14 and 0.15$),{ }^{54,55}$ when comparing haloperidol with second-generation antipsychotics. (SOE: Low)

\section{Second-Generation Antipsychotics Versus Second-Generation Antipsychotics}

One three-arm RCT, with unclear risk of bias and enrolling non-critically ill inpatients, compared risperidone with olanzapine. ${ }^{54}$ This RCT reported no statistically significant effect on the Mini-Mental State Exam score for risperidone compared with olanzapine. ${ }^{54}$ We were unable to draw a conclusion based upon this single small study. (SOE: Insufficient)

\section{Haloperidol Versus Other Therapies}

One three-arm RCT, with unclear risk of bias and enrolling medical inpatients with acquired immunodeficiency syndrome, compared lorazepam with haloperidol. ${ }^{51}$ This RCT reported no statistically significant effect on the Mini-Mental State Exam score for lorazepam compared with haloperidol. We were unable to draw a conclusion based upon this single small study. (SOE: Insufficient)

\section{Other Comparisons}

We found no delirium treatment trials evaluating cognitive functioning in patients with delirium for the following comparisons:

- Haloperidol versus placebo

- Second-generation antipsychotics versus other therapies

\section{Strength of Evidence}

Table 18 summarizes the strength of evidence of the effects of antipsychotics on cognitive functioning in patients with delirium. 
Table 18. Strength of evidence domains for trials evaluating antipsychotics for the treatment of delirium in terms of cognitive functioning

\begin{tabular}{|c|c|c|c|c|c|c|c|c|}
\hline Comparison & $\begin{array}{l}\text { Number Of } \\
\text { Trials (N) }\end{array}$ & $\begin{array}{c}\text { Study } \\
\text { Limitations } \\
\end{array}$ & Directness & Consistency & Precision & $\begin{array}{c}\text { Reporting } \\
\text { Bias }\end{array}$ & $\begin{array}{l}\text { Strength Of } \\
\text { Evidence }\end{array}$ & Summary \\
\hline $\begin{array}{l}\text { Haloperidol vs. } \\
\text { placebo }\end{array}$ & No trials & & & & & & & \\
\hline $\begin{array}{l}\text { Second- } \\
\text { generation } \\
\text { antipsychotics } \\
\text { vs. placebo }\end{array}$ & $1^{63}(42)$ & Low & Indirect & $\begin{array}{l}\text { Consistency } \\
\text { unknown } \\
\text { (single study) }\end{array}$ & Imprecise & Undetected & Insufficient & $\begin{array}{l}\text { We were unable to draw a } \\
\text { conclusion. }\end{array}$ \\
\hline $\begin{array}{l}\text { Haloperidol vs. } \\
\text { second- } \\
\text { generation } \\
\text { antipsychotics }\end{array}$ & $2^{54,55}(127)$ & Medium & Indirect & Consistent & Imprecise & Undetected & Low & $\begin{array}{l}\text { RCTs of inpatients reported no } \\
\text { clinically important or } \\
\text { statistically significant difference } \\
\text { in MMSE scores (mean } \\
\text { differences } 0.14 \text { and } 0.15 \text { ). }\end{array}$ \\
\hline $\begin{array}{l}\text { Second- } \\
\text { generation } \\
\text { antipsychotics } \\
\text { vs. second- } \\
\text { generation } \\
\text { antipsychotics }\end{array}$ & $1^{54}(64)$ & Medium & Indirect & $\begin{array}{l}\text { Consistency } \\
\text { unknown } \\
\text { (single study) }\end{array}$ & Imprecise & Undetected & Insufficient & $\begin{array}{l}\text { We were unable to draw a } \\
\text { conclusion. }\end{array}$ \\
\hline $\begin{array}{l}\text { Haloperidol vs. } \\
\text { other therapies }\end{array}$ & $1^{51}(30)$ & Medium & Indirect & $\begin{array}{l}\text { Consistency } \\
\text { unknown } \\
\text { (single study) }\end{array}$ & Imprecise & Undetected & Insufficient & $\begin{array}{l}\text { We were unable to draw a } \\
\text { conclusion. }\end{array}$ \\
\hline $\begin{array}{l}\text { Second- } \\
\text { generation } \\
\text { antipsychotics } \\
\text { vs. other } \\
\text { therapies } \\
\end{array}$ & No trials & & & & & & & \\
\hline
\end{tabular}

MMSE = Mini-Mental State Exam; RCT = randomized controlled trials. 


\section{Other Final Health or Patient-Centered Outcomes - Overall}

We found no trials enrolling patients with delirium that compared an antipsychotic with a placebo, another antipsychotic, or another treatment and evaluated the following final health or patient-centered outcomes:

- Quality of life

- Institutionalization

- Caregiver burden/strain

- Falls

- Memory of patient distress

\section{Resource Utilization Outcomes}

\section{Readmission to Intensive Care Unit - Overall}

\section{Haloperidol Versus Placebo}

Two RCTs, with low risk of bias, compared haloperidol with placebo in critically ill medical and surgical patients. ${ }^{45}, 53$ Both RCTs reported no effect on readmission to the intensive care unit. The evidence was consistent but imprecise.

\section{Second-Generation Antipsychotics Versus Placebo}

One three-arm RCT, with low risk of bias, compared ziprasidone with placebo in critically ill medical and surgical patients. ${ }^{53}$ This RCT reported no statistically significant effect on readmission to the intensive care unit (RR, 0.76; 95\% CI, 0.42 to 1.36).

\section{Haloperidol Versus Second-Generation Antipsychotics}

One three-arm RCT, with low risk of bias, included a comparison of ziprasidone with haloperidol in critically ill medical and surgical patients. ${ }^{53}$ This RCT reported no statistically significant effect on readmission to the intensive care unit.

\section{Haloperidol Versus Other Therapies}

One RCT, with an unclear risk of bias, compared haloperidol with morphine in post-cardiac surgery patients with hyperactive delirium and reported no statistically significant effect on readmission to the intensive care unit. ${ }^{49}$

\section{Other Comparisons}

We found no delirium treatment trials evaluating readmission to the intensive care unit in patients with delirium for the following comparisons:

- Second-generation antipsychotics versus second-generation antipsychotics

- Second-generation antipsychotics versus other therapies 


\section{Length of Stay in Hospital - Overall}

\section{Haloperidol Versus Placebo}

Three RCTs, with low risk of bias, compared haloperidol with placebo and assessed length of stay in hospital in medical and surgical patients in intensive care units. ${ }^{44,45,53}$ We did not conduct a meta-analysis because these trials generally provided results as median values and the data for length of stay are skewed, precluding conversion or transformation of the data. While the two smaller RCTs ( $\mathrm{N}=101$ and $\mathrm{N}=141$ ) each reported clinically important effects, the largest trial ( $\mathrm{N}=566)$ found no clinically important effect, and all three RCTs reported no statistically significant effect on length of stay in hospital. Haloperidol did not shorten or lengthen the length of stay in hospital in patients in intensive care units. (SOE: Moderate)

\section{Second-Generation Antipsychotics Versus Placebo}

Three RCTs, with low risk of bias, compared a second-generation antipsychotic with placebo and assessed length of stay in hospital in medical and surgical patients in the intensive care units. ${ }^{44,52,53}$ We did not conduct a meta-analysis because these trials generally provided results as median values and the data for length of stay are skewed, precluding conversion or transformation of the data. While the two smaller RCTs $(\mathrm{N}=101$ and $\mathrm{N}=36)$ each reported potentially clinically important effects (about a 2-day difference), the largest trial $(\mathrm{N}=566)$ found no clinically important effect, and all three RCTs reported no statistically significant effect on length of stay in hospital. Second-generation antipsychotics did not shorten or lengthen length of stay in hospital in medical and surgical patients in intensive care units. (SOE: Moderate)

\section{Haloperidol Versus Second-Generation Antipsychotics}

Two RCTs, with low risk of bias, compared ziprasidone with haloperidol and assessed length of stay in hospital in medical and surgical patients in intensive care units. ${ }^{44,53}$ We did not conduct a meta-analysis because these trials generally provided results as median values and the data for length of stay are skewed, precluding conversion or transformation of the data. There was no clinically important or statistically significant effect on length of stay in hospital for haloperidol compared with second-generation antipsychotics. (SOE: Moderate)

\section{Haloperidol Versus Other Therapies}

One RCT, with an unclear risk of bias, compared haloperidol with morphine and assessed length of stay in hospital in post-cardiac surgery patients with hyperactive delirium. This RCT reported no statistically significant effect for haloperidol compared with morphine. ${ }^{49}$ We were unable to draw conclusions on the effect of haloperidol compared with other therapies on length of stay based on this single small study. (SOE: Insufficient)

\section{Other Comparisons}

We found no delirium treatment trials evaluating length of stay in hospital in patients with delirium for the following comparisons:

- Second-generation antipsychotics versus second-generation antipsychotics

- Second-generation antipsychotics versus other therapies 


\section{Strength of Evidence}

Table 19 summarizes the strength of evidence of the effects of antipsychotics on length of stay in hospital in patients with delirium. 
Table 19. Strength of evidence domains for trials evaluating antipsychotics for the treatment of delirium in terms of length of stay in hospital

\begin{tabular}{|c|c|c|c|c|c|c|c|c|}
\hline Comparison & $\begin{array}{l}\text { Number Of } \\
\text { Trials (N) }\end{array}$ & $\begin{array}{c}\text { Study } \\
\text { Limitations }\end{array}$ & Directness & Consistency & Precision & $\begin{array}{c}\text { Reporting } \\
\text { Bias }\end{array}$ & $\begin{array}{l}\text { Strength Of } \\
\text { Evidence }\end{array}$ & Summary \\
\hline $\begin{array}{l}\text { Haloperidol vs. } \\
\text { placebo }\end{array}$ & $\begin{array}{l}3^{44,45,53} \\
(808)\end{array}$ & Low & Indirect* & Consistent & Precise & Undetected & Moderate & $\begin{array}{l}\text { RCTs, conducted exclusively } \\
\text { in critically ill patients, reported } \\
\text { no statistically significant } \\
\text { difference, with the largest } \\
\text { RCT reporting no clinically } \\
\text { important difference. }\end{array}$ \\
\hline $\begin{array}{l}\text { Second- } \\
\text { generation } \\
\text { antipsychotics } \\
\text { vs. placebo }\end{array}$ & $\begin{array}{l}3^{44,52,53} \\
(703)\end{array}$ & Low & Indirect* & Consistent & Precise & Undetected & Moderate & $\begin{array}{l}\text { RCTs, conducted exclusively } \\
\text { in critically ill patients, reported } \\
\text { no statistically significant } \\
\text { difference, with the largest } \\
\text { RCT reporting no clinically } \\
\text { important difference. }\end{array}$ \\
\hline $\begin{array}{l}\text { Haloperidol vs. } \\
\text { second- } \\
\text { generation } \\
\text { antipsychotics }\end{array}$ & $2^{44,53}(667)$ & Low & Indirect* $^{*}$ & Consistent & Precise & Undetected & Moderate & $\begin{array}{l}\text { RCTs, conducted exclusively } \\
\text { in critically ill patients, reported } \\
\text { no clinically important or } \\
\text { statistically significant } \\
\text { difference. }\end{array}$ \\
\hline $\begin{array}{l}\text { Second- } \\
\text { generation } \\
\text { antipsychotics } \\
\text { vs. second- } \\
\text { generation } \\
\text { antipsychotics }\end{array}$ & No trials & & & & & & & \\
\hline $\begin{array}{l}\text { Haloperidol vs. } \\
\text { other therapies }\end{array}$ & $1^{49}(53)$ & High & Indirect & $\begin{array}{l}\text { Consistency } \\
\text { unknown } \\
\text { (single study) }\end{array}$ & Imprecise & Undetected & Insufficient & $\begin{array}{l}\text { We were unable to draw a } \\
\text { conclusion. }\end{array}$ \\
\hline $\begin{array}{l}\text { Second- } \\
\text { generation } \\
\text { antipsychotics } \\
\text { vs. other } \\
\text { therapies }\end{array}$ & No trials & & & & & & & \\
\hline
\end{tabular}

* Indirect rating owing to a single patient population in all RCTs and results may not be generalizable to other patients with delirium.

$\mathrm{RCT}=$ randomized controlled trial. 


\section{Length of Stay in Intensive Care Unit - Overall}

\section{Haloperidol Versus Placebo}

Three RCTs, with low risk of bias, compared haloperidol with placebo and assessed length of stay in intensive care unit in medical and surgical critically ill patients. ${ }^{44,45,53}$ We did not conduct a meta-analysis because these RCTs generally provided results as median values and the data for length of stay are skewed, precluding conversion or transformation of the data. There was inconsistent and imprecise evidence of little or no effect on length of stay in the intensive care unit for haloperidol compared with placebo.

\section{Second-Generation Antipsychotics Versus Placebo}

Three RCTs, with low risk of bias, compared a second-generation antipsychotic with placebo and assessed length of stay in the intensive care unit in critically ill medical and surgical patients. $^{44,52,53}$ We did not conduct a meta-analysis because these trials generally provided results as median values and the data for length of stay are skewed, precluding conversion or transformation of the data. There was inconsistent and imprecise evidence of little or no effect on length of stay in the intensive care unit for a second-generation antipsychotic compared with placebo.

\section{Haloperidol Versus Second-Generation Antipsychotics}

Two RCTs, with low risk of bias, compared haloperidol with ziprasidone and assessed length of stay in the intensive care unit in critically ill medical and surgical patients. ${ }^{44,53}$ We did not conduct a meta-analysis because these trials generally provided results as median values and the data for length of stay are skewed, precluding conversion or transformation of the data. There was inconsistent evidence of no statistically significant difference on length of stay in the intensive care unit for haloperidol compared with ziprasidone.

\section{Haloperidol Versus Other Therapies}

One RCT, with unclear risk of bias, compared haloperidol with morphine and assessed length of stay in the intensive care unit in post-cardiac surgery patients with hyperactive delirium. ${ }^{49}$ This RCT reported no statistically significant effect for haloperidol compared with morphine.

\section{Other Comparisons}

We found no delirium treatment trials evaluating length of stay in the intensive care unit in patients with delirium for the following comparisons:

- Second-generation antipsychotics versus second-generation antipsychotics

- Second-generation antipsychotics versus other therapies

\section{Other Resource Utilization Outcomes - Overall}

We found no trials enrolling patients with delirium that compared an antipsychotic with a placebo, another antipsychotic, or another treatment and evaluated the following resource utilization outcomes:

- Readmission to hospital

- Length of stay in skilled nursing facility

- Patient safety attendant use 
- Hospice enrollment

\section{Adverse Effects}

\section{Sedation - Overall}

\section{Haloperidol Versus Placebo}

Two RCTs, with low risk of bias, compared haloperidol with placebo in critically ill medical and surgical patients. ${ }^{45,53}$ The smaller trial $(\mathrm{N}=141)$ reported a potentially clinically important but not statistically significant effect on oversedation for haloperidol compared with placebo. However, the larger trial $(\mathrm{N}=566)$ reported no clinically important or statistically significant difference. Overall, there was no clinically important or statistically significant effect of haloperidol compared with placebo on sedation. (SOE: Low)

We did not find any observational studies or non-randomized trials that compared haloperidol with placebo and reported on sedation.

\section{Second-Generation Antipsychotics Versus Placebo}

Three RCTs, with low risk of bias, compared second-generation antipsychotics with placebo in medical and surgical inpatients with and without critical illness. ${ }^{52,53,63}$ Our meta-analysis demonstrated no clinically important or statistically significant between-group difference in the onset of sedation (pooled RR, 1.10; 95\% CI, 0.78 to 1.53; I-squared, 0.0\%) (Figure 23). (SOE: Moderate) We tested the effect of each individual trial on the combined point estimate. No single trial influenced the pooled results. No substantial heterogeneity was identified.

We did not find any observational studies or non-randomized trials that compared a secondgeneration antipsychotic with placebo and reported on sedation.

\section{Haloperidol Versus Second-Generation Antipsychotics}

Six RCTs, ${ }^{33,54,56,58,62,64}$ with varying risk of bias, plus five observational studies with comparison groups ${ }^{66,68,75,84,89}$ enrolled medical and surgical inpatients with and without critical illness. These studies reported no clinically important or statistically significant differences in sedation-related effects in haloperidol compared with second-generation antipsychotics. (SOE: Moderate)

The six RCTs compared four different second-generation antipsychotics (ziprasidone, olanzapine, quetiapine, and risperidone) with haloperidol in medical and surgical inpatients with and without critical illness on sedation-related effects. Four of the six RCTs had low risk of bias; ${ }^{53,54,62,64}$ one RCT had unclear risk of bias; ${ }^{56}$ and one RCT had high risk of bias, primarily owing to a lack of blinding and missing outcome data. ${ }^{58} \mathrm{~A}$ meta-analysis of the six RCTs found no statistically significant between-group difference in the onset of sedation (pooled RR, 1.26; 95\% CI, 0.92 to 1.72; I-squared, 0.0\%) (Figure 24). ${ }^{53,54,56,58,62,64}$ We tested the effect of each individual trial on the combined point estimate. No single trial influenced the pooled results. No substantial heterogeneity was identified. 
Figure 23. Meta-analysis of sedation in trials comparing second-generation antipsychotics with placebo in patients with delirium

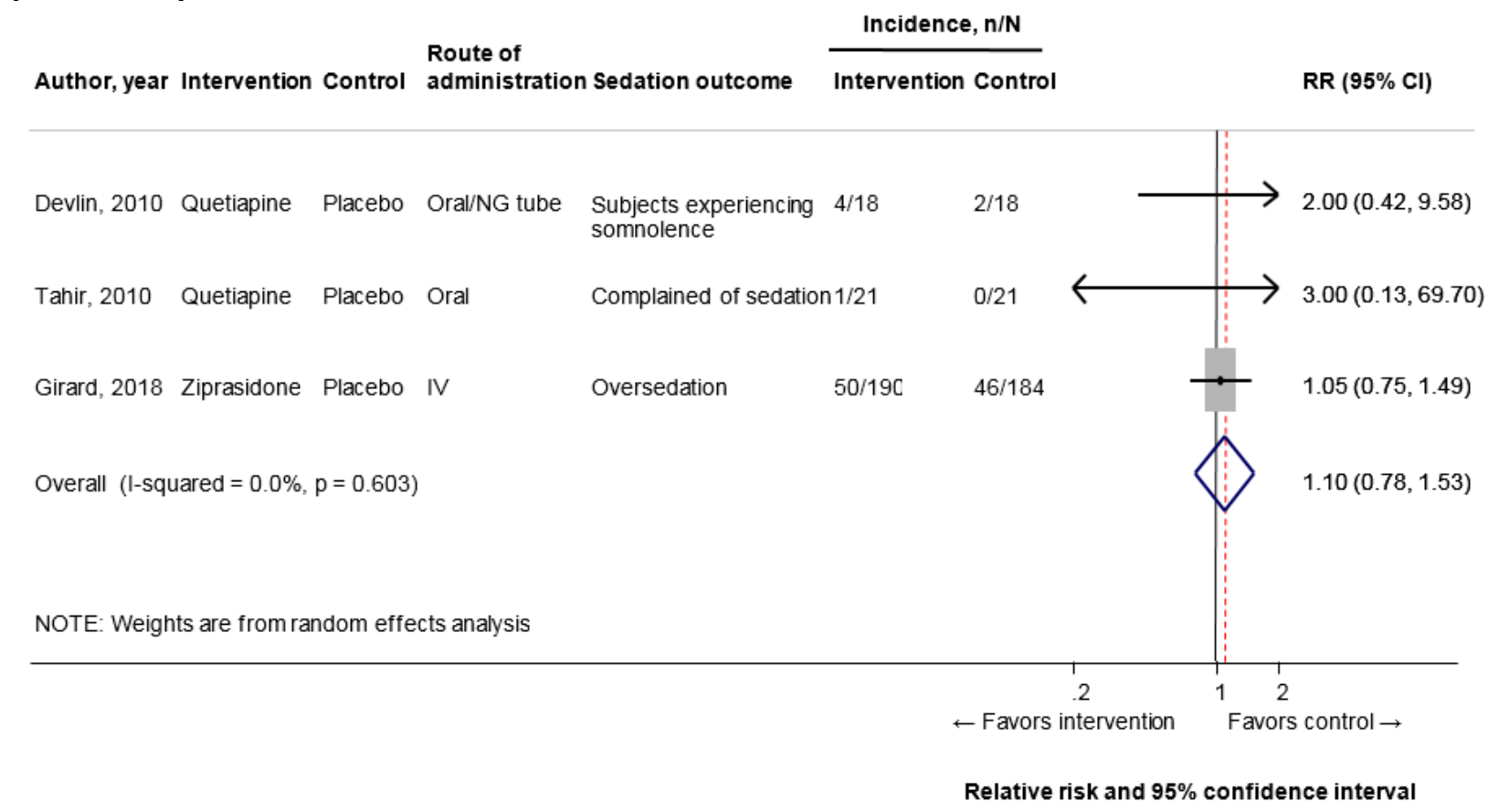

$\mathrm{CI}=$ confidence interval; $\mathrm{IV}=$ intravenous; $\mathrm{N}=$ total arm population; $\mathrm{n}=$ incidence within $\mathrm{arm}$; $\mathrm{NG}=$ nasogastric tube; $\mathrm{p}=\mathrm{p}$ value, $\mathrm{RR}=$ relative risk.

Figure 24. Meta-analysis of sedation outcomes in trials comparing second-generation antipsychotics with haloperidol

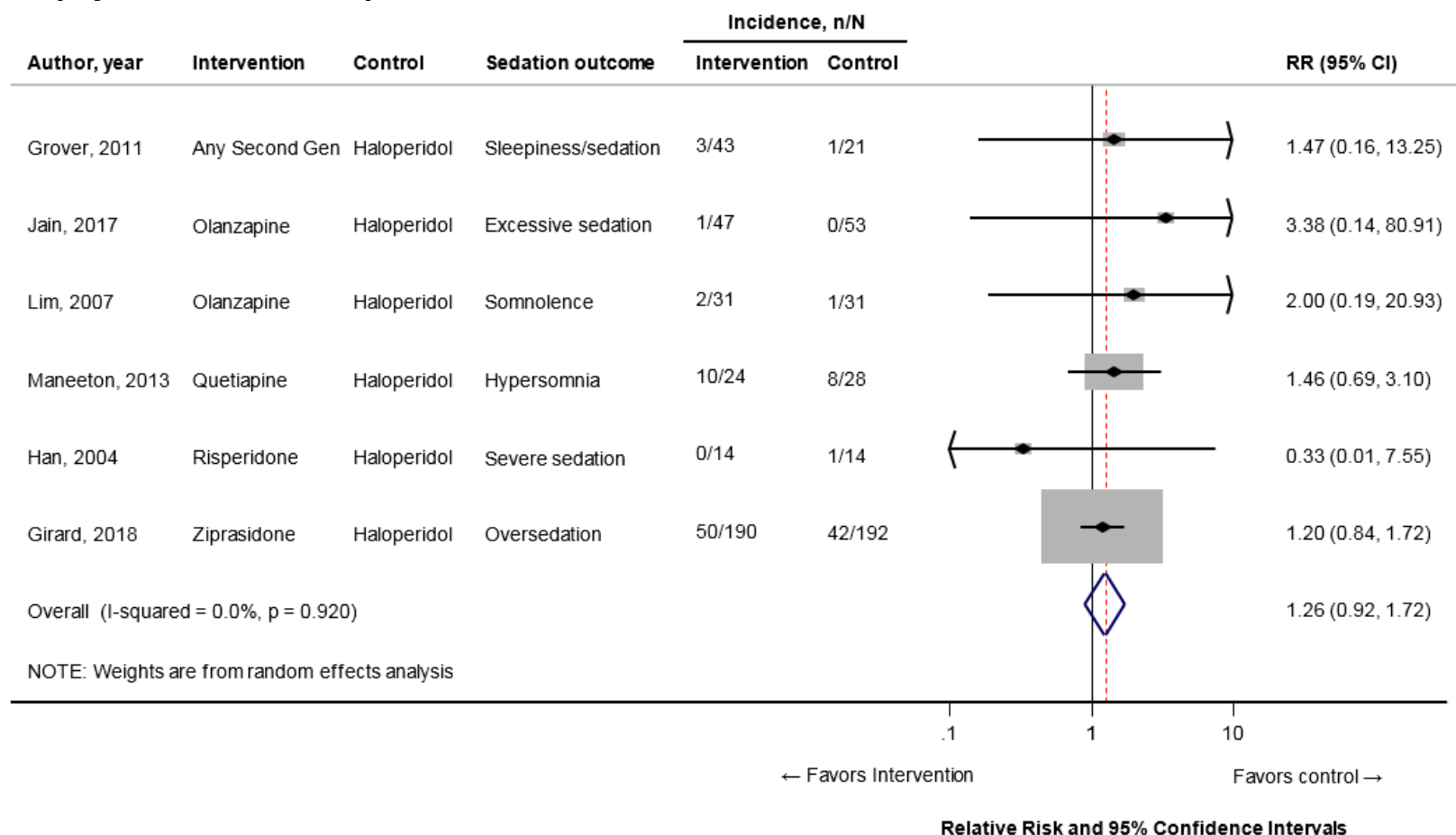

$\mathrm{CI}=$ confidence interval; $\mathrm{IV}=$ intravenous; $\mathrm{N}$ = total arm population; $\mathrm{n}=$ incidence within arm; $\mathrm{p}=\mathrm{p}$-value; $\mathrm{RR}=$ relative risk. 
We also identified five observational studies evaluating inpatients without critical illness (sample size range, 22 to 84) for sedation-related effects. One study had a moderate risk of bias, ${ }^{68}$ and the others all had potential for serious risk of bias. ${ }^{66,75,84,89}$ Four different secondgeneration antipsychotics (quetiapine, risperidone, olanzapine, and aripiprazole) were compared with haloperidol, with no study reporting a statistically significant difference in sedation-related effects.

\section{Second-Generation Antipsychotics Versus Second-Generation Antipsychotics}

Three RCTs ${ }^{54,59,60}$ and four observational studies ${ }^{66,68,69,89}$ evaluated sedation-related effects in inpatients without critical illness for second-generation antipsychotics compared with secondgeneration antipsychotics. The trials were small and, owing to unclear and high risk of bias and imprecise findings, we were unable to draw conclusions. (SOE: Insufficient)

These three RCTs included one trial with unclear risk of bias ${ }^{54}$ and two trials with high risk of bias, ${ }^{59,60}$ with sample sizes ranging from 31 to 64 inpatients without critical illness. Two RCTs compared risperidone with olanzapine, ${ }^{54,59}$ and one RCT compared amisulpride with quetiapine. ${ }^{60}$ All three RCTs reported no statistically significant differences in the comparisons. One RCT reported no oversedation events in either group, ${ }^{60}$ and the other RCTs reported imprecise effects (RR, 8.00; 95\% CI, 0.44 to 146$)^{54}$ and (RR, 0.88; 95\% CI, 0.32 to 2.46). ${ }^{59}$

The four observational studies with comparison groups had moderate ${ }^{68,69}$ or serious ${ }^{66,89}$ risk of bias. These four studies evaluated inpatients (sample size range, 40 to 84) with and without critical illness. Comparisons were made between five different second-generation antipsychotics (risperidone, olanzapine, quetiapine, aripiprazole, and lurasidone); no study reported a statistically significant difference in sedation-related outcomes.

\section{Other Comparisons}

We found no studies of delirium treatment evaluating sedation in patients with delirium for the following comparisons:

- Haloperidol versus other therapies

- Second-generation antipsychotics versus other therapies

\section{Studies With No Comparison Group}

Thirteen observational studies without a comparison group evaluated sedation-related side effects of second-generation antipsychotics in a total of 331 patients who were predominantly medical and surgical inpatients without critical illness. Twelve of the studies reported a range of 0 percent to 30 percent of patients experiencing a sedation-related effect. ${ }^{67,71-74,76,78,80-83,87}$ One study of 17 inpatients ${ }^{77}$ reported, without any explanation for the finding, that 76 percent of patients had daytime sleepiness.

\section{Strength of Evidence}

Table 20 summarizes the strength of evidence findings for the effects of antipsychotics on sedation in patients with delirium. 
Table 20. Strength of evidence domains for trials evaluating antipsychotics for the treatment of delirium in terms of sedation

\begin{tabular}{|c|c|c|c|c|c|c|c|c|}
\hline Comparison & $\begin{array}{l}\text { Number Of } \\
\text { Studies (N) }\end{array}$ & $\begin{array}{c}\text { Study } \\
\text { Limitations }\end{array}$ & Directness & Consistency & Precision & $\begin{array}{c}\text { Reporting } \\
\text { Bias }\end{array}$ & $\begin{array}{c}\text { Strength Of } \\
\text { Evidence }\end{array}$ & Summary \\
\hline $\begin{array}{l}\text { Haloperidol vs. } \\
\text { placebo }\end{array}$ & $2^{45,53}(707)$ & Low & Indirect* & Consistent & Imprecise & Undetected & Low & $\begin{array}{l}\text { In patients with critical illness, } \\
\text { there was no clinically } \\
\text { important or statistically } \\
\text { significant difference in } \\
\text { sedation. }\end{array}$ \\
\hline $\begin{array}{l}\text { Second- } \\
\text { generation } \\
\text { antipsychotics } \\
\text { vs. placebo }\end{array}$ & $3^{52,53,63}(644)$ & Low & Indirect* & Consistent & Precise & Undetected & Moderate & $\begin{array}{l}\text { In patients with critical illness, } \\
\text { there was no clinically } \\
\text { important or statistically } \\
\text { significant difference in } \\
\text { sedation (pooled RR, } 1.10 \text {; } \\
95 \% \mathrm{Cl}, 0.78 \text { to } 1.53 \text { ). }\end{array}$ \\
\hline $\begin{array}{l}\text { Haloperidol vs. } \\
\text { second- } \\
\text { generation } \\
\text { antipsychotics }\end{array}$ & $\begin{array}{l}6 \text { RCTs53, 54, } \\
56,58,62,64 \\
\text { (872) [and } 5 \\
\text { observational } \\
\text { studies with } \\
\text { comparison } \\
\text { group } 66,68,75, \\
84,89(444) \text { ] }\end{array}$ & Low & Indirect* & Consistent & Precise & Undetected & Moderate & $\begin{array}{l}\text { In patients with critical illness, } \\
\text { there was no clinically } \\
\text { important statistically } \\
\text { significant difference in } \\
\text { sedation (pooled RR, 1.26; } \\
95 \% \mathrm{Cl}, 0.92 \text { to } 1.72 \text { ). }\end{array}$ \\
\hline $\begin{array}{l}\text { Second- } \\
\text { generation } \\
\text { antipsychotics } \\
\text { vs. second- } \\
\text { generation } \\
\text { antipsychotics }\end{array}$ & $\begin{array}{l}3 \text { RCT }^{54,59,} \\
60 \text { (127) [and } \\
4 \\
\text { observational } \\
\text { studies with } \\
\text { comparison } \\
\text { group } 66,68,69, \\
89(318)] \\
\end{array}$ & High & Indirect & Inconsistent & Imprecise & Undetected & Insufficient & $\begin{array}{l}\text { We were unable to draw a } \\
\text { conclusion. }\end{array}$ \\
\hline $\begin{array}{l}\text { Haloperidol vs. } \\
\text { other therapies }\end{array}$ & No studies & & & & & & & \\
\hline $\begin{array}{l}\text { Second- } \\
\text { generation } \\
\text { antipsychotics } \\
\text { vs. other } \\
\text { therapies } \\
\end{array}$ & No studies & & & & & & & \\
\hline
\end{tabular}

* Indirect rating owing to data entirely or predominantly coming from a single patient population across the RCTs such that the results may not be generalizable to other patients with delirium.

$\mathrm{CI}=$ confidence interval; RCT = randomized controlled trial; RR = relative risk. 


\section{Cardiac Effects - Overall}

\section{Haloperidol Versus Placebo}

Three RCTs, ${ }^{44,45,53}$ with low risk of bias, and one observational study, ${ }^{79}$ with potential for serious risk of bias, compared haloperidol with placebo for a variety of cardiac effects. All of the studies evaluated critically ill patients. In each of these studies, there was no statistically significant difference in a variety of cardiac effects for haloperidol compared with placebo. However, different cardiac effects tended to occur more frequently in the haloperidol group which can be potentially clinically important.

For the specific cardiac effect of prolongation of the corrected QT interval, all the studies reported data. ${ }^{44,45,53,79}$ We conducted a meta-analysis of the three RCTs and found no statistically significant between-group difference for haloperidol compared with placebo (pooled RR, 1.13; 95\% CI, 0.62 to 2.05; I-squared, 0\%) (Figure 25). We tested the effect of each individual trial on the combined point estimate. No single trial influenced the pooled results. No substantial heterogeneity was identified. The observational study, enrolling 925 patients with and without delirium in the coronary care unit, reported on prolongation of the corrected QT interval at multiple time points. ${ }^{79}$ This observational study reported no clinically important or statistically significant difference for haloperidol compared with placebo in patients with delirium. Additional cardiac effects evaluated in the three RCTs are reported below.

For the specific cardiac effect of hypotension, one RCT compared haloperidol with placebo in 141 critically ill patients receiving mechanical ventilation enrolled within 72 hours of intensive care unit admission. ${ }^{45}$ This RCT reported no statistically significant difference in hypotension for haloperidol (4\%) compared with placebo (3\%).

For the specific cardiac effect of arrhythmia, all three RCTs reported data. One three-arm RCT, enrolling patients receiving mechanical ventilation, reported no instances of ventricular arrhythmia events in any study group. ${ }^{44}$ Another RCT, enrolling critically ill patients, reported no instances of permanent discontinuation of the study drug owing to suspected torsades de pointes. ${ }^{53}$ Another RCT, enrolling critically ill patients receiving mechanical ventilation within 72 hours of intensive care unit admission, reported no statistically significant difference in the incidence of atrial fibrillation, bradycardia, and supraventricular tachycardia for haloperidol compared with placebo. ${ }^{45}$

\section{Second-Generation Antipsychotics Versus Placebo}

Three RCTs, ${ }^{44,52,53}$ with low risk of bias, and one observational study, ${ }^{79}$ with potential for serious risk of bias, compared second-generation antipsychotics with placebo for a variety of cardiac effects. All four studies evaluated critically ill patients. Across all of these studies and the variety of cardiac effects, there was no statistically significant difference in second-generation antipsychotics compared with placebo. However, there was a potential increase in QT interval prolongation for second-generation antipsychotics compared with placebo.

For the specific cardiac effect of prolongation of the corrected QT interval, all four of these studies reported data. ${ }^{44,52,53,79}$ We conducted a meta-analysis of the three RCTs, two of which compared ziprasidone with placebo, ${ }^{44,53}$ and one that compared quetiapine with placebo. ${ }^{52}$ Our meta-analysis demonstrated a potentially clinically important, but not statistically significant, between-group difference for second-generation antipsychotics compared with placebo (pooled RR, 1.57; 95\% CI, 0.90 to 2.76; I-squared, 0\%) (Figure 25). Exclusion of one of the three trials ${ }^{52}$ 
suggested a potentially clinically important and statistically significant effect on prolongation of the corrected QT interval for second-generation antipsychotics compared with placebo (pooled RR with Devlin 2010 removed, 1.95; 95\% CI, 1.03 to 3.71). This trial was not qualitatively different, so we retained it in the overall meta-analysis. The one observational study, enrolling 925 patients with and without delirium in the coronary care unit, compared haloperidol with quetiapine and reported on prolongation of the corrected QT interval at multiple time points. ${ }^{79}$ This study reported no clinically important or statistically significant difference for quetiapine compared with placebo in patients with delirium. ${ }^{79}$ Additional cardiac effects evaluated in the three RCTs are reported below.

For the specific cardiac effect of hypotension, one RCT compared quetiapine with placebo in patients admitted to a medical or surgical intensive care unit and reported no statistically significant difference in hypotension for quetiapine (6\%) compared with placebo $(0 \%) .{ }^{52}$

All three RCTs reported data on the specific cardiac effect of arrhythmia. Two of the three RCTs, enrolling critically ill patients, compared ziprasidone with placebo. These RCTs reported no instance of ventricular arrhythmia ${ }^{44}$ or permanent discontinuation of the study drug owing to suspected torsades de pointes ${ }^{53}$ in either of the trial groups. Another RCT compared quetiapine with placebo in patients admitted to a medical or surgical intensive care unit and reported no instances of torsades de pointes in either group. ${ }^{52}$

Figure 25. Meta-analysis of cardiac outcomes in trials comparing haloperidol or secondgeneration antipsychotics with placebo in patients with delirium

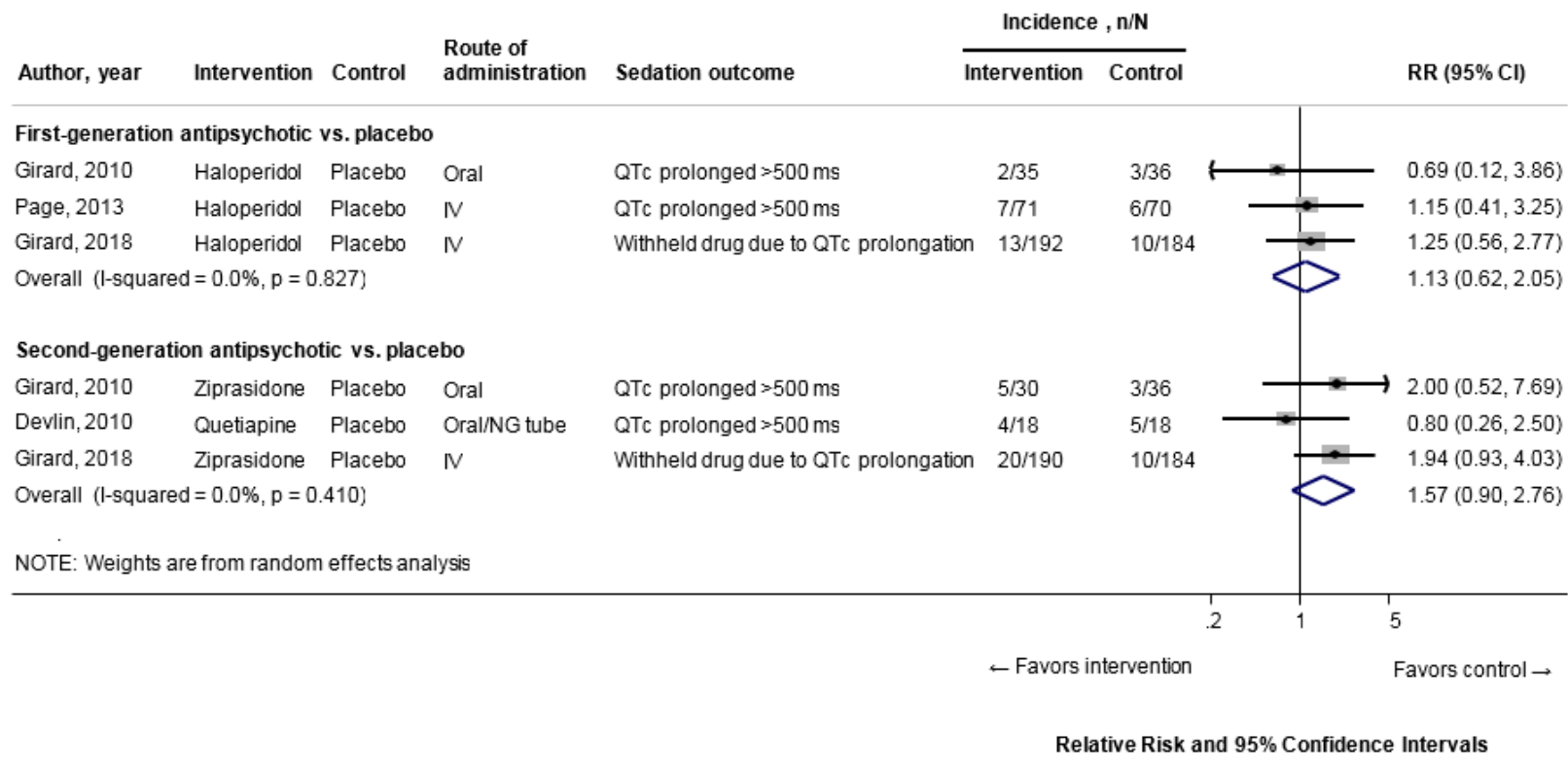

$\mathrm{CI}=$ confidence interval; $\mathrm{IV}=$ intravenous; $\mathrm{N}=$ total arm population; $\mathrm{n}=$ incidence within arm; $\mathrm{NG}=$ nasogastric tube; $\mathrm{p}=\mathrm{p}$ value; $\mathrm{QTc}=$ corrected $\mathrm{QT}$ interval; $\mathrm{RR}$ = relative risk

\section{Haloperidol Versus Second-Generation Antipsychotics}

Four RCTs, ${ }^{44,53,62,64}$ with low risk of bias, and three observational studies ${ }^{68,70,79}$ compared haloperidol with five different second-generation antipsychotics (quetiapine, olanzapine, risperidone, ziprasidone, and aripiprazole) for a variety of cardiac effects. All of the studies evaluated medical and surgical inpatients with and without critical illness. Across all of these studies and the variety of cardiac effects, there was consistent but imprecise evidence of no statistically significant difference in second-generation antipsychotics compared with 
haloperidol. However, there was a potentially clinically important increase in episodes of QT interval prolongation for ziprasidone.

For the specific cardiac effect of prolongation of the corrected QT interval, three RCTs, ${ }^{44,53,64}$ with low risk of bias, and one observational study, ${ }^{79}$ with potential for serious risk of bias, reported data. One RCT reported no statistically significant difference in the incidence of prolongation of the corrected QT interval to more than 500 milliseconds for ziprasidone (17\%) compared with haloperidol (6\%). Another RCT, a three-arm trial enrolling critically ill patients, reported no statistically significant difference in the incidence of temporary discontinuation of the study drug owing to prolongation of the corrected QT interval for ziprasidone (11\%) compared with haloperidol (7\%). ${ }^{53}$ This RCT also reported no incidence of permanent discontinuation of the study drug owing to suspected torsades de pointes in either of the randomization groups. One RCT of inpatients reported no incidence of QT interval prolongation in either group. ${ }^{64}$ One observational study, with potential for serious risk of bias, enrolled patients with and without delirium in the coronary care unit. ${ }^{79}$ This observational study compared quetiapine with haloperidol and reported on prolongation of the corrected QT interval at multiple time points. This study reported no clinically important or statistically significant difference for quetiapine compared with haloperidol in patients with delirium.

For the specific cardiac effect of hypotension, two observational studies with comparison groups reported data. ${ }^{68,70}$ One study, with moderate risk of bias, reported no statistically significant difference in the incidence of orthostatic hypotension for quetiapine (3\%) compared with haloperidol (0\%). This study also reported no orthostatic hypotension in the risperidone group. ${ }^{68}$ Another observational study, with potential for serious risk of bias, compared four different second-generation antipsychotics (aripiprazole, olanzapine, quetiapine, and risperidone) with haloperidol in inpatients. This study reported no statistically significant difference in the incidence of rapid decline in systolic blood pressure for quetiapine $(0.1 \%)$ or risperidone $(0.1 \%)$ compared with haloperidol (0\%). ${ }^{70}$ This study also reported no hypotension in the quetiapine and aripiprazole groups.

For the specific cardiac effect of arrhythmia, three RCTs, ${ }^{44,62,64}$ with low risk of bias, and one observational study, ${ }^{68}$ with moderate risk of bias, reported data. Two of the three RCTs reported no incidence of ventricular arrhythmia in either group. ${ }^{44,64}$ One RCT, enrolling inpatients with hyperactive delirium, reported no statistically significant difference in the incidence of study drug withdrawal owing to atrioventricular block for quetiapine (0\%) compared with haloperidol (3.6\%). ${ }^{62}$ One three-arm observational study, ${ }^{68}$ with moderate risk of bias and enrolling a total of 56 inpatients, reported no statistically significant difference in the incidence of arrhythmias for quetiapine $(0 \%)$ or risperidone $(7 \%)$ compared with haloperidol $(9 \%)$.

\section{Second-Generation Antipsychotics Versus Second-Generation Antipsychotics}

Three observational studies, enrolling inpatients with and without critical illness, compared second-generation antipsychotics with second-generation antipsychotics. ${ }^{68-70}$ These studies reported no statistically significant differences in the incidence of different types of cardiac effects.

One observational study, with moderate risk of bias and enrolling critically ill patients, reported no difference in the incidence of a corrected QT interval increase of more than 60 milliseconds from baseline for quetiapine (10\%) compared with lurasidone (10\%). ${ }^{69}$ This study also reported no statistically significant difference in discontinuation of the study medication 
owing to prolonged QT interval for quetiapine (5\%) compared with lurasidone ( $0 \%)$. The evidence was consistent but imprecise, due to a very low number of events.

For the specific cardiac effect of hypotension, two observational studies reported data. ${ }^{68,70}$ One three-arm study, with moderate risk of bias, compared risperidone with quetiapine and reported no statistically significant difference in the instances of orthostatic hypotension. ${ }^{68} \mathrm{~A}$ five-arm study, with potential for high risk of bias, compared four different second-generation antipsychotics (aripiprazole, olanzapine, quetiapine, and risperidone) with haloperidol in a total of 2,453 inpatients. ${ }^{70}$ This study reported no statistically significant difference in the incidence of rapid decline in systolic blood pressure for quetiapine $(0.1 \%)$ compared with aripiprazole $(0 \%)$, or olanzapine $(0 \%)$, and for risperidone $(0.1 \%)$ compared with aripiprazole $(0 \%)$, olanzapine $(0 \%)$, or quetiapine $(0.1 \%)$.

For the specific cardiac effect of arrhythmia, two observational studies, with moderate risk of bias, reported data. One three-arm observational study, with moderate risk of bias and enrolling a total of 56 patients, reported no statistically significant difference in the incidence of arrhythmia for risperidone (7\%) compared with quetiapine (0\%). ${ }^{68}$ Another study, enrolling 40 critically ill patients, compared quetiapine with lurasidone and reported no instances of life-threatening arrhythmia or torsades de pointes in either group. ${ }^{69}$

\section{Haloperidol Versus Other Therapies}

One three-arm RCT, with low risk of bias, compared haloperidol with ondansetron and dexmedetomidine in trauma patients in an intensive care unit. ${ }^{50}$ This trial reported no incidence of hypotension, bradycardia, or prolongation of corrected QT interval in any of the study groups.

We did not find any observational studies or non-randomized trials that compared haloperidol with other therapies and reported on cardiac outcomes.

\section{Second-Generation Antipsychotics Versus Other Therapies}

We found no studies evaluating cardiac effects in patients with delirium that compared second-generation antipsychotics with other therapies.

\section{Studies With No Comparison Group}

Five observational studies, without comparison groups and with variable risk of biases, evaluated various cardiac effects of three different second-generation antipsychotics (aripiprazole, quetiapine, and risperidone) in a total of 77 inpatients with and without critical illness. $^{74,77,78,80,86}$ The results varied across these studies.

Two studies reported the specific cardiac effect of prolongation of the corrected QT interval. ${ }^{78,86}$ One observational study, with potential for serious risk of bias and enrolling 14 inpatients receiving aripiprazole, reported three (21\%) instances of corrected QT interval prolongation. ${ }^{86}$ Another observational study, with moderate risk of bias and enrolling 10 inpatients receiving risperidone, reported no change in the corrected QT interitale ${ }^{7}$ studies reported data about the specific cardiac effect of hypotension. ${ }^{77,78,80}$ Two of these studies, with potential for high risk of bias and enrolling a total of 41 inpatients receiving quetiapine, reported data. One study reported two instances (12\%) of hypotension, ${ }^{77}$ the other study reported no hypotension. ${ }^{80}$ One observational study, with moderate risk of bias, and enrolling 10 inpatients receiving risperidone, reported one instance (10\%) of discontinuation of the study drug owing to hypotension. ${ }^{78}$ 
Two studies reported the specific cardiac effects of arrhythmia or myocardial infarction. ${ }^{74,86}$ One observational study, with moderate risk of bias and enrolling 12 critically ill patients receiving quetiapine, reported one (8\%) fatal acute myocardial infarction. ${ }^{74}$ Another observational study, with potential for serious risk of bias and enrolling 14 inpatients receiving aripiprazole, reported one (7\%) cardiac arrest and no instance of ventricular arrhythmia. ${ }^{86}$

\section{Neurologic Effects - Overall}

\section{Haloperidol Versus Placebo}

Four RCTs, with low risk of bias, compared haloperidol with placebo for a number of neurologic effects. ${ }^{44,45,48,53}$ Three of the four trials evaluated critically ill patients, ${ }^{44,45,53}$ and the fourth evaluated patients receiving palliative care. ${ }^{48}$ Overall, across a variety of neurologic effects, including extrapyramidal symptoms and neuroleptic malignant syndrome, only the single RCT enrolling patients receiving palliative care reported a statistically significant increase in extrapyramidal symptoms for haloperidol compared with placebo.

For the neurologic effect of extrapyramidal symptoms, we conducted a meta-analysis with three of the RCTs, all of which evaluated critically ill patients. This meta-analysis demonstrated no between-group difference in extrapyramidal symptoms comparing haloperidol with placebo (pooled RR, 0.77; 95\% CI, 0.29 to 2.02; I-squared, 0\%) (Figure 26). ${ }^{44,45,53}$ We tested the effect of each individual trial on the combined point estimate. No single trial influenced the pooled results. No substantial statistical heterogeneity was identified. One of the three RCTs, a threearm trial enrolling a total of 566 critically ill patients, also reported no instances of temporarily holding the study drug owing to extrapyramidal symptoms. ${ }^{53}$ In addition, the fourth RCT, a three-arm trial comparing haloperidol with placebo in a total of 249 patients receiving palliative care, reported increased extrapyramidal symptoms (evaluated using the Extra Pyramidal Symptom Rating Scale score) in the haloperidol group (mean difference, 0.8; 95\% CI, 0.2 to 1.4; $P=0.01) .^{48}$

For the specific neurologic effect of akathisia, one three-arm RCT enrolling patients receiving mechanical ventilation, reported a non-statistically significant increase in the incidence of akathisia for haloperidol (29\%) compared with placebo (19\%). ${ }^{44}$

For the specific neurologic effects of muscle stiffness, torticollis, and dystonia, two RCTs reported data. One RCT evaluated patients receiving mechanical ventilation and enrolled within 72 hours of intensive care unit admission. This trial reported no statistically significant effect on muscle stiffness for haloperidol (1.4\%) compared with placebo (1.4\%) and on torticollis for haloperidol (0\%) compared with placebo (1.4\%). ${ }^{45}$ Another RCT, a three-arm trial enrolling critically ill patients, reported no instances of temporarily holding the study drug owing to dystonia. ${ }^{53}$

Two three-arm RCTs reported data on the neurologic effect of neuroleptic malignant syndrome. One RCT, enrolling patients receiving mechanical ventilation, reported no incidence of neuroleptic malignant syndrome in the haloperidol or placebo groups. ${ }^{44}$ Another RCT, a threearm trial enrolling critically ill patients, reported no incidence of permanent discontinuation of the study drug owing to suspected neuroleptic malignant syndrome. ${ }^{53}$

We did not find any observational studies or non-randomized trials that compared haloperidol with placebo and reported on neurologic effects. 
Figure 26. Meta-analysis of neurological outcomes in trials comparing haloperidol with placebo in patients with delirium

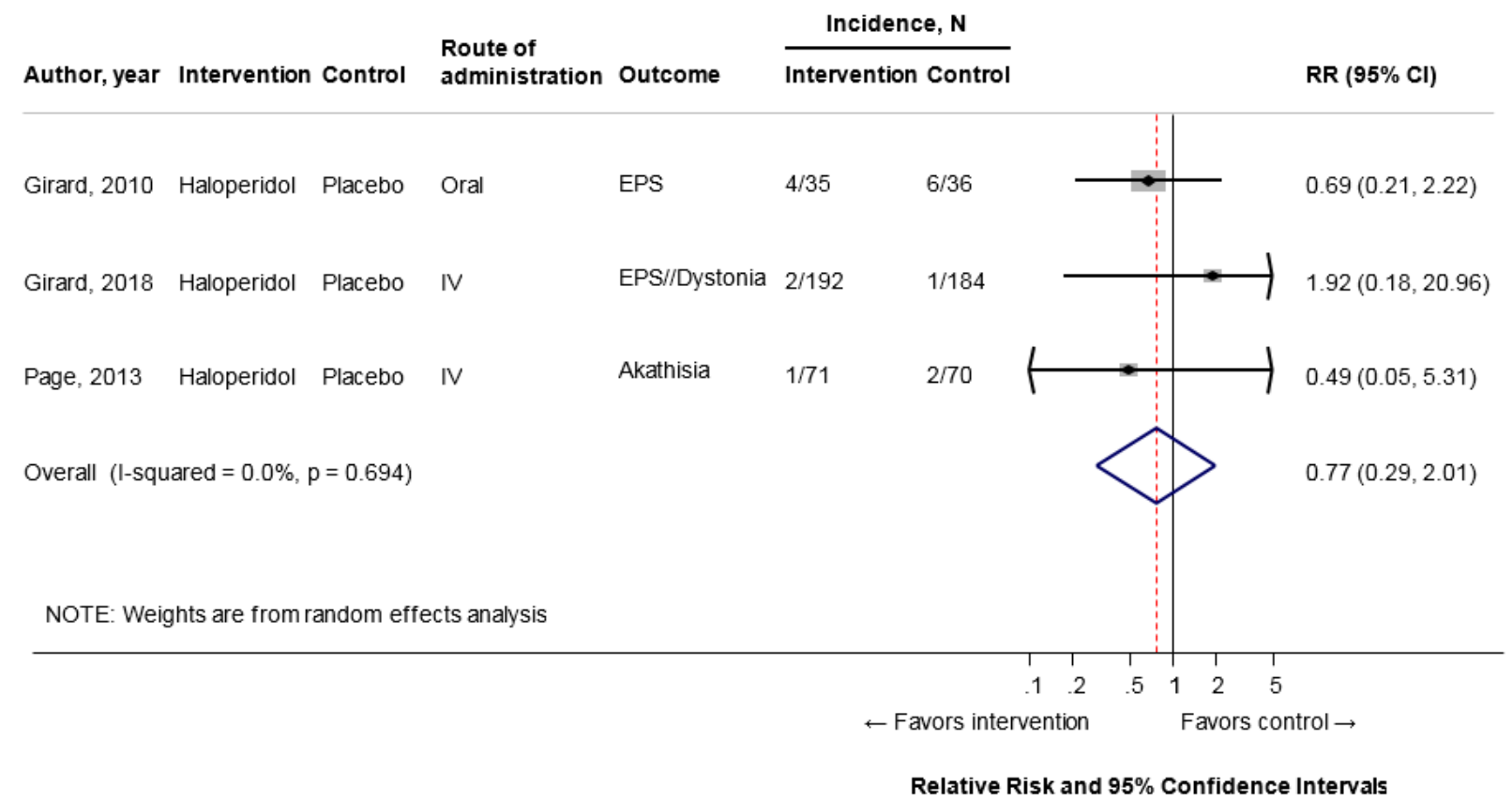

$\mathrm{CI}=$ confidence interval; EPS = extrapyramidal symptoms; IV = intravenous; $\mathrm{N}=$ total arm population; $\mathrm{n}=$ incidence within arm; $\mathrm{p}=\mathrm{p}$-value; $\mathrm{RR}=$ relative risk

\section{Second-Generation Antipsychotics Versus Placebo}

Five RCTs, with low risk of bias, compared second-generation antipsychotics with placebo for a number of different neurologic effects. ${ }^{44,48,52,53,63}$ Three of the five trials evaluated critically ill patients, one evaluated inpatients without critical illness, and one evaluated patients receiving palliative care. Overall, across a variety of neurologic effects, including extrapyramidal symptoms and neuroleptic malignant syndrome, only the single RCT that enrolled patients receiving palliative care reported a statistically significant increase in extrapyramidal symptoms for risperidone compared with placebo.

For the neurologic effect of extrapyramidal symptoms, we conducted a meta-analysis with three RCTs that enrolled inpatients with and without critical illness. ${ }^{44,53,63}$ This meta-analysis demonstrated no increase in extrapyramidal symptoms comparing second-generation antipsychotics with placebo (pooled RR, 0.44; 95\% CI, 0.14 to 1.38; I-squared, 0\%) (Figure 27). We tested the effect of each individual trial on the combined point estimate. No single trial influenced the pooled results. No substantial statistical heterogeneity was identified. One of the three RCTs, a three-arm trial enrolling a total of 566 critically ill patients, also reported no instances of temporarily holding the study drug owing to extrapyramidal symptoms. ${ }^{53}$ Another RCT, evaluating quetiapine compared with placebo in 36 medical and surgical critically ill patients, reported no incidence of extra pyramidal symptoms in either randomization group. ${ }^{52}$ In addition, a three-arm RCT, comparing risperidone and placebo in a total of 249 patients receiving palliative care, reported increased symptoms (evaluated using the Extra Pyramidal Symptom Rating Scale score) in the risperidone group (mean difference, 0.7; 95\% CI, 0.1 to 1.4; $P=0.03) .^{48}$ 
For the specific neurological effect of akathisia, one three-arm RCT, enrolling patients receiving mechanical ventilation, reported no statistically significant effect in incidence of akathisia for ziprasidone (20\%) compared with placebo (19\%). ${ }^{44}$

For the specific neurological effects of dystonia, one three-arm RCT, enrolling critically ill patients, reported no instances of temporarily holding the study drug owing to dystonia. ${ }^{53}$

For the neurological effect of neuroleptic malignant syndrome, two three-arm RCTs reported data. One RCT, enrolling patients receiving mechanical ventilation, reported no incidence of neuroleptic malignant syndrome in the ziprasidone or placebo groups. ${ }^{44}$ Another three-arm RCT, enrolling critically ill patients, reported no incidence of permanent discontinuation of the study drug owing to suspected neuroleptic malignant syndrome. ${ }^{53}$

We did not find any observational studies or non-randomized trials that compared a secondgeneration antipsychotic with placebo and reported on neurologic effects.

Figure 27. Meta-analysis of neurologic outcomes in trials comparing second-generation antipsychotics with placebo

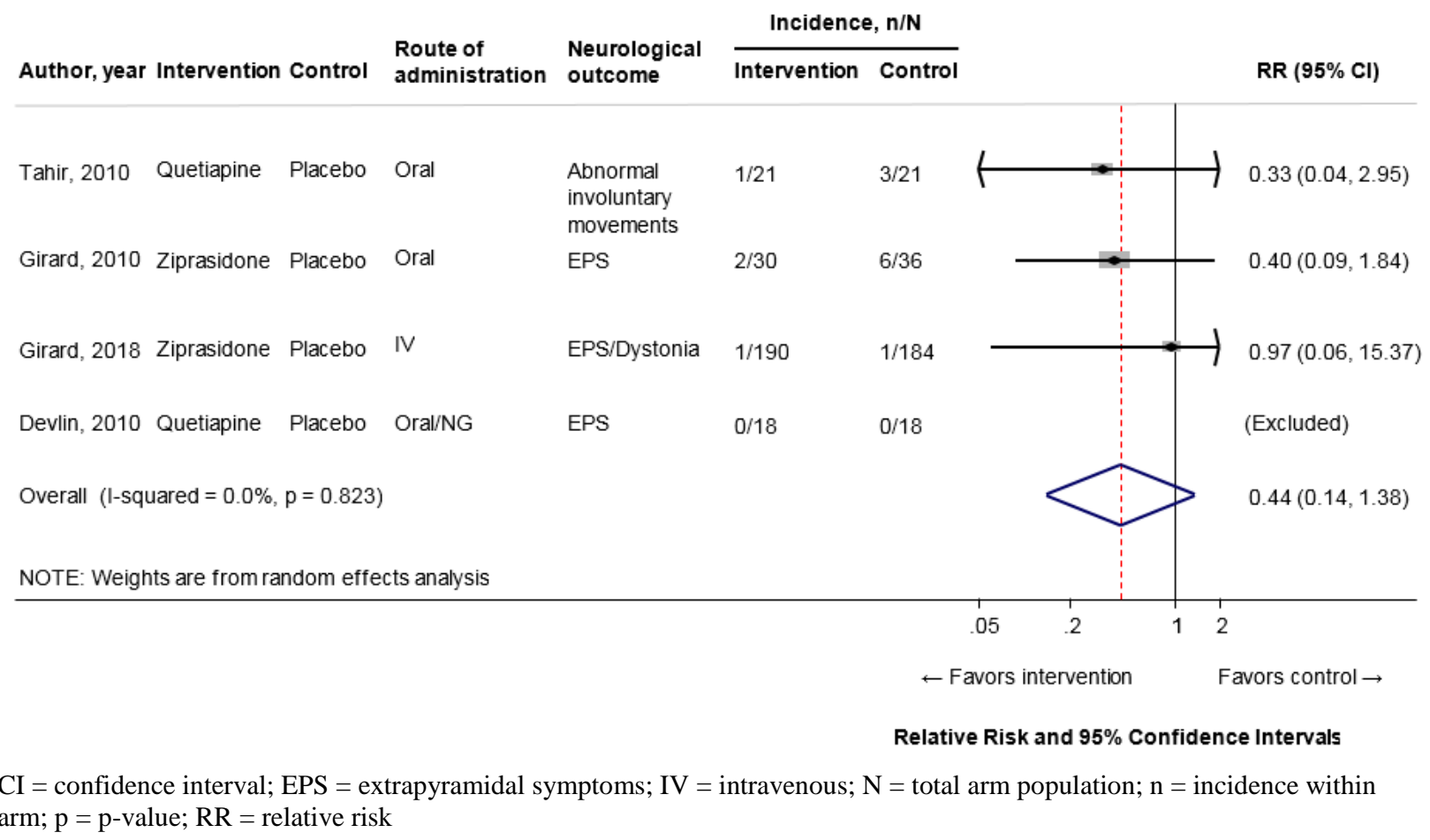

\section{Haloperidol Versus Second-Generation Antipsychotics}

Eight RCTs ${ }^{44,53,54,56,58,61,62,64}$ and eight observational studies with a comparison group ${ }^{65,66 \text {, }}$ 68, 70, 75, 84, 85, 89 compared second-generation antipsychotics with haloperidol for a number of different neurological effects. One RCT evaluated patients receiving palliative care, ${ }^{61}$ four RCTs

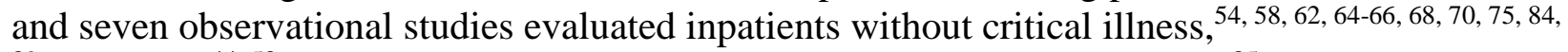
${ }^{89}$ two RCTs ${ }^{44,53}$ and one observational study evaluated critically ill patients, ${ }^{85}$ and one RCT evaluated inpatients with and without critical illness. ${ }^{56}$ Overall, across a variety of neurologic effects, including extrapyramidal symptoms and neuroleptic malignant syndrome, no RCT or observational study reported a statistically significant difference when comparing secondgeneration antipsychotics with haloperidol. 
For the neurologic effect of extrapyramidal symptoms, we conducted a meta-analysis of the six RCTs that evaluated inpatients with and without critical illness. ${ }^{44,53,54,56,62,64}$ For the purpose of this meta-analysis, we combined the olanzapine and risperidone groups to compare them with the haloperidol group in one study. ${ }^{54}$ Four of the six RCTs had low risk of bias, 44, 53, 62, 64 and two RCTs had unclear risk of bias. ${ }^{54,56}$ The meta-analysis showed a potentially clinically important but not statistically significant between-group difference in the incidence of extrapyramidal symptoms in second-generation antipsychotics compared with haloperidol (pooled RR, 0.45; 95\% CI, 0.20 to 1.01; I-squared, 0\%) (Figure 28). We tested the effect of each individual trial on the combined point estimate. No single trial influenced the pooled results. No substantial statistical heterogeneity was identified. One of the five RCTs, enrolling inpatients with hyperactive delirium, also evaluated extrapyramidal symptoms using the Modified Simpson Angus Scale. ${ }^{62}$ This RCT reported no statistically significant effect for quetiapine compared with haloperidol.

Seven RCTs ${ }^{44,53,54,58,61,62,64}$ and eight observational studies with a comparison group ${ }^{65,66,68,}$ 70, 75, 84, 85, 89 evaluated various neurologic effects related to extrapyramidal symptoms: akathisia; bradykinesia; drug-induced parkinsonism; dystonia; rigidity; tremors; tics; abnormal movements of jaw, tongue, lips, and perioral region; and withdrawal of study drug owing to extrapyramidal symptoms. Of the seven RCTs, two had potential for high risk of bias, primarily owing to a lack of blinding and missing outcome data; ${ }^{58,61}$ four were low risk of bias; ${ }^{44,}$ 53, 62, 64 and one had unclear risk of bias. ${ }^{54}$ Of the eight observational studies, one had a low risk of bias, ${ }^{85}$ one had a moderate risk of bias, ${ }^{68}$ and the rest had potential for serious risk of bias. ${ }^{65,66,70,75,84,89}$ Sample sizes for these observational studies with a comparison group ranged from 22 to 2,453. Five different second-generation antipsychotics (aripiprazole, olanzapine, quetiapine, risperidone, and ziprasidone) were compared with haloperidol, and no trial or observational study with a comparison group reported statistically significant differences in various neurologic effects related to extrapyramidal symptoms.

For the neurologic effect of neuroleptic malignant syndrome, two three-arm RCTs, with low risk of bias, reported data. ${ }^{44,53}$ One RCT, enrolling patients receiving mechanical ventilation, reported no incidence of neuroleptic malignant syndrome in the second-generation antipsychotics or haloperidol groups. ${ }^{44}$ One three-arm trial, enrolling critically ill patients, reported no incidence of permanent discontinuation of the study drug owing to suspected neuroleptic malignant syndrome. ${ }^{53}$

One RCT, with low risk of bias and enrolling inpatients with hyperactive delirium, reported no statistically significant difference in withdrawal of drug owing to seizures for quetiapine (4\%) compared to haloperidol (0\%). ${ }^{62}$ 
Figure 28. Meta-analysis of neurologic outcomes in trials comparing haloperidol with secondgeneration antipsychotics in patients with delirium

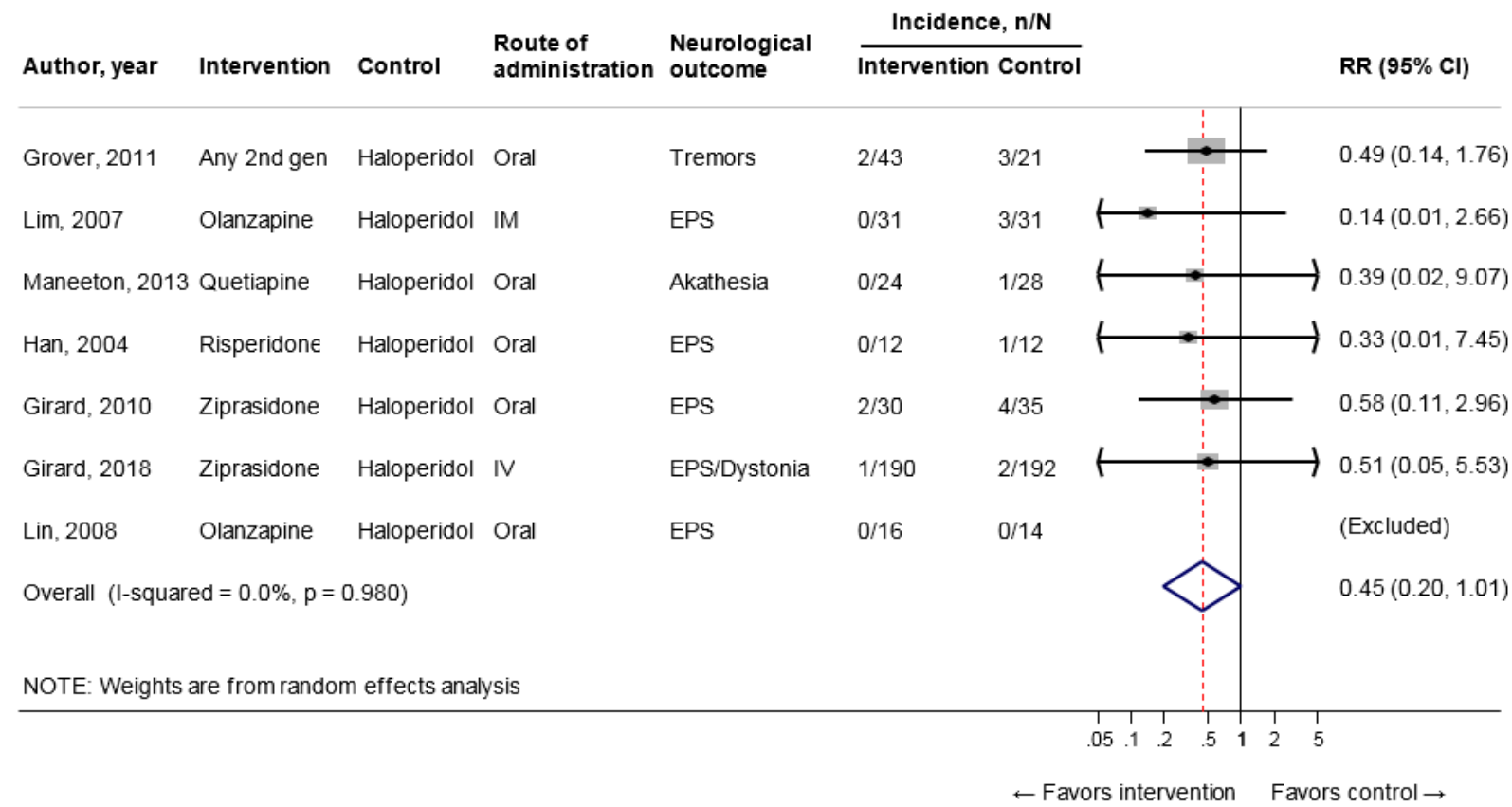

Relative Risk and $\mathbf{9 5 \%}$ Confidence Intervals

$\mathrm{CI}=$ confidence interval; EPS = extrapyramidal symptoms; IM = intramuscular; $\mathrm{IV}=$ intravenous; $\mathrm{N}=$ total arm population; $\mathrm{n}=$ incidence within arm; $\mathrm{p}=\mathrm{p}$-value; $\mathrm{RR}=$ relative risk

\section{Haloperidol Versus Other Therapies}

One three-arm RCT, with an unclear risk of bias, compared haloperidol with lorazepam in medical inpatients with acquired immunodeficiency syndrome and reported no statistically significant between-group difference in the Extra Pyramidal Symptom Rating Scale. ${ }^{51}$

We did not find any observational studies or non-randomized trials that compared haloperidol with other therapies and reported on neurologic effects.

\section{Second-Generation Antipsychotics Versus Second-Generation Antipsychotics}

Three RCTs ${ }^{54,59,60}$ and four observational studies with a comparison group ${ }^{66,68,70,89}$ compared second-generation antipsychotics with second-generation antipsychotics for a number of different neurologic effects in inpatients without critical illness. Of the three RCTs, two had high risk of bias ${ }^{59,60}$ and one had unclear risk of bias. ${ }^{54}$ Of the four observational studies, one study had moderate risk of bias ${ }^{68}$ and three studies had potential for serious risk of bias. ${ }^{66,70,89}$ Five different second-generation antipsychotics were compared with each other (aripiprazole, olanzapine, quetiapine, risperidone, and amisulpride) for various neurological effects related to extrapyramidal symptoms: akathisia; bradykinesia; drug-induced parkinsonism; dystonia; dyskinesia; rigidity; tremors; and abnormal movements of jaw, tongue, lips, and perioral region. No RCT or observational study with a comparison group reported statistically significant differences in extrapyramidal symptoms. The evidence was consistent but imprecise.

\section{Studies With No Comparison Group}

Twelve observational studies without a comparison group, with a total of 251 patients who were predominantly medical and surgical inpatients without critical illness, evaluated various 
neurologic effects of four different second-generation antipsychotics (aripiprazole, quetiapine, risperidone, and paliperidone). ${ }^{71,72,74,76-78,80-83,86,88}$ The results varied across these studies.

For the neurologic effect of extrapyramidal symptoms, four studies reported no incidence, ${ }^{74}$, 76, 80, 81 and two studies reported no statistically significant increase comparing baseline and final assessments of extrapyramidal symptoms scores. ${ }^{78,83}$ In contrast, four studies reported a range from 5 percent to 13 percent of patients experiencing extrapyramidal symptoms. ${ }^{71,72,77,88}$

For the neurologic effect of cerebrovascular accidents, two studies reported no incidence of such events. ${ }^{82,86}$

\section{Aspiration Pneumonia - Overall}

\section{Haloperidol Versus Second-Generation Antipsychotics}

One cohort study, with potential for high risk of bias, compared aripiprazole, olanzapine, quetiapine, and risperidone with haloperidol in inpatients. ${ }^{70}$ This study demonstrated no statistically significant effect on the incidence of aspiration pneumonia for aripiprazole compared with haloperidol, olanzapine compared with haloperidol, quetiapine compared with haloperidol, and risperidone compared with haloperidol.

\section{Second-Generation Antipsychotics Versus Second-Generation Antipsychotics}

One cohort study, with potential for high risk of bias and enrolling inpatients, compared aripiprazole, olanzapine, quetiapine, and risperidone. ${ }^{70}$ This study reported no statistically significant effect on the incidence of aspiration pneumonia for olanzapine compared with aripiprazole, quetiapine compared with aripiprazole, risperidone compared with aripiprazole, risperidone compared with quetiapine, quetiapine compared with olanzapine, and risperidone compared with olanzapine.

\section{Other Comparisons}

We found no studies of delirium treatment evaluating aspiration pneumonia in patients with delirium for the following comparisons:

- Haloperidol versus placebo

- Second-generation antipsychotics versus placebo

- Haloperidol versus other therapies

- Second-generation antipsychotics versus other therapies

\section{Studies With No Comparison Group}

One single-arm cohort study, enrolling 14 medically ill patients with delirium treated with aripiprazole, reported aspiration pneumonia in one patient (7\%). ${ }^{86}$

\section{Adverse Effects - Overall}

We found no studies in patients with delirium that compared an antipsychotic with a placebo, another antipsychotic, or another treatment and evaluated the following adverse events:

- Weight gain

- Changes in appetite

- Hypersensitivity reactions

- Inappropriate continuation of antipsychotics

- Swallowing difficulties 


\title{
Key Question 2a. Benefits and Harms of Antipsychotics To Treat Delirium in Persons Aged 65 Years and Older
}

\author{
Key Points - Aged 65 Years and Older
}

\section{Delirium Severity}

- We were unable to draw a conclusion regarding the effects of antipsychotics on cognitive functioning for the following comparisons:

o Haloperidol versus placebo (insufficient evidence)

o Second-generation antipsychotics versus placebo (insufficient evidence)

o Second-generation antipsychotics versus second-generation antipsychotics (insufficient evidence)

o Haloperidol versus other therapies (insufficient evidence)

o Second-generation antipsychotics versus other therapies (no trials)

\section{Cognitive Functioning, Falls, Inappropriate Continuation of Antipsychotics, or Sedation}

- We found no studies evaluating cognitive functioning, falls, inappropriate continuation of antipsychotics, or sedation in patients aged 65 years and older with delirium that compared an antipsychotic with a placebo, another antipsychotic, or another treatment.

\section{Intermediate Outcomes}

\section{Delirium Severity - Aged 65 Years and Older}

\section{Haloperidol Versus Placebo}

One three-arm RCT, with unclear risk of bias and enrolling inpatients aged 65 years and older, compared haloperidol with placebo. ${ }^{57}$ This RCT evaluated delirium severity at day seven, using the Delirium Rating Scale, with a lower score for haloperidol compared with placebo. Those patients randomized to haloperidol had a greater reduction in delirium severity score than patients randomized to placebo.

This RCT also reported lower delirium severity at day seven, evaluated using the Clinical Global Impression scale, for haloperidol compared with placebo. Using the Clinical Global Impression scale and 7-day followup, the RCT reported a statistically significant reduction in delirium severity for haloperidol compared with placebo. This trial also reported a statistically significant increase in the number of patients with a "significant improvement" or "complete alleviation" for haloperidol (88\%) compared with placebo (31\%). ${ }^{57}$ We were unable to draw conclusions about the effect of haloperidol compared with placebo on delirium severity based upon this single study. (SOE: Insufficient)

\section{Second-Generation Antipsychotics Versus Placebo}

One three-arm RCT, with unclear risk of bias and enrolling inpatients aged 65 years and older, compared olanzapine with placebo. ${ }^{57}$ This RCT evaluated delirium severity at day seven, using the Delirium Rating Scale, with a lower score for olanzapine compared with placebo. 
This RCT also reported lower delirium severity at day seven, evaluated using the Clinical Global Impression scale, for olanzapine compared with placebo. Using the Clinical Global Impression scale and 7-day followup, the RCT reported a statistically significant reduction in delirium severity for olanzapine compared with placebo. This trial also reported a statistically significant increase in the number of patients with a "significant improvement" or "complete alleviation” for olanzapine (82\%) compared with placebo (31\%). ${ }^{57}$ We were unable to draw conclusions based upon this single trial. (SOE: Insufficient)

\section{Haloperidol Versus Second-Generation Antipsychotics}

One three-arm RCT, with unclear risk of bias and enrolling inpatients aged 65 years and older, compared haloperidol with olanzapine. ${ }^{57}$ This RCT evaluated delirium severity at day seven, using the Delirium Rating Scale, with a lower score for olanzapine compared with haloperidol. There was no statistically significant difference in reduction from baseline between treatment groups.

This RCT also reported higher delirium severity at day seven, evaluated using the Clinical Global Impression scale, for olanzapine compared with haloperidol. Using the Clinical Global Impression scale and 7-day followup, the RCT reported no statistically significant difference in reduction in delirium severity for olanzapine compared with haloperidol and a non-statistically significant difference in the number of patients with a "significant improvement" or "complete alleviation" for olanzapine (82\%) compared with haloperidol (88\%). ${ }^{57}$ We were unable to draw conclusions based upon this single trial. (SOE: Insufficient)

\section{Other Comparisons}

We found no delirium treatment trials evaluating delirium severity in patients aged 65 years and older with delirium for the following comparisons:

- Second-generation antipsychotics versus second-generation antipsychotics

- Haloperidol versus other therapies

- Second-generation antipsychotics versus other therapies

\section{Strength of Evidence}

Table 21 summarizes the strength of evidence findings for the effects of antipsychotics on delirium severity in patients aged 65 years and older with delirium. 
Table 21. Strength of evidence domains for trials evaluating antipsychotics for the treatment of delirium in terms of delirium severity in patients aged 65 years and older

\begin{tabular}{|c|c|c|c|c|c|c|c|c|}
\hline Comparison & $\begin{array}{l}\text { Number Of } \\
\text { Trials (N) }\end{array}$ & $\begin{array}{c}\text { Study } \\
\text { Limitations }\end{array}$ & Directness & Consistency & Precision & $\begin{array}{l}\text { Reporting } \\
\text { Bias }\end{array}$ & $\begin{array}{l}\text { Strength Of } \\
\text { Evidence }\end{array}$ & Summary \\
\hline $\begin{array}{l}\text { Haloperidol vs. } \\
\text { placebo }\end{array}$ & $1^{57}(175)$ & High & Direct & $\begin{array}{l}\text { Consistency } \\
\text { unknown } \\
\text { (single study) }\end{array}$ & Imprecise & Undetected & Insufficient & $\begin{array}{l}\text { We were unable to draw a } \\
\text { conclusion. }\end{array}$ \\
\hline $\begin{array}{l}\text { Second- } \\
\text { generation } \\
\text { antipsychotics } \\
\text { vs. placebo }\end{array}$ & $1^{57}(175)$ & High & Direct & $\begin{array}{l}\text { Consistency } \\
\text { unknown } \\
\text { (single study) }\end{array}$ & Imprecise & Undetected & Insufficient & $\begin{array}{l}\text { We were unable to draw a } \\
\text { conclusion. }\end{array}$ \\
\hline $\begin{array}{l}\text { Haloperidol vs. } \\
\text { second- } \\
\text { generation } \\
\text { antipsychotics }\end{array}$ & $1^{57}(175)$ & High & Direct & $\begin{array}{l}\text { Consistency } \\
\text { unknown } \\
\text { (single study) }\end{array}$ & Imprecise & Undetected & Insufficient & $\begin{array}{l}\text { We were unable to draw a } \\
\text { conclusion. }\end{array}$ \\
\hline $\begin{array}{l}\text { Second- } \\
\text { generation } \\
\text { antipsychotics } \\
\text { vs. second- } \\
\text { generation } \\
\text { antipsychotics }\end{array}$ & No trials & & & & & & & \\
\hline $\begin{array}{l}\text { Haloperidol vs. } \\
\text { other therapies }\end{array}$ & No trials & & & & & & & \\
\hline $\begin{array}{l}\text { Second- } \\
\text { generation } \\
\text { antipsychotics } \\
\text { vs. other } \\
\text { therapies }\end{array}$ & No trials & & & & & & & \\
\hline
\end{tabular}




\section{Other Intermediate Outcomes - Aged 65 Years and Older}

We found no trials enrolling patients aged 65 years and older with delirium that compared an antipsychotic with a placebo, another antipsychotic, or another treatment and evaluated the following intermediate outcomes:

- Delirium incidence

- Short-term delirium symptoms

- Delirium- and coma-free days alive

- Duration of delirium

- Patient distress

- Use of rescue therapy

- Use of physical restraint

\section{Final Health or Patient-Centered Outcomes - Aged 65 Years and Older}

We found no trials enrolling patients aged 65 years and older with delirium that compared an antipsychotic with a placebo, another antipsychotic, or another treatment and evaluated the following final health or patient-centered outcomes:

- Mortality

- Quality of life

- Cognitive functioning

- Institutionalization

- Caregiver burden/strain

- Falls

- Memory of patient distress

\section{Resource Utilization Outcomes - Aged 65 Years and Older}

We found no trials enrolling patients aged 65 years and older with delirium that compared an antipsychotic with a placebo, another antipsychotic, or another treatment and evaluated the following resource utilization outcomes:

- Readmission to hospital

- Readmission to intensive care unit

- Length of stay in hospital

- Length of stay in intensive care unit

- Length of stay in skilled nursing facility

- Patient safety attendant use

- Hospice enrollment

\section{Adverse Effects - Aged 65 Years and Older}

We found no studies enrolling patients aged 65 years and older with delirium that compared an antipsychotic with a placebo, another antipsychotic, or another treatment and evaluated the following adverse events:

- Sedation 
- Weight gain

- Changes in appetite

- Cardiac effects

- Neurologic effects

- Hypersensitivity reactions

- Inappropriate continuation of antipsychotics

- Swallowing difficulties

- Aspiration pneumonia

\section{Key Question 2b. Benefits and Harms of Antipsychotics To Treat Delirium in Persons With Dementia}

We found no studies evaluating antipsychotics for the treatment of delirium in persons with dementia.

\section{Key Question 2c. Benefits and Harms of Antipsychotics To Treat Delirium in Patients in an Intensive Care Unit}

\section{Key Points - Intensive Care Unit}

\section{Length of Stay in Hospital}

- All RCTs reported no statistically significant difference in length of stay in hospital for critically ill patients for the following comparisons:

o Haloperidol with placebo (SOE: Moderate)

o Second-generation antipsychotics with placebo (SOE: Moderate)

o Haloperidol with second-generation antipsychotics (SOE: Moderate)

\section{Sedation}

- There was no clinically important or statistically significant difference in sedation for critically ill patients for the following comparisons:

o Haloperidol with placebo (SOE: Moderate)

o Second-generation antipsychotics with placebo (SOE: Moderate)

o Haloperidol with second-generation antipsychotics (SOE: Moderate)

- We are unable to draw a conclusion regarding the effects of antipsychotics on sedation in critically ill patients for the following comparisons:

o Second-generation antipsychotics with second-generation antipsychotics (insufficient evidence)

o Haloperidol with other therapies (no studies)

o Second-generation antipsychotics versus other therapies (no studies)

\section{Delirium Severity, Cognitive Functioning, and Inappropriate Continuation of Antipsychotics}

- We found no trials evaluating delirium severity, cognitive functioning, or inappropriate continuation of antipsychotics in critically ill patients with delirium that compared an antipsychotic with a placebo, another antipsychotic, or another treatment. 


\section{Intermediate Outcomes}

\section{Delirium Incidence - Intensive Care Unit}

\section{Second-Generation Antipsychotics Versus Placebo}

The findings presented in the "Delirium Incidence - Overall" results section are wholly represented by this subgroup. Please refer to the "Delirium Incidence - Overall” results section.

\section{Other Comparisons}

We found no delirium treatment trials evaluating delirium incidence in critically ill patients with delirium for the following comparisons:

- Haloperidol versus placebo

- Haloperidol versus second-generation antipsychotics

- Second-generation antipsychotics versus second-generation antipsychotics

- Haloperidol versus other therapies

- Second-generation antipsychotics versus other therapies.

\section{Short-Term Delirium Symptoms - Intensive Care Unit}

\section{Haloperidol Versus Placebo}

The findings presented in the "Short-Term Delirium Symptoms - Overall” results section are wholly represented by this subgroup. Please refer to the "Short-Term Delirium Symptoms Overall” results section.

\section{Second-Generation Antipsychotics Versus Placebo}

One RCT, with low risk of bias, compared quetiapine with placebo in medical and surgical critically ill patients. ${ }^{52}$ This RCT reported on agitation, defined as the number of hours with a Sedation-Agitation Scale score of equal to or greater than 5, and reported fewer hours of agitation for patients receiving quetiapine compared with placebo.

\section{Other Comparisons}

We found no delirium treatment trials evaluating short-term delirium symptoms in critically ill patients with delirium for the following comparisons:

- Haloperidol versus second-generation antipsychotics

- Second-generation antipsychotics versus second-generation antipsychotics

- Haloperidol versus other therapies

- Second-generation antipsychotics versus other therapies

\section{Delirium- and Coma-Free Days Alive - Intensive Care Unit}

\section{Haloperidol Versus Placebo}

The findings presented in the "Delirium- and Coma-Free Days Alive - Overall” results section are wholly represented by this subgroup. Please refer to the "Delirium- and Coma-Free Days Alive - Overall” results section. 


\section{Second-Generation Antipsychotics Versus Placebo}

The findings presented in the "Delirium- and Coma-Free Days Alive - Overall" results section are wholly represented by this subgroup. Please refer to the "Delirium- and Coma-Free Days Alive - Overall” results section.

\section{Haloperidol Versus Second-Generation Antipsychotics}

The findings presented in the "Delirium- and Coma-Free Days Alive - Overall" results section are wholly represented by this subgroup. Please refer to the "Delirium- and Coma-Free Days Alive - Overall” results section.

\section{Other Comparisons}

We found no delirium treatment trials evaluating delirium- and coma-free days alive in critically ill patients with delirium for the following comparisons:

- Second-generation antipsychotics versus second-generation antipsychotics

- Haloperidol versus other therapies

- Second-generation antipsychotics versus other therapies

\section{Duration of Delirium - Intensive Care Unit}

\section{Haloperidol Versus Placebo}

The findings presented in the "Duration of Delirium - Overall" results section are wholly represented by this subgroup. Please refer to the "Duration of Delirium - Overall" results section.

\section{Second-Generation Antipsychotics Versus Placebo}

The findings presented in the "Duration of Delirium - Overall" results section are wholly represented by this subgroup. Please refer to the "Duration of Delirium - Overall" results section.

\section{Haloperidol Versus Second-Generation Antipsychotics}

Two RCTs, with low risk of bias, compared ziprasidone with haloperidol and assessed delirium duration in medical and surgical patients in intensive care units. ${ }^{44,53}$ There was consistent and precise evidence from these well-conducted trials of no difference in delirium duration for second-generation antipsychotics compared with haloperidol. We did not conduct a meta-analysis because these trials provided results as median values and delirium duration data may be skewed, precluding conversion or transformation of the data.

\section{Other Comparisons}

We found no delirium treatment trials evaluating delirium duration in critically ill patients with delirium for the following comparisons:

- Second-generation antipsychotics versus second-generation antipsychotics

- Haloperidol versus other therapies

- Second-generation antipsychotics versus other therapies 


\section{Use of Rescue Therapy - Intensive Care Unit}

\section{Haloperidol Versus Placebo}

The findings presented in the "Use of Rescue Therapy - Overall” results section are wholly represented by this subgroup. Please refer to the "Use of Rescue Therapy - Overall” results section.

\section{Second-Generation Antipsychotics Versus Placebo}

The findings presented in the "Use of Rescue Therapy - Overall" results section are wholly represented by this subgroup. Please refer to the "Use of Rescue Therapy - Overall” results section.

\section{Haloperidol Versus Second-Generation Antipsychotics}

Two RCTs, with low risk of bias, compared ziprasidone with haloperidol and assessed the use of rescue therapy, defined as the use of any open-label antipsychotic. ${ }^{44,53}$ Both of these trials, enrolling medical and surgical patients in intensive care units, reported no statistically significant effect on the use of rescue therapy. The evidence was consistent but imprecise.

\section{Other Comparisons}

We found no delirium treatment trials evaluating the use of rescue therapy in critically ill patients with delirium for the following comparisons:

- Second-generation antipsychotics versus second-generation antipsychotics

- Haloperidol versus other therapies

- Second-generation antipsychotics versus other therapies

\section{Other Intermediate Outcomes - Intensive Care Unit}

We found no trials enrolling critically ill patients with delirium that compared an antipsychotic with a placebo, another antipsychotic, or another treatment and evaluated the following intermediate outcomes:

- Delirium severity

- Patient distress

- Use of physical restraint

\section{Final Health or Patient-Centered Outcomes}

\section{Mortality - Intensive Care Unit}

\section{Haloperidol Versus Placebo}

Three RCTs, with low risk of bias and enrolling medical and surgical critically ill patients, compared haloperidol with placebo. All three RCTs reported no between-group difference in short-term mortality, defined as mortality while in hospital or up to 30 days after randomization (pooled RR, 1.08; 95\% CI, 0.85 to 1.36; I-squared, 0\%) (Figure 29). ${ }^{44,45,53}$ We tested the effect of each individual trial on the combined point estimate. No single trial influenced the pooled results. No substantial statistical heterogeneity was identified. There was consistent but imprecise evidence of no difference for short-term mortality. 
Of these three RCTs, the largest trial was a three-arm RCT, with low risk of bias, that also evaluated 90-day mortality in a total of 566 critically ill patients. The results of this evaluation were consistent with the results of our meta-analysis, with no between-group difference in shortterm mortality when comparing haloperidol with placebo. ${ }^{53}$

\section{Second-Generation Antipsychotics Versus Placebo}

Three RCTs, with low risk of bias and enrolling medical and surgical critically ill patients, compared a second-generation antipsychotic with placebo. All three RCTs reported no betweengroup difference in short-term mortality, defined as mortality while in hospital or up to 30 days after randomization (pooled RR, 0.98; 95\% CI, 0.75 to 1.28; I-squared, 0.0\%) (Figure 29). ${ }^{44,52,53}$ Two of the RCTs evaluated ziprasidone, and one RCT evaluated quetiapine. We tested the effect of each individual trial on the combined point estimate. No single trial influenced the pooled results. No substantial statistical heterogeneity was identified. The evidence was consistent but imprecise.

Of these three RCTs, the largest trial was a three-arm RCT, with low risk of bias, that also evaluated 90-day mortality in a total of 566 critically ill patients. The results of this evaluation were consistent with the results of our meta-analysis, with no between-group difference reported for short-term mortality comparing risperidone with placebo. ${ }^{53}$

Figure 29. Meta-analysis of mortality in trials with an intensive care unit population comparing haloperidol or second-generation antipsychotics with placebo

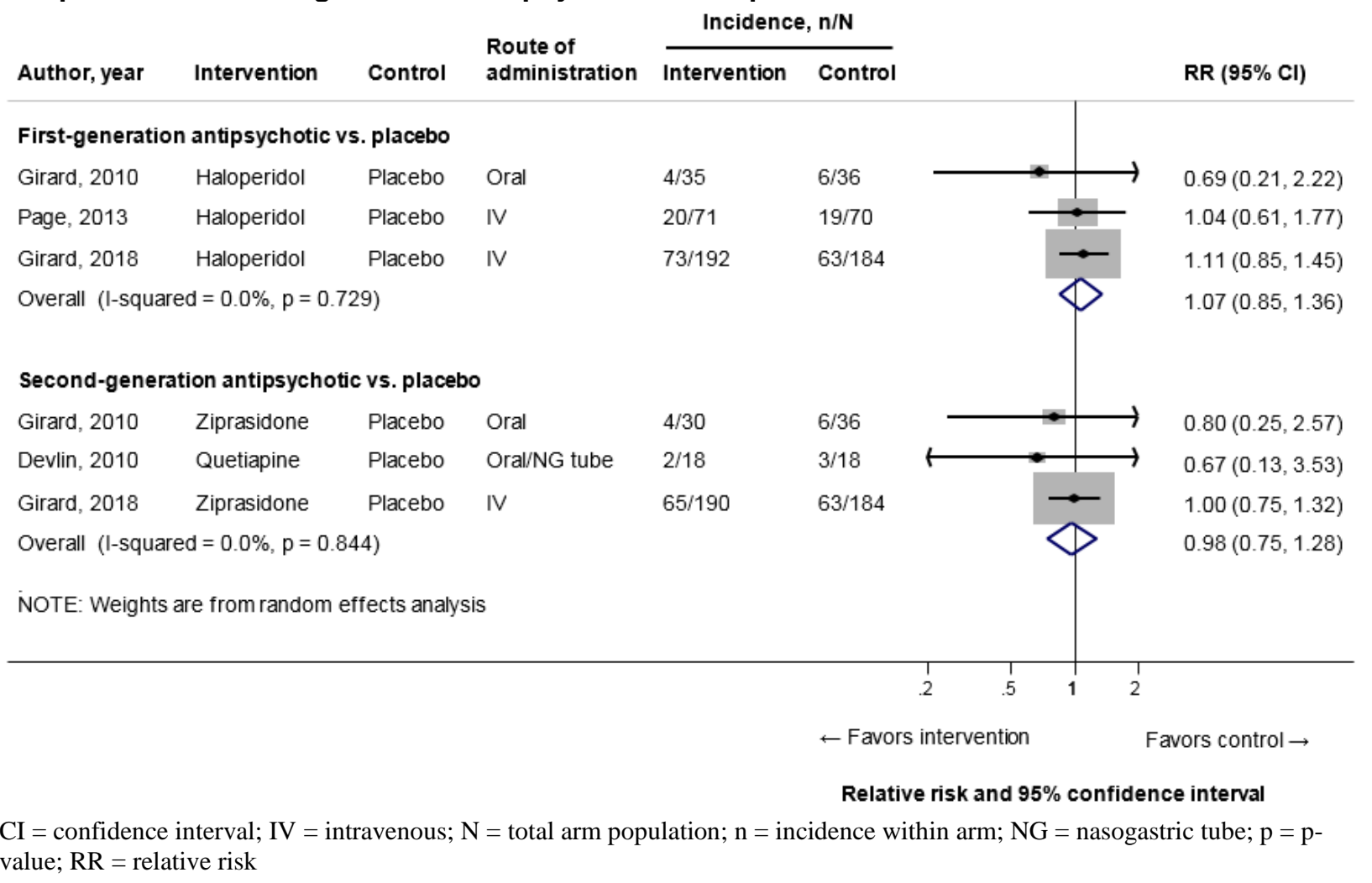

\section{Haloperidol Versus Second-Generation Antipsychotics}

Two RCTs, with low risk of bias and enrolling medical and surgical critically ill patients, compared haloperidol with ziprasidone. ${ }^{44,53}$ These RCTs assessed short-term mortality, defined 
as mortality while in hospital or up to 30 days after randomization. There was consistent but imprecise evidence of no effect on short-term mortality.

\section{Other Comparisons}

We found no delirium treatment trials evaluating mortality in critically ill patients with delirium for the following comparisons:

- Second-generation antipsychotics versus second-generation antipsychotics

- Haloperidol versus other therapies

- Second-generation antipsychotics versus other therapies

\section{Other Final Health or Patient-Centered Outcomes - Intensive Care Unit}

We found no trials enrolling critically ill patients with delirium that compared an antipsychotic with a placebo, another antipsychotic, or another treatment and evaluated the following final health or patient-centered outcomes:

- Quality of life

- Cognitive functioning

- Institutionalization

- Caregiver burden/strain

- Falls

- Memory of patient distress

\section{Resource Utilization Outcomes}

\section{Readmission to Intensive Care Unit - Intensive Care Unit}

\section{Haloperidol Versus Placebo}

The findings presented in the "Readmission to Intensive Care Unit - Overall” results section are wholly represented by this subgroup. Please refer to the "Readmission to Intensive Care Unit - Overall” results section.

\section{Second-Generation Antipsychotics Versus Placebo}

The findings presented in the "Readmission to Intensive Care Unit - Overall" results section are wholly represented by this subgroup. Please refer to the "Readmission to Intensive Care Unit - Overall” results section.

\section{Haloperidol Versus Second-Generation Antipsychotics}

The findings presented in the "Readmission to Intensive Care Unit - Overall” results section are wholly represented by this subgroup. Please refer to the "Readmission to Intensive Care Unit - Overall” results section.

\section{Other Comparisons}

We found no delirium treatment trials evaluating readmission to the intensive care unit in critically ill patients with delirium for the following comparisons:

- Second-generation antipsychotics versus second-generation antipsychotics 
- Haloperidol versus other therapies

- Second-generation antipsychotics versus other therapies

\section{Length of Stay in Hospital - Intensive Care Unit}

\section{Haloperidol Versus Placebo}

The findings presented in the "Length of Stay in Hospital - Overall" results section are wholly represented by this subgroup. Please refer to the "Length of Stay in Hospital - Overall" results section. (SOE for patients in the intensive care unit: Moderate)

\section{Second-Generation Antipsychotics Versus Placebo}

The findings presented in the "Length of Stay in Hospital - Overall" results section are wholly represented by this subgroup. Please refer to the "Length of Stay in Hospital - Overall" results section. (SOE for patients in the intensive care unit: Moderate)

\section{Haloperidol Versus Second-Generation Antipsychotics}

The findings presented in the "Length of Stay in Hospital - Overall" results section are wholly represented by this subgroup. Please refer to the "Length of Stay in Hospital - Overall" results section. (SOE for patients in the intensive care unit: Moderate)

\section{Other Comparisons}

We found no delirium treatment trials evaluating length of stay in hospital for critically ill patients with delirium for the following comparisons:

- Second-generation antipsychotics versus second-generation antipsychotics

- Haloperidol versus other therapies

- Second-generation antipsychotics versus other therapies

\section{Strength of Evidence}

Table 22 summarizes the strength of evidence findings for length of stay in hospital for critically ill patients with delirium. 
Table 22. Strength of evidence domains for trials evaluating antipsychotics for the treatment of delirium in terms of length of stay in hospital for patients in an intensive care unit

\begin{tabular}{|c|c|c|c|c|c|c|c|c|}
\hline Comparison & $\begin{array}{c}\text { Number Of } \\
\text { Trials (N) }\end{array}$ & $\begin{array}{c}\text { Study } \\
\text { Limitations }\end{array}$ & Directness & Consistency & Precision & $\begin{array}{c}\text { Reporting } \\
\text { Bias }\end{array}$ & $\begin{array}{c}\text { Strength Of } \\
\text { Evidence }\end{array}$ & Summary \\
\hline $\begin{array}{l}\text { Haloperidol vs. } \\
\text { placebo }\end{array}$ & $\begin{array}{l}3^{44,45,53} \\
(808)\end{array}$ & Low & Direct & Consistent & Precise & Undetected & Moderate & $\begin{array}{l}\text { All RCTs reported no } \\
\text { statistically significant } \\
\text { difference. }\end{array}$ \\
\hline $\begin{array}{l}\text { Second- } \\
\text { generation } \\
\text { antipsychotics } \\
\text { vs. placebo }\end{array}$ & $\begin{array}{l}3^{44,52,53} \\
(703)\end{array}$ & Low & Direct & Consistent & Precise & Undetected & Moderate & $\begin{array}{l}\text { All RCTs reported no } \\
\text { statistically significant } \\
\text { difference. }\end{array}$ \\
\hline $\begin{array}{l}\text { Haloperidol vs. } \\
\text { second- } \\
\text { generation } \\
\text { antipsychotics }\end{array}$ & $2^{44,53}(667)$ & Low & Direct & Consistent & Precise & Undetected & Moderate & $\begin{array}{l}\text { All RCTs reported no } \\
\text { statistically significant } \\
\text { difference. }\end{array}$ \\
\hline $\begin{array}{l}\text { Second- } \\
\text { generation } \\
\text { antipsychotics } \\
\text { vs. second- } \\
\text { generation } \\
\text { antipsychotics }\end{array}$ & No trials & & & & & & & \\
\hline $\begin{array}{l}\text { Haloperidol vs. } \\
\text { other therapies }\end{array}$ & No trials & & & & & & & \\
\hline $\begin{array}{l}\text { Second- } \\
\text { generation } \\
\text { antipsychotics } \\
\text { vs. other } \\
\text { therapies }\end{array}$ & No trials & & & & & & & \\
\hline
\end{tabular}

Abbreviations: RCT = randomized controlled trial. 


\section{Length of Stay in Intensive Care Unit - Intensive Care Unit}

\section{Haloperidol Versus Placebo}

The findings presented in the "Length of Stay in Intensive Care Unit - Overall” results section are wholly represented by this subgroup. Please refer to the "Length of Stay in Intensive Care Unit - Overall” results section.

\section{Second-Generation Antipsychotics Versus Placebo}

The findings presented in the "Length of Stay in Intensive Care Unit - Overall" results section are wholly represented by this subgroup. Please refer to the "Length of Stay in Intensive Care Unit - Overall” results section.

\section{Haloperidol Versus Second-Generation Antipsychotics}

The findings presented in the "Length of Stay in Intensive Care Unit - Overall" results section are wholly represented by this subgroup. Please refer to the "Length of Stay in Intensive Care Unit - Overall” results section.

\section{Other Comparisons}

We found no delirium treatment trials evaluating length of stay in the intensive care unit for critically ill patients with delirium for the following comparisons:

- Second-generation antipsychotics versus second-generation antipsychotics

- Haloperidol versus other therapies

- Second-generation antipsychotics versus other therapies

\section{Other Resource Utilization Outcomes - Intensive Care Unit}

We found no trials enrolling critically ill patients with delirium that compared an antipsychotic with a placebo, another antipsychotic, or another treatment and evaluated the following resource utilization outcomes:

- Readmission to hospital

- Length of stay in skilled nursing facility

- Patient safety attendant use

- Hospice enrollment

\section{Adverse Effects}

\section{Sedation - Intensive Care Unit}

\section{Haloperidol Versus Placebo}

The findings presented in the "Sedation - Overall" results section are wholly represented by this subgroup. Please refer to the "Sedation - Overall" results section. (SOE for patients in the intensive care unit: Moderate) 


\section{Second-Generation Antipsychotics Versus Placebo}

Two RCTs, with low risk of bias, compared second-generation antipsychotics with placebo in inpatients with critical illness. ${ }^{52,53}$ The smaller trial $(\mathrm{N}=36)$ reported a potentially clinically important but not statistically significant effect on sedation-related effects for quetiapine compared with placebo. However, the larger trial $(\mathrm{N}=566)$ reported no clinically important or statistically significant difference for ziprasidone compared with placebo. Overall, there was no clinically important or statistically significant effect of haloperidol compared with placebo on sedation. (SOE for patients in the intensive care unit: Moderate)

\section{Haloperidol Versus Second-Generation Antipsychotics}

One three-arm RCT, with low risk of bias, compared ziprasidone with haloperidol in critically ill patients and reported on the instances of temporarily holding the study drug owing to oversedation. This RCT reported no clinically important or statistically significant difference in the ziprasidone group compared with the haloperidol group. ${ }^{53}$ (SOE for patients in the intensive care unit: Moderate)

\section{Second-Generation Antipsychotics Versus Second-Generation Antipsychotics}

One observational study that compared quetiapine with lurasidone in critically ill patients reported on the instances of discontinuation of the study drug owing to increased sedation. This observational study, with moderate risk of bias, reported no statistically significant difference for the quetiapine group (20\%) compared with the lurasidone group (10\%). ${ }^{69}$ This study also reported no statistically significant difference in the percentage of time patients were oversedated, defined as a Richmond Agitation-Sedation Scale lower than or equal to -3, in the quetiapine group compared with the lurasidone group. We were unable to draw conclusions regarding sedation when comparing second-generation antipsychotics in patients in the intensive care unit. (SOE for patients in the intensive care unit: Insufficient)

\section{Other Comparisons}

We found no studies of delirium treatment evaluating sedation in critically ill patients with delirium for the following comparisons:

- Haloperidol versus other therapies

- Second-generation antipsychotics versus other therapies

\section{Strength of Evidence}

Table 23 summarizes the strength of evidence findings for antipsychotics in terms of sedation in critically ill patients. 
Table 23. Strength of evidence domains for studies evaluating antipsychotics for the treatment of delirium in terms of sedation in patients in the intensive care unit

\begin{tabular}{|c|c|c|c|c|c|c|c|c|}
\hline Comparison & $\begin{array}{l}\text { Number Of } \\
\text { Studies (N) }\end{array}$ & $\begin{array}{c}\text { Study } \\
\text { Limitations }\end{array}$ & Directness & Consistency & Precision & $\begin{array}{l}\text { Reporting } \\
\text { Bias }\end{array}$ & $\begin{array}{l}\text { Strength Of } \\
\text { Evidence }\end{array}$ & Summary \\
\hline $\begin{array}{l}\text { Haloperidol vs. } \\
\text { placebo }\end{array}$ & $2^{45,53}(707)$ & Low & Direct & Consistent & Imprecise & Undetected & Moderate & $\begin{array}{l}\text { In critically ill patients, there } \\
\text { was no clinically important } \\
\text { or statistically significant } \\
\text { difference in sedation. }\end{array}$ \\
\hline $\begin{array}{l}\text { Second- } \\
\text { generation } \\
\text { antipsychotics } \\
\text { vs. placebo }\end{array}$ & $2^{52,53}(602)$ & Low & Direct & Consistent & Precise & Undetected & Moderate & $\begin{array}{l}\text { There was no clinically } \\
\text { important or statistically } \\
\text { significant difference. }\end{array}$ \\
\hline $\begin{array}{l}\text { Haloperidol vs. } \\
\text { second- } \\
\text { generation } \\
\text { antipsychotics }\end{array}$ & $1^{53}(566)$ & Low & Direct & $\begin{array}{l}\text { Consistency } \\
\text { unknown } \\
\text { (single study) }\end{array}$ & Precise & Undetected & Moderate & $\begin{array}{l}\text { There was no clinically } \\
\text { important or statistically } \\
\text { significant difference. }\end{array}$ \\
\hline $\begin{array}{l}\text { Second- } \\
\text { generation } \\
\text { antipsychotics } \\
\text { vs. second- } \\
\text { generation } \\
\text { antipsychotics }\end{array}$ & $\begin{array}{l}1 \text { observational } \\
\text { study with } \\
\text { comparison } \\
\text { group }^{69}(40)\end{array}$ & Medium & Direct & $\begin{array}{l}\text { Consistency } \\
\text { unknown } \\
\text { (single study) }\end{array}$ & Imprecise & Undetected & Insufficient & $\begin{array}{l}\text { We were unable to draw a } \\
\text { conclusion. }\end{array}$ \\
\hline $\begin{array}{l}\text { Haloperidol vs. } \\
\text { other therapies }\end{array}$ & No studies & & & & & & & \\
\hline $\begin{array}{l}\text { Second- } \\
\text { generation } \\
\text { antipsychotics } \\
\text { vs. other } \\
\text { therapies }\end{array}$ & No studies & & & & & & & \\
\hline
\end{tabular}

Abbreviations: $\mathrm{CI}$ = confidence interval; $\mathrm{RR}=$ relative risk 


\section{Cardiac Effects - Intensive Care Unit}

\section{Haloperidol Versus Placebo}

The findings presented in the "Cardiac Effects - Overall" results section are wholly represented by this subgroup. Please refer to the "Cardiac Effects - Overall” results section.

\section{Second-Generation Antipsychotics Versus Placebo}

The findings presented in the "Cardiac Effects - Overall" results section are wholly represented by this subgroup. Please refer to the "Cardiac Effects - Overall” results section.

\section{Haloperidol Versus Second-Generation Antipsychotics}

Two RCTs, with low risk of bias, and one observational study, with a comparison group and potential for serious risk of bias, evaluated different cardiac events in patients receiving haloperidol compared with second-generation antipsychotics. ${ }^{44,53,79}$ All three studies enrolled critically ill patients. Across all three studies and types of cardiac events, there were no statistically significant differences in haloperidol compared with second-generation antipsychotics. The evidence was consistent but imprecise.

One three-arm RCT, enrolling patients receiving mechanical ventilation with and without delirium, reported no statistically significant difference in the incidence of prolongation of the corrected QT interval to more than 500 milliseconds for ziprasidone (17\%) compared with haloperidol (6\%). ${ }^{44}$ This RCT also reported no incidence of ventricular arrhythmia in either randomization group.

Another three-arm RCT, enrolling critically ill patients, reported no statistically significant difference in the incidence of temporary discontinuation of the study drug owing to prolongation of the corrected QT interval for ziprasidone (11\%) compared with haloperidol (7\%). ${ }^{53}$ This RCT also reported no incidence of permanent discontinuation of the study drug owing to suspected torsades de pointes in either randomization group.

One observational study, with comparison groups and potential for serious risk of bias, enrolled a total of 925 patients with and without delirium in the coronary care unit. This observational study compared haloperidol with quetiapine on prolongation of the corrected QT interval at multiple time points. This study reported no clinically important or statistically significant difference for haloperidol compared with quetiapine in patients with delirium. ${ }^{79}$

\section{Second-Generation Antipsychotics Versus Second-Generation Antipsychotics}

One observational study, with moderate risk of bias, enrolled medical and cardiac critically ill patients. ${ }^{69}$ This study reported no statistically significant difference in a corrected QT interval increase of more than 60 milliseconds from baseline for quetiapine (10\%) compared with lurasidone (10\%). This observational study also reported no statistically significant difference in discontinuation of study medication owing to prolonged QT interval for quetiapine (5\%) compared with lurasidone (0\%).

\section{Other Comparisons}

We found no studies of delirium treatment evaluating cardiac effects in critically ill patients with delirium for the following comparisons:

- Haloperidol versus other therapies 
- Second-generation antipsychotics versus other therapies

\section{Neurologic Effects - Intensive Care Unit}

\section{Haloperidol Versus Placebo}

Three RCTs, with low risk of bias, compared haloperidol with placebo for a number of different neurologic effects in critically ill patients. ${ }^{44,45}, 53$ Overall, across a variety of neurologic effects, including extrapyramidal symptoms and neuroleptic malignant syndrome, no RCT demonstrated a statistically significant difference for haloperidol compared with placebo. The evidence was consistent but imprecise.

For the neurologic effect of extrapyramidal symptoms, we conducted a meta-analysis of the three RCTs ${ }^{44,45,53}$ which demonstrated no between-group difference in extrapyramidal symptoms comparing haloperidol with placebo (pooled RR, 0.77; 95\% CI, 0.29 to 2.02; Isquared, 0\%) (Figure 30). We tested the effect of each individual trial on the combined point estimate. No single trial influenced the pooled results. No substantial statistical heterogeneity was identified. One of the three RCTs, a three-arm trial enrolling critically ill patients, also reported no instances of temporarily holding the study drug owing to extrapyramidal symptoms. ${ }^{53}$

For the specific neurologic effect of akathisia, one three-arm RCT, enrolling patients receiving mechanical ventilation, reported a non-statistically significant increase in the incidence of akathisia for haloperidol (29\%) compared with placebo (19\%). ${ }^{44}$

For the specific neurologic effects of muscle stiffness, torticollis, and dystonia, two RCTs reported data. One RCT evaluated patients receiving mechanical ventilation enrolled within 72 hours of intensive care unit admission. This trial reported no statistically significant effect on muscle stiffness for haloperidol (1\%) compared with placebo (1.4\%) and no statistically significant effect on torticollis for haloperidol (0\%) compared with placebo (1.4\%). ${ }^{45}$ Another RCT, a three-arm trial enrolling critically ill patients, reported no instances of temporarily holding the study drug owing to dystonia. ${ }^{53}$

For the neurologic effect of neuroleptic malignant syndrome, two three-arm RCTs reported data. One RCT, enrolling patients receiving mechanical ventilation, reported no incidence of neuroleptic malignant syndrome in the haloperidol or placebo groups ${ }^{44}$ Another RCT, a threearm trial enrolling critically ill patients, reported no incidence of permanent discontinuation of the study drug owing to suspected neuroleptic malignant syndrome. ${ }^{53}$ 
Figure 30. Meta-analysis of neurological extrapyramidal symptoms outcomes in trials with an intensive care unit population comparing haloperidol with placebo

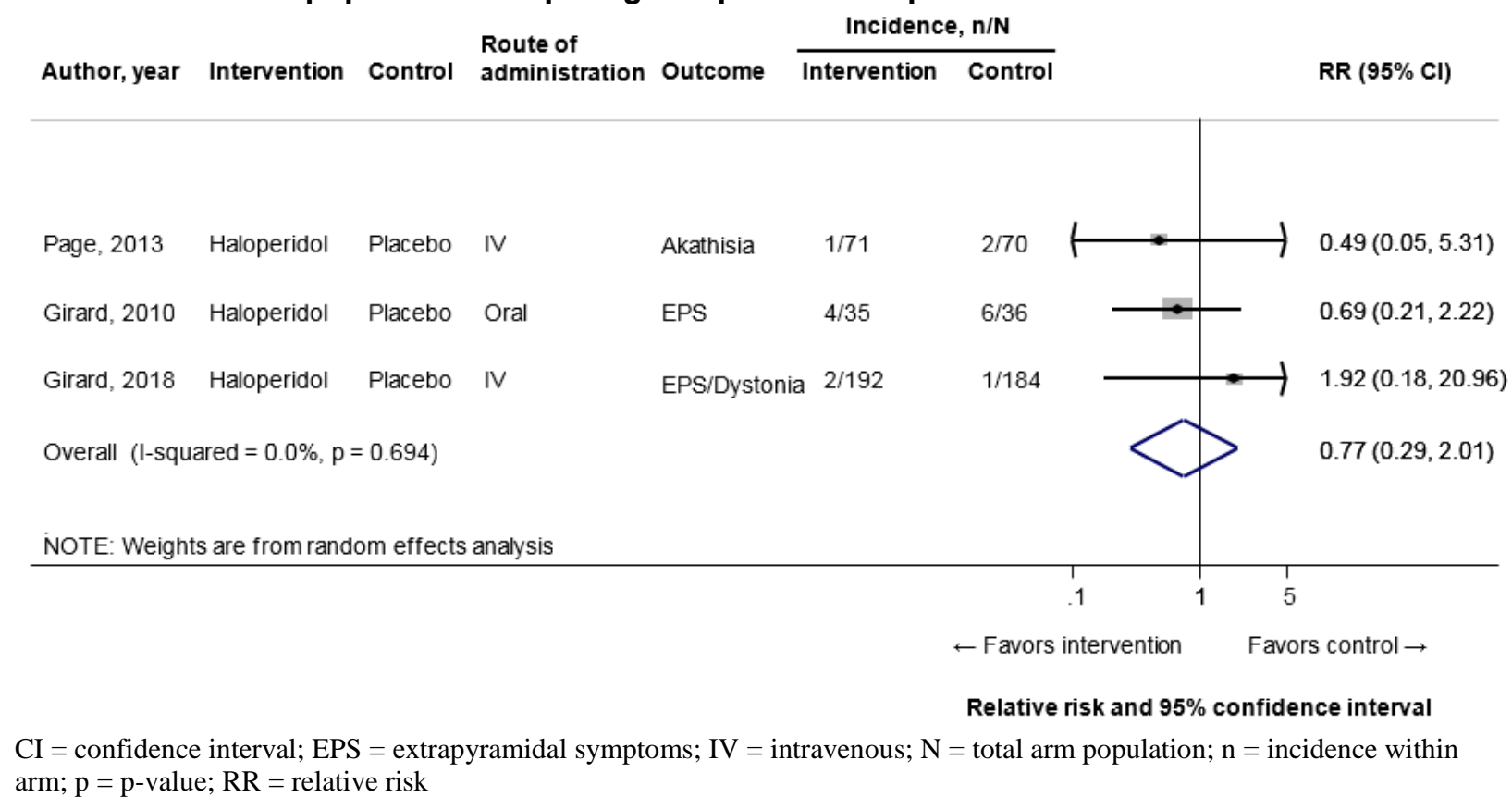

\section{Second-Generation Antipsychotics Versus Placebo}

Three RCTs, with low risk of bias, compared two second-generation antipsychotics (ziprasidone and quetiapine) with placebo for a number of different neurologic effects in critically ill patients. ${ }^{44,52,53}$ Overall, across a variety of neurologic effects, including extrapyramidal symptoms and neuroleptic malignant syndrome, no RCT demonstrated a statistically significant difference in second-generation antipsychotics compared with placebo. ${ }^{44,}$ 52,53

\section{Haloperidol Versus Second-Generation Antipsychotics}

Two RCTs ${ }^{44,53}$, with low risk of bias, and one observational study with a comparison group ${ }^{85}$ compared second-generation antipsychotics (ziprasidone and olanzapine) with haloperidol for a number of different neurologic effects in critically ill patients. Overall, across a variety of neurologic effects, including extrapyramidal symptoms and neuroleptic malignant syndrome, neither of the two trials ${ }^{44,53}$ nor the observational study ${ }^{85}$ demonstrated a statistically significant difference in second-generation antipsychotics compared with haloperidol. The evidence was consistent but imprecise.

\section{Other Comparisons}

We found no studies of delirium treatment evaluating neurologic effects in critically ill patients with delirium for the following comparisons:

- Second-generation antipsychotics versus second-generation antipsychotics

- Haloperidol versus other therapies

- Second-generation antipsychotics versus other therapies 


\section{Other Adverse Effects - Intensive Care Unit}

We found no studies enrolling critically ill patients with delirium that compared an antipsychotic with a placebo, another antipsychotic, or another treatment and evaluated the following adverse events:

- Weight gain

- Changes in appetite

- Hypersensitivity reactions

- Inappropriate continuation of antipsychotics

- Swallowing difficulties

- Aspiration pneumonia

\section{Key Question 2d. Benefits and Harms of Antipsychotics To Treat Delirium in Patients in a Post-Acute Care Facility}

We found no studies evaluating antipsychotics for the treatment of delirium in patients in a post-acute care facility.

\section{Key Question 2e. Benefits and Harms of Antipsychotics To Treat Delirium in Patients in Palliative or Hospice Care}

\section{Key Points - Palliative or Hospice Care}

\section{Delirium Severity}

- In patients in palliative or hospice care, there was less improvement with haloperidol than placebo in delirium severity by day three using two severity measures (mean betweengroup difference for the delirium symptom score, 0.2; 95\% CI, 0.1 to 0.4 , and for the Memorial Delirium Assessment Scale, 0.8; 95\% CI, -0.03 to 1.5), but the magnitude of this difference may not be clinically important. (SOE: Low)

- In patients in palliative or hospice care, there was less improvement with risperidone than placebo in delirium severity by day three using two severity measures (mean betweengroup difference for the delirium symptom score, 0.5; 95\% CI, 0.1 to 0.9, and for the Memorial Delirium Assessment Scale, 1.0; 95\% CI, 0.2 to 1.8), but the magnitude of this difference may not be clinically important. (SOE: Low)

- We were unable to draw a conclusion regarding the effects of antipsychotics on delirium severity in patients in palliative or hospice care for the following comparisons:

o Haloperidol versus second-generation antipsychotics (insufficient evidence)

o Second-generation antipsychotics versus second-generation antipsychotics (no studies)

o Haloperidol versus other therapies (no studies)

o Second-generation antipsychotics versus other therapies (no studies)

\section{Caregiver Burden/Strain and Sedation}

- We found no studies evaluating caregiver burden/strain or sedation in patients in palliative or hospice care with delirium that compared an antipsychotic with a placebo, another antipsychotic, or another treatment. 


\section{Intermediate Outcomes}

\section{Delirium Severity - Palliative or Hospice Care}

\section{Haloperidol Versus Placebo}

One three-arm RCT, with low risk of bias, enrolled patients receiving palliative care. ${ }^{48}$ This RCT reported less improvement in delirium severity by day three for haloperidol compared with placebo when evaluated using a delirium symptom score. However, this difference is of uncertain clinical importance. When severity was evaluated using the Memorial Delirium Assessment Scale, this RCT reported no statistically significant difference in improvement in delirium severity by day three. We concluded that haloperidol compared to placebo has no clinically important effect on delirium severity in palliative care patients. (SOE in patients in palliative or hospice care: Low)

\section{Second-Generation Antipsychotics Versus Placebo}

One three-arm RCT, with low risk of bias, enrolled patients receiving palliative care. ${ }^{48}$ This RCT reported less improvement in delirium severity by day three for risperidone compared with placebo when evaluated using both a delirium symptom score and the Memorial Delirium Assessment Scale. ${ }^{48}$ (SOE in patients in palliative or hospice care: Low)

\section{Haloperidol Versus Second-Generation Antipsychotics}

One RCT, with high risk of bias primarily owing to a lack of blinding and missing outcome data, compared olanzapine with haloperidol in patients receiving palliative care. ${ }^{61}$ This RCT reported a lower delirium severity score at day seven, as evaluated using the Delirium Rating Scale, for olanzapine compared with haloperidol but the between-group difference in reduction of delirium severity from baseline during the 7 day followup was not statistically significant.. This RCT also reported no difference in delirium severity score at day seven or in reduction of delirium severity from baseline during the 7 day followup, as evaluated using the Clinical Global Impression scale for olanzapine compared with haloperidol. ${ }^{61}$ We were unable to draw conclusions from this single study. (SOE in patients in palliative or hospice care: Insufficient)

\section{Other Comparisons}

We found no delirium treatment trials evaluating delirium severity in patients in palliative or hospice care with delirium for the following comparisons:

- Second-generation antipsychotics versus second-generation antipsychotics

- Haloperidol versus other therapies

- Second-generation antipsychotics versus other therapies

\section{Strength of Evidence}

Table 24 summarizes the strength of evidence findings for antipsychotics in terms of delirium severity in patients in palliative or hospice care. 
Table 24. Strength of evidence domains for trials evaluating antipsychotics for the treatment of delirium in terms of delirium severity in patients in palliative or hospice care

\begin{tabular}{|c|c|c|c|c|c|c|c|c|}
\hline Comparison & $\begin{array}{c}\text { Number Of } \\
\text { Trials (N) }\end{array}$ & $\begin{array}{c}\text { Study } \\
\text { Limitations }\end{array}$ & Directness & Consistency & Precision & $\begin{array}{c}\text { Reporting } \\
\text { Bias }\end{array}$ & $\begin{array}{c}\text { Strength Of } \\
\text { Evidence }\end{array}$ & Summary \\
\hline $\begin{array}{l}\text { Haloperidol vs. } \\
\text { placebo }\end{array}$ & $1^{48}(249)$ & Low & Direct & $\begin{array}{l}\text { Consistency } \\
\text { unknown } \\
\text { (single study) }\end{array}$ & Imprecise & Undetected & Low $^{*}$ & $\begin{array}{l}\text { Haloperidol had less improvement } \\
\text { in delirium severity by day } 3 \text { using } \\
\text { two severity measures (mean } \\
\text { between group difference for } \\
\text { delirium symptom score: } 0.2 ; 95 \% \\
\mathrm{Cl}, 0.1 \text { to } 0.4 \text {, and for Memorial } \\
\text { Delirium Assessment Scale: } 0.8 \text {; } \\
95 \% \mathrm{Cl},-0.03 \text { to } 1.5 \text { ), but the } \\
\text { magnitude of this difference may } \\
\text { not be clinically important. }\end{array}$ \\
\hline $\begin{array}{l}\text { Second- } \\
\text { generation } \\
\text { antipsychotics } \\
\text { vs. placebo }\end{array}$ & $1^{48}(249)$ & Low & Direct & $\begin{array}{l}\text { Consistency } \\
\text { unknown } \\
\text { (single study) }\end{array}$ & Imprecise & Undetected & Low $^{*}$ & $\begin{array}{l}\text { Risperidone had less improvement } \\
\text { in delirium severity by day } 3 \text { using } \\
\text { two severity measures (mean } \\
\text { between group difference for } \\
\text { delirium symptom score: } 0.5 ; 95 \% \\
\mathrm{Cl}, 0.1 \text { to } 0.9 \text {, and for Memorial } \\
\text { Delirium Assessment Scale: } 1.0 ; \\
95 \% \mathrm{Cl}, 0.2 \text { to } 1.8 \text { ), but the } \\
\text { magnitude of this difference may } \\
\text { not be clinically important. }\end{array}$ \\
\hline $\begin{array}{l}\text { Haloperidol vs. } \\
\text { second- } \\
\text { generation } \\
\text { antipsychotics }\end{array}$ & $1^{61}(30)$ & High & Direct & $\begin{array}{l}\text { Consistency } \\
\text { unknown } \\
\text { (single study) }\end{array}$ & Imprecise & Undetected & Insufficient & $\begin{array}{l}\text { We were unable to draw a } \\
\text { conclusion. }\end{array}$ \\
\hline $\begin{array}{l}\text { Second- } \\
\text { generation } \\
\text { antipsychotics } \\
\text { vs. second- } \\
\text { generation } \\
\text { antipsychotics }\end{array}$ & No trials & & & & & & & \\
\hline $\begin{array}{l}\text { Haloperidol vs. } \\
\text { other therapies }\end{array}$ & No trials & & & & & & & \\
\hline $\begin{array}{l}\text { Second- } \\
\text { generation } \\
\text { antipsychotics } \\
\text { vs. other } \\
\text { therapies }\end{array}$ & No trials & & & & & & & \\
\hline
\end{tabular}

* Rating based on a single multi-site study in Australia with imprecise results; additional trials are needed to determine if results are stable and generalizable.

$\mathrm{CI}=$ confidence interval 


\section{Use of Rescue Therapy - Palliative or Hospice Care}

\section{Haloperidol and Second-Generation Antipsychotics Versus Placebo}

The findings presented in the "Use of Rescue Therapy - Overall” results section are wholly represented by this subgroup. Please refer to the "Use of Rescue Therapy - Overall” results section.

\section{Haloperidol Versus Second-Generation Antipsychotics}

One RCT, with high risk of bias primarily owing to a lack of blinding and missing outcome data, compared olanzapine with haloperidol in patients receiving palliative care. ${ }^{61}$ This RCT reported no statistically significant difference in the use of rescue therapy, defined as the use of as needed midazolam.

\section{Other Comparisons}

We found no delirium treatment trials evaluating the use of rescue therapy in patients in palliative or hospice care with delirium for the following comparisons:

- Haloperidol versus placebo

- Second-generation antipsychotics versus placebo

- Second-generation antipsychotics versus second-generation antipsychotics

- Haloperidol versus other therapies

- Second-generation antipsychotics versus other therapies

\section{Other Intermediate Outcomes - Palliative or Hospice Care}

We found no trials in patients in palliative or hospice care with delirium that compared an antipsychotic with a placebo, another antipsychotic, or another treatment and evaluated the following intermediate outcomes:

- Delirium incidence

- Short-term delirium symptoms

- Delirium- and coma-free days alive

- Duration of delirium

- Patient distress

- Use of physical restraint

\section{Final Health or Patient-Centered Outcomes}

\section{Mortality - Palliative or Hospice Care}

\section{Haloperidol Versus Placebo}

One three-arm RCT, with low risk of bias and enrolling patients receiving palliative care, compared haloperidol with placebo. ${ }^{48}$ In an analysis of mortality associated with the 72-hour treatment duration, this RCT reported no statistically significant effect when comparing haloperidol with placebo. However, the RCT also performed a time-to-event analysis showing decreased survival for the haloperidol group compared with the placebo group. It is unclear how 
much of the longer-term mortality risk is attributable to haloperidol use during the relatively brief 72-hour study period.

\section{Second-Generation Antipsychotics Versus Placebo}

One three-arm RCT, with low risk of bias and enrolling patients receiving palliative care, compared risperidone with placebo. ${ }^{48}$ In an analysis of mortality associated with the 72-hour treatment duration, the RCT reported no statistically significant effect when comparing risperidone with placebo. The RCT performed a time-to-event analysis that demonstrated a nonstatistically significant decrease in survival for the risperidone group compared with the placebo group.

\section{Haloperidol Versus Second-Generation Antipsychotics}

One three-arm RCT, with low risk of bias and enrolling patients receiving palliative care, compared haloperidol with second-generation antipsychotics. ${ }^{48}$ In an analysis of mortality associated with the 72-hour treatment duration, the RCT reported no statistically significant effect when comparing risperidone with haloperidol.

\section{Other Comparisons}

We found no delirium treatment trials evaluating mortality in patients in palliative or hospice care with delirium for the following comparisons:

- Second-generation antipsychotics versus second-generation antipsychotics

- Haloperidol versus other therapies

- Second-generation antipsychotics versus other therapies

\section{Other Final Health or Patient-Centered Outcomes - Palliative or Hospice Care}

We found no trials enrolling patients in palliative or hospice care with delirium that compared an antipsychotic with a placebo, another antipsychotic, or another treatment and evaluated the following final health or patient-centered outcomes:

- Quality of life

- Cognitive functioning

- Institutionalization

- Caregiver burden/strain

- Falls

- Memory of patient distress

\section{Resource Utilization Outcomes - Palliative or Hospice Care}

We found no trials enrolling patients in palliative or hospice care with delirium that compared an antipsychotic with a placebo, another antipsychotic, or another treatment and evaluated the following resource utilization outcomes:

- Readmission to hospital

- Readmission to intensive care unit

- Length of stay in hospital

- Length of stay in intensive care unit

- Length of stay in skilled nursing facility 
- Patient safety attendant use

- Hospice enrollment

\section{Adverse Effects}

\section{Neurologic Effects - Palliative or Hospice Care}

\section{Haloperidol Versus Placebo}

One three-arm RCT, with low risk of bias and enrolling patients receiving palliative care, compared haloperidol with placebo. ${ }^{48}$ This RCT reported a statistically significant difference of more extrapyramidal symptoms, evaluated using the Extra Pyramidal Symptom Rating Scale, for haloperidol compared with placebo.

We did not find any observational studies or non-randomized trials that compared haloperidol with placebo and reported on neurologic effects.

\section{Second-Generation Antipsychotics Versus Placebo}

One three-arm RCT, with low risk of bias and enrolling patients receiving palliative care, compared risperidone with placebo. ${ }^{48}$ This RCT reported a statistically significant difference of more extrapyramidal symptoms, evaluated using the Extra Pyramidal Symptom Rating Scale, for risperidone compared with placebo.

We did not find any observational studies or non-randomized trials that compared a secondgeneration antipsychotic with placebo and reported on neurologic effects.

\section{Haloperidol Versus Second-Generation Antipsychotics}

One RCT, with high risk of bias, primarily owing to a lack of blinding and missing outcome data, and enrolling patients receiving palliative care, compared olanzapine with haloperidol. ${ }^{61}$ This RCT reported no extrapyramidal symptoms in either group.

We did not find any observational studies or non-randomized trials that compared haloperidol with a second-generation antipsychotic and reported on neurologic effects.

\section{Other Comparisons}

We found no studies of delirium treatment evaluating neurologic effects in patients receiving palliative care with delirium for the following comparisons:

- Second-generation antipsychotics versus second-generation antipsychotics

- Haloperidol versus other therapies

- Second-generation antipsychotics versus other therapies

\section{Other Adverse Effects - Palliative or Hospice Care}

We found no studies enrolling patients in palliative or hospice care with delirium that compared an antipsychotic with a placebo, another antipsychotic, or another treatment and evaluated the following adverse events:

- Sedation

- Weight gain

- Changes in appetite

- Cardiac effects 
- Hypersensitivity reactions

- Inappropriate continuation of antipsychotics

- Swallowing difficulties

- Aspiration pneumonia

\section{Key Question 2f. Benefits and Harms of Antipsychotics To Treat Delirium in Patients in Postoperative Care}

\section{Key Points - Postoperative Care}

\section{Length of Stay in Hospital}

- We were unable to draw a conclusion regarding the effects of antipsychotics on length of stay in hospital for patients in postoperative care for the following comparisons:

o Haloperidol versus placebo (no trials)

o Second-generation antipsychotics versus placebo (no trials)

o Haloperidol versus second-generation antipsychotics (no trials)

o Second-generation antipsychotics versus second-generation antipsychotics (no trials)

o Haloperidol versus other therapies (insufficient evidence)

o Second-generation antipsychotics versus other therapies (no trials)

\section{Delirium Severity, Cognitive Functioning, Inappropriate Continuation of Antipsychotics}

- We found no trials evaluating delirium severity, cognitive functioning, or inappropriate continuation of antipsychotics in postoperative patients with delirium that compared an antipsychotic with a placebo, another antipsychotic, or another treatment.

\section{Intermediate Outcomes}

\section{Duration of Delirium - Postoperative}

\section{Haloperidol Versus Other Therapies}

The findings presented in the "Duration of Delirium - Overall" results section are wholly represented by this subgroup. Please refer to the "Duration of Delirium - Overall” results section.

\section{Other Comparisons}

We found no delirium treatment trials evaluating delirium duration in postoperative patients with delirium for the following comparisons:

- Haloperidol versus placebo

- Second-generation antipsychotics versus placebo

- Haloperidol versus second-generation antipsychotics

- Second-generation antipsychotics versus second-generation antipsychotics

- Second-generation antipsychotics versus other therapies 


\section{Other Intermediate Outcomes - Postoperative}

We found no trials enrolling postoperative patients with delirium that compared an antipsychotic with a placebo, another antipsychotic, or another treatment and evaluated the following intermediate outcomes:

- Delirium incidence

- Short-term delirium symptoms

- Delirium severity

- Delirium- and coma-free days alive

- Patient distress

- Use of rescue therapy

- Use of physical restraint

\section{Final Health or Patient-Centered Outcomes}

\section{Mortality - Postoperative}

\section{Haloperidol Versus Other Therapies}

One RCT, with unclear risk of bias and enrolling 53 post-cardiac surgery patients with hyperactive delirium, compared haloperidol with morphine. This trial reported no statistically significant between-group difference (RR, 2.08; 95\% CI, 0.20 to 21.55). ${ }^{49}$

\section{Other Comparisons}

We found no delirium treatment trials evaluating mortality in postoperative patients with delirium for the following comparisons:

- Haloperidol versus placebo

- Second-generation antipsychotics versus placebo

- Haloperidol versus second-generation antipsychotics

- Second-generation antipsychotics versus second-generation antipsychotics

- Second-generation antipsychotics versus other therapies.

\section{Resource Utilization Outcomes}

\section{Readmission to Intensive Care Unit - Postoperative}

\section{Haloperidol Versus Other Therapies}

The findings presented in the "Readmission to the Intensive Care Unit - Overall" results section are wholly represented by this subgroup. Please refer to the "Readmission to the Intensive Care Unit - Overall” results section.

\section{Other Comparisons}

We found no delirium treatment trials evaluating readmission to intensive care unit in postoperative patients with delirium for the following comparisons:

- Haloperidol versus placebo

- Second-generation antipsychotics versus placebo

- Haloperidol versus second-generation antipsychotics 
- Second-generation antipsychotics versus second-generation antipsychotics

- Second-generation antipsychotics versus other therapies

\section{Length of Stay in Hospital - Postoperative}

\section{Haloperidol Versus Other Therapies}

The findings presented in the "Length of Stay in Hospital - Overall" results section are wholly represented by this subgroup. Please refer to the "Length of Stay in Hospital - Overall" results section. (SOE in patients in postoperative care: Insufficient)

\section{Other Comparisons}

We found no delirium treatment trials evaluating length of stay in hospital in postoperative patients with delirium for the following comparisons:

- Haloperidol versus placebo

- Second-generation antipsychotics versus placebo

- Haloperidol versus second-generation antipsychotics

- Second-generation antipsychotics versus second-generation antipsychotics

- Second-generation antipsychotics versus other therapies

\section{Strength of Evidence}

Table 25 summarizes the strength of evidence findings for length of stay in hospital in patients in postoperative care. 
Table 25. Strength of evidence domains for trials evaluating antipsychotics for the treatment of delirium in terms of length of stay in hospital for postoperative patients

\begin{tabular}{|c|c|c|c|c|c|c|c|c|}
\hline Comparison & $\begin{array}{c}\text { Number Of } \\
\text { Trials (N) }\end{array}$ & $\begin{array}{c}\text { Study } \\
\text { Limitations }\end{array}$ & Directness & Consistency & Precision & $\begin{array}{c}\text { Reporting } \\
\text { Bias }\end{array}$ & $\begin{array}{l}\text { Strength of } \\
\text { Evidence }\end{array}$ & Summary \\
\hline $\begin{array}{l}\text { Haloperidol vs. } \\
\text { placebo }\end{array}$ & No trials & & & & & & & \\
\hline $\begin{array}{l}\text { Second- } \\
\text { generation } \\
\text { antipsychotics } \\
\text { vs. placebo }\end{array}$ & No trials & & & & & & & \\
\hline $\begin{array}{l}\text { Haloperidol vs. } \\
\text { second- } \\
\text { generation } \\
\text { antipsychotics }\end{array}$ & No trials & & & & & & & \\
\hline $\begin{array}{l}\text { Second- } \\
\text { generation } \\
\text { antipsychotics } \\
\text { vs. second- } \\
\text { generation } \\
\text { antipsychotics }\end{array}$ & No trials & & & & & & & \\
\hline $\begin{array}{l}\text { Haloperidol vs. } \\
\text { other therapies }\end{array}$ & $1^{49}(53)$ & High & Indirect & $\begin{array}{l}\text { Consistency } \\
\text { unknown } \\
\text { (single study) }\end{array}$ & Imprecise & Undetected & Insufficient & $\begin{array}{l}\text { We were unable to draw a } \\
\text { conclusion. }\end{array}$ \\
\hline $\begin{array}{l}\text { Second- } \\
\text { generation } \\
\text { antipsychotics } \\
\text { vs. other } \\
\text { therapies } \\
\end{array}$ & No trials & & & & & & & \\
\hline
\end{tabular}




\section{Length of Stay in Intensive Care Unit - Postoperative}

\section{Haloperidol Versus Other Therapies}

The findings presented in the "Length of Stay in Intensive Care Unit - Overall” results section are wholly represented by this subgroup. Please refer to the "Length of Stay in Intensive Care Unit - Overall” results section.

\section{Other Comparisons}

We found no delirium treatment trials evaluating length of stay in the intensive care unit in postoperative patients with delirium for the following comparisons:

- Haloperidol versus placebo

- Second-generation antipsychotics versus placebo

- Haloperidol versus second-generation antipsychotics

- Second-generation antipsychotics versus second-generation antipsychotics

- Second-generation antipsychotics versus other therapies

\section{Other Resource Utilization Outcomes - Postoperative}

We found no trials enrolling postoperative patients with delirium that compared an antipsychotic with a placebo, another antipsychotic, or another treatment and evaluated the following resource utilization outcomes:

- Readmission to hospital

- Length of stay in skilled nursing facility

- Patient safety attendant use

- Hospice enrollment

\section{Other Final Health or Patient-Centered Outcomes - Postoperative}

We found no trials enrolling postoperative patients with delirium that compared an antipsychotic with a placebo, another antipsychotic, or another treatment and evaluated the following final health or patient-centered outcomes:

- Quality of life

- Cognitive functioning

- Institutionalization

- Caregiver burden/strain

- Falls

- Memory of patient distress

\section{Adverse Effects - Postoperative}

We found no studies enrolling postoperative patients with delirium that compared an antipsychotic with a placebo, another antipsychotic, or another treatment and evaluated the following adverse events:

- Sedation

- Weight gain

- Changes in appetite

- Cardiac effects 
- Neurologic effects

- Hypersensitivity reactions

- Inappropriate continuation of antipsychotics

- Swallowing difficulties

- Aspiration pneumonia 


\section{Discussion}

\section{Key Findings and Strength of Evidence}

We identified 57 studies evaluating the effectiveness and harms of antipsychotics for the prevention (15 studies) and treatment (44 studies) ( 2 studies were classified as both prevention and treatment) of delirium. The trials for the prevention of delirium evaluated delirium incidence, length of stay in hospital, sedation, and severity, with most of the studies focused on postoperative and/or intensive care unit-based populations. The treatment trials primarily evaluated length of stay in hospital and sedation effects, with most of the studies focused on inpatients, particularly those with critical illness.

In the prevention of delirium across all populations, trials of haloperidol versus placebo demonstrated no effect on delirium incidence or length of stay in hospital (high strength of evidence); there was insufficient evidence to determine the effect of haloperidol versus placebo on delirium severity or sedation. Our meta-analysis found a statistically significant difference in the relative risk of delirium favoring second-generation antipsychotics versus placebo for the prevention of delirium. However, there was no effect on the length of stay in hospital (low strength of evidence) and insufficient evidence to determine the impact on the severity of delirium for second-generation antipsychotics compared with placebo. We were unable to draw conclusions for any type of drug-to-drug comparisons between second-generation antipsychotics or comparisons with any other types of therapies (i.e., other than antipsychotics) owing to the absence of studies or insufficient evidence.

In the treatment of delirium, trials using haloperidol or second-generation antipsychotics demonstrated no effect on length of stay in hospital (moderate strength of evidence) or sedation (low strength of evidence for haloperidol and moderate strength of evidence for secondgeneration antipsychotics) compared with placebo. The comparison of haloperidol with secondgeneration antipsychotics demonstrated no statistically significant differences for cognitive functioning (low strength of evidence), delirium severity, length of stay in hospital, and sedation (all moderate strength of evidence). We were unable to draw conclusions for any type of drug-todrug comparisons between second-generation antipsychotics or comparisons with any other types of therapies (i.e., other than antipsychotics) owing to the absence of studies or insufficient evidence.

We specifically evaluated cardiac and neurological harms. Most or all of the studies assessing cardiac effects included critically ill patients who may be at a higher risk of cardiac events compared with other patient populations. In all randomized controlled trials (RCTs) and observational studies evaluating haloperidol versus placebo, second-generation antipsychotics versus placebo, haloperidol versus second-generation antipsychotics, and second-generation antipsychotics versus second-generation antipsychotics, there were no statistically significant differences in the occurrence of any of type of cardiac effects reported; however, we cannot exclude a potentially important increase in cardiac events for antipsychotics compared to placebo, particularly for QT interval prolongation for second-generation antipsychotics compared to haloperidol or placebo.

Studies that reported neurological harms included critically ill patients who may be at a higher risk of neurological events compared with other patient populations. Monitoring for neuroleptic malignant syndrome and various manifestations of extrapyramidal symptoms were the most commonly reported neurological effects. Symptom reporting within the extrapyramidal designation was heterogeneous and likely represented a wide variety of measurement methods. 
Furthermore, studies included a variety of doses, frequencies and routes of antipsychotics, potentially obscuring the true frequencies of adverse neurologic side effects at the higher antipsychotic exposures. A single RCT evaluating patients receiving palliative care demonstrated a statistically significant increase in extrapyramidal symptoms for both haloperidol compared with placebo and for second-generation antipsychotics compared with placebo. Otherwise, across the larger body of evidence in all other patient populations, no study reported a statistically significant increase in any neurological effect for haloperidol compared with placebo, or secondgeneration antipsychotics compared with placebo, haloperidol compared with second-generation antipsychotics and second-generation antipsychotics compared with second-generation antipsychotics.

\section{Findings in Relationship to What Is Already Known}

Several systematic reviews have examined the risks and benefits of antipsychotic treatments for delirium but none of these existing reviews had the same scope as ours. For instance, we considered prevention and treatment studies separately and examined the evidence for specific subpopulations, in addition to looking at the data overall.

Kishi et al., reviewed antipsychotics for the treatment, but not prevention, of delirium in adult patients, with no subgroup analysis in older adults. ${ }^{13}$ A major conclusion of this review was that antipsychotics are superior to placebo/usual care. That finding contradicts our findings and those of other more recent systematic reviews and guidelines that have concluded that there is no evidence that antipsychotics improve the outcomes among hospitalized adults with delirium. ${ }^{12,90}$ Although we included all of the published studies reviewed by Kishi et al., recently completed trials were also added to our synthesis and contributed to our conclusions.

Neufeld et al., conducted a systematic review of antipsychotics as part of an American Geriatrics Society clinical practice guideline. ${ }^{12}$ This systematic review focused on antipsychotics for delirium prevention or treatment in adult surgical and medical inpatients, including the critically ill. The review combined populations and did not examine the results for subpopulations. ${ }^{12}$ The review concluded "current evidence does not support the use of antipsychotics for prevention or treatment of delirium.” The search for this systematic review ended in 2013; consequently, it excluded important, newer studies included in this report.

The Society for Critical Care Medicine recently published clinical practice guidelines for the prevention and management of pain, agitation/sedation, delirium, immobility, and sleep disruption in adult patients in the intensive care unit. ${ }^{91}$ Notably, this guideline focused on critically ill patients only. Also a systematic review was not conducted to support the guideline, in that there was no duplicate, independent process for the screening and data abstraction. This guideline suggested not using haloperidol or a second-generation antipsychotic to prevent or treat delirium in critically ill adults (conditional recommendation, very low to low quality of evidence).

A recently published Cochrane systematic review included RCTs evaluating pharmacological or non-pharmacological interventions for preventing delirium in critically ill patients. ${ }^{92}$ In terms of antipsychotics, this review reported on only two RCTs comparing haloperidol with placebo, with no reporting on any trials of second-generation antipsychotics. ${ }^{42,} 45$ Both of these RCTs are included in our review. With respect to the comparison of haloperidol with placebo, the conclusions from this Cochrane review ("There is probably little or no difference between haloperidol and placebo for preventing ICU delirium”) are consistent with the findings in our review. 
Another Cochrane review evaluating pharmacologic and non-pharmacologic interventions in non-intensive care unit hospitalized adults concluded, from a pre-planned subgroup analysis of surgical patients, that there was no evidence for the effectiveness of first-generation antipsychotics, such as haloperidol. ${ }^{90}$ However, delirium incidence was reported to be lower for postoperative patients treated with a second-generation antipsychotic (olanzapine) compared with placebo and adjudicated to be derived from moderate-quality evidence. Our review came to the same conclusion; however we also concluded that there were no between-group differences for second-generation antipsychotics versus placebo in length of stay in hospital (low strength of evidence) and insufficient evidence to understand the impact on severity of delirium symptoms for both the postoperative subgroup and across all populations.

\section{Applicability}

The greatest challenge to the applicability of this body of evidence is related to the populations and outcomes studied. In terms of study population, existing trials were often conducted in medical and surgical critically ill patients. Hence, the overall results of this report may not be directly applicable to other populations, including postoperative patients, older inpatients, and patients with dementia.

Critically ill patients may have differing pathophysiological etiologies of delirium compared with other populations, as well as more severe physiological and metabolic derangements. They also routinely receive relatively high doses of deliriogenic medications, such as sedatives (e.g., benzodiazepines and propofol). For similar reasons, critically ill patients may be more susceptible to the potentially harmful effects of antipsychotics. Hence, any benefit and risk of antipsychotics for prevention or treatment of delirium within the context of critical illness may not be generalizable to other populations.

\section{Implications for Clinical and Policy Decisionmaking}

There is no Food and Drug Administration-approved medication for the prevention or treatment of delirium. Our findings do not support the use of antipsychotics for the routine treatment of delirium. While the use of second-generation antipsychotic medications may be associated with decreased incidence of postoperative delirium, this review did not identify improved outcomes associated with the use of these agents.

Notably, across a range of cardiac and neurological effects evaluated, there was little evidence of increased serious harms related to antipsychotics compared with placebo or with other antipsychotics (i.e., drug-to-drug comparisons), even in critically ill patients who may be at greater risk of harmful side effects. However, across different types of cardiac events, the absolute number tended to be greater for antipsychotics compared to placebo, which may be clinically relevant. Moreover, inappropriate continuation of antipsychotics and associated longterm harms from inadvertent prolonged use were not evaluated in any studies that met our inclusion criteria. Given that observational studies of routine clinical practice demonstrate frequent inappropriate continuation of antipsychotics, this is an important issue. ${ }^{93-95}$

Given the lack of effectiveness of antipsychotics for the treatment of delirium, particularly in critically ill adults, and the lack of improved outcomes associated with the use of secondgeneration antipsychotics in preventing postoperative delirium, the use of other nonpharmacological therapies with low cost, burden, and harms warrants consideration for clinical practice as recommended by existing clinical practice guidelines. ${ }^{91,96}$ 


\section{Limitations of the Systematic Review Process}

The systematic review process, despite its rigor, had some limitations: our review may have missed studies that are currently in progress as well as studies not in the peer-reviewed literature. Owing to heterogeneity in the populations, interventions, and outcome measures, we were unable to conduct a network meta-analysis. Such an analysis could potentially provide important drugto-drug comparison information that the current trials do not provide.

\section{Limitations of the Evidence Base}

There was insufficient or no evidence for many comparisons and outcomes owing to the paucity of studies. The body of evidence with the most studies and the highest strength of evidence (i.e., moderate) was generally from studies of critically ill adults. There was insufficient evidence comparing haloperidol with second-generation antipsychotics and for drug-to-drug comparisons within the class of second-generation antipsychotics. Inadequate reporting meant that the risk of bias assessment of RCTs was frequently unclear, especially regarding sequence generation and allocation concealment.

Moreover, there was frequent unclear risk of bias related to missing outcome data and selective outcome reporting. There was inconsistency in the use of measurement instruments and approaches to statistical analysis and reporting, even in evaluating the same outcome domain, such as delirium severity.

Many studies were underpowered, with short duration. Many studies were of insufficient duration to adequately assess longer-term clinical outcomes, particularly related to cognitive impairment—a well-known sequela of delirium.

Finally, study designs were fairly heterogeneous, using different dosing routes, and a range of doses and frequencies of different antipsychotics. Combining heterogeneous treatment and prevention approaches may bias the findings toward the null hypothesis. So, too, combining a range of dosing exposures may obscure adverse outcomes associated with higher doses of medications.

\section{Research Recommendations}

Studies evaluating pharmacologic prevention and treatment strategies should evaluate patient groups that are anticipated to have similar delirium risk factor(s) and associated pathophysiology, including anticholinergic agents and other medications taken before delirium occurs, given that these factors may affect response to therapy. With the exception of critically ill patients, there were either no RCTs or insufficient evidence from existing studies that evaluated the effect of antipsychotics on treatment outcomes in all patient subpopulations. Research that is focused on more homogeneous patient groups within each subpopulation is needed. This also applies to postoperative patient populations, where larger, well-controlled trials of secondgeneration antipsychotics in the prevention of delirium are needed to clarify whether there is any beneficial role for the perioperative setting.

The outcomes of currently published RCTs on the use of antipsychotics on the treatment and prevention of delirium focus predominantly on the length of stay in hospital and sedation. Other critical outcomes, including delirium severity, were reported in a minority of the studies and, often, the measures used were heterogeneous, making it difficult to synthesize results and conduct meta-analyses. For example, median lengths of stay data were reported in many studies. Since such data are often skewed, length of stay measures could not be pooled. Important 
outcomes such as impact on cognitive functioning, caregiver burden, and inappropriate continuation of medications were absent in the current literature.

Heterogeneity of outcome domains and measurement instruments emphasize the need for greater standardization. Such standardization would assist with comparison, synthesis, and metaanalysis of studies and would reduce omission of critical outcomes in future trials (which would also reduce research waste). In particular, careful identification of the outcomes of greatest importance to clinicians, patients/caregivers, and researchers would advance future research. The field would benefit from the development of standardized, clinically meaningful measures of the following outcomes: patient agitation and distress, subsequent memories of delirium, caregiver burden and distress, inappropriate continuation of antipsychotics, and long-term cognitive and functional outcomes. Research currently underway to define core outcomes for delirium, core measurement instruments, and crosswalks to directly compare different delirium severity measures are important advances for the field. ${ }^{97}$ The National Institute on Aging-funded delirium research network, known as the Network for Investigation of Delirium: Unifying Scientists (NIDUS; https://deliriumnetwork.org/), aims to help facilitate some of these research recommendations. ${ }^{98}$

Delirium assessment methods for use in both prevention and treatment trials require ongoing refinement and scrutiny. A recent multinational interdisciplinary perspective calls for the development and validation of objective tools to screen for delirium and diagnose the condition in critically ill patients by including the electroencephalogram, or computer-based applications. The delirium field would benefit from better phenotyping of delirium subtypes, through adoption and development of more detailed standardized measurement tools. The NIDUS group is currently developing web-based resources for delirium measurement instruments and is building infrastructure related to delirium measurement methods.

\section{(https://deliriumnetwork.org/measurement/)}

Given the lack of evidence to support antipsychotics for treatment of delirium, and weak evidence for limited benefits of the prevention of postoperative delirium, both pharmacological and non-pharmacological therapies should continue to be evaluated in RCTs with well-powered designs that include the study of more homogenous populations of patients. A striking finding resulting from this review was the lack of investigation of many important patient and care-giver centered outcomes in the study of delirium prevention and treatment. Much more research is needed to study questions such as the comparison between the pharmacologic and nonpharmacologic approaches, quality of life outcomes and best treatment approaches in populations of patients such as those with pre-existing dementia. Studies answering these questions will have important implications for policy and treatment recommendations for patients within our healthcare system.

\section{Conclusions}

Haloperidol or second-generation antipsychotics, compared to placebo, used for the prevention or treatment of delirium did not improve clinically important outcomes. In a subgroup of postoperative patients, second-generation antipsychotics may decrease delirium incidence but this evidence is limited and requires more study. We did not detect neurological harms associated with haloperidol or second-generation antipsychotics used for the prevention or treatment of delirium, but cardiac effects tended to occur more frequently in antipsychotics compared with placebo. 


\section{References}

1. American Psychiatric Association. DSM-5 Task Force. Diagnostic and Statistical Manual of Mental Disorders. DSM-5. 5th ed. Arlington, VA: American Psychiatric Association; 2013.

2. Zaal IJ, Devlin JW, Peelen LM, et al. A systematic review of risk factors for delirium in the ICU. Crit Care Med. 2015 Jan;43(1):40-7. doi: 10.1097/ccm.0000000000000625. PMID: 25251759.

3. McNicoll L, Pisani MA, Zhang Y, et al. Delirium in the intensive care unit: occurrence and clinical course in older patients. J Am Geriatr Soc. 2003 May;51(5):591-8. PMID: 12752832.

4. Pisani MA, Murphy TE, Araujo KL, et al. Benzodiazepine and opioid use and the duration of intensive care unit delirium in an older population. Crit Care Med. 2009 Jan;37(1):17783. doi: 10.1097/CCM.0b013e318192fcf9. PMID: 19050611.

5. Leslie DL, Marcantonio ER, Zhang Y, et al. Oneyear health care costs associated with delirium in the elderly population. Arch Intern Med. 2008 Jan 14;168(1):27-32. doi:

10.1001/archinternmed.2007.4. PMID: 18195192.

6. Pandharipande PP, Girard TD, Jackson JC, et al. Long-term cognitive impairment after critical illness. N Engl J Med. 2013 Oct 3;369(14):130616. doi: 10.1056/NEJMoa1301372. PMID: 24088092.

7. Sakusic A, O'Horo JC, Dziadzko M, et al. Potentially Modifiable Risk Factors for LongTerm Cognitive Impairment After Critical Illness: A Systematic Review. Mayo Clin Proc. 2018 Jan;93(1):68-82. doi: 10.1016/j.mayocp.2017.11.005. PMID: 29304923.

8. Davis DH, Muniz Terrera G, Keage H, et al. Delirium is a strong risk factor for dementia in the oldest-old: a population-based cohort study. Brain. 2012 Sep;135(Pt 9):2809-16. doi: 10.1093/brain/aws190. PMID: 22879644.

9. Davis DH, Muniz-Terrera G, Keage HA, et al. Association of Delirium With Cognitive Decline in Late Life: A Neuropathologic Study of 3 Population-Based Cohort Studies. JAMA Psychiatry. 2017 Mar 1;74(3):244-51. doi: 10.1001/jamapsychiatry.2016.3423. PMID: 28114436.
10. Devlin JW, Bhat S, Roberts RJ, et al. Current perceptions and practices surrounding the recognition and treatment of delirium in the intensive care unit: a survey of 250 critical care pharmacists from eight states. Ann Pharmacother. 2011 Oct;45(10):1217-29. doi: 10.1345/aph.1Q332. PMID: 21934036.

11. Seeman P. Atypical antipsychotics: mechanism of action. Can J Psychiatry. 2002 Feb;47(1):27-38. PMID: 11873706.

12. Neufeld KJ, Yue J, Robinson TN, et al. Antipsychotic Medication for Prevention and Treatment of Delirium in Hospitalized Adults: A Systematic Review and Meta-Analysis. J Am Geriatr Soc. 2016 Apr;64(4):705-14. doi: 10.1111/jgs.14076. PMID: 27004732.

13. Kishi T, Hirota T, Matsunaga S, et al. Antipsychotic medications for the treatment of delirium: a systematic review and meta-analysis of randomised controlled trials. J Neurol Neurosurg Psychiatry. 2016 Jul;87(7):767-74. doi: 10.1136/jnnp-2015-311049. PMID: 26341326.

14. Yang C, Hao Z, Tian J, et al. Does antipsychotic drug use increase the risk of long term mortality? A systematic review and meta-analysis of observational studies. Oncotarget. 2018 Mar 13;9(19):15101-10. doi: 10.18632/oncotarget.24120. PMID: 29599930.

15. Chen WY, Chen LY, Liu HC, et al. Antipsychotic medications and stroke in schizophrenia: A casecrossover study. PLoS One. 2017;12(6):e0179424. doi: 10.1371/journal.pone.0179424. PMID: 28614417.

16. Wu CS, Wu KY, Lo YR, et al. Psychotropic use and risk of stroke among patients with bipolar disorders: 10-year nationwide population based study. J Affect Disord. 2018 Jan 15;226:77-84. doi: 10.1016/j.jad.2017.09.020. PMID: 28964996.

17. Methods Guide for Effectiveness and Comparative Effectiveness Reviews. AHRQ Publication No. 10(14)-EHC063-EF. Agency for Healthcare Research and Quality. Rockville, MD. January 2014. 2014. www.effectivehealthcare.ahrq.gov.

18. Hospital Elder Life Program. Delirium Bibliography. 2018. http://www.hospitalelderlifeprogram.org/forclinicians/bibliography/. Accessed on May 29 2018. 
19. NIDUS-Network for Investigation of Delirium: Unifying Scientists. Delirium Research Catalog. 2018. https://deliriumnetwork.org/deliriumresearch-catalog/. Accessed on May 292018.

20. Mobilization Network. Publications. 2018. http://www.mobilizationnetwork.org/Network/Publications.html. Accessed on May 292018.

21. NIDUS-Network for Investigation of Delirium: Unifying Scientists. Delirium Measurement Info Cards. 2018.

https://deliriumnetwork.org/measurement/deliriu m-info-cards/. Accessed on May 282018.

22. Oh ES, Fong TG, Hshieh TT, et al. Delirium in Older Persons: Advances in Diagnosis and Treatment. JAMA. 2017 Sep 26;318(12):1161-74. doi: 10.1001/jama.2017.12067. PMID: 28973626.

23. Higgins JPT, Green S. Cochrane handbook for systemic reviews of interventions Version 5.1.0. Oxford, England: The Cochrane Collaboration; 2011. handbook.cochrane.org. Accessed on 11/4 2013.

24. Sterne JA, Hernan MA, Reeves BC, et al. ROBINS-I: a tool for assessing risk of bias in non-randomised studies of interventions. BMJ. 2016 Oct 12;355:i4919. doi: 10.1136/bmj.i4919. PMID: 27733354.

25. DerSimonian R, Laird N. Meta-analysis in clinical trials. Control Clin Trials. 1986 Sep;7(3):177-88. PMID: 3802833.

26. Cornell JE, Mulrow CD, Localio R, et al. Random-effects meta-analysis of inconsistent effects: a time for change. Ann Intern Med. 2014 Feb 18;160(4):267-70. doi: 10.7326/m13-2886. PMID: 24727843.

27. Michiels S, Piedbois P, Burdett S, et al. Metaanalysis when only the median survival times are known: a comparison with individual patient data results. Int J Technol Assess Health Care. 2005 Winter;21(1):119-25. PMID: 15736523.

28. Gross AL, Tommet D, D'Aquila M, et al. Harmonization of delirium severity instruments: a comparison of the DRS-R-98, MDAS, and CAM$\mathrm{S}$ using item response theory. BMC Med Res Methodol. 2018 Sep 10;18(1):92. doi: 10.1186/s12874-018-0552-4. PMID: 30200896.
29. Network for Investigation of Delirium: Unifying Scientists. Crosswalk for Delirium Severity Measures. 2018.

https://deliriumnetwork.org/measurement/deliriu m-severity-crosswalk-tool/. Accessed on November 292018.

30. Begg CB, Mazumdar M. Operating characteristics of a rank correlation test for publication bias. Biometrics. 1994 Dec;50(4):1088-101. PMID: 7786990.

31. Egger M, Davey Smith G, Schneider M, et al. Bias in meta-analysis detected by a simple, graphical test. BMJ. 1997 Sep 13;315(7109):62934. PMID: 9310563.

32. Owens DK, Lohr KN, Atkins D, et al. AHRQ series paper 5: grading the strength of a body of evidence when comparing medical interventions-agency for healthcare research and quality and the effective health-care program. J Clin Epidemiol. 2010 May;63(5):513-23. doi: 10.1016/j.jclinepi.2009.03.009. PMID: 19595577.

33. Abdelgalel EF. Dexmedetomidine versus haloperidol for prevention of delirium during non-invasive mechanical ventilation. Egyptian Journal of Anaesthesia. 2016;32(4):473-81. doi: 10.1016/j.egja.2016.05.008.

34. Al-Qadheeb NS, Skrobik Y, Schumaker G, et al. Preventing ICU Subsyndromal Delirium Conversion to Delirium With Low-Dose IV Haloperidol: A Double-Blind, Placebo-Controlled Pilot Study. Crit Care Med. 2016 Mar;44(3):58391. doi: 10.1097/ccm.0000000000001411. PMID: 26540397.

35. Fukata S, Kawabata Y, Fujisiro K, et al. Haloperidol prophylaxis does not prevent postoperative delirium in elderly patients: a randomized, open-label prospective trial. Surg Today. 2014 Dec;44(12):2305-13. doi: 10.1007/s00595-014-0859-7. PMID: 24532143.

36. Hakim SM, Othman AI, Naoum DO. Early treatment with risperidone for subsyndromal delirium after on-pump cardiac surgery in the elderly: a randomized trial. Anesthesiology. 2012 May;116(5):987-97. doi: 10.1097/ALN.0b013e31825153cc. PMID: 22436797.

37. Kalisvaart KJ, de Jonghe JF, Bogaards MJ, et al. Haloperidol prophylaxis for elderly hip-surgery patients at risk for delirium: a randomized placebo-controlled study. J Am Geriatr Soc. 2005 Oct;53(10):1658-66. doi: 10.1111/j.15325415.2005.53503.x. PMID: 16181163. 
38. Kaneko T, Cai J, Ishikura T, et al. Prophylactic consecutive administration of haloperidol can reduce the occurrence of postoperative delirium in gastrointestinal surgery. Yonago Acta Medica. 1999;42(3):179-84.

39. Larsen KA, Kelly SE, Stern TA, et al. Administration of olanzapine to prevent postoperative delirium in elderly jointreplacement patients: a randomized, controlled trial. Psychosomatics. 2010 Sep-Oct;51(5):40918. doi: 10.1176/appi.psy.51.5.409. PMID: 20833940.

40. Prakanrattana U, Prapaitrakool S. Efficacy of risperidone for prevention of postoperative delirium in cardiac surgery. Anaesth Intensive Care. 2007 Oct;35(5):714-9. PMID: 17933157.

41. Schrijver EJM, de Vries OJ, van de Ven PM, et al. Haloperidol versus placebo for delirium prevention in acutely hospitalised older at risk patients: a multi-centre double-blind randomised controlled clinical trial. Age Ageing. 2018 Jan 1;47(1):48-55. doi: 10.1093/ageing/afx124. PMID: 28985255.

42. van den Boogaard M, Slooter AJC, Bruggemann RJM, et al. Effect of Haloperidol on Survival Among Critically Ill Adults With a High Risk of Delirium: The REDUCE Randomized Clinical Trial. JAMA. 2018 Feb 20;319(7):680-90. doi: 10.1001/jama.2018.0160. PMID: 29466591.

43. Wang W, Li HL, Wang DX, et al. Haloperidol prophylaxis decreases delirium incidence in elderly patients after noncardiac surgery: a randomized controlled trial*. Crit Care Med. 2012 Mar;40(3):731-9. doi: 10.1097/CCM.0b013e3182376e4f. PMID: 22067628.

44. Girard TD, Pandharipande PP, Carson SS, et al. Feasibility, efficacy, and safety of antipsychotics for intensive care unit delirium: the MIND randomized, placebo-controlled trial. Crit Care Med. 2010 Feb;38(2):428-37. PMID: 20095068.

45. Page VJ, Ely EW, Gates S, et al. Effect of intravenous haloperidol on the duration of delirium and coma in critically ill patients (HopeICU): a randomised, double-blind, placebocontrolled trial. Lancet Respir Med. 2013 Sep;1(7):515-23. doi: 10.1016/s22132600(13)70166-8. PMID: 24461612.
46. Khan BA, Perkins AJ, Campbell NL, et al. Preventing Postoperative Delirium After Major Noncardiac Thoracic Surgery-A Randomized Clinical Trial. J Am Geriatr Soc. 2018 Dec;66(12):2289-97. doi: 10.1111/jgs.15640. PMID: 30460981.

47. van den Boogaard M, Schoonhoven L, van Achterberg T, et al. Haloperidol prophylaxis in critically ill patients with a high risk for delirium. Crit Care. 2013 Jan 17;17(1):R9. doi: 10.1186/cc11933. PMID: 23327295.

48. Agar MR, Lawlor PG, Quinn S, et al. Efficacy of Oral Risperidone, Haloperidol, or Placebo for Symptoms of Delirium Among Patients in Palliative Care: A Randomized Clinical Trial. JAMA Intern Med. 2017 Jan 1;177(1):34-42. doi: 10.1001/jamainternmed.2016.7491. PMID: 27918778.

49. Atalan N, Efe Sevim M, Akgun S, et al. Morphine is a reasonable alternative to haloperidol in the treatment of postoperative hyperactive-type delirium after cardiac surgery. J Cardiothorac Vasc Anesth. 2013 Oct;27(5):933-8. doi: 10.1053/j.jvca.2013.01.017. PMID: 23791495.

50. Bakri MH, Ismail EA, Ibrahim A. Comparison of dexmedetomidine or ondansetron with haloperidol for treatment of postoperative delirium in trauma patients admitted to intensive care unit: Randomized controlled trial. Anaesthesia, Pain and Intensive Care. 2015;19(2):118-23.

51. Breitbart W, Marotta R, Platt MM, et al. A double-blind trial of haloperidol, chlorpromazine, and lorazepam in the treatment of delirium in hospitalized AIDS patients. Am J Psychiatry. 1996 Feb;153(2):231-7. doi: 10.1176/ajp.153.2.231. PMID: 8561204.

52. Devlin JW, Roberts RJ, Fong JJ, et al. Efficacy and safety of quetiapine in critically ill patients with delirium: a prospective, multicenter, randomized, double-blind, placebo-controlled pilot study. Crit Care Med. 2010 Feb;38(2):41927. doi: 10.1097/CCM.0b013e3181b9e302. PMID: 19915454.

53. Girard TD, Exline MC, Carson SS, et al. Haloperidol and Ziprasidone for Treatment of Delirium in Critical Illness. N Engl J Med. 2018 Oct 22. doi: 10.1056/NEJMoa1808217. PMID: 30346242. 
54. Grover S, Kumar V, Chakrabarti S. Comparative efficacy study of haloperidol, olanzapine and risperidone in delirium. J Psychosom Res. 2011 Oct;71(4):277-81. doi:

10.1016/j.jpsychores.2011.01.019. PMID: 21911107.

55. Grover S, Mahajan S, Chakrabarti S, et al. Comparative effectiveness of quetiapine and haloperidol in delirium: A single blind randomized controlled study. World J Psychiatry. 2016 Sep 22;6(3):365-71. doi:

10.5498/wjp.v6.i3.365. PMID: 27679777.

56. Han CS, Kim YK. A double-blind trial of risperidone and haloperidol for the treatment of delirium. Psychosomatics. 2004 JulAug;45(4):297-301. doi: 10.1016/s00333182(04)70170-x. PMID: 15232043.

57. Hu H, Deng W, Yang H, et al. Olanzapine and haloperidol for senile delirium: A randomized controlled observation. Chinese Journal of Clinical Rehabilitation. 2006;10(42):188-90.

58. Jain R, Arun P, Sidana A, et al. Comparison of efficacy of haloperidol and olanzapine in the treatment of delirium. Indian J Psychiatry. 2017 Oct-Dec;59(4):451-6. doi: 10.4103/psychiatry.IndianJPsychiatry_59_17. PMID: 29497187.

59. Kim SW, Yoo JA, Lee SY, et al. Risperidone versus olanzapine for the treatment of delirium. Hum Psychopharmacol. 2010 Jun-Jul;25(4):298302. doi: 10.1002/hup.1117. PMID: 20521319.

60. Lee KU, Won WY, Lee HK, et al. Amisulpride versus quetiapine for the treatment of delirium: a randomized, open prospective study. Int Clin Psychopharmacol. 2005 Nov;20(6):311-4. PMID: 16192839.

61. Lin CJ, Sun FJ, Fang CK, et al. An open trial comparing haloperidol with olanzapine for the treatment of delirium in palliative and hospice center cancer patients. Journal of Internal Medicine of Taiwan. 2008;19(4):346-54.

62. Maneeton B, Maneeton N, Srisurapanont M, et al. Quetiapine versus haloperidol in the treatment of delirium: a double-blind, randomized, controlled trial. Drug Des Devel Ther. 2013;7:657-67. doi: 10.2147/dddt.s45575. PMID: 23926422.
63. Tahir TA, Eeles E, Karapareddy V, et al. A randomized controlled trial of quetiapine versus placebo in the treatment of delirium. J Psychosom Res. 2010 Nov;69(5):485-90. doi: 10.1016/j.jpsychores.2010.05.006. PMID: 20955868.

64. Lim HK, Paik IH, Oh K, et al. Comparison of the Clinical Efficacy and Safety between Intramuscular Olanzapine and Intramuscular Haloperidol Injection in the Treatment of Delirium. In Korean journal of psychopharmacology

65. Boettger S, Breitbart W, Passik S. Haloperidol and risperidone in the treatment of delirium and its subtypes. European Journal of Psychiatry. 2011;25(2):59-67.

66. Boettger S, Jenewein J, Breitbart W. Haloperidol, risperidone, olanzapine and aripiprazole in the management of delirium: A comparison of efficacy, safety, and side effects. Palliative \& supportive care. 2015;13(4):1079-85. doi: $10.1017 / \mathrm{s} 1478951514001059$.

67. Breitbart W, Tremblay A, Gibson C. An open trial of olanzapine for the treatment of delirium in hospitalized cancer patients. Psychosomatics. 2002 May-Jun;43(3):175-82. doi: 10.1176/appi.psy.43.3.175. PMID: 12075032.

68. Charoenporn V. Efficacy and safety of antipsychotic medications in the treatment of delirium. Journal of the Medical Association of Thailand. 2018;101(3):361-6.

69. Fox MA, Elefritz JL, Huang BM, et al. Comparison of Lurasidone Versus Quetiapine for the Treatment of Delirium in Critically Ill Patients. J Intensive Care Med. 2018 Jan 1:885066617754187. doi: 10.1177/0885066617754187. PMID: 29357776.

70. Hatta K, Kishi Y, Wada K, et al. Antipsychotics for delirium in the general hospital setting in consecutive 2453 inpatients: a prospective observational study. Int J Geriatr Psychiatry. 2014 Mar;29(3):253-62. doi: 10.1002/gps.3999. PMID: 23801358.

71. Horikawa N, Yamazaki T, Miyamoto K, et al. Treatment for delirium with risperidone: results of a prospective open trial with 10 patients. Gen Hosp Psychiatry. 2003 Jul-Aug;25(4):289-92. PMID: 12850662. 
72. Ikezawa K, Canuet L, Ishii R, et al. Efficacy of risperidone in the treatment of delirium in elderly patients. Psychogeriatrics. 2008;8(2):62-5. doi: 10.1111/j.1479-8301.2008.00227.x.

73. Kim KS, Pae CU, Chae JH, et al. An open pilot trial of olanzapine for delirium in the Korean population. Psychiatry Clin Neurosci. 2001 Oct;55(5):515-9. doi: 10.1046/j.14401819.2001.00898.x. PMID: 11555348.

74. Kim KY, Bader GM, Kotlyar V, et al. Treatment of delirium in older adults with quetiapine. $\mathrm{J}$ Geriatr Psychiatry Neurol. 2003 Mar;16(1):29-31. doi: 10.1177/0891988702250533. PMID: 12641370 .

75. Kim JY, Jung IK, Han C, et al. Antipsychotics and dopamine transporter gene polymorphisms in delirium patients. Psychiatry Clin Neurosci. 2005 Apr;59(2):183-8. doi: 10.1111/j.14401819.2005.01355.x. PMID: 15823165.

76. Kishi Y, Kato M, Okuyama T, et al. Treatment of delirium with risperidone in cancer patients. Psychiatry Clin Neurosci. 2012 Aug;66(5):411-7. doi: 10.1111/j.1440-1819.2012.02346.x. PMID: 22834659 .

77. Maneeton B, Maneeton N, Srisurapanont M. An open-label study of quetiapine for delirium. $\mathrm{J}$ Med Assoc Thai. 2007 Oct;90(10):2158-63. PMID: 18041437.

78. Mittal D, Jimerson NA, Neely EP, et al. Risperidone in the treatment of delirium: results from a prospective open-label trial. J Clin Psychiatry. 2004 May;65(5):662-7. PMID: 15163252.

79. Naksuk N, Thongprayoon C, Park JY, et al. Editor's Choice-Clinical impact of delirium and antipsychotic therapy: 10-Year experience from a referral coronary care unit. Eur Heart J Acute Cardiovasc Care. 2017 Sep;6(6):560-8. doi: 10.1177/2048872615592232. PMID: 26124454.

80. Omura K, Amano N. Clinical experience of quetiapine in 24 elderly patients with delirium. Psychogeriatrics. 2003;3(2):69-72. doi: 10.1046/j.1479-8301.2003.00012.x. PMID: 200419733-005.

81. Pae CU, Lee SJ, Lee CU, et al. A pilot trial of quetiapine for the treatment of patients with delirium. Hum Psychopharmacol. 2004 Mar;19(2):125-7. doi: 10.1002/hup.559. PMID: 14994323.
82. Parellada E, Baeza I, de Pablo J, et al. Risperidone in the treatment of patients with delirium. J Clin Psychiatry. 2004 Mar;65(3):34853. PMID: 15096074.

83. Sasaki Y, Matsuyama T, Inoue S, et al. A prospective, open-label, flexible-dose study of quetiapine in the treatment of delirium. J Clin Psychiatry. 2003 Nov;64(11):1316-21. PMID: 14658945.

84. Sipahimalani A, Masand PS. Olanzapine in the treatment of delirium. Psychosomatics. 1998 SepOct;39(5):422-30. doi: 10.1016/s00333182(98)71301-5. PMID: 9775699.

85. Skrobik YK, Bergeron N, Dumont M, et al. Olanzapine vs haloperidol: treating delirium in a critical care setting. Intensive Care Med. 2004 Mar;30(3):444-9. doi: 10.1007/s00134-003-21170. PMID: 14685663.

86. Straker DA, Shapiro PA, Muskin PR. Aripiprazole in the treatment of delirium. Psychosomatics. 2006 Sep-Oct;47(5):385-91. doi: 10.1176/appi.psy.47.5.385. PMID: 16959926.

87. Toda H, Kusumi I, Sasaki Y, et al. Relationship between plasma concentration levels of risperidone and clinical effects in the treatment of delirium. Int Clin Psychopharmacol. 2005 Nov;20(6):331-3. PMID: 16192843.

88. Yoon HK, Kim YK, Han C, et al. Paliperidone in the treatment of delirium: results of a prospective open-label pilot trial. Acta Neuropsychiatr. 2011 Aug;23(4):179-83. doi: 10.1111/j.16015215.2011.00568.x. PMID: 25379796.

89. Yoon HJ, Park KM, Choi WJ, et al. Efficacy and safety of haloperidol versus atypical antipsychotic medications in the treatment of delirium. BMC Psychiatry. 2013 Sep 30;13:240. doi: 10.1186/1471-244x-13-240. PMID: 24074357.

90. Siddiqi N, Harrison JK, Clegg A, et al. Interventions for preventing delirium in hospitalised non-ICU patients. Cochrane Database Syst Rev. 2016 Mar 11;3:CD005563. doi: 10.1002/14651858.CD005563.pub3. PMID: 26967259.

91. Devlin JW, Skrobik Y, Gelinas C, et al. Clinical Practice Guidelines for the Prevention and Management of Pain, Agitation/Sedation, Delirium, Immobility, and Sleep Disruption in Adult Patients in the ICU. Crit Care Med. 2018 Sep;46(9):e825-e73. doi: 10.1097/ccm.0000000000003299. PMID: 30113379. 
92. Herling SF, Greve IE, Vasilevskis EE, et al. Interventions for preventing intensive care unit delirium in adults. Cochrane Database Syst Rev. 2018 Nov 23;11:CD009783. doi:

10.1002/14651858.CD009783.pub2. PMID: 30484283.

93. Tomichek JE, Stollings JL, Pandharipande PP, et al. Antipsychotic prescribing patterns during and after critical illness: a prospective cohort study. Crit Care. 2016 Nov 24;20(1):378. doi: 10.1186/s13054-016-1557-1. PMID: 27881149.

94. Herzig SJ, Rothberg MB, Guess JR, et al. Antipsychotic medication utilization in nonpsychiatric hospitalizations. J Hosp Med. 2016 Aug;11(8):543-9. doi: 10.1002/jhm.2596. PMID: 27130311.

95. Marshall J, Herzig SJ, Howell MD, et al. Antipsychotic utilization in the intensive care unit and in transitions of care. J Crit Care. 2016 Jun;33:119-24. doi: 10.1016/j.jcrc.2015.12.017. PMID: 26818629.

96. Postoperative delirium in older adults: Best practice statement from the American geriatrics society. Journal of the American College of Surgeons. 2015;220(2):136-48.e1. doi: 10.1016/j.jamcollsurg.2014.10.019.

97. Rose L, Agar M, Burry LD, et al. Development of core outcome sets for effectiveness trials of interventions to prevent and/or treat delirium (Del-COrS): study protocol. BMJ Open. 2017 Sep 18;7(9):e016371. doi: 10.1136/bmjopen-2017016371. PMID: 28928181.

98. NIDUS-Network for Investigation of Delirium: Unifying Scientists. NIDUS-Network for Investigation of Delirium: Unifying Scientists. 2018. https://deliriumnetwork.org/. Accessed on December 142018. 
AGS

AHRQ

AIDS

APACHE II

ASA

bid

BMT

BPRS

BUN

CAM-ICU

CAM-S

CCU

CENTRAL

CGI

CGI-GI

CI

CINAHL

d

DRS

DRS-R-98

DSM-IV

EPC

EPS

ESRS

g

HR

ICDSC

ICU

IQR

IV

JHU

MDAS

$\mathrm{MeSH}$

$\mathrm{mg}$

mg/day

$\mathrm{mg} / \mathrm{hr}$

\section{Abbreviations and Acronyms}

American Geriatric Society

Agency for Healthcare Research and Quality

acquired immunodeficiency syndrome

Acute Physiology and Chronic Health Evaluation 2

American Society of Anesthesiologists

twice daily

bone marrow transplant

Brief Psychiatric Rating Scale

blood urea nitrogen ratio

Confusion Assessment Method for the ICU

Confusion Assessment Method-Severity

coronary care unit

Cochrane Central Register of Controlled Trials

Clinical Global Impressions Scale

Clinical Global Impressions Scale-Global Impression

confidence interval

Cumulative Index to Nursing and Allied Health Literature

days

Delirium Rating Scale

Delirium Rating Scale Revised-98

Diagnostic and Statistical Manual of Mental Disorders, 4th ed.

Evidence-based Practice Center

extrapyramidal symptoms

Extrapyramidal Symptom Rating Scale

gram

Hazard ratio

Intensive Care Delirium Screening Checklist

intensive care unit

interquartile range

intravenous

Johns Hopkins University

Memorial Delirium Assessment Scale

medical subject headings

milligram

milligram per day

milligram per hour 


\begin{tabular}{|c|c|}
\hline $\min$ & minutes \\
\hline $\mathrm{ml}$ & millimeter \\
\hline $\mathrm{ml} / \mathrm{hr}$ & millimeter per hour \\
\hline MMSE & Mini-Mental State Examination \\
\hline MV & mechanical ventilation \\
\hline $\mathrm{N}$ & sample size \\
\hline $\mathrm{NaCl}$ & sodium chloride \\
\hline NCGG & National Center for Geriatrics and Gerontology \\
\hline NEECHAM & Neelon and Champagne Confusion Scale \\
\hline NG & nadogastric tube \\
\hline NuDESC & Nursing Delirium Screening Scale \\
\hline OR & odds ratio \\
\hline $\mathrm{p}$ & p-value \\
\hline qd & once daily \\
\hline qid & four times a day \\
\hline QT & $\mathrm{Q}$ and $\mathrm{T}$ wave interval \\
\hline QTc & corrected QT interval \\
\hline RASS & Richmond Agitation and Sedation Scale \\
\hline RCT & randomized controlled trial \\
\hline ROBINS-I & Risk of Bias in Non-Randomized Studies of Interventions \\
\hline $\mathrm{RR}$ & risk ratio \\
\hline SAS & Sedation Agitated Scale \\
\hline SCID & severe combined immunodeficiency \\
\hline SD & standard deviation \\
\hline SE & standard error \\
\hline SEADS & Supplemental Evidence and Data for Systematic Reviews \\
\hline SNF & skilled nursing facility \\
\hline T3 & time point three \\
\hline TEP & Technical Expert Panel \\
\hline tid & three times daily \\
\hline ug/kg & microgram per kilogram \\
\hline UKU & Udvalg for Kliniske Undersøgelser Scale \\
\hline US & United States \\
\hline
\end{tabular}




\section{Appendix A. Detailed Search Strategy}

PubMed Search String, run on June 4, 2018

\begin{tabular}{|c|c|c|}
\hline \# & String & Hits \\
\hline 1 & $\begin{array}{l}\text { Confusion[mh] OR delirium*[tiab] OR delerium*[tiab] OR deliria*[tiab] OR delirious[tiab] } \\
\text { OR delerious[tiab] OR confusion*[tiab] OR "agitated emergence"[tiab] OR "altered } \\
\text { consciousness"[tiab] OR "dis orientation"[tiab] OR "dis oriented"[tiab] OR "dis } \\
\text { orientations"[tiab] OR "emergence agitation"[tiab] OR "emergence excitement"[tiab] OR } \\
\text { "postanaesthetic excitement"[tiab] OR "postanesthetic excitement"[tiab] OR } \\
\text { disorient*[tiab] OR hallucinat*[tiab] OR illusion*[tiab] OR illusor*[tiab] }\end{array}$ & 72,336 \\
\hline 2 & Antipsychotic agents[mh] OR antipsychotic*[tiab] OR neuroleptic*[tiab] & 72,597 \\
\hline 3 & $\begin{array}{l}\text { Chlorpromazine[mh] OR chlorpromazine*[tiab] OR Droperidol[mh] OR droperidol*[tiab] } \\
\text { OR Fluphenazine[mh] OR fluphenazine*[tiab] OR Haloperidol[mh] OR haloperidol*[tiab] } \\
\text { OR Loxapine[mh] OR loxapine*[tiab] OR Molindone[mh] OR molindone*[tiab] OR } \\
\text { Perphenazine[mh] OR perphenazine*[tiab] OR Pimozide[mh] OR pimozide*[tiab] OR } \\
\text { Prochlorperazine[mh] OR prochlorperazine*[tiab] OR Thiothixene[mh] OR } \\
\text { thiothixene*[tiab] OR tiotixene*[tiab] OR Thioridazine[mh] OR thioridazine*[tiab] OR } \\
\text { Trifluoperazine[mh] OR trifluoperazine*[tiab] }\end{array}$ & 55,379 \\
\hline 4 & $\begin{array}{l}\text { Aripiprazole[mh] OR aripiprazole*[tiab] OR Asenapine[nm] OR asenapine*[tiab] OR } \\
\text { Brexpiprazole[nm] OR brexpiprazole*[tiab] OR Cariprazine[nm] OR cariprazine*[tiab] OR } \\
\text { Clozapine[mh] OR clozapine*[tiab] OR Iloperidone[nm] OR iloperidone*[tiab] OR } \\
\text { Lurasidone hydrochloride[mh] OR lurasidone*[tiab] OR Olanzapine[nm] OR } \\
\text { olanzapine*[tiab] OR Paliperidone palmitate[mh] OR paliperidone*[tiab] OR Quetiapine } \\
\text { fumarate[mh] OR quetiapine*[tiab] OR Risperidone[mh] OR risperidone*[tiab] OR } \\
\text { Ziprasidone[nm] OR ziprasidone*[tiab] }\end{array}$ & 28,785 \\
\hline 5 & $\# 1$ AND (\#2 OR \#3 OR \#4) & 3881 \\
\hline 6 & Animals[mh] NOT humans[mh] & $4,460,022$ \\
\hline 7 & \#5 NOT \#6 & 3837 \\
\hline 8 & $\begin{array}{l}\text { Addresses[ptyp] OR News[ptyp] OR Patient Education Handout[ptyp] OR } \\
\text { Bibliography[ptyp] OR Dictionary[ptyp] OR Directory[ptyp] OR Legal Cases[ptyp] OR } \\
\text { Legislation[ptyp] OR Newspaper Article[ptyp] OR Periodical Index[ptyp] }\end{array}$ & 257,594 \\
\hline 9 & \#7 NOT \#8 & 3834 \\
\hline
\end{tabular}

\section{Embase Search String, run on June 6, 2018}

\begin{tabular}{|c|c|c|}
\hline \# & String & Hits \\
\hline 1 & $\begin{array}{l}\text { 'delirium'/exp OR delirium:ti,ab OR delerium*:ti,ab OR deliria*:ti,ab OR delirious:ti,ab OR } \\
\text { delirious:ti,ab OR confusion*:ti,ab OR “agitated emergence":ti,ab OR “altered } \\
\text { consciousness":ti,ab OR “dis orientation":ti,ab OR “dis oriented":ti,ab OR “dis } \\
\text { orientations":ti,ab OR "emergence agitation":ti,ab OR "emergence excitement":ti,ab OR } \\
\text { "postanaesthetic excitement":ti,ab OR “postanesthetic excitement”:ti,ab OR } \\
\text { disorient*:ti,ab OR hallucinate*:ti,ab OR illusion*:ti,ab OR illusor*:ti,ab }\end{array}$ & 94,891 \\
\hline 2 & ‘Neuroleptic agent'/exp OR antipsychotic*:ti,ab OR neuroleptic*ti,ab & 287,109 \\
\hline 3 & 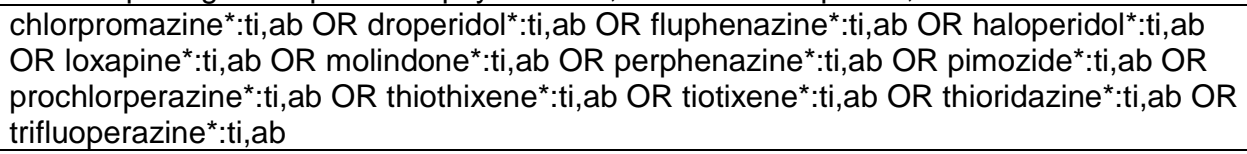 & 53,228 \\
\hline 4 & 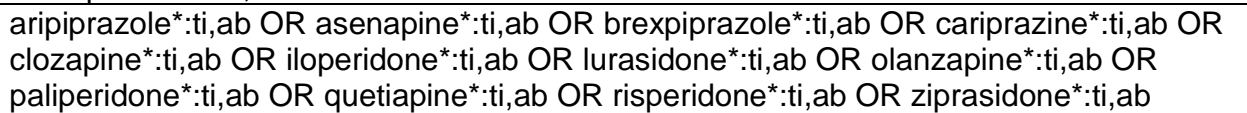 & 39,504 \\
\hline 5 & $\# 1$ AND (\#2 OR \#3 OR \#4) & 8274 \\
\hline 6 & Animals/exp NOT humans/exp & $5,290,387$ \\
\hline 7 & \#5 NOT \#6 & 8,219 \\
\hline 8 & $\begin{array}{l}\text { [conference abstract]/lim OR [conference paper]/lim OR [conference review]/lim OR } \\
\text { [editorial]/lim OR [erratum]/lim OR [letter]/lim OR [note]/lim OR 'case report'/exp }\end{array}$ & $8,222,104$ \\
\hline 9 & \#7 not \#8 & 4,853 \\
\hline
\end{tabular}


Cochrane Search String, run on June 8, 2018

\begin{tabular}{|c|c|c|}
\hline$\#$ & String & Hits \\
\hline 1 & 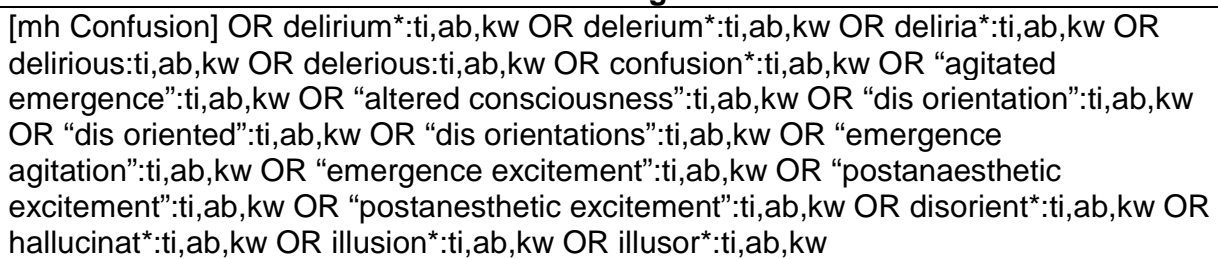 & 6055 \\
\hline 2 & antipsychotic*:ti,ab,kw OR neuroleptic*:ti,ab,kw & 9669 \\
\hline 3 & $\begin{array}{l}\text { [mh Chlorpromazine] OR chlorpromazine*:ti,ab,kw OR [mh Droperidol] OR } \\
\text { droperidol*:ti,ab,kw OR [mh Fluphenazine] OR fluphenazine*:ti,ab,kw OR [mh } \\
\text { Haloperidol] OR haloperidol*:ti,ab,kw OR [mh Loxapine] OR loxapine*:ti,ab,kw OR [mh } \\
\text { Molindone] OR molindone*:ti,ab,kw OR [mh Perphenazine] OR perphenazine*:ti,ab,kw } \\
\text { OR [mh Pimozide] OR pimozide*:ti,ab,kw OR [mh Prochlorperazine] OR } \\
\text { prochlorperazine*:ti,ab,kw OR [mh Thiothixene] OR thiothixene*:ti,ab,kw OR } \\
\text { tiotixene*:ti,ab,kw OR [mh Thioridazine] OR thioridazine*:ti,ab,kw OR [mh } \\
\text { Trifluoperazine] OR trifluoperazine*:ti,ab,kw }\end{array}$ & 6631 \\
\hline 4 & 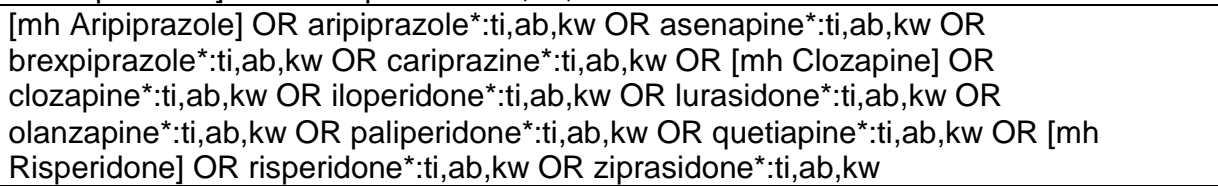 & 8273 \\
\hline 5 & $\begin{array}{l}\# 1 \text { AND (\#2 OR \#3 OR \#4) } \\
\text { Limit to Trials }\end{array}$ & $\begin{array}{l}793 \\
710\end{array}$ \\
\hline
\end{tabular}

\section{CINAHL Search String, run on June 8, 2018}

\begin{tabular}{|c|c|c|}
\hline \# & String & Hits \\
\hline 1 & 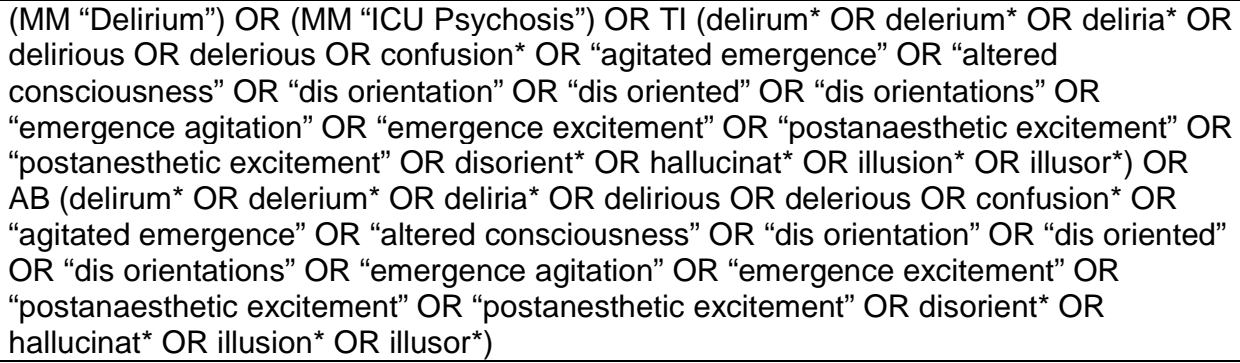 & 12,245 \\
\hline 2 & $\begin{array}{l}\text { (MM “Antipsychotic Agents+") OR TI (antipsychotic* OR neuroleptic*) OR AB } \\
\text { (antipsychotic* OR neuroleptic*) }\end{array}$ & 9447 \\
\hline 3 & 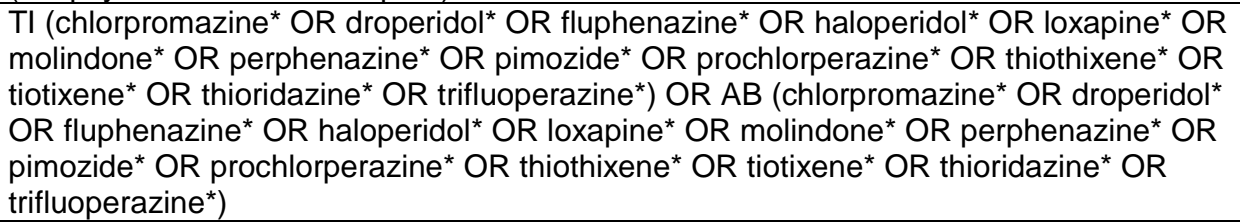 & 1317 \\
\hline 4 & 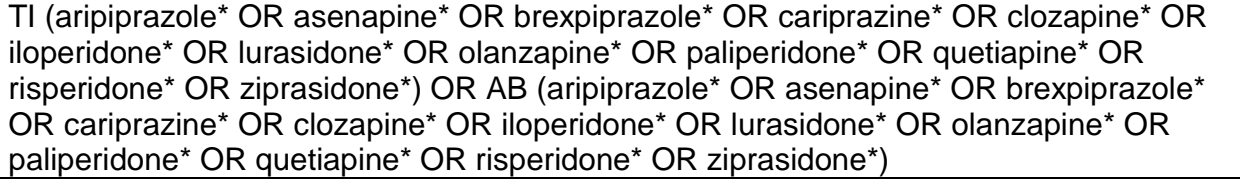 & 3399 \\
\hline 5 & S1 AND (S2 OR S3 OR S4) & 475 \\
\hline
\end{tabular}


PsycINFO Search String, run on June 8, 2018

\begin{tabular}{|c|c|c|}
\hline \# & String & Hits \\
\hline 1 & $\begin{array}{l}\text { (MM "Delirium") OR (MM "ICU Psychosis") OR TI (delirum* OR delerium* OR deliria* OR } \\
\text { delirious OR delerious OR confusion* OR "agitated emergence" OR "altered } \\
\text { consciousness" OR "dis orientation" OR "dis oriented" OR "dis orientations" OR } \\
\text { "emergence agitation" OR "emergence excitement" OR "postanaesthetic excitement" OR } \\
\text { "postanesthetic excitement" OR disorient* OR hallucinat* OR illusion* OR illusor*) OR } \\
\text { AB (delirum* OR delerium* OR deliria* OR delirious OR delerious OR confusion* OR } \\
\text { "agitated emergence" OR "altered consciousness" OR "dis orientation" OR "dis oriented" } \\
\text { OR "dis orientations" OR "emergence agitation" OR "emergence excitement" OR } \\
\text { "postanaesthetic excitement" OR "postanesthetic excitement" OR disorient* OR } \\
\text { hallucinat* OR illusion* OR illusor*) }\end{array}$ & 52,283 \\
\hline 2 & $\begin{array}{l}\text { (MM “Antipsychotic Agents+") OR TI (antipsychotic* OR neuroleptic*) OR AB } \\
\text { (antipsychotic* OR neuroleptic*) }\end{array}$ & 36,343 \\
\hline 3 & 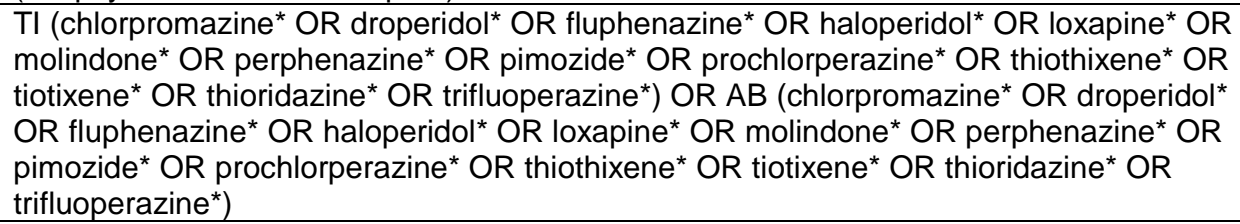 & 13,995 \\
\hline 4 & 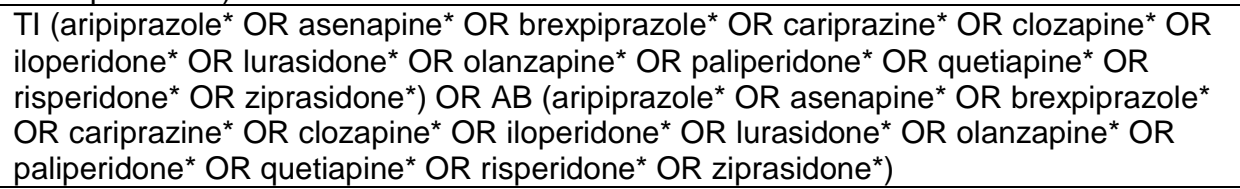 & 18770 \\
\hline 5 & $\begin{array}{l}\text { S1 AND (S2 OR S3 OR S4) } \\
\text { Limits: Academic journals }\end{array}$ & $\begin{array}{l}2583 \\
2471\end{array}$ \\
\hline
\end{tabular}




\section{Appendix B. Screening and Data Abstraction Forms}

Figure B-1. Abstract screening form

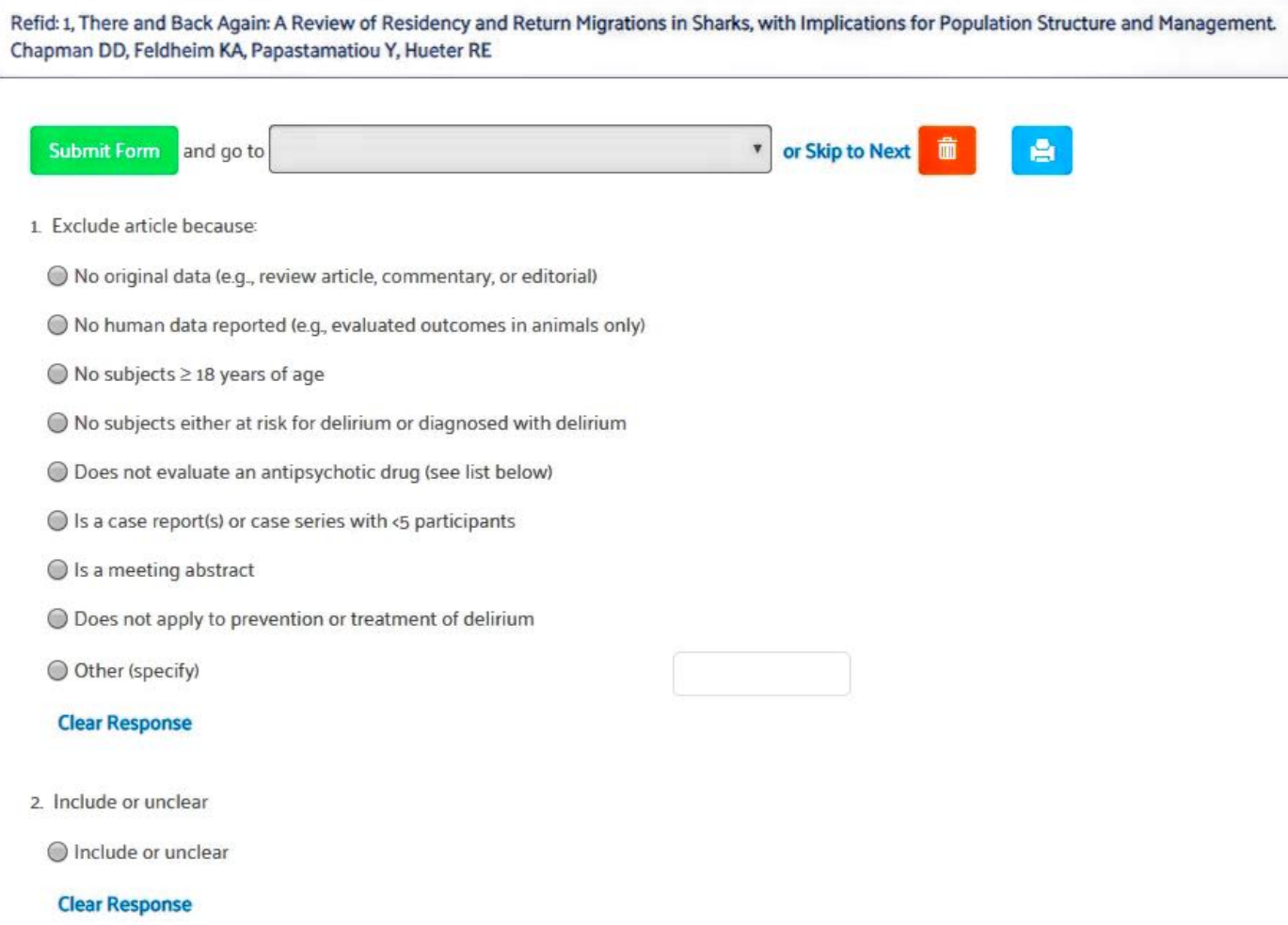




\section{Figure B-2. Article screening form}

Refid: 1, There and Back Again: A Review of Residency and Return Migrations in Sharks, with Implications for Population Structure and Management.

Chapman DD, Feldheim KA, Papastamatiou Y, Hueter RE

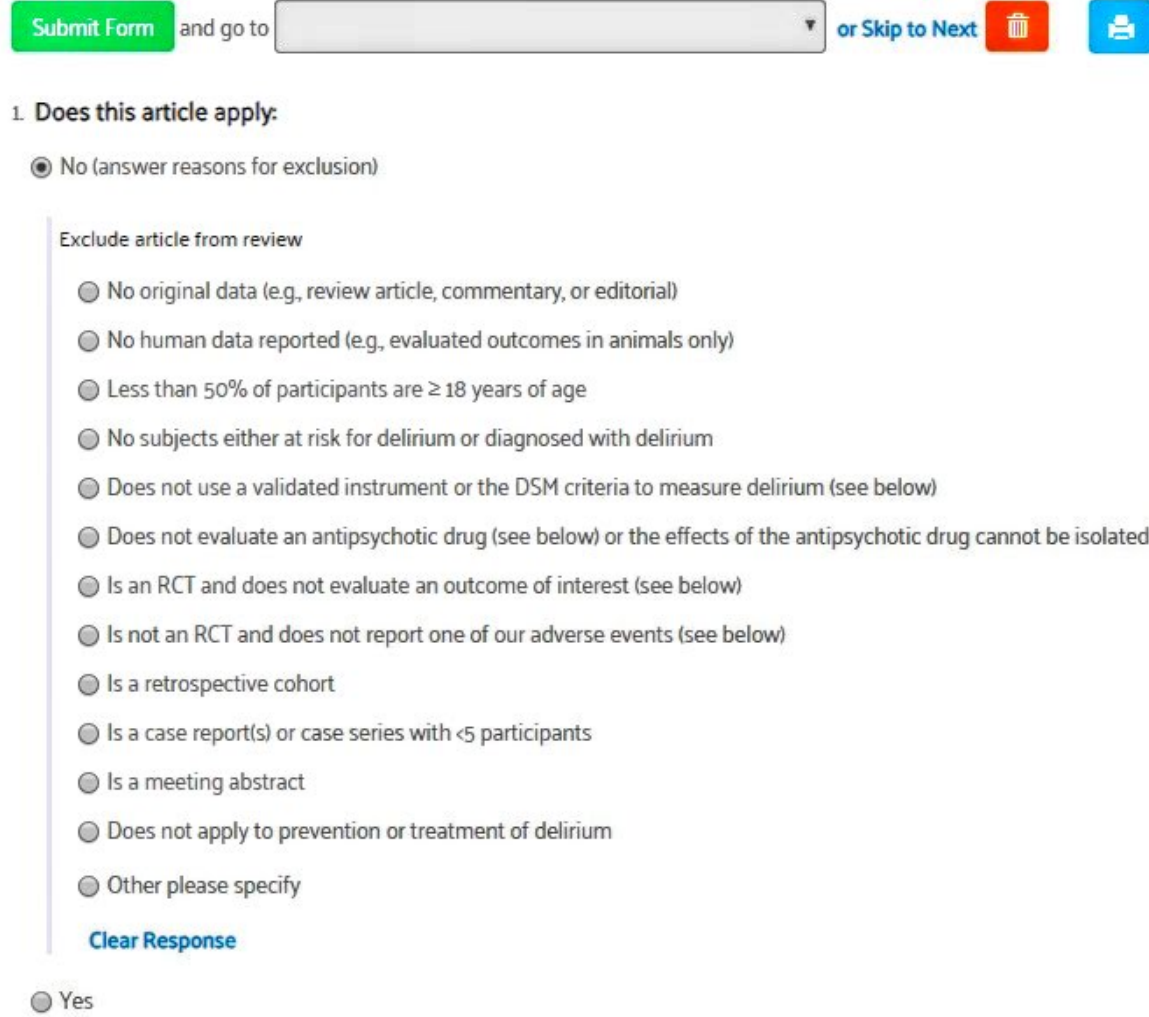




\section{Figure B-3. Study design}

Refid: 1, There and Back Again: A Review of Residency and Return Migrations in Sharks, with Implications for Population Structure and Management. Chapman DD, Feldheim KA, Papastamatiou Y, Hueter RE

Submit Form and go to

EPC Antipsychotics for the Prevention and Treatment of Delirium Study Design Form

1 Study design

Select an Answer

2. Does the study evaluate the prevention or treatment of delirium?

Select an Answer

\footnotetext{
3. Does the study apply to one of our subgroups of interest? (Check all that apply)

$\square$ Persons aged 65 or older

$\square$ Persons with dementia

$\square$ Patients in an intensive care unit

$\square$ Patients in a post-acute care facility

$\square$ Patients in palliative or hospice care

$\square$ Patients in post-operative care

$\square$ None of the above
} 
4. Specify the patient population (provide a simple summary (e.g, cardlac surgery patients))

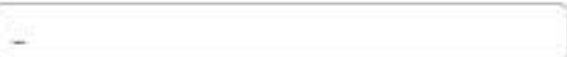

5. Country (check all that apply and then fill in specific countries)

$\square$ United States

$\square$ North America (not US)

$\square$ South America

$\square$ Europe

$\square$ Asia

$\square$ Africa

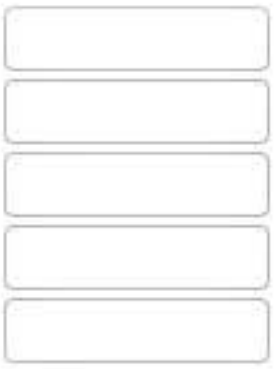

$\square$ Australia

$\square$ Not reported

6. Study start year (enter 4-digit number)

Start year

Not reported

Clear Response

7. Funding (check all that apply)

$\square$ industry

$\square$ Government

Nonprofit

$\square$ other

$\square$ None

$\square$ Not reported 
8. Tool used to diagnose delirium (check all that apply; use the text box to identify the threshold used to diagnose, if specified)

a. 3-Minute Diagnostic Confusion Assessment Method (3D-CAM)

b. 4AT Rapid Clinical Test for Delirium (4AT)

c. Brief Confusion Assessment Method (bCAM)

$\square$ d. Confusion Assessment Method (CAM)

$\square$ e. Confusion Assessment Method - Severity Scale (CAM-S)

f. Confusion Assessment Method for the Intensive Care Unit (CAM-ICU)

g. Communication Capacity Scale and Agitation Distress Scale (CCS \& ADS)

h. Confusional State Evaluation (CSE)

i. Cognitive Test for Delirium (CTD)

$\square$ j. Delirium Index (DI, DIDX)

$\square$ k. Delirium-O-Meter (DOM)

$\square$ L. Delirium Observation Screening Scale (DOSS)

$\square$ m. Delirium Rating Scale - Revised-98 (DRS-R-98)

n. Delirium Symptom Interview (DSI)

$\square$ o. Diagnostic and Statistical Manual of Mental Disorders (DSM)

$\square$ p. Family Confusion Assessment Method (FAM-CAM)

$\square$ q. Intensive Care Delirium Screening Checklist (ICDSC)

$\square$ r. Memorial Delirium Assessment Scale (MDAS)

s. The Neelon and Champagne Confusion Scale (NEECHAM)

$\square$ t. Nursing Delirium Screening Checklist (Nu-DESC)

$\square$ u. Recognizing Acute Delirium as Part of Your Routine (RADAR)

$\square$ v. Recoverable Cognitive Dysfunction Scale epérage Actif du Delirium Adapté à la Routine] (RCDS)

$\square$ w. Single Question in Delirium (SQiD)

x. Other (in addition to one of the tools marked above)

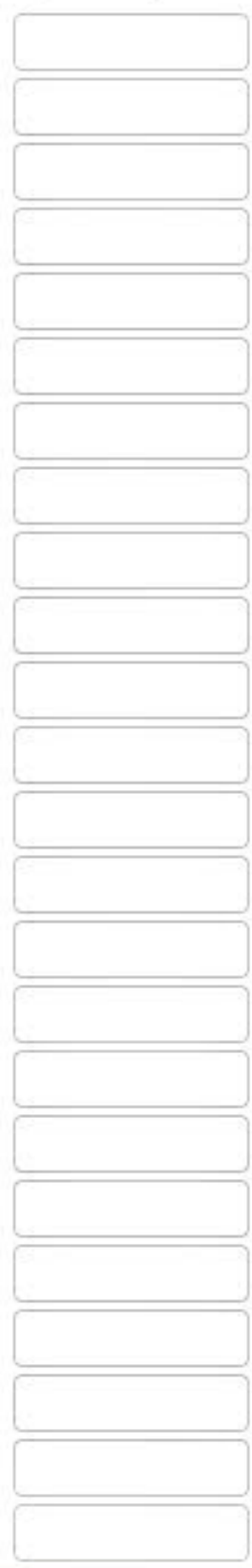




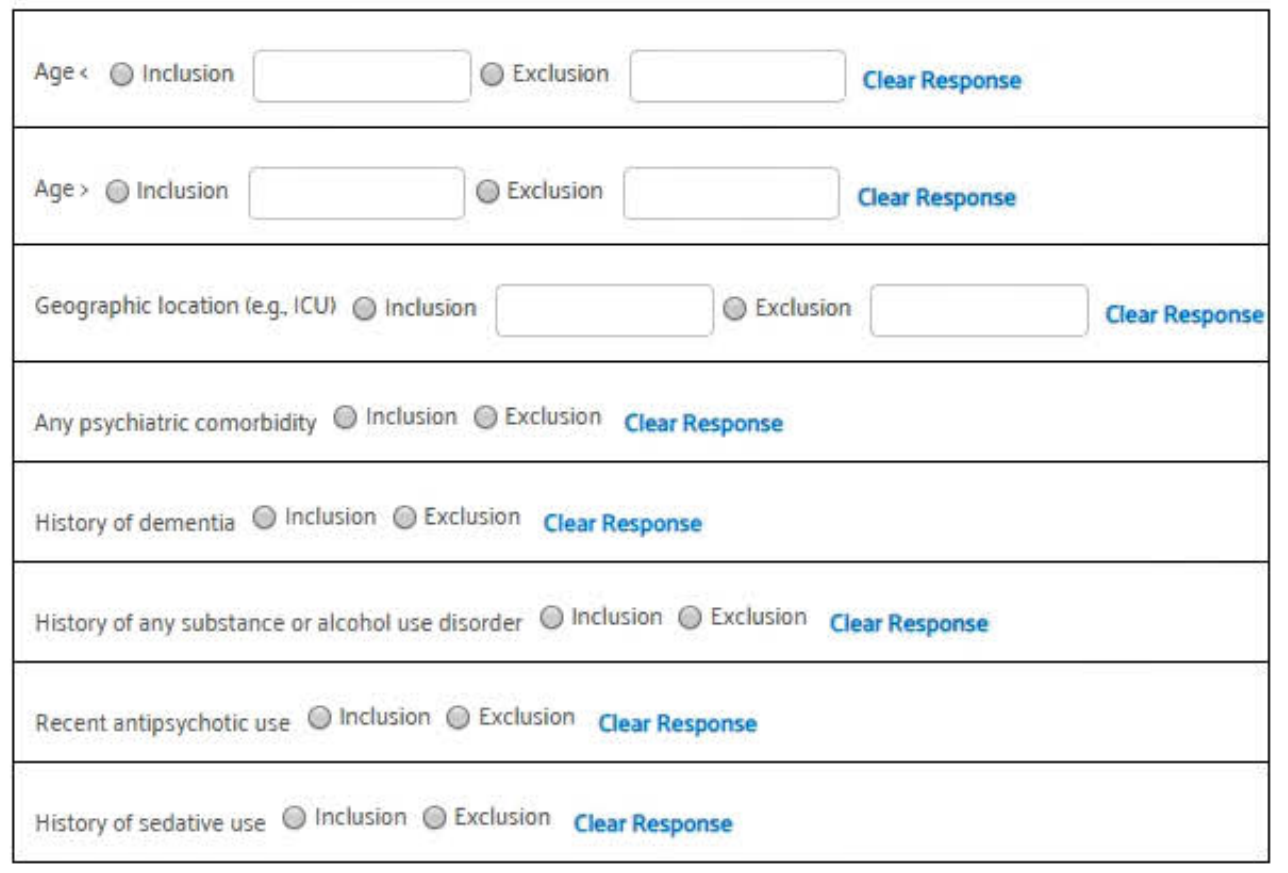

17. Has the study been registered in a clinical trials registry?

Select an Answer

18. Comments (limit 250 characters) 
Figure B-4. Intervention form

1 Identify the group being reported on this form (Mandatory question)

Select an Answer

$\checkmark$

Complete this table for pharmacologic interventions.

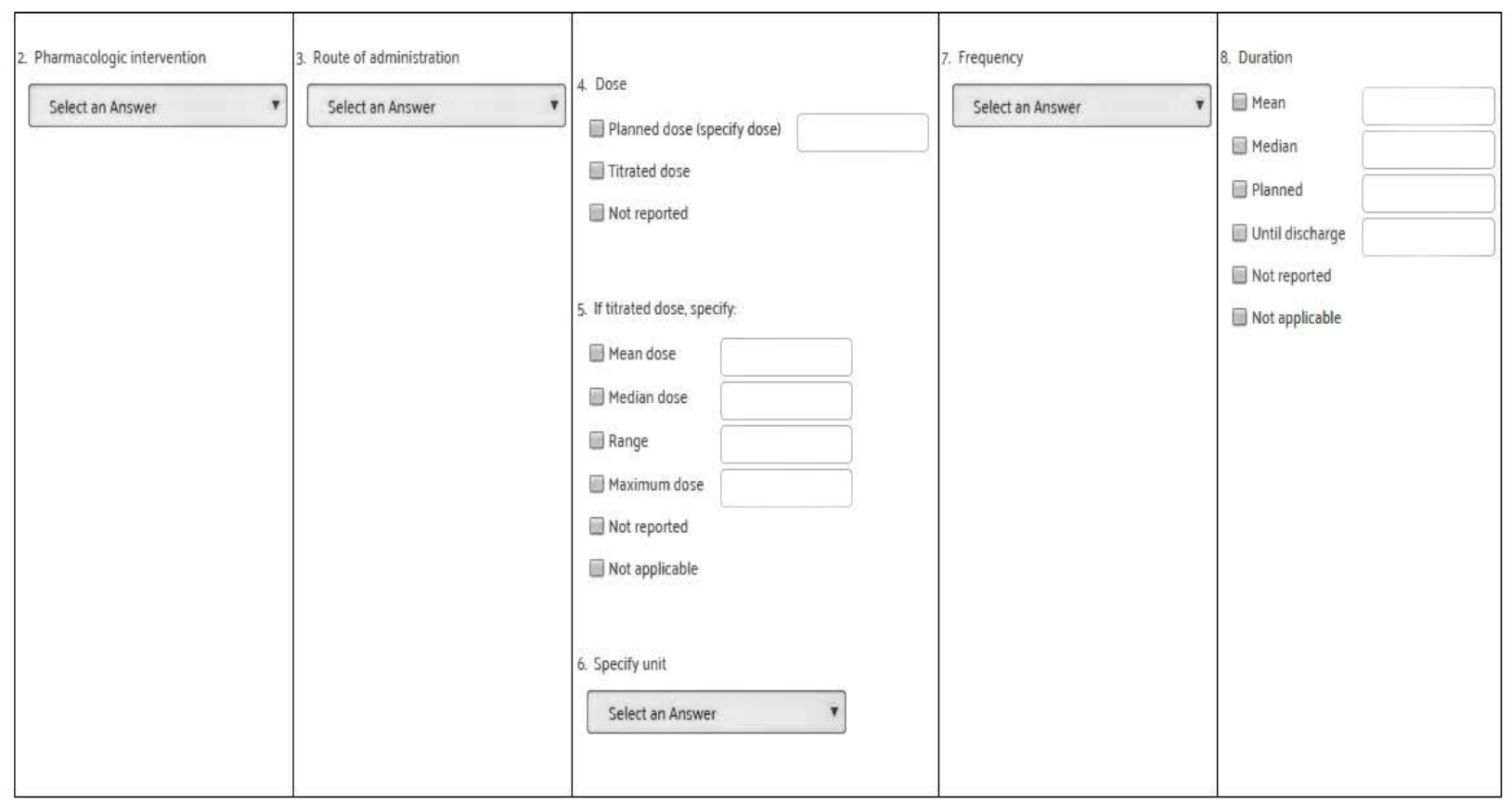

$-\ldots \ldots . ., . \ldots$. 


\begin{tabular}{|c|c|}
\hline $\begin{array}{l}\square \text { Orientation and therapeutic activities } \\
\square \text { Fluid repletion } \\
\square \text { Early mobilization } \\
\square \text { Feeding assistance } \\
\square \text { Vision and hearing } \\
\square \text { Sleep enhancement } \\
\square \text { Infection prevention } \\
\square \text { Pain management } \\
\square \text { Hypoxia protocol } \\
\square \text { Psychoactive medication protocol }\end{array}$ & 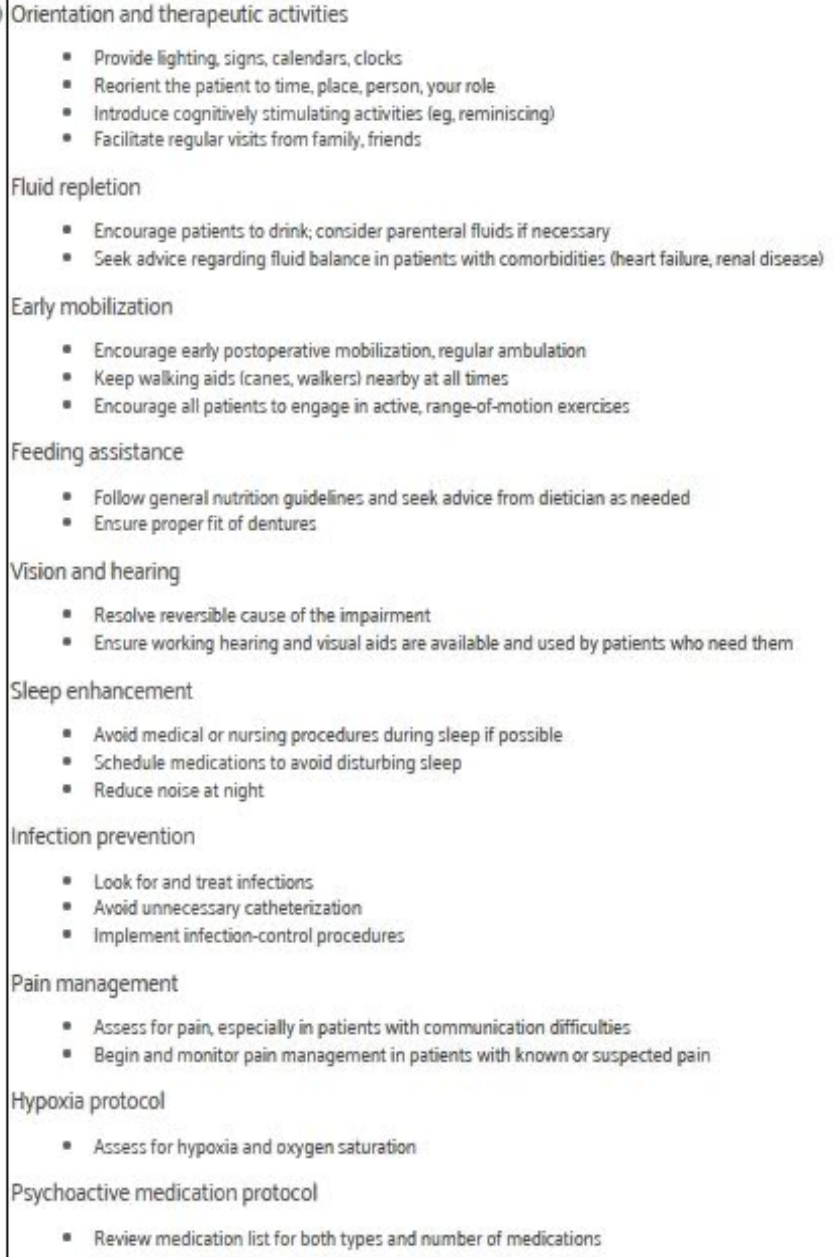 \\
\hline
\end{tabular}


Figure B-5. Population characteristics form

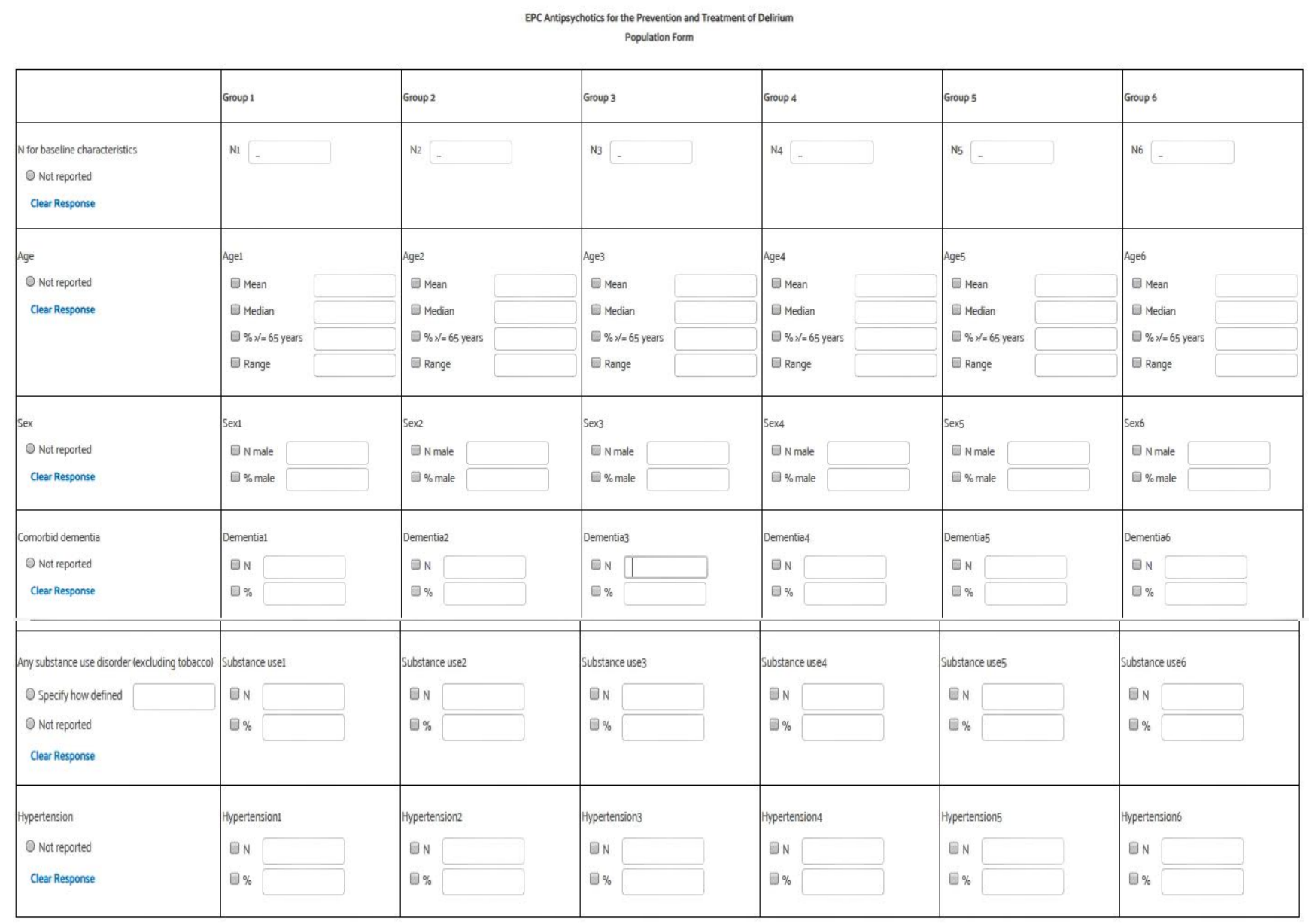

43. Comments limit 250 characters) 
Figure B-6. Outcomes definition form (short-term delirium specific)

Please flll out one form for each outcome indicate the outcome being reported on this form

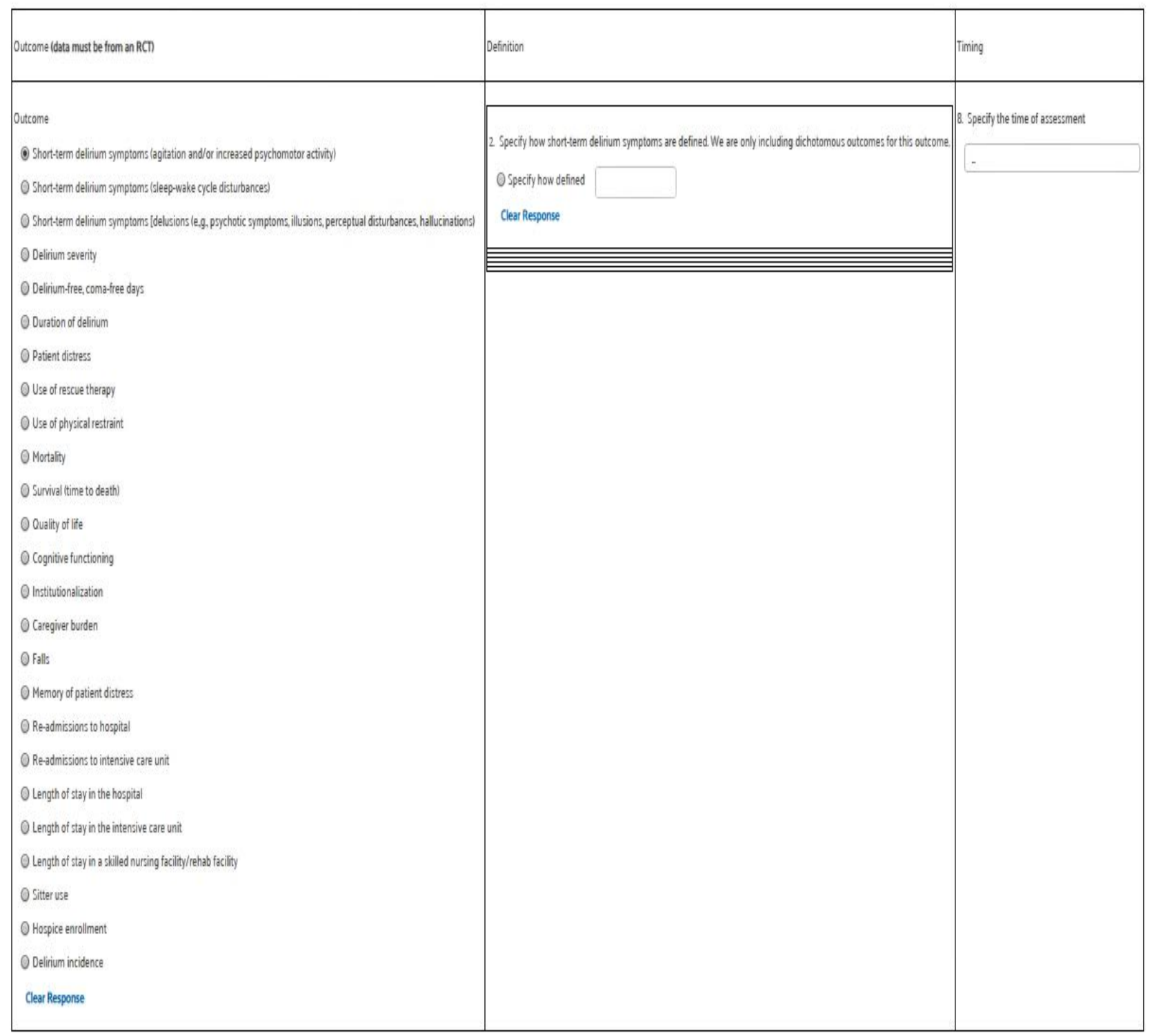


Figure B-7. Outcomes definition form (rescue therapy specific)

Please fill out one form for each outcome Indicate the outcome being reported on this form.

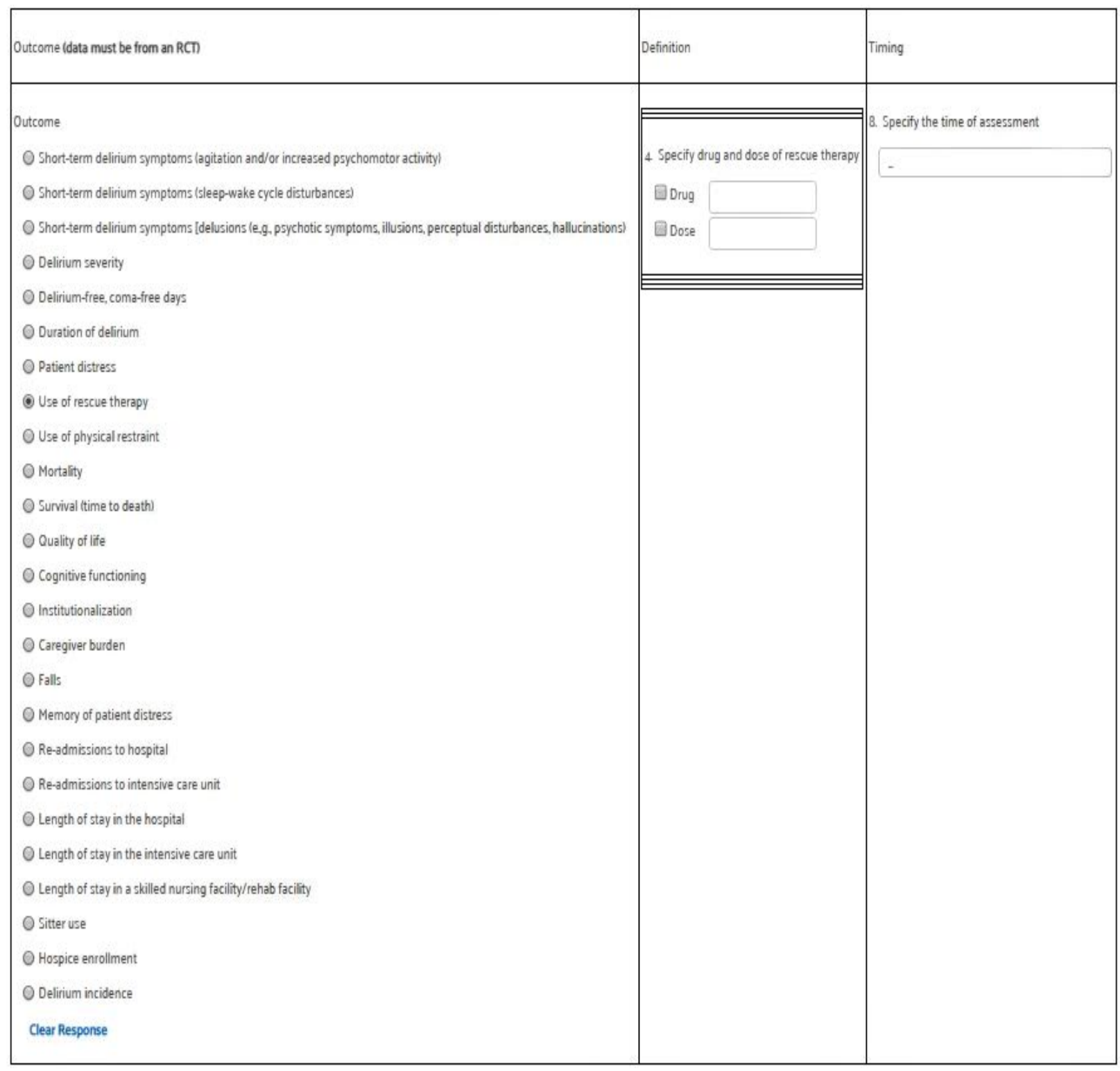


Figure B-8. Outcomes definition form (quality of life specific)

Please flll out one form for each outcome. Indicate the outcome being reported on this form.

\begin{tabular}{|c|c|c|}
\hline Outcome (data must be from an RCT) & Definition & Timing \\
\hline Outcome & \multirow[b]{2}{*}{ 5. Specify how quality of life is defined } & 8. Specify the time of assessment \\
\hline Short-term delirium symptoms (agitation and/or increased psychomotor activity) & & - \\
\hline Short-term delirium symptoms (sleep-wake cycle disturbances) & OSF-36 & \\
\hline O Short-term delinum symptoms (delusions le.g. psychotic symptoms, illusions, perceptual disturbances, hallucinationst) & $O E Q-50$ & \\
\hline Delirium severity & Oother & \\
\hline Delirium-free, coma-free days & \multirow{2}{*}{ Clear Response } & \\
\hline Duration of delifium & & \\
\hline Patient distress & & \\
\hline Use of rescue therapy & & \\
\hline Use of physical restraint & & \\
\hline O. Mortality & & \\
\hline O Survival (time to death) & & \\
\hline (1) Quality of life & & \\
\hline Cognitive functioning & & \\
\hline Institutionalization & & \\
\hline C Caregiver burden & & \\
\hline O Falls & & \\
\hline Memory of patient distress & & \\
\hline Re-admissions to hospital & & \\
\hline O Re-admissions to intensive care unit & & \\
\hline Length of stay in the hospital & & \\
\hline Length of stay in the intensive care unit & & \\
\hline Length of stay in a skilled nursing facility/rehab facility & & \\
\hline O sitter use & & \\
\hline Hospice enrollment & & \\
\hline Delirium incidence & & \\
\hline Clear Response & & \\
\hline
\end{tabular}


Figure B-9. Outcomes definition form (cognitive function specific)

Please flli out one form for each outcome. Indicate the outcome being reported on this form.

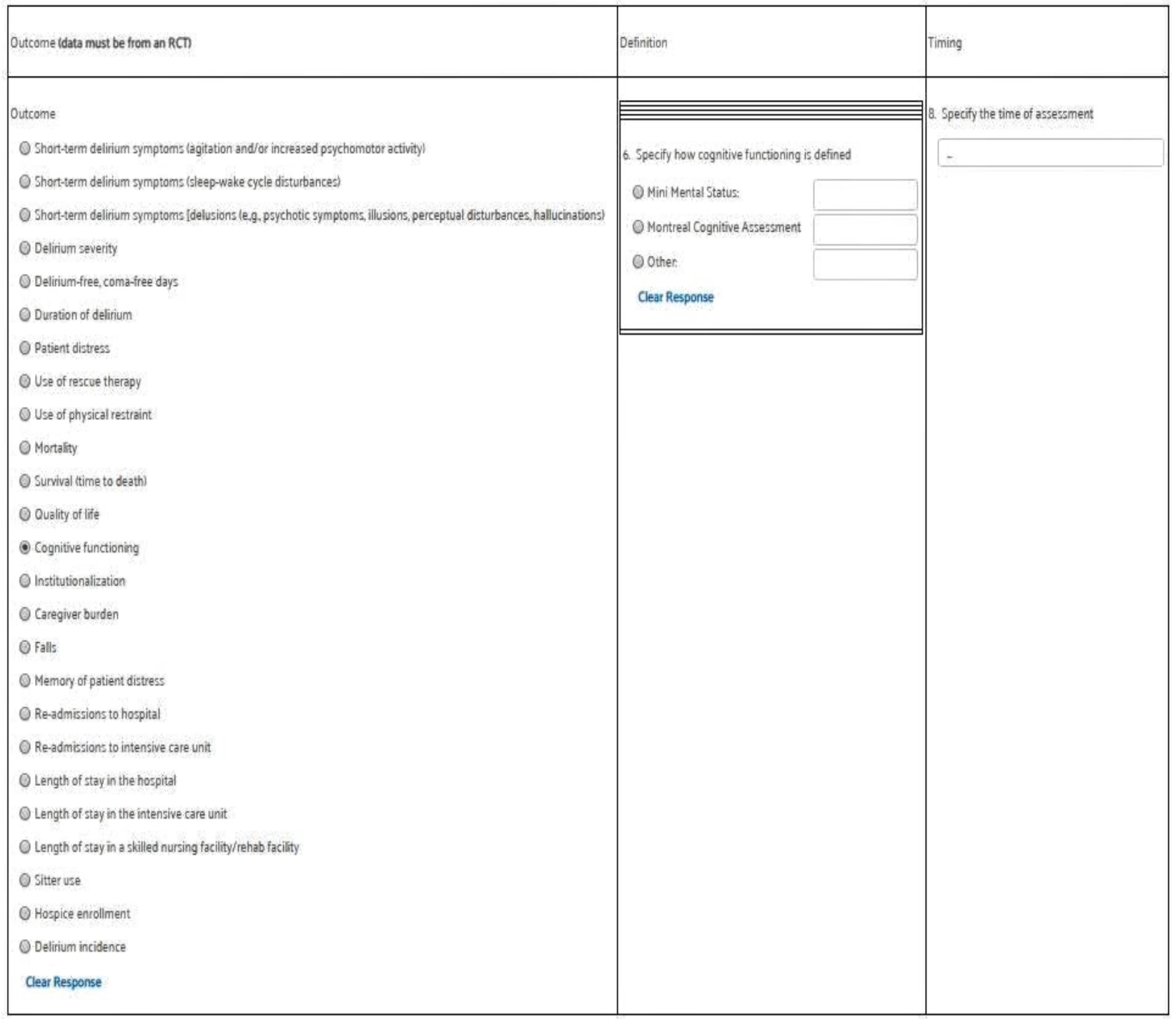


Figure B-10. Outcomes definition form (delirium severity specific)

Please flll out one form for each outcome. Indicate the outcome being reported on this form

\begin{tabular}{|c|c|c|}
\hline Outcome (data must be from an RCT) & Definition & Timing \\
\hline 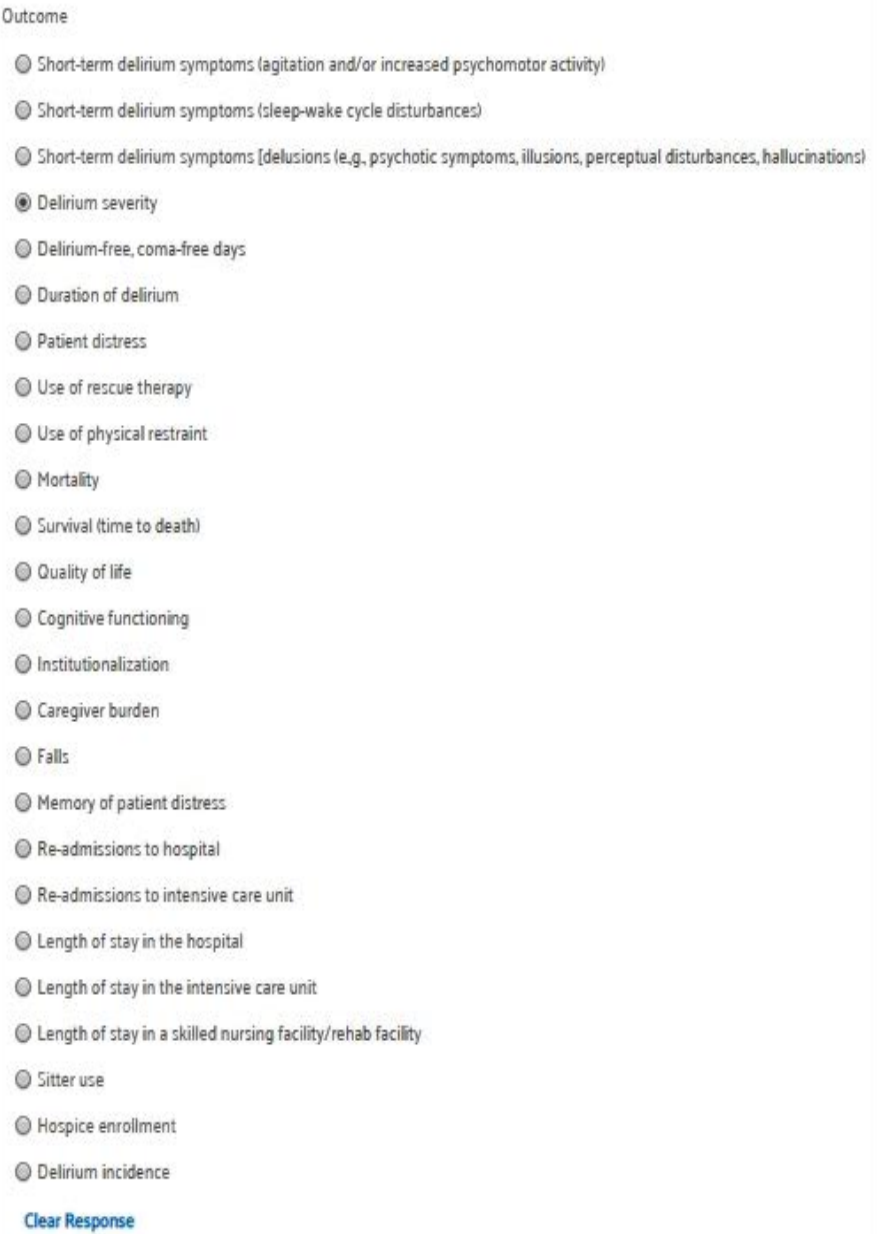 & $\begin{array}{l}\text { 3. Specify how delirium severity is defined } \\
\text { O DRS-R-98 } \\
\text { O Visual analog scale } \\
\text { Other } \\
\text { Clear Response }\end{array}$ & 8. Specify the time of assessment \\
\hline
\end{tabular}




\section{Figure B-11. Outcomes definition form (general)}

Please flll out one form for each outcome. Indicate the outcome being reported on this form.

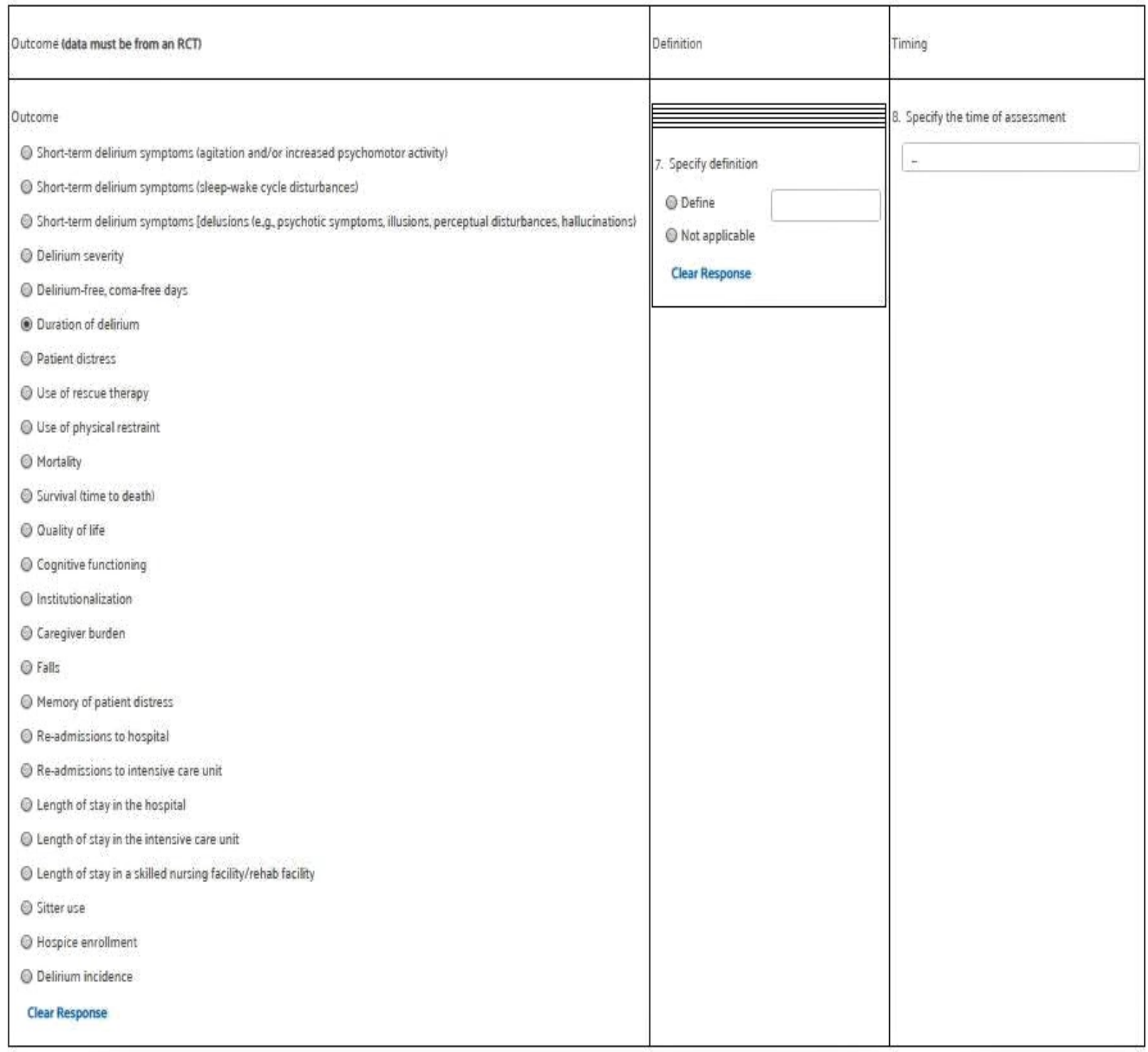


Figure B-12. Outcomes definition form (adverse events: appetite change specific)

\begin{tabular}{|c|c|c|}
\hline Adverse everit table (data can be from an RCT, controlled clinical trial, or prospective cohort with or without a comparison & Definition & Timing of outcome \\
\hline $\begin{array}{l}\text { 9. Adverse event } \\
\text { O Sedation } \\
\text { O Weight } \\
\text { O Change in appetite } \\
\text { O Cardiac effects } \\
\text { O Neurologic effects } \\
\text { O Hypersensitivity reactions } \\
\text { O Inappropriate continuation of antipsychotic medication } \\
\text { O Swallowing difficulties } \\
\text { O Aspiration pneumonia } \\
\text { Clear Response }\end{array}$ & \begin{tabular}{||l} 
11. Specify change in appetite outcome \\
O Increase in appetite \\
O Decrease in appetite \\
Clear Response
\end{tabular} & 15. Specify the time of assessment \\
\hline
\end{tabular}

Figure B-13. Outcomes definition form (adverse events: cardiac effects specific)

\begin{tabular}{|c|c|c|}
\hline Adverse event table (data can be from an RCT, controlled clinical trial, or prospective cohort with or without a comparison & Definition & Timing of outcome \\
\hline 9. Adverse event & \multirow[b]{2}{*}{ 12. Specify cardiac outcome } & 15. Specify the time of assersment \\
\hline O Sedation & & \multirow[t]{10}{*}{ - } \\
\hline O Weight & OProlongation in the OTC intervat & \\
\hline C Change in appetite & OProlongation in the OT interval & \\
\hline - Cardiac effects & O Other & \\
\hline O Neurologic effects & Clear Response & \\
\hline O Hypersensthivity reactions. & & \\
\hline O Inappropriate continuation of antipsychotic medication & & \\
\hline O Swallowing difficulties & & \\
\hline OAspiration pneumonia & & \\
\hline Clear Response & & \\
\hline
\end{tabular}

Figure B-14. Outcomes definition form (adverse events: neurologic effects specific)

\begin{tabular}{|c|c|c|}
\hline Adverse event table (data can be from an $\mathrm{RCT}$, controlled clinical trial, or prospective cohort with or without a comparison & Definition & Timing of outcome \\
\hline $\begin{array}{l}\text { 9. Adverse event } \\
\text { O Sedation } \\
\text { O Weight } \\
\text { O Change in appetite } \\
\text { O Cardiac effects } \\
\text { O Neurologic effects } \\
\text { O Hypersensitivity reactions } \\
\text { O inappropriate continuation of antipsychotic medication } \\
\text { Swallowing difficulties } \\
\text { Aspiration pneumonia } \\
\text { Clear Response }\end{array}$ & $\mid$\begin{tabular}{||l} 
13. Specify neurologic outcome \\
O Extrapyramidal symptoms \\
O Other \\
Clear Response
\end{tabular} & 15. Specify the time of assessment \\
\hline
\end{tabular}


Figure B-15. Outcomes definition form (adverse events: weight specific)

\begin{tabular}{|c|c|c|}
\hline Adverse event table (data can be from an RCT, controlled clinical trial, or prospective cohort with or without a comparison & Definition & Timing of outcome \\
\hline $\begin{array}{l}\text { 9. Adverse event } \\
\text { Sedation } \\
\text { - Weight } \\
\text { Change in appetite } \\
\text { Cardiac effects } \\
\text { Neurologic effects } \\
\text { Hypersensitivity reactions } \\
\text { Inappropriate continuation of antipsychotic medication } \\
\text { Swallowing difficulties } \\
\text { Aspiration pneumonia } \\
\text { Clear Response }\end{array}$ & $\begin{array}{l}\text { 10. Specify weight outcome } \\
\text { O Weight gain } \\
\text { Weight loss } \\
\text { Clear Response }\end{array}$ & 15. Specify the time of assessment \\
\hline
\end{tabular}

Figure B-16. Outcomes definition form (general adverse events)

\begin{tabular}{|c|c|c|}
\hline Adverse event table (data can be from an RCT, controlled clinical trial, or prospective cohort with or without a comparison & Definition & Timing of outcome \\
\hline $\begin{array}{l}\text { 9. Adverse event } \\
\text { O Sedation } \\
\text { O Weight } \\
\text { O Change in appetite } \\
\text { O Cardiac effects } \\
\text { O Neurologic effects } \\
\text { O Hypersensitivity reactions } \\
\text { O Inappropriate continuation of antipsychotic medication } \\
\text { O Swallowing difficulties } \\
\text { O Aspiration pneumonia } \\
\text { Clear Response }\end{array}$ & || $\mid$\begin{tabular}{l||} 
14. Specify outcome definition \\
O Define \\
O Not applicable \\
Clear Response
\end{tabular} & 15. Specify the time of assessment \\
\hline
\end{tabular}




\section{Figure B-17. Outcomes data extraction form}

Continuous outcomes

Table L.Mean difference from other intervention group measures of outcome between-group differencel When flliling out this table, please note that the point estimate should reflect this formula (final- Baselline for the intevention group) - (final - Baseline for the reference groupl. You do not need to calculate this

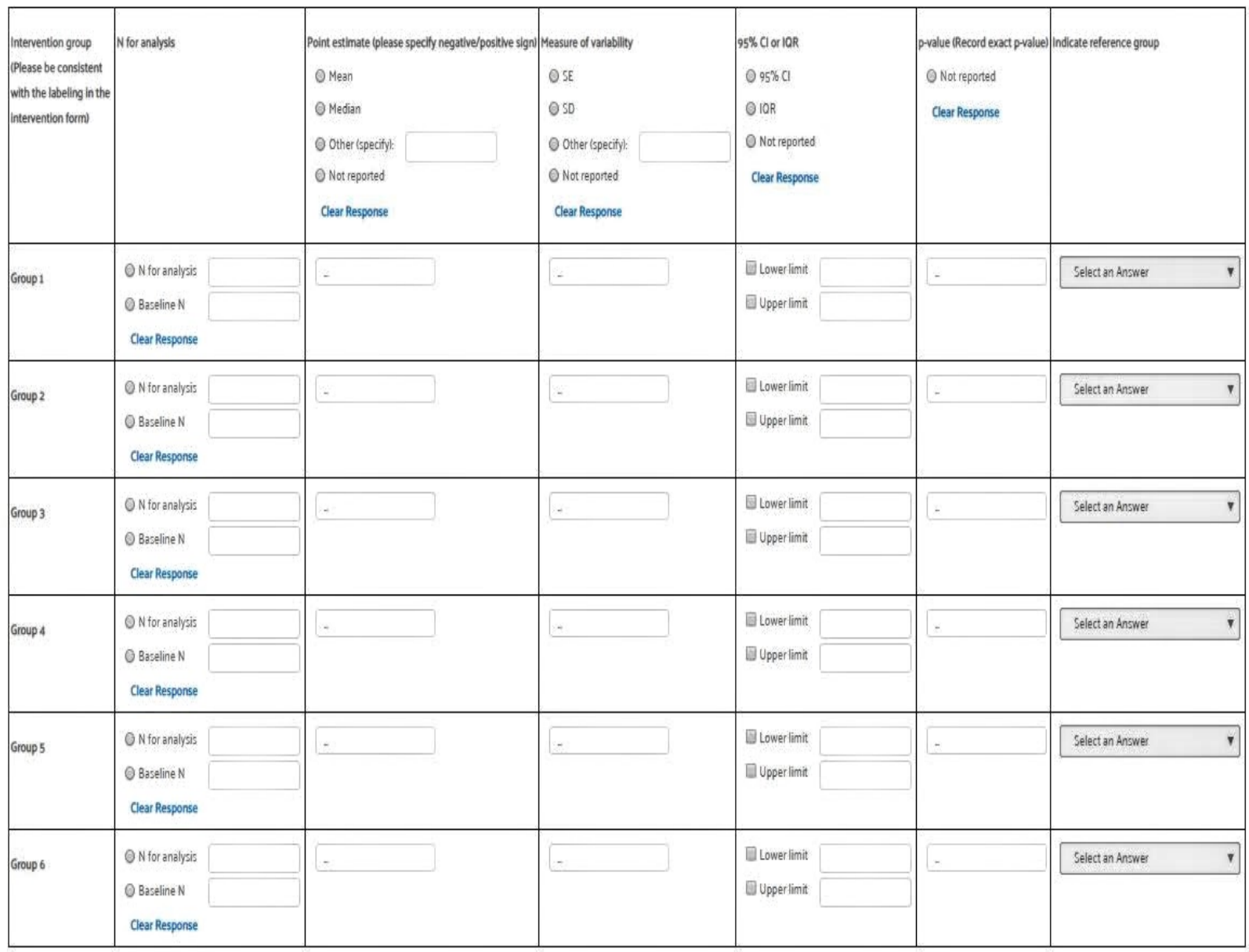


Table IL Mean difference from baseline to final measures of outcome iwithin-group difference) This should be final - Baseline. If values decrease, be sure to indicate with a negative sign $\mathrm{fH}$.

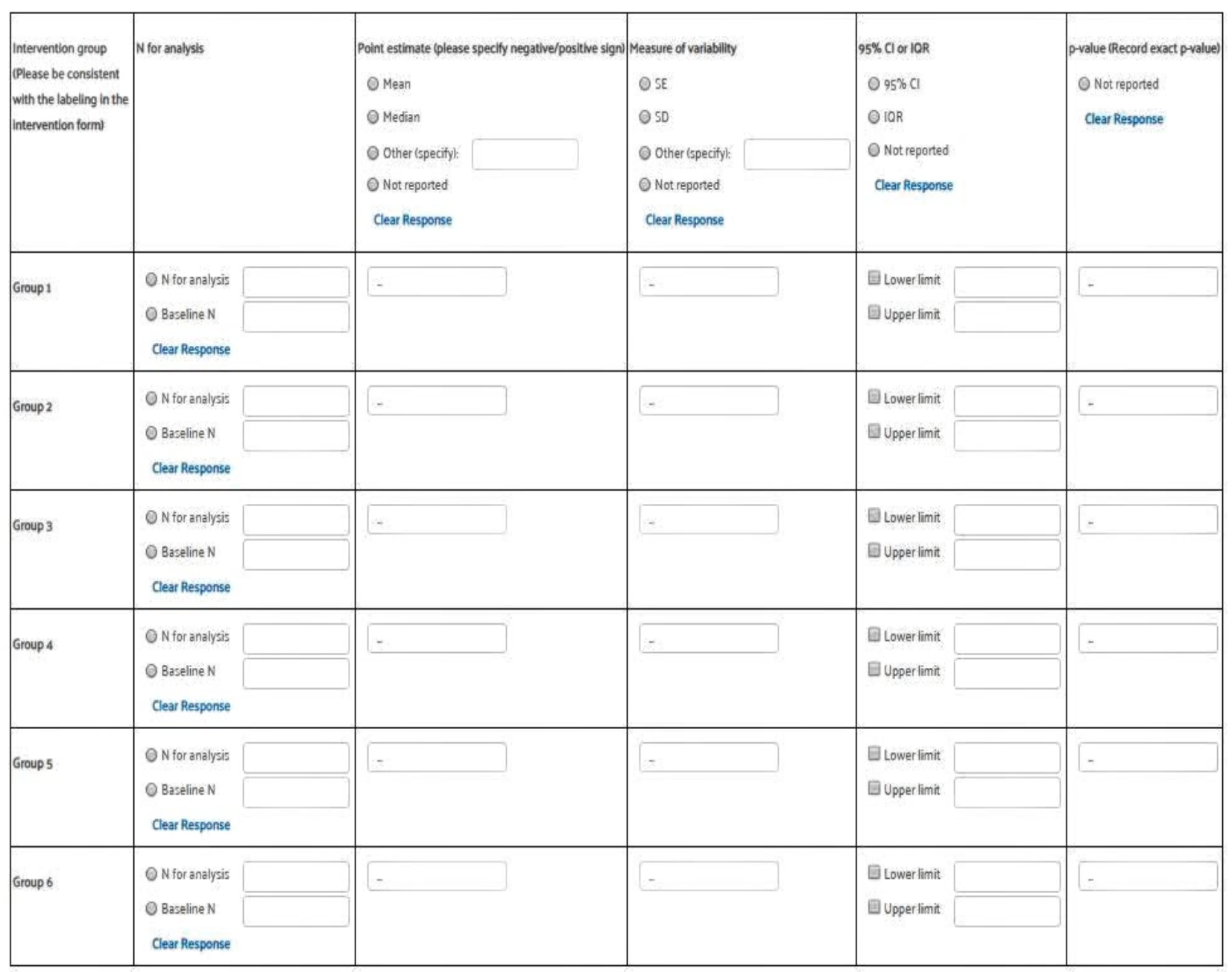


Table lli. Baseline measures of cohort

\begin{tabular}{|c|c|c|c|c|c|c|}
\hline \multirow{5}{*}{$\begin{array}{l}\text { Intervention group } \\
\text { (Please be consistent } \\
\text { with the labeling in the } \\
\text { intervention form) }\end{array}$} & \multirow{5}{*}{ N for analysis } & \multirow{3}{*}{$\begin{array}{l}\text { Point estimate (please specily negative/positive sign) } \\
\text { O Mean } \\
\text { O Median }\end{array}$} & \multirow{3}{*}{$\begin{array}{l}\text { Measure of variability } \\
\text { OSE } \\
\text { OSD }\end{array}$} & \multirow{5}{*}{$\begin{array}{l}95 \% \text { Cl or laR } \\
\text { O } 95 \% \mathrm{Cl} \\
\text { O } 10 \mathrm{R} \\
\text { O Not reported } \\
\text { Clear Response }\end{array}$} & \multirow{5}{*}{$\begin{array}{l}\text { p-value (Record exact p-value) } \\
\text { O Not reponted } \\
\text { Clear Response }\end{array}$} & \multirow{5}{*}{ Indicate reference group } \\
\hline & & & & & & \\
\hline & & & & & & \\
\hline & & O Other (specityl: & O Other (specify): & & & \\
\hline & & $\begin{array}{l}\text { O Not reported } \\
\text { Clear Response }\end{array}$ & $\begin{array}{l}\text { O Not reported } \\
\text { Clear Response }\end{array}$ & & & \\
\hline \multirow[t]{3}{*}{ Group 1} & O N for analysis & - & - & \multirow{3}{*}{$\begin{array}{l}\text { DLower limit } \\
\text { Gupper limit }\end{array}$} & \multirow[t]{3}{*}{-} & \multirow[t]{3}{*}{ Select an Answer: } \\
\hline & O Baseline N & & & & & \\
\hline & Clear Response & & & & & \\
\hline \multirow[t]{3}{*}{ Group 2} & O N for analysis & - & - & \multirow{3}{*}{$\begin{array}{l}\text { G Lower limit } \\
\text { G Upper limit }\end{array}$} & \multirow[t]{3}{*}{-} & \multirow[t]{3}{*}{ Select an Answer } \\
\hline & O Baseline $\mathrm{N}$ & & & & & \\
\hline & Clear Response & & & & & \\
\hline \multirow[t]{3}{*}{ Group 3} & O N for analysis. & - & - & \multirow{3}{*}{$\begin{array}{l}\text { G Lower limit } \\
\text { Gupper limit }\end{array}$} & \multirow[t]{3}{*}{-} & \multirow[t]{3}{*}{ Select an Answer } \\
\hline & O Baseline $\mathrm{N}$ & & & & & \\
\hline & Clear Response & & & & & \\
\hline \multirow[t]{3}{*}{ Group 4} & O N for analyyis & - & - & \multirow{3}{*}{$\begin{array}{l}\text { Q Lower limit } \\
\text { 『Upper limit }\end{array}$} & \multirow[t]{3}{*}{-} & \multirow[t]{3}{*}{ Select an Answer } \\
\hline & O Baseline N & & & & & \\
\hline & Clear Response & & & & & \\
\hline \multirow[t]{3}{*}{ Group 5} & Q N for analysis & - & - & \multirow{3}{*}{$\begin{array}{l}\text { Lower limit } \\
\text { Eupper limit }\end{array}$} & \multirow[t]{3}{*}{ - } & \multirow[t]{3}{*}{ Select an Answer } \\
\hline & O Baseline N & & & & & \\
\hline & Clear Response & & & & & \\
\hline \multirow[t]{3}{*}{ Group 6} & O N for analysis & - & - & \multirow{3}{*}{$\begin{array}{l}\text { E Lower limit } \\
\text { Gupper limit }\end{array}$} & \multirow[t]{3}{*}{-} & \multirow[t]{3}{*}{ Select an Answer } \\
\hline & O Baseline $\mathrm{N}$ & & & & & \\
\hline & Clear Response & & & & & \\
\hline
\end{tabular}




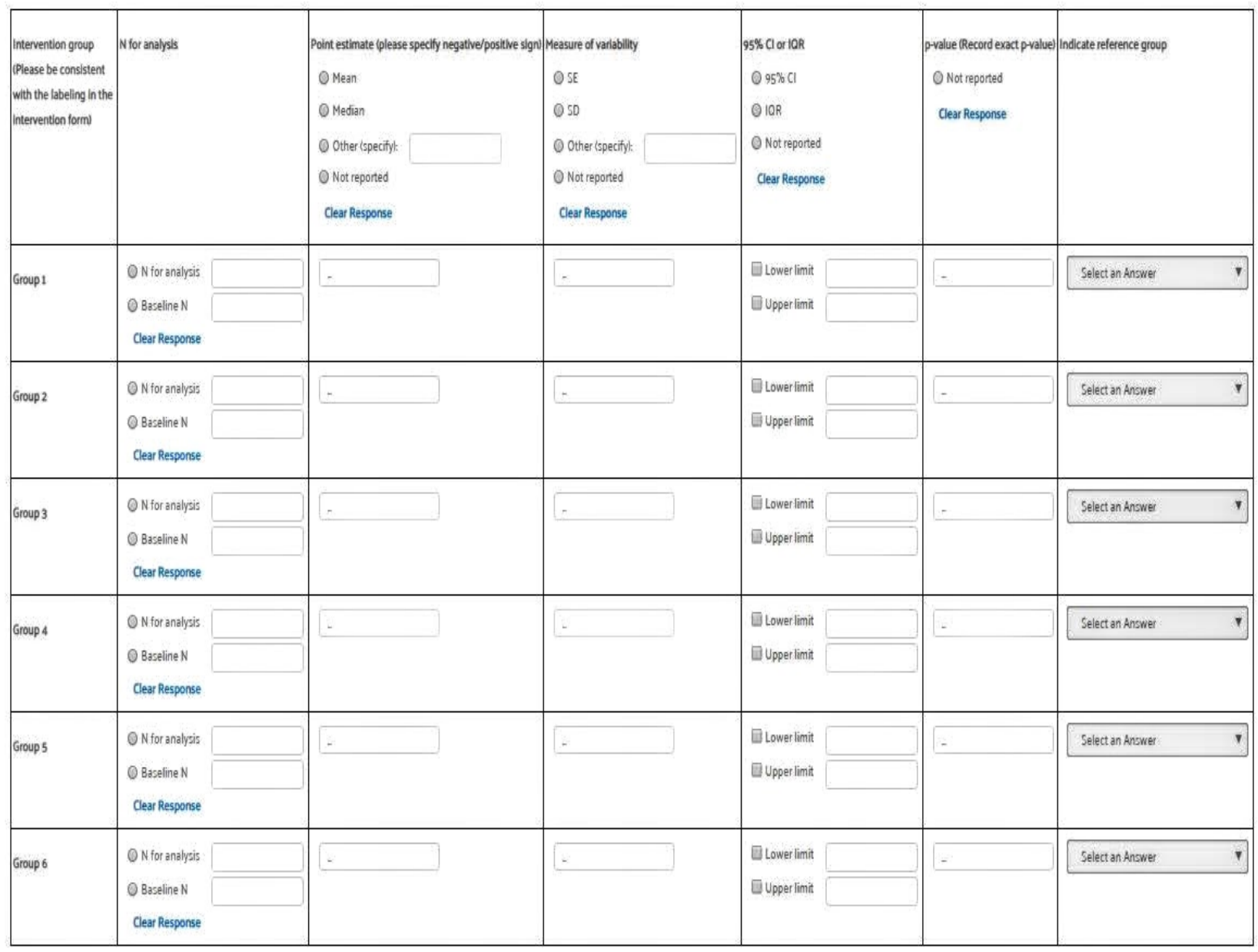


Dichotomous Outcomes

Table V. Incidence of the outcome by intervention group

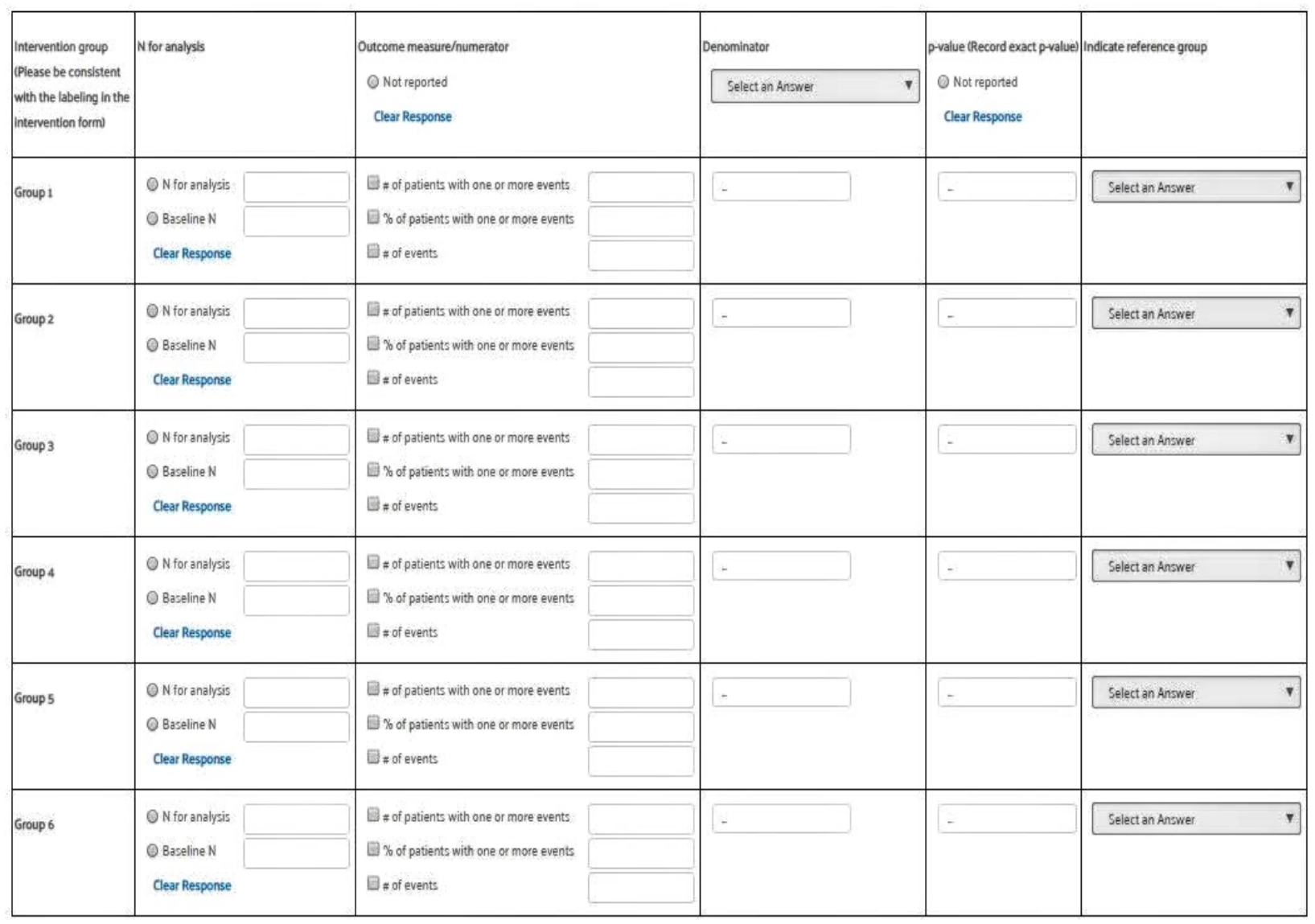


Table V. Measures of association for comparison of outcome between intervention groups

\begin{tabular}{|c|c|c|c|c|c|c|}
\hline $\begin{array}{l}\text { Intervention group } \\
\text { (Please be consistent } \\
\text { with the labeling in the } \\
\text { intervention form) }\end{array}$ & N for analysis & $\begin{array}{l}\text { Point estimate } \\
\text { O Relative risk } \\
\text { O Relative hazard } \\
\text { O Odds ratio } \\
\text { O Risk difference } \\
\text { O Other: } \\
\text { O Not reported } \\
\text { Clear Response }\end{array}$ & $\begin{array}{l}\text { Measure of variablilty } \\
\text { O SE } \\
\text { OSD } \\
\text { O Not reported } \\
\text { Clear Response }\end{array}$ & $\begin{array}{l}95 \% \text { Conflidence interval } \\
\text { O Not reported } \\
\text { Clear Response }\end{array}$ & $\begin{array}{l}\text { p-value (Record exact p-value) } \\
\text { O Not reported } \\
\text { Clear Response }\end{array}$ & Indicate reference group \\
\hline Group 1 & $\begin{array}{l}\text { O N for analysis } \\
\text { O Baseline N } \\
\text { Clear Response }\end{array}$ & - & - & $\begin{array}{l}\text { G Lower limit } \\
\text { EUpper limit }\end{array}$ & - & Select an Answer \\
\hline Group 2 & $\begin{array}{l}\text { ON for analysis } \\
\text { O Baseline } \mathrm{N} \\
\text { Clear Response }\end{array}$ & - & - & $\begin{array}{l}\text { DLower limit } \\
\text { Dupper limit }\end{array}$ & - & Select an Answer \\
\hline Group 3 & $\begin{array}{l}\text { O N for analysis } \\
\text { O Baseline N } \\
\text { Clear Response }\end{array}$ & - & - & $\begin{array}{l}\text { DLower limit } \\
\text { EUpper limit }\end{array}$ & - & Select an Answer \\
\hline Group 4 & $\begin{array}{l}\text { O N for analysis } \\
\text { Baseline N } \\
\text { Clear Response }\end{array}$ & - & - & $\begin{array}{l}\text { E Lower limit } \\
\text { —upper limit }\end{array}$ & - & Select an Answer \\
\hline Group 5 & $\begin{array}{l}\text { O N for analysis } \\
\text { O Baseline } \mathrm{N} \\
\text { Clear Response }\end{array}$ & - & - & $\begin{array}{l}\text { G Lower limit } \\
\text { [Upper limit }\end{array}$ & - & Select an Answer \\
\hline Group 6 & $\begin{array}{l}\text { O N for analysis } \\
\text { O Baseline N } \\
\text { Clear Response }\end{array}$ & - & - & $\begin{array}{l}\text { ELower limit } \\
\text { EUpper limit }\end{array}$ & - & Select an Answer \\
\hline
\end{tabular}




\section{Figure B-18. Risk of bias for randomized controlled trials}

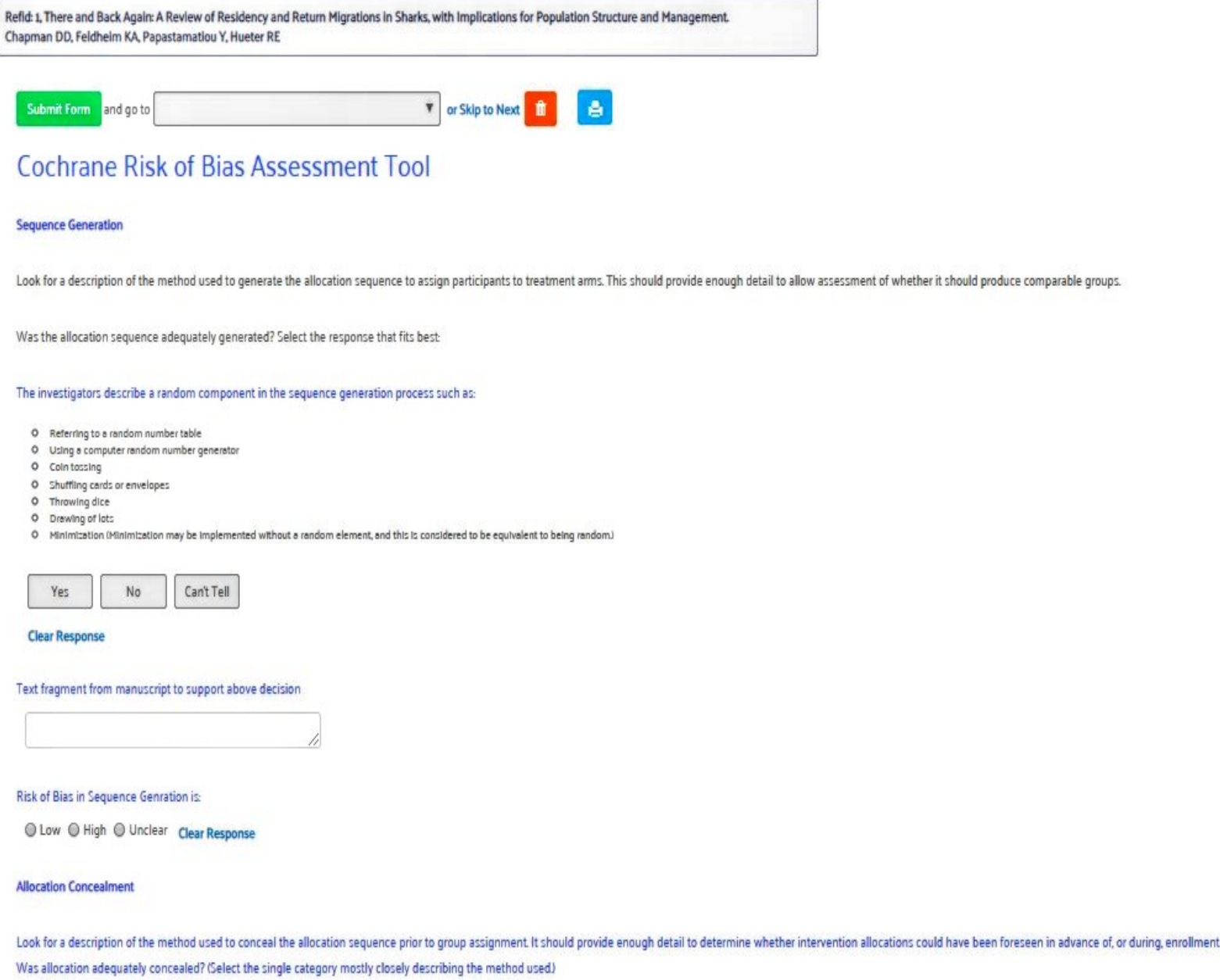




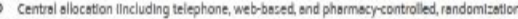

- Sequentislily numbered drug contaliner: of identical sppesance

- Sequentilily numbered, opsque, sesied emvelopes:

\section{Ye:}

Clear Response

Text fragment from manuscript to support above decision

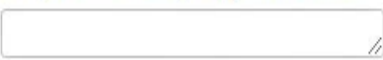

Risk of Bias in Allocation Concealment is:

O Low O High O Unclear Clear Response

Blinding of participants, personnel and outcome assessors

Look for a description of all measures used, if any, to blind study participants, personnel and outcome assessors from knowledge of which intervention a participant received. Note any information on whether the intended blinding was successful

Blinding for outcomes for each population group:

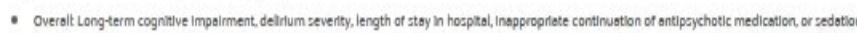

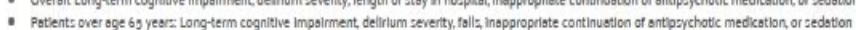

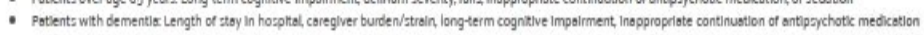

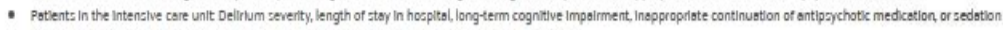

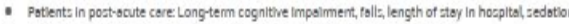

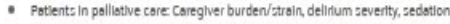

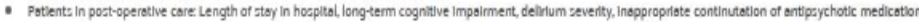

Was knowledge of the allocated intervention adequately prevented during this study? Select the option most closely describing the status of blinding:

Any one of the following is considered adequate blinding:

- No binding, but the review outhora judge that the cutcome and the outcome messurement ore not likely to be influenced by lock of blinding

Blinding of particlponts and key ztudy personnel enzured, ond it t un unikely that the blinding could have been broken.

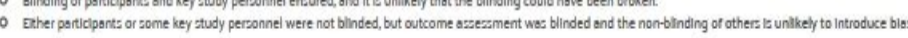

Adequate blinding demonstrated

No

Cant Tel

Clear Response

Text fragment from manuscript to support above decision 
Risk of Bias in binding of participants, personnel and outcomes assessors is:

O Low $O$ High O Unclear Clear Response

Incomplete Outcome Data

Primary Outcome: Describe the completeness of outcome data for the primary outcome, including attrition and exclusions from the analysis.

Were attrition and exclusions reported?

Yes No

Clear Response

Were incomplete outcome data adequately addressed? Select the statement below that best characterizes the status of incomplete outcome data.

No missing outcome data

Reasons for missing outcome data unlikely to be related to true outcome (for survival data, censoring unlikely to be introducing bias) because:

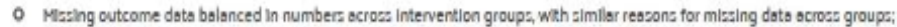

- For dichotomous outcome data, the proportion of mizzing outcomes compared with obreved event ritk not enough to hove a clinically relevant impoct on the intervention effect eztimate;

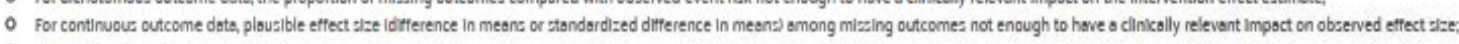

- Mashng dato have been imputed ualing appropriate method:

No missing outcome data

Potentially missing outcome data

Unclear

Clear Response

Provide text fragments from the manuscript or other evidence to support your decision:

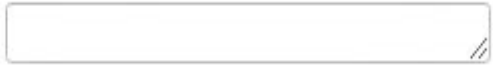

23. Risk of bias from missing outcome data

Low O High O Unclear Clear Response

(Instructions to review coordinator: repeat these questions for any additional main outcome or class of outcomes.)

Selective Outcome Reporting

Are reports of the study free of suggestion of selective outcome reporting? Select the statement below that best characterizes the completeness of outcome reporting. 
- The study protocol is avalloble and all of the studys pre-specined (primary and seconderyl outcomes that are of interest in the review have been reported in the pre-specified way.

- The study protocol is not avalioble but it is clear that the published report include oll expected outcomes, including those that were pre-specined iNote to reter. convincing text of this noture may be uncommoni.

Yes No Unclear

\section{Clear Response}

Provide text fragments from the manuscript or other evidence to support your decision:

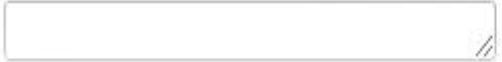

Risk of Bials from Selective Outcome Reporting

Low O High O Unclear Clear Response

Unstructions to review coordinator: repeat these questions for any additional main outcome or class of outcomes.)

Other Sources of Blas

State any important concerns about bias not addressed in the other domains in the tool. Was the study apparently free of other problems that could put it at a high risk of bias? Select the responses that apply:

The study appears to be free of other sources of bias

Yes No Unclear

\section{Clear Response}

Provide text fragments from the manuscript or other evidence to support your decision:

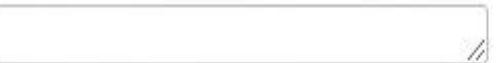

Submit Form and go to




\section{Figure B-19. Risk of bias for observational studies}

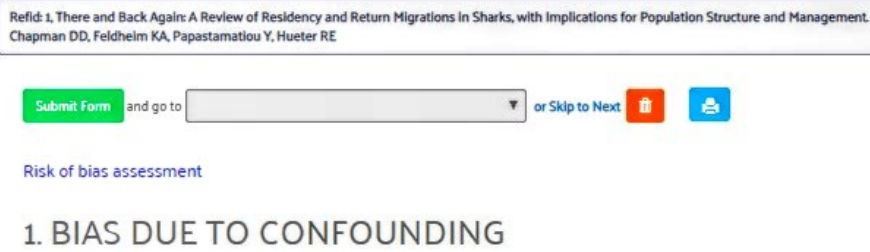

\begin{tabular}{|c|c|c|}
\hline Signalling question & Description & Location in text or source \\
\hline $\begin{array}{l}1.1 \text { Is there potential for confounding of the effect of intervention in this study? } \\
\text { If N/PN to ort the study can be considered to be at low risk of bias due to confounding and } \\
\text { no further signalling questions need be considered } \\
\text { O Yes O Probably yes O Probably no O No clear Response }\end{array}$ & 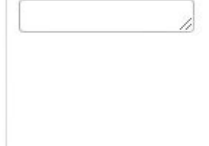 & 11 \\
\hline $\begin{array}{l}\text { If Y/PY to 1.1: determine whether there is a need to assess time-varying confounding: } \\
\text { 1.2. Was the analysis based on splitting participants' follow up time according to } \\
\text { intervention received? } \\
\text { If N/PN, answer questions relating to baseline confounding (1.4 to 1.6) } \\
\text { If Y/PY, proceed to question } 1.3 \text {. } \\
\text { O Yes O Probably yes O Probably no O No O No information clear Response }\end{array}$ & & \\
\hline $\begin{array}{l}\text { 1.3. Were intervention discontinuations or switches likely to be related to factors that are } \\
\text { prognostic for the outcome? } \\
\text { If N/PN, answer questions relating to baseline confounding ( } 1.4 \text { to } 1.6 \text { ) } \\
\text { If Y/PY, answer questions relating to both baseline and time-varying confounding ( } 1.7 \text { and } 1.8 \text { ) } \\
\text { O Yes O Probably yes O Probably no O No O No information Clear Response }\end{array}$ & & \\
\hline
\end{tabular}




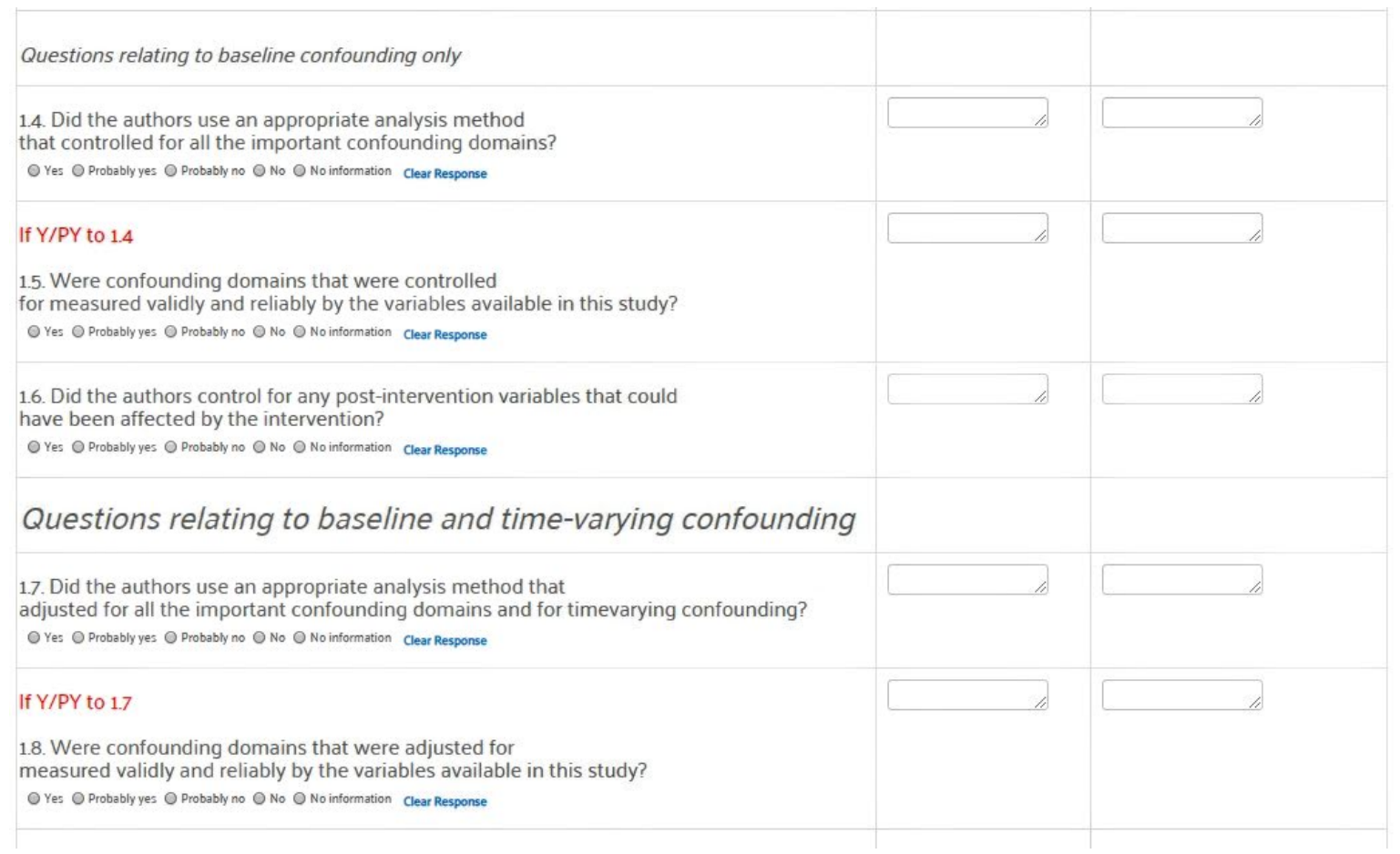


DOMAIN 1: RISK OF BIAS JUDGEMENT

O Lo

O Moderate

O Serious

O critical

O No information

clear Response
Support for judgement

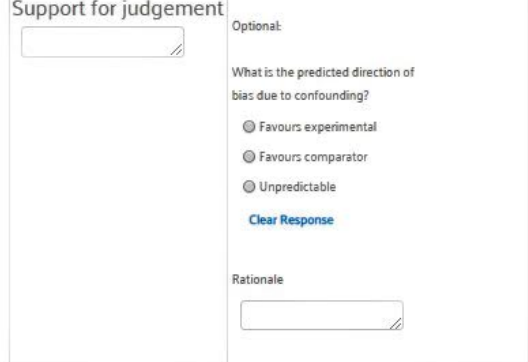

\section{BIAS IN SELECTION OF PARTICIPANTS INTO THE STUDY}

\begin{tabular}{|c|c|c|}
\hline Signalling question & $\begin{array}{l}\text { Description } \\
\text { Plus location in text or source } \\
\text { (reference, } \mathrm{pg} \& \text { \%/fig/table/other) }\end{array}$ & Location in text or source \\
\hline $\begin{array}{l}\text { If Y/PY to } 2.1 \\
\text { 2.2. Were the postintervention variables that influenced } \\
\text { selection likely to be associated with intervention? } \\
\text { O Yes O Probably yes O Probably no O No O No information Clear Response }\end{array}$ & 4 & 10 \\
\hline
\end{tabular}




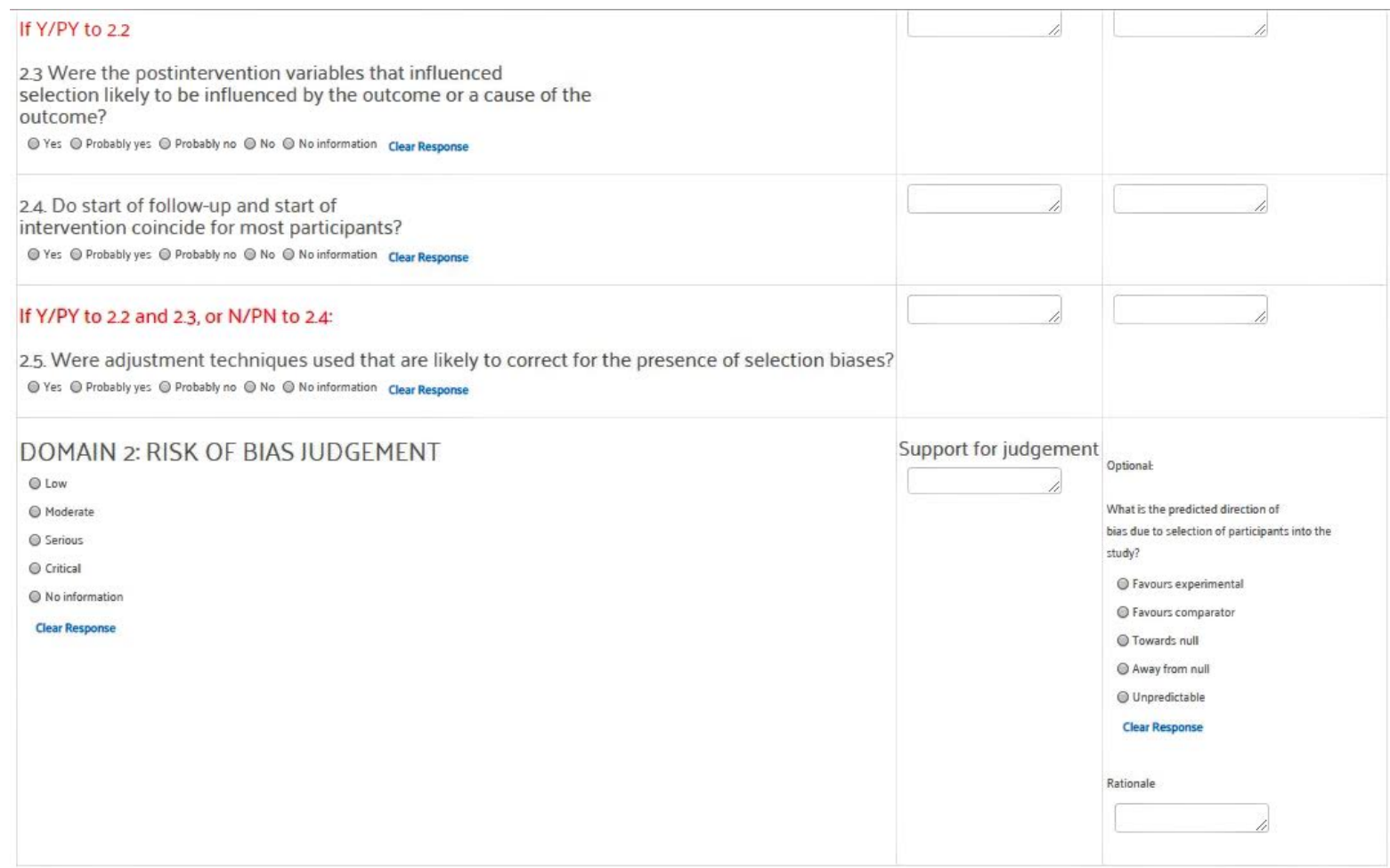

B-31 


\section{BIAS IN CLASSIFICATION OF INTERVENTIONS}

\begin{tabular}{|c|c|c|}
\hline Signalling question & $\begin{array}{l}\text { Description } \\
\text { Plus location in text or source } \\
\text { (reference, pg \& } \pi / \text { /fig/table/other) }\end{array}$ & 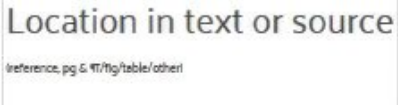 \\
\hline $\begin{array}{l}\text { 3.1 Were intervention groups clearly defined? } \\
\text { O Yes O Probably yes O Probably no O No O No information Clear Response }\end{array}$ & 11 & 11 \\
\hline $\begin{array}{l}3.2 \text { Was the information used to define intervention groups } \\
\text { recorded at the start of the intervention? } \\
\text { O Yes O Probably yes O Probably no O No O No information Clear Response }\end{array}$ & 11 & 11 \\
\hline $\begin{array}{l}\text { 3.3 Could classification of intervention status have been } \\
\text { affected by knowledge of the outcome or risk of the outcome? } \\
\text { O Yes O Probably yes O Probably no O No O No information Clear Response }\end{array}$ & & \\
\hline $\begin{array}{l}\text { DOMAIN 3: RISK OF BIAS JUDGEMENT } \\
\text { O Low } \\
\text { O Moderate } \\
\text { O Serious } \\
\text { O critical } \\
\text { O No information } \\
\text { Clear Response }\end{array}$ & Support for judgement & $\begin{array}{l}\text { Optionat } \\
\text { What is the predicted direction of } \\
\text { bias due to measurement of outcomes or } \\
\text { interventions? } \\
\text { O Favours experimental } \\
\text { O Favours comparator } \\
\text { O Towards null } \\
\text { O Away from null } \\
\text { O Unpredictable } \\
\text { Clear Response } \\
\text { Rationale }\end{array}$ \\
\hline
\end{tabular}




\section{BIAS DUE TO DEVIATIONS FROM INTENDED INTERVENTIONS}

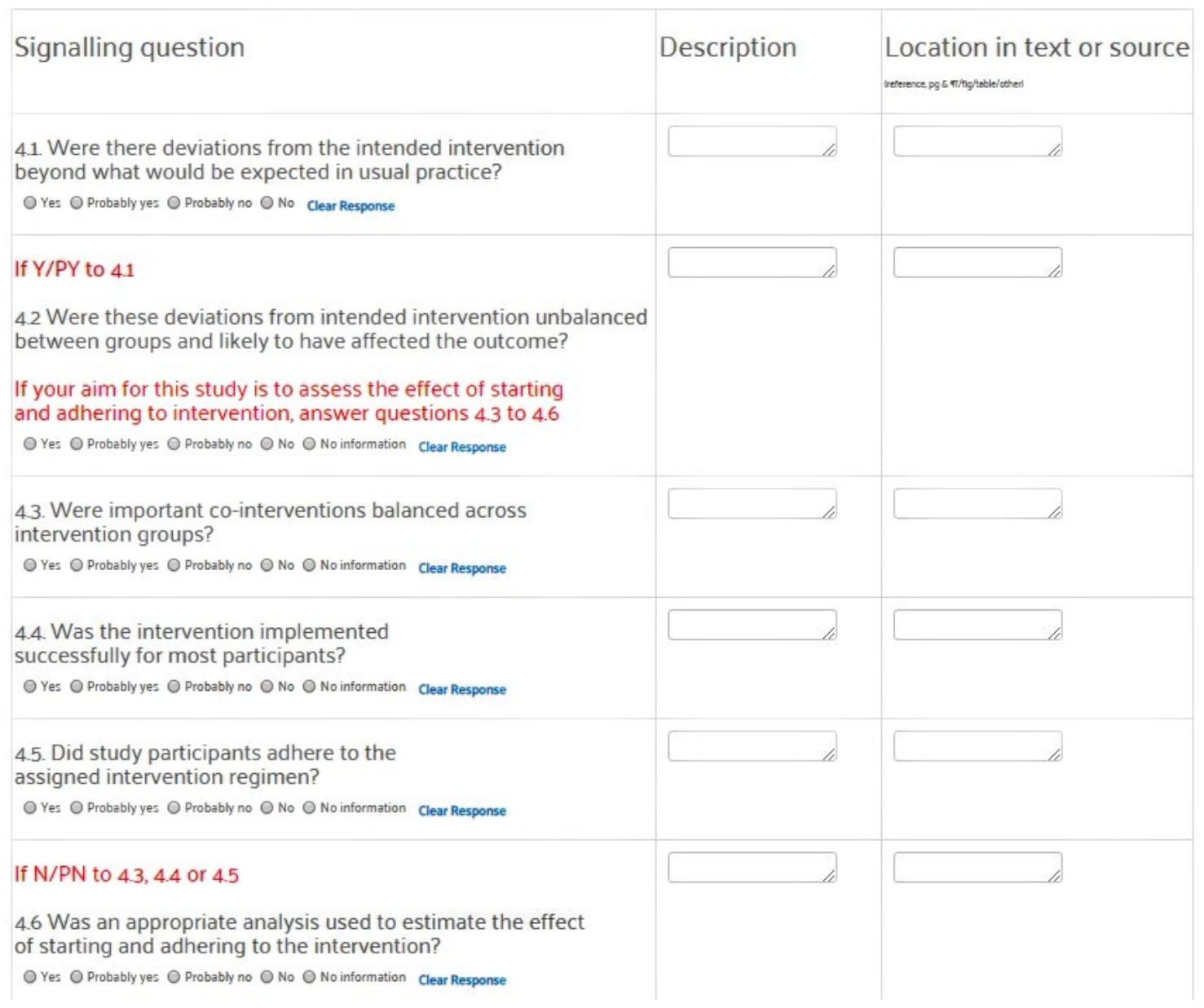




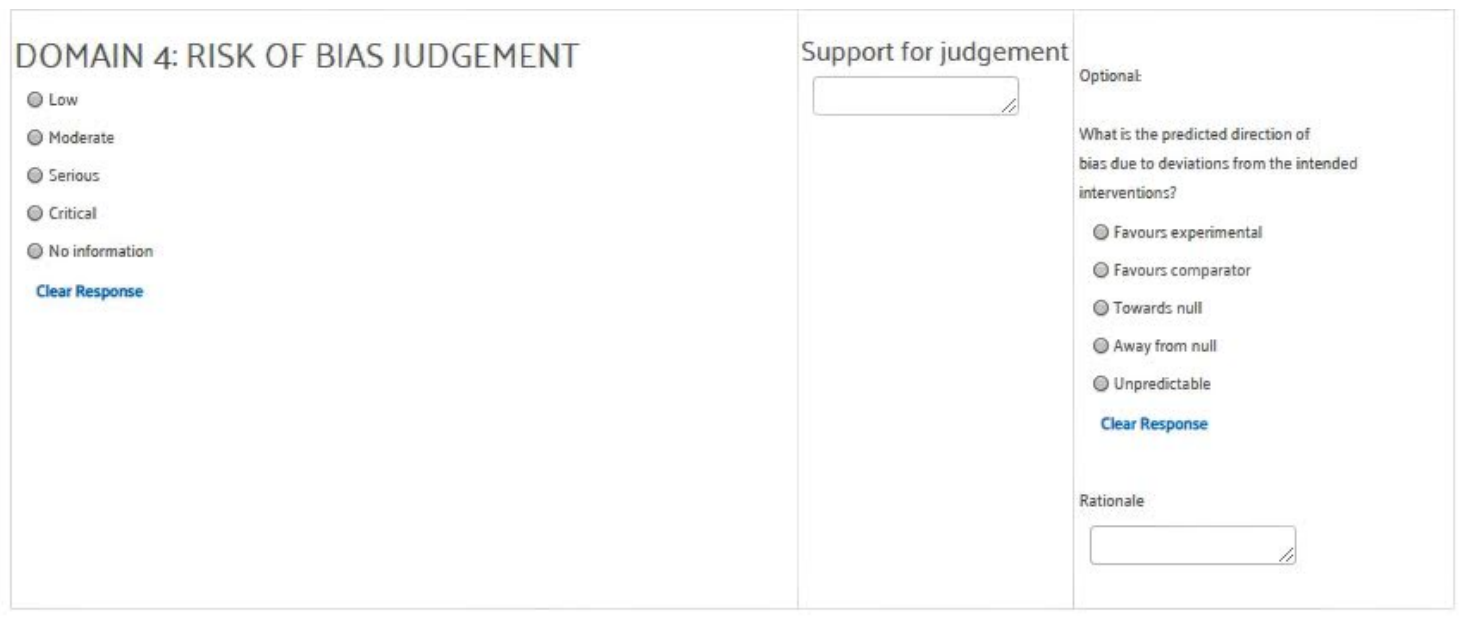

\section{BIAS DUE TO MISSING DATA}

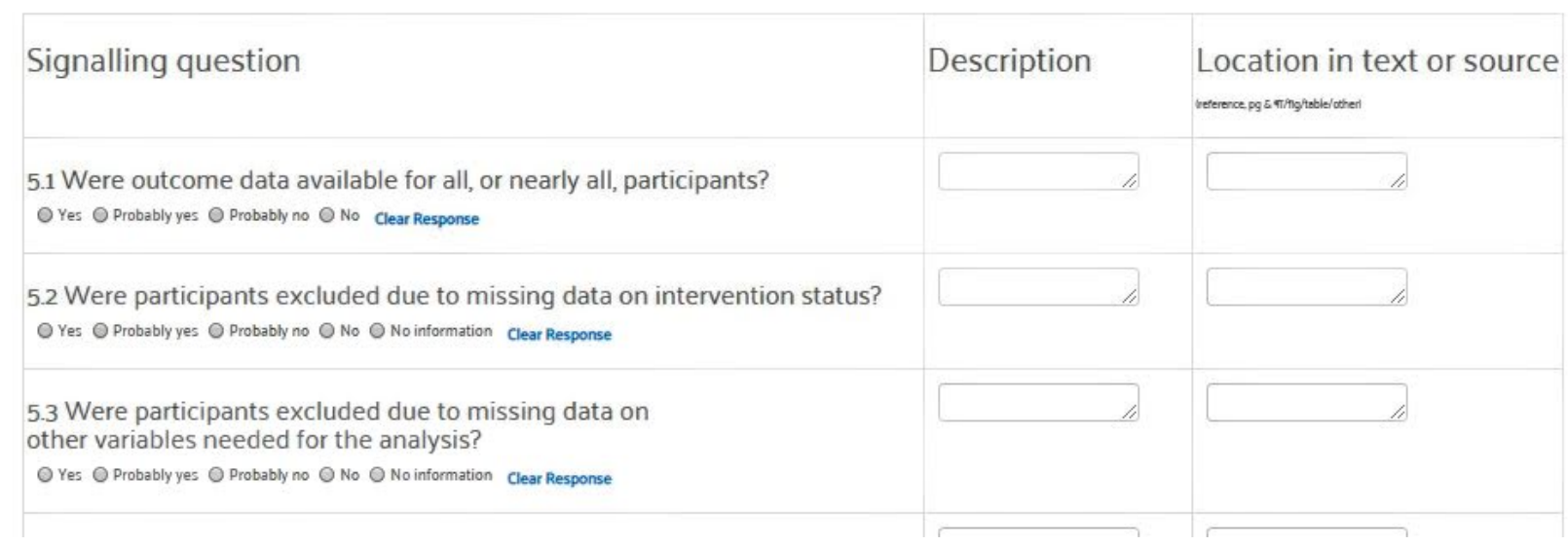




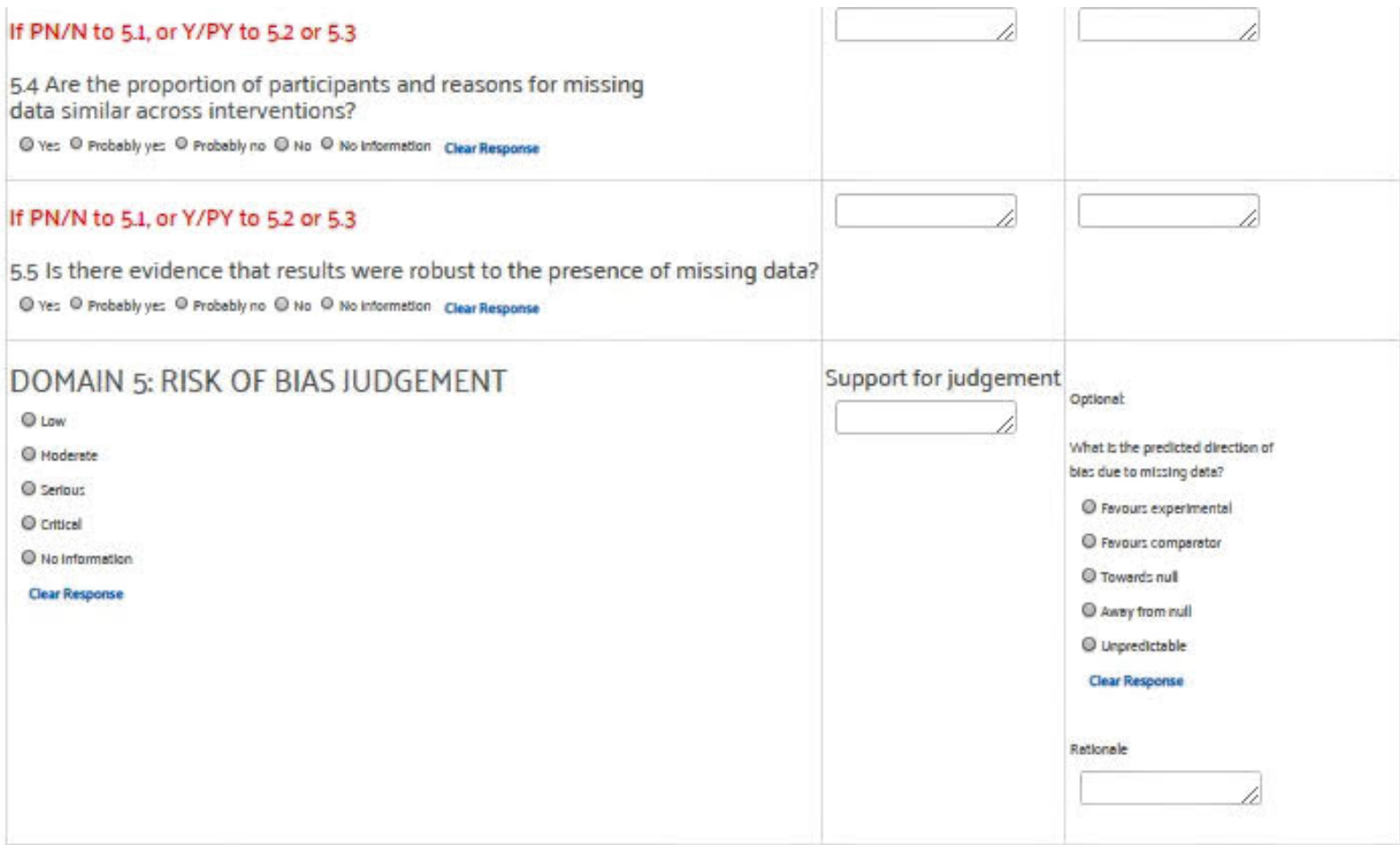

\section{BIAS IN MEASUREMENT OF OUTCOMES}

\begin{tabular}{|c|c|c|}
\hline Signalling question & Description & Location in text or source \\
\hline $\begin{array}{l}6.1 \text { Could the outcome measure have been influenced by } \\
\text { knowledge of the intervention received? } \\
\text { Y ves O Probobly ye O Probobly no } O \text { No Cear Response }\end{array}$ & 10 & \\
\hline $\begin{array}{l}6.2 \text { Were outcome assessors aware of the intervention } \\
\text { received by study participants? } \\
\text { O yez O Probobly yes O Probobly no } 0 \text { No No ntoemetion Clear Response }\end{array}$ & & \\
\hline $\begin{array}{l}\text { 6.3 Were the methods of outcome assessment comparable } \\
\text { across intervention groups? } \\
\text { O yes O Proboblyye } 0 \text { probobly no } O \text { No } O \text { No intometon Clear Respome }\end{array}$ & 10 & \\
\hline
\end{tabular}




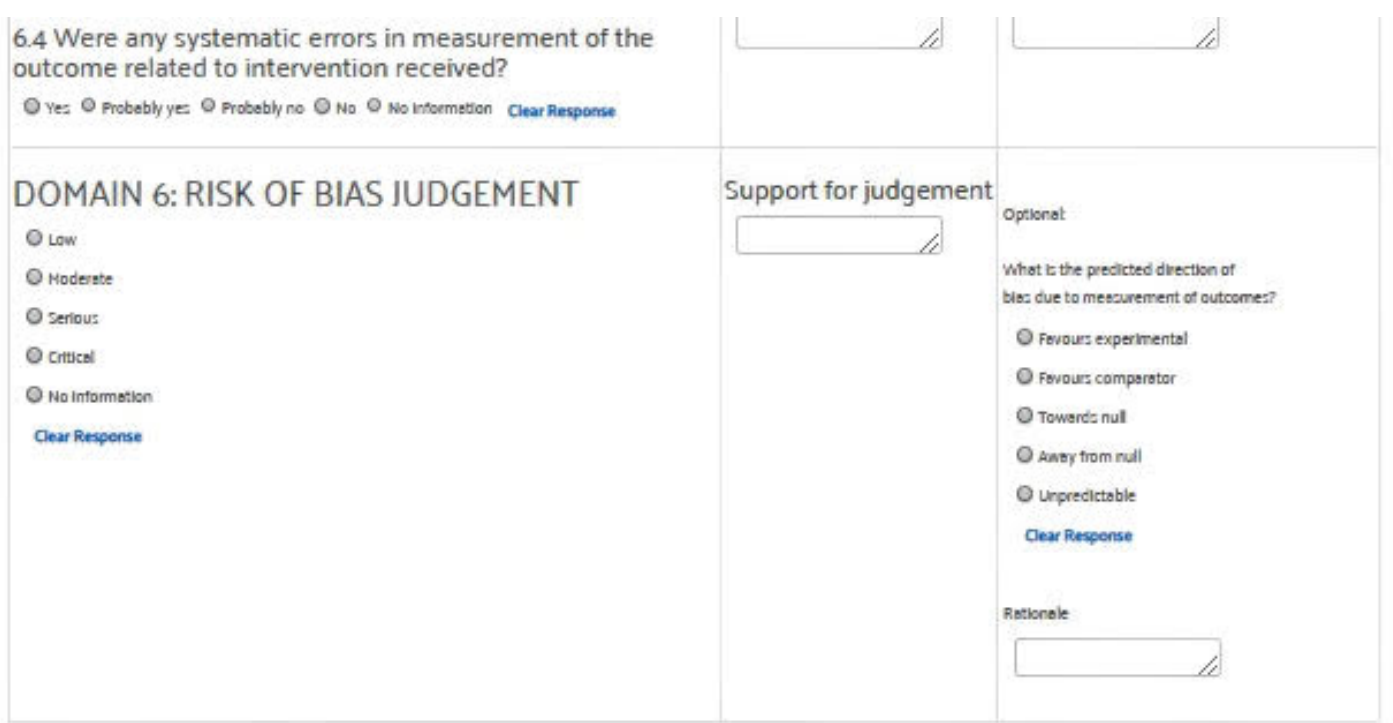

\section{BIAS IN SELECTION OF REPORTED RESULT}

\begin{tabular}{|c|c|c|}
\hline Signalling question & 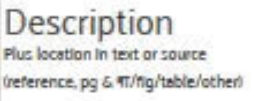 & Location in text or source \\
\hline $\begin{array}{l}\text { Is the reported effect estimate likely to be selected, on the } \\
\text { basis of the results, from } \\
7.1 \text { - multiple outcome measurements within the outcome domain? } \\
\text { O ves O probobly ye O Probobly no O No O No ntommotion clear nesponse }\end{array}$ & 10 & $1 /$ \\
\hline $\begin{array}{l}7.2 \text { _ multiple analyses of the intervention-outcome } \\
\text { relationship? } \\
\text { O yez } O \text { probably ye } O \text { probobly no } O \text { No } O \text { No intormation Clear Response }\end{array}$ & $\angle$ & \\
\hline $\begin{array}{l}7.3 \text { - different subgroups? } \\
\text { O yez O Probebly ye O Probably no O No O No intoemetion Clear Respoense }\end{array}$ & & \\
\hline
\end{tabular}




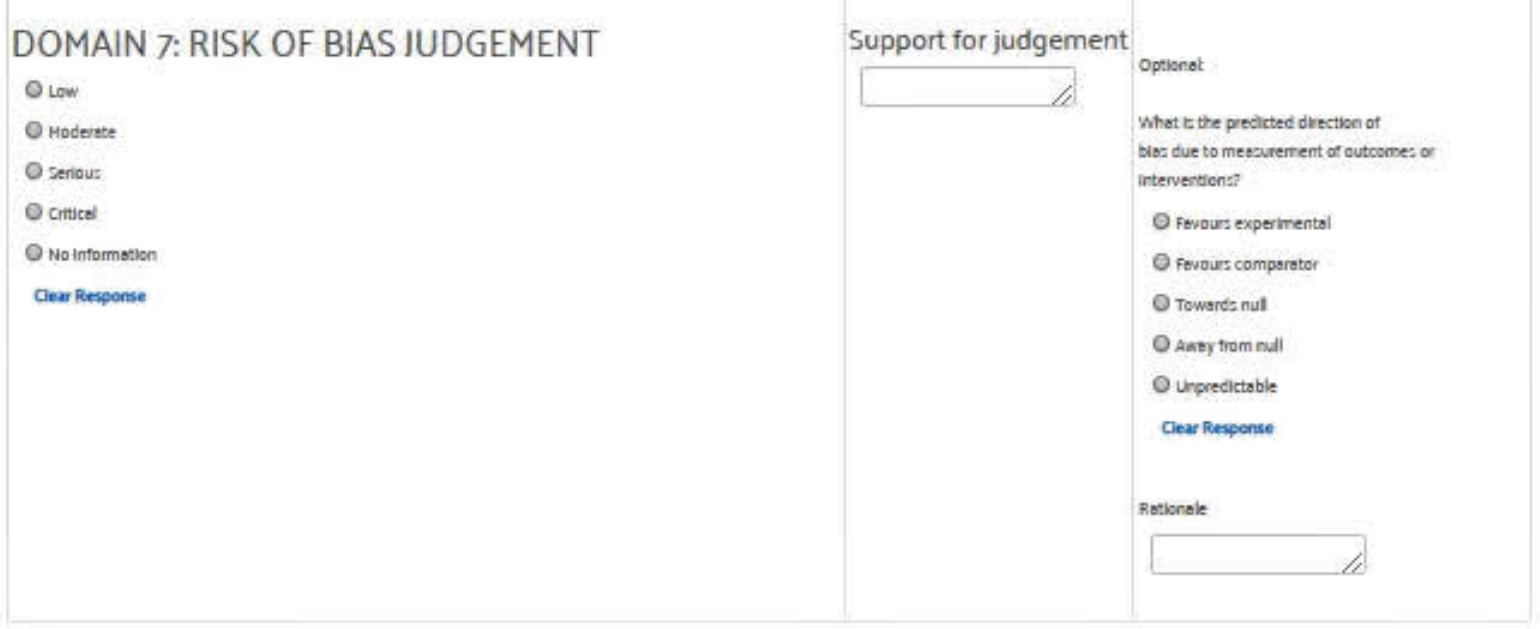

\section{OVERALL BIAS}

\section{RISK OF BIAS JUDGEMENT \\ L Low \\ OModente \\ Oserious \\ Octrices \\ O No intamerton \\ Clear Response}

Support for judgement
Optienst
What $t$ the presicted divestion of
bles due to mesasrement of outcomes o:
intervertion:?
O Fovous experimental
- Fevour comperator
O Towerdz nul
O Anay trom null
- Unpredictoble
Clear Respense

Fistionale 


\section{Appendix C. Included and Excluded Articles}

\section{Included Articles}

1. Abdelgalel EF. Dexmedetomidine versus haloperidol for prevention of delirium during non-invasive mechanical ventilation. Egyptian Journal of Anaesthesia. 2016;32(4):473-81. doi:

10.1016/j.egja.2016.05.008.

2. Agar MR, Lawlor PG, Quinn S, et al. Efficacy of Oral Risperidone, Haloperidol, or Placebo for Symptoms of Delirium Among Patients in Palliative Care: A Randomized Clinical Trial. JAMA Intern Med. 2017 Jan 1;177(1):34-42. doi: 10.1001/jamainternmed.2016.7491. PMID: 27918778.

3. Al-Qadheeb NS, Skrobik Y, Schumaker G, et al. Preventing ICU Subsyndromal Delirium Conversion to Delirium With Low-Dose IV Haloperidol: A Double-Blind, Placebo-Controlled Pilot Study. Crit Care Med. 2016 Mar;44(3):58391. doi: 10.1097/ccm.0000000000001411. PMID: 26540397.

4. Atalan N, Efe Sevim M, Akgun S, et al. Morphine is a reasonable alternative to haloperidol in the treatment of postoperative hyperactive-type delirium after cardiac surgery. J Cardiothorac Vasc Anesth. 2013 Oct;27(5):933-8. doi: 10.1053/j.jvca.2013.01.017. PMID: 23791495.
5. Bakri MH, Ismail EA, Ibrahim A. Comparison of dexmedetomidine or ondansetron with haloperidol for treatment of postoperative delirium in trauma patients admitted to intensive care unit: Randomized controlled trial. Anaesthesia, Pain and Intensive Care. 2015;19(2):118-23.

6. Boettger S, Breitbart W, Passik S. Haloperidol and risperidone in the treatment of delirium and its subtypes. European Journal of Psychiatry. 2011;25(2):59-67.

7. Boettger S, Jenewein J, Breitbart W. Haloperidol, risperidone, olanzapine and aripiprazole in the management of delirium: A comparison of efficacy, safety, and side effects. Palliative \& supportive care.

2015;13(4):1079-85. doi: 10.1017/s1478951514001059.

8. Breitbart W, Marotta R, Platt MM, et al. A double-blind trial of haloperidol, chlorpromazine, and lorazepam in the treatment of delirium in hospitalized AIDS patients. Am J Psychiatry. 1996 Feb;153(2):231-7. doi: 10.1176/ajp.153.2.231. PMID: 8561204.

9. Breitbart W, Tremblay A, Gibson C. An open trial of olanzapine for the treatment of delirium in hospitalized cancer patients. Psychosomatics. 2002 May-Jun;43(3):17582. doi: 10.1176/appi.psy.43.3.175. PMID: 12075032. 
10. Carrasco G, Baeza N, Cabre L, et al. Dexmedetomidine for the Treatment of Hyperactive Delirium Refractory to Haloperidol in Nonintubated ICU Patients: A Nonrandomized Controlled Trial. Crit Care Med. 2016 Jul;44(7):1295-306. doi: 10.1097/ccm.0000000000001622. PMID: 26925523.

11. Charoenporn V. Efficacy and safety of antipsychotic medications in the treatment of delirium. Journal of the Medical Association of Thailand. 2018;101(3):361-6.

12. Devlin JW, Roberts RJ, Fong JJ, et al. Efficacy and safety of quetiapine in critically ill patients with delirium: a prospective, multicenter, randomized, double-blind, placebo-controlled pilot study. Crit Care Med. 2010 Feb;38(2):419-27. doi: 10.1097/CCM.0b013e3181b9e302. PMID: 19915454.

13. Drinkwater B, Billyard T. Effect of intravenous haloperidol on the duration of delirium and coma in critically ill patients (hope-ICU). Journal of the Intensive Care Society. 2014;15(2):167-8. doi: 10.1177/175114371401500218.

14. Fox MA, Elefritz JL, Huang BM, et al. Comparison of Lurasidone Versus Quetiapine for the Treatment of Delirium in Critically Ill Patients. J Intensive Care Med. 2018 Jan 1:885066617754187. doi: 10.1177/0885066617754187. PMID: 29357776.
15. Fukata S, Kawabata Y, Fujisiro K, et al. Haloperidol prophylaxis does not prevent postoperative delirium in elderly patients: a randomized, open-label prospective trial.

Surg Today. 2014 Dec;44(12):2305-13. doi: 10.1007/s00595-014-0859-7. PMID: 24532143.

16. Girard TD, Exline MC, Carson SS, et al. Haloperidol and Ziprasidone for Treatment of Delirium in Critical Illness. N Engl J Med. 2018 Oct 22doi:

10.1056/NEJMoa1808217. PMID: 30346242.

17. Girard TD, Pandharipande PP, Carson SS, et al. Feasibility, efficacy, and safety of antipsychotics for intensive care unit delirium: the MIND randomized, placebo-controlled trial. Crit Care Med. 2010 Feb;38(2):428-37. PMID: 20095068.

18. Grover S, Kumar V, Chakrabarti S. Comparative efficacy study of haloperidol, olanzapine and risperidone in delirium. J Psychosom Res. 2011 Oct;71(4):277-81. doi: 10.1016/j.jpsychores.2011.01.019. PMID: 21911107.

19. Grover S, Mahajan S, Chakrabarti S, et al. Comparative effectiveness of quetiapine and haloperidol in delirium: A single blind randomized controlled study. World J Psychiatry. 2016 Sep 22;6(3):36571. doi: 10.5498/wjp.v6.i3.365. PMID: 27679777. 
20. Hakim SM, Othman AI, Naoum DO. Early treatment with risperidone for subsyndromal delirium after on-pump cardiac surgery in the elderly: a randomized trial. Anesthesiology. 2012 May;116(5):98797. doi: 10.1097/ALN.0b013e31825153cc. PMID: 22436797.

21. Han CS, Kim YK. A double-blind trial of risperidone and haloperidol for the treatment of delirium. Psychosomatics. 2004 Jul-Aug;45(4):297-301. doi: 10.1016/s00333182(04)70170-x. PMID: 15232043.

22. Hatta K, Kishi Y, Wada K, et al. Antipsychotics for delirium in the general hospital setting in consecutive 2453 inpatients: a prospective observational study. Int J Geriatr Psychiatry. 2014 Mar;29(3):253-62. doi: 10.1002/gps.3999. PMID: 23801358.

23. Horikawa N, Yamazaki T, Miyamoto $\mathrm{K}$, et al. Treatment for delirium with risperidone: results of a prospective open trial with 10 patients. Gen Hosp Psychiatry. 2003 Jul-Aug;25(4):289-92. PMID: 12850662.

24. Hu H, Deng W, Yang H, et al. Olanzapine and haloperidol for senile delirium: A randomized controlled observation. Chinese Journal of Clinical Rehabilitation. 2006;10(42):188-90.
25. Hui D, Frisbee-Hume S, Wilson A, et al. Effect of Lorazepam With Haloperidol vs Haloperidol Alone on Agitated Delirium in Patients With Advanced Cancer Receiving Palliative Care: A Randomized Clinical Trial. JAMA. 2017 Sep 19;318(11):1047-56. doi: 10.1001/jama.2017.11468. PMID: 28975307.

26. Ikezawa K, Canuet L, Ishii R, et al. Efficacy of risperidone in the treatment of delirium in elderly patients. Psychogeriatrics. 2008;8(2):62-5. doi: 10.1111/j.1479-8301.2008.00227.x.

27. Jain R, Arun P, Sidana A, et al. Comparison of efficacy of haloperidol and olanzapine in the treatment of delirium. Indian J Psychiatry. 2017 OctDec;59(4):451-6. doi: 10.4103/psychiatry.IndianJPsychiatry_59_1 7. PMID: 29497187.

28. Kalisvaart KJ, de Jonghe JF, Bogaards MJ, et al. Haloperidol prophylaxis for elderly hip-surgery patients at risk for delirium: a randomized placebo-controlled study. J Am Geriatr Soc. 2005 Oct;53(10):1658-66. doi: 10.1111/j.15325415.2005.53503.x. PMID: 16181163.

29. Kaneko T, Cai J, Ishikura T, et al. Prophylactic consecutive administration of haloperidol can reduce the occurrence of postoperative delirium in gastrointestinal surgery. Yonago Acta Medica. 1999;42(3):179-84. 
30. Khan BA, Perkins AJ, Campbell NL, et al. Preventing Postoperative Delirium After Major Noncardiac Thoracic Surgery-A Randomized Clinical Trial. J Am Geriatr Soc. 2018 Dec;66(12):2289-97. doi: 10.1111/jgs.15640. PMID: 30460981.

31. Kim JY, Jung IK, Han C, et al. Antipsychotics and dopamine transporter gene polymorphisms in delirium patients. Psychiatry Clin Neurosci. 2005 Apr;59(2):183-8. doi: 10.1111/j.14401819.2005.01355.x. PMID: 15823165.

32. Kim KS, Pae CU, Chae JH, et al. An open pilot trial of olanzapine for delirium in the Korean population. Psychiatry Clin Neurosci. 2001 Oct;55(5):515-9. doi: 10.1046/j.1440-1819.2001.00898.x. PMID: 11555348.

33. Kim KY, Bader GM, Kotlyar V, et al. Treatment of delirium in older adults with quetiapine. J Geriatr Psychiatry Neurol. 2003 Mar;16(1):29-31. doi: 10.1177/0891988702250533. PMID: 12641370 .

34. Kim SW, Yoo JA, Lee SY, et al. Risperidone versus olanzapine for the treatment of delirium. Hum Psychopharmacol. 2010 Jun-Jul;25(4):298302. doi: 10.1002/hup.1117. PMID: 20521319.

35. Kishi Y, Kato M, Okuyama T, et al. Treatment of delirium with risperidone in cancer patients. Psychiatry Clin Neurosci. 2012 Aug;66(5):411-7. doi: 10.1111/j.14401819.2012.02346.x. PMID: 22834659.
36. Konkayev AK, Bekmagambetova NV. [Comparison of sedation with dexmedetomidine and haloperidol in patients with delirium after femoral neck fractures]. Anesteziol Reanimatol. 2015 JanFeb;60(1):8-11. PMID: 26027216.

37. Larsen KA, Kelly SE, Stern TA, et al. Administration of olanzapine to prevent postoperative delirium in elderly jointreplacement patients: a randomized, controlled trial. Psychosomatics. 2010 SepOct;51(5):409-18. doi: 10.1176/appi.psy.51.5.409. PMID: 20833940.

38. Lee KU, Won WY, Lee HK, et al. Amisulpride versus quetiapine for the treatment of delirium: a randomized, open prospective study. Int Clin Psychopharmacol. 2005 Nov;20(6):311-4. PMID: 16192839.

39. Lim HK, Paik IH, Oh K, et al. Comparison of the Clinical Efficacy and Safety between Intramuscular Olanzapine and Intramuscular Haloperidol Injection in the Treatment of Delirium. Korean journal of psychopharmacology; 2007. p. 423-8.

40. Lin CJ, Sun FJ, Fang CK, et al. An open trial comparing haloperidol with olanzapine for the treatment of delirium in palliative and hospice center cancer patients. Journal of Internal Medicine of Taiwan. 2008;19(4):346-54.

41. Maneeton B, Maneeton N, Srisurapanont M. An open-label study of quetiapine for delirium. J Med Assoc Thai. 2007 Oct;90(10):2158-63. PMID: 18041437. 
42. Maneeton B, Maneeton N, Srisurapanont $\mathrm{M}$, et al. Quetiapine versus haloperidol in the treatment of delirium: a double-blind, randomized, controlled trial. Drug Des Devel Ther. 2013;7:657-67. doi: 10.2147/dddt.s45575. PMID: 23926422.

43. Mittal D, Jimerson NA, Neely EP, et al. Risperidone in the treatment of delirium: results from a prospective open-label trial. J Clin Psychiatry. 2004 May;65(5):662-7. PMID: 15163252.

44. Naksuk N, Thongprayoon C, Park JY, et al. Editor's Choice-Clinical impact of delirium and antipsychotic therapy: 10-Year experience from a referral coronary care unit. Eur Heart J Acute Cardiovasc Care. 2017 Sep;6(6):560-8. doi: 10.1177/2048872615592232. PMID: 26124454.

45. Omura K, Amano N. Clinical experience of quetiapine in 24 elderly patients with delirium. Psychogeriatrics. 2003;3(2):69-72. doi: 10.1046/j.14798301.2003.00012.x. PMID: 2004-19733005.

46. Pae CU, Lee SJ, Lee CU, et al. A pilot trial of quetiapine for the treatment of patients with delirium. Hum Psychopharmacol. 2004 Mar;19(2):125-7. doi: 10.1002/hup.559. PMID: 14994323.
47. Page VJ, Ely EW, Gates S, et al. Effect of intravenous haloperidol on the duration of delirium and coma in critically ill patients (Hope-ICU): a randomised, double-blind, placebo-controlled trial. Lancet Respir Med. 2013 Sep;1(7):515-23. doi: 10.1016/s2213-2600(13)70166-8.

PMID: 24461612.

48. Parellada E, Baeza I, de Pablo J, et al. Risperidone in the treatment of patients with delirium. J Clin Psychiatry. 2004 Mar;65(3):348-53. PMID: 15096074.

49. Prakanrattana U, Prapaitrakool S. Efficacy of risperidone for prevention of postoperative delirium in cardiac surgery. Anaesth Intensive Care. 2007 Oct;35(5):714-9. PMID: 17933157.

50. Sasaki Y, Matsuyama T, Inoue S, et al. A prospective, open-label, flexible-dose study of quetiapine in the treatment of delirium. J Clin Psychiatry. 2003 Nov;64(11):1316-21. PMID: 14658945.

51. Schrijver EJM, de Vries OJ, van de Ven PM, et al. Haloperidol versus placebo for delirium prevention in acutely hospitalised older at risk patients: a multicentre double-blind randomised controlled clinical trial. Age Ageing. 2018 Jan 1;47(1):48-55. doi: 10.1093/ageing/afx124. PMID: 28985255.

52. Schrijver EJM, Verstraaten M, Van De Ven PM, et al. Low dose oral haloperidol does not prolong QTc interval in older acutely hospitalised adults: Results fromA subanalysis of a randomised double-blind placebo-controlled study. Journal of Geriatric Cardiology. 2018;15(6):401-7. 
53. Sipahimalani A, Masand PS.

Olanzapine in the treatment of delirium. Psychosomatics. 1998 Sep-Oct;39(5):42230. doi: 10.1016/s0033-3182(98)71301-5. PMID: 9775699.

54. Skrobik YK, Bergeron N, Dumont $\mathrm{M}$, et al. Olanzapine vs haloperidol: treating delirium in a critical care setting. Intensive Care Med. 2004 Mar;30(3):444-9. doi: 10.1007/s00134-003-2117-0. PMID: 14685663.

55. Straker DA, Shapiro PA, Muskin $\mathrm{PR}$. Aripiprazole in the treatment of delirium. Psychosomatics. 2006 SepOct;47(5):385-91. doi: 10.1176/appi.psy.47.5.385. PMID: 16959926.

56. Tahir TA, Eeles E, Karapareddy V, et al. A randomized controlled trial of quetiapine versus placebo in the treatment of delirium. J Psychosom Res. 2010 Nov;69(5):485-90. doi: 10.1016/j.jpsychores.2010.05.006. PMID: 20955868.

57. Toda H, Kusumi I, Sasaki Y, et al. Relationship between plasma concentration levels of risperidone and clinical effects in the treatment of delirium. Int Clin Psychopharmacol. 2005 Nov;20(6):331-3. PMID: 16192843.
58. van den Boogaard M, Schoonhoven L, van Achterberg T, et al. Haloperidol prophylaxis in critically ill patients with a high risk for delirium. Crit Care. 2013 Jan 17;17(1):R9. doi: 10.1186/cc11933. PMID: 23327295.

59. van den Boogaard M, Slooter AJC, Bruggemann RJM, et al. Effect of Haloperidol on Survival Among Critically Ill Adults With a High Risk of Delirium: The REDUCE Randomized Clinical Trial. JAMA. 2018 Feb 20;319(7):680-90. doi: 10.1001/jama.2018.0160. PMID: 29466591.

60. Wang W, Li HL, Wang DX, et al. Haloperidol prophylaxis decreases delirium incidence in elderly patients after noncardiac surgery: a randomized controlled trial*. Crit Care Med. 2012 Mar;40(3):731-9. doi: 10.1097/CCM.0b013e3182376e4f. PMID: 22067628.

61. Yoon HJ, Park KM, Choi WJ, et al. Efficacy and safety of haloperidol versus atypical antipsychotic medications in the treatment of delirium. BMC Psychiatry. 2013 Sep 30;13:240. doi: 10.1186/1471244x-13-240. PMID: 24074357.

62. Yoon HK, Kim YK, Han C, et al. Paliperidone in the treatment of delirium: results of a prospective open-label pilot trial. Acta Neuropsychiatr. 2011 Aug;23(4):17983. doi: 10.1111/j.1601-5215.2011.00568.x. PMID: 25379796. 


\section{Excluded Articles}

1. Anon. Antipsychotics in the treatment of delirium in the palliative phase? Geneesmiddelenbulletin. 2017;51(2):23-4. -No original data

2. Anon. Effect of haloperidol on survival among critically ill adults with a high risk of delirium: The REDUCE randomised controlled trial. J Intensive Care Soc. 2019 Feb;20(1):74-6. doi: 10.1177/1751143718799920. PMID: 30792766. -No original data

3. Anon. Haloperidol or Placebo? Similar Outcomes for Treating Delirium. AACN Bold Voices. 2014;6(1):13-. PMID: 104135047. Language: English. Entry Date: 20140110. Revision Date: 20150710. Publication Type: Journal Article. No original data

4. Anon. In patients receiving palliative care, risperidone or haloperidol increased delirium symptoms vs placebo. Annals of internal medicine. 2017;166(6):JC32. doi:

10.7326/acpjc-2017-166-6-032. PMID: CN-01616421. -Meeting abstract

5. Anon. Quetiapine + haloperidol $=$ faster resolution of ICU delirium. Journal of the National Medical Association. 2010;102(7):662. Meeting abstract

6. Adams F, Fernandez F, Andersson BS. Emergency pharmacotherapy of delirium in the critically ill cancer patient. Psychosomatics. 1986 Jan;27(1 Suppl):33-8. PMID: 3952253. -No intervention of interest
7. Adams F. Emergency intravenous sedation of the delirious, medically ill patient. J Clin Psychiatry. 1988 Dec;49 Suppl:22-7. PMID: 2461929. -No original data

8. Adams F. Neuropsychiatric evaluation and treatment of delirium in the critically ill cancer patient. Cancer Bulletin. 1984;36(3):156-60. -No original data

9. Addesi D, Maio R, Smirne N, et al. Prevalence of Delirium in a Population of Elderly Outpatients with Dementia: A Retrospective Study. J Alzheimers Dis. 2018;61(1):251-7. doi: 10.3233/jad170339. PMID: 29171993. -

\section{Retrospective cohort}

10. Agar M, Lawlor P, Quinn S, et al. Phase iii randomized double-blind controlled trial of oral risperidone, haloperidol or placebo with rescue subcutaneous midazolam for delirium management in palliative care. Journal of the american geriatrics society.; 2015. p. S99. Meeting abstract

11. Agar M, Lawlor P, Quinn S, et al. Phase III randomized double-blind controlled trial of oral risperidone, haloperidol or placebo with rescue subcutaneous midazolam for delirium management in palliative care. Palliative medicine; 2016. p. 228. -Meeting abstract 
12. Agar MR, Lawlor P, Draper B, et al. Randomised control trial of oral risperidone, oral haloperidol, and oral placebo with rescue subcutaneous midazolam in the management of delirium in palliative care inpatients. Palliative medicine.; 2014. p. 570-1. -Meeting abstract

13. Ahc M. Antipsychotics Do Not Shorten the Duration of ICU Delirium. Critical Care Alert. 2019;26(10):0-. PMID: 133598840. Language: English. Entry Date: 20181220. Revision Date: 20181231. Publication Type: Article. Original Study: Girard TD. Haloperidol and ziprasidone for treatment of delirium in critical illness. (N Engl J Med) 2018 Oct 22.;. Journal Subset: Biomedical. -No original data

14. Akechi T, Uchitomi Y, Okamura H, et al. Usage of haloperidol for delirium in cancer patients. Support Care Cancer. 1996 Sep;4(5):390-2. PMID: 8883234. - Does not use a validated instrument or the DSM criteria to measure delirium

15. Allen KR, Fosnight SM, Wilford R, et al. Implementation of a systemwide quality improvement project to prevent delirium in hospitalized patients. Journal of Clinical Outcomes Management. 2011;18(6):253-8. - Does not apply to prevention or treatment of delirium

16. Al-Qadheeb N, Skrobik Y, Schumaker G, et al. Preventing ssd conversion to delirium with IV haloperidol: a D-B, placebocontrolled, pilot study. Critical care medicine.; 2013. p. A112. -Meeting abstract
17. Al-Samarrai S, Dunn J, Newmark T, et al. Quetiapine for treatmentresistant delirium. Psychosomatics. 2003 Jul-Aug;44(4):350-1. doi: 10.1176/appi.psy.44.4.350. PMID: 12832605. -Case report

18. Al-Shahri MZ, Sroor MY, Ghareeb WA, et al. Using Neuroleptics to Treat Delirium in Dying Cancer Patients at a Cancer Center in Saudi Arabia. J Pain Palliat Care Pharmacother. 2015;29(4):365-9. doi:

10.3109/15360288.2015.1101638. PMID: 26654409. -Retrospective cohort

19. Asano S, Kunii Y, Hoshino H, et al. The efficacy of antipsychotics for prolonged delirium with renal dysfunction. Neuropsychiatr Dis Treat. 2017;13:2823-8. doi: 10.2147/ndt.s147701. PMID: 29180868. -Retrospective cohort

20. Bange C, Lauxerois G. [Follow-up of a group of 40 chronically delirious patients discharged after treatment with neuroleptics: clinical \& statistical study]. J Med Lyon. 1958 Jul 5;39(924):593-7. PMID: 13564077. - Is not an RCT and does not report one of our adverse events

21. Barres P, Laboucarie J, Rascol A. [Action of chlorpromazine in the treatment of systematized delirious psychoses and schizophrenia]. Encephale. 1956;45(4):691-7. PMID: 13384286. -Other

22. Basaglia F. [The effects of chlorpromazine on a state of primary delirium]. Riv Sper Freniatr Med Leg Alien Ment. 1957 Sep 30;81(3):787-98. PMID: 13506504. -No original data 
23. Benoit Y, Chadenson O, Ducloux B. The indications of tiapride in emergency traumatology and orthopedics. Annales de Chirurgie. 1982;36(9):745-8. -No original data

24. Blitzstein SM, Brandt GT.

Extrapyramidal symptoms from intravenous haloperidol in the treatment of delirium. Am J Psychiatry. 1997 Oct;154(10):14745. PMID: 9326840. -No original data

25. Boettger S, Jenewein J, Breitbart W. Delirium management in patients with cancer: Dosing of antipsychotics in the delirium subtypes and response to psychopharmacological management. German Journal of Psychiatry. 2014;17(1):10-8. - Is not an RCT and does not report one of our adverse events

26. Boettger S, Jenewein J. Placebo might be superior to antipsychotics in management of delirium in the palliative care setting. Evid Based Med. 2017 Aug;22(4):152-3. doi: 10.1136/ebmed-2017-110723. PMID: 28637684. -No original data

27. Boettger S, Knopfel S, Schubert M, et al. Pipamperone and delirium: a preliminary evaluation of its effectiveness in the management of delirium and its subtypes. Swiss Med Wkly. 2017 Jul 27;147:w14471. doi: 10.4414/smw.2017.14471. PMID: 28750419. -Retrospective cohort
28. Bogner J, Barrett RS, Hammond FM, et al. Predictors of Agitated Behavior During Inpatient Rehabilitation for Traumatic Brain Injury. Arch Phys Med Rehabil. 2015 Aug;96(8 Suppl):S274-81 e4. doi: 10.1016/j.apmr.2015.04.020. PMID: 26212403. - Does not apply to prevention or treatment of delirium

29. Borger van der Burg BLS. Randomised Double-Blind PlaceboControlled Study of Post-Operative Haloperidol Versus Placebo for Prevention of Post-Operative Delirium After Acute Hip Surgery. Http://wwwclinicaltrialsgov/; 2005. Other

30. Borger vand der Burg B. Postoperative Haloperidol versus Placebo for prevention of post-operative delirium after acute hip surgery. Clinicaltrials.gov [http://clinicaltrials.gov]; 2006. Other

31. Bourgeois JA, Hilty DM. Prolonged delirium managed with risperidone. Psychosomatics. 2005 JanFeb;46(1):90-1. doi: 10.1176/appi.psy.46.1.90. PMID: 15765831. -Case report

32. Briskman I, Dubinski R, Barak Y. Treating delirium in a general hospital: a descriptive study of prescribing patterns and outcomes. Int Psychogeriatr. 2010 Mar;22(2):328-31. doi: 10.1017/s1041610209990986. PMID: 19785917. -Retrospective cohort 
33. Burkhart CS, Birkner-Binder D, Steiner LA. [Delirium in the intensive care unit]. Ther Umsch. 2010 Feb;67(2):75-8. doi: 10.1024/0040-5930/a000014. PMID: 20131215. -No original data

34. Burry LD, Williamson DR, Mehta S, et al. Delirium and exposure to psychoactive medications in critically ill adults: A multi-centre observational study. J Crit Care. 2017 Dec;42:268-74. doi: 10.1016/j.jcrc.2017.08.003. PMID: 28806561. - Is not an RCT and does not report one of our adverse events

35. Buyse G. Acute psychiatric syndromes and their emergency treatment (Dutch). Tijdschrift voor Geneeskunde. 1975;31(16):789-93. No original data

36. Cameron D, Bridge D, BlitzLindeque J. Use of sedation to relieve refractory symptoms in dying patients. S Afr Med J. 2004 Jun;94(6):445-9. PMID: 15250458. - Does not apply to prevention or treatment of delirium

37. Campbell NL, Khan BA, Farber M, et al. Improving delirium care in the intensive care unit: the design of a pragmatic study. Trials. 2011 Jun 6;12:139. doi: 10.1186/1745-621512-139. PMID: 21645330. -No original data

38. Chaudhury S, Augustine M. Psychosis following ocular surgery: Review with five case reports. Indian Journal of Clinical Psychology. 1991;18(1):25-9. PMID: 199406966-001. - Is not an RCT and does not report one of our adverse events
39. Cheung ENM, Benjamin S, Heckman G, et al. Clinical characteristics associated with the onset of delirium among long-term nursing home residents. BMC Geriatr. 2018 Feb 2;18(1):39. doi: 10.1186/s12877-018-0733-3. PMID: 29394886. -Retrospective cohort

40. Cheung ENM, Benjamin S, Heckman G, et al. Clinical characteristics associated with the onset of delirium among long-term nursing home residents. BMC geriatrics. 2018;18(1):39. doi: 10.1186/s12877-018-0733-3. -

\section{Retrospective cohort}

41. Choi JY, Kim JM, Kwon CH, et al. Use of Dexmedetomidine in Liver Transplant Recipients With Postoperative Agitated Delirium. Transplant Proc. 2016 May;48(4):1063-6. doi: 10.1016/j.transproceed.2016.01.020. PMID: 27320557. -Retrospective cohort

42. Christensen DB, Benfield WR. Alprazolam as an alternative to lowdose haloperidol in older, cognitively impaired nursing facility patients. $\mathrm{J}$ Am Geriatr Soc. 1998 May;46(5):620-5. PMID: 9588378. -

Does not use a validated instrument or the DSM criteria to measure delirium

43. Christensen P, Modvig KM.

Physostigmine and the anticholinergic syndrome. Treatment of post-anaesthetic delirium and stupor and certain types of intoxication. Ugeskrift for Laeger. 1979;141(5):287-9. -No original data 
44. Collet MO, Caballero J, Sonneville $\mathrm{R}$, et al. Prevalence and risk factors related to haloperidol use for delirium in adult intensive care patients: the multinational AID-ICU inception cohort study. Intensive Care Med. 2018 May 16doi: 10.1007/s00134-018-5204-y. PMID: 29767323. - Is not an RCT and does not report one of our adverse events

45. Collet MO, Woien H, Schandl A, et al. Pharmacological interventions for delirium in intensive care unit (AIDICU): an international inception cohort study. Intensive care medicine experimental. Conference: 30th annual congress of the european society of intensive care medicine, ESICM 2017. Austria; 2017.

46. Craven JL. Postoperative organic mental syndromes in lung transplant recipients. Toronto Lung Transplant Group. J Heart Transplant. 1990 Mar-Apr;9(2):129-32. PMID: 2319371. -Retrospective cohort

47. Crawford GB, Agar MM, Quinn SJ, et al. Pharmacovigilance in hospice/palliative care: net effect of haloperidol for delirium. J Palliat Med. 2013 Nov;16(11):1335-41. doi: 10.1089/jpm.2013.0230. PMID: 24138282. - Does not use a validated instrument or the DSM criteria to measure delirium

48. D'Angelo RG, Rincavage M, Tata AL, et al. Impact of an Antipsychotic Discontinuation Bundle During Transitions of Care in Critically Ill Patients. J Intensive Care Med. 2016 Jan 1:885066616686741. doi: 10.1177/0885066616686741. PMID: 28049388. -Retrospective cohort
49. Daniels LM, Nelson SB, Frank RD, et al. Pharmacologic Treatment of Intensive Care Unit Delirium and the Impact on Duration of Delirium, Length of Intensive Care Unit Stay, Length of Hospitalization, and 28Day Mortality. Mayo Clin Proc. 2018 Dec;93(12):1739-48. doi: 10.1016/j.mayocp.2018.06.022. PMID: 30292426. -Retrospective cohort

50. Dautzenberg PL, Mulder LJ, Olde Rikkert MG, et al. Adding rivastigmine to antipsychotics in the treatment of a chronic delirium. Age Ageing. 2004 Sep;33(5):516-7. doi: 10.1093/ageing/afh125. PMID: 15315924. -Retrospective cohort

51. Dave MB, Dave UM, Lagali RS, et al. Post operative delirium in elderly patients undergoing hip surgery: Diazepam versus haloperidol. International Journal of Pharmaceutical Sciences and Research. 2017;8(7):2965-7. doi: 10.13040/ijpsr.0975-8232.8(7).296567. -Retrospective cohort

52. de Jonghe JF, Kalisvaart KJ, Dijkstra $\mathrm{M}$, et al. Early symptoms in the prodromal phase of delirium: a prospective cohort study in elderly patients undergoing hip surgery. Am J Geriatr Psychiatry. 2007 Feb;15(2):112-21. doi: 10.1097/01.JGP.0000241002.86410. c2. PMID: 17272731. - Is not an RCT and does not report one of our adverse events

53. Delay J, Pichot $\mathrm{P}$, Lemperière $\mathrm{T}$, et al. The action of haloperidol on psychoses. Acta Neurologica Belgica. 1960;60(1):21-38. - Does not use a validated instrument or the DSM criteria to measure delirium 
54. Denke C, Balzer F, Menk M, et al. Long-term sequelae of acute respiratory distress syndrome caused by severe community-acquired pneumonia: Delirium-associated cognitive impairment and posttraumatic stress disorder. J Int Med Res. 2018 Jan 1:300060518762040. doi: $10.1177 / 0300060518762040$. PMID: 29609489. - Does not apply to prevention or treatment of delirium

55. Devlin JW, Skrobik Y, Riker R, et al. Impact of quetiapine on the resolution of individual delirium symptoms: an a priori-designed analysis of a randomized, doubleblind, placebo-controlled study. Pharmacotherapy; 2011. p. 327e. -Meeting abstract

56. Dotson B. Comparing Dexmedetomidine With Haloperidol for the Treatment of Hyperactive Delirium in Nonintubated ICU Patients. Crit Care Med. 2016 Dec;44(12):e1259-e60. doi: 10.1097/ccm.0000000000002049. PMID: 27858832. -Meeting abstract

57. Duffy JD, Kant R. Clinical utility of clozapine in 16 patients with neurological disease. $\mathrm{J}$

Neuropsychiatry Clin Neurosci. 1996 Winter;8(1):92-6. doi: 10.1176/jnp.8.1.92. PMID: 8845709.

\section{-Retrospective cohort}

58. Duprey M, Boogaard M, Hoeven J, et al. Icu delirium, its duration, and coma/ delirium days: association with 28-and 90-day mortality. Critical care medicine. Conference: 47th society of critical care medicine critical care congress, SCCM 2018. United states; 2018. p. 368. -

\section{Meeting abstract}

59. Duprey MS, Al-Qadheeb N, Roberts $\mathrm{R}$, et al. The use of low-dose IV haloperidol is not associated with QTc prolongation: post hoc analysis of a randomized, placebo-controlled trial. Intensive Care Med. 2016 Nov;42(11):1818-9. doi: 10.1007/s00134-016-4512-3. PMID: 27637718. -No original data

60. Elie M, Boss K, Cole MG, et al. A retrospective, exploratory, secondary analysis of the association between antipsychotic use and mortality in elderly patients with delirium. Int Psychogeriatr. 2009 Jun;21(3):58892. doi: 10.1017/s1041610209008977. PMID: 19368755. -Retrospective cohort

61. Elsayem A, Bush SH, Munsell MF, et al. Subcutaneous olanzapine for hyperactive or mixed delirium in patients with advanced cancer: a preliminary study. J Pain Symptom Manage. 2010 Nov;40(5):774-82. doi: 10.1016/j.jpainsymman.2010.02.017. PMID: 20728301. - Is not an RCT and does not report one of our adverse events

62. Ely EW. Delirium in the ICU: a prospective, randomized, trial of placebo vs haloperidol vs ziprasidone. Clinicaltrials.gov [http://clinicaltrials.gov]; 2004. Other

63. Eremenko AA, Chernova EV. [Treatment of delirium in the early postoperative period after cardiac surgery]. Anesteziol Reanimatol. 2014 May-Jun(3):30-4. PMID: 25306681. -Other 
64. Euctr DK. Pharmacological treatment of organic psychosis in critically ill adults. 2017. -Other

65. Euctr NL. Effectiveness of haloperidol for the treatment of acute confusional state (delirium) in patients treated at the intensive care. 2017. -Other

66. Ey H, Bohard F. [Results of drug therapy in chronic delirium. Association of prochlorperazine (Tementil) and of levopromazine (Nozinan)]. Evol Psychiatr (Paris). 1970 Jan-Mar;35(1):251-95. PMID: 5421008. -Other

67. Fainsinger RL, Landman W, Hoskings M, et al. Sedation for uncontrolled symptoms in a South African hospice. J Pain Symptom Manage. 1998 Sep;16(3):145-52. PMID: 9769616. -Retrospective cohort

68. Fernandez F, Levy JK, Mansell PW. Management of delirium in terminally ill AIDS patients. Int $\mathrm{J}$ Psychiatry Med. 1989;19(2):165-72. PMID: 2681028. - Is not an RCT and does not report one of our adverse events

69. Ferraz Goncalves JA, Almeida A, Costa I, et al. Comparison of Haloperidol Alone and in Combination with Midazolam for the Treatment of Acute Agitation in an Inpatient Palliative Care Service. J Pain Palliat Care Pharmacother. 2016 Dec;30(4):284-8. doi: 10.1080/15360288.2016.1231733. PMID: 27749141. - Does not apply to prevention or treatment of delirium
70. Fontaine GV, Mortensen W, Guinto KM, et al. Newly Initiated InHospital Antipsychotics Continued at Discharge in Non-psychiatric Patients. Hospital Pharmacy. 2018((Fontaine G.V., fontaine.gabe@gmail.com; Mortensen W.; Guinto K.M.; Scott D.M.; Miller R.R.) Intermountain Healthcare, Salt Lake City, UT, USA)doi: 10.1177/0018578717750095. -

\section{Retrospective cohort}

71. Fox MA, Elefritz JL, Huang BM, et al. Comparison of Lurasidone Versus Quetiapine for the Treatment of Delirium in Critically Ill Patients...Kleinman RA. Comparison of Lurasidone and Quetiapine in Critically Ill Patients. J Intensive Care Med. 2019;34(2):172. Journal of Intensive Care Medicine (Sage Publications Inc.). 2019;34(2):172-. doi: 10.1177/0885066618788018. PMID: 133860393. Language: English. Entry Date: 20190105. Revision Date: 20190108. Publication Type: Article. Journal Subset: Biomedical. -No original data

72. Frankenburg FR, Ely EW, Dittus RS, et al. Pharmacological treatment of delirium in the intensive care unit...Ely EW, Shintani A, Truman B et al. Delirium as a predictor of mortality in mechanically ventilated patients in the intensive care unit. JAMA. 2004;291:1753-1762. Chicago, Illinois: American Medical Association; 2004. p. 168-9. -

Meeting abstract 
73. Fruensgaard K. Withdrawal psychosis after drugs. Report of a consecutive material. Ugeskrift for Laeger. 1977;139(29):1719-22. - No subjects either at risk for delirium or diagnosed with delirium

74. Frye MA, Coudreaut MF, Hakeman SM, et al. Continuous droperidol infusion for management of agitated delirium in an intensive care unit. Psychosomatics. 1995 MayJun;36(3):301-5. doi: 10.1016/s00333182(95)71670-x. PMID: 7638318. Case report

75. Fuchita M, Khan SH, Perkins AJ, et al. Perioperative Risk Factors for Postoperative Delirium in Patients Undergoing Esophagectomy. Ann Thorac Surg. 2019 Feb 25doi: 10.1016/j.athoracsur.2019.01.040. PMID: 30817919. - Is not an RCT and does not report one of our adverse events

76. Furuya M, Miyaoka T, Yasuda H, et al. Ramelteon as adjunctive therapy for delirium referred to a consultation-liaison psychiatry service: a retrospective analysis. Int J Geriatr Psychiatry. 2015 Sep;30(9):994-5. doi: 10.1002/gps.4280. PMID: 26220878.

-Retrospective cohort

77. Gayral LF, Escande MM, Fournie $\mathrm{HC}$, et al. Differential study of two long acting neuroleptics, fluphenazine enanthate and pipothiazine undecylenate. Therapie. 1973;28(3):619-25. - Does not apply to prevention or treatment of delirium
78. Gelfand SB, Indelicato J, Benjamin J. Using intravenous haloperidol to control delirium. Hosp Community Psychiatry. 1992 Mar;43(3):215. PMID: 1555816. -No original data

79. Gonski PN, Moon I. Outcomes of a behavioral unit in an acute aged care service. Arch Gerontol Geriatr. 2012 Jul-Aug;55(1):60-5. doi: 10.1016/j.archger.2011.06.013. PMID: 21763009. -Retrospective cohort

80. Gonzalez-Seijo JC, Hidalgo Rodrigo I. Study of delirium in a General Hospital. Anales de Psiquiatria. 1994;10(2):81-4. - Does not use a validated instrument or the DSM criteria to measure delirium

81. Gorski S, Piotrowicz K, Rewiuk K, et al. Nonpharmacological Interventions Targeted at Delirium Risk Factors, Delivered by Trained Volunteers (Medical and Psychology Students), Reduced Need for Antipsychotic Medications and the Length of Hospital Stay in Aged Patients Admitted to an Acute Internal Medicine Ward: Pilot Study. Biomed Res Int. 2017;2017:1297164. doi: 10.1155/2017/1297164. PMID: 28164113. -Retrospective cohort

82. Gupta N, Sharma P, Mattoo SK. Effectiveness of risperidone in delirium. Can J Psychiatry. 2005 Jan;50(1):75. doi: 10.1177/070674370505000117. PMID: 15754670. -No original data 
83. Hale GM, Kane-Gill SL, Groetzinger L, et al. An Evaluation of Adverse Drug Reactions Associated With Antipsychotic Use for the Treatment of Delirium in the Intensive Care Unit. J Pharm Pract. 2016

Aug;29(4):355-60. doi: 10.1177/0897190014566313. PMID: 25609664. -No intervention of interest

84. Haloperidol vs olanzapine for the management of ICU delirium. Clinicaltrials.gov [http://clinicaltrials.gov]; 2008. Other

85. Hashimoto K, Uemura H, Morikawa $\mathrm{M}$, et al. Investigation of patients with delirium in the Department of Psychiatric Service in Higashiosaka City General Hospital. Journal of Nara Medical Association. 2007;58(2-3):83-6. -Retrospective cohort

86. Hasuo H, Kanbara K, Fujii R, et al. Factors Associated with the Effectiveness of Intravenous Administration of Chlorpromazine for Delirium in Patients with Terminal Cancer. J Palliat Med. 2018 May 14doi:

10.1089/jpm.2017.0669. PMID: 29757064. -Retrospective cohort

87. Hattori H, Matsumoto M, Morimoto

$\mathrm{S}$, et al. Effects of low-dose quetiapine on psychotic symptoms in elderly patients with physical illnesses: Report of eight cases. Psychogeriatrics. 2003;3(1):39-44. doi: 10.1046/j.14798301.2003.00005.x. PMID: 200419732-007. - Is not an RCT and does not report one of our adverse events
88. Hawkins WA, Phan SV, Campbell SL. Effect of various antipsychotic regimens on incidence of delirium in critically ill adults. J Crit Care. 2018 Apr;44:289-93. doi: 10.1016/j.jcrc.2017.11.032. PMID: 29223744. -Retrospective cohort

89. Herman E, Hovorka J, Doubek P, et al. A group of patients with delirium in the "Na Františku" Hospital in 2002-2004. Ceska a Slovenska Neurologie a Neurochirurgie. 2006;69(SUPPL. 1):20-3. - Is not an RCT and does not report one of our adverse events

90. Herzig SJ, LaSalvia MT, Naidus E, et al. Antipsychotics and the Risk of Aspiration Pneumonia in Individuals Hospitalized for Nonpsychiatric Conditions: A Cohort Study. J Am Geriatr Soc. 2017 Dec;65(12):25806. doi: 10.1111/jgs.15066. PMID: 29095482. -Retrospective cohort

91. Herzig SJ, Rothberg MB, Guess JR, et al. Antipsychotic Use in Hospitalized Adults: Rates, Indications, and Predictors. J Am Geriatr Soc. 2016 Feb;64(2):299305. doi: 10.1111/jgs.13943. PMID: 26889839. -Retrospective cohort

92. Heymann A, Radtke F, Schiemann A, et al. Delayed treatment of delirium increases mortality rate in intensive care unit patients. Journal of International Medical Research. 2010;38(5):1584-95. - Does not use a validated instrument or the DSM criteria to measure delirium 
93. Hui D, Bush SH, Gallo LE, et al. Neuroleptic dose in the management of delirium in patients with advanced cancer. J Pain Symptom Manage. 2010 Feb;39(2):186-96. doi: 10.1016/j.jpainsymman.2009.07.009. PMID: 20152585. -Retrospective cohort

94. Hui D, Frisbee-Hume S, Wilson A, et al. Lorazepam as an adjuvant to haloperidol for agitated delirium at the end-of-life: a double-blind randomized controlled trial. Supportive care in cancer. Conference: 2017 international MASCC/ISOO symposium: supportive care in cancer. United states; 2017. p. S192-s3. -Meeting abstract

95. Hui D, Reddy A, Palla S, et al. Neuroleptic prescription pattern for delirium in patients with advanced cancer. J Palliat Care. 2011 Summer;27(2):141-7. PMID: 21805949. -Retrospective cohort

96. Ikeshita K, Okumura K, Nakatani N, et al. Cases of delirium in Tenri Hospital. Journal of Nara Medical Association. 2005;56(5-6):225-8. -

\section{Retrospective cohort}

97. Inouye SK, Bogardus ST, Jr., Charpentier PA, et al. A multicomponent intervention to prevent delirium in hospitalized older patients. N Engl J Med. 1999 Mar 4;340(9):669-76. doi: 10.1056/nejm199903043400901. PMID: 10053175. -No intervention of interest
98. Inouye SK, Marcantonio ER, Metzger ED. Doing Damage in Delirium: The Hazards of Antipsychotic Treatment in Elderly Persons. Lancet Psychiatry. 2014 Sep 1;1(4):312-5. doi: 10.1016/s2215-0366(14)70263-9. PMID: 25285270. -No original data

99. Jacobson SA. Delirium in the elderly. Psychiatr Clin North Am. 1997 Mar;20(1):91-110. PMID: 9139298. -No original data

100. Jain FA, Brooks JO, 3rd, Larsen KA, et al. Individual risk profiles for postoperative delirium after joint replacement surgery.

Psychosomatics. 2011 Sep-

Oct;52(5):410-6. doi:

10.1016/j.psym.2011.03.011. PMID: 21907058. -No outcome of interest

101. Jasiak KD, Middleton EA, Camamo JM, et al. Evaluation of discontinuation of atypical antipsychotics prescribed for ICU delirium. J Pharm Pract. 2013 Jun;26(3):253-6. doi: 10.1177/0897190012465987. PMID: 23184410. -Retrospective cohort

102. Joyce C, Witcher R, Herrup E, et al. Evaluation of the Safety of Quetiapine in Treating Delirium in Critically Ill Children: A Retrospective Review. J Child Adolesc Psychopharmacol. 2015 Nov;25(9):666-70. doi: 10.1089/cap.2015.0093. PMID: 26469214. -Retrospective cohort

103. Jung HY, Meucci M, Unruh MA, et al. Antipsychotic use in nursing home residents admitted with hip fracture. J Am Geriatr Soc. 2013 Jan;61(1):101-6. doi: 10.1111/jgs.12043. PMID: 23252409. -Retrospective cohort 
104. Jung SW, Kim KS. A randomized controlled, pilot study of aripiprazole in the treatment of delirium.

European neuropsychopharmacology.; 2009. p. S553-s4. -Meeting abstract

105. Jung S-W. Ziprasidone for the treatment of delirium: Case studies. Clinical Psychopharmacology and Neuroscience. 2010;8(2):111-4. PMID: 2010-18714-009. -Case report

106. Kalisvaart KJ, Jonghe J, Bogaards $\mathrm{M}$, et al. A placebo-controlled study of haloperidol prophylaxis for postoperative delirium in elderly hipsurgery patients. International psychogeriatrics; 2003. -Meeting abstract

107. Karamchandani K, Schoaps RS, Printz J, et al. Gender differences in the use of atypical antipsychotic medications for ICU delirium. Crit Care. 2018 Sep 21;22(1):220. doi: 10.1186/s13054-018-2143-5. PMID: 30236134. -Retrospective cohort

108. Karmacharya R, England ML, Ongur D. Delirious mania: clinical features and treatment response. J Affect Disord. 2008 Aug;109(3):312-6. doi: 10.1016/j.jad.2007.12.001. PMID: 18191210. -Retrospective cohort

109. Kat MG, de Jonghe JF, Vreeswijk R, et al. Mortality associated with delirium after hip-surgery: a 2-year follow-up study. Age Ageing. 2011 May;40(3):312-8. doi: 10.1093/ageing/afr014. PMID: 21414946. - Is not an RCT and does not report one of our adverse events
110. Khan BA, Perkins AJ, Campbell NL, et al. Pharmacological Management of Delirium in the Intensive Care Unit: A Randomized Pragmatic Clinical Trial. J Am Geriatr Soc. 2019 Jan 25doi: 10.1111/jgs.15781. PMID: 30681720. -No intervention of interest

111. Kim HW, Kim HG. The effectiveness of combination therapy with quetapine and valproic acid in delirium with aggressive tendency: a prospective, controlled trial study. European neuropsychopharmacology.; 2015. p. S591-s2. -Meeting abstract

112. Kim Y, Kim H, Lee S, et al. Efficacy of low dose prophylactic quetiapine on delirium prevention in critically ill patients: a prospective, randomized, double-blind, placebocontrolled study. American journal of respiratory and critical care medicine. Conference: american thoracic society international conference, ATS 2018. United states. 2018;197(MeetingAbstracts) (no pagination) PMID: CN-01619230. Meeting abstract

113. Kirven LE, Montero EF. Comparison of thioridazine and diazepam in the control of nonpsychotic symptoms associated with senility: double-blind study. J Am Geriatr Soc. 1973 Dec;21(12):546-51. PMID: 4584169. - Does not apply to prevention or treatment of delirium

114. Kleinman RA. Comparison of Lurasidone and Quetiapine in Critically Ill Patients. J Intensive Care Med. 2019 Feb;34(2):171. doi: 10.1177/0885066618788009. PMID: 30021490. -No original data 
115. Kolanowski A, Mogle J, Fick DM, et al. Anticholinergic Exposure during Rehabilitation: Cognitive and Physical Function Outcomes in Patients with Delirium Superimposed on Dementia. American Journal of Geriatric Psychiatry. 2015;23(12):1250-8. doi: 10.1016/j.jagp.2015.07.004. -Other

116. Koponen H. Acute confusional states in the elderly. Nordisk Psykiatrisk Tidsskrift. 1985;39(4):313-7. doi: 10.3109/08039488509101912. PMID: 1987-35395-001. - Is not an RCT and does not report one of our adverse events

117. Kram BL, Kram SJ, Brooks KR. Implications of atypical antipsychotic prescribing in the intensive care unit. J Crit Care. 2015 Aug;30(4):814-8. doi: 10.1016/j.jcrc.2015.03.030. PMID: 25887805. -Retrospective cohort

118. Kuşçu MK, Topçuoğlu V, Altunel Ö, et al. Deliryum tanisiyla takip edilen hastalarin izlem sonuçlari. $=$ Clinical presentations and follow up outcomes of the delirium patients. Anadolu Psikiyatri Dergisi. 2004;5(1):16-21. PMID: 200500217-003. -Retrospective cohort

119. Kuznetsov ON. Perphenazine in treatment of resistant cases of schizophrenia. Zh. Nevropat. Psikhiat. Korsakov. 1964;64(9):1381-5. -Other

120. La MK, Thompson Bastin ML, Gisewhite JT, et al. Impact of restarting home neuropsychiatric medications on sedation outcomes in medical intensive care unit patients. J Crit Care. 2018 Feb;43:102-7. doi: 10.1016/j.jcrc.2017.07.046. PMID: 28865338. -Retrospective cohort
121. Lee CS, Rim GM, Hahn KH, et al. Comparison of Efficacy between Aripiprazole and Haloperidol in the Treatment of Patients with Delirium. Korean journal of psychopharmacology; 2007. p. 2405. - Is not an RCT and does not report one of our adverse events

122. Lee SH, Park SJ. Comparison of efficacy and side effects between aripiprazole and haloperidol in the treatment of delirium. International psychogeriatrics.; 2013. p. S132-s3. Meeting abstract

123. Lee Y, Lee J, Rim HD, et al. A comparative study of haloperidol and quetiapine in the treatment of delirium: a preliminary randomized open label, flexible dose trial. European Neuropsychopharmacology. 2006 Sep;16:S488-S9. doi: 10.1016/s0924-977x(06)70656-3. PMID: WOS:000240771302063. Meeting abstract

124. Lee YJ, Jung HY, Lee SI, et al. Comparison on the Efficacy of Quetiapine Versus Haloperidol in the Treatment of Delirium: prospective, Randomized Trial. Korean journal of biological psychiatry; 2009. p. 1524. - Is not an RCT and does not report one of our adverse events

125. Leso L, Schwartz TL. Ziprasidone treatment of delirium.

Psychosomatics. 2002 Jan-

Feb;43(1):61-2. doi: 10.1176/appi.psy.43.1.61. PMID: 11927760. -Case report 
126. Leung JG, Dare FY, Flowers LM, et al. Thiothixene in the Management of Delirium: A Case Series.

Psychosomatics. 2015 Sep-

Oct;56(5):542-6. doi:

10.1016/j.psym.2015.02.003. PMID: 26002225. -Retrospective cohort

127. Lilburn JK, Dundee JW, Nair SG, et al. Ketamine sequelae. Evaluation of the ability of various premedicants to attenuate its psychic actions.

Anaesthesia. 1978 Apr;33(4):307-11. PMID: 27120. - Is not an RCT and does not report one of our adverse events

128. Liperoti R, Fusco D, Cipriani MC, et al. Balancing the risks and benefits of antipsychotic medications for symptom management in older patients with cancer. Journal of Geriatric Oncology. 2018;9(6):6935. doi: 10.1016/j.jgo.2018.07.007. No original data

129. Litvineneko IV, Odinak MM, Khlystov Iu V, et al. [Efficacy and safety of rivastigmine (exelon) in the confusion syndrome in the acute phase of ischemic stroke]. Zh Nevrol Psikhiatr Im S S Korsakova. 2010;110(11 Pt 2):36-41. PMID: 21389938. -Other

130. Liu CY, Juang YY, Liang HY, et al. Efficacy of risperidone in treating the hyperactive symptoms of delirium. Int Clin Psychopharmacol. 2004 May;19(3):165-8. PMID: 15107660. -Retrospective cohort

131. Lixouriotis C, Peritogiannis V. Delirium in the primary care setting. Psychiatry and Clinical Neurosciences. 2011;65(1):102-4. doi: $10.1111 /$ j.14401819.2010.02165.x. -Retrospective cohort
132. Lund R, Ruther E. A comparison of three-time-daily and single dose regime of haloperidol. Annual review of chronopharmacology. VOL; 1984. p. 49-52. - Is not an RCT and does not report one of our adverse events

133. Maikin M, Hirsch AC.

Postanesthetic delirium and its treatment. Preuminary report. Oral. Surg. 1966;21(6). -Less than $\mathbf{5 0 \%}$ adult

134. Mangan KC, McKinzie BP, Deloney LP, et al. Evaluating the risk profile of quetiapine in treating delirium in the intensive care adult population: A retrospective review. J Crit Care. 2018 Oct;47:169-72. doi: 10.1016/j.jcrc.2018.07.005. PMID: 30005303. -Retrospective cohort

135. Mansouri P, Javadpour S, Zand F, et al. Implementation of a protocol for integrated management of pain, agitation, and delirium can improve clinical outcomes in the intensive care unit: A randomized clinical trial. Journal of Critical Care.

2013;28(6):918-22. doi: 10.1016/j.jcrc.2013.06.019. -No intervention of interest

136. Mark BZ, Kunkel EJ, Fabi MB, et al. Pimozide is effective in delirium secondary to hypercalcemia when other neuroleptics fail.

Psychosomatics. 1993 SepOct;34(5):446-50. PMID: 8140195.

\section{-Case report}

137. Matsuoka Y, Okamoto Y, Sueda K, et al. The clinical study of delirious cancer patients: Focusing on the clinical course of delirium. Seishin Igaku (Clinical Psychiatry). 1997;39(8):831-5. PMID: 199730130-004. -Retrospective cohort 
138. Menza MA, Murray GB, Holmes VF, et al. Controlled study of extrapyramidal reactions in the management of delirious, medically ill patients: intravenous haloperidol versus intravenous haloperidol plus benzodiazepines. Heart Lung. 1988 May;17(3):238-41. PMID: 2896642.

- Does not use a validated instrument or the DSM criteria to measure delirium

139. Meyers KJ, Upadhyaya HP, Landry JL, et al. Postinjection delirium/sedation syndrome in patients with schizophrenia receiving olanzapine long-acting injection: results from a large observational study. BJPsych Open. 2017 Jul;3(4):186-92. doi: 10.1192/bjpo.bp.116.004382. PMID: 28811926. - Does not apply to prevention or treatment of delirium

140. Michaud CJ, Bullard HM, Harris SA, et al. Impact of Quetiapine Treatment on Duration of Hypoactive Delirium in Critically Ill Adults: A Retrospective Analysis. Pharmacotherapy. 2015 Aug;35(8):731-9. doi: 10.1002/phar.1619. PMID: 26238778. -Retrospective cohort

141. Michaud CJ, Thomas WL, McAllen KJ. Early pharmacological treatment of delirium may reduce physical restraint use: a retrospective study. Ann Pharmacother. 2014 Mar;48(3):328-34. doi: 10.1177/1060028013513559. PMID: 24259659. -Retrospective cohort
142. Miyaji S, Yamamoto K, Hoshino S, et al. Comparison of the risk of adverse events between risperidone and haloperidol in delirium patients. Psychiatry Clin Neurosci. 2007 Jun;61(3):275-82. doi: 10.1111/j.1440-1819.2007.01655.x. PMID: 17472596. -Retrospective cohort

143. Mo Y, Zimmermann AE, Thomas MC. Practice patterns and opinions on current clinical practice guidelines regarding the management of delirium in the intensive care unit. Journal of Pharmacy Practice. 2017;30(2):16271. doi: 10.1177/0897190015625396. -Other

144. Moore DP. Rapid treatment of delirium in critically ill patients. Am J Psychiatry. 1977

Dec;134(12):1431-2. doi: 10.1176/ajp.134.12.1431. PMID: 920848. -Case report

145. Moulaert P. Treatment of acute nonspecific delirium with i.v. haloperidol in surgical intensive care patients. Acta Anaesthesiol Belg. 1989;40(3):183-6. PMID: 2816247. - Does not use a validated instrument or the DSM criteria to measure delirium

146. Murphy TE, Van Ness PH, Araujo KL, et al. An Empirical Method of Detecting Time-Dependent Confounding: An Observational Study of Next Day Delirium in a Medical ICU. Int J Stat Med Res. 2016;5(1):41-7. doi: 10.6000/19296029.2016.05.01.4. PMID: 26798411. - Does not apply to prevention or treatment of delirium 
147. Muzyk AJ, Rayfield A, Revollo JY, et al. Examination of baseline risk factors for QTc interval prolongation in patients prescribed intravenous haloperidol. Drug Safety. 2012;35(7):547-53. doi: 10.2165/11599960-00000000000000. -Retrospective cohort

148. Naber D, Greenspan A, Schreiner A. Efficacy and safety of risperidone in the treatment of elderly patients suffering from organic brain disease (organic brain syndrome): Results from a double-blind, randomized, placebo-controlled clinical trail. Psychopharmacology. 2007;191(4):1027-9. doi: 10.1007/s00213-007-0747-6. PMID: 2007-06461-018. - Does not use a validated instrument or the DSM criteria to measure delirium

149. Nakamura J, Uchimura N, Yamada $\mathrm{S}$, et al. Does plasma free-3methoxy-4hydroxyphenyl(ethylene)glycol increase in the delirious state? A comparison of the effects of mianserin and haloperidol on delirium. Int Clin Psychopharmacol. 1997 May;12(3):147-52. PMID: 9248871. - Is not an RCT and does not report one of our adverse events

150. Nakamura J, Uchimura N, Yamada

$\mathrm{S}$, et al. Effects of mianserin hydrochloride on delirium: Comparison with the effects of oxypertine and haloperidol. Japanese Journal of Psychopharmacology. 1994;14(4):269-77. - Is not an RCT and does not report one of our adverse events
151. Nakamura J, Uchimura N, Yamada $\mathrm{S}$, et al. The effect of mianserin hydrochloride on delirium. Human Psychopharmacology. 1995;10(4):289-97. - Is not an RCT and does not report one of our adverse events

152. Navines R, Gomez E, Franco JG, et al. [Delirium in a consultation liaison psychiatry unit of a general hospital]. Actas Esp Psiquiatr. 2001 MayJun;29(3):159-64. PMID: 11412490. -Retrospective cohort

153. Navinés R, Gómez E, Franco JG, et al. Delirium en la interconsulta psiquiátrica de un hospital general. = Delirium in a consultation liaison psychiatry unit of a general hospital. Actas Españolas de Psiquiatría. 2001;29(3):159-64. PMID: 200101707-004. -Other

154. Nguyen PVQ, Malachane A, Minh TTV. Antipsychotic prescription patterns in the management of delirium symptoms in hospitalized elderly patients. Proceedings of Singapore Healthcare. 2017;26(4):230-4. doi: 10.1177/2010105817697733. -

\section{Retrospective cohort}

155. Niu WB, Li ZY, Zhang XN, et al. Postoperative delirium in elderly patients with colorectal cancer: Risk factors and treatment. World Chinese Journal of Digestology. 2014;22(34):5381-4. doi: 10.11569/wcjd.v22.i34.5381. Retrospective cohort 
156. O’Regan NA, Mohamad MM, Meagher DJ. Impact of an educational workshop upon psychiatrists' attitudes towards delirium care. Irish Journal of Psychological Medicine. 2017((O’Regan N.A.; Mohamad M.M.; Meagher D.J., david.meagher@ul.ie)Cognitive Impairment Research Group, Graduate Entry Medical School, University of Limerick, Limerick, Ireland):1-10. doi: 10.1017/ipm.2016.47. -No outcome of interest

157. Ogawa A, Okumura Y, Fujisawa D, et al. Quality of care in hospitalized cancer patients before and after implementation of a systematic prevention program for delirium: the DELTA exploratory trial. Supportive Care in Cancer. 2019;27(2):557-65. doi: 10.1007/s00520-018-4341-8. No intervention of interest

158. Okuyama T, Yoshiuchi K, Ogawa A, et al. Current Pharmacotherapy Does Not Improve Severity of Hypoactive Delirium in Patients with Advanced Cancer: Pharmacological Audit Study of Safety and Efficacy in Real World (Phase-R). Oncologist. 2019 Jan 4doi:

10.1634/theoncologist.2018-0242. PMID: 30610009. - Does not apply to prevention or treatment of delirium

159. Olofsson SM, Weitzner MA, Valentine AD, et al. A retrospective study of the psychiatric management and outcome of delirium in the cancer patient. Support Care Cancer. 1996 Sep;4(5):351-7. PMID: 8883228. -Retrospective cohort
160. Otto MA. Haloperidol does not prevent delirium in ICU patients. Chest Physician. 2018;13(4):16-7. PMID: 129488610. Language: English. Entry Date: 20180518. Revision Date: 20180518. Publication Type: Article. -No original data

161. Page VJ, Casarin A. Use of antipsychotics for the treatment of intensive care unit delirium. Rev Bras Ter Intensiva. 2014 AprJun;26(2):86-8. PMID: 25028943. No original data

162. Page VJ, Ely EW, Gates S, et al. A randomised, double-blind, placebocontrolled trial to compare the early administration of intravenous haloperidol versus placebo in the prevention and treatment of delirium in critically ill ventilated patients (hope-ICU). American journal of respiratory and critical care medicine; 2013. -Meeting abstract

163. Page VJ, Marti J, McAuley DF, et al. Health evaluation and costeffectiveness analysis from a randomized trial of haloperidol in the management of delirium in the critically ill (hope-ICU trial). American journal of respiratory and critical care medicine; 2015. Meeting abstract

164. Park HJ, Kim JY. Incidence of Neutropenia With Valproate and Quetiapine Combination Treatment in Subjects With Acquired Brain Injuries. Arch Phys Med Rehabil. 2016 Feb;97(2):183-8. doi: 10.1016/j.apmr.2015.09.004. PMID: 26427579. -Retrospective cohort 
165. Parker RO, King AB, Hughes CG. Dexmedetomidine for the treatment of hyperactive delirium refractory to haloperidol in non-intubated patients. J Thorac Dis. 2016 Jul;8(7):E596-8. doi: 10.21037/jtd.2016.05.47. PMID: 27501127. -No original data

166. Pasina L, Colzani L, Cortesi L, et al. Relation Between Delirium and Anticholinergic Drug Burden in a Cohort of Hospitalized Older Patients: An Observational Study. Drugs Aging. 2018 Nov 27doi: 10.1007/s40266-018-0612-9. PMID: 30484239. -No intervention of interest

167. Pinkhasov A, James SA, Fazzari M, et al. Role of Ramelteon in Reduction of As-Needed Antipsychotics in Elderly Patients with Delirium in a General Hospital Setting. Clin Drug Investig. 2017 Dec;37(12):1137-41. doi: 10.1007/s40261-017-0573-5. PMID: 28933013. -Retrospective cohort

168. Pisani MA, Araujo KL, Murphy TE. Association of cumulative dose of haloperidol with next-day delirium in older medical ICU patients. Crit Care Med. 2015 May;43(5):996-1002. doi:

10.1097/ccm.0000000000000863. PMID: 25746748. - Does not apply to prevention or treatment of delirium

169. Pisani MA, Murphy TE, Araujo KL, et al. Factors associated with persistent delirium after intensive care unit admission in an older medical patient population. J Crit Care. 2010 Sep;25(3):540 e1-7. doi: 10.1016/j.jcrc.2010.02.009. PMID: 20413252. - Is not an RCT and does not report one of our adverse events
170. Pitkala KH, Laurila JV, Strandberg TE, et al. Multicomponent geriatric intervention for elderly inpatients with delirium: a randomized, controlled trial. J Gerontol A Biol Sci Med Sci. 2006 Feb;61(2):176-81. PMID: 16510862. -No intervention of interest

171. Porteus SD. Specific changes in behaviour under the effect of chlorpromazine. Revue de Psychologie Appliques. 1956;6(3):187-202. - Does not apply to prevention or treatment of delirium

172. Prado BL, Gomes DBD, Uson Junior PLS, et al. Continuous palliative sedation for patients with advanced cancer at a tertiary care cancer center. BMC Palliat Care. 2018 Jan 4;17(1):13. doi: 10.1186/s12904017-0264-2. PMID: 29301574. -

\section{Retrospective cohort}

173. Radha Krishna LK, Poulose VJ, Goh C. The use of midazolam and haloperidol in cancer patients at the end of life. Singapore Med J. 2012 Jan;53(1):62-6. PMID: 22252186. Retrospective cohort

174. Rais AR, Williams K, Rais T, et al. Use of intramuscular ziprasidone for the control of acute psychosis or agitation in an inpatient geriatric population: an open-label study. Psychiatry (Edgmont). 2010 Jan;7(1):17-24. PMID: 20386633. No subjects either at risk for delirium or diagnosed with delirium 
175. Raiten JM, Gutsche JT. Use of risperidone in cardiac surgery patients with subsyndromal delirium. Anesthesiology. 2012 Nov;117(5):1141; author reply -3. doi: 10.1097/ALN.0b013e31826f8c89. PMID: 23095545. -Meeting abstract

176. Reade MC, Finfer S. Sedation and delirium in the intensive care unit. New England Journal of Medicine. 2014;370(5):444-54. doi: 10.1056/NEJMra1208705. -No original data

177. Reade MC, O'Sullivan K, Bates S, et al. Dexmedetomidine vs. haloperidol in delirious, agitated, intubated patients: a randomised open-label trial. Crit Care. 2009;13(3):R75. doi: 10.1186/cc7890. PMID: 19454032. Other

178. Reddy A, Hui D, Palla S, et al. Neuroleptic doses in the management of delirium in patients with advanced cancer...18th International Congress on Palliative Care, October 5-8, 2010 - Palais Des Congres, Montreal, Canada. Journal of Palliative Care. 2010;26(3):242-. PMID: 105008038. Language: English. Entry Date: 20101210. Revision Date: 20150711.

Publication Type: Journal Article. Meeting abstract
179. Riegger H, Hollinger A, Seifert B, et al. Baden Prevention and Reduction of Incidence of Postoperative Delirium Trial (PRIDe): a phase IV multicenter, randomized, placebocontrolled, double-blind clinical trial of ketamine versus haloperidol for prevention of postoperative delirium. Trials. 2018 Feb 26;19(1):142. doi: 10.1186/s13063-018-2498-6. PMID: 29482596. -No original data

180. Robinson BR, Mueller EW, Henson $\mathrm{K}$, et al. An analgesia-deliriumsedation protocol for critically ill trauma patients reduces ventilator days and hospital length of stay. $\mathrm{J}$ Trauma. 2008 Sep;65(3):517-26. doi: 10.1097/TA.0b013e318181b8f6. PMID: 18784563. -Retrospective cohort

181. Rokhlenko SZ. The treatment of acute delirium. Zh. Nevropat. Psikhiat. 1961;61(3):426-7. - Does

not apply to prevention or treatment of delirium

182. Rosenthal LJ, Francis BA, Beaumont JL, et al. Agitation, Delirium, and Cognitive Outcomes in Intracerebral Hemorrhage. Psychosomatics. 2017;58(1):19-27. doi: 10.1016/j.psym.2016.07.004. - Is not an RCT and does not report one of our adverse events

183. Ruíz-Dangú DG, Tamayo-Illescas AJ, Vargas-Ayala G, et al. Efficacy of prophylaxis with haloperidol vs placebo in the prevention of delirium in patients with risk of suffering it hospitalized in an Internal Medicine service. Medicina Interna de Mexico. 2017;33(3):310-22. - Is not an RCT and does not report one of our adverse events 
184. Sanders KM, Minnema AM, Murray GB. Low incidence of extrapyramidal symptoms in treatment of delirium with intravenous haloperidol and lorazepam in the intensive care unit. Journal of Intensive Care Medicine. 1989;4(5):201-4. -Retrospective cohort

185. Sanders KM, Stern TA, O'Gara PT, et al. Delirium during intra-aortic balloon pump therapy: Incidence and management. Psychosomatics: Journal of Consultation and Liaison Psychiatry. 1992 Win 1992;33(1):3544. doi: 10.1016/s00333182(92)72019-2. PMID: 199224676-001. -Retrospective cohort

186. Sanders KM, Stern TA. Management of delirium associated with use of the intra-aortic balloon pump. Am J Crit Care. 1993 Sep;2(5):371-7. PMID: 8220668. - Is not an RCT and does not report one of our adverse events

187. Sauer AC, Slooter AJ, Veldhuijzen DS, et al. Intraoperative dexamethasone and delirium after cardiac surgery: a randomized clinical trial. Critical care.; 2013. p. S148. -Meeting abstract

188. Sauer AM, Slooter AJ, Veldhuijzen DS, et al. Intraoperative dexamethasone and delirium after cardiac surgery: a randomized clinical trial. Anesth Analg. 2014 Nov;119(5):1046-52. doi: 10.1213/ane.0000000000000248. PMID: 24810262. -No intervention of interest
189. Savaskan E, Baumgartner M, Georgescu D, et al. [Not Available]. Praxis (Bern 1994). 2016 Aug;105(16):941-52. doi: 10.1024/1661-8157/a002433. PMID: 27524166. -No original data

190. Scharko AM, Panzer J, McIntyre $\mathrm{CM}$. Treatment of delirium in the context of anti-N-methyl-D-aspartate receptor antibody encephalitis. J Am Acad Child Adolesc Psychiatry. 2015 Mar;54(3):233-4. doi: 10.1016/j.jaac.2014.12.014. PMID: 25721189. -No original data

191. Schildmann E, Pornbacher S, Kalies $\mathrm{H}$, et al. 'Palliative sedation'? A retrospective cohort study on the use and labelling of continuously administered sedatives on a palliative care unit. Palliat Med. 2018 Mar 1:269216318764095. doi: 10.1177/0269216318764095. PMID: 29557260. -Retrospective cohort

192. Schrijver EJ, de Vries OJ, Verburg A, et al. Efficacy and safety of haloperidol prophylaxis for delirium prevention in older medical and surgical at-risk patients acutely admitted to hospital through the emergency department: study protocol of a multicenter, randomised, double-blind, placebocontrolled clinical trial. BMC Geriatr. 2014 Aug 28;14:96. doi: 10.1186/1471-2318-14-96. PMID: 25168927. -Other 
193. Schrijver EJ, Verstraaten M, van de Ven PM, et al. Low dose oral haloperidol does not prolong QTc interval in older acutely hospitalised adults: a subanalysis of a randomised double-blind placebo-controlled study. J Geriatr Cardiol. 2018

Jun;15(6):401-7. doi: 10.11909/j.issn.1671-

5411.2018.06.003. PMID: 30108611. -Other

194. Schrijver EJM, Verstraaten M, Van De Ven PM, et al. Low dose oral haloperidol does not prolong QTC interval in older acutely hospitalised adults: Results fromA subanalysis of a randomised double-blind placebocontrolled study. Journal of Geriatric Cardiology. 2018;15(6):401-7. Does not apply to prevention or treatment of delirium

195. Schroder Pedersen S, Kirkegaard T, Balslev Jorgensen M, et al. Effects of a screening and treatment protocol with haloperidol on post-cardiotomy delirium: a prospective cohort study. Interact Cardiovasc Thorac Surg. 2014 Apr;18(4):438-45. doi: 10.1093/icvts/ivt501. PMID: 24357472. - Is not an RCT and does not report one of our adverse events

196. Schwartz TL, Masand PS. Treatment of Delirium With Quetiapine. Prim Care Companion J Clin Psychiatry. 2000 Feb;2(1):10-2. PMID: 15014661. -Retrospective cohort
197. Seitz DP, Gill SS. Neuroleptic malignant syndrome complicating antipsychotic treatment of delirium or agitation in medical and surgical patients: case reports and a review of the literature. Psychosomatics. 2009 Jan-Feb;50(1):8-15. doi: 10.1176/appi.psy.50.1.8. PMID: 19213967. - Does not use a validated instrument or the DSM criteria to measure delirium

198. Shehabi Y, Chan L, Kadiman S, et al. Sedation depth and long-term mortality in mechanically ventilated critically ill adults: A prospective longitudinal multicentre cohort study. Intensive Care Medicine. 2013;39(5):910-8. doi: 10.1007/s00134-013-2830-2. -No intervention of interest

199. Shehabi Y, Nakae H, Hamond N, et al. The effect of dexmedetomidine on agitation during weaning of mechanical ventilation in critically ill patients. Anaesthesia and Intensive Care. 2010;38(1):82-90. -No

intervention of interest

200. Shin SH, Hui D, Chisholm G, et al. Frequency and Outcome of Neuroleptic Rotation in the Management of Delirium in Patients with Advanced Cancer. Cancer Res Treat. 2015 Jul;47(3):399-405. doi: 10.4143/crt.2013.229. PMID: 25648094. -Retrospective cohort

201. Shinno H, Matsuoka T, Yamamoto $\mathrm{O}$, et al. Successful treatment with quetiapine for delirium in terminally ill cancer patients. Psychogeriatrics. 2007;7(2):64-8. doi: 10.1111/j.14798301.2007.00182.x. -Retrospective cohort 
202. Shrikant Kulkarni N. Lorazepam Added to Haloperidol Effective for Agitated Delirium in End-of-Life Cancer Patients. Am Fam Physician. 2018 Feb 15;97(4):Online. PMID: 29671522. -No original data

203. Shuster J. Heart failure with sunitinib: Prolonged QT interval, syncope, and delirium with galantamine - Memantine-induced hepatitis. Cabergoline-associated erythema nodosum: "Sleep driving" and the use of nonbenzodiazepine hypnotics - Atypical fractures in postmenopausal women taking alendronate. Drug-related problems in patients with human immunodeficiency virus. Hospital Pharmacy. 2008;43(6):449-52. -

\section{Meeting abstract}

204. Sigwald J, Henne M. The use of the neuroplegic properties of chlorpromazine in the treatment of several cases of chronic and subacute deliriant psychoses. Sem. Hop. Paris. 1954;30(50-51):2978-84. - Is not an RCT and does not report one of our adverse events

205. Simoni-Wastila L, Wei YJ, Lucas JA, et al. Mortality Risk of Antipsychotic Dose and Duration in Nursing Home Residents with Chronic or Acute Indications. J Am Geriatr Soc. 2016 May;64(5):973-80. doi: 10.1111/jgs.14111. PMID: 27166586. -Retrospective cohort

206. Sipahimalani A, Sime RM, Masand PS. Treatment of delirium with risperidone. International Journal of Geriatric Psychopharmacology. 1997;1(1):24-6. - Does not use a validated instrument or the DSM criteria to measure delirium
207. Slor CJ, de Jonghe JF, Vreeswijk R, et al. Anesthesia and postoperative delirium in older adults undergoing hip surgery. J Am Geriatr Soc. 2011 Jul;59(7):1313-9. doi: 10.1111/j.1532-5415.2011.03452.x. PMID: 21718258. - Is not an RCT and does not report one of our adverse events

208. Spiegler P. Dazed and Confused in the ICU. Clinical Pulmonary Medicine. 2010;17(3):149-50. doi: 10.1097/CPM.0b013e3181dabff2. No original data

209. Spisla C, Bunter M. [Treatment with haloperidol of clozapine-triggered drug-induced delirium]. Psychiatr Prax. 1997 Nov;24(6):308. PMID: 9490450. -Meeting abstract

210. Stiefel F, Fainsinger R, Bruera E. Acute confusional states in patients with advanced cancer. J Pain Symptom Manage. 1992 Feb;7(2):94-8. PMID: 1573291. Retrospective cohort

211. Strik J, Schieveld JNM. Prophylactic Haloperidol for Critically Ill Adults. JAMA. 2018 Jul 17;320(3):303. doi: 10.1001/jama.2018.6041. PMID: 30027242. -No original data

212. Sullinger D, Gilmer A, Jurado L, et al. Development, Implementation, and Outcomes of a Delirium Protocol in the Surgical Trauma Intensive Care Unit. Ann Pharmacother. 2017 Jan;51(1):5-12. doi: 10.1177/1060028016668627. PMID: 27630190. -Retrospective cohort 
213. Suusi C, Malato G. Combination of insulin and neuroleptic •sente in psychiatric treatment (therapeutic study). Ospedpsichiat. 1969;37((Suusi C.; Malato G.) Osp. Psichiat Prov. L. Bianchi, Napoli):145-56. -Other

214. Suzuki S, Ichida Y, Fujisawa D, et al. [Hyperglycemia due to atypical antipsychotics in cancer patients with delirium]. Gan To Kagaku Ryoho. 2012 Oct;39(10):1523-6. PMID: 23064064. -Retrospective cohort

215. Swan JT, Fitousis K, Hall JB, et al. Antipsychotic use and diagnosis of delirium in the intensive care unit. Crit Care. 2012 May 16;16(3):R84. doi: 10.1186/cc11342. PMID: 22591601. -Retrospective cohort

216. Szobor A. Clinical experiments with haloperidol (R 1625) in psychiatry. Acta Neurologica Belgica. 1962;62(6):662-76. - No subjects either at risk for delirium or diagnosed with delirium

217. Szyguła-Jurkiewicz B, Szymik M, Mrozowska B, et al. Psychological consequences of acute myocardial infarction. Psychotropic drugs in patients after acute myocardial infarction. Psychiatria. 2012;9(2):60-

7. -Other

218. Tagarakis GI, Voucharas C, Tsolaki $F$, et al. Ondasetron versus haloperidol for the treatment of postcardiotomy delirium: a prospective, randomized, doubleblinded study. J Cardiothorac Surg. 2012 Mar 21;7:25. doi: 10.1186/1749-8090-7-25. PMID: 22436170. - Does not use a validated instrument or the DSM criteria to measure delirium
219. Tahir T, Eeles E, Reddy V, et al. A randomized controlled trial of quetiapine versus placebo in the treatment of delirium. Journal of psychosomatic research [11th annual scientific meeting of the european association for consultation-liaison psychiatry and psychosomatics (EACLPP) and the 27th european conference on psychosomatic research (ECPR)]; 2008. p. 677. Meeting abstract

220. Tahir T. Quetiapine versus placebo in the treatment of delirium: a randomized controlled trial. European neuropsychopharmacology.; 2009. p. S358. -Meeting abstract

221. Takahashi H, Yoshida K, Sugita T, et al. Quetiapine treatment of psychotic symptoms and aggressive behavior in patients with dementia with Lewy bodies: a case series. Prog Neuropsychopharmacol Biol Psychiatry. 2003 May;27(3):549-53. doi: 10.1016/s0278-5846(03)00040x. PMID: 12691793. - Is not an RCT and does not report one of our adverse events

222. Takahashi S, Takagi T, Yonemura K, et al. Delirium in aged ophthalmic inpatients. Seishin Igaku (Clinical Psychiatry). 1995;37(8):869-76. PMID: 1996-86386-001. -

\section{Retrospective cohort}

223. Takashi T, Tomoaki Y, Hidekazu M, et al. The usefulness of atypical antipsychotics in the medication of delirium. Seishin Igaku (Clinical Psychiatry). 2007;49(8):821-8. PMID: 2007-14937-002. Retrospective cohort 
224. Tampi RR, Tampi DJ, Ghori AK. Acetylcholinesterase Inhibitors for Delirium in Older Adults. Am J Alzheimers Dis Other Demen. 2016 Jun;31(4):305-10. doi: 10.1177/1533317515619034. PMID: 26646113. -No original data

225. Tanimukai H, Tsujimoto H, Matsuda Y, et al. Novel Therapeutic Strategies for Delirium in Patients With Cancer: A Preliminary Study. Am J Hosp Palliat Care. 2016 Jun;33(5):456-62. doi: 10.1177/1049909114565019. PMID: 25550442. - Is not an RCT and does not report one of our adverse events

226. Tctr. Quetiapine prevention for postoperative delirium in cardiac surgery: a randomized controlled study. 2018. -Other

227. Tctr. The randomized controlled trial of risperidone in reduction for the incidence of postoperative delirium in patients undergoing vascular surgery. 2017. -Other

228. Thiboutot Z, Perreault MM, Williamson DR, et al. Antipsychotic Drug Use and Screening for Delirium in Mechanically Ventilated Patients in Canadian Intensive Care Units: An Observational Study. Can J Hosp Pharm. 2016 MarApr;69(2):107-13. PMID: 27168631. - Does not apply to prevention or treatment of delirium
229. Thom RP, Bui MP, Rosner B, et al. A Comparison of Early, Late, and No Treatment of Intensive Care Unit Delirium With Antipsychotics: A Retrospective Cohort Study. Prim Care Companion CNS Disord. 2018 Nov 15;20(6)doi: 10.4088/PCC.18m02320. PMID: 30476373. -Retrospective cohort

230. Thomas H, Jr., Schwartz E, Petrilli R. Droperidol versus haloperidol for chemical restraint of agitated and combative patients. Ann Emerg Med. 1992 Apr;21(4):407-13. PMID: 1554179. - Does not apply to prevention or treatment of delirium

231. Torres R, Mittal D, Kennedy R. Use of quetiapine in delirium: case reports. Psychosomatics. 2001 JulAug;42(4):347-9. doi: 10.1176/appi.psy.42.4.347. PMID: 11496025. -Case report

232. Travale I, Drake K. Haloperidol prophylaxis in cardiac surgery for patients at risk of delirium: a randomized placebocontrolled pilot study. Canadian journal of cardiology. Conference: 69th annual meeting of the canadian cardiovascular society. Canada. Conference start: 20161022. Conference end: 20161025; 2016. p. S315. -Meeting abstract

233. Travale IL, Drake KA, Power P, et al. Haloperidol prophylaxis in cardiac surgery for patients at risk for delirium: a randomized placebocontrolled pilot study. Canadian journal of cardiology. Conference: 69th annual meeting of the canadian cardiovascular society. Canada. Conference start: 20161022. Conference end: 20161025; 2016. p. S231. -Meeting abstract 
234. Tsuang MM, Lu LM, Stotsky BA, et al. Haloperidol versus thioridazine for hospitalized psychogeriatric patients: double-blind study. J Am Geriatr Soc. 1971 Jul;19(7):593-600. PMID: 4937658. - Does not use a validated instrument or the DSM criteria to measure delirium

235. Tune L. The role of antipsychotics in treating delirium. Curr Psychiatry Rep. 2002 Jun;4(3):209-12. PMID: 12003684. -No original data

236. Ueda S, Takeuchi J, Okubo Y. Successful use of olanzapine for catatonia following delirium. Psychiatry Clin Neurosci. 2012 Aug;66(5):465. doi: 10.1111/j.14401819.2012.02368.x. PMID: 22834674. -Case report

237. Ushijima M, Yokoyama S, Sugiyama E, et al. Contribution of perospirone and risperidone to reduce delirium in senile patients. Psychogeriatrics. 2008;8(1):4-7. doi: 10.1111/j.14798301.2007.00206.x. -Retrospective cohort

238. van den Boogaard M, Pickkers $\mathrm{P}$, van der Hoeven $\mathrm{H}$, et al. Implementation of a delirium assessment tool in the ICU can influence haloperidol use. Crit Care. 2009;13(4):R131. doi:

10.1186/cc7991. PMID: 19664260. Is not an RCT and does not report one of our adverse events
239. van Eijk MM, Roes KC, Honing ML, et al. Effect of rivastigmine as an adjunct to usual care with haloperidol on duration of delirium and mortality in critically ill patients: a multicentre, double-blind, placebocontrolled randomised trial. Lancet. 2010 Nov 27;376(9755):1829-37. doi: 10.1016/s0140-6736(10)618557. PMID: 21056464. - Is not an RCT and does not report one of our adverse events

240. van Keulen K, Knol W, Schrijver EJM, et al. Prophylactic Use of Haloperidol and Changes in Glucose Levels in Hospitalized Older Patients. J Clin Psychopharmacol. 2018 Feb;38(1):51-4. doi:

10.1097/jcp.0000000000000812. PMID: 29210808. -No outcome of interest

241. Vochteloo AJ, Moerman S, van der Burg BL, et al. Delirium risk screening and haloperidol prophylaxis program in hip fracture patients is a helpful tool in identifying high-risk patients, but does not reduce the incidence of delirium. BMC Geriatr. 2011 Aug 11;11:39. doi: 10.1186/1471-231811-39. PMID: 21834991. - Is not an RCT and does not report one of our adverse events

242. Wada K, Morita Y, Iwamoto T, et al. First- and second-line pharmacological treatment for delirium in general hospital settingRetrospective analysis. Asian J Psychiatr. 2018 Feb;32:50-3. doi: 10.1016/j.ajp.2017.11.028. PMID: 29216606. -Retrospective cohort 
243. Wahid N, Chin G, Turner AH, et al. Clinical response of clozapine as a treatment for delirious mania. Ment Illn. 2017 Oct 19;9(2):7182. doi: 10.4081/mi.2017.7182. PMID: 29142662. -Case report

244. Wan RY, Kasliwal M, McKenzie $\mathrm{CA}$, et al. Quetiapine in refractory hyperactive and mixed intensive care delirium: a case series. Crit Care. 2011 Jun 28;15(3):R159. doi: 10.1186/cc10294. PMID: 21711554. -Retrospective cohort

245. Wang K, Li C, Shi J, et al. [Effects of patient-controlled intravenous analgesia with dexmedetomidine and sufentanil on postoperative cognition in elderly patients after spine surgery]. Zhonghua Yi Xue Za Zhi. 2015 Aug 11;95(30):2437-41. PMID: 26711204. - Does not apply to prevention or treatment of delirium

246. Watanabe S, Fukatsu T, Kanemoto K. Risk of hospitalization associated with anticholinergic medication for patients with dementia.

Psychogeriatrics. 2018 Jan;18(1):5763. doi: 10.1111/psyg.12291. PMID: 29372598. -Retrospective cohort

247. Weaver CB, Kane-Gill SL, Gunn $\mathrm{SR}$, et al. A retrospective analysis of the effectiveness of antipsychotics in the treatment of ICU delirium. J Crit Care. 2017 Oct;41:234-9. doi: 10.1016/j.jcrc.2017.05.034. PMID: 28595082. -Retrospective cohort
248. Weiss A, Scheeringa MS. Psychopharmacological treatment of delirium: does earlier treatment and scheduled dosing improve outcomes? J La State Med Soc. 2014 Nov-Dec;166(6):242-7. PMID: 25978660. -Retrospective cohort

249. Wolters AE, van Dijk D, Pasma W, et al. Long-term outcome of delirium during intensive care unit stay in survivors of critical illness: a prospective cohort study. Crit Care. 2014 Jun 18;18(3):R125. doi: 10.1186/cc13929. PMID: 24942154. - Does not apply to prevention or treatment of delirium

250. Yang J, Choi W, Ko YH, et al. Bright light therapy as an adjunctive treatment with risperidone in patients with delirium: a randomized, open, parallel group study. Gen Hosp Psychiatry. 2012 Sep-Oct;34(5):54651. doi:

10.1016/j.genhosppsych.2012.05.003 . PMID: 22717090. - Is not an RCT and does not report one of our adverse events

251. Young CC, Lujan E. Intravenous ziprasidone for treatment of delirium in the intensive care unit.

Anesthesiology. 2004

Sep;101(3):794-5. PMID: 15329607. -Case report

252. Zirker W, Dorokhine I, Knapp CM, et al. Haloperidol overdosing in the treatment of agitated hospitalized older people with delirium: a retrospective chart review from a community teaching hospital. Drugs Aging. 2013 Aug;30(8):639-44. doi: 10.1007/s40266-013-0087-7. PMID: 23653156. -Retrospective cohort 


\section{Appendix D. Evidence Tables}

Evidence Table D-1. Study design characteristics for studies comparing interventions to prevent development of delirium

\begin{tabular}{|c|c|c|c|c|c|c|}
\hline Author, year & Study design & Location & Subgroup & Patient population & Start year & Funding \\
\hline Abdelgalel, $2016^{1}$ & $\mathrm{RCT}$ & Africa: Egypt & ICU & $\begin{array}{l}\text { Adult intensive care patients of } \\
\text { ASA physical status III and IV } \\
\text { aged between } 26 \text { and } 70 \text { years }\end{array}$ & 2014 & Not reported \\
\hline Al-Qadheeb, $2016^{2}$ & RCT & United States & ICU & $\begin{array}{l}\text { MV patients with subsyndromal } \\
\text { delirium }\end{array}$ & Not reported & Government \\
\hline Fukata, 201433 & RCT & Asia: Japan & Aged 65+, post-operative & $\begin{array}{l}\text { Delirium after abdominal or } \\
\text { orthopedic surgery in elderly } \\
\text { patients }\end{array}$ & 2007 & $\begin{array}{l}\text { Government, Other: } \\
\text { National Center for } \\
\text { Geriatrics and } \\
\text { Gerontology (NCGG), }\end{array}$ \\
\hline Girard, $2010^{4}$ & RCT & United States & ICU, post-operative & $\begin{array}{l}>18 \text { years MV medical and } \\
\text { surgical ICU patients }\end{array}$ & 2005 & $\begin{array}{l}\text { Industry, Government, } \\
\text { Nonprofit, Other: The } \\
\text { Hartford Geriatrics } \\
\text { Health Outcomes } \\
\text { Research Scholars } \\
\text { Award Program }\end{array}$ \\
\hline Hakim, $2012^{5}$ & RCT & Africa: Egypt & Aged $65+$, post-operative & $\begin{array}{l}\text { Patients } 65 \text { years or older } \\
\text { experiencing subsyndromal } \\
\text { delirium after on-pump cardiac } \\
\text { surgery }\end{array}$ & 2007 & Not reported \\
\hline Kalisvaart, $2005^{6}$ & RCT & Europe: Netherlands & Post-operative & $\begin{array}{l}\text { Acute or elective hip surgery } \\
\text { patients } \geq 70 \text { years is at risk of } \\
\text { delirium: MMSE between less } \\
\text { than or equal to } 24 ; \\
\text { dehydration=BUN/creatinine } \\
\text { greater than or equal to } 18, \text { low } \\
\text { visual acuity and/or increased } \\
\text { severity of illness on APACHE II } \\
\text { of } 16 \text { or greater. }\end{array}$ & 2000 & $\begin{array}{l}\text { Other: Medical Center } \\
\text { Alkmaar }\end{array}$ \\
\hline Kaneko, $1999^{7}$ & RCT & Asia: Japan & ICU, post-operative & $\begin{array}{l}\text { Scheduled for elective } \\
\text { gastrointestinal surgery and } \\
\text { admitted to ICU }\end{array}$ & 1995 & Not reported \\
\hline Khan, $2018^{8}$ & RCT & United States & ICU, post-operative & $\begin{array}{l}\text { English speaking individuals } \\
\text { undergoing thoracic surgery }\end{array}$ & 2013 & Government \\
\hline Larsen, $2010^{9}$ & RCT & United States & Aged 65+, post-operative & $\begin{array}{l}\text { Post-operative elderly joint } \\
\text { replacement surgery patients }\end{array}$ & 2005 & Nonprofit \\
\hline Page, $2013^{10}$ & RCT & Europe: UK & ICU & $\begin{array}{l}\text { ICU patients needing MV within } \\
72 \text { hours of admission were } \\
\text { enrolled }\end{array}$ & 2010 & Government \\
\hline
\end{tabular}




\begin{tabular}{|c|c|c|c|c|c|c|}
\hline Author, year & Study design & $\begin{array}{l}\text { Location } \\
\end{array}$ & Subgroup & $\begin{array}{l}\text { Patient population } \\
\end{array}$ & Start year & $\begin{array}{l}\text { Funding } \\
\end{array}$ \\
\hline Prakanrattana, $2007^{11}$ & RCT & Asia: Thailand & Post-operative & $\begin{array}{l}\text { Elective Cardiac Surgery with } \\
\text { cardiopulmonary bypass }\end{array}$ & Not reported & $\begin{array}{l}\text { Other: Sriraj Grant for } \\
\text { Research Development }\end{array}$ \\
\hline Schrijver, $2018^{12}$ & RCT & Europe: Netherlands & Aged 65+ & Medical and surgical patients & 2012 & $\begin{array}{l}\text { Other: Dutch Hospital } \\
\text { Patient Safety Program }\end{array}$ \\
\hline Schrijver, 2018 & RCT & Europe: Netherlands & Aged 65+ & $\begin{array}{l}\text { Medical and surgical patients age } \\
70 \text { and above, with at least three } \\
\text { ECGs }\end{array}$ & 2012 & $\begin{array}{l}\text { Other: Dutch Hospital } \\
\text { Patient Safety Program }\end{array}$ \\
\hline van den Boogaard, $2013^{14}$ & $\begin{array}{l}\text { Prospective cohort with a } \\
\text { comparison group, } \\
\text { analyzed as a single group }\end{array}$ & Europe: the Netherlands & ICU & $\begin{array}{l}\text { Patients in the ICU who are at a } \\
\text { high risk of delirium }\end{array}$ & 2010 & Not reported \\
\hline van den Boogaard, $2018^{15}$ & $\mathrm{RCT}$ & Europe: Netherlands & ICU & $\begin{array}{l}\text { ICU patients at high risk of } \\
\text { delirium }\end{array}$ & 2013 & Nonprofit \\
\hline Wang, $2012^{16}$ & RCT & Asia: China & Aged $65+$, ICU & $\begin{array}{l}\text { Patients } 65 \text { or older admitted to } \\
\text { the ICU after noncardiac surgery }\end{array}$ & 2009 & Not reported \\
\hline
\end{tabular}

APACHE II= Acute Physiology and Chronic Health Evaluation 2; ASA=American Society of Anesthesiologists; BUN=blood urea nitrogen ratio; ICU=intensive care unit; MMSE=Mini-Mental State Examination; MV=mechanical ventilation; NCGG=National Center for Geriatrics and Gerontology; RCT=randomized controlled trial 
Evidence Table D-2. Study design characteristics for studies comparing interventions to treat delirium

\begin{tabular}{|c|c|c|c|c|c|c|}
\hline Author, year & Study design & Location & Subgroup & Patient population & $\begin{array}{l}\text { Start } \\
\text { year }\end{array}$ & Funding \\
\hline Agar, $2017^{17}$ & RCT & Australia: Australia & Palliative & Patients in Hospice and palliative care with delirium & 2008 & Government \\
\hline Atalan, $2013^{18}$ & RCT & Asia: Turkey & $\begin{array}{l}\text { Post- } \\
\text { operative }\end{array}$ & Post cardiac surgery patients with hyperactive type delirium & 2010 & Not reported \\
\hline Bakri, $2015^{19}$ & RCT & Asia: Saudi Arabia & ICU & $\begin{array}{l}\text { ICU trauma patients without severe injury, comatose, or } \\
\text { moribund patients were excluded }\end{array}$ & $\begin{array}{l}\text { Not } \\
\text { reported }\end{array}$ & None \\
\hline $\begin{array}{l}\text { Boettger, } \\
2011^{20}\end{array}$ & $\begin{array}{l}\text { Cohort w/ } \\
\text { comparison group }\end{array}$ & United States & None & Cancer patients & 2004 & None \\
\hline $\begin{array}{l}\text { Boettger, } \\
2015^{21}\end{array}$ & $\begin{array}{l}\text { Cohort w/ } \\
\text { comparison group }\end{array}$ & United States & None & Cancer patients & 2000 & None \\
\hline $\begin{array}{l}\text { Breitbart, } \\
1996^{22}\end{array}$ & RCT & United States & None & $\begin{array}{l}\text { Medically hospitalized adult patients with AIDS and a DRS score } \\
\text { of } 13 \text { or greater (delirium) }\end{array}$ & $\begin{array}{l}\text { Not } \\
\text { reported }\end{array}$ & Government \\
\hline $\begin{array}{l}\text { Breitbart, } \\
2002^{23}\end{array}$ & $\begin{array}{l}\text { Cohort w/o } \\
\text { comparison group }\end{array}$ & United States & None & Cancer patients & 2000 & Not reported \\
\hline $\begin{array}{l}\text { Carrasco, } \\
2016^{24}\end{array}$ & $\begin{array}{l}\text { Cohort w/ } \\
\text { comparison group }\end{array}$ & Europe: Spain & $\begin{array}{l}\text { Aged 65+, } \\
\text { ICU }\end{array}$ & Non-intubated ICU patients refectory to haloperidol & 2013 & Other: no external funding \\
\hline $\begin{array}{l}\text { Charoenporn, } \\
2018^{25}\end{array}$ & $\begin{array}{l}\text { Cohort w/ } \\
\text { comparison group }\end{array}$ & Asia: Thailand & None & Physically ill patients & 2015 & None \\
\hline Devlin, $2010^{26}$ & $\mathrm{RCT}$ & $\begin{array}{l}\text { North America (not } \\
\text { US): United States, } \\
\text { Canada }\end{array}$ & ICU & Patients admitted to Medical \& Surgical ICU with delirium. & 2006 & Industry, Nonprofit \\
\hline $\begin{array}{l}\text { Drinkwater, } \\
2014^{27}\end{array}$ & $\mathrm{RCT}$ & Europe: UK & ICU & $\begin{array}{l}\text { Adult patients requiring mechanical ventilation within } 72 \text { hours of } \\
\text { ICU admission }\end{array}$ & $\begin{array}{l}\text { Not } \\
\text { reported }\end{array}$ & Not reported \\
\hline Fox, $2018^{28}$ & $\begin{array}{l}\text { Cohort w/ } \\
\text { comparison group }\end{array}$ & United States & ICU & Critically ill patients in the medical and cardiovascular ICU & 2014 & None \\
\hline Girard, $2010^{4}$ & RCT & United States & $\begin{array}{l}\text { ICU, post- } \\
\text { operative }\end{array}$ & $>18$ years MV medical and surgical ICU patients & 2005 & $\begin{array}{l}\text { Industry, Government, Nonprofit, Other: The } \\
\text { Hartford Geriatrics Health Outcomes Research } \\
\text { Scholars Award Program }\end{array}$ \\
\hline Girard, $2018^{29}$ & RCT & United States & ICU & $\begin{array}{l}\text { Patients over } 18 \text { years of age in the medical or surgical ICU, with } \\
\text { delirium }\end{array}$ & 2011 & Government \\
\hline Grover, $2011^{30}$ & RCT & Asia: India & None & $\begin{array}{l}\text { Consecutive patients }>-18 \text { yo referred to psychiatry and } \\
\text { diagnosed with delirium }\end{array}$ & $\begin{array}{l}\text { Not } \\
\text { reported }\end{array}$ & Government \\
\hline Grover, $2016^{31}$ & RCT & Asia: India & None & $\begin{array}{l}\text { Patients }>18 \text { years old referred to Psychiatry who met DSM-IV } \\
\text { criteria for delirium }\end{array}$ & $\begin{array}{l}\text { Not } \\
\text { reported }\end{array}$ & Not reported \\
\hline Han, 200432 & RCT & Asia: South Korea & None & $\begin{array}{l}\text { All patients presenting with altered mental status (from both ICU } \\
\text { and non-ICU units) who were referred to the consulting } \\
\text { psychiatry division were evaluated. }\end{array}$ & $\begin{array}{l}\text { Not } \\
\text { reported }\end{array}$ & Government \\
\hline Hatta, 201433 & $\begin{array}{l}\text { Cohort w/ } \\
\text { comparison group }\end{array}$ & Asia: Japan & None & $\begin{array}{l}\text { Patients in a general hospital who developed delirium and were } \\
\text { managed by a psychiatrist }\end{array}$ & 2011 & Nonprofit \\
\hline
\end{tabular}




\begin{tabular}{|c|c|c|c|c|c|c|}
\hline Author, year & Study design & Location & Subgroup & $\begin{array}{ll}\text { Patient population } \\
\end{array}$ & Start year & Funding \\
\hline $\begin{array}{l}\text { Horikawa, } \\
2003^{34}\end{array}$ & Cohort w/o comparison group & Asia: Japan & None & Medical and surgical inpatients & 2001 & Not reported \\
\hline $\mathrm{Hu}, 2006^{35}$ & RCT & Asia: China & Aged 65+ & Inpatients with senile delirium aged 65-99 & 2001 & Not reported \\
\hline Hui, $2017^{36}$ & $\begin{array}{l}\text { Randomized controlled trial, analyzed } \\
\text { as cohort }\end{array}$ & United States & Palliative & $\begin{array}{l}\text { Advanced cancer patients with hyperactive or mixed delirium in the acute } \\
\text { palliative care unit }\end{array}$ & 2014 & $\begin{array}{l}\text { Government, } \\
\text { Nonprofit }\end{array}$ \\
\hline $\begin{array}{l}\text { Ikezawa, } \\
2008^{37}\end{array}$ & Open-label trial & Asia: Japan & None & Elderly patients with hyperactive-hypervalent delirium & 1998 & Not reported \\
\hline Jain, $2017^{38}$ & RCT & Asia: India & None & Medical and surgical inpatients & 2011 & None \\
\hline Kim, $2001^{39}$ & Open label study & $\begin{array}{l}\text { Asia: South } \\
\text { Korea }\end{array}$ & None & Medico-surgical patients from Neurology, Neurosurgery and BMT unit & $\begin{array}{l}\text { Not } \\
\text { reported }\end{array}$ & Not reported \\
\hline Kim, $2003^{40}$ & Cohort w/o comparison group & United States & None & Patients in the acute medical units & $\begin{array}{l}\text { Not } \\
\text { reported }\end{array}$ & Industry \\
\hline Kim, $2005^{41}$ & Cohort w/ comparison group & $\begin{array}{l}\text { Asia: South } \\
\text { Korea }\end{array}$ & None & Hospital patients referred to the psychiatry division & $\begin{array}{l}\text { Not } \\
\text { reported }\end{array}$ & Not reported \\
\hline Kim, $2010^{42}$ & RCT & Asia: Korea & None & General hospital inpatients who met DSM-IV diagnostic criteria for delirium & $\begin{array}{l}\text { Not } \\
\text { reported }\end{array}$ & Nonprofit \\
\hline Kishi, $2012^{43}$ & Cohort w/o comparison group & Asia: Japan & None & Delirious cancer patients & $\begin{array}{l}\text { Not } \\
\text { reported }\end{array}$ & Not reported \\
\hline $\begin{array}{l}\text { Konkayev, } \\
2015^{44}\end{array}$ & Cohort w/ comparison group & $\begin{array}{l}\text { Asia: } \\
\text { Kazakhstan }\end{array}$ & $\begin{array}{l}\text { Aged } 65+, \text { ICU, post- } \\
\text { operative }\end{array}$ & $\begin{array}{l}\text { Patients who needed surgery for a traumatology or orthopedic condition and } \\
\text { had to stay in the ICU }\end{array}$ & 2012 & Not reported \\
\hline Lee, $2005^{45}$ & RCT & $\begin{array}{l}\text { Asia: South } \\
\text { Korea }\end{array}$ & None & $\begin{array}{l}\text { Patients referred to the Psychiatric Consultation that met DSM-IV criteria for } \\
\text { delirium }\end{array}$ & $\begin{array}{l}\text { Not } \\
\text { reported }\end{array}$ & Not reported \\
\hline Lim, $2007^{46}$ & RCT & Asia: Korea & None & $\begin{array}{l}\text { Patients from internal medicine, PMR, neurology, neurosurgery, orthopedic } \\
\text { surgery, or general surgery that were referred to the psychiatric department }\end{array}$ & 2006 & Not reported \\
\hline Lin, $2008^{47}$ & RCT & Asia: Taiwan & Palliative & $\begin{array}{l}\text { Patients receiving hospice or palliative care with advanced cancer and met } \\
\text { DSM-IV criteria for delirium }\end{array}$ & 2003 & Not reported \\
\hline $\begin{array}{l}\text { Maneeton, } \\
2007^{48}\end{array}$ & Open-label study & Asia: Thailand & None & All physically ill in-patients whose pcp consulted psychiatrists & 2005 & None \\
\hline $\begin{array}{l}\text { Maneeton, } \\
2013^{49}\end{array}$ & $\mathrm{RCT}$ & Asia: Thailand & None & $\begin{array}{l}\text { General adult population (age 18-75) with hyperactive delirium, referred to } \\
\text { psychiatry consult liaison service at tertiary care hospital in Thailand }\end{array}$ & 2009 & Nonprofit \\
\hline Mittal, $2004^{50}$ & Cohort w/o comparison group & United States & None & Hospitalized patients & 2000 & Industry \\
\hline $\begin{array}{l}\text { Naksuk, } \\
2017^{51}\end{array}$ & $\begin{array}{l}\text { Case control analyzed as cohort with } \\
\text { comparison group }\end{array}$ & United States & ICU & Patients admitted to the coronary care unit & 2004 & None \\
\hline Omura, $2003^{52}$ & Cohort w/o comparison group & Asia: Japan & Aged 65+ & Hospitalized patients & 2001 & Not reported \\
\hline Pae, $2004^{53}$ & Cohort w/o comparison group & Asia: S. Korea & None & $\begin{array}{l}\text { Delirious patients recruited from the departments of neurosurgery, } \\
\text { orthopedic surgery, and oncology }\end{array}$ & $\begin{array}{l}\text { Not } \\
\text { reported }\end{array}$ & Industry \\
\hline Page, $2013^{10}$ & RCT & Europe: UK & $\mathrm{ICU}$ & ICU patients needing MV within 72 hours of admission were enrolled & 2010 & Government \\
\hline $\begin{array}{l}\text { Parellada, } \\
2004^{54}\end{array}$ & Cohort w/o comparison group & Europe: Spain & None & General hospital patients & 2000 & Industry \\
\hline
\end{tabular}




\begin{tabular}{|c|c|c|c|c|c|c|}
\hline Author, year & $\begin{array}{l}\text { Study design } \\
\end{array}$ & $\begin{array}{l}\text { Location } \\
\end{array}$ & Subgroup & Patient population & Start year & Funding \\
\hline Sasaki, $2003^{55}$ & $\begin{array}{l}\text { Cohort w/o comparison } \\
\text { group }\end{array}$ & Asia: Japan & None & Inpatients and outpatients & 2001 & $\begin{array}{l}\text { Not } \\
\text { reported }\end{array}$ \\
\hline $\begin{array}{l}\text { Sipahimalani, } \\
1998^{56}\end{array}$ & Cohort w/ comparison group & United States & None & Hospital patients & $\begin{array}{l}\text { Not } \\
\text { reported }\end{array}$ & $\begin{array}{l}\text { Not } \\
\text { reported }\end{array}$ \\
\hline Skrobik, $2004^{57}$ & Cohort w/ comparison group & $\begin{array}{l}\text { North America (not US): } \\
\text { Canada }\end{array}$ & ICU & Medical-surgical ICU & 2000 & Industry \\
\hline Straker, $2006^{58}$ & $\begin{array}{l}\text { Cohort w/o comparison } \\
\text { group }\end{array}$ & Not reported & None & Medically ill delirium patients & 2004 & $\begin{array}{l}\text { Not } \\
\text { reported }\end{array}$ \\
\hline Tahir, $2010^{59}$ & RCT & Europe: Wales & None & $\begin{array}{l}\text { Patients in medical, surgical and orthopedic wards, who met the DSM-IV } \\
\text { criteria for delirium on the same day if they had a DRS-R-98 total score of } \\
15 \text { or more }\end{array}$ & 2003 & Industry \\
\hline Toda, $2005^{60}$ & $\begin{array}{l}\text { Cohort w/o comparison } \\
\text { group }\end{array}$ & Asia: Japan & None & Hospital patients with delirium & $\begin{array}{l}\text { Not } \\
\text { reported }\end{array}$ & $\begin{array}{l}\text { Not } \\
\text { reported }\end{array}$ \\
\hline Yoon, $2011^{61}$ & Open-label pilot trial & Asia: Korea & None & $\begin{array}{l}\text { Patients who were referred to psychiatrists at Korea University Ansan } \\
\text { Hospital }\end{array}$ & 2009 & $\begin{array}{l}\text { Not } \\
\text { reported }\end{array}$ \\
\hline Yoon, $2013^{62}$ & Cohort w/ comparison group & Asia: Korea & None & $\begin{array}{l}\text { Referred to consultation-liaison psychiatric service for a mental status } \\
\text { change }\end{array}$ & $\begin{array}{l}\text { Not } \\
\text { reported }\end{array}$ & $\begin{array}{l}\text { Not } \\
\text { reported }\end{array}$ \\
\hline
\end{tabular}


Evidence Table D-3. Patient characteristics for studies comparing interventions to prevent development of delirium

\begin{tabular}{|c|c|c|c|c|c|c|c|c|}
\hline Author, year & Arm & $\mathbf{N}$ & Age, mean & Male, n (\%) & Dementia, n (\%) & $\begin{array}{l}\text { Substance use } \\
\text { disorder, } \mathrm{n}(\%)\end{array}$ & Hypertension, n (\%) & Comments \\
\hline \multirow[t]{3}{*}{ Abdelgalel, 2016 } & 1 & 30 & 49.1 & $21(70)$ & $0(0)$ & Not reported & Not reported & \\
\hline & 2 & 30 & 51 & $22(73)$ & $0(0)$ & Not reported & Not reported & \\
\hline & 3 & 30 & 51.1 & $24(80)$ & $0(0)$ & Not reported & Not reported & \\
\hline \multirow[t]{2}{*}{ Al-Qadheeb, $2016^{2}$} & 1 & 34 & 59.3 & $20(58.8)$ & $0(0)$ & $\begin{array}{l}\text { Alcohol use - } \\
\text { moderate: } 16 \text { (47.1) }\end{array}$ & Not reported & \\
\hline & 2 & 34 & 61.7 & $18(52.9)$ & $0(0)$ & $\begin{array}{l}\text { Alcohol use - } \\
\text { moderate: } 14 \text { (41.1) }\end{array}$ & Not reported & \\
\hline \multirow[t]{2}{*}{ Fukata, $2014^{3}$} & 1 & 62 & 80.2 & $32(52)$ & Not reported & Not reported & Not reported & $\begin{array}{l}\text { Considered high risk for postoperative delirium groups as } \\
\text { preoperative MMSE }<25 \text { and preoperative NEECHAM }<27\end{array}$ \\
\hline & 2 & 59 & 80.5 & $32(54)$ & Not reported & Not reported & Not reported & $\begin{array}{l}\text { Considered high risk for postoperative delirium groups as } \\
\text { preoperative MMSE }<25 \text { and preoperative NEECHAM }<27\end{array}$ \\
\hline \multirow[t]{3}{*}{ Girard, $2010^{4}$} & 1 & 36 & Median: 56 & $22(61)$ & Not reported & Not reported & Not reported & \\
\hline & 2 & 35 & Median: 51 & $20(57)$ & Not reported & Not reported & Not reported & \\
\hline & 3 & 30 & Median: 54 & $21(70)$ & Not reported & Not reported & Not reported & \\
\hline \multirow[t]{2}{*}{ Hakim, $2012^{5}$} & 1 & 50 & $\begin{array}{l}\text { Percent over } \\
\text { age 65: } 100\end{array}$ & $36(72)$ & $0(0)$ & Not reported & Not reported & \\
\hline & 2 & 51 & $\begin{array}{l}\text { Percent over } \\
\text { age } 65: 100\end{array}$ & $33(65)$ & $0(0)$ & Not reported & Not reported & \\
\hline \multirow[t]{2}{*}{ Kalisvaart, $2005^{6}$} & 1 & 218 & 79.57 & $47(21.6)$ & Not reported & Not reported & Not reported & \\
\hline & 2 & 212 & 78.71 & 40 (18.9) & Not reported & Not reported & Not reported & \\
\hline \multirow[t]{2}{*}{ Kaneko, $1999^{7}$} & 1 & 40 & 73.1 & $26(65)$ & Not reported & Not reported & $12(30)$ & \\
\hline & 2 & 40 & 72.4 & $24(60)$ & Not reported & Not reported & $13(34.2)$ & \\
\hline \multirow[t]{2}{*}{ Khan, $2018^{8}$} & 1 & 67 & $\begin{array}{l}\text { Median: } 62.3 \\
\text { (IQR 52.6 to } \\
69.2 \text { ) }\end{array}$ & $54(80.6)$ & Not reported & Not reported & Not reported & \\
\hline & 2 & 68 & $\begin{array}{l}\text { Median: 60 } \\
\text { (IQR to 51.8- } \\
\text { 68) }\end{array}$ & $46(67.6)$ & Not reported & Not reported & Not reported & \\
\hline \multirow[t]{2}{*}{ Larsen, $2010^{9}$} & 1 & 204 & 74 & $81(39.7)$ & $0(0)$ & Not reported & Not reported & 123 in placebo group and 94 in Olanzapine group are females \\
\hline & 2 & 196 & 73.4 & $102(52)$ & $0(0)$ & Not reported & Not reported & 123 in placebo group and 94 in Olanzapine group are females \\
\hline \multirow[t]{2}{*}{ Page, $2013^{10}$} & 1 & 70 & 68.7 & $45(64)$ & $0(0)$ & Not reported & Not reported & \\
\hline & 2 & 71 & 67.9 & $37(52)$ & $0(0)$ & Not reported & Not reported & \\
\hline \multirow[t]{2}{*}{ Prakanrattana, $2007^{11}$} & 1 & 63 & 60.7 & $38(60)$ & Not reported & Not reported & $26(41)$ & \\
\hline & $\frac{1}{2}$ & 63 & 61.3 & $36(57)$ & Not reported & Not reported & $27(43)$ & \\
\hline \multirow[t]{2}{*}{ Schrijver, $2018^{12}$} & 1 & 124 & 83.4 & $51(41)$ & Not reported & Not reported & Not reported & \\
\hline & 2 & 118 & 83.5 & $57(48)$ & Not reported & Not reported & Not reported & \\
\hline \multirow[t]{2}{*}{ Schrijver, $2018^{13}$} & 1 & 34 & 93.7 & $13(38.2)$ & Not reported & Not reported & $20(58.8)$ & \\
\hline & 2 & 38 & 81.6 & $20(52.6)$ & Not reported & Not reported & $22(57.9)$ & \\
\hline $\begin{array}{l}\text { van den Boogaard, } \\
2013^{14}\end{array}$ & 1 & 177 & 63 & $115(65)$ & $2(1)$ & $\begin{array}{l}\text { Alcohol abuse: } 20 \\
\text { (11) }\end{array}$ & Not reported & \\
\hline
\end{tabular}




\begin{tabular}{|c|c|c|c|c|c|c|c|c|}
\hline Author, year & Arm & $\mathbf{N}$ & Age, mean & Male, n (\%) & Dementia, $\mathrm{n}$ (\%) & $\begin{array}{l}\text { Substance use } \\
\text { disorder, n (\%) }\end{array}$ & Hypertension, n (\%) & Comments \\
\hline \multirow{3}{*}{$\begin{array}{l}\text { van den Boogaard, } \\
2018^{15}\end{array}$} & 1 & 707 & 67 & $434(61)$ & $0(0)$ & Not reported & Not reported & \\
\hline & 2 & 350 & 66.1 & $206(59)$ & $0(0)$ & Not reported & Not reported & \\
\hline & 3 & 732 & 66.7 & $459(63)$ & $0(0)$ & Not reported & Not reported & \\
\hline \multirow[t]{2}{*}{ Wang, $2012^{16}$} & 1 & 228 & 74.4 & $143(62.7)$ & Not reported & Alcoholism: 19 (8.3) & $125(54.8)$ & \\
\hline & 2 & 229 & 74 & $145(63.3)$ & Not reported & Alcoholism: 14 (6.1) & $128(55.9)$ & \\
\hline
\end{tabular}

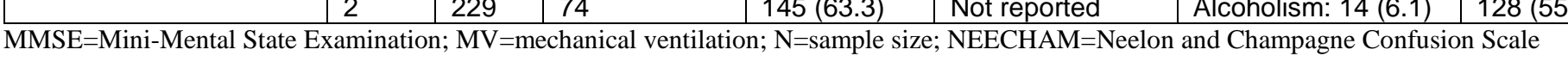


Evidence Table D-4. Patient characteristics for studies comparing interventions to treat delirium

\begin{tabular}{|c|c|c|c|c|c|c|c|c|}
\hline Author, year & Arm & $\mathbf{N}$ & Age, mean & Male, $n(\%)$ & Dementia, $\mathbf{n}(\%)$ & $\begin{array}{c}\text { Substance use disorder, } \mathbf{n} \\
(\%)\end{array}$ & $\begin{array}{l}\text { Hypertension, } \\
\text { n (\%) }\end{array}$ & Comments \\
\hline \multirow[t]{3}{*}{ Agar, $2017^{17}$} & 1 & 84 & $\begin{array}{l}73.8 \text { (Percent over age 65: } \\
\text { 80) }\end{array}$ & $57(68)$ & Not reported & Not reported & Not reported & \\
\hline & 2 & 81 & $\begin{array}{l}76.5 \text { (Percent over age } 65: \\
90)\end{array}$ & $48(59)$ & Not reported & Not reported & Not reported & \\
\hline & 3 & 82 & $\begin{array}{l}74.5 \text { (Percent over age 65: } \\
\text { 78) }\end{array}$ & $57(70)$ & Not reported & Not reported & Not reported & \\
\hline \multirow[t]{2}{*}{ Atalan, $2013^{18}$} & 1 & & & & Not reported & Not reported & $16(61.5)$ & \\
\hline & 2 & 26 & 66 & $21(80.2)$ & $0(0)$ & Not reported & Not reported & \\
\hline \multirow[t]{3}{*}{ Bakri, $2015^{19}$} & 1 & 32 & 31 & $29(91)$ & Not reported & Not reported & Not reported & \\
\hline & 2 & 32 & 30 & $28(88)$ & Not reported & Not reported & Not reported & \\
\hline & 3 & 32 & 32 & $30(94)$ & Not reported & Not reported & Not reported & \\
\hline \multirow[t]{2}{*}{ Boettger, $2011^{20}$} & 1 & 32 & 61.97 & $20(62.5)$ & Not reported & Not reported & Not reported & \\
\hline & 2 & 32 & 67.5 & $20(62.5)$ & Not reported & Not reported & Not reported & \\
\hline \multirow[t]{4}{*}{ Boettger, $2015^{21}$} & 1 & 21 & 69.6 (Range: 36 to 85 ) & $(47.6)$ & $(28.6)$ & Not reported & Not reported & \\
\hline & 2 & 21 & 64 (Range: 36 to 79 ) & $(38.1)$ & $(23.8)$ & Not reported & Not reported & \\
\hline & 3 & 21 & 65.6 (Range: 51 to 84 ) & $(38.1)$ & $(28.6)$ & Not reported & Not reported & \\
\hline & 4 & 21 & 67.2 (Range: 29 to 84 ) & $(47.6)$ & $(23.8)$ & Not reported & Not reported & \\
\hline \multirow[t]{3}{*}{ Breitbart, $1996^{22}$} & 1 & 13 & Not reported & Not reported & Not reported & Not reported & Not reported & $\begin{array}{l}\text { Demographic data given as overall } \\
\text { sample: Age: } 39.2 \text { (Range } 23-56) ; \mathrm{N}=23 \\
\text { ( } 77 \% \text { ) men; dementia, substance use } \\
\text { disorder, and hypertension data not } \\
\text { reported. }\end{array}$ \\
\hline & 2 & 11 & Not reported & Not reported & Not reported & Not reported & Not reported & $\begin{array}{l}\text { Demographic data given as overall } \\
\text { sample: Age: } 39.2 \text { (Range } 23-56) ; \mathrm{N}=23 \\
\text { ( } 77 \% \text { ) men; dementia, substance use } \\
\text { disorder, and hypertension data not } \\
\text { reported. }\end{array}$ \\
\hline & 3 & 6 & Not reported & Not reported & Not reported & Not reported & Not reported & $\begin{array}{l}\text { Demographic data given as overall } \\
\text { sample: Age: } 39.2 \text { (Range } 23-56 \text { ); } \mathrm{N}=23 \\
\text { ( } 77 \% \text { ) men; dementia, substance use } \\
\text { disorder, and hypertension data not } \\
\text { reported. }\end{array}$ \\
\hline
\end{tabular}




\begin{tabular}{|c|c|c|c|c|c|c|c|c|}
\hline Author, year & Arm & $\mathbf{N}$ & Age, mean & $\begin{array}{c}\text { Male, } \mathbf{n} \\
(\%)\end{array}$ & Dementia, $\mathrm{n}$ (\%) & $\begin{array}{c}\text { Substance use disorder, } \mathbf{n} \\
(\%)\end{array}$ & $\begin{array}{l}\text { Hypertension, } \\
\text { n (\%) }\end{array}$ & Comments \\
\hline Breitbart, $2002^{23}$ & 1 & 79 & 60.6 (Range: 19 to 89 ) & $40(51)$ & $14(17)$ & Not reported & Not reported & \\
\hline Carrasco, 2016 24 & 1 & 86 & 71.3 & $77(90)$ & Not reported & Not reported & Not reported & \\
\hline \multirow[t]{3}{*}{ Charoenporn, $2018^{25}$} & 1 & 11 & 70.5 & $8(72.7)$ & $2(18.2)$ & Not reported & Not reported & \\
\hline & 2 & 31 & 76.4 & $13(41.9)$ & $10(32.3)$ & Not reported & Not reported & \\
\hline & 3 & 14 & 69.4 & $6(42.9)$ & $1(7.1)$ & Not reported & Not reported & \\
\hline \multirow[t]{2}{*}{ Devlin, $2010^{26}$} & 1 & 18 & 63.6 & $(56)$ & $0(0)$ & Not reported & Not reported & \\
\hline & 2 & 18 & 62.4 & $(56)$ & $0(0)$ & Not reported & Not reported & \\
\hline \multirow[t]{2}{*}{ Drinkwater, $2014^{27}$} & 1 & 71 & Not reported & $\begin{array}{l}\text { Not } \\
\text { reported }\end{array}$ & Not reported & Not reported & Not reported & \\
\hline & 2 & 71 & Not reported & $\begin{array}{l}\text { Not } \\
\text { reported }\end{array}$ & Not reported & Not reported & Not reported & \\
\hline \multirow[t]{2}{*}{ Fox, $2018^{28}$} & 1 & 20 & 67.2 & $10(50)$ & Not reported & Not reported & Not reported & \\
\hline & 2 & 20 & 66 & $11(55)$ & Not reported & Not reported & Not reported & \\
\hline \multirow[t]{3}{*}{ Girard, $2010^{4}$} & 1 & 36 & Median: 56 & $22(61)$ & Not reported & Not reported & Not reported & \\
\hline & 2 & 35 & Median: 51 & $20(57)$ & Not reported & Not reported & Not reported & \\
\hline & 3 & 30 & Median: 54 & $21(70)$ & Not reported & Not reported & Not reported & \\
\hline \multirow[t]{3}{*}{ Girard, $2018^{29}$} & 1 & 184 & 59 & $107(58)$ & Not reported & Not reported & Not reported & Severe dementia excluded \\
\hline & 2 & 192 & 61 & $108(56)$ & Not reported & Not reported & Not reported & Severe dementia excluded \\
\hline & 3 & 190 & 61 & $108(57)$ & Not reported & Not reported & Not reported & Severe dementia excluded \\
\hline \multirow[t]{3}{*}{ Grover, 201130 } & 1 & 21 & $\begin{array}{l}44.09 \text { (Percent over age } 65: 14 \text { ) } \\
\text { (Range: } 20 \text { to } 72 \text { ) }\end{array}$ & $13(62)$ & $0(0)$ & Not reported & Not reported & \\
\hline & 2 & 23 & $\begin{array}{l}45.39 \text { (Percent over age } 65: 26 \text { ) } \\
\text { (Range: } 19 \text { to } 78 \text { ) }\end{array}$ & $14(61)$ & $0(0)$ & Not reported & Not reported & \\
\hline & 3 & 20 & $\begin{array}{l}46.5 \text { (Percent over age } 65: 10 \text { ) } \\
\text { (Range: } 27 \text { to } 78 \text { ) }\end{array}$ & $18(90)$ & $0(0)$ & Not reported & Not reported & \\
\hline \multirow[t]{2}{*}{ Grover, $2016^{31}$} & 1 & 32 & 44.4 (Range: 18 to 76$)$ & $28(87.5)$ & $0(0)$ & Not reported & Not reported & \\
\hline & 2 & 31 & 48.51 (Range: 18 to 85 ) & $21(67.74)$ & $0(0)$ & Not reported & Not reported & \\
\hline
\end{tabular}




\begin{tabular}{|c|c|c|c|c|c|c|c|c|}
\hline Author, year & $\begin{array}{l}\mathbf{A r} \\
\mathbf{m}\end{array}$ & $\mathbf{N}$ & Age, mean & $\begin{array}{c}\text { Male, } n \\
(\%)\end{array}$ & Dementia, $n$ (\%) & $\begin{array}{c}\text { Substance use disorder, } \mathbf{n} \\
(\%)\end{array}$ & $\begin{array}{l}\text { Hypertension, } \\
\text { n (\%) }\end{array}$ & Comments \\
\hline \multirow[t]{2}{*}{ Han, $2004^{32}$} & 1 & 12 & 66.5 & $7(58)$ & $0(0)$ & Not reported & Not reported & $\begin{array}{l}\text { Patients with any type of dementia or } \\
\text { other psychiatric diagnosis determined } \\
\text { with the SCID were excluded. }\end{array}$ \\
\hline & 2 & 12 & 65.6 & $6(50)$ & $0(0)$ & Not reported & Not reported & $\begin{array}{l}\text { Patients with any type of dementia or } \\
\text { other psychiatric diagnosis determined } \\
\text { with the SCID were excluded. }\end{array}$ \\
\hline \multirow[t]{5}{*}{ Hatta, $2014^{33}$} & 1 & 61 & 70.2 & $29(48)$ & $15(25)$ & Not reported & Not reported & $\begin{array}{l}\text { Age, gender, and presence of comorbid } \\
\text { dementia were not balanced across } \\
\text { groups. }\end{array}$ \\
\hline & 2 & 480 & 71.9 & $322(67)$ & $95(20)$ & Not reported & Not reported & $\begin{array}{l}\text { Age, gender, and presence of comorbid } \\
\text { dementia were not balanced across } \\
\text { groups. }\end{array}$ \\
\hline & 3 & 87 & 66.6 & $53(61)$ & $17(20)$ & Not reported & Not reported & $\begin{array}{l}\text { Age, gender, and presence of comorbid } \\
\text { dementia were not balanced across } \\
\text { groups. }\end{array}$ \\
\hline & 4 & 779 & 74.4 & $475(61)$ & $261(34)$ & Not reported & Not reported & $\begin{array}{l}\text { Age, gender, and presence of comorbid } \\
\text { dementia were not balanced across } \\
\text { groups. }\end{array}$ \\
\hline & 5 & 835 & 73.5 & $544(65)$ & $259(31)$ & Not reported & Not reported & $\begin{array}{l}\text { Age, gender, and presence of comorbid } \\
\text { dementia were not balanced across } \\
\text { groups. }\end{array}$ \\
\hline Horikawa, $2003^{34}$ & 1 & 10 & 56.8 (Range: 22 to 81 ) & $6(60)$ & Not reported & $\begin{array}{l}\text { history of substance abuse: } \\
0(0)\end{array}$ & Not reported & \\
\hline \multirow[t]{3}{*}{$\mathrm{Hu}, 2006^{35}$} & 1 & 29 & 73 & $18(62.1)$ & Not reported & Not reported & Not reported & \\
\hline & 2 & 72 & 74 & $48(66.7)$ & Not reported & Not reported & Not reported & \\
\hline & 3 & 74 & 74 & $45(60.8)$ & Not reported & Not reported & Not reported & \\
\hline Hui, $2017^{36}$ & 1 & 29 & 64 (Range: 30 to 88 ) & $13(44.8)$ & $0(0)$ & Not reported & Not reported & \\
\hline Ikezawa, $2008^{37}$ & 1 & 22 & 73.6 (Range: 60 to 86 ) & $8(36.4)$ & Not reported & Not reported & Not reported & Substance use already excluded \\
\hline \multirow[t]{2}{*}{ Jain, $2017^{38}$} & 1 & $\begin{array}{l}\text { Not } \\
\text { reporte } \\
d\end{array}$ & Not reported & $\begin{array}{l}\text { Not } \\
\text { reported }\end{array}$ & Not reported & Not reported & Not reported & \\
\hline & 2 & $\begin{array}{l}\text { Not } \\
\text { reporte } \\
\text { d }\end{array}$ & Not reported & $\begin{array}{l}\text { Not } \\
\text { reported }\end{array}$ & Not reported & Not reported & Not reported & \\
\hline Kim, $2001^{39}$ & 1 & 20 & 45.8 (Range: 19 to 74 ) & $15(75)$ & $1(5)$ & Not reported & Not reported & \\
\hline Kim, $2003^{40}$ & 1 & 12 & 74 & $12(100)$ & $2(17)$ & Not reported & Not reported & \\
\hline
\end{tabular}




\begin{tabular}{|c|c|c|c|c|c|c|c|c|}
\hline Author, year & Arm & $\mathbf{N}$ & Age, mean & $\begin{array}{c}\text { Male, } n \\
(\%)\end{array}$ & Dementia, n (\%) & $\begin{array}{l}\text { Substance use disorder, } \mathbf{n} \\
(\%)\end{array}$ & $\begin{array}{l}\text { Hypertension, } \\
\text { n (\%) }\end{array}$ & Comments \\
\hline \multirow[t]{2}{*}{ Kim, $2005^{41}$} & 1 & 24 & Not reported & $\begin{array}{l}\text { Not } \\
\text { reported }\end{array}$ & $0(0)$ & Not reported & Not reported & $\begin{array}{l}\text { Mean age overall: } 71.26 \text { years; } 25 \text { were } \\
\text { male }\end{array}$ \\
\hline & 2 & 18 & Not reported & $\begin{array}{l}\text { Not } \\
\text { reported }\end{array}$ & $0(0)$ & Not reported & Not reported & $\begin{array}{l}\text { Mean age overall: } 71.26 \text { years; } 25 \text { were } \\
\text { male }\end{array}$ \\
\hline \multirow[t]{2}{*}{ Kim, $2010^{42}$} & 1 & 15 & 68.3 & $9(60)$ & $0(0)$ & Not reported & Not reported & Reported female gender \\
\hline & 2 & 17 & 66.7 & $9(53)$ & $0(0)$ & Not reported & Not reported & Reported female gender \\
\hline Kishi, $2012^{43}$ & 1 & 29 & 68.9 & $20(69)$ & $(0)$ & Not reported & Not reported & \\
\hline \multirow[t]{2}{*}{ Konkayev, $2015^{44}$} & 1 & 30 & $\begin{array}{l}\text { 82.7 (Percent over age 65: } \\
100)\end{array}$ & $(33.3)$ & $0(0)$ & $0(0)$ & $22(73)$ & \\
\hline & 2 & 43 & $\begin{array}{l}80.3 \text { (Percent over age 65: } \\
100)\end{array}$ & $(25.5)$ & $0(0)$ & $0(0)$ & $26(60.5)$ & \\
\hline \multirow[t]{2}{*}{ Lee, $2005^{45}$} & 1 & 15 & 63.1 & $8(53.3)$ & Not reported & Not reported & Not reported & \\
\hline & 2 & 16 & 60.8 & $12(75)$ & Not reported & Not reported & Not reported & \\
\hline \multirow[t]{2}{*}{ Lim, $2007^{46}$} & 1 & 31 & 67.3 & $15(48.4)$ & Not reported & Not reported & Not reported & \\
\hline & 2 & 31 & 66.2 & $17(55.8)$ & Not reported & Not reported & Not reported & \\
\hline \multirow[t]{2}{*}{ Lin, $2008^{47}$} & 1 & 14 & 68 (Range: 39 to 87 ) & $4(29)$ & Not reported & Not reported & Not reported & \\
\hline & 2 & 16 & 61.13 (Range: 23 to 80 ) & $9(56)$ & Not reported & Not reported & Not reported & \\
\hline Maneeton, $2007^{48}$ & 1 & 17 & 55.6 & $10(58.8)$ & Not reported & Not reported & Not reported & $\begin{array}{l}\text { Mean (SD) age } 55.6(18.6) ; \text { none of } \\
\text { patients had a history of psychiatric } \\
\text { illness }\end{array}$ \\
\hline \multirow[t]{2}{*}{ Maneeton, $2013^{49}$} & 1 & 28 & 57 & $20(71.4)$ & Not reported & Not reported & Not reported & \\
\hline & 2 & 24 & 56.6 & $15(62.5)$ & Not reported & Not reported & Not reported & \\
\hline Mittal, 200450 & 1 & 10 & 64.7 & $8(80)$ & $0(0)$ & Not reported & Not reported & \\
\hline \multirow[t]{3}{*}{ Naksuk, $2017^{51}$} & 1 & 681 & Not reported & $\begin{array}{l}\text { Not } \\
\text { reported }\end{array}$ & Not reported & Not reported & Not reported & $\begin{array}{l}\text { Population characteristics were reported } \\
\text { for patients with delirium who received } \\
\text { vs. did not receive antipsychotics. }\end{array}$ \\
\hline & 2 & 124 & Not reported & $\begin{array}{l}\text { Not } \\
\text { reported }\end{array}$ & Not reported & Not reported & Not reported & $\begin{array}{l}\text { Population characteristics were reported } \\
\text { for patients with delirium who received } \\
\text { vs. did not receive antipsychotics. }\end{array}$ \\
\hline & 3 & 59 & Not reported & $\begin{array}{l}\text { Not } \\
\text { reported }\end{array}$ & Not reported & Not reported & Not reported & $\begin{array}{l}\text { Population characteristics were reported } \\
\text { for patients with delirium who received } \\
\text { vs. did not receive antipsychotics. }\end{array}$ \\
\hline Omura, $2003^{52}$ & 1 & 24 & 76.5 & $10(42)$ & $12(50)$ & Not reported & Not reported & \\
\hline Pae, $2004^{53}$ & 1 & 22 & 69.1 (Range: 48 to 85 ) & $13(59)$ & $7(32)$ & Not reported & Not reported & \\
\hline \multirow[t]{2}{*}{ Page, $2013^{10}$} & 1 & 70 & 68.7 & $45(64)$ & $0(0)$ & Not reported & Not reported & \\
\hline & 2 & 71 & 67.9 & $37(52)$ & $0(0)$ & Not reported & Not reported & \\
\hline Parellada, $2004^{54}$ & 1 & 64 & 67.3 & $40(62.5)$ & Not reported & Not reported & Not reported & \\
\hline
\end{tabular}




\begin{tabular}{|c|c|c|c|c|c|c|c|c|}
\hline Author, year & Arm & $\mathbf{N}$ & Age, mean & Male, $n$ (\%) & Dementia, n (\%) & $\begin{array}{l}\text { Substance use disorder, } \mathbf{n} \\
(\%)\end{array}$ & $\begin{array}{l}\text { Hypertension, } \\
\text { n (\%) }\end{array}$ & Comments \\
\hline Sasaki, $2003^{55}$ & 1 & 12 & 67.3 & $10(83)$ & Not reported & Not reported & Not reported & \\
\hline \multirow{2}{*}{$\begin{array}{l}\text { Sipahimalani, } \\
1998^{56}\end{array}$} & 1 & 11 & 64.5 & $10(91)$ & $3(27)$ & alcohol dependence: 0 (0) & $1(9)$ & \\
\hline & 2 & 11 & 63.5 & $6(55)$ & $3(27)$ & alcohol dependence: 2 (18) & $1(9)$ & \\
\hline \multirow[t]{2}{*}{ Skrobik, $2004^{57}$} & 1 & 45 & 63.26 & $31(69)$ & Not reported & Not reported & Not reported & \\
\hline & 2 & 28 & 67.5 & $22(79)$ & Not reported & Not reported & Not reported & \\
\hline Straker, $2006^{58}$ & 1 & 14 & 70.9 & $6(43)$ & $2(14)$ & Not reported & $10(71)$ & $\begin{array}{l}\text { Hypertension data obtained from Table } \\
1 .\end{array}$ \\
\hline \multirow[t]{2}{*}{ Tahir, $2010^{59}$} & 1 & 21 & 84.3 (Range: 71 to 98 ) & $6(28.6)$ & $0(0)$ & $0(0)$ & Not reported & \\
\hline & 2 & 21 & 84.1 (Range: 58 to 95 ) & $6(28.6)$ & $0(0)$ & $0(0)$ & Not reported & \\
\hline Toda, $2005^{60}$ & 1 & 10 & 61.1 & $5(50)$ & $1(10)$ & Not reported & Not reported & \\
\hline Yoon, $2011^{61}$ & 1 & 15 & 66.09 & $8(53.3)$ & $0(0)$ & $0(0)$ & Not reported & $\begin{array}{l}\text { Age : } 66.09 \text { (SD 20.69); dementia and } \\
\text { substance were excluded }\end{array}$ \\
\hline \multirow[t]{4}{*}{ Yoon, $2013^{62}$} & 1 & 23 & 74 & $12(52.2)$ & $0(0)$ & Not reported & Not reported & \\
\hline & 2 & 18 & 69.5 & $8(44.4)$ & $0(0)$ & Not reported & Not reported & \\
\hline & 3 & 18 & 73.3 & $8(44.4)$ & $0(0)$ & Not reported & Not reported & \\
\hline & 4 & 21 & 70.1 & $8(38.1)$ & $0(0)$ & Not reported & Not reported & \\
\hline
\end{tabular}




\begin{tabular}{|c|c|c|c|c|c|c|c|c|}
\hline $\begin{array}{l}\text { Author, } \\
\text { year }\end{array}$ & Arm & Drug & $\begin{array}{c}\text { Route of } \\
\text { Administration }\end{array}$ & Dose & Frequency & Duration & $\begin{array}{c}\text { Non- } \\
\begin{array}{c}\text { pharmaceutical } \\
\text { intervention }\end{array}\end{array}$ & Comments \\
\hline \multirow[t]{3}{*}{$\begin{array}{l}\text { Abdelgalel, } \\
2016^{1}\end{array}$} & 1 & Placebo & Intravenous & $\begin{array}{l}\text { Planned dose: } 2-8 \mathrm{ml} \text {, loading dose } \\
10 \mathrm{ml} \text { over } 10 \mathrm{~min} \text { if needed (if RASS } \\
>2+\text { ) }\end{array}$ & $\begin{array}{l}\text { Per hour per } \\
\text { day }\end{array}$ & Not reported & None & \\
\hline & 2 & Haloperidol & Intravenous & $\begin{array}{l}\text { Planned dose: } 0.5-2 \mathrm{mg} / \mathrm{h} \\
\text { preceded by a loading dose of } 2.5 \\
\mathrm{mg} \text { intravenously over } 10 \mathrm{~min} \text { if } \\
\text { needed (if RASS }>2+\text { ) }\end{array}$ & Per hour & Not reported & None & \\
\hline & 3 & Dexmedetomidine & Intravenous & $\begin{array}{l}\text { Planned dose: } 0.2-0.7 \mathrm{mcg} / \mathrm{kg} / \mathrm{h} \\
\text { preceded by a loading dose of } 1.0 \\
\text { ug } / \mathrm{kg} \text { intravenously over } 10 \mathrm{~min} \text { if } \\
\text { needed (if RASS }>2+\text { ) }\end{array}$ & Per hour & Not reported & None & \\
\hline \multirow[t]{2}{*}{$\begin{array}{l}\text { Al-Qadheeb, } \\
2016^{2}\end{array}$} & 1 & Placebo & Intravenous & Planned dose: $5 \%$ dextrose & $\begin{array}{l}\text { Four times a } \\
\text { day (qid) }\end{array}$ & $\begin{array}{l}\text { Planned } \\
\text { duration: } 10 \\
\text { days or until } \\
\text { discharge }\end{array}$ & $\begin{array}{l}\text { Early mobilization, } \\
\text { Pain management }\end{array}$ & $\begin{array}{l}\text { An early mobilization protocol was implemented in } \\
\text { one of the three study ICUs part-way through the } \\
\text { study }\end{array}$ \\
\hline & 2 & Haloperidol & Intravenous & Planned dose: $1 \mathrm{mg}$ & $\begin{array}{l}\text { Four times a } \\
\text { day (qid) }\end{array}$ & $\begin{array}{l}\text { Planned } \\
\text { duration: } 10 \\
\text { days or until } \\
\text { discharge }\end{array}$ & $\begin{array}{l}\text { Early mobilization, } \\
\text { Pain management }\end{array}$ & $\begin{array}{l}1 \mathrm{mg} \text { every } 6 \text { hours until delirium or therapy } \geq 10 \\
\text { days or ICU discharged occurred; An early } \\
\text { mobilization protocol was implemented in one of } \\
\text { the three study ICUs part-way through the study; }\end{array}$ \\
\hline \multirow[t]{2}{*}{$\begin{array}{l}\text { Fukata, } \\
2014^{3}\end{array}$} & 1 & Placebo & Not reported & Not reported & Not reported & Not reported & None & $\begin{array}{l}\text { The non-intervention group did not receive } \\
\text { preventive treatment, and delirium was evaluated } \\
\text { in the same way as in the intervention group }\end{array}$ \\
\hline & 2 & Haloperidol & Intravenous & Planned dose: $2.5 \mathrm{mg}$ & $\begin{array}{l}\text { Once a day } \\
\text { (qd) }\end{array}$ & $\begin{array}{l}\text { Planned } \\
\text { duration: } 3 \text { days }\end{array}$ & None & \\
\hline \multirow[t]{3}{*}{$\begin{array}{l}\text { Girard, } \\
2010^{4}\end{array}$} & 1 & Placebo & Oral & Planned dose: $5 \mathrm{ml}$ & $\begin{array}{l}\text { Twice a day } \\
\text { (bid) }\end{array}$ & $\begin{array}{l}\text { Median: } 5 \text { (IQR } \\
3 \text { to } 7 \text { ) days }\end{array}$ & None & \\
\hline & 2 & Haloperidol & Oral & $\begin{array}{l}\text { Planned dose: } 5 \mathrm{mg} \text { Median: } 15 \mathrm{mg} \\
\text { (Range } 10.8 \text { to } 17 \mathrm{mg} \text { ) }\end{array}$ & Per Day & $\begin{array}{l}\text { Median: } 7 \text { (4 to } \\
\text { 10) }\end{array}$ & None & \\
\hline & 3 & Ziprasidone & Oral & $\begin{array}{l}\text { Planned dose: } 40 \mathrm{mg} \text { Median: } \\
\text { 113mg (Range } 81 \text { to } 140 \mathrm{mg} \text { ) }\end{array}$ & Per Day & $\begin{array}{l}\text { Median: } 4 \text { (3 to } \\
\text { 10) }\end{array}$ & None & \\
\hline
\end{tabular}




\begin{tabular}{|c|c|c|c|c|c|c|c|c|}
\hline $\begin{array}{c}\text { Author, } \\
\text { year }\end{array}$ & Arm & Drug & $\begin{array}{c}\text { Route of } \\
\text { Administration } \\
\end{array}$ & Dose & Frequency & Duration & $\begin{array}{c}\text { Non-pharmaceutical } \\
\text { intervention }\end{array}$ & Comments \\
\hline \multirow[t]{2}{*}{$\begin{array}{l}\text { Hakim, } \\
2012^{5}\end{array}$} & 1 & Placebo & Oral & $\begin{array}{l}\text { Planned dose: } \\
\text { given every } 12 \\
\text { hours }\end{array}$ & $\begin{array}{l}\text { Twice a day } \\
\text { (bid) }\end{array}$ & Not reported & Pain management & \\
\hline & 2 & Risperidone & Oral & $\begin{array}{l}\text { Planned dose: } \\
0.5 \mathrm{mg}\end{array}$ & $\begin{array}{l}\text { Twice a day } \\
\text { (bid) }\end{array}$ & Not reported & Pain management & \\
\hline \multirow[t]{2}{*}{$\begin{array}{l}\text { Kalisvaart, } \\
2005^{6}\end{array}$} & 1 & Placebo & Oral & $\begin{array}{l}\text { Not reported: } \\
\text { Not applicable }\end{array}$ & $\begin{array}{l}\text { Three times a } \\
\text { day (tid) }\end{array}$ & $\begin{array}{l}\text { Planned duration: Trial medication was } \\
\text { started on admission and continued } \\
\text { until } 3 \text { days after surgery. A maximum } \\
\text { delay for surgery of } 72 \text { hours was } \\
\text { permitted. }\end{array}$ & $\begin{array}{l}\text { Orientation and therapeutic } \\
\text { activities, Fluid repletion, Sleep } \\
\text { enhancement, Pain } \\
\text { management }\end{array}$ & $\begin{array}{l}\text { Enhancement of orientation and cognition; } \\
\text { sensory and mobility-improving advice; } \\
\text { attention to pain and sleeping problems; } \\
\text { extra attention to fluid and food intake }\end{array}$ \\
\hline & 2 & Haloperidol & Oral & $\begin{array}{l}\text { Planned dose: } \\
0.5 \mathrm{mg}\end{array}$ & $\begin{array}{l}\text { Three times a } \\
\text { day (tid) }\end{array}$ & $\begin{array}{l}\text { Planned duration: Trial medication was } \\
\text { started on admission and continued } \\
\text { until } 3 \text { days after surgery. A maximum } \\
\text { delay for surgery of } 72 \text { hours was } \\
\text { permitted. }\end{array}$ & $\begin{array}{l}\text { Orientation and therapeutic } \\
\text { activities, Fluid repletion, Sleep } \\
\text { enhancement, Pain } \\
\text { management }\end{array}$ & $\begin{array}{l}\text { Enhancement of orientation and cognition; } \\
\text { sensory and mobility-improving advice; } \\
\text { attention to pain and sleeping problems; } \\
\text { extra attention to fluid and food intake }\end{array}$ \\
\hline \multirow[t]{2}{*}{$\begin{array}{l}\text { Kaneko, } \\
1999^{7}\end{array}$} & 1 & Placebo & Intravenous & $\begin{array}{l}\text { Planned dose: } \\
1 \mathrm{ml}(0.9 \% \\
\text { normal saline) }\end{array}$ & $\begin{array}{l}\text { Once a day } \\
\text { (qd) }\end{array}$ & Planned duration: 5 days post surgery & None & \\
\hline & 2 & Haloperidol & Intravenous & $\begin{array}{l}\text { Planned dose: } \\
5 \mathrm{mg}\end{array}$ & $\begin{array}{l}\text { Once a day } \\
\text { (qd) }\end{array}$ & Planned duration: 5 days post surgery & None & \\
\hline \multirow[t]{2}{*}{$\begin{array}{l}\text { Khan, } \\
2018^{8}\end{array}$} & 1 & Placebo & Intravenous & $\begin{array}{l}\text { Planned dose: } \\
\text { 5mg }\end{array}$ & 3 times daily & $\begin{array}{l}\begin{array}{l}\text { Planned duration: } 4 \text { days (11 doses } \\
\text { total) }\end{array} \\
\text { tola }\end{array}$ & None & $\begin{array}{l}\text { groups received non-pharma as part of } \\
\text { the rescue }\end{array}$ \\
\hline & 2 & Haloperidol & Intravenous & $\begin{array}{l}\text { Planned dose: } \\
\text { 5mg }\end{array}$ & 3 times daily & $\begin{array}{l}\text { Planned duration: } 4 \text { days (11 doses } \\
\text { total) }\end{array}$ & None & $\begin{array}{l}\text { groups received non-pharma as part of } \\
\text { the rescue }\end{array}$ \\
\hline \multirow[t]{2}{*}{$\begin{array}{l}\text { Larsen, } \\
2010^{9}\end{array}$} & 1 & Placebo & Oral & $\begin{array}{l}\text { Planned dose: } \\
\text { 5mg }\end{array}$ & $\begin{array}{l}\text { Once before } \\
\text { and once after } \\
\text { surgery }\end{array}$ & $\begin{array}{l}\text { Planned duration: Once before and } \\
\text { once after surgery }\end{array}$ & Pain management & \\
\hline & 2 & Olanzapine & Oral & $\begin{array}{l}\text { Planned dose: } \\
\text { 5mg }\end{array}$ & $\begin{array}{l}\text { Once before } \\
\text { and once after } \\
\text { surgery }\end{array}$ & $\begin{array}{l}\text { Planned duration: Once before and } \\
\text { once after surgery }\end{array}$ & Pain management & \\
\hline
\end{tabular}




\begin{tabular}{|c|c|c|c|c|c|c|c|c|}
\hline Author, year & Arm & Drug & $\begin{array}{c}\text { Route of } \\
\text { Administration }\end{array}$ & Dose & Frequency & Duration & $\begin{array}{c}\text { Non- } \\
\text { pharmaceutical } \\
\text { intervention }\end{array}$ & Comments \\
\hline \multirow[t]{2}{*}{ Page, $2013^{10}$} & 1 & Placebo & Intravenous & $\begin{array}{l}\text { Planned dose: } \\
0.5 \mathrm{ml}\end{array}$ & $\begin{array}{l}\text { Three times } \\
\text { a day (tid) }\end{array}$ & $\begin{array}{l}\text { Planned duration: } 14 \text { days } \\
\text { or } 2 \text { consecutive days of } \\
\text { delirium free coma free days } \\
\text { or until discharge }\end{array}$ & $\begin{array}{l}\text { Early mobilization, } \\
\text { Pain management }\end{array}$ & $\begin{array}{l}\text { Study drug was discontinued in all patients on ICU discharge, } \\
\text { when the patient was delirium-free for two consecutive days, or } \\
\text { after a maximum of } 14 \text { days treatment, whichever came first.; } \\
\text { The same volumes administered IV: } 0.5 \text { ml of study drug ( } 2.5 \\
\text { mg dissolved) and placebo; }\end{array}$ \\
\hline & 2 & Haloperidol & Intravenous & $\begin{array}{l}\text { Planned dose: } \\
2.5 \mathrm{mg}\end{array}$ & $\begin{array}{l}\text { Three times } \\
\text { a day (tid) }\end{array}$ & $\begin{array}{l}\text { Planned duration: } 14 \text { days } \\
\text { or } 2 \text { consecutive days of } \\
\text { delirium free coma free days } \\
\text { or until discharge }\end{array}$ & $\begin{array}{l}\text { Early mobilization, } \\
\text { Pain management }\end{array}$ & $\begin{array}{l}\text { Study drug was discontinued in all patients on ICU discharge, } \\
\text { when the patient was delirium-free for two consecutive days, or } \\
\text { after a maximum of } 14 \text { days treatment, whichever came first; } \\
\text { The same volumes administered IV: } 0.5 \text { ml of study drug ( } 2.5 \\
\text { mg dissolved) and placebo; }\end{array}$ \\
\hline \multirow[t]{2}{*}{$\begin{array}{l}\text { Prakanrattana, } \\
2007^{11}\end{array}$} & 1 & Placebo & Sublingual & $\begin{array}{l}\text { Planned dose: } \\
\text { Not applicable }\end{array}$ & $\begin{array}{l}\text { Once a day } \\
\text { (qd) }\end{array}$ & Planned duration: 1 day & Pain management & $\begin{array}{l}\text { Only one dose of risperidone or placebo after the patient wakes } \\
\text { up in the ICU post-operatively; Note that the placebo was a } \\
\text { listerine strip. }\end{array}$ \\
\hline & 2 & Risperidone & $\begin{array}{l}\text { Orally } \\
\text { disintegrating } \\
\text { tablet }\end{array}$ & $\begin{array}{l}\text { Planned dose: } \\
1 \mathrm{mg}\end{array}$ & $\begin{array}{l}\text { Once a day } \\
\text { (qd) }\end{array}$ & Planned duration: 1 day & Pain management & \\
\hline \multirow[t]{2}{*}{ Schrijver, $2018^{12}$} & 1 & Placebo & Oral & Not reported & $\begin{array}{l}\text { Twice a day } \\
\text { (bid) }\end{array}$ & Mean: 7 days & None & \\
\hline & 2 & Haloperidol & Oral & $\begin{array}{l}\text { Planned dose: } \\
\text { 1mg }\end{array}$ & $\begin{array}{l}\text { Twice a day } \\
\text { (bid) }\end{array}$ & Mean: 7 days & None & \\
\hline \multirow[t]{2}{*}{ Schrijver, $2018^{13}$} & 1 & Placebo & Oral & Not reported & $\begin{array}{l}\text { Twice a day } \\
\text { (bid) }\end{array}$ & Mean: 7 days & None & \\
\hline & 2 & Haloperidol & Oral & $\begin{array}{l}\text { Planned dose: } \\
\text { 1mg }\end{array}$ & $\begin{array}{l}\text { Twice a day } \\
\text { (bid) }\end{array}$ & Mean: 7 days & None & \\
\hline $\begin{array}{l}\text { van den } \\
\text { Boogaard, } \\
2013^{14}\end{array}$ & 1 & Haloperidol & Intravenous & $\begin{array}{l}\text { Planned dose: } \\
\text { 1mg Median: } \\
\text { 2mg }\end{array}$ & $\begin{array}{l}\text { Three times } \\
\text { a day (tid) }\end{array}$ & Mean: 5 & None & $\begin{array}{l}\text { Or a lower dose of } 0.5 \mathrm{mg} / 8 \mathrm{~h} \text { when then were } 80 \text { years or } \\
\text { older, had a body weight }<50 \mathrm{~kg} \text {, had a serum creatinine level } \\
>150 \text { umol/L or had a serum bilirubin level }>50 \text { umol/L. }\end{array}$ \\
\hline
\end{tabular}




\begin{tabular}{|c|c|c|c|c|c|c|c|c|}
\hline $\begin{array}{l}\text { Author, } \\
\text { year }\end{array}$ & Arm & Drug & $\begin{array}{c}\text { Route of } \\
\text { Administration }\end{array}$ & Dose & Frequency & Duration & $\begin{array}{c}\text { Non-pharmaceutical } \\
\text { intervention }\end{array}$ & Comments \\
\hline \multirow[t]{3}{*}{$\begin{array}{l}\text { van den } \\
\text { Boogaard, } \\
2018^{15}\end{array}$} & 1 & Placebo & Intravenous & Planned dose: $0.9 \% \mathrm{NaCl}$ & $\begin{array}{l}\text { Three times a day } \\
\text { (tid) }\end{array}$ & $\begin{array}{l}\text { Planned } \\
\text { duration: } 28 \\
\text { days }\end{array}$ & $\begin{array}{l}\text { Early mobilization, } \\
\text { Vision and hearing, } \\
\text { Sleep enhancement }\end{array}$ & $\begin{array}{l}\text { Nonpharmacological delirium interventions are the part of daily } \\
\text { ICU care in this study and prophylactic pharmacological study } \\
\text { was conducted in addition to this.; Other nonpharmacological } \\
\text { interventions reported are reduction of sedation and } \\
\text { benzodiazepines, awakening trials.; }\end{array}$ \\
\hline & 2 & Haloperidol & Intravenous & Planned dose: $1 \mathrm{mg}$ & $\begin{array}{l}\text { Three times a day } \\
\text { (tid) }\end{array}$ & $\begin{array}{l}\text { Planned } \\
\text { duration: } 28 \\
\text { days }\end{array}$ & $\begin{array}{l}\text { Early mobilization, } \\
\text { Vision and hearing, } \\
\text { Sleep enhancement }\end{array}$ & $\begin{array}{l}\text { Dose of study medication was reduced by } 50 \% \text { of the patients } \\
\text { are } 80 \text { years or older, had body weight of } 50 \mathrm{~kg} \text { or less or had } \\
\text { liver failure at the time of inclusion }\end{array}$ \\
\hline & 3 & Haloperidol & Intravenous & Planned dose: $2 \mathrm{mg}$ & $\begin{array}{l}\text { Three times a day } \\
\text { (tid) }\end{array}$ & $\begin{array}{l}\text { Planned } \\
\text { duration: } 28 \\
\text { days }\end{array}$ & $\begin{array}{l}\text { Early mobilization, } \\
\text { Vision and hearing, } \\
\text { Sleep enhancement }\end{array}$ & $\begin{array}{l}\text { Lower dose is given for patients aged } 80 \text { years or older, had a } \\
\text { body weight of } 50 \mathrm{~kg} \text { or less or had liver failure }\end{array}$ \\
\hline \multirow[t]{2}{*}{$\begin{array}{l}\text { Wang, } \\
2012^{16}\end{array}$} & 1 & Placebo & Intravenous & $\begin{array}{l}\text { Planned dose: Normal } \\
\text { saline }\end{array}$ & $\begin{array}{l}\text { Three times a day } \\
\text { (tid) }\end{array}$ & $\begin{array}{l}\text { Not } \\
\text { applicable }\end{array}$ & Pain management & Normal saline was used as placebo \\
\hline & 2 & Haloperidol & Intravenous & $\begin{array}{l}\text { Planned dose: } 0.5 \mathrm{mg} \\
\text { followed by continuous } \\
\text { infusion at a rate of } 1 \\
\mathrm{~mL} / \mathrm{hr}(0.1 \mathrm{mg} / \mathrm{hr} \\
\text { haloperidol })\end{array}$ & $\begin{array}{l}1 \text { hour after } \\
\text { enrollment and } \\
\text { continued for } 12 \\
\text { hours }\end{array}$ & $\begin{array}{l}\text { Planned } \\
\text { duration: } 12 \\
\text { hours }\end{array}$ & Pain management & \\
\hline
\end{tabular}


Evidence Table D-6. Intervention characteristics for studies comparing interventions to treat delirium

\begin{tabular}{|c|c|c|c|c|c|c|c|c|}
\hline $\begin{array}{c}\text { Author, } \\
\text { year }\end{array}$ & Arm & Drug & $\begin{array}{c}\text { Route of } \\
\text { Administration }\end{array}$ & Dose & Frequency & Duration & $\begin{array}{c}\begin{array}{c}\text { Non-pharmaceutical } \\
\text { intervention }\end{array} \\
\end{array}$ & Comments \\
\hline \multirow[t]{3}{*}{$\begin{array}{l}\text { Agar, } \\
2017^{17}\end{array}$} & 1 & Placebo & Oral & $\begin{array}{l}\text { Titrated dose: } \\
\text { Maximum } 4 \\
\mathrm{mg} / \mathrm{d}\end{array}$ & $\begin{array}{l}\text { Twice a day } \\
\text { (bid) }\end{array}$ & $\begin{array}{l}\text { Planned } \\
\text { duration: } 3 \\
\text { days }\end{array}$ & $\begin{array}{l}\text { Orientation and therapeutic } \\
\text { activities, Fluid repletion, } \\
\text { Vision and hearing }\end{array}$ & $\begin{array}{l}\text { A } 0.5 \mathrm{mg} \text { loading dose administered with the first dose of } 0.5 \mathrm{mg} \text {, then } 0.5 \mathrm{mg} \\
\text { maintenance doses every } 12 \text { hours. Doses could be titrated by } 0.25 \mathrm{mg} \text { on day } \\
1 \text { and by } 0.5 \mathrm{mg} \text { thereafter to a maximum dose of } 4 \mathrm{mg} / \mathrm{d} \text {; for those patients } \\
\text { over the age of } 65 \text { the loading, initial and max doses were one half that listed } \\
\text { for the remainder of the population. }\end{array}$ \\
\hline & 2 & Haloperidol & Oral & $\begin{array}{l}\text { Titrated dose: } \\
\text { Maximum } 4 \\
\mathrm{mg} / \mathrm{d}\end{array}$ & $\begin{array}{l}\text { Twice a day } \\
\text { (bid) }\end{array}$ & $\begin{array}{l}\text { Planned } \\
\text { duration: } 3 \\
\text { days }\end{array}$ & $\begin{array}{l}\text { Orientation and therapeutic } \\
\text { activities, Fluid repletion, } \\
\text { Vision and hearing }\end{array}$ & $\begin{array}{l}\text { A } 0.5 \mathrm{mg} \text { loading dose administered with the first dose of } 0.5 \mathrm{mg} \text {, then } 0.5 \mathrm{mg} \\
\text { maintenance doses every } 12 \text { hours. Doses could be titrated by } 0.25 \mathrm{mg} \text { on day } \\
1 \text { and by } 0.5 \mathrm{mg} \text { thereafter to a maximum dose of } 4 \mathrm{mg} / \mathrm{d} \text {; for those patients } \\
\text { over the age of } 65 \text { the loading, initial and max doses were one half that listed } \\
\text { for the remainder of the population. }\end{array}$ \\
\hline & 3 & Risperidone & Oral & $\begin{array}{l}\text { Titrated dose: } \\
\text { Maximum } 4 \\
\mathrm{mg} / \mathrm{d}\end{array}$ & $\begin{array}{l}\text { Twice a day } \\
\text { (bid) }\end{array}$ & $\begin{array}{l}\text { Planned } \\
\text { duration: } 3 \\
\text { days }\end{array}$ & $\begin{array}{l}\text { Orientation and therapeutic } \\
\text { activities, Fluid repletion, } \\
\text { Vision and hearing }\end{array}$ & $\begin{array}{l}\text { A } 0.5 \mathrm{mg} \text { loading dose administered with the first dose of } 0.5 \mathrm{mg} \text {, then } 0.5 \mathrm{mg} \\
\text { maintenance doses every } 12 \text { hours. Doses could be titrated by } 0.25 \mathrm{mg} \text { on day } \\
1 \text { and by } 0.5 \mathrm{mg} \text { thereafter to a maximum dose of } 4 \mathrm{mg} / \mathrm{d} \text {; for those patients } \\
\text { over the age of } 65 \text { the loading, initial and max doses were one half that listed } \\
\text { for the remainder of the population. }\end{array}$ \\
\hline \multirow[t]{2}{*}{$\begin{array}{l}\text { Atalan, } \\
2013^{18}\end{array}$} & 1 & Haloperidol & Intramuscular & $\begin{array}{l}\text { Titrated dose: } \\
\text { Maximum } \\
\text { 20mg }\end{array}$ & Per day & $\begin{array}{l}\text { Planned } \\
\text { duration: Until } \\
\text { discharge }\end{array}$ & Pain management & $\begin{array}{l}\text { Postoperative analgesia was achieved by providing } 1 \mathrm{~g} \text { of paracetamol } \\
\text { intravenously every } 8 \text { hours and } 50 \mathrm{mg} \text { of dexketoprofen intravenously twice a } \\
\text { day. Postoperative pain was recorded once a day using the verbal version of } \\
\text { the visual analog scale. }\end{array}$ \\
\hline & 2 & $\begin{array}{l}\text { morphine } \\
\text { sulfate }\end{array}$ & Intramuscular & $\begin{array}{l}\text { Titrated dose: } \\
\text { Maximum } \\
\text { 20mg }\end{array}$ & Per day & $\begin{array}{l}\text { Planned } \\
\text { duration: Until } \\
\text { discharge }\end{array}$ & Pain management & $\begin{array}{l}\text { Postoperative analgesia was achieved by providing } 1 \mathrm{~g} \text { of paracetamol } \\
\text { intravenously every } 8 \text { hours and } 50 \mathrm{mg} \text { of dexketoprofen intravenously twice a } \\
\text { day. Postoperative pain was recorded once a day using the verbal version of } \\
\text { the visual analog scale. }\end{array}$ \\
\hline
\end{tabular}




\begin{tabular}{|c|c|c|c|c|c|c|c|c|}
\hline $\begin{array}{l}\text { Author, } \\
\text { year }\end{array}$ & Arm & Drug & $\begin{array}{c}\text { Route of } \\
\text { Administration }\end{array}$ & Dose & Frequency & Duration & $\begin{array}{c}\text { Non-pharmaceutical } \\
\text { intervention }\end{array}$ & Comments \\
\hline \multirow[t]{3}{*}{$\begin{array}{l}\text { Bakri, } \\
2015^{19}\end{array}$} & 1 & Dexmedetomidine & Intravenous & Planned dose: 1 ug/kg & $\begin{array}{l}\text { Twice a day } \\
\text { (bid) }\end{array}$ & $\begin{array}{l}\text { Planned } \\
\text { duration: } 3 \\
\text { days }\end{array}$ & None & \\
\hline & 2 & Haloperidol & Intravenous & Planned dose: $5 \mathrm{mg}$ & $\begin{array}{l}\text { Twice a day } \\
\text { (bid) }\end{array}$ & $\begin{array}{l}\text { Planned } \\
\text { duration: } 3 \\
\text { days }\end{array}$ & None & \\
\hline & 3 & Ondansetron & Intravenous & Planned dose: 8 mg & $\begin{array}{l}\text { Twice a day } \\
\text { (bid) }\end{array}$ & $\begin{array}{l}\text { Planned } \\
\text { duration: } 3 \\
\text { days }\end{array}$ & None & \\
\hline \multirow[t]{2}{*}{$\begin{array}{l}\text { Boettger, } \\
2011^{20}\end{array}$} & 1 & Haloperidol & Not reported & $\begin{array}{l}\text { Titrated dose: Mean } \\
4.6 \mathrm{mg} \text { (Range: } 1 \text { to } \\
\text { 10mg) }\end{array}$ & Not reported & Not reported & None & $\begin{array}{l}\text { Mean dose was } 4.6 \mathrm{mg} \text { (range } 1 \text { to } 10 \mathrm{mg} \text { ) at baseline, } 4.9 \mathrm{mg} \\
\text { (range } 1 \text { to } 10 \mathrm{mg} \text { ) at T2 (days } 2 \text { to } 3 \text { ), and } 4.8 \mathrm{mg} \text { (range } 1 \text { to } \\
16 \mathrm{mg} \text { ) at T3 (4 to } 7 \text { days) }\end{array}$ \\
\hline & 2 & Risperidone & Not reported & $\begin{array}{l}\text { Titrated dose: Mean } \\
1.0 \text { (Range: } 0.25 \text { to } 2 \text { ) }\end{array}$ & Not reported & Not reported & None & $\begin{array}{l}\text { Mean dose was } 1.0 \mathrm{mg}(0.25 \text { to } 2 \mathrm{mg} \text { ) at baseline, } 1.2 \mathrm{mg} \\
\text { (range } 0.25 \text { to } 3 \mathrm{mg} \text { ) at T2 (days } 2 \text { to } 3 \text { ), and } 1.3 \mathrm{mg}(0.25 \text { to } 3 \\
\mathrm{mg} \text { ) at T3 (days } 4 \text { to } 7 \text { ). }\end{array}$ \\
\hline \multirow[t]{4}{*}{$\begin{array}{l}\text { Boettger, } \\
2015^{21}\end{array}$} & 1 & Aripiprazole & Not reported & $\begin{array}{l}\text { Titrated dose: Mean } \\
\text { 15.2mg (Range: } 5 \text { to } \\
\text { 30mg) }\end{array}$ & Not reported & Not reported & None & $\begin{array}{l}\text { Mean dose was } 15.2 \mathrm{mg} \text { (range } 5 \text { to } 30 \mathrm{mg} \text { ) at baseline, } 16.0 \\
\mathrm{mg} \text { (range } 10 \text { to } 30 \mathrm{mg} \text { ) at T2 ( } 2 \text { to } 3 \text { days), and } 18.3 \mathrm{mg} \text { (range } \\
10 \text { to } 30 \mathrm{mg} \text { ) at T3 (4 to } 7 \text { days). }\end{array}$ \\
\hline & 2 & Haloperidol & Not reported & $\begin{array}{l}\text { Titrated dose: Mean } \\
\text { 4.9mg (Range: } 1.5 \text { to } \\
16 \mathrm{mg})\end{array}$ & Not reported & Not reported & None & $\begin{array}{l}\text { Mean dose was } 4.9 \mathrm{mg} \text { (range } 1,5 \text { to } 16 \mathrm{mg} \text { ) at baseline, } 5.5 \\
\mathrm{mg} \text { (range } 1.5 \text { to } 16 \mathrm{mg} \text { ) at T2 (2 to } 3 \text { days), and } 5.5 \mathrm{mg} \text { (range } \\
1.5 \text { to } 16 \mathrm{mg}) \text { at T3 (4 } 4 \text { to days). }\end{array}$ \\
\hline & 3 & Olanzapine & Not reported & $\begin{array}{l}\text { Titrated dose: Mean } \\
\text { 3.5mg (Range: } 2.5 \text { to } \\
\text { 10mg) }\end{array}$ & Not reported & Not reported & None & $\begin{array}{l}\text { Mean dose was } 3.5 \mathrm{mg} \text { (range } 2.5 \text { to } 10 \mathrm{mg} \text { ) at baseline, } 5.2 \\
\mathrm{mg} \text { (range } 2.5 \text { to } 15 \mathrm{mg} \text { ) at T2 (2 to } 3 \text { days), and } 7.1 \mathrm{mg} \text { (range } \\
2.5 \text { to } 20 \mathrm{mg} \text { ) at T3 (4 to } 7 \text { days). }\end{array}$ \\
\hline & 4 & Risperidone & Not reported & $\begin{array}{l}\text { Titrated dose: Mean } \\
0.9 \mathrm{mg} \text { (Range: } 0.5 \text { to } \\
2 \mathrm{mg} \text { ) }\end{array}$ & Not reported & Not reported & None & $\begin{array}{l}\text { Mean dose was } 0.9 \mathrm{mg} \text { (range } 0.5 \text { to } 2 \mathrm{mg} \text { ) at baseline, } 1.1 \mathrm{mg} \\
\text { (range } 0.5 \text { to } 3 \mathrm{mg} \text { ) at T2 (2 to } 3 \text { days), and } 1.3 \mathrm{mg} \text { (range } 0.5 \\
\text { to } 3 \mathrm{mg} \text { ) at T3 ( } 4 \text { to } 7 \text { days). }\end{array}$ \\
\hline
\end{tabular}




\begin{tabular}{|c|c|c|c|c|c|c|c|c|}
\hline Author, year & Arm & Drug & $\begin{array}{c}\text { Route of } \\
\text { Administration }\end{array}$ & Dose & Frequency & Duration & $\begin{array}{c}\text { Non-pharmaceutical } \\
\text { intervention }\end{array}$ & Comments \\
\hline \multirow[t]{3}{*}{$\begin{array}{l}\text { Breitbart, } \\
1996^{22}\end{array}$} & 1 & Chlorpromazine & $\begin{array}{l}\text { Oral or } \\
\text { intramuscular }\end{array}$ & $\begin{array}{l}\text { Titrated dose: Oral: } 10 \text { to } \\
\text { 200mg, Intramuscular: } 5 \text { to } \\
\text { 100mg }\end{array}$ & Per day & $\begin{array}{l}\text { Planned } \\
\text { duration: } 6 \\
\text { days }\end{array}$ & None & $\begin{array}{l}\text { "The maintenance dose was equal to one to half of the } \\
\text { first } 24 \text { hour dose requirement, given twice a day } \\
\text { regimen." }\end{array}$ \\
\hline & 2 & Haloperidol & $\begin{array}{l}\text { Oral or } \\
\text { intramuscular }\end{array}$ & $\begin{array}{l}\text { Titrated dose: oral: } 0.25 \text { to } \\
5.0 \mathrm{mg} \text {, Intramuscular: } 0.125 \\
\text { to } 3.0 \mathrm{mg}\end{array}$ & Per day & $\begin{array}{l}\text { Planned } \\
\text { duration: } 6 \\
\text { days }\end{array}$ & None & $\begin{array}{l}\text { "The maintenance dose was equal to one to half of the } \\
\text { first } 24 \text { hour dose requirement, given twice a day } \\
\text { regimen." }\end{array}$ \\
\hline & 3 & Lorazepam & $\begin{array}{l}\text { Oral or } \\
\text { intramuscular }\end{array}$ & $\begin{array}{l}\text { Titrated dose: Oral: } 0.50 \text { to } \\
4.0 \mathrm{mg}, \text { Intramuscular: } 0.2 \text { to } \\
2.0 \mathrm{mg}\end{array}$ & Per day & $\begin{array}{l}\text { Planned } \\
\text { duration: } 6 \\
\text { days }\end{array}$ & None & $\begin{array}{l}\text { "The maintenance dose was equal to one to half of the } \\
\text { first } 24 \text { hour dose requirement, given twice a day } \\
\text { regimen." }\end{array}$ \\
\hline $\begin{array}{l}\text { Breitbart, } \\
2002^{23}\end{array}$ & 1 & Olanzapine & Oral & $\begin{array}{l}\text { Titrated dose: Mean } 3.0 \mathrm{mg} \\
\text { (Range: } 2.5 \text { to } 10 \mathrm{mg} \text { ) }\end{array}$ & $\begin{array}{l}\text { either once or } \\
\text { twice a day }\end{array}$ & Not reported & None & $\begin{array}{l}\text { Mean dose was } 3.0 \mathrm{mg} \text { (range } 2.5 \text { to } 10 \mathrm{mg} \text { ) at } \\
\text { baseline, } 4.6 \mathrm{mg} \text { (range } 2.5 \text { to } 15 \mathrm{mg} \text { ) at T2 (days } 2 \text { to } \\
\text { 3), and } 6.3 \mathrm{mg} \text { (range } 2.5 \text { to } 20 \mathrm{mg} \text { ) at T3 (days } 4 \text { to } 7 \text { ). }\end{array}$ \\
\hline $\begin{array}{l}\text { Carrasco, } \\
2016^{24}\end{array}$ & 1 & Haloperidol & Intravenous & $\begin{array}{l}\text { Titrated dose: (Range: } 0.5 \text { to } \\
1 \mathrm{mg} / \mathrm{hr} \text { ) }\end{array}$ & Not reported & Not reported & None & \\
\hline \multirow[t]{3}{*}{$\begin{array}{l}\text { Charoenporn, } \\
2018^{25}\end{array}$} & 1 & Haloperidol & Oral & $\begin{array}{l}\text { Titrated dose: Mean } 0.9 \mathrm{mg} \\
\text { Median } 0.5 \mathrm{mg}\end{array}$ & Not reported & $\begin{array}{l}\text { Planned } \\
\text { duration: } 7 \\
\text { days }\end{array}$ & None & \\
\hline & 2 & Quetiapine & Oral & $\begin{array}{l}\text { Titrated dose: Mean 26.7; } \\
\text { Median } 25\end{array}$ & Not reported & $\begin{array}{l}\text { Planned } \\
\text { duration: } 7 \\
\text { days }\end{array}$ & None & \\
\hline & 3 & Risperidone & Oral & $\begin{array}{l}\text { Titrated dose: Mean } 0.6 \mathrm{mg} ; \\
\text { Median } 0.5 \mathrm{mg}\end{array}$ & Not reported & $\begin{array}{l}\text { Planned } \\
\text { duration: } 7 \\
\text { days }\end{array}$ & None & \\
\hline
\end{tabular}




\begin{tabular}{|c|c|c|c|c|c|c|c|c|}
\hline $\begin{array}{l}\text { Author, } \\
\text { year }\end{array}$ & Arm & Drug & $\begin{array}{c}\text { Route of } \\
\text { Administration }\end{array}$ & Dose & Frequency & Duration & $\begin{array}{c}\text { Non- } \\
\begin{array}{c}\text { pharmaceutical } \\
\text { intervention }\end{array} \\
\end{array}$ & Comments \\
\hline \multirow[t]{2}{*}{$\begin{array}{l}\text { Devlin, } \\
2010^{26}\end{array}$} & 1 & Placebo & $\begin{array}{l}\text { either orally or via } \\
\text { NG tube }\end{array}$ & $\begin{array}{l}\text { Titrated dose: Mean 50; } \\
\text { Median 210mg (IQR } 116 \\
\text { to 293); Maximum } \\
\text { 200mg }\end{array}$ & $\begin{array}{l}\text { Twice a day } \\
\text { (bid) }\end{array}$ & $\begin{array}{l}\text { Median: } 186 \text { (IQR } 108 \text { to } 228 \text { ) hours; } \\
\text { Planned dose: } 10 \text { days or earlier if shows } \\
\text { no signs of delirium or if ICU discharge } \\
\text { prior to } 10 \text { days of therapy }\end{array}$ & None & \\
\hline & 2 & Quetiapine & $\begin{array}{l}\text { Either orally or } \\
\text { NG tube }\end{array}$ & $\begin{array}{l}\text { Titrated dose: Mean 50; } \\
\text { Median 110mg (IQR } 88 \\
\text { to 191); Maximum } \\
\text { 200mg }\end{array}$ & $\begin{array}{l}\text { Twice a day } \\
\text { (bid) }\end{array}$ & $\begin{array}{l}\text { Median: } 102 \text { (IQR } 84 \text { to } 168 \text { ) hours; } \\
\text { Planned dose: } 10 \text { days or earlier if shows } \\
\text { no signs of delirium or If ICU discharge } \\
\text { prior to } 10 \text { days of therapy }\end{array}$ & None & \\
\hline \multirow[t]{2}{*}{$\begin{array}{l}\text { Drinkwater, } \\
2014^{27}\end{array}$} & 1 & Placebo & Intravenous & $\begin{array}{l}\text { Planned dose: } 5 \mathrm{ml}(0.9 \% \\
\text { saline)ml }\end{array}$ & 8 hourly & $\begin{array}{l}\text { Planned duration: until patient delirium } \\
\text { free for } 48 \text { hours (CAM to ICU)until } \\
\text { discharged or after } 14 \text { days }\end{array}$ & None & $\begin{array}{l}\text { For non to resolving agitation, open to } \\
\text { label haloperidol could be administered in } \\
2.5 \text { to } 5 \mathrm{mg} \text { doses up to } 10 \mathrm{mg} \text { in a } 24 \text { to } \\
\text { hour period }\end{array}$ \\
\hline & 2 & Haloperidol & Intravenous & Planned dose: $2.5 \mathrm{mg}$ & 8 hourly & $\begin{array}{l}\text { Planned duration: delirium free for } 48 \\
\text { hours (CAM to ICU) or until } 14 \text { days or } \\
\text { until discharged }\end{array}$ & None & \\
\hline \multirow[t]{2}{*}{ Fox, $2018^{28}$} & 1 & Lurasidone & Not reported & $\begin{array}{l}\text { Titrated dose: Mean } \\
42.6 \mathrm{mg}\end{array}$ & Not reported & Not reported & None & $\begin{array}{l}\text { Overall duration of atypical antipsychotic } \\
\text { treatment during the hospital stay was } \\
\text { similar }(6.0[5.0 \text { to } 11.5] \text { vs } 11.0 \text { [ } 7.0 \text { to } \\
16.0] \text { days, } P=0.28) \text {. }\end{array}$ \\
\hline & 2 & Quetiapine & Not reported & $\begin{array}{l}\text { Titrated dose: Mean } \\
\text { 153.8mg }\end{array}$ & $\begin{array}{l}\text { Twice a day } \\
\text { (bid) }\end{array}$ & Not reported & None & $\begin{array}{l}\text { Overall duration of atypical antipsychotic } \\
\text { treatment during the hospital stay was } \\
\text { similar ( } 6.0[5.0 \text { to } 11.5] \text { vs } 11.0 \text { [ } 7.0 \text { to } \\
16.0] \text { days, } P=0.28) \text {. }\end{array}$ \\
\hline
\end{tabular}




\begin{tabular}{|c|c|c|c|c|c|c|c|c|}
\hline $\begin{array}{c}\text { Author, } \\
\text { year }\end{array}$ & Arm & Drug & $\begin{array}{c}\text { Route of } \\
\text { Administration }\end{array}$ & Dose & Frequency & Duration & $\begin{array}{c}\text { Non- } \\
\text { pharmaceutical } \\
\text { intervention }\end{array}$ & Comments \\
\hline \multirow[t]{3}{*}{$\begin{array}{l}\text { Girard, } \\
2010^{4}\end{array}$} & 1 & Placebo & Oral & $\begin{array}{l}\text { Planned dose: } \\
5 \mathrm{ml}\end{array}$ & $\begin{array}{l}\text { Twice a day } \\
\text { (bid) }\end{array}$ & $\begin{array}{l}\text { Median: } 5 \text { (IQR } 3 \\
\text { to 7) days }\end{array}$ & None & \\
\hline & 2 & Haloperidol & Oral & $\begin{array}{l}\text { Planned dose: } \\
\text { 5mg; Median: } \\
\text { 15mg (10.8 to } \\
17)\end{array}$ & Per day & $\begin{array}{l}\text { Median: } 7 \text { (4 to } \\
\text { 10) }\end{array}$ & None & $\begin{array}{l}\text { After enrollment } 5 \mathrm{mg} \text { haloperidol as a solution, if no temporary gastric access IM } \\
\text { (no more than } 8 \text { total doses). Second dose to } 12 \text { hour, then subsequent doses } \\
\text { every } 6 \text { hours. Frequency reduced to every } 8 \text { hour when CAM to ICU negative on } \\
2 \text { consecutive assessments; reduced to every } 12 \text { hours when patients were } \\
\text { delirium/coma to free on three consecutive assessments, and discontinued when } \\
\text { patients were delirium/coma to free on four consecutive assessments. Frequency } \\
\text { was reduced in a similar manner when patients were over sedated (RASS } \geq 2 \\
\text { levels deeper than target RASS). Dosing frequency was increased when over } \\
\text { sedation resolved. All study drug was discontinued on study day } 14 \text {. }\end{array}$ \\
\hline & 3 & Ziprasidone & Oral & $\begin{array}{l}\text { Planned dose: } \\
\text { 40mg; Median: } \\
\text { 113mg (81 to } \\
140)\end{array}$ & Per day & $\begin{array}{l}\text { Median: } 4 \text { (3 to } \\
\text { 10) }\end{array}$ & None & \\
\hline \multirow[t]{3}{*}{$\begin{array}{l}\text { Girard, } \\
2018^{29}\end{array}$} & 1 & Placebo & Intravenous & $\begin{array}{l}\text { Titrated dose: } \\
\text { Not reported }\end{array}$ & $\begin{array}{l}\text { Twice a day } \\
\text { (bid) }\end{array}$ & $\begin{array}{l}\text { Median: } 4 ; \\
\text { Planned dose: } \\
14 \text { days or until } \\
\text { discharge }\end{array}$ & None & $\begin{array}{l}\text { First dose for }<70: 0.5 \mathrm{ml} \text { placebo. First dose for } \geq 70: 0.25 \mathrm{ml} \text {. Placebo } \\
\text { administered every } 12 \text { hours }\end{array}$ \\
\hline & 2 & Haloperidol & Intravenous & $\begin{array}{l}\text { Titrated dose: } \\
\text { Mean 11; } \\
\text { Median ; } \\
\text { Maximum 10mg }\end{array}$ & $\begin{array}{l}\text { Twice a day } \\
\text { (bid) }\end{array}$ & $\begin{array}{l}\text { Median: } 4 \\
\text { Planned dose: } \\
14 \text { days or until } \\
\text { discharge }\end{array}$ & None & $\begin{array}{l}\text { First dose for }<70: 2.5 \mathrm{mg} \text { per } 0.5 \mathrm{ml} \text { haloperidol. First dose for } \geq 70: 1.25 \mathrm{mg} \text { per } \\
0.25 \mathrm{ml} \text { haloperidol. Administered every } 12 \text { hours }\end{array}$ \\
\hline & 3 & Ziprasidone & Intravenous & $\begin{array}{l}\text { Titrated dose: } \\
\text { Mean 20; } \\
\text { Median; } \\
\text { Maximum 20mg }\end{array}$ & $\begin{array}{l}\text { Twice a day } \\
\text { (bid) }\end{array}$ & $\begin{array}{l}\text { Median: 4; } \\
\text { Planned dose: } \\
\text { 14 days or until } \\
\text { discharge }\end{array}$ & None & $\begin{array}{l}\text { First dose for }<70: 5 \mathrm{mg} \text { per } 0.5 \mathrm{ml} \text { ziprasidone. First dose for } \geq 70: 2.5 \mathrm{mg} \text { per } \\
0.25 \mathrm{ml} \text { ziprasidone. Administered every } 12 \text { hours }\end{array}$ \\
\hline
\end{tabular}




\begin{tabular}{|c|c|c|c|c|c|c|c|c|}
\hline $\begin{array}{c}\text { Author, } \\
\text { year }\end{array}$ & Arm & Drug & $\begin{array}{c}\text { Route of } \\
\text { Administration }\end{array}$ & Dose & Frequency & Duration & Non-pharmaceutical intervention & Comments \\
\hline \multirow[t]{3}{*}{$\begin{array}{l}\text { Grover, } \\
2011^{30}\end{array}$} & 1 & Haloperidol & Oral & $\begin{array}{l}\text { Titrated dose: Mean } \\
0.88 \mathrm{mg} \text { (Range: } 0.25 \text { to } \\
\text { 5mg per day) }\end{array}$ & Per day & $\begin{array}{l}\text { Planned } \\
\text { duration: } 6 \\
\text { days }\end{array}$ & $\begin{array}{l}\text { Orientation and therapeutic activities, } \\
\text { Vision and hearing, Psychoactive } \\
\text { medication protocol }\end{array}$ & \\
\hline & 2 & Olanzapine & Oral & $\begin{array}{l}\text { Titrated dose: Mean } \\
\text { 3.05mg (Range: } 1.25 \text { to } \\
10 \mathrm{mg} \text { per day) }\end{array}$ & Per day & $\begin{array}{l}\text { Planned } \\
\text { duration: } 6 \\
\text { days }\end{array}$ & $\begin{array}{l}\text { Orientation and therapeutic activities, } \\
\text { Vision and hearing, Psychoactive } \\
\text { medication protocol }\end{array}$ & \\
\hline & 3 & Risperidone & Oral & $\begin{array}{l}\text { Titrated dose: Mean } \\
0.95 \mathrm{mg} \text { (Range: } 0.5 \text { to } \\
2.0 \mathrm{mg} \text { per day) }\end{array}$ & per day & $\begin{array}{l}\text { Planned } \\
\text { duration: } 6 \\
\text { days }\end{array}$ & $\begin{array}{l}\text { Orientation and therapeutic activities, } \\
\text { Vision and hearing, Psychoactive } \\
\text { medication protocol }\end{array}$ & \\
\hline \multirow[t]{2}{*}{$\begin{array}{l}\text { Grover, } \\
2016^{31}\end{array}$} & 1 & Haloperidol & Oral & $\begin{array}{l}\text { Titrated dose: Mean } 0.67 \\
\pm 0.35 \mathrm{mg} \text { (Range: } 0.25 \text { to } \\
1.25 \mathrm{mg} \text { ) }\end{array}$ & per day & $\begin{array}{l}\text { Planned } \\
\text { duration: } 6 \\
\text { days }\end{array}$ & $\begin{array}{l}\text { Orientation and therapeutic activities, } \\
\text { Vision and hearing, Psychoactive } \\
\text { medication protocol }\end{array}$ & \\
\hline & 2 & Quetiapine & Oral & $\begin{array}{l}\text { Titrated dose: Mean } 31.83 \\
\pm 4.10 \mathrm{mg} \text { (Range: } 12.5 \text { to } \\
75 \mathrm{mg})\end{array}$ & Per day & $\begin{array}{l}\text { Planned } \\
\text { duration: } 6 \\
\text { days }\end{array}$ & $\begin{array}{l}\text { Orientation and therapeutic activities, } \\
\text { Vision and hearing, Psychoactive } \\
\text { medication protocol }\end{array}$ & $\begin{array}{l}\text { Note that mean/SD for seroquel differs between } \\
\text { text (results on page } 368 \text { ) and Table } 126.63 \\
+/ 15.61 \text {, but the range is same }\end{array}$ \\
\hline
\end{tabular}




\begin{tabular}{|c|c|c|c|c|c|c|c|c|}
\hline $\begin{array}{l}\text { Author, } \\
\text { year }\end{array}$ & Arm & Drug & $\begin{array}{c}\text { Route of } \\
\text { Administration }\end{array}$ & Dose & Frequency & Duration & $\begin{array}{c}\text { Non- } \\
\text { pharmaceutical } \\
\text { intervention }\end{array}$ & Comments \\
\hline \multirow[t]{2}{*}{$\begin{array}{l}\text { Han, } \\
2004^{32}\end{array}$} & 1 & Haloperidol & Oral & $\begin{array}{l}\text { Titrated dose: } \\
\text { Mean } 1.7 \mathrm{mg} \\
\text { (Range: } 1.0 \text { to } \\
\text { 3.0mg) }\end{array}$ & $\begin{array}{l}\text { Twice a day } \\
\text { (bid) }\end{array}$ & $\begin{array}{l}\text { Planned } \\
\text { duration: } 7 \\
\text { days }\end{array}$ & None & $\begin{array}{l}\text { At the end of the study (the seventh day), the mean dose of the haloperidol group was } \\
1.71 \mathrm{mg} \text { (SD0.84, range1.0-3.0); The initial starting dose of each drug was } 0.75 \mathrm{mg} \\
\text { (haloperidol) or } 0.5 \mathrm{mg} \text { (risperidone) twice a day. The dosage was increased } \\
\text { depending on the status of delirium during the } 7 \text { days. }\end{array}$ \\
\hline & 2 & Risperidone & Oral & $\begin{array}{l}\text { Titrated dose: } \\
\text { Mean } 1.02 \mathrm{mg} \\
\text { (Range: } 0.5 \text { to } \\
\text { 2.0mg) }\end{array}$ & $\begin{array}{l}\text { Twice a day } \\
\text { (bid) }\end{array}$ & $\begin{array}{l}\text { Planned } \\
\text { duration: } 7 \\
\text { days }\end{array}$ & None & $\begin{array}{l}\text { The initial starting dose of each drug was } 0.75 \mathrm{mg} \text { (haloperidol) or } 0.5 \mathrm{mg} \\
\text { (risperidone) twice a day. The dosage was increased depending on the status of } \\
\text { delirium during the } 7 \text { days. At the end of the study (the seventh day), the mean dose } \\
\text { of the haloperidol group was } 1.71 \mathrm{mg} \text { (SD0.84, range1.0-3.0), and the mean dose of } \\
\text { the risperidone group was } 1.02 \mathrm{mg} \text { (SD0.41, range0.5-2.0). }\end{array}$ \\
\hline \multirow[t]{2}{*}{$\begin{array}{l}\text { Hatta, } \\
2014^{33}\end{array}$} & 1 & Aripiprazole & Not reported & $\begin{array}{l}\text { Titrated dose: } \\
\text { Maximum } \\
7.23 \mathrm{mg}\end{array}$ & $\begin{array}{l}\text { Not } \\
\text { reported }\end{array}$ & $\begin{array}{l}\text { Not } \\
\text { reported }\end{array}$ & None & $\begin{array}{l}43 \% \text { of patients took this drug for } 1 \text { week or less; } 25 \% \text { for } 2 \text { weeks or less, and } 33 \% \\
\text { for over } 2 \text { weeks; There was a priority for non to pharmacological management for } \\
\text { delirium, which included minimize drug side effects, fluid repletion, orientation and } \\
\text { therapeutic activities, limit sensory underload or overload, and early mobilization. }\end{array}$ \\
\hline & 2 & Haloperidol & Intravenous & $\begin{array}{l}\text { Titrated dose: } \\
\text { Maximum } \\
6.40 \mathrm{mg}\end{array}$ & $\begin{array}{l}\text { Not } \\
\text { reported }\end{array}$ & $\begin{array}{l}\text { Not } \\
\text { reported }\end{array}$ & None & $\begin{array}{l}52 \% \text { of patients took this drug for } 1 \text { week or less; } 19 \% \text { for } 2 \text { weeks or less, and } 29 \% \\
\text { for over } 2 \text { weeks; There was a priority for non to pharmacological management for } \\
\text { delirium, which included minimize drug side effects, fluid repletion, orientation and } \\
\text { therapeutic activities, limit sensory underload or overload, and early mobilization. }\end{array}$ \\
\hline
\end{tabular}




\begin{tabular}{|c|c|c|c|c|c|c|c|c|}
\hline Author, year & Arm & Drug & $\begin{array}{c}\text { Route of } \\
\text { Administration }\end{array}$ & Dose & Frequency & Duration & $\begin{array}{c}\text { Non- } \\
\text { pharmaceutical } \\
\text { intervention }\end{array}$ & Comments \\
\hline \multirow[t]{3}{*}{$\begin{array}{l}\text { Hatta, 201433 } \\
\text { (continued) }\end{array}$} & 3 & Olanzapine & Not reported & $\begin{array}{l}\text { Titrated dose: } \\
\text { Maximum } \\
10.2 \mathrm{mg}\end{array}$ & $\begin{array}{l}\text { Not } \\
\text { reported }\end{array}$ & Not reported & None & $\begin{array}{l}67 \% \text { of patients took this drug for } 1 \text { week or less; } 17 \% \text { for } 2 \text { weeks or less, and } \\
18 \% \text { for over } 2 \text { weeks; There was a priority for non to pharmacological } \\
\text { management for delirium, which included minimize drug side effects, fluid } \\
\text { repletion, orientation and therapeutic activities, limit sensory underload or } \\
\text { overload, and early mobilization. }\end{array}$ \\
\hline & 4 & Quetiapine & Not applicable & $\begin{array}{l}\text { Titrated dose: } \\
\text { Maximum } \\
\text { 71.8mg }\end{array}$ & $\begin{array}{l}\text { Not } \\
\text { reported }\end{array}$ & Not reported & None & $\begin{array}{l}60 \% \text { of patients took this drug for } 1 \text { week or less; } 19 \% \text { for } 2 \text { weeks or less, and } \\
21 \% \text { for over } 2 \text { weeks; There was a priority for non to pharmacological } \\
\text { management for delirium, which included minimize drug side effects, fluid } \\
\text { repletion, orientation and therapeutic activities, limit sensory underload or } \\
\text { overload, and early mobilization. }\end{array}$ \\
\hline & 5 & Risperidone & Not applicable & $\begin{array}{l}\text { Titrated dose: } \\
\text { Maximum } \\
1.35 \mathrm{mg}\end{array}$ & $\begin{array}{l}\text { Not } \\
\text { reported }\end{array}$ & Not reported & None & $\begin{array}{l}49 \% \text { of patients took this drug for } 1 \text { week or less; } 25 \% \text { for } 2 \text { weeks or less, and } \\
26 \% \text { for over } 2 \text { weeks; There was a priority for non to pharmacological } \\
\text { management for delirium, which included minimize drug side effects, fluid } \\
\text { repletion, orientation and therapeutic activities, limit sensory underload or } \\
\text { overload, and early mobilization. }\end{array}$ \\
\hline $\begin{array}{l}\text { Horikawa, } \\
2003^{34}\end{array}$ & 1 & Risperidone & Oral & $\begin{array}{l}\text { Titrated dose: } \\
0.5 \mathrm{mg}\end{array}$ & $\begin{array}{l}\text { Once a day } \\
\text { (qd) }\end{array}$ & $\begin{array}{l}\text { Mean: } 19.4 \\
\text { days }\end{array}$ & None & $\begin{array}{l}\text { Haloperidol at a dose of } 0.75 \text { to } 5.0 \mathrm{mg} / \text { day had been used in all patients, but not } \\
\text { continuously, without any effect. }\end{array}$ \\
\hline \multirow[t]{3}{*}{$\mathrm{Hu}, 2006^{35}$} & 1 & None & Not applicable & Not reported & $\begin{array}{l}\text { Not } \\
\text { reported }\end{array}$ & $\begin{array}{l}\text { Planned } \\
\text { duration: } 1 \\
\text { week }\end{array}$ & None & $\begin{array}{l}\text { Given somatic treatment aiming at delirium, not any drug for central nervous } \\
\text { system was used }\end{array}$ \\
\hline & 2 & Haloperidol & Intramuscular & $\begin{array}{l}\text { Titrated dose: } \\
\text { (Range: } 2.5 \text { to } \\
\text { 10mg) }\end{array}$ & $\begin{array}{l}\text { Once a day } \\
\text { (qd) }\end{array}$ & $\begin{array}{l}\text { Planned } \\
\text { duration: } 1 \\
\text { week }\end{array}$ & None & \\
\hline & 3 & Olanzapine & $\begin{array}{l}\text { Oral or } \\
\text { sublingually }\end{array}$ & $\begin{array}{l}\text { Titrated dose: } \\
\text { (Range: } 1.25 \\
\text { to 20mg) }\end{array}$ & $\begin{array}{l}\text { Once a day } \\
\text { (qd) }\end{array}$ & $\begin{array}{l}\text { Planned } \\
\text { duration: } 1 \\
\text { week }\end{array}$ & None & \\
\hline
\end{tabular}




\begin{tabular}{|c|c|c|c|c|c|c|c|c|}
\hline $\begin{array}{c}\text { Author, } \\
\text { year }\end{array}$ & Arm & Drug & $\begin{array}{c}\text { Route of } \\
\text { Administration }\end{array}$ & Dose & Frequency & Duration & $\begin{array}{c}\text { Non-pharmaceutical } \\
\text { intervention }\end{array}$ & Comments \\
\hline Hui, $2017^{36}$ & 1 & Haloperidol & Intravenous & $\begin{array}{l}\text { Titrated dose: Not } \\
\text { reported }\end{array}$ & $\begin{array}{l}2 \mathrm{mg} \text { every } 4 \text { hours, and } \\
\text { another } 2 \mathrm{mg} \text { every hour as } \\
\text { needed for agitation }\end{array}$ & $\begin{array}{l}\text { Planned } \\
\text { duration: Until } \\
\text { discharge }\end{array}$ & None & \\
\hline $\begin{array}{l}\text { Ikezawa, } \\
2008^{37}\end{array}$ & 1 & Risperidone & Oral & $\begin{array}{l}\text { Titrated dose: Mean } 1.5 \\
(0.7) \text { (Range: } 0.5 \text { to } 3 \mathrm{mg} \text { ) }\end{array}$ & Once or twice & Mean: 11.7 (3.5) & None & \\
\hline \multirow[t]{2}{*}{$\begin{array}{l}\text { Jain, } \\
2017^{38}\end{array}$} & 1 & Haloperidol & Oral & $\begin{array}{l}\text { Titrated dose: Mean } \\
\text { 2.10mg }\end{array}$ & Once a day (qd) & Mean: 3.37 days & None & \\
\hline & 2 & Olanzapine & Oral & $\begin{array}{l}\text { Titrated dose: Mean } \\
\text { 5.49mg }\end{array}$ & Once a day (qd) & Mean: 3.57 days & None & \\
\hline $\begin{array}{l}\text { Kim, } \\
2001^{39}\end{array}$ & 1 & Olanzapine & Oral & $\begin{array}{l}\text { Titrated dose: Mean } 5.9 \\
\text { (1.5); Median ; Maximum } \\
8.8(2.2)\end{array}$ & Not reported & Mean: 6.6 (1.7) & None & \\
\hline $\begin{array}{l}\text { Kim, } \\
2003^{40}\end{array}$ & 1 & Quetiapine & Not reported & Titrated dose: $25 \mathrm{mg}$ & Twice a day (bid) & $\begin{array}{l}\text { Planned } \\
\text { duration: } 3 \\
\text { months }\end{array}$ & None & $\begin{array}{l}\text { Mean dose is the mean dose when patients } \\
\text { were maximally stabilized. }\end{array}$ \\
\hline \multirow[t]{2}{*}{$\begin{array}{l}\text { Kim, } \\
2005^{41}\end{array}$} & 1 & Haloperidol & Not reported & $\begin{array}{l}\text { Titrated dose: Not } \\
\text { reported }\end{array}$ & Not reported & Mean: 8.5 days & None & $\begin{array}{l}\text { Mean starting dose: } 2.67 \mathrm{mg} \text { (range } 0.75 \text { to } \\
10.0 \mathrm{mg} / \text { day); Mean ending dose } 1.67 \mathrm{mg} \\
\text { (range } 0.50 \text { to } 2.50 \mathrm{mg} / \mathrm{day} \text { ). }\end{array}$ \\
\hline & 2 & Risperidone & Not reported & $\begin{array}{l}\text { Titrated dose: Not } \\
\text { reported }\end{array}$ & Not reported & Mean: 4.8 days & None & $\begin{array}{l}\text { Mean starting dose } 0.97 \mathrm{mg} \text { (range } 0.5 \text { to } 5.0 \\
\mathrm{mg} / \text { day); mean ending dose } 1.19 \text { (range } 0.5 \\
\text { to } 5.0 \mathrm{mg} / \text { day) }\end{array}$ \\
\hline \multirow[t]{2}{*}{$\begin{array}{l}\text { Kim, } \\
2010^{42}\end{array}$} & 1 & Olanzapine & Not reported & $\begin{array}{l}\text { Titrated dose: Mean } 2.4 \pm \\
1.7 \mathrm{mg} \text { (Range: } 1.25 \text { to } \\
7.5 \mathrm{mg})\end{array}$ & Per day & $\begin{array}{l}\text { Planned } \\
\text { duration: } 7 \text { days }\end{array}$ & None & $\begin{array}{l}\text { The mean doses at last observation } 2.4 \pm 1.7 \\
\text { for olanzapine group }\end{array}$ \\
\hline & 2 & Risperidone & Not reported & $\begin{array}{l}\text { Titrated dose: Mean } 0.9 \pm \\
0.6 \mathrm{mg} \text { (Range: } 0.25 \text { to } \\
2 \mathrm{mg} \text { ) }\end{array}$ & Per day & $\begin{array}{l}\text { Planned } \\
\text { duration: } 7 \text { days }\end{array}$ & None & $\begin{array}{l}\text { The mean doses of last observation were } 0.9 \\
\pm 0.6 \mathrm{mg} / \mathrm{day}\end{array}$ \\
\hline $\begin{array}{l}\text { Kishi, } \\
2012^{43}\end{array}$ & 1 & Risperidone & Oral & $\begin{array}{l}\text { Titrated dose: Mean } \\
\text { 1.4mg }\end{array}$ & Once a day (qd) & $\begin{array}{l}\text { Planned } \\
\text { duration: } 7 \text { days }\end{array}$ & None & \\
\hline
\end{tabular}




\begin{tabular}{|c|c|c|c|c|c|c|c|c|}
\hline $\begin{array}{c}\text { Author, } \\
\text { year }\end{array}$ & Arm & Drug & $\begin{array}{c}\text { Route of } \\
\text { Administration }\end{array}$ & Dose & Frequency & Duration & $\begin{array}{c}\text { Non- } \\
\text { pharmaceutical } \\
\text { intervention }\end{array}$ & Comments \\
\hline \multirow[t]{2}{*}{$\begin{array}{l}\text { Konkayev, } \\
2015^{44}\end{array}$} & 1 & Dexmedetomidine & Infusion & $\begin{array}{l}\text { Titrated dose: Median } \\
0.93 \mathrm{mg} / \mathrm{kg} / \mathrm{hour} \text { (Range: } \\
0.2 \text { to } 1.4 \mathrm{mg} / \mathrm{kg} / \text { hour) }\end{array}$ & Continuous & Not reported & Pain management & $\begin{array}{l}\text { Fentanyl and propofol were also used to manage symptoms, if } \\
\text { necessary; Less people in the dexmedetomidine group }(10 \%) \\
\text { were on propofol than in the haloperidol }(42 \%) \text {. More people in } \\
\text { the haloperidol group ( } 72 \%) \text { were on fentanyl than in the } \\
\text { dexmedetomidine group (43\%). }\end{array}$ \\
\hline & 2 & Haloperidol & $\begin{array}{l}\text { Intravenous } \\
\text { infusion }\end{array}$ & $\begin{array}{l}\text { Titrated dose: Median } \\
0.12 \mathrm{mg} / \mathrm{kg} / \mathrm{h}(\text { Range: } \\
0.10 \text { to } 0.15 \mathrm{mg} / \mathrm{kg} / \mathrm{h})\end{array}$ & Continuous & Not reported & Pain management & \\
\hline \multirow[t]{2}{*}{ Lee, $2005^{45}$} & 1 & Quetiapine & Not reported & $\begin{array}{l}\text { Titrated dose: Mean } 113 \\
\pm 85.5 \mathrm{mg} \text { (Range: } 50 \text { to } \\
\text { 300mg) }\end{array}$ & Per day & $\begin{array}{l}\text { Planned } \\
\text { duration: or } \\
\text { into remission }\end{array}$ & None & \\
\hline & 2 & Amisulpride & Not reported & $\begin{array}{l}\text { Titrated dose: Mean } \\
156.4 \pm 97.5 \mathrm{mg} \text { (Range: } \\
50 \text { to } 800 \mathrm{mg} \text { ) }\end{array}$ & Per day & $\begin{array}{l}\text { Planned } \\
\text { duration: or } \\
\text { remission }\end{array}$ & None & \\
\hline \multirow[t]{2}{*}{ Lim, $2007^{46}$} & 1 & Haloperidol & Intramuscular & Titrated (5 to $20 \mathrm{mg}$ ) & $\begin{array}{l}\text { Other: More } \\
\text { than } 2 \text { hours } \\
\text { apart }\end{array}$ & Not reported & None & \\
\hline & 2 & Olanzapine & Intramuscular & Titrated (5 to $20 \mathrm{mg}$ ) & $\begin{array}{l}\text { Other: More } \\
\text { than } 2 \text { hours } \\
\text { apart }\end{array}$ & Not reported & None & \\
\hline \multirow[t]{2}{*}{ Lin, $2008^{47}$} & 1 & Haloperidol & Oral & $\begin{array}{l}\text { Titrated dose: start dose } \\
5 \mathrm{mg}\end{array}$ & $\begin{array}{l}\text { Once a day } \\
\text { (qd) }\end{array}$ & $\begin{array}{l}\text { Planned } \\
\text { duration: } 7 \\
\text { days }\end{array}$ & None & \\
\hline & 2 & Olanzapine & Oral & $\begin{array}{l}\text { Titrated dose: start dose } \\
5 \mathrm{mg}\end{array}$ & $\begin{array}{l}\text { Once a day } \\
\text { (qd) }\end{array}$ & $\begin{array}{l}\text { Planned } \\
\text { duration: } 7 \\
\text { days }\end{array}$ & None & \\
\hline $\begin{array}{l}\text { Maneeton, } \\
2007^{48}\end{array}$ & 1 & Quetiapine & Oral & $\begin{array}{l}\text { Titrated dose: Mean } 45.7 \\
\text { (28.7); Maximum } 100 \mathrm{mg} \\
\text { (Range: } 25 \text { to } 100 \mathrm{mg} \text { ) }\end{array}$ & $\begin{array}{l}\text { Once or twice } \\
\text { per day }\end{array}$ & $\begin{array}{l}\text { Planned } \\
\text { duration: } 7 \\
\text { days }\end{array}$ & None & $\begin{array}{l}\text { Dose between } 25 \text { to } 100 \mathrm{mg} / \text { day; Mean start dose (SD) is } 36.8 \\
\text { (24.8); During the present study, lorazepam at the maximum } \\
\text { dose of } 2 \mathrm{mg} / \text { day was an additional medication that could } \\
\text { be used if needed but never used for any of patients } \\
\text { Mean (SD) }\end{array}$ \\
\hline \multirow[t]{2}{*}{$\begin{array}{l}\text { Maneeton, } \\
2013^{49}\end{array}$} & 1 & Haloperidol & Oral & $\begin{array}{l}\text { Titrated dose: Mean } 0.8 \\
\text { (SD 0.3) (Range: } 0.5 \text { to } \\
\text { 2.0mg) }\end{array}$ & Per day & $\begin{array}{l}\text { Planned } \\
\text { duration: } 7 \\
\text { days }\end{array}$ & $\begin{array}{l}\text { Orientation and } \\
\text { therapeutic } \\
\text { activities }\end{array}$ & \\
\hline & 2 & Quetiapine & Oral & $\begin{array}{l}\text { Titrated dose: Mean } 67.6 \\
\text { (SD 9.7) (Range: } 25 \text { to } \\
\text { 100mg) }\end{array}$ & per day & $\begin{array}{l}\text { Planned } \\
\text { duration: } 7 \\
\text { days }\end{array}$ & $\begin{array}{l}\text { Orientation and } \\
\text { therapeutic } \\
\text { activities }\end{array}$ & \\
\hline
\end{tabular}




\begin{tabular}{|c|c|c|c|c|c|c|c|c|}
\hline $\begin{array}{l}\text { Author, } \\
\text { year }\end{array}$ & Arm & Drug & $\begin{array}{c}\text { Route of } \\
\text { Administration }\end{array}$ & Dose & Frequency & Duration & $\begin{array}{c}\text { Non- } \\
\text { pharmaceutical } \\
\text { intervention }\end{array}$ & Comments \\
\hline $\begin{array}{l}\text { Mittal, } \\
2004^{50}\end{array}$ & 1 & Risperidone & Not reported & $\begin{array}{l}\text { Titrated dose: } \\
\text { Mean } 0.75 \mathrm{mg}\end{array}$ & Twice a day (bid) & Planned duration: 5 days & None & $\begin{array}{l}\text { Mean dosage on day } 1 \text { was } 1.35 \mathrm{mg} / \text { day (range } 1.0 \text { to } 2.0 \\
\mathrm{mg} / \text { day). Mean maintenance dosage was } 0.75 \mathrm{mg} / \mathrm{day} \\
\text { (range } 0.5 \text { to } 1.50 \mathrm{mg} / \mathrm{day} \text { ). }\end{array}$ \\
\hline \multirow[t]{3}{*}{$\begin{array}{l}\text { Naksuk, } \\
2017^{51}\end{array}$} & 1 & None & & Not reported & & Not reported & None & $\begin{array}{l}\text { This group developed delirium, but did not receive } \\
\text { antipsychotics. }\end{array}$ \\
\hline & 2 & Haloperidol & Not reported & $\begin{array}{l}\text { Titrated dose: } \\
\text { Median 5.0mg } \\
\text { (Range: } 1.0 \text { to } \\
\text { 57.5mg) }\end{array}$ & Not reported & Median: 1.0 & None & $\begin{array}{l}\text { Duration obtained from Table } 2 \text {. Range in duration as } 1 \text { to } \\
10 \text { days; Dose is average dose per day, obtained from } \\
\text { Table } 2 \text {. }\end{array}$ \\
\hline & 3 & Quetiapine & Not reported & $\begin{array}{l}\text { Titrated dose: } \\
\text { Median } 25.0 \mathrm{mg} \\
\text { (Range: } 4.2 \text { to } \\
\text { 150.0mg) }\end{array}$ & Not reported & Median: 2.0 & None & $\begin{array}{l}\text { Median duration obtained from Table } 2 . \text { Range in duration } \\
\text { was } 1.0 \text { to } 8.0 \text { days; Dose is average dose per day, } \\
\text { obtained from Table } 2 \text {. }\end{array}$ \\
\hline $\begin{array}{l}\text { Omura, } \\
2003^{52}\end{array}$ & 1 & Quetiapine & Not reported & $\begin{array}{l}\text { Titrated dose: } \\
\text { Mean } 54.7 \mathrm{mg} \\
\text { (Range: } 25 \text { to } \\
\text { 125mg) }\end{array}$ & $\begin{array}{l}\text { once a day ( } 13 \\
\text { patients), twice a day } \\
\text { ( } 5 \text { patients), } 3 \text { times } \\
\text { per day }(6 \text { patients) }\end{array}$ & Not reported & None & \\
\hline $\begin{array}{l}\text { Pae, } \\
2004^{53}\end{array}$ & 1 & Quetiapine & Oral & $\begin{array}{l}\text { Titrated dose: } \\
\text { Mean } 127.1 \mathrm{mg}\end{array}$ & Not reported & Mean: 8.5 days & None & \\
\hline \multirow[t]{2}{*}{$\begin{array}{l}\text { Page, } \\
2013^{10}\end{array}$} & 1 & Placebo & Intravenous & $\begin{array}{l}\text { Planned dose: } \\
0.5 \mathrm{ml}\end{array}$ & Three times a day (tid) & $\begin{array}{l}\text { Planned duration: } 14 \text { days } \\
\text { or } 2 \text { consecutive days of } \\
\text { delirium free coma free } \\
\text { days or until discharge }\end{array}$ & $\begin{array}{l}\text { Early mobilization, } \\
\text { Pain management }\end{array}$ & $\begin{array}{l}\text { Study drug was discontinued in all patients on ICU } \\
\text { discharge, when the patient was delirium to free for two } \\
\text { consecutive days, or after a maximum of } 14 \text { days } \\
\text { treatment, whichever came first.; The same volumes } \\
\text { administered IV: } 0.5 \mathrm{ml} \text { of study drug ( } 2.5 \mathrm{mg} \text { dissolved) } \\
\text { and placebo }\end{array}$ \\
\hline & 2 & Haloperidol & Intravenous & $\begin{array}{l}\text { Planned dose: } \\
2.5 \mathrm{mg}\end{array}$ & Three times a day (tid) & $\begin{array}{l}\text { Planned duration: } 14 \text { days } \\
\text { or } 2 \text { consecutive days of } \\
\text { delirium free coma free } \\
\text { days or until discharge }\end{array}$ & $\begin{array}{l}\text { Early mobilization, } \\
\text { Pain management }\end{array}$ & $\begin{array}{l}\text { Study drug was discontinued in all patients on ICU } \\
\text { discharge, when the patient was delirium to free for two } \\
\text { consecutive days, or after a maximum of } 14 \text { days } \\
\text { treatment, whichever came first; The same volumes } \\
\text { administered IV: } 0.5 \text { ml of study drug ( } 2.5 \text { mg dissolved) } \\
\text { and placebo }\end{array}$ \\
\hline $\begin{array}{l}\text { Parellada, } \\
2004^{54}\end{array}$ & 1 & Risperidone & Oral & $\begin{array}{l}\text { Titrated dose: } \\
\text { Mean day } 1,2.6 \text {; } \\
\text { day } 3,2.6 ; \text { day } 7 \text {, } \\
1.5 \mathrm{mg}\end{array}$ & Twice a day (bid) & Planned duration: 7 days & None & \\
\hline
\end{tabular}




\begin{tabular}{|c|c|c|c|c|c|c|c|c|}
\hline Author, year & Arm & Drug & Route of Administration & Dose & Frequency & Duration & $\begin{array}{c}\text { Non-pharmaceutical } \\
\text { intervention }\end{array}$ & Comments \\
\hline Sasaki, $2003^{55}$ & 1 & Quetiapine & Oral & $\begin{array}{l}\text { Titrated dose: Mean } \\
\text { 44.9; Median ; } \\
\text { Maximum 63.5mg }\end{array}$ & $\begin{array}{l}\text { Once a day } \\
\text { (qd) }\end{array}$ & $\begin{array}{l}\text { Mean: } 4.8 \\
\text { days }\end{array}$ & None & \\
\hline \multirow[t]{2}{*}{$\begin{array}{l}\text { Sipahimalani, } \\
1998^{56}\end{array}$} & 1 & Haloperidol & $\begin{array}{l}\text { Orally, except one patient } \\
\text { received haloperidol } \\
\text { intravenously }\end{array}$ & $\begin{array}{l}\text { Titrated dose: (Range: } \\
1.5 \text { to } 10 \mathrm{mg})\end{array}$ & Not reported & $\begin{array}{l}\text { Mean: } 14.6 \\
\text { days }\end{array}$ & None & \\
\hline & 2 & Olanzapine & Not reported & $\begin{array}{l}\text { Titrated dose: Mean } \\
\text { 8.2; Median ; Maximum } \\
\text { 15mg }\end{array}$ & $\begin{array}{l}\text { Once a day } \\
\text { (qd) }\end{array}$ & $\begin{array}{l}\text { Mean: } 23.6 \\
\text { days }\end{array}$ & None & \\
\hline \multirow[t]{2}{*}{ Skrobik, $2004^{57}$} & 1 & Haloperidol & Oral & $\begin{array}{l}\text { Titrated dose: Mean } \\
6.5 \mathrm{mg} \text { (Range: } 1 \text { to } \\
\text { 28mg) }\end{array}$ & per day & Not reported & None & $\begin{array}{l}\text { Duration of therapy is not mentioned, but the study } \\
\text { data is collected for } 5 \text { days, so can we assume the } \\
\text { duration of therapy as } 5 \text { days. }\end{array}$ \\
\hline & 2 & Olanzapine & Oral & $\begin{array}{l}\text { Titrated dose: Mean } \\
4.54 \mathrm{mg} \text { (Range: } 2.5 \text { to } \\
13.5 \mathrm{mg} \text { ) }\end{array}$ & per day & Not reported & None & \\
\hline Straker, $2006^{58}$ & 1 & Aripiprazole & Not reported & $\begin{array}{l}\text { Titrated dose: Mean } \\
\text { 8.9mg (Range: } 5 \text { to } \\
\text { 15mg) }\end{array}$ & Not reported & $\begin{array}{l}\text { Planned } \\
\text { duration: } 7 \\
\text { days }\end{array}$ & None & $\begin{array}{l}\text { Only two patients received a dose higher than } 10 \mathrm{mg} \text {; } \\
\text { Four patients also received as to needed haloperidol } \\
\text { in the first few days of treatment. }\end{array}$ \\
\hline \multirow[t]{2}{*}{ Tahir, $2010^{59}$} & 1 & Placebo & Oral & $\begin{array}{l}\text { Titrated dose: Mean 25; } \\
\text { Maximum 175mg }\end{array}$ & $\begin{array}{l}\text { Once a day } \\
\text { (qd) }\end{array}$ & $\begin{array}{l}\text { Planned } \\
\text { duration: } 10 \\
\text { days }\end{array}$ & None & \\
\hline & 2 & Quetiapine & Oral & $\begin{array}{l}\text { Titrated dose: Mean } \\
\text { 40mg (Range: } 25 \text { to } \\
\text { 175mg) }\end{array}$ & $\begin{array}{l}\text { Once a day } \\
\text { (qd) }\end{array}$ & $\begin{array}{l}\text { Planned } \\
\text { duration: } 10 \\
\text { days }\end{array}$ & None & \\
\hline
\end{tabular}




\begin{tabular}{|c|c|c|c|c|c|c|c|c|}
\hline $\begin{array}{c}\text { Author, } \\
\text { year }\end{array}$ & Arm & Drug & $\begin{array}{c}\text { Route of } \\
\text { Administration }\end{array}$ & Dose & Frequency & Duration & $\begin{array}{c}\text { Non- } \\
\text { pharmaceutical } \\
\text { intervention }\end{array}$ & Comments \\
\hline $\begin{array}{l}\text { Toda, } \\
2005^{60}\end{array}$ & 1 & Risperidone & Oral & $\begin{array}{l}\text { Titrated dose: } \\
\text { Mean 0.92; } \\
\text { Median ; } \\
\text { Maximum } 1.38\end{array}$ & $\begin{array}{l}\text { Once a day } \\
\text { (qd) }\end{array}$ & $\begin{array}{l}\text { Planned duration: } \\
\text { until remission, } \\
\text { which ranged from } \\
1 \text { to } 48 \text { days }\end{array}$ & None & \\
\hline $\begin{array}{l}\text { Yoon, } \\
2011^{61}\end{array}$ & 1 & Paliperidone & Oral & $\begin{array}{l}\text { Titrated dose: } \\
\text { Mean 3.75 } \\
\text { (1.06); Maximum } \\
9 \mathrm{mg} \\
\end{array}$ & $\begin{array}{l}\text { Once a day } \\
\text { (qd) }\end{array}$ & $\begin{array}{l}\text { Planned duration: } 7 \\
\text { days }\end{array}$ & None & Start dose was $3 \mathrm{mg} / \mathrm{d}$ \\
\hline \multirow[t]{4}{*}{$\begin{array}{l}\text { Yoon, } \\
2013^{62}\end{array}$} & 1 & Haloperidol & Not reported & $\begin{array}{l}\text { Titrated dose: } \\
\text { Mean } 1.2 \mathrm{mg} \\
\text { (Range: } 0.5 \text { to } \\
\text { 10mg) }\end{array}$ & $\begin{array}{l}\text { Not } \\
\text { reported }\end{array}$ & $\begin{array}{l}\text { Mean duration: } 4.7 \\
\text { days; Planned } \\
\text { duration: } 6 \text { days }\end{array}$ & None & $\begin{array}{l}\text { The initial starting dose was determined on the basis of age, degree of severity } \\
\text { of delirium, and the general medical or postsurgical condition of the individual } \\
\text { subject. Dose was adjusted according to clinical judgment based on daily } \\
\text { overall impression of delirium over } 6 \text { days primarily, and on clinical } \\
\text { assessments of the degree of improvement in delirium symptoms and the }+/ \text { to } \\
\text { of adverse events. }\end{array}$ \\
\hline & 2 & Olanzapine & Not reported & $\begin{array}{l}\text { Titrated dose: } \\
\text { Mean 2.9mg } \\
\text { (Range: } 1 \text { to } \\
\text { 20mg) }\end{array}$ & $\begin{array}{l}\text { Not } \\
\text { reported }\end{array}$ & $\begin{array}{l}\text { Mean duration: 5.3; } \\
\text { Planned duration: } 6 \\
\text { days }\end{array}$ & None & \\
\hline & 3 & Quetiapine & Not reported & $\begin{array}{l}\text { Titrated dose: } \\
\text { Mean } 47.9 \mathrm{mg} \\
\text { (Range: } 25 \text { to } \\
\text { 200mg) }\end{array}$ & $\begin{array}{l}\text { Not } \\
\text { reported }\end{array}$ & $\begin{array}{l}\text { Mean duration: } 4.8 \\
\text { days; Planned } \\
\text { duration: } 6 \text { days }\end{array}$ & None & \\
\hline & 4 & Risperidone & Not reported & $\begin{array}{l}\text { Titrated dose: } \\
\text { Mean 1.1mg } \\
\text { (Range: } 0.25 \text { to } \\
4 \mathrm{mg} \text { ) }\end{array}$ & $\begin{array}{l}\text { Not } \\
\text { reported }\end{array}$ & $\begin{array}{l}\text { Mean duration: } 5.1 \\
\text { days; Planned } \\
\text { duration: } 6 \text { days }\end{array}$ & None & \\
\hline
\end{tabular}

bid=twice daily; bid=twice daily; g=gram; IQR=interquartile range; mg/d=milligram per day; mg/hr=milligram per hour; mg=milligram; ml=millimeter; NG=nadogastric tube; qd=once daily; SD=standard deviation; T3=time point three; $\mathrm{ug} / \mathrm{kg}=$ =microgram per kilogram 
Evidence Table D-7. Short-term continuous delirium symptom outcomes for studies comparing interventions to prevent development of delirium

\begin{tabular}{|c|c|c|c|c|c|c|c|c|}
\hline Author, year & Population & Intervention group, $\mathbf{n}$ & Control group, $\mathbf{n}$ & $\begin{array}{c}\text { Route of } \\
\text { administration }\end{array}$ & $\begin{array}{l}\text { Outcome } \\
\text { definition }\end{array}$ & $\begin{array}{c}\text { Mean (SD) sleep } \\
\text { time, intervention } \\
\text { group }\end{array}$ & $\begin{array}{l}\text { Mean (SD) sleep } \\
\text { time, control } \\
\text { group }\end{array}$ & $\begin{array}{l}\text { Mean difference }(95 \% \mathrm{Cl}) \text { in } \\
\text { sleep time }\end{array}$ \\
\hline \multicolumn{9}{|l|}{$\begin{array}{l}\text { First-generation } \\
\text { antipsychotic vs. } \\
\text { placebo }\end{array}$} \\
\hline Kaneko, $1999^{7}$ & $\begin{array}{l}\text { Scheduled for } \\
\text { elective } \\
\text { gastrointestinal } \\
\text { surgery and } \\
\text { admitted to ICU }\end{array}$ & $\begin{array}{l}\text { Haloperidol (Planned } \\
\text { dose: } 5 \mathrm{mg} \text { ), } 38\end{array}$ & $\begin{array}{l}\text { Placebo (Planned } \\
\text { dose: } 1 \mathrm{ml}(0.9 \% \\
\text { normal saline)ml), } \\
40\end{array}$ & Intravenous & $\begin{array}{l}\text { Night time } \\
\text { sleeping (min) }\end{array}$ & $500(55.38)$ & 418.46 (112.3) & 81.54 (42.24 to 120.84$)$ \\
\hline Kaneko, $1999^{7}$ & $\begin{array}{l}\text { Scheduled for } \\
\text { elective } \\
\text { gastrointestinal } \\
\text { surgery and } \\
\text { admitted to ICU }\end{array}$ & $\begin{array}{l}\text { Haloperidol (Planned } \\
\text { dose: } 5 \mathrm{mg} \text { ), } 38\end{array}$ & $\begin{array}{l}\text { Placebo (Planned } \\
\text { dose: } 1 \mathrm{ml}(0.9 \% \\
\text { normal saline)ml), } \\
40\end{array}$ & Intravenous & $\begin{array}{l}\text { Daytime } \\
\text { sleeping (min) }\end{array}$ & $260(73.85)$ & $289.2(76.95)$ & $-29.2(-62.67$ to 4.27$)$ \\
\hline
\end{tabular}

$\mathrm{CI=confidence} \mathrm{interval;} \mathrm{ICU=intensive} \mathrm{care} \mathrm{unit;} \mathrm{mg=milligram;} \mathrm{min=minutes;} \mathrm{ml=millimeter;} \mathrm{N}=$ =sample size; $\mathrm{SD}=$ =standard deviation 


\begin{tabular}{|c|c|c|c|c|c|c|c|c|c|}
\hline Author, year & $\begin{array}{l}\text { Population } \\
\end{array}$ & $\begin{array}{l}\text { Intervention } \\
\text { group }\end{array}$ & Control group & $\begin{array}{c}\text { Route of } \\
\text { administration }\end{array}$ & $\begin{array}{l}\text { Outcome } \\
\text { definition }\end{array}$ & Time of assessment & $\begin{array}{c}\mathrm{n} / \mathrm{N} \text { (\%), } \\
\text { control group }\end{array}$ & $\begin{array}{c}n / N(\%) \\
\text { intervention } \\
\text { group }\end{array}$ & Relative risk $(95 \% \mathrm{Cl})$ \\
\hline \multicolumn{10}{|l|}{$\begin{array}{l}\text { First-generation } \\
\text { antipsychotic } \\
\text { vs. placebo }\end{array}$} \\
\hline Page, $2013^{10}$ & $\begin{array}{l}\text { ICU patients needing } \\
\text { MV within } 72 \text { hours of } \\
\text { admission were enrolled }\end{array}$ & $\begin{array}{l}\text { Haloperidol } \\
\text { (Planned dose: } \\
2.5 \mathrm{mg} \text { ) }\end{array}$ & $\begin{array}{l}\text { Placebo } \\
\text { (Planned dose: } \\
0.5 \mathrm{ml} \text { ) }\end{array}$ & Intravenous & RASS +2 & 14 days & $20 / 70(28.6)$ & 13/71 (18.3) & Not reported, $\mathrm{p}=0.0075$ \\
\hline
\end{tabular}


Evidence Table D-9. Continuous delirium severity outcomes for studies comparing interventions to prevent development of delirium

\begin{tabular}{|c|c|c|c|c|c|c|c|c|}
\hline Author, year & Population & Intervention group, $\mathbf{n}$ & Control group, $\mathbf{n}$ & $\begin{array}{c}\text { Route of } \\
\text { administration }\end{array}$ & $\begin{array}{l}\text { Outcome } \\
\text { definition }\end{array}$ & $\begin{array}{l}\text { Mean (SD) delirium } \\
\text { severity score, } \\
\text { intervention group }\end{array}$ & $\begin{array}{c}\text { Mean (SD) } \\
\text { delirium severity } \\
\text { score, control } \\
\text { group } \\
\end{array}$ & $\begin{array}{l}\text { Mean between-group } \\
\text { difference }(95 \% \mathrm{Cl}) \text { in } \\
\text { delirium severity score }\end{array}$ \\
\hline \multicolumn{9}{|l|}{$\begin{array}{l}\text { First-generation } \\
\text { antipsychotic vs. } \\
\text { placebo }\end{array}$} \\
\hline Kalisvaart, $2005^{6}$ & $\begin{array}{l}\text { Acute or elective hip } \\
\text { surgery patients, } \geq 70 \\
\text { years, at risk of delirium: } \\
\text { MMSE between less } \\
\text { than or equal to } 24 ; \\
\text { dehydration=BUN/creatin } \\
\text { ine> or = to } 18 \text {, low } \\
\text { visual acuity and/or } \\
\text { increased severity of } \\
\text { illness on APACHE II of } \\
16 \text { or greater. }\end{array}$ & $\begin{array}{l}\text { Haloperidol (Planned } \\
\text { dose: } 0.5 \mathrm{mg} \text { ), } 36\end{array}$ & $\begin{array}{l}\text { Placebo (Not } \\
\text { applicable), } 32\end{array}$ & Oral & DRS-R-98 & $14.4(3.4)$ & $18.41(4.4)$ & $-4.01(-5.88$ to -2.14$)$ \\
\hline Khan, $2018^{8}$ & $\begin{array}{l}\text { English speaking } \\
\text { individuals undergoing } \\
\text { thoracic surgery }\end{array}$ & $\begin{array}{l}\text { Haloperidol (Planned } \\
\text { dose: } 0.5 \mathrm{mg} \text {, Planned } \\
\text { duration: } 4 \text { days (11 } \\
\text { doses total)), } 68\end{array}$ & $\begin{array}{l}\text { Placebo (Planned } \\
\text { dose: NR, } \\
\text { Planned duration: } \\
4 \text { days (11 doses } \\
\text { total)), } 67\end{array}$ & Intravenous & $\begin{array}{l}\text { DRS-R-98, } \\
\text { total } \\
\text { population }\end{array}$ & $\begin{array}{l}\text { Baseline: Mean } 1.81 \text { (SD } \\
2.11) \\
\text { Final: Mean } 1.19 \text { (SD } \\
1.52)\end{array}$ & $\begin{array}{l}\text { Baseline: Mean } \\
2.9 \text { (SD 4) } \\
\text { Final: Mean } 1.5 \\
\text { (SD 2.18) }\end{array}$ & $-0.31(-0.94$ to 0.32$)$ \\
\hline Khan, $2018^{8}$ & $\begin{array}{l}\text { English speaking } \\
\text { individuals undergoing } \\
\text { thoracic surgery }\end{array}$ & $\begin{array}{l}\text { Haloperidol (Planned } \\
\text { dose: } 0.5 \mathrm{mg} \text {, Planned } \\
\text { duration: } 4 \text { days (11 } \\
\text { doses total)), } 7\end{array}$ & $\begin{array}{l}\text { Placebo (Planned } \\
\text { dose: NR, } \\
\text { Planned duration: } \\
4 \text { days (11 doses } \\
\text { total)), } 17\end{array}$ & Intravenous & $\begin{array}{l}\text { DRS-R-98, } \\
\text { only patients } \\
\text { with delirium }\end{array}$ & $\begin{array}{l}\text { Baseline: Mean } 4.1 \text { (SD } \\
\text { 3.4) } \\
\text { Final: Mean } 3 \text { (SD 2.4) }\end{array}$ & $\begin{array}{l}\text { Baseline: Mean } \\
6.5 \text { (SD 6.2) } \\
\text { Final: Mean } 2.6 \\
\text { (SD 2.8) }\end{array}$ & 0.4 (-1.69 to 2.49$)$ \\
\hline Schrijver, $2018^{12}$ & $\begin{array}{l}\text { Medical and surgical } \\
\text { patients }\end{array}$ & $\begin{array}{l}\text { Haloperidol (Planned } \\
\text { dose: } 1 \mathrm{mg}), 23\end{array}$ & Placebo, 18 & Oral & $\begin{array}{l}\text { Maximum } \\
\text { DRS-R-98 } \\
\text { score }\end{array}$ & $15.1(7.6)$ & $12.67(13.5)$ & $2.43(-4.27$ to 9.14$)$ \\
\hline
\end{tabular}




\begin{tabular}{|c|c|c|c|c|c|c|c|c|}
\hline Author, year & Population & Intervention group, $\mathbf{n}$ & Control group, $\mathbf{n}$ & $\begin{array}{c}\text { Route of } \\
\text { administration }\end{array}$ & $\begin{array}{l}\text { Outcome } \\
\text { definition }\end{array}$ & $\begin{array}{l}\text { Mean (SD) delirium } \\
\text { severity score, } \\
\text { intervention group }\end{array}$ & $\begin{array}{c}\text { Mean (SD) } \\
\text { delirium severity } \\
\text { score, control } \\
\text { group } \\
\end{array}$ & $\begin{array}{l}\text { Mean between-group } \\
\text { difference }(95 \% \mathrm{Cl}) \text { in } \\
\text { delirium severity score }\end{array}$ \\
\hline \multicolumn{9}{|l|}{$\begin{array}{l}\text { Second-generation } \\
\text { antipsychotic vs. } \\
\text { placebo }\end{array}$} \\
\hline Hakim, $2012^{5}$ & $\begin{array}{l}\text { Patients } 65 \text { years or } \\
\text { older experiencing } \\
\text { subsyndromal delirium } \\
\text { after on-pump cardiac } \\
\text { surgery }\end{array}$ & $\begin{array}{l}\text { Risperidone (Planned } \\
\text { dose: } 0.5 \mathrm{mg}), 7\end{array}$ & $\begin{array}{l}\text { Placebo (Planned } \\
\text { dose: given every } \\
12 \text { hours), } 17\end{array}$ & Oral & $\begin{array}{l}\text { Highest score } \\
\text { on the ICDSC }\end{array}$ & Median: 6 (IQR 5 to 7 ) & $\begin{array}{l}\text { Median: } 5 \text { (IQR } 4 \\
\text { to 5) }\end{array}$ & $\mathrm{p}=0.234$ \\
\hline Larsen, $2010^{9}$ & $\begin{array}{l}\text { Post-operative elderly } \\
\text { joint replacement surgery } \\
\text { patients }\end{array}$ & $\begin{array}{l}\text { Olanzapine (Planned } \\
\text { dose: } 5 \mathrm{mg}), 28\end{array}$ & $\begin{array}{l}\text { Placebo (Planned } \\
\text { dose: } 5 \mathrm{mg}), 82\end{array}$ & Oral & DRS-R-98 & $16.4(3.7)$ & $14.5(2.7)$ & $1.9(0.69$ to 3.11$)$ \\
\hline
\end{tabular}

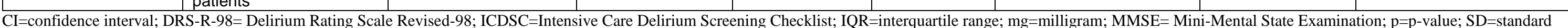
deviation 
Evidence Table D-10. Delirium free days outcomes for studies comparing interventions to prevent development of delirium

\begin{tabular}{|c|c|c|c|c|c|c|c|c|}
\hline Author, year & Population & Intervention group, $\mathbf{n}$ & Control group, $\mathbf{n}$ & $\begin{array}{c}\text { Route of } \\
\text { administration }\end{array}$ & Outcome definition & $\begin{array}{l}\text { Median (IQR) } \\
\text { days, } \\
\text { intervention } \\
\text { group }\end{array}$ & $\begin{array}{l}\text { Median (IQR), } \\
\text { days, control } \\
\text { group }\end{array}$ & $\begin{array}{c}\text { Measure of } \\
\text { association (95\% } \\
\mathrm{Cl})\end{array}$ \\
\hline \multicolumn{9}{|l|}{$\begin{array}{l}\text { First-generation } \\
\text { antipsychotic vs. } \\
\text { placebo }\end{array}$} \\
\hline Girard, $2010^{4}$ & $\begin{array}{l}>18 \text { years MV } \\
\text { medical and surgical } \\
\text { ICU patients }\end{array}$ & $\begin{array}{l}\text { Haloperidol (Planned dose: } 5 \text {, } \\
\text { Median dose: } 15 \mathrm{mg}(0.8- \\
17 \mathrm{mg}) \text { ), } 35\end{array}$ & $\begin{array}{l}\text { Placebo (Planned dose: } \\
5 \mathrm{ml}), 36\end{array}$ & Oral & $\begin{array}{l}\text { Delirium/coma-free } \\
\text { days during the } 21 \text {-day } \\
\text { study period. }\end{array}$ & 14 (6 to 18$)$ & $12.5(1.2$ to 17.2$)$ & $\begin{array}{l}P=0.66 \text { across } \\
\text { the three groups }\end{array}$ \\
\hline Page, $2013^{10}$ & $\begin{array}{l}\text { ICU patients needing } \\
\text { MV within } 72 \text { hours } \\
\text { of admission were } \\
\text { enrolled }\end{array}$ & $\begin{array}{l}\text { Haloperidol (Planned dose: } \\
2.5 \mathrm{mg}), 71\end{array}$ & $\begin{array}{l}\text { Placebo (Planned dose: } \\
0.5 \mathrm{ml}), 70\end{array}$ & Intravenous & $\begin{array}{l}\text { Alive, delirium free, } \\
\text { and coma free days in } \\
\text { first } 14 \text { days, median } \\
\text { (IQR) }\end{array}$ & 5 (0 to 10$)$ & 6 (0 to 11$)$ & $\begin{array}{l}\text { Mean difference - } \\
0.48 \text { (95\% Cl: - } \\
2.08 \text { to } 1.21) \\
p=0.53\end{array}$ \\
\hline Page, $2013^{10}$ & $\begin{array}{l}\text { ICU patients needing } \\
\text { MV within } 72 \text { hours } \\
\text { of admission were } \\
\text { enrolled }\end{array}$ & $\begin{array}{l}\text { Haloperidol (Planned dose: } \\
2.5 \mathrm{mg}), 71\end{array}$ & $\begin{array}{l}\text { Placebo (Planned dose: } \\
0.5 \mathrm{ml}), 70\end{array}$ & Intravenous & $\begin{array}{l}\text { Alive, delirium free, } \\
\text { and coma free days in } \\
\text { first } 28 \text { days }\end{array}$ & 19 (0 to 24$)$ & 19.5 (0 to 25$)$ & $\begin{array}{l}\text { Mean difference - } \\
0.26 \text { (95\% Cl: - } \\
3.72 \text { to } 3.46) \\
p=0.57\end{array}$ \\
\hline $\begin{array}{l}\text { van den Boogaard, } \\
2018^{15}\end{array}$ & $\begin{array}{l}\text { ICU patients at high } \\
\text { risk of delirium }\end{array}$ & $\begin{array}{l}\text { Haloperidol (Planned dose: } \\
2 \mathrm{mg}), 608\end{array}$ & $\begin{array}{l}\text { Placebo (Planned dose: } \\
0.9 \% \mathrm{NaCl}), 599\end{array}$ & Intravenous & $\begin{array}{l}\text { Number of delirium- } \\
\text { and coma-free, median } \\
\text { (IQR), db }\end{array}$ & 26 (17 to 28 ) & 26 (19 to 28$)$ & $\begin{array}{l}\text { Mean difference } 0 \\
(95 \% \mathrm{Cl}: 0 \text { to } 0)\end{array}$ \\
\hline $\begin{array}{l}\text { van den Boogaard, } \\
2018^{15}\end{array}$ & $\begin{array}{l}\text { ICU patients at high } \\
\text { risk of delirium }\end{array}$ & $\begin{array}{l}\text { Haloperidol (Planned dose: } \\
\text { 1mg), } 299\end{array}$ & $\begin{array}{l}\text { Placebo (Planned dose: } \\
0.9 \% \mathrm{NaCl}), 599\end{array}$ & Intravenous & $\begin{array}{l}\text { Number of delirium- } \\
\text { and coma-free, median } \\
\text { (IQR), db }\end{array}$ & 26 (17 to 28 ) & 26 (19 to 28$)$ & Not reported \\
\hline Wang, $2012^{16}$ & $\begin{array}{l}\text { Patients } 65 \text { or older } \\
\text { admitted to the ICU } \\
\text { after non-cardiac } \\
\text { surgery }\end{array}$ & $\begin{array}{l}\text { Haloperidol (Planned dose: } \\
0.5 \mathrm{mg} \text { followed by } \\
\text { continuous infusion at a rate } \\
\text { of } 1 \mathrm{~mL} / \mathrm{hr}(0.1 \mathrm{mg} / \mathrm{hr} \\
\text { haloperidol), } 35\end{array}$ & $\begin{array}{l}\text { Placebo (Planned dose: } \\
\text { Normal), } 53\end{array}$ & Intravenous & $\begin{array}{l}\text { Number of delirium } \\
\text { free days, in patients } \\
\text { who developed post- } \\
\text { operative delirium }\end{array}$ & $\begin{array}{l}\text { Mean } 5.7(\mathrm{SD} \\
0.7)\end{array}$ & Mean 5.6 (SD 1) & $\begin{array}{l}\text { Mean difference } \\
0.1(95 \% \mathrm{Cl}:-0.26 \\
\text { to } 0.46)\end{array}$ \\
\hline
\end{tabular}




\begin{tabular}{|c|c|c|c|c|c|c|c|c|}
\hline Author, year & Population & Intervention group, $\mathbf{n}$ & Control group, $n$ & $\begin{array}{c}\text { Route of } \\
\text { administration }\end{array}$ & Outcome definition & $\begin{array}{c}\text { Median (IQR) } \\
\text { days, } \\
\text { intervention } \\
\text { group } \\
\end{array}$ & $\begin{array}{l}\text { Median (IQR), } \\
\text { days, control } \\
\text { group }\end{array}$ & $\begin{array}{c}\text { Measure of } \\
\text { association }(95 \% \\
\mathrm{Cl})\end{array}$ \\
\hline Wang, $2012^{16}$ & $\begin{array}{l}\text { Patients } 65 \text { or older } \\
\text { admitted to the ICU } \\
\text { after non-cardiac } \\
\text { surgery }\end{array}$ & $\begin{array}{l}\text { Haloperidol (Planned dose: } \\
0.5 \mathrm{mg} \text { followed by } \\
\text { continuous infusion at a rate } \\
\text { of } 1 \mathrm{~mL} / \mathrm{hr}(0.1 \mathrm{mg} / \mathrm{hr} \\
\text { haloperidol), } 229\end{array}$ & $\begin{array}{l}\text { Placebo (Planned dose: } \\
\text { Normal saline), } 228\end{array}$ & Intravenous & $\begin{array}{l}\text { Coma and delirium } \\
\text { free days for all the } \\
\text { patients in the group }\end{array}$ & $\begin{array}{l}\text { Mean } 6.8(\text { SD } \\
0.7)\end{array}$ & Mean 6.7 (SD 0.9) & $\begin{array}{l}\text { Mean difference } \\
0.1(95 \% \mathrm{Cl}:-0.05 \\
\text { to } 0.25)\end{array}$ \\
\hline \multicolumn{9}{|c|}{$\begin{array}{l}\text { Second-generation } \\
\text { antipsychotic vs. } \\
\text { placebo }\end{array}$} \\
\hline Girard, $2010^{4}$ & $\begin{array}{l}>18 \text { years MV } \\
\text { medical and surgical } \\
\text { ICU patients }\end{array}$ & $\begin{array}{l}\text { Ziprasidone (Planned dose: } \\
40 \text { Median dose: } 113 \mathrm{mg}(81 \\
\text { to } 140 \mathrm{mg}) \text { ), } 30\end{array}$ & $\begin{array}{l}\text { Placebo (Planned dose: } \\
5 \mathrm{ml}), 36\end{array}$ & Oral & $\begin{array}{l}\text { Delirium/coma-free } \\
\text { days during the } 21 \text {-day } \\
\text { study period. }\end{array}$ & 15 (9.1 to 18$)$ & 12.5 (1.2 to 17.2$)$ & $\begin{array}{l}P=0.66 \text { across } \\
\text { the three groups }\end{array}$ \\
\hline \multicolumn{9}{|c|}{$\begin{array}{l}\text { First-generation } \\
\text { antipsychotic vs. } \\
\text { second-generation } \\
\text { antipsychotic }\end{array}$} \\
\hline Girard, $2010^{4}$ & $\begin{array}{l}>18 \text { years MV } \\
\text { medical and surgical } \\
\text { ICU patients }\end{array}$ & $\begin{array}{l}\text { Ziprasidone (Planned dose: } \\
40 \text { Median dose: } 113 \mathrm{mg}(81 \\
\text { to } 140 \mathrm{mg}) \text { ), } 30\end{array}$ & $\begin{array}{l}\text { Haloperidol (Planned } \\
\text { dose: } 5 \mathrm{mg}, \text { Median } \\
\text { dose: } 15 \mathrm{mg}(10.8-17) \text {, } \\
35\end{array}$ & Oral & $\begin{array}{l}\text { Delirium/coma-free } \\
\text { days during the 21-day } \\
\text { study period. }\end{array}$ & $15(9.1$ to 18$)$ & 14 (6 to 18$)$ & $\begin{array}{l}P=0.66 \text { across } \\
\text { the three groups }\end{array}$ \\
\hline
\end{tabular}

CI=confidence interval; ICU=intensive care unit; IQR=interquartile range; Mg/hr=milligram per hour; ml/hr=millimeter per hour; ml=millimeter; $\mathrm{MV=mechanical} \mathrm{ventilation;} \mathrm{N}=$ sample size; $\mathrm{NaCl=sodium} \mathrm{chloride;} \mathrm{p}=\mathrm{p}$-value; $\mathrm{SD}=$ standard deviation 
Evidence Table D-11. Duration of delirium outcomes for studies comparing interventions to prevent development of delirium

\begin{tabular}{|c|c|c|c|c|c|c|c|c|}
\hline Author, year & Population & $\begin{array}{l}\text { Intervention } \\
\text { group, } \mathrm{n}\end{array}$ & $\begin{array}{l}\text { Control } \\
\text { group, } n\end{array}$ & $\begin{array}{c}\text { Route of } \\
\text { administration }\end{array}$ & $\begin{array}{l}\text { Outcome } \\
\text { definition }\end{array}$ & $\begin{array}{l}\text { Mean (SD) days, } \\
\text { intervention } \\
\text { group }\end{array}$ & $\begin{array}{l}\text { Mean (SD) days, } \\
\text { control group }\end{array}$ & $\begin{array}{c}\text { Mean between-group } \\
\text { difference }(95 \% \mathrm{Cl})\end{array}$ \\
\hline \multicolumn{9}{|l|}{$\begin{array}{l}\text { First-generation } \\
\text { antipsychotic vs. } \\
\text { placebo }\end{array}$} \\
\hline Al-Qadheeb, $2016^{2}$ & $\begin{array}{l}\text { MV patients with subsyndromal } \\
\text { delirium }\end{array}$ & $\begin{array}{l}\text { Haloperidol } \\
\text { (Planned dose: } \\
\text { 1mg), } 34\end{array}$ & $\begin{array}{l}\text { Placebo } \\
\text { (Planned } \\
\text { dose: } 5 \% \\
\text { dextrose), } 34\end{array}$ & Intravenous & $\begin{array}{l}\text { Duration of first } \\
\text { episode of } \\
\text { delirium (d) }\end{array}$ & $1.67(0.8)$ & $3(1.6)$ & $-1.33(-1.93$ to -0.73$)$ \\
\hline Fukata, $2014^{3}$ & $\begin{array}{l}\text { Delirium after abdominal or } \\
\text { orthopedic surgery in elderly } \\
\text { patients }\end{array}$ & $\begin{array}{l}\text { Haloperidol } \\
\text { (Planned dose: } \\
\text { 2.5mg), } 59\end{array}$ & Placebo, 60 & Intravenous & $\begin{array}{l}\text { Persistence of } \\
\text { delirium }\end{array}$ & $1.38(0.9)$ & $1.1(0.8)$ & $0.28(-0.03$ to 0.59$)$ \\
\hline Girard, $2010^{4}$ & $\begin{array}{l}>18 \text { years MV medical and } \\
\text { surgical ICU patients }\end{array}$ & $\begin{array}{l}\text { Haloperidol } \\
\text { (Planned dose: } 5 \\
\text { Median dose: } 15 \\
(10.8-17), 35\end{array}$ & $\begin{array}{l}\text { Placebo } \\
\text { (Planned } \\
\text { dose: } 5 \mathrm{ml}), 36\end{array}$ & Oral & Delirium days & $4.33(3.9)$ & $4(3.2)$ & 0.33 (-1.33 to 1.99$)$ \\
\hline Kalisvaart, $2005^{6}$ & $\begin{array}{l}\text { Acute or elective hip surgery } \\
\text { patients } \geq 70 \text { years, at risk of } \\
\text { delirium: MMSE between less than } \\
\text { or equal to } 24 ; \\
\text { dehydration=BUN/creatinine> or = } \\
\text { to } 18 \text {, low visual acuity and/or } \\
\text { increased severity of illness on } \\
\text { APACHE II of } 16 \text { or greater. }\end{array}$ & $\begin{array}{l}\text { Haloperidol } \\
\text { (Planned dose: } \\
0.5 \mathrm{mg}), 32\end{array}$ & $\begin{array}{l}\text { Placebo (Not } \\
\text { applicable), } 36\end{array}$ & Oral & $\begin{array}{l}\text { Delirium } \\
\text { duration, days }\end{array}$ & $5.4(4.9)$ & $11.8(7.5)$ & $-6.4(-9.41$ to -3.39$)$ \\
\hline
\end{tabular}




\begin{tabular}{|c|c|c|c|c|c|c|c|c|}
\hline Author, year & Population & $\begin{array}{l}\text { Intervention } \\
\text { group, } \mathrm{n}\end{array}$ & $\begin{array}{c}\text { Control } \\
\text { group, } n\end{array}$ & $\begin{array}{c}\text { Route of } \\
\text { administration }\end{array}$ & $\begin{array}{l}\text { Outcome } \\
\text { definition }\end{array}$ & $\begin{array}{l}\text { Mean (SD) days, } \\
\text { intervention } \\
\text { group }\end{array}$ & $\begin{array}{l}\text { Mean (SD) days, } \\
\text { control group }\end{array}$ & $\begin{array}{l}\text { Mean between-group } \\
\text { difference }(95 \% \mathrm{Cl})\end{array}$ \\
\hline Khan, $2018^{8}$ & $\begin{array}{l}\text { English speaking individuals } \\
\text { undergoing thoracic surgery }\end{array}$ & $\begin{array}{l}\text { Haloperidol } \\
\text { (Planned dose: } \\
0.5 \mathrm{mg}, \text { Planned } \\
\text { duration: } 4 \text { days } \\
(11 \text { doses total)), } \\
68\end{array}$ & $\begin{array}{l}\text { Placebo } \\
\text { (Planned } \\
\text { dose: NR, } \\
\text { Planned } \\
\text { duration: } 4 \\
\text { days (11 } \\
\text { doses total)), } \\
67\end{array}$ & Intravenous & Delirium days & $\begin{array}{l}\text { Median: } 1 \text { (IQR } 1 \\
\text { to 2) }\end{array}$ & $\begin{array}{l}\text { Median: } 1 \text { (IQR } 1 \text { to } \\
\text { 2) }\end{array}$ & $p=0.71$ \\
\hline Page, $2013^{10}$ & $\begin{array}{l}\text { ICU patients needing MV within } 72 \\
\text { hours of admission were enrolled }\end{array}$ & $\begin{array}{l}\text { Haloperidol } \\
\text { (Planned dose: } \\
2.5 \mathrm{mg}), 71\end{array}$ & $\begin{array}{l}\text { Placebo } \\
\text { (Planned } \\
\text { dose: } 0.5 \mathrm{ml} \text { ), } \\
70\end{array}$ & Intravenous & Days in delirium & $5(4.7)$ & $4.67(5.6)$ & 0.33 (-1.38 to 2.04$)$ \\
\hline Page, $2013^{10}$ & $\begin{array}{l}\text { ICU patients needing MV within } 72 \\
\text { hours of admission were enrolled }\end{array}$ & $\begin{array}{l}\text { Haloperidol } \\
\text { (Planned dose: } \\
2.5 \mathrm{mg}), 71\end{array}$ & $\begin{array}{l}\text { Placebo } \\
\text { (Planned } \\
\text { dose: } 0.5 \mathrm{ml} \text { ), } \\
70\end{array}$ & Intravenous & Days in delirium & $5.67(6.3)$ & $5(6.3)$ & 0.67 (-1.41 to 2.75$)$ \\
\hline Schrijver, $2018^{12}$ & Medical and surgical patients & $\begin{array}{l}\text { Haloperidol } \\
\text { (Planned dose: } \\
\text { 1mg), } 23\end{array}$ & Placebo, 18 & Oral & Days & $3.67(2.4)$ & $3.33(4)$ & 0.34 (-1.68 to 2.36$)$ \\
\hline
\end{tabular}




\begin{tabular}{|c|c|c|c|c|c|c|c|c|}
\hline Author, year & Population & $\begin{array}{l}\text { Intervention } \\
\text { group, } \mathrm{n}\end{array}$ & $\begin{array}{l}\text { Control } \\
\text { group, } n\end{array}$ & $\begin{array}{c}\text { Route of } \\
\text { administration }\end{array}$ & $\begin{array}{l}\text { Outcome } \\
\text { definition }\end{array}$ & $\begin{array}{l}\text { Mean (SD) days, } \\
\text { intervention } \\
\text { group }\end{array}$ & $\begin{array}{l}\text { Mean (SD) days, } \\
\text { control group }\end{array}$ & $\begin{array}{l}\text { Mean between-group } \\
\text { difference }(95 \% \mathrm{Cl})\end{array}$ \\
\hline \multicolumn{9}{|c|}{$\begin{array}{l}\text { Second-generation } \\
\text { antipsychotic vs. placebo }\end{array}$} \\
\hline Girard, $2010^{4}$ & $\begin{array}{l}>18 \text { years MV medical and } \\
\text { surgical ICU patients }\end{array}$ & $\begin{array}{l}\text { Ziprasidone } \\
\text { (Planned dose: } 40 \\
\text { Median dose: } \\
\text { 113mg (81- } \\
\text { 140mg)), } 30\end{array}$ & $\begin{array}{l}\text { Placebo } \\
\text { (Planned } \\
\text { dose: } 5 \mathrm{ml}), 36\end{array}$ & Oral & Delirium days & $4.67(4.7)$ & $4(3.2)$ & 0.67 (-1.27 to 2.61$)$ \\
\hline Hakim, $2012^{5}$ & $\begin{array}{l}\text { Patients } 65 \text { years or older } \\
\text { experiencing subsyndromal } \\
\text { delirium after on-pump } \\
\text { cardiac surgery }\end{array}$ & $\begin{array}{l}\text { Risperidone } \\
\text { (Planned dose: } \\
0.5 \mathrm{mg})\end{array}$ & $\begin{array}{l}\text { Placebo } \\
\text { (Planned } \\
\text { dose: given } \\
\text { every } 12 \\
\text { hours), }\end{array}$ & Oral & $\begin{array}{l}\text { Failure to treat } \\
\text { subsyndromal } \\
\text { delirium with } \\
\text { risperidone }\end{array}$ & & & $\begin{array}{l}\text { Subhazard ratio: } 3.83 \text { (SE } \\
\text { 1.67) (95\% Cl: } 1.63 \text { to } \\
\text { 8.98), } p=0.002\end{array}$ \\
\hline Hakim, $2012^{5}$ & $\begin{array}{l}\text { Patients } 65 \text { years or older } \\
\text { experiencing subsyndromal } \\
\text { delirium after on-pump } \\
\text { cardiac surgery }\end{array}$ & $\begin{array}{l}\text { Risperidone } \\
\text { (Planned dose: } \\
0.5 \mathrm{mg}), 7\end{array}$ & $\begin{array}{l}\text { Placebo } \\
\text { (Planned } \\
\text { dose: given } \\
\text { every } 12 \\
\text { hours), } 17\end{array}$ & Oral & $\begin{array}{l}\text { Duration of } \\
\text { clinical delirium, } \\
\text { for affected } \\
\text { patients, days }\end{array}$ & $3(1.6)$ & $3.33(0.8)$ & $-0.33(-1.34$ to 0.68$)$ \\
\hline Larsen, $2010^{9}$ & $\begin{array}{l}\text { Post-operative elderly joint } \\
\text { replacement surgery patients }\end{array}$ & $\begin{array}{l}\text { Olanzapine } \\
\text { (Planned dose: } \\
\text { 5mg), } 28\end{array}$ & $\begin{array}{l}\text { Placebo } \\
\text { (Planned } \\
\text { dose: } 5 \mathrm{mg}), 82\end{array}$ & Oral & $\begin{array}{l}\text { Duration of } \\
\text { delirium }\end{array}$ & $2.2(1.3)$ & $1.6(0.7)$ & 0.6 (0.21 to 0.99$)$ \\
\hline \multicolumn{9}{|c|}{$\begin{array}{l}\text { First-generation } \\
\text { antipsychotic vs. second- } \\
\text { generation antipsychotic }\end{array}$} \\
\hline Girard, $2010^{4}$ & $\begin{array}{l}>18 \text { years MV medical and } \\
\text { surgical ICU patients }\end{array}$ & $\begin{array}{l}\text { Ziprasidone } \\
\text { (Planned dose: } 40 \\
\text { Median dose: } \\
\text { 113mg (81- } \\
\text { 140mg)), } 30\end{array}$ & $\begin{array}{l}\text { Haloperidol } \\
\text { (Planned } \\
\text { dose: } 5 \\
\text { Median dose: } \\
\text { 15mg (10.8- } \\
\text { 17mg)), } 35\end{array}$ & Oral & Delirium days & $4.67(4.7)$ & $4.33(4)$ & 0.34 (-1.78 to 2.46$)$ \\
\hline
\end{tabular}




\begin{tabular}{|c|c|c|c|c|c|c|c|c|}
\hline Author, year & $\begin{array}{l}\text { Population } \\
\end{array}$ & Intervention group & Control group & $\begin{array}{c}\text { Route of } \\
\text { administration }\end{array}$ & Outcome definition & $\begin{array}{l}\mathrm{n} / \mathrm{N}(\%) \\
\text { intervention } \\
\text { group }\end{array}$ & $\begin{array}{l}\text { n I N (\%), } \\
\text { control } \\
\text { group }\end{array}$ & $\begin{array}{l}\text { Relative risk } \\
\quad(95 \% \mathrm{Cl})\end{array}$ \\
\hline \multicolumn{9}{|c|}{$\begin{array}{l}\text { First-generation } \\
\text { antipsychotic vs. placebo }\end{array}$} \\
\hline Abdelgalel, $2016^{1}$ & $\begin{array}{l}\text { Adult intensive care patients } \\
\text { of ASA physical status III and } \\
\text { IV aged between } 26 \text { and } 70 \\
\text { years }\end{array}$ & $\begin{array}{l}\text { Haloperidol (Planned } \\
\text { dose: } 0.5-2 \mathrm{mg} / \mathrm{h} \\
\text { preceded by a loading } \\
\text { dose of } 2.5 \mathrm{mg} \\
\text { intravenously over } 10 \\
\text { min if needed (if RASS } \\
>2+\text { ) }\end{array}$ & $\begin{array}{l}\text { Placebo (Planned } \\
\text { dose: } 2-8 \mathrm{ml}, \\
\text { loading dose } 10 \mathrm{ml} \\
\text { over } 10 \mathrm{~min} \text { if } \\
\text { needed (if RASS > } \\
2+\text { ) }\end{array}$ & Intravenous & $\begin{array}{l}\text { Midazolam Mean total } \\
\text { dose ranged from } 5.5 \text { to } \\
30 \mathrm{mg})\end{array}$ & $8 / 30(26.7 \%)$ & $\begin{array}{l}11 / 30 \\
(36.7 \%)\end{array}$ & $\begin{array}{l}0.73(0.34 \text { to } \\
1.55)\end{array}$ \\
\hline Abdelgalel, $2016^{1}$ & $\begin{array}{l}\text { Adult intensive care patients } \\
\text { of ASA physical status III and } \\
\text { IV aged between } 26 \text { and } 70 \\
\text { years }\end{array}$ & $\begin{array}{l}\text { Haloperidol (Planned } \\
\text { dose: } 0.5-2 \mathrm{mg} / \mathrm{h} \\
\text { preceded by a loading } \\
\text { dose of } 2.5 \mathrm{mg} \\
\text { intravenously over } 10 \\
\text { min if needed (if RASS } \\
>2+\text { ) }\end{array}$ & $\begin{array}{l}\text { Placebo (Planned } \\
\text { dose: } 2-8 \mathrm{ml}, \\
\text { loading dose } 10 \mathrm{ml} \\
\text { over } 10 \text { min if } \\
\text { needed (if RASS > } \\
2+\text { ) }\end{array}$ & Intravenous & $\begin{array}{l}\text { Fentanyl Range in } \\
\text { mean total dose, } 100.5 \\
\text { to } 480.1 \mathrm{mg}\end{array}$ & $8 / 30(26.7 \%)$ & $\begin{array}{l}10 / 30 \\
(33.3 \%)\end{array}$ & $\begin{array}{l}0.80(0.37 \text { to } \\
1.74)\end{array}$ \\
\hline Abdelgalel, $2016^{1}$ & $\begin{array}{l}\text { Adult intensive care patients } \\
\text { of ASA physical status III and } \\
\text { IV aged between } 26 \text { and } 70 \\
\text { years }\end{array}$ & $\begin{array}{l}\text { Haloperidol (Planned } \\
\text { dose: } 0.5-2 \mathrm{mg} / \mathrm{h} \\
\text { preceded by a loading } \\
\text { dose of } 2.5 \mathrm{mg} \\
\text { intravenously over } 10 \\
\text { min if needed (if RASS } \\
>2+\text { ) }\end{array}$ & $\begin{array}{l}\text { Placebo (Planned } \\
\text { dose: 2-8ml, } \\
\text { loading dose 10ml } \\
\text { over } 10 \text { min if } \\
\text { needed (if RASS > } \\
2+\text { ) }\end{array}$ & Intravenous & $\begin{array}{l}\text { Propofol range in total } \\
\text { mean dose, } 320.2 \text { to } \\
1151.4 \mathrm{mg}\end{array}$ & $10 / 30(33.3 \%)$ & $\begin{array}{l}13 / 30 \\
(43.3 \%)\end{array}$ & $\begin{array}{l}0.77(0.40 \text { to } \\
1.47)\end{array}$ \\
\hline Girard, 20104 & $\begin{array}{l}>18 \text { years MV medical and } \\
\text { surgical ICU patients }\end{array}$ & $\begin{array}{l}\text { Haloperidol (Planned } \\
\text { dose: } 5 \mathrm{mg} \text {; Median } \\
\text { dose: } 15 \mathrm{mg} \text { (10.8-17) }\end{array}$ & $\begin{array}{l}\text { Placebo (Planned } \\
\text { dose: } 5 \mathrm{ml} \text { ), }\end{array}$ & Oral & $\begin{array}{l}\text { Haloperidol varies for } \\
\text { each group }\end{array}$ & $6 / 35(17 \%)$ & 14 / 36 (39\%) & $\begin{array}{l}0.44(0.19 \text { to } \\
1.02)\end{array}$ \\
\hline Page, $2013^{10}$ & $\begin{array}{l}\text { ICU patients needing MV } \\
\text { within } 72 \text { hours of admission } \\
\text { were enrolled }\end{array}$ & $\begin{array}{l}\text { Haloperidol (Planned } \\
\text { dose: } 2.5 \mathrm{mg} \text { ) }\end{array}$ & $\begin{array}{l}\text { Placebo (Planned } \\
\text { dose: } 0.5 \mathrm{ml} \text { ) }\end{array}$ & Intravenous & Any anti-psychotic & $8 / 71(11 \%)$ & $18 / 70(26 \%)$ & $\begin{array}{l}0.44(0.20 \text { to } \\
0.94)\end{array}$ \\
\hline
\end{tabular}




\begin{tabular}{|c|c|c|c|c|c|c|c|c|}
\hline Author, year & Population & Intervention group & Control group & $\begin{array}{c}\text { Route of } \\
\text { administration }\end{array}$ & Outcome definition & $\begin{array}{c}\mathrm{n} / \mathrm{N}(\%) \\
\text { intervention } \\
\text { group }\end{array}$ & $\begin{array}{c}\text { n I N (\%) } \\
\text { control } \\
\text { group }\end{array}$ & $\begin{array}{c}\text { Relative risk } \\
(95 \% \mathrm{Cl})\end{array}$ \\
\hline Page, $2013^{10}$ & $\begin{array}{l}\text { ICU patients needing MV } \\
\text { within } 72 \text { hours of admission } \\
\text { were enrolled }\end{array}$ & $\begin{array}{l}\text { Haloperidol (Planned } \\
\text { dose: } 2.5 \mathrm{mg} \text { ) }\end{array}$ & $\begin{array}{l}\text { Placebo (Planned } \\
\text { dose: } 0.5 \mathrm{ml})\end{array}$ & Intravenous & Open Label Haloperidol & $6 / 71(8 \%)$ & $15 / 70(21 \%)$ & $\begin{array}{l}0.39(0.16 \text { to } \\
0.96)\end{array}$ \\
\hline Schrijver, 2018 & Medical and surgical patients & $\begin{array}{l}\text { Haloperidol (Planned } \\
\text { dose: } 1 \mathrm{mg} \text { ) }\end{array}$ & Placebo & Oral & $\begin{array}{l}\text { Haloperidol mean daily } \\
\text { dose ranged from } 1.3 \text { to } \\
1.4 \mathrm{mg} \text { in the } \\
\text { haloperidol and placebo } \\
\text { groups. }\end{array}$ & $\begin{array}{l}15 / 118 \\
(12.7 \%)\end{array}$ & $\begin{array}{l}12 / 124 \\
(9.7 \%)\end{array}$ & $\begin{array}{l}1.31(0.64 \text { to } \\
2.69)\end{array}$ \\
\hline Wang, $2012^{16}$ & $\begin{array}{l}\text { Patients } 65 \text { or older admitted } \\
\text { to the ICU after noncardiac } \\
\text { surgery }\end{array}$ & $\begin{array}{l}\text { Haloperidol (Planned } \\
\text { dose: } 0.5 \mathrm{mg} \text { followed } \\
\text { by continuous infusion } \\
\text { at a rate of } 1 \mathrm{~mL} / \mathrm{hr}(0.1 \\
\mathrm{mg} / \mathrm{hr} \text { haloperidol) }\end{array}$ & $\begin{array}{l}\text { Placebo (Planned } \\
\text { dose: Normal } \\
\text { saline) }\end{array}$ & Intravenous & Haloperidol & $3 / 229(1.3 \%)$ & $\begin{array}{l}6 / 228 \\
(2.6 \%)\end{array}$ & $\begin{array}{l}0.50(0.13 \text { to } \\
1.97)\end{array}$ \\
\hline \multicolumn{9}{|c|}{$\begin{array}{l}\text { Second-generation } \\
\text { antipsychotic vs. placebo }\end{array}$} \\
\hline Girard, $2010^{4}$ & $\begin{array}{l}>18 \text { years MV medical and } \\
\text { surgical ICU patients }\end{array}$ & $\begin{array}{l}\text { Ziprasidone (Planned } \\
\text { dose: } 40 \text { Median dose: } \\
113 \text { (81-140) }\end{array}$ & $\begin{array}{l}\text { Placebo (Planned } \\
\text { dose: } 5 \mathrm{ml} \text { ) }\end{array}$ & Oral & $\begin{array}{l}\text { Haloperidol varies for } \\
\text { each group }\end{array}$ & $9 / 30(30 \%)$ & $14 / 36(39 \%)$ & $\begin{array}{l}0.77(0.39 \text { to } \\
1.53)\end{array}$ \\
\hline \multicolumn{9}{|c|}{$\begin{array}{l}\text { First-generation } \\
\text { antipsychotic vs. second- } \\
\text { generation antipsychotic }\end{array}$} \\
\hline Girard, $2010^{4}$ & $\begin{array}{l}>18 \text { years MV medical and } \\
\text { surgical ICU patients }\end{array}$ & $\begin{array}{l}\text { Ziprasidone (Planned } \\
\text { dose: } 40 \text { Median dose: } \\
113 \text { (81-140) }\end{array}$ & $\begin{array}{l}\text { Haloperidol } \\
\text { (Planned dose: } 5 \\
\text { Median dose: } 15 \\
(10.8-17)\end{array}$ & Oral & $\begin{array}{l}\text { Haloperidol varies for } \\
\text { each group }\end{array}$ & $9 / 30(30 \%)$ & $6 / 35(17 \%)$ & $\begin{array}{l}1.75(0.70 \text { to } \\
4.35)\end{array}$ \\
\hline
\end{tabular}




\begin{tabular}{|c|c|c|c|c|c|c|c|c|}
\hline Author, year & Population & Intervention group & Control group & $\begin{array}{c}\text { Route of } \\
\text { administration }\end{array}$ & Outcome definition & $\begin{array}{c}\mathrm{n} / \mathrm{N}(\%) \\
\text { intervention } \\
\text { group }\end{array}$ & $\begin{array}{c}\text { n I N (\%), } \\
\text { control } \\
\text { group }\end{array}$ & $\begin{array}{c}\text { Relative risk } \\
(95 \% \mathrm{Cl})\end{array}$ \\
\hline \multicolumn{9}{|l|}{$\begin{array}{l}\text { First-generation } \\
\text { antipsychotic vs. other }\end{array}$} \\
\hline Abdelgalel, $2016^{1}$ & $\begin{array}{l}\text { Adult intensive care patients } \\
\text { of ASA physical status III and } \\
\text { IV aged between } 26 \text { and } 70 \\
\text { years }\end{array}$ & $\begin{array}{l}\text { Haloperidol (Planned } \\
\text { dose: } 0.5-2 \mathrm{mg} / \mathrm{h} \\
\text { preceded by a loading } \\
\text { dose of } 2.5 \mathrm{mg} \\
\text { intravenously over } 10 \\
\text { min if needed (if RASS } \\
>2+\text { ) }\end{array}$ & $\begin{array}{l}\text { Dexmedetomidine } \\
\text { (Planned dose: } \\
0.2-0.7 \mathrm{mcg} / \mathrm{kg} / \mathrm{h} \\
\text { preceded by a } \\
\text { loading dose of } 1.0 \\
\text { ug } / \mathrm{kg} \text { intravenously } \\
\text { over } 10 \text { min if } \\
\text { needed (if RASS > } \\
2+\text { ) }\end{array}$ & Intravenous & $\begin{array}{l}\text { Midazolam Mean total } \\
\text { dose ranged from } 5.5 \text { to } \\
30 \mathrm{mg} \text { ) }\end{array}$ & $8 / 30(26.7 \%)$ & $2 / 30(6.7 \%)$ & $\begin{array}{l}4.00(0.92 \text { to } \\
17.30)\end{array}$ \\
\hline Abdelgalel, 2016 ${ }^{1}$ & $\begin{array}{l}\text { Adult intensive care patients } \\
\text { of ASA physical status III and } \\
\text { IV aged between } 26 \text { and } 70 \\
\text { years }\end{array}$ & $\begin{array}{l}\text { Haloperidol (Planned } \\
\text { dose: } 0.5-2 \mathrm{mg} / \mathrm{h} \\
\text { preceded by a loading } \\
\text { dose of } 2.5 \mathrm{mg} \\
\text { intravenously over } 10 \\
\text { min if needed (if RASS } \\
>2+\text { ) }\end{array}$ & $\begin{array}{l}\text { Dexmedetomidine } \\
\text { (Planned dose: } \\
0.2-0.7 \mathrm{mcg} / \mathrm{kg} / \mathrm{h} \\
\text { preceded by a } \\
\text { loading dose of } 1.0 \\
\text { ug } / \mathrm{kg} \text { intravenously } \\
\text { over } 10 \mathrm{~min} \text { if } \\
\text { needed (if RASS > } \\
2+\text { ) }\end{array}$ & Intravenous & $\begin{array}{l}\text { Fentanyl Range in } \\
\text { mean total dose, } 100.5 \\
\text { to } 480.1 \mathrm{mg}\end{array}$ & $8 / 30(26.7 \%)$ & $2 / 30(6.7 \%)$ & $\begin{array}{l}4.00(0.92 \text { to } \\
17.30)\end{array}$ \\
\hline Abdelgalel, $2016^{1}$ & $\begin{array}{l}\text { Adult intensive care patients } \\
\text { of ASA physical status III and } \\
\text { IV aged between } 26 \text { and } 70 \\
\text { years }\end{array}$ & $\begin{array}{l}\text { Haloperidol (Planned } \\
\text { dose: } 0.5-2 \mathrm{mg} / \mathrm{h} \\
\text { preceded by a loading } \\
\text { dose of } 2.5 \mathrm{mg} \\
\text { intravenously over } 10 \\
\text { min if needed (if RASS } \\
>2+\text { ) }\end{array}$ & $\begin{array}{l}\text { Dexmedetomidine } \\
\text { (Planned dose: } \\
0.2-0.7 \mathrm{mcg} / \mathrm{kg} / \mathrm{h} \\
\text { preceded by a } \\
\text { loading dose of } 1.0 \\
\text { ug/kg intravenously } \\
\text { over } 10 \text { min if } \\
\text { needed (if RASS > } \\
2+\text { ) }\end{array}$ & Intravenous & $\begin{array}{l}\text { Propofol Range in total } \\
\text { mean dose, } 320.2 \text { to } \\
1151.4 \mathrm{mg}\end{array}$ & $10 / 30$ (33.3\%) & $3 / 30(10 \%)$ & $\begin{array}{l}3.33(1.02 \text { to } \\
10.92)\end{array}$ \\
\hline
\end{tabular}

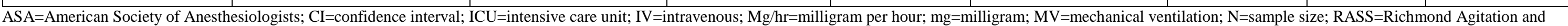
Sedation Scale 
Evidence Table D-13. Use of physical restraint outcomes in studies comparing interventions to prevent development of delirium

\begin{tabular}{|c|c|c|c|c|c|c|c|c|}
\hline Author, year & Population & $\begin{array}{l}\text { Intervention } \\
\text { group }\end{array}$ & $\begin{array}{l}\text { Control } \\
\text { group }\end{array}$ & $\begin{array}{c}\text { Route of } \\
\text { administration }\end{array}$ & $\begin{array}{l}\text { Outcome } \\
\text { definition }\end{array}$ & $\begin{array}{c}\mathrm{n} / \mathrm{N}(\%), \text { intervention } \\
\text { group }\end{array}$ & $\begin{array}{l}\text { n I N (\%), control } \\
\text { group }\end{array}$ & $\begin{array}{l}\text { Relative risk (95\% } \\
\text { Cl) }\end{array}$ \\
\hline \multicolumn{9}{|l|}{$\begin{array}{l}\text { First-generation antipsychotic vs. } \\
\text { placebo }\end{array}$} \\
\hline van den Boogaard, 2018 ${ }^{15}$ & $\begin{array}{l}\text { ICU patients at } \\
\text { high risk of } \\
\text { delirium }\end{array}$ & $\begin{array}{l}\text { Haloperidol } \\
\text { (Planned dose: } \\
\text { 1mg) }\end{array}$ & $\begin{array}{l}\text { Placebo } \\
\text { (Planned } \\
\text { dose: } 0.9 \% \\
\mathrm{NaCl} \text { ) }\end{array}$ & Intravenous & $\begin{array}{l}\text { Incidence, No. } \\
(\%)\end{array}$ & $102 / 350(30 \%)$ & $169 / 707(24.8 \%)$ & 1.22 (0.98 to 1.50$)$ \\
\hline van den Boogaard, $2018^{15}$ & $\begin{array}{l}\text { ICU patients at } \\
\text { high risk of } \\
\text { delirium }\end{array}$ & $\begin{array}{l}\text { Haloperidol } \\
\text { (Planned dose: } \\
\text { 2mg) }\end{array}$ & $\begin{array}{l}\text { Placebo } \\
\text { (Planned } \\
\text { dose: } 0.9 \% \\
\mathrm{NaCl} \text { ) }\end{array}$ & Intravenous & $\begin{array}{l}\text { Incidence, No. } \\
(\%)\end{array}$ & $191 / 732(27 \%)$ & $169 / 707$ (24.8\%) & 1.09 (0.91 to 1.31$)$ \\
\hline Khan, $2018^{8}$ & $\begin{array}{l}\text { English } \\
\text { speaking } \\
\text { individuals } \\
\text { undergoing } \\
\text { thoracic surgery }\end{array}$ & $\begin{array}{l}\text { Haloperidol } \\
\text { (Planned dose: } \\
\text { 0.5mg, Planned } \\
\text { duration: } 4 \text { days } \\
(11 \text { doses total)), }\end{array}$ & $\begin{array}{l}\text { Placebo } \\
\text { (Planned } \\
\text { dose: NR, } \\
\text { Planned } \\
\text { duration: } 4 \\
\text { days (11 } \\
\text { doses total)), }\end{array}$ & Intravenous & $\begin{array}{l}\text { Physically } \\
\text { restrained }\end{array}$ & 24 / 68 (35.3\%) & $25 / 67$ (37.3\%) & $\begin{array}{l}\text { RR } 0.95(95 \% \mathrm{Cl} \text { : } \\
0.60 \text { to } 1.48) \\
p=0.513\end{array}$ \\
\hline \multicolumn{9}{|l|}{$\begin{array}{l}\text { Second-generation antipsychotic } \\
\text { vs. placebo }\end{array}$} \\
\hline Larsen, $2010^{9}$ & $\begin{array}{l}\text { Post-operative } \\
\text { elderly joint } \\
\text { replacement } \\
\text { surgery patients }\end{array}$ & $\begin{array}{l}\text { Olanzapine } \\
\text { (Planned dose: } \\
\text { 5mg) }\end{array}$ & $\begin{array}{l}\text { Placebo } \\
\text { (Planned } \\
\text { dose: } 5 \mathrm{mg} \text { ) }\end{array}$ & Oral & & $5 / 196(2.6 \%)$ & $0 / 204(\%)$ & $\begin{array}{l}11.45(0.64 \text { to } \\
205.64)\end{array}$ \\
\hline
\end{tabular}

CI=confidence interval; $\mathrm{N}=$ sample size; $\mathrm{NaCl}=$ sodium chloride 


\begin{tabular}{|c|c|c|c|c|c|c|c|c|}
\hline Author, year & Population & Intervention group & Control group & $\begin{array}{c}\text { Route of } \\
\text { administration }\end{array}$ & $\begin{array}{l}\text { Outcome } \\
\text { definition }\end{array}$ & $\begin{array}{c}\text { n / N (\%), intervention } \\
\text { group }\end{array}$ & $\begin{array}{c}\text { n / N (\%), control } \\
\text { group }\end{array}$ & $\begin{array}{c}\text { Relative risk } \\
(95 \% \mathrm{Cl})\end{array}$ \\
\hline \multicolumn{9}{|c|}{$\begin{array}{l}\text { First-generation } \\
\text { antipsychotic vs. placebo }\end{array}$} \\
\hline Abdelgalel, $2016^{1}$ & $\begin{array}{l}\text { Adult intensive } \\
\text { care patients of } \\
\text { ASA physical } \\
\text { status III and IV } \\
\text { aged between } \\
26 \text { and } 70 \text { years }\end{array}$ & $\begin{array}{l}\text { Haloperidol (Planned dose: } 0.5-2 \\
\mathrm{mg} / \mathrm{h} \text { preceded by a loading dose of } \\
2.5 \mathrm{mg} \text { intravenously over } 10 \mathrm{~min} \text { if } \\
\text { needed (if RASS }>2+\text { ) }\end{array}$ & $\begin{array}{l}\text { Placebo (Planned } \\
\text { dose: } 2-8 \mathrm{ml}, \\
\text { loading dose 10ml } \\
\text { over } 10 \text { min if } \\
\text { needed (if RASS > } \\
2+) \text { ) }\end{array}$ & Intravenous & Mortality & $2 / 30(6.7 \%)$ & $3 / 30(10 \%)$ & $\begin{array}{l}0.67(0.12 \text { to } \\
3.71)\end{array}$ \\
\hline Al-Qadheeb, $2016^{2}$ & $\begin{array}{l}\text { MV patients } \\
\text { with } \\
\text { subsyndromal } \\
\text { delirium }\end{array}$ & Haloperidol (Planned dose: 1mg) & $\begin{array}{l}\text { Placebo (Planned } \\
\text { dose: } 5 \% \\
\text { dextrose) }\end{array}$ & Intravenous & Died in ICU & $9 / 34(26.5 \%)$ & $7 / 34(20.6 \%)$ & $\begin{array}{l}1.29(0.54 \text { to } \\
3.06)\end{array}$ \\
\hline Al-Qadheeb, $2016^{2}$ & $\begin{array}{l}\text { MV patients } \\
\text { with } \\
\text { subsyndromal } \\
\text { delirium }\end{array}$ & Haloperidol (Planned dose: 1mg), & $\begin{array}{l}\text { Placebo (Planned } \\
\text { dose: } 5 \% \\
\text { dextrose) }\end{array}$ & Intravenous & $\begin{array}{l}\text { Died in } \\
\text { hospital }\end{array}$ & $9 / 34(26.5 \%)$ & $7 / 34(20.6 \%)$ & $\begin{array}{l}1.29(0.54 \text { to } \\
3.06)\end{array}$ \\
\hline Girard, $2010^{4}$ & $\begin{array}{l}>18 \text { years MV } \\
\text { medical and } \\
\text { surgical ICU } \\
\text { patients }\end{array}$ & $\begin{array}{l}\text { Haloperidol (Planned dose: } 5 \text { Median } \\
\text { dose: } 15 \text { (10.8-17) }\end{array}$ & $\begin{array}{l}\text { Placebo (Planned } \\
\text { dose: } 5 \mathrm{ml})\end{array}$ & Oral & $\begin{array}{l}\text { 21-day } \\
\text { mortality, n } \\
(\%)\end{array}$ & $4 / 35(11 \%)$ & $6 / 36(17 \%)$ & $\begin{array}{l}0.69(0.21 \text { to } \\
2.22)\end{array}$ \\
\hline Khan, $2018^{8}$ & $\begin{array}{l}\text { English } \\
\text { speaking } \\
\text { individuals } \\
\text { undergoing } \\
\text { thoracic surgery }\end{array}$ & $\begin{array}{l}\text { Haloperidol (Planned dose: } 0.5 \mathrm{mg} \text {, } \\
\text { Planned duration: } 4 \text { days (11 doses } \\
\text { total)), } 68\end{array}$ & $\begin{array}{l}\text { Placebo (Planned } \\
\text { dose: NR, Planned } \\
\text { duration: } 4 \text { days } \\
(11 \text { doses total)) } \\
67\end{array}$ & Intravenous & $\begin{array}{l}\text { In-hospital } \\
\text { mortality }\end{array}$ & $0 / 68(0 \%)$ & $0 / 67(0 \%)$ & \\
\hline Page, $2013^{10}$ & $\begin{array}{l}\text { ICU patients } \\
\text { needing MV } \\
\text { within } 72 \text { hours } \\
\text { of admission } \\
\text { were enrolled }\end{array}$ & Haloperidol (Planned dose: $2.5 \mathrm{mg}$ ), & $\begin{array}{l}\text { Placebo (Planned } \\
\text { dose: } 0.5 \mathrm{ml} \text { ) }\end{array}$ & Intravenous & & $20 / 71(28.2 \%)$ & $19 / 70(27.1 \%)$ & $\begin{array}{l}1.04(0.61 \text { to } \\
1.77)\end{array}$ \\
\hline Schrijver, $2018^{12}$ & $\begin{array}{l}\text { Medical and } \\
\text { surgical patients }\end{array}$ & Haloperidol (Planned dose: 1mg) & Placebo & Oral & Not applicable & $6 / 118(5.1 \%)$ & $8 / 124(6.5 \%)$ & $\begin{array}{l}0.79(0.28 \text { to } \\
2.20)\end{array}$ \\
\hline
\end{tabular}




\begin{tabular}{|c|c|c|c|c|c|c|c|c|}
\hline Author, year & Population & Intervention group & Control group & $\begin{array}{c}\text { Route of } \\
\text { administration }\end{array}$ & $\begin{array}{l}\text { Outcome } \\
\text { definition }\end{array}$ & $\begin{array}{l}\text { n I N (\%), intervention } \\
\text { group }\end{array}$ & $\begin{array}{l}\text { n I N (\%), control } \\
\text { group }\end{array}$ & $\begin{array}{c}\text { Relative risk } \\
(95 \% \mathrm{Cl})\end{array}$ \\
\hline Schrijver, $2018^{12}$ & $\begin{array}{l}\text { Medical and } \\
\text { surgical patients }\end{array}$ & Haloperidol (Planned dose: 1mg) & Placebo & Oral & $\begin{array}{l}\text { In-hospital } \\
\text { mortality }\end{array}$ & $4 / 118(3.5 \%)$ & $3 / 124(2.5 \%)$ & $\begin{array}{l}1.40(0.32 \text { to } \\
6.13)\end{array}$ \\
\hline Schrijver, $2018^{12}$ & $\begin{array}{l}\text { Medical and } \\
\text { surgical patients }\end{array}$ & Haloperidol (Planned dose: 1mg) & Placebo & Oral & Not applicable & $11 / 118(9.9 \%)$ & $15 / 124$ (12.5\%) & $\begin{array}{l}0.77(0.37 \text { to } \\
1.61)\end{array}$ \\
\hline Schrijver, $2018^{12}$ & $\begin{array}{l}\text { Medical and } \\
\text { surgical patients }\end{array}$ & Haloperidol (Planned dose: $1 \mathrm{mg}$ ) & Placebo & Oral & Not applicable & $18 / 118(17.3 \%)$ & $20 / 124(17.5 \%)$ & $\begin{array}{l}0.95(0.53 \text { to } \\
1.70)\end{array}$ \\
\hline van den Boogaard, 2018 & $\begin{array}{l}\text { ICU patients at } \\
\text { high risk of } \\
\text { delirium }\end{array}$ & Haloperidol (Planned dose: $2 \mathrm{mg}$ ) & $\begin{array}{l}\text { Placebo (Planned } \\
\text { dose: } 0.9 \% \mathrm{NaCl} \text { ) }\end{array}$ & Intravenous & $\begin{array}{l}\text { Number of } \\
\text { patients died } \\
\text { by the time of } \\
\text { assessment at } \\
90 \text { days }\end{array}$ & $153 / 732(20.9 \%)$ & $151 / 707$ (21.4\%) & $\begin{array}{l}0.98(0.80 \text { to } \\
1.20)\end{array}$ \\
\hline van den Boogaard, 2018 ${ }^{15}$ & $\begin{array}{l}\text { ICU patients at } \\
\text { high risk of } \\
\text { delirium }\end{array}$ & Haloperidol (Planned dose: $1 \mathrm{mg}$ ) & $\begin{array}{l}\text { Placebo (Planned } \\
\text { dose: } 0.9 \% \mathrm{NaCl} \text { ) }\end{array}$ & Intravenous & $\begin{array}{l}\text { Number of } \\
\text { patients died } \\
\text { by the time of } \\
\text { assessment at } \\
90 \text { days }\end{array}$ & 74 / $350(21.1 \%)$ & $151 / 707(21.4 \%)$ & $\begin{array}{l}0.99 \text { (0.77 to } \\
1.27)\end{array}$ \\
\hline van den Boogaard, $2018^{15}$ & $\begin{array}{l}\text { ICU patients at } \\
\text { high risk of } \\
\text { delirium }\end{array}$ & Haloperidol (Planned dose: $2 \mathrm{mg}$ ) & $\begin{array}{l}\text { Placebo (Planned } \\
\text { dose: } 0.9 \% \mathrm{NaCl} \text { ) }\end{array}$ & Intravenous & $\begin{array}{l}\text { Number of } \\
\text { patients died } \\
\text { at } 28 \text { days } \\
\text { assessment }\end{array}$ & $122 / 732(16.7 \%)$ & $122 / 707(17.3 \%)$ & $\begin{array}{l}0.97(0.77 \text { to } \\
1.21)\end{array}$ \\
\hline
\end{tabular}




\begin{tabular}{|c|c|c|c|c|c|c|c|c|}
\hline Author, year & Population & Intervention group & Control group & $\begin{array}{c}\text { Route of } \\
\text { administration }\end{array}$ & $\begin{array}{l}\text { Outcome } \\
\text { definition }\end{array}$ & $\begin{array}{l}\text { n I N (\%), intervention } \\
\text { group }\end{array}$ & $\begin{array}{c}\text { n I N (\%), control } \\
\text { group }\end{array}$ & $\begin{array}{l}\text { Relative risk } \\
(95 \% \mathrm{Cl})\end{array}$ \\
\hline van den Boogaard, 2018 & $\begin{array}{l}\text { ICU patients at } \\
\text { high risk of } \\
\text { delirium }\end{array}$ & Haloperidol (Planned dose: 1mg) & $\begin{array}{l}\text { Placebo (Planned } \\
\text { dose: } 0.9 \% \mathrm{NaCl})\end{array}$ & Intravenous & $\begin{array}{l}\text { Number of } \\
\text { patients died at } \\
28 \text { days } \\
\text { assessment }\end{array}$ & $64 / 350(18.3 \%)$ & $122 / 707(17.3 \%)$ & $\begin{array}{l}1.06(0.81 \text { to } \\
1.39)\end{array}$ \\
\hline Wang, $2012^{16}$ & $\begin{array}{l}\text { Patients } 65 \text { or } \\
\text { older admitted } \\
\text { to the ICU after } \\
\text { noncardiac } \\
\text { surgery }\end{array}$ & $\begin{array}{l}\text { Haloperidol (Planned dose: } 0.5 \mathrm{mg} \\
\text { followed by continuous infusion at a } \\
\text { rate of } 1 \mathrm{~mL} / \mathrm{hr}(0.1 \mathrm{mg} / \mathrm{hr} \\
\text { haloperidol)) }\end{array}$ & $\begin{array}{l}\text { Placebo (Planned } \\
\text { dose: Normal } \\
\text { saline) }\end{array}$ & Intravenous & $\begin{array}{l}\text { All-cause } \\
\text { mortality for all } \\
\text { the patients in } \\
\text { the groups }\end{array}$ & $2 / 229(0.9 \%)$ & $6 / 228(2.6 \%)$ & $\begin{array}{l}0.33(0.07 \text { to } \\
1.63)\end{array}$ \\
\hline Wang, 2012 $2^{16}$ & $\begin{array}{l}\text { Patients } 65 \text { or } \\
\text { older admitted } \\
\text { to the ICU after } \\
\text { noncardiac } \\
\text { surgery }\end{array}$ & $\begin{array}{l}\text { Haloperidol (Planned dose: } 0.5 \mathrm{mg} \\
\text { followed by continuous infusion at a } \\
\text { rate of } 1 \mathrm{~mL} / \mathrm{hr}(0.1 \mathrm{mg} / \mathrm{hr} \\
\text { haloperidol)) }\end{array}$ & $\begin{array}{l}\text { Placebo (Planned } \\
\text { dose: Normal } \\
\text { saline) }\end{array}$ & Intravenous & $\begin{array}{l}\text { All-cause } \\
\text { mortality, in } \\
\text { patients who } \\
\text { developed } \\
\text { post-operative } \\
\text { delirium }\end{array}$ & $0 / 35(0 \%)$ & $4 / 53(7.5 \%)$ & $\begin{array}{l}0.17(0.01 \text { to } \\
3.00)\end{array}$ \\
\hline \multicolumn{9}{|l|}{$\begin{array}{l}\text { Second-generation } \\
\text { antipsychotic vs. placebo }\end{array}$} \\
\hline Girard, $2010^{4}$ & $\begin{array}{l}>18 \text { years MV } \\
\text { medical and } \\
\text { surgical ICU } \\
\text { patients }\end{array}$ & $\begin{array}{l}\text { Ziprasidone (Planned dose: } 40 \\
\text { Median dose: } 113 \text { (81-140)) }\end{array}$ & $\begin{array}{l}\text { Placebo (Planned } \\
\text { dose: } 5 \mathrm{ml} \text { ) }\end{array}$ & Oral & $\begin{array}{l}\text { 21-day } \\
\text { mortality, n (\%) }\end{array}$ & $4 / 30(13 \%)$ & $6 / 36(17 \%)$ & $\begin{array}{l}0.80(0.25 \text { to } \\
2.57)\end{array}$ \\
\hline Hakim, $2012^{5}$ & $\begin{array}{l}\text { Patients } 65 \\
\text { years or older } \\
\text { experiencing } \\
\text { subsyndromal } \\
\text { delirium after } \\
\text { on-pump } \\
\text { cardiac surgery }\end{array}$ & Risperidone (Planned dose: 0.5mg), & $\begin{array}{l}\text { Placebo (Planned } \\
\text { dose: given every } \\
12 \text { hours) }\end{array}$ & Oral & $\begin{array}{l}\text { Died of } \\
\text { cardiogenic } \\
\text { shock }\end{array}$ & $2 / 51(3.9 \%)$ & $1 / 50(2 \%)$ & $\begin{array}{l}1.96(0.18 \text { to } \\
20.95)\end{array}$ \\
\hline
\end{tabular}




\begin{tabular}{|c|c|c|c|c|c|c|c|c|}
\hline Author, year & Population & Intervention group & Control group & $\begin{array}{c}\text { Route of } \\
\text { administration }\end{array}$ & $\begin{array}{l}\text { Outcome } \\
\text { definition }\end{array}$ & $\begin{array}{l}\text { n I N (\%), intervention } \\
\text { group }\end{array}$ & $\begin{array}{l}\text { n I N (\%), control } \\
\text { group }\end{array}$ & $\begin{array}{c}\text { Relative risk } \\
(95 \% \mathrm{Cl})\end{array}$ \\
\hline \multicolumn{9}{|c|}{$\begin{array}{l}\text { First-generation } \\
\text { antipsychotic vs. second- } \\
\text { generation antipsychotic }\end{array}$} \\
\hline Girard, $2010^{4}$ & $\begin{array}{l}>18 \text { years MV } \\
\text { medical and } \\
\text { surgical ICU } \\
\text { patients }\end{array}$ & $\begin{array}{l}\text { Ziprasidone (Planned dose: } 40 \\
\text { Median dose: } 113 \text { (81-140)) }\end{array}$ & $\begin{array}{l}\text { Haloperidol } \\
\text { (Planned dose: } 5 \\
\text { Median dose: } 15 \\
(10.8-17))\end{array}$ & Oral & $\begin{array}{l}\text { 21-day } \\
\text { mortality, n } \\
(\%)\end{array}$ & $4 / 30(13 \%)$ & $4 / 35(11 \%)$ & $\begin{array}{l}1.17(0.32 \text { to } \\
4.27)\end{array}$ \\
\hline \multicolumn{9}{|l|}{$\begin{array}{l}\text { First-generation } \\
\text { antipsychotic vs. other }\end{array}$} \\
\hline Abdelgalel, $2016^{1}$ & $\begin{array}{l}\text { Adult intensive } \\
\text { care patients of } \\
\text { ASA physical } \\
\text { status III and IV } \\
\text { aged between } \\
26 \text { and } 70 \text { years }\end{array}$ & $\begin{array}{l}\text { Haloperidol (Planned dose: } 0.5-2 \\
\mathrm{mg} / \mathrm{h} \text { preceded by a loading dose of } \\
2.5 \mathrm{mg} \text { intravenously over } 10 \mathrm{~min} \text { if } \\
\text { needed (if RASS }>2+\text { ) }\end{array}$ & $\begin{array}{l}\text { Dexmedetomidine } \\
\text { (Planned dose: } \\
0.2-0.7 \mathrm{mcg} / \mathrm{kg} / \mathrm{h} \\
\text { preceded by a } \\
\text { loading dose of } 1.0 \\
\text { ug } / \mathrm{kg} \\
\text { intravenously over } \\
10 \text { min if needed } \\
\text { (if RASS }>2+\text { ) }\end{array}$ & Intravenous & Mortality & $2 / 30(6.7 \%)$ & $2 / 30(6.7 \%)$ & $\begin{array}{l}1.00(0.15 \text { to } \\
6.64)\end{array}$ \\
\hline
\end{tabular}

ASA=American Society of Anesthesiologists; CI=confidence interval; ICU=intensive care unit; Mg/hr=milligram per hour; mg=milligram; MV=mechanical ventilation; $\mathrm{n}=$ sample size; NaCl=sodium chloride; RASS=Richmond Agitation and Sedation Scale 
Evidence Table D-15. Survival outcomes in studies comparing interventions to prevent development of delirium

\begin{tabular}{|c|c|c|c|c|c|c|c|c|}
\hline Author, year & Population & $\begin{array}{c}\text { Intervention } \\
\text { group, } \mathrm{n}\end{array}$ & Control group, $\mathbf{n}$ & $\begin{array}{c}\text { Route of } \\
\text { administration }\end{array}$ & $\begin{array}{l}\text { Outcome } \\
\text { definition }\end{array}$ & $\begin{array}{l}\text { Median (IQR) days, } \\
\text { intervention group }\end{array}$ & $\begin{array}{l}\text { Median (IQR), days, } \\
\text { control group }\end{array}$ & $\begin{array}{c}\text { Difference in Medians } \\
(95 \% \mathrm{Cl})\end{array}$ \\
\hline \multicolumn{9}{|l|}{$\begin{array}{l}\text { First-generation } \\
\text { antipsychotic vs. } \\
\text { placebo }\end{array}$} \\
\hline $\begin{array}{l}\text { van den } \\
\text { Boogaard, } 2018^{15}\end{array}$ & $\begin{array}{l}\text { ICU patients at } \\
\text { high risk of } \\
\text { delirium }\end{array}$ & $\begin{array}{l}\text { Haloperidol } \\
\text { (Planned dose: } \\
\text { 1mg), } 350\end{array}$ & $\begin{array}{l}\text { Placebo (Planned } \\
\text { dose: } 0.9 \% \mathrm{NaCl} \text {, } \\
707\end{array}$ & Intravenous & $\begin{array}{l}\text { Median number of } \\
\text { days patients } \\
\text { survived at the } \\
\text { time of } 28 \text { days } \\
\text { assessment }\end{array}$ & 28 (28 to 28 ) & 28 (28 to 28 ) & $0(0$ to 0$)$ \\
\hline $\begin{array}{l}\text { van den } \\
\text { Boogaard, } 2018^{15}\end{array}$ & $\begin{array}{l}\text { ICU patients at } \\
\text { high risk of } \\
\text { delirium }\end{array}$ & $\begin{array}{l}\text { Haloperidol } \\
\text { (Planned dose: } \\
\text { 2mg), } 732\end{array}$ & $\begin{array}{l}\text { Placebo (Planned } \\
\text { dose: } 0.9 \% \mathrm{NaCl} \text {, } \\
707\end{array}$ & Intravenous & $\begin{array}{l}\text { Median number of } \\
\text { days patients } \\
\text { survived at the } \\
\text { time of } 28 \text { days } \\
\text { assessment }\end{array}$ & 28 (28 to 28 ) & 28 (28 to 28 ) & 0 (0 to 0$)$ \\
\hline $\begin{array}{l}\text { van den } \\
\text { Boogaard, } 2018^{15}\end{array}$ & $\begin{array}{l}\text { ICU patients at } \\
\text { high risk of } \\
\text { delirium }\end{array}$ & $\begin{array}{l}\text { Haloperidol } \\
\text { (Planned dose: } \\
\text { 1mg), } 350\end{array}$ & $\begin{array}{l}\text { Placebo (Planned } \\
\text { dose: } 0.9 \% \mathrm{NaCl} \text {, } \\
707\end{array}$ & Intravenous & $\begin{array}{l}\text { Median number of } \\
\text { days patients } \\
\text { survived at the } \\
\text { time of } 90 \text { days } \\
\text { assessment }\end{array}$ & 90 (90 to 90$)$ & 90 (90 to 90 ) & 0 (0 to 0$)$ \\
\hline $\begin{array}{l}\text { van den } \\
\text { Boogaard, } 2018^{15}\end{array}$ & $\begin{array}{l}\text { ICU patients at } \\
\text { high risk of } \\
\text { delirium }\end{array}$ & $\begin{array}{l}\text { Haloperidol } \\
\text { (Planned dose: } \\
\text { 2mg), } 732\end{array}$ & $\begin{array}{l}\text { Placebo (Planned } \\
\text { dose: } 0.9 \% \mathrm{NaCl} \text {, } \\
707\end{array}$ & Intravenous & $\begin{array}{l}\text { Median number of } \\
\text { days patients } \\
\text { survived at the } \\
\text { time of } 90 \text { days } \\
\text { assessment }\end{array}$ & 90 (90 to 90$)$ & 90 (90 to 90$)$ & 0 (0 to 0$)$ \\
\hline
\end{tabular}

CI=confidence interval; ICU=intensive care unit; IQR=interquartile range; $\mathrm{Mg} / \mathrm{hr}=$ =milligram per hour; mg=milligram; $\mathrm{MV}=$ =mechanical ventilation; $\mathrm{n}=\mathrm{sample}$ size; NaCl=sodium chloride 
Evidence Table D-16. Institutionalization outcomes in studies comparing interventions to prevent development of delirium

\begin{tabular}{|c|c|c|c|c|c|c|c|c|}
\hline Author, year & Population & Intervention group & Control group & $\begin{array}{c}\text { Route of } \\
\text { administration }\end{array}$ & Outcome definition & $\begin{array}{c}\mathrm{n} / \mathrm{N}(\%), \text { intervention } \\
\text { group }\end{array}$ & $\begin{array}{c}\text { n / N (\%), control } \\
\text { group }\end{array}$ & $\begin{array}{c}\text { Relative risk (95\% } \\
\text { Cl) }\end{array}$ \\
\hline \multicolumn{9}{|l|}{$\begin{array}{l}\text { First-generation } \\
\text { antipsychotic vs. } \\
\text { placebo }\end{array}$} \\
\hline Schrijver, $2018^{12}$ & $\begin{array}{l}\text { Medical and } \\
\text { surgical patients }\end{array}$ & $\begin{array}{l}\text { Haloperidol } \\
\text { (Planned dose: } \\
\text { 1mg) }\end{array}$ & Placebo & Oral & $\begin{array}{l}\text { New } \\
\text { institutionalization }\end{array}$ & $13 / 118(18.3 \%)$ & $8 / 124(11.9 \%)$ & $1.71(0.73$ to 3.97$)$ \\
\hline Schrijver, $2018^{12}$ & $\begin{array}{l}\text { Medical and } \\
\text { surgical patients }\end{array}$ & $\begin{array}{l}\text { Haloperidol } \\
\text { (Planned dose: } \\
\text { 1mg), }\end{array}$ & Placebo & Oral & Not applicable & $7 / 118$ (6.1\%) & $6 / 124(5 \%)$ & $1.23(0.42$ to 3.54$)$ \\
\hline Schrijver, $2018^{12}$ & $\begin{array}{l}\text { Medical and } \\
\text { surgical patients }\end{array}$ & $\begin{array}{l}\text { Haloperidol } \\
\text { (Planned dose: } \\
\text { 1mg) }\end{array}$ & Placebo & Oral & $\begin{array}{l}\text { New } \\
\text { institutionalization }\end{array}$ & $10 / 118(16.1 \%)$ & $8 / 124(11.9 \%)$ & $1.31(0.54$ to 3.21$)$ \\
\hline \multicolumn{9}{|l|}{$\begin{array}{l}\text { Second-generation } \\
\text { antipsychotic vs. } \\
\text { placebo }\end{array}$} \\
\hline Larsen, $2010^{9}$ & $\begin{array}{l}\text { Post-operative } \\
\text { elderly joint } \\
\text { replacement } \\
\text { surgery patients }\end{array}$ & $\begin{array}{l}\text { Olanzapine } \\
\text { (Planned dose: } \\
5 \mathrm{mg} \text { ) }\end{array}$ & $\begin{array}{l}\text { Placebo (Planned } \\
\text { dose: } 5 \mathrm{mg} \text { ) }\end{array}$ & Oral & $\begin{array}{l}\text { Discharge to } \\
\text { rehabilitation facility } \\
\text { (SNF or acute } \\
\text { [hospital-level]) care }\end{array}$ & $116 / 196(59.2 \%)$ & 143 / 204 (70.1\%) & 0.84 (0.73 to 0.98$)$ \\
\hline
\end{tabular}

CI=confidence interval; ICU=intensive care unit; mg=milligram; $\mathrm{MV}=$ =mechanical ventilation; $\mathrm{n}=$ sample size; $\mathrm{SNF}=$ skilled nursing facility 
Evidence Table D-17. Fall outcomes in studies comparing interventions to prevent development of delirium

Author, year $\quad$ Population

Route of

Outcome definition

Futhor, year

Population

Intervention group

Control group

administration

n / N (\%), intervention group

n / N (\%), control group

Relative risk (95\%

antipsychotic vs.

placebo

Fukata, $2014^{3}$

\section{Delirium after}

abdominal or

orthopedic surgery

in elderly patients

\begin{tabular}{|l|l|l|l|}
$\begin{array}{l}\text { Haloperidol } \\
\text { (Planned dose: } \\
2.5 \mathrm{mg}), 59\end{array}$ & Placebo, 60 & Intravenous & $\begin{array}{l}\text { Number of patients } \\
\text { fell }\end{array}$ \\
& & & \\
\hline
\end{tabular}

$1 / 59(2 \%)$

\begin{tabular}{|l|l|}
\hline $0 / 60(0 \%)$ & $3.05(0.13$ to 73.39$)$ \\
& \\
\hline
\end{tabular}

CI=confidence interval; ICU=intensive care unit; $\mathrm{mg}=$ =milligram; $\mathrm{n}=$ sample size 
Evidence Table D-18. Hospital readmission outcomes in studies comparing interventions to prevent development of delirium

\begin{tabular}{|c|c|c|c|c|c|c|c|c|}
\hline Author, year & Population & $\begin{array}{c}\text { Intervention group, } \\
\mathrm{n}\end{array}$ & Control group, $n$ & $\begin{array}{c}\text { Route of } \\
\text { administration }\end{array}$ & Outcome definition & $\begin{array}{c}\mathrm{n} / \mathrm{N}(\%), \text { intervention } \\
\text { group }\end{array}$ & $\begin{array}{c}\text { n I N (\%), control } \\
\text { group }\end{array}$ & $\begin{array}{l}\text { Relative risk (95\% } \\
\text { Cl) }\end{array}$ \\
\hline \multicolumn{9}{|l|}{$\begin{array}{l}\text { First-generation } \\
\text { antipsychotic vs. } \\
\text { placebo }\end{array}$} \\
\hline Schrijver, $2018^{12}$ & $\begin{array}{l}\text { Medical and } \\
\text { surgical patients }\end{array}$ & $\begin{array}{l}\text { Haloperidol } \\
\text { (Planned dose: } \\
\text { 1mg), } 118\end{array}$ & Placebo, 124 & Oral & Not applicable & $27 / 118(33.8 \%)$ & 22 / $124(27.2 \%)$ & $1.29(0.78$ to 2.13$)$ \\
\hline Schrijver, $2018^{12}$ & $\begin{array}{l}\text { Medical and } \\
\text { surgical patients }\end{array}$ & $\begin{array}{l}\text { Haloperidol } \\
\text { (Planned dose: } \\
\text { 1mg), } 118\end{array}$ & Placebo, 124 & Oral & Not applicable & $17 / 118(24.6 \%)$ & 14 / $124(18.2 \%)$ & $1.28(0.66$ to 2.47$)$ \\
\hline
\end{tabular}

CI=confidence interval; mg=milligram; n=sample size 
Evidence Table D-19. Intensive care unit readmission outcomes in studies comparing interventions to prevent development of delirium

\begin{tabular}{|c|c|c|c|c|c|c|c|c|}
\hline Author, year & Population & $\begin{array}{c}\text { Intervention group, } \\
\mathrm{n}\end{array}$ & Control group, $\mathbf{n}$ & $\begin{array}{c}\text { Route of } \\
\text { administration }\end{array}$ & Outcome definition & $\begin{array}{c}\mathrm{n} / \mathrm{N}(\%), \text { intervention } \\
\text { group }\end{array}$ & $\begin{array}{c}\text { n I N (\%), control } \\
\text { group }\end{array}$ & $\begin{array}{l}\text { Relative risk (95\% } \\
\text { Cl) }\end{array}$ \\
\hline \multicolumn{9}{|l|}{$\begin{array}{l}\text { First-generation } \\
\text { antipsychotic vs. } \\
\text { placebo }\end{array}$} \\
\hline Page, $2013^{10}$ & $\begin{array}{l}\text { ICU patients } \\
\text { needing MV within } \\
72 \text { hours of } \\
\text { admission were } \\
\text { enrolled }\end{array}$ & $\begin{array}{l}\text { Haloperidol } \\
\text { (Planned dose: } \\
2.5 \mathrm{mg}), 71\end{array}$ & $\begin{array}{l}\text { Placebo (Planned } \\
\text { dose: } 0.5 \mathrm{ml}), 70\end{array}$ & Intravenous & $\begin{array}{l}\text { Readmission to ICU } \\
\text { with sepsis }\end{array}$ & $1 / 71(3 \%)$ & $1 / 70(1 \%)$ & 0.99 (0.06 to 15.45$)$ \\
\hline $\begin{array}{l}\text { van den Boogaard, } \\
2018^{15}\end{array}$ & $\begin{array}{l}\text { ICU patients at high } \\
\text { risk of delirium }\end{array}$ & $\begin{array}{l}\text { Haloperidol } \\
\text { (Planned dose: } \\
2 \mathrm{mg}), 732\end{array}$ & $\begin{array}{l}\text { Placebo (Planned } \\
\text { dose: } 0.9 \% \mathrm{NaCl} \text {, } \\
707\end{array}$ & Intravenous & $\begin{array}{l}\text { ICU readmission, } \\
\text { No. }(\%)\end{array}$ & $65 / 732$ (8.9\%) & 68 / 707 (9.6\%) & 0.92 (0.67 to 1.28$)$ \\
\hline $\begin{array}{l}\text { van den Boogaard, } \\
2018^{15}\end{array}$ & $\begin{array}{l}\text { ICU patients at high } \\
\text { risk of delirium }\end{array}$ & $\begin{array}{l}\text { Haloperidol } \\
\text { (Planned dose: } \\
\text { 1mg), } 350\end{array}$ & $\begin{array}{l}\text { Placebo (Planned } \\
\text { dose: } 0.9 \% \mathrm{NaCl} \text {, } \\
707\end{array}$ & Intravenous & $\begin{array}{l}\text { ICU readmission, } \\
\text { No. (\%) }\end{array}$ & 36 / $350(10.3 \%)$ & 68 / 707 (9.6\%) & 1.07 (0.73 to 1.57$)$ \\
\hline
\end{tabular}

CI=confidence interval; ICU=intensive care unit; mg=milligram; MV=mechanical ventilation; n=sample size; NaCl=sodium chloride 


\begin{tabular}{|c|c|c|c|c|c|c|c|c|}
\hline Author, year & $\begin{array}{l}\text { Population } \\
\end{array}$ & Intervention group, $\mathbf{n}$ & Control group, $n$ & $\begin{array}{c}\text { Route of } \\
\text { administration }\end{array}$ & $\begin{array}{l}\text { Outcome } \\
\text { definition }\end{array}$ & $\begin{array}{l}\text { Mean (SD) } \\
\text { days, } \\
\text { intervention } \\
\text { group }\end{array}$ & $\begin{array}{l}\text { Mean (SD) } \\
\text { days, } \\
\text { control } \\
\text { group }\end{array}$ & $\begin{array}{l}\text { Mean } \\
\text { between- } \\
\text { group } \\
\text { difference } \\
(95 \% \mathrm{Cl}) \\
\end{array}$ \\
\hline \multicolumn{9}{|l|}{$\begin{array}{l}\text { First-generation } \\
\text { antipsychotic vs. } \\
\text { placebo }\end{array}$} \\
\hline Abdelgalel, 2016 ${ }^{1}$ & $\begin{array}{l}\text { Adult intensive care patients of ASA } \\
\text { physical status III and IV aged between } 26 \\
\text { and } 70 \text { years }\end{array}$ & $\begin{array}{l}\text { Haloperidol (Planned dose: } \\
0.5-2 \mathrm{mg} / \mathrm{h} \text { preceded by a } \\
\text { loading dose of } 2.5 \mathrm{mg} \\
\text { intravenously over } 10 \mathrm{~min} \text { if } \\
\text { needed (if RASS }>2+\text { ), } 30\end{array}$ & $\begin{array}{l}\text { Placebo (Planned dose: } \\
2-8 \mathrm{ml} \text {, loading dose } \\
10 \mathrm{ml} \text { over } 10 \mathrm{~min} \text { if } \\
\text { needed (if RASS }>2+) \text { ), } \\
30\end{array}$ & Intravenous & $\begin{array}{l}\text { Length of stay in } \\
\text { hospital (days) }\end{array}$ & $13.5(2)$ & $15.5(2.5)$ & $\begin{array}{l}-2(-3.15 \text { to } \\
-0.85)\end{array}$ \\
\hline Girard, $2010^{4}$ & $\begin{array}{l}>18 \text { years MV medical and surgical ICU } \\
\text { patients }\end{array}$ & $\begin{array}{l}\text { Haloperidol (Planned dose: } \\
\text { 5mg Median dose: } 15 \mathrm{mg} \\
(10.8-17 \mathrm{mg}), 35\end{array}$ & $\begin{array}{l}\text { Placebo (Planned dose: } \\
5 \mathrm{ml}), 36\end{array}$ & Oral & $\begin{array}{l}\text { Length of stay, } \\
\text { days in hospital }\end{array}$ & $\begin{array}{l}\text { Median } 13.8 \\
\text { (IQR } 9.4 \text { to } \\
\text { NA) }\end{array}$ & $\begin{array}{l}\text { Median } 15.4 \\
\text { (IQR } 8.9 \text { to } \\
\text { NA) }\end{array}$ & NA \\
\hline Kalisvaart, $2005^{6}$ & $\begin{array}{l}\text { Acute or elective hip surgery patients } \geq 70 \\
\text { years is at risk of delirium: MMSE between } \\
\text { less than or equal to } 24 ; \\
\text { dehydration=BUN/creatinine }>\text { or }=\text { to } 18, \\
\text { low visual acuity and/or increased severity } \\
\text { of illness on APACHE II of } 16 \text { or greater. }\end{array}$ & $\begin{array}{l}\text { Haloperidol (Planned dose: } \\
0.5 \mathrm{mg} \text { ), } 32\end{array}$ & $\begin{array}{l}\text { Placebo (Not } \\
\text { applicable), } 36\end{array}$ & Oral & Hospital Days & $17.1(11.1)$ & $22.6(16.7)$ & $\begin{array}{l}-5.5(- \\
12.24 \text { to } \\
1.24)\end{array}$ \\
\hline Khan, $2018^{8}$ & $\begin{array}{l}\text { English speaking individuals undergoing } \\
\text { thoracic surgery }\end{array}$ & $\begin{array}{l}\text { Haloperidol (Planned dose: } \\
0.5 \mathrm{mg} \text {, Planned duration: } 4 \\
\text { days (11 doses total)), } 68\end{array}$ & $\begin{array}{l}\text { Placebo (Planned dose: } \\
\text { NR, Planned duration: } 4 \\
\text { days (11 doses total)), } \\
67\end{array}$ & Intravenous & $\begin{array}{l}\text { Hospital length of } \\
\text { stay, median, days }\end{array}$ & $\begin{array}{l}\text { Median: } 10 \\
\text { (IQR } 8 \text { to } \\
11.5 \text { ) }\end{array}$ & $\begin{array}{l}\text { Median: } 10 \\
\text { (IQR } 8 \text { to } 12 \text { ) }\end{array}$ & $\mathrm{p}=0.4$ \\
\hline Page, $2013^{10}$ & $\begin{array}{l}\text { ICU patients needing MV within } 72 \text { hours } \\
\text { of admission were enrolled }\end{array}$ & $\begin{array}{l}\text { Haloperidol (Planned dose: } \\
2.5 \mathrm{mg}), 42\end{array}$ & $\begin{array}{l}\text { Placebo (Planned dose: } \\
0.5 \mathrm{ml}), 47\end{array}$ & Intravenous & $\begin{array}{l}\text { Length of hospital } \\
\text { stay, Excluding } \\
\text { patients who died } \\
\text { in hospital } \\
\end{array}$ & $20.5(14.9)$ & $27(19.8)$ & $\begin{array}{l}-6.5(- \\
13.78 \text { to } \\
0.78)\end{array}$ \\
\hline Schrijver, $2018^{12}$ & Medical and surgical patients & $\begin{array}{l}\text { Haloperidol (Planned dose: } \\
1 \mathrm{mg}), 118\end{array}$ & Placebo, 124 & Oral & Not applicable & $7.1(4.9)$ & $7.93(5.4)$ & $\begin{array}{l}-0.83(- \\
2.13 \text { to } \\
0.47)\end{array}$ \\
\hline $\begin{array}{l}\text { van den Boogaard, } \\
2018^{15}\end{array}$ & ICU patients at high risk of delirium & $\begin{array}{l}\text { Haloperidol (Planned dose: } \\
\text { 1mg), } 350\end{array}$ & $\begin{array}{l}\text { Placebo (Planned dose: } \\
0.9 \% \mathrm{NaCl}), 707\end{array}$ & Intravenous & $\begin{array}{l}\text { Length of stay, } \\
\text { median (IQR), } \\
\text { days }\end{array}$ & $18.67(17.3)$ & $16.67(13.5)$ & $\begin{array}{l}2(0.13 \text { to } \\
3.87)\end{array}$ \\
\hline $\begin{array}{l}\text { van den Boogaard, } \\
2018^{15}\end{array}$ & ICU patients at high risk of delirium & $\begin{array}{l}\text { Haloperidol (Planned dose: } \\
2 \mathrm{mg}), 732\end{array}$ & $\begin{array}{l}\text { Placebo (Planned dose: } \\
0.9 \% \mathrm{NaCl}), 707\end{array}$ & Intravenous & $\begin{array}{l}\text { Length of stay, } \\
\text { median (IQR), } \\
\text { days }\end{array}$ & $17.33(14.9)$ & $16.67(13.5)$ & $\begin{array}{l}0.66(-0.81 \\
\text { to } 2.13)\end{array}$ \\
\hline
\end{tabular}




\begin{tabular}{|c|c|c|c|c|c|c|c|c|}
\hline Author, year & Population & Intervention group, $\mathbf{n}$ & Control group, $n$ & $\begin{array}{c}\text { Route of } \\
\text { administration }\end{array}$ & $\begin{array}{l}\text { Outcome } \\
\text { definition }\end{array}$ & $\begin{array}{l}\text { Mean (SD) } \\
\text { days, } \\
\text { intervention } \\
\text { group }\end{array}$ & $\begin{array}{l}\text { Mean (SD) } \\
\text { days, } \\
\text { control } \\
\text { group }\end{array}$ & $\begin{array}{l}\text { Mean } \\
\text { between- } \\
\text { group } \\
\text { difference } \\
(95 \% \mathrm{Cl})\end{array}$ \\
\hline Wang, $2012^{16}$ & $\begin{array}{l}\text { Patients } 65 \text { or older admitted to the ICU } \\
\text { after noncardiac surgery }\end{array}$ & $\begin{array}{l}\text { Haloperidol (Planned dose: } \\
0.5 \mathrm{mg} \text { followed by } \\
\text { continuous infusion at a rate } \\
\text { of } 1 \mathrm{~mL} / \mathrm{hr}(0.1 \mathrm{mg} / \mathrm{hr} \\
\text { haloperidol)), } 35\end{array}$ & $\begin{array}{l}\text { Placebo (Planned dose: } \\
\text { Normal saline), } 53\end{array}$ & Intravenous & $\begin{array}{l}\text { Median Length of } \\
\text { stay in hospital } \\
\text { after surgery, in } \\
\text { patients who } \\
\text { developed post- } \\
\text { operative delirium } \\
\text { in days }\end{array}$ & $14(5.3)$ & $12(2.5)$ & $\begin{array}{l}2(0.27 \text { to } \\
3.73)\end{array}$ \\
\hline Wang, $2012^{16}$ & $\begin{array}{l}\text { Patients } 65 \text { or older admitted to the ICU } \\
\text { after noncardiac surgery }\end{array}$ & $\begin{array}{l}\text { Haloperidol (Planned dose: } \\
0.5 \mathrm{mg} \text { followed by } \\
\text { continuous infusion at a rate } \\
\text { of } 1 \mathrm{~mL} / \mathrm{hr}(0.1 \mathrm{mg} / \mathrm{hr} \\
\text { haloperidol)), } 229\end{array}$ & $\begin{array}{l}\text { Placebo (Planned dose: } \\
\text { Normal saline), } 228\end{array}$ & Intravenous & $\begin{array}{l}\text { Median length of } \\
\text { stay in hospital } \\
\text { after surgery for all } \\
\text { the patients in the } \\
\text { group }\end{array}$ & $11(1.4)$ & $11(1.3)$ & $\begin{array}{l}0(-0.25 \text { to } \\
0.25)\end{array}$ \\
\hline \multicolumn{9}{|l|}{$\begin{array}{l}\text { Second-generation } \\
\text { antipsychotic vs. } \\
\text { placebo }\end{array}$} \\
\hline Girard, $2010^{4}$ & $\begin{array}{l}>18 \text { years MV medical and surgical ICU } \\
\text { patients }\end{array}$ & $\begin{array}{l}\text { Ziprasidone (Planned dose: } \\
\text { 40mg Median dose: 113mg } \\
\text { (81-140mg) }\end{array}$ & $\begin{array}{l}\text { Placebo (Planned dose: } \\
\text { 5ml), }\end{array}$ & Oral & $\begin{array}{l}\text { Length of stay, } \\
\text { days in Hospital }\end{array}$ & Not reported & Not reported & $\begin{array}{l}\text { Median } \\
15.4 \text { (IQR } \\
8.9 \text { to NA) } \\
\end{array}$ \\
\hline Hakim, $2012^{5}$ & $\begin{array}{l}\text { Patients } 65 \text { years or older experiencing } \\
\text { subsyndromal delirium after on-pump } \\
\text { cardiac surgery }\end{array}$ & $\begin{array}{l}\text { Risperidone (Planned dose: } \\
0.5 \mathrm{mg}), 51\end{array}$ & $\begin{array}{l}\text { Placebo (Planned dose: } \\
\text { given every } 12 \text { hours), } \\
50\end{array}$ & Oral & In study group & $6(1.6)$ & $6.33(2.4)$ & $\begin{array}{l}-0.33(- \\
1.13 \text { to } \\
0.47)\end{array}$ \\
\hline Prakanrattana, $2007^{11}$ & $\begin{array}{l}\text { Elective Cardiac Surgery with } \\
\text { cardiopulmonary bypass }\end{array}$ & $\begin{array}{l}\text { Risperidone (Planned dose: } \\
1 \mathrm{mg}), 63\end{array}$ & $\begin{array}{l}\text { Placebo (Not } \\
\text { applicable), } 63\end{array}$ & $\begin{array}{l}\text { Orally } \\
\text { disintegrating } \\
\text { tablet }\end{array}$ & Not applicable & $10.5(6.1)$ & $10.3(4.4)$ & $\begin{array}{l}0.2(-1.66 \\
\text { to } 2.06)\end{array}$ \\
\hline \multicolumn{9}{|l|}{$\begin{array}{l}\text { First-generation } \\
\text { antipsychotic vs. } \\
\text { second-generation } \\
\text { antipsychotic }\end{array}$} \\
\hline Girard, $2010^{4}$ & $\begin{array}{l}>18 \text { years MV medical and surgical ICU } \\
\text { patients }\end{array}$ & $\begin{array}{l}\text { Ziprasidone (Planned dose: } \\
\text { 40mg Median dose: 113mg } \\
\text { (81-140mg) }\end{array}$ & $\begin{array}{l}\text { Haloperidol (Planned } \\
\text { dose: } 5 \mathrm{mg} \text { Median } \\
\text { dose: } 15 \mathrm{mg}(10.8-17)\end{array}$ & Oral & $\begin{array}{l}\text { Length of stay, } \\
\text { days in Hospital }\end{array}$ & $\begin{array}{l}\text { Median } 13.5 \\
\text { (IQR } 9.3 \text { to } \\
\text { Not reported) }\end{array}$ & $\begin{array}{l}\text { Median } 13.8 \\
\text { (IQR } 9.4 \text { to } \\
\text { Not reported) }\end{array}$ & $p=0.68$ \\
\hline
\end{tabular}




\begin{tabular}{|c|c|c|c|c|c|c|c|c|}
\hline Author, year & Population & Intervention group, $\mathbf{n}$ & Control group, $n$ & $\begin{array}{c}\text { Route of } \\
\text { administration }\end{array}$ & $\begin{array}{l}\text { Outcome } \\
\text { definition }\end{array}$ & $\begin{array}{l}\text { Mean (SD) } \\
\text { days, } \\
\text { intervention } \\
\text { group }\end{array}$ & $\begin{array}{c}\text { Mean (SD) } \\
\text { days, } \\
\text { control } \\
\text { group }\end{array}$ & $\begin{array}{c}\text { Mean } \\
\text { between- } \\
\text { group } \\
\text { difference } \\
(95 \% \mathrm{Cl})\end{array}$ \\
\hline \multicolumn{9}{|c|}{$\begin{array}{l}\text { First-generation } \\
\text { antipsychotic vs. other }\end{array}$} \\
\hline Abdelgalel, $2016^{1}$ & $\begin{array}{l}\text { Adult intensive care patients of ASA } \\
\text { physical status III and IV aged between } 26 \\
\text { and } 70 \text { years }\end{array}$ & $\begin{array}{l}\text { Dexmedetomidine (Planned } \\
\text { dose: } 0.2-0.7 \mathrm{ug} / \mathrm{kg} / \mathrm{h} \\
\text { preceded by a loading dose } \\
\text { of } 1.0 \mathrm{ug} / \mathrm{kg} \text { intravenously } \\
\text { over } 10 \mathrm{~min} \text { if needed (if } \\
\text { RASS }>2+\text { )), } 30\end{array}$ & $\begin{array}{l}\text { Haloperidol (Planned } \\
\text { dose: } 0.5-2 \mathrm{mg} / \mathrm{h} \\
\text { preceded by a loading } \\
\text { dose of } 2.5 \mathrm{mg} \\
\text { intravenously over } 10 \\
\text { min if needed (if RASS } \\
>2+), 30\end{array}$ & Intravenous & $\begin{array}{l}\text { Length of stay in } \\
\text { hospital (days) }\end{array}$ & $6.2(0.9)$ & $13.5(2)$ & $\begin{array}{l}-7.3(-8.08 \\
\text { to }-6.52)\end{array}$ \\
\hline
\end{tabular}

APACHE II= Acute Physiology and Chronic Health Evaluation 2; BUN=blood urea nitrogen ratio; ICU=intensive care unit; ICU=intensive care unit; Mg/hr=milligram per hour; Ml/hr=milliliter per hour; MV=mechanical ventilation; $\mathrm{NaCl}=$ sodium chloride; RASS=Richmond Agitation and Sedation Scale; SD=standard deviation; Ug/kg/h=microgram per kilogram per hour 
Evidence Table D-21. Length of intensive care unit stay outcomes in studies comparing interventions to prevent development of delirium

\begin{tabular}{|c|c|c|c|c|c|c|c|c|}
\hline Author, year & Population & Intervention group, $\mathbf{n}$ & Control group, $\mathbf{n}$ & $\begin{array}{c}\text { Route of } \\
\text { administration }\end{array}$ & Outcome definition & $\begin{array}{l}\text { Mean (SD) } \\
\text { days, } \\
\text { intervention } \\
\text { group }\end{array}$ & $\begin{array}{l}\text { Mean (SD) } \\
\text { days, } \\
\text { control } \\
\text { group }\end{array}$ & $\begin{array}{l}\text { Mean } \\
\text { between- } \\
\text { group } \\
\text { difference } \\
(95 \% \mathrm{Cl}) \\
\end{array}$ \\
\hline \multicolumn{9}{|l|}{$\begin{array}{l}\text { First-generation } \\
\text { antipsychotic vs. } \\
\text { placebo }\end{array}$} \\
\hline Abdelgalel, $2016^{1}$ & $\begin{array}{l}\text { Adult intensive care } \\
\text { patients of ASA } \\
\text { physical status III and } \\
\text { IV aged between } 26 \\
\text { and } 70 \text { years }\end{array}$ & $\begin{array}{l}\text { Haloperidol (Planned dose: } 0.5- \\
2 \mathrm{mg} / \mathrm{h} \text { preceded by a loading } \\
\text { dose of } 2.5 \mathrm{mg} \text { intravenously over } \\
10 \mathrm{~min} \text { if needed (if RASS }>2+\text { ), } \\
30\end{array}$ & $\begin{array}{l}\text { Placebo (Planned dose: } 2- \\
8 \mathrm{ml}, \text { loading dose } 10 \mathrm{ml} \text { over } \\
10 \text { min if needed (if RASS > } \\
2+) \text { ), } 30\end{array}$ & Intravenous & $\begin{array}{l}\text { Length of ICU stays } \\
\text { (days) }\end{array}$ & $6.5(1)$ & $6.9(1.2)$ & $\begin{array}{l}-0.4(-0.96 \text { to } \\
0.16)\end{array}$ \\
\hline Al-Qadheeb, $2016^{2}$ & $\begin{array}{l}\text { MV patients with } \\
\text { subsyndromal delirium }\end{array}$ & $\begin{array}{l}\text { Haloperidol (Planned dose: } 1 \mathrm{mg} \text { ), } \\
34\end{array}$ & $\begin{array}{l}\text { Placebo (Planned dose: } 5 \% \\
\text { dextroseml), } 34\end{array}$ & Intravenous & $\begin{array}{l}\text { Duration of ICU stay in } \\
\text { days }\end{array}$ & $6.17(3.1)$ & $6.67(4)$ & $\begin{array}{l}-0.5(-2.2 \text { to } \\
1.2)\end{array}$ \\
\hline Girard, $2010^{4}$ & $\begin{array}{l}>18 \text { years MV medical } \\
\text { and surgical ICU } \\
\text { patients }\end{array}$ & $\begin{array}{l}\text { Haloperidol (Planned dose: } 5 \\
\text { Median dose: } 15 \text { (10.8-17), } 35\end{array}$ & $\begin{array}{l}\text { Placebo (Planned dose: } \\
5 \mathrm{ml}), 36\end{array}$ & Oral & $\begin{array}{l}\text { Length of stay, days in } \\
\text { ICU }\end{array}$ & $10.67(8.7)$ & $8.1(6)$ & $\begin{array}{l}2.57(-0.91 \text { to } \\
6.05)\end{array}$ \\
\hline Khan, 2018 & $\begin{array}{l}\text { English speaking } \\
\text { individuals undergoing } \\
\text { thoracic surgery }\end{array}$ & $\begin{array}{l}\text { Haloperidol (Planned dose: } \\
0.5 \mathrm{mg} \text {, Planned duration: } 4 \text { days } \\
(11 \text { doses total)), } 68\end{array}$ & $\begin{array}{l}\text { Placebo (Planned dose: NR, } \\
\text { Planned duration: } 4 \text { days } \\
(11 \text { doses total)), } 67\end{array}$ & Intravenous & $\begin{array}{l}\text { ICU length of stay, } \\
\text { median, days }\end{array}$ & $\begin{array}{l}\text { Median: } 2.2 \\
\text { (IQR } 1 \text { to } 3.3 \text { ) }\end{array}$ & $\begin{array}{l}\text { Median: } 2.3 \\
\text { (IQR } 1 \text { to } 4 \text { ) }\end{array}$ & $p=0.29$ \\
\hline Page, $2013^{10}$ & $\begin{array}{l}\text { ICU patients needing } \\
\text { MV within } 72 \text { hours of } \\
\text { admission were } \\
\text { enrolled }\end{array}$ & $\begin{array}{l}\text { Haloperidol (Planned dose: } \\
2.5 \mathrm{mg}), 50\end{array}$ & $\begin{array}{l}\text { Placebo (Planned dose: } \\
0.5 \mathrm{ml}), 52\end{array}$ & Intravenous & $\begin{array}{l}\text { Length of critical care } \\
\text { stay, excluding } \\
\text { patients who died in } \\
\text { ICU }\end{array}$ & $9.5(7.1)$ & $\begin{array}{l}10.67 \\
(10.3)\end{array}$ & $\begin{array}{l}-1.17(-4.6 \text { to } \\
2.26)\end{array}$ \\
\hline $\begin{array}{l}\text { van den Boogaard, } \\
2018^{15}\end{array}$ & $\begin{array}{l}\text { ICU patients at high } \\
\text { risk of delirium }\end{array}$ & $\begin{array}{l}\text { Haloperidol (Planned dose: } 2 \mathrm{mg} \text { ), } \\
732\end{array}$ & $\begin{array}{l}\text { Placebo (Planned dose: } \\
0.9 \% \mathrm{NaCl}), 707\end{array}$ & Intravenous & $\begin{array}{l}\text { Length of stay, median } \\
\text { (IQR), days }\end{array}$ & $5.33(5.5)$ & $5(5.6)$ & $\begin{array}{l}0.33(-0.24 \text { to } \\
0.9)\end{array}$ \\
\hline $\begin{array}{l}\text { van den Boogaard, } \\
2018^{15}\end{array}$ & $\begin{array}{l}\text { ICU patients at high } \\
\text { risk of delirium }\end{array}$ & $\begin{array}{l}\text { Haloperidol (Planned dose: } 1 \mathrm{mg} \text { ), } \\
350\end{array}$ & $\begin{array}{l}\text { Placebo (Planned dose: } \\
0.9 \% \mathrm{NaCl}), 707\end{array}$ & Intravenous & $\begin{array}{l}\text { Length of stay, median } \\
\text { (IQR), days }\end{array}$ & $5(5.5)$ & $5(5.6)$ & $\begin{array}{l}0(-0.67 \text { to } \\
0.67)\end{array}$ \\
\hline Wang, $2012^{16}$ & $\begin{array}{l}\text { Patients } 65 \text { or older } \\
\text { admitted to the ICU } \\
\text { after noncardiac } \\
\text { surgery }\end{array}$ & $\begin{array}{l}\text { Haloperidol (Planned dose: } 0.5 \\
\mathrm{mg} \text { followed by continuous } \\
\text { infusion at a rate of } 1 \mathrm{~mL} / \mathrm{hr}(0.1 \\
\mathrm{mg} / \mathrm{hr} \text { haloperidol)), } 229\end{array}$ & $\begin{array}{l}\text { Placebo (Planned dose: } \\
\text { Normal saline), } 228\end{array}$ & Intravenous & $\begin{array}{l}\text { Median Length of stay } \\
\text { in ICU for all the } \\
\text { patients in the group in } \\
\text { hours }\end{array}$ & $0.89(0.06)$ & $0.96(0.14)$ & $\begin{array}{l}-0.07(-0.09 \text { to } \\
-0.05)\end{array}$ \\
\hline Wang, $2012^{16}$ & $\begin{array}{l}\text { Patients } 65 \text { or older } \\
\text { admitted to the ICU } \\
\text { after noncardiac } \\
\text { surgery }\end{array}$ & $\begin{array}{l}\text { Haloperidol (Planned dose: } 0.5 \\
\mathrm{mg} \text { followed by continuous } \\
\text { infusion at a rate of } 1 \mathrm{~mL} / \mathrm{hr}(0.1 \\
\mathrm{mg} / \mathrm{hr} \text { haloperidol)), } 35\end{array}$ & $\begin{array}{l}\text { Placebo (Planned dose: } \\
\text { Normal saline), } 53\end{array}$ & Intravenous & $\begin{array}{l}\text { Median Length of stay } \\
\text { in ICU, in patients who } \\
\text { developed post- } \\
\text { operative delirium, in } \\
\text { hours }\end{array}$ & $0.82(0.22)$ & $1.73(0.14)$ & $\begin{array}{l}-0.91(-0.99 \text { to } \\
-0.83)\end{array}$ \\
\hline
\end{tabular}




\begin{tabular}{|c|c|c|c|c|c|c|c|c|}
\hline Author, year & Population & Intervention group, $\mathbf{n}$ & Control group, $n$ & $\begin{array}{c}\text { Route of } \\
\text { administration }\end{array}$ & Outcome definition & $\begin{array}{l}\text { Mean (SD) } \\
\text { days, } \\
\text { intervention } \\
\text { group }\end{array}$ & $\begin{array}{l}\text { Mean (SD) } \\
\text { days, } \\
\text { control } \\
\text { group }\end{array}$ & $\begin{array}{l}\text { Mean } \\
\text { between- } \\
\text { group } \\
\text { difference } \\
(95 \% \mathrm{Cl}) \\
\end{array}$ \\
\hline \multicolumn{9}{|l|}{$\begin{array}{l}\text { Second-generation } \\
\text { antipsychotic vs. } \\
\text { placebo } \\
\end{array}$} \\
\hline Girard, $2010^{4}$ & $\begin{array}{l}>18 \text { years MV medical } \\
\text { and surgical ICU } \\
\text { patients }\end{array}$ & $\begin{array}{l}\text { Ziprasidone (Planned dose: } 40 \\
\text { Median dose: } 113 \text { (81-140), } 30\end{array}$ & $\begin{array}{l}\text { Placebo (Planned dose: } \\
\text { 5ml), } 36\end{array}$ & Oral & $\begin{array}{l}\text { Length of stay, days in } \\
\text { ICU }\end{array}$ & $9.3(8.4)$ & $8.1(6)$ & $\begin{array}{l}1.2(-2.32 \text { to } \\
4.72)\end{array}$ \\
\hline Hakim, $2012^{5}$ & $\begin{array}{l}\text { Patients } 65 \text { years or } \\
\text { older experiencing } \\
\text { subsyndromal delirium } \\
\text { after on-pump cardiac } \\
\text { surgery }\end{array}$ & $\begin{array}{l}\text { Risperidone (Planned dose: } \\
0.5 \mathrm{mg}), 51\end{array}$ & $\begin{array}{l}\text { Placebo (Planned dose: } \\
\text { given every } 12 \text { hours), } 50\end{array}$ & Oral & In study group & $2.33(0.8)$ & $2.67(0.8)$ & $\begin{array}{l}-0.34(-0.65 \text { to } \\
-0.03)\end{array}$ \\
\hline Prakanrattana, $2007^{11}$ & $\begin{array}{l}\text { Elective Cardiac } \\
\text { Surgery with } \\
\text { cardiopulmonary } \\
\text { bypass }\end{array}$ & $\begin{array}{l}\text { Risperidone (Planned dose: } \\
1 \mathrm{mg}), 63\end{array}$ & Placebo (Not applicable), 63 & $\begin{array}{l}\text { Orally } \\
\text { disintegrating } \\
\text { tablet }\end{array}$ & Not applicable & $3.3(2.3)$ & $3.2(1.9)$ & $\begin{array}{l}0.1(-0.64 \text { to } \\
0.84)\end{array}$ \\
\hline \multicolumn{9}{|l|}{$\begin{array}{l}\text { First-generation } \\
\text { antipsychotic vs. } \\
\text { second-generation } \\
\text { antipsychotic } \\
\end{array}$} \\
\hline Girard, $2010^{4}$ & $\begin{array}{l}>18 \text { years MV medical } \\
\text { and surgical ICU } \\
\text { patients }\end{array}$ & $\begin{array}{l}\text { Ziprasidone (Planned dose: } 40 \\
\text { Median dose: } 113 \text { (81-140), } 30\end{array}$ & $\begin{array}{l}\text { Haloperidol (Planned dose: } \\
5 \text { Median dose: } 15 \text { (10.8- } \\
17), 35\end{array}$ & Oral & $\begin{array}{l}\text { Length of stay, days in } \\
\text { ICU }\end{array}$ & $9.3(8.4)$ & $10.67(8.8)$ & $\begin{array}{l}-1.37(-5.55 \text { to } \\
2.81)\end{array}$ \\
\hline \multicolumn{9}{|l|}{$\begin{array}{l}\text { First-generation } \\
\text { antipsychotic vs. other }\end{array}$} \\
\hline Abdelgalel, $2016^{1}$ & $\begin{array}{l}\text { Adult intensive care } \\
\text { patients of ASA } \\
\text { physical status III and } \\
\text { IV aged between } 26 \\
\text { and } 70 \text { years }\end{array}$ & $\begin{array}{l}\text { Haloperidol (Planned dose: } 0.5- \\
2 \mathrm{mg} / \mathrm{h} \text { preceded by a loading } \\
\text { dose of } 2.5 \mathrm{mg} \text { intravenously over } \\
10 \mathrm{~min} \text { if needed (if RASS }>2+\text { ), } \\
30\end{array}$ & $\begin{array}{l}\text { Dexmedetomidine (Planned } \\
\text { dose: } 0.2-0.7 \mathrm{mcg} / \mathrm{kg} / \mathrm{h} \\
\text { preceded by a loading dose } \\
\text { of } 1.0 \mathrm{ug} / \mathrm{kg} \text { intravenously } \\
\text { over } 10 \mathrm{~min} \text { if needed (if } \\
\text { RASS }>2+) \text { ), } 30\end{array}$ & Intravenous & $\begin{array}{l}\text { Length of ICU stays } \\
\text { (days) }\end{array}$ & $3.1(0.4)$ & $6.5(1)$ & $\begin{array}{l}-3.4(-3.79 \text { to }- \\
3.01)\end{array}$ \\
\hline
\end{tabular}

and Sedation Scale; $\mathrm{SD}=$ standard deviation 
Evidence Table D-22. Sitter use outcomes in studies comparing interventions to prevent development of delirium

\begin{tabular}{|c|c|c|c|c|c|c|c|c|}
\hline Author, year & Population & Intervention group, $\mathbf{n}$ & Control group, $\mathbf{n}$ & $\begin{array}{c}\text { Route of } \\
\text { administration }\end{array}$ & Outcome definition & $\begin{array}{c}\mathrm{n} / \mathrm{N}(\%) \text {, intervention } \\
\text { group }\end{array}$ & $\begin{array}{c}\text { n I N (\%), control } \\
\text { group }\end{array}$ & $\begin{array}{l}\text { Relative risk (95\% } \\
\text { CI) }\end{array}$ \\
\hline \multicolumn{9}{|l|}{$\begin{array}{l}\text { Second-generation } \\
\text { antipsychotic vs. } \\
\text { placebo }\end{array}$} \\
\hline Larsen, $2010^{9}$ & $\begin{array}{l}\text { Post-operative } \\
\text { elderly joint } \\
\text { replacement } \\
\text { surgery patients }\end{array}$ & $\begin{array}{l}\text { Olanzapine (Planned } \\
\text { dose: } 5 \mathrm{mg}), 196\end{array}$ & $\begin{array}{l}\text { Placebo (Planned } \\
\text { dose: } 5 \mathrm{mg}), 204\end{array}$ & Oral & Sitter use & 9/ 196 (4.6\%) & $4 / 204(2 \%)$ & $2.34(0.73$ o 7.48$)$ \\
\hline
\end{tabular}

$\mathrm{CI}=$ confidence interval; $\mathrm{Mg}=$ =milligram; $\mathrm{N}=$ sample size 
Evidence Table D-23. Incidence of delirium outcomes in studies comparing interventions to prevent development of delirium

\begin{tabular}{|c|c|c|c|c|c|c|c|c|}
\hline Author, year & $\begin{array}{l}\text { Population } \\
\end{array}$ & Intervention group, $\mathrm{n}$ & Control group, $\mathbf{n}$ & $\begin{array}{c}\text { Route of } \\
\text { administration }\end{array}$ & $\begin{array}{l}\text { Outcome } \\
\text { definition }\end{array}$ & $\begin{array}{c}\mathrm{n} / \mathrm{N}(\%) \\
\text { intervention } \\
\text { group }\end{array}$ & $\begin{array}{c}\mathrm{n} / \mathrm{N}(\%) \\
\text { control group }\end{array}$ & $\begin{array}{l}\text { Relative risk } \\
(95 \% \mathrm{Cl})\end{array}$ \\
\hline \multicolumn{9}{|l|}{$\begin{array}{l}\text { First-generation } \\
\text { antipsychotic vs. } \\
\text { placebo }\end{array}$} \\
\hline Abdelgalel, $2016^{1}$ & $\begin{array}{l}\text { Adult intensive care patients of ASA physical } \\
\text { status III and IV aged between } 26 \text { and } 70 \\
\text { years }\end{array}$ & $\begin{array}{l}\text { Haloperidol (Planned } \\
\text { dose: } 0.5-2 \mathrm{mg} / \mathrm{h} \\
\text { preceded by a loading } \\
\text { dose of } 2.5 \mathrm{mg} \\
\text { intravenously over } 10 \\
\text { min if needed (if RASS > } \\
2+), 30\end{array}$ & $\begin{array}{l}\text { Placebo (Planned } \\
\text { dose: } 2-8 \mathrm{ml} \text {, } \\
\text { loading dose } \\
10 \mathrm{ml} \text { over } 10 \mathrm{~min} \\
\text { if needed (if } \\
\text { RASS > 2+)), } 30\end{array}$ & Intravenous & $\begin{array}{l}\text { Incidence of } \\
\text { delirium based } \\
\text { on CAM-ICU } \\
\text { threshold }\end{array}$ & $10 / 30(33.3 \%)$ & $13 / 30(43.3 \%)$ & $\begin{array}{l}0.77(0.40 \text { to } \\
1.47)\end{array}$ \\
\hline Al-Qadheeb, $2016^{2}$ & MV patients with subsyndromal delirium & $\begin{array}{l}\text { Haloperidol (Planned } \\
\text { dose: } 1 \mathrm{mg} \text { ), } 34\end{array}$ & $\begin{array}{l}\text { Placebo (Planned } \\
\text { dose: } 5 \% \\
\text { dextroseml), } 34\end{array}$ & Intravenous & $\begin{array}{l}\text { During study } \\
\text { drug } \\
\text { administration }\end{array}$ & $12 / 34(35.3 \%)$ & $8 / 34(23.5 \%)$ & $\begin{array}{l}1.50(0.70 \text { to } \\
3.20)\end{array}$ \\
\hline Al-Qadheeb, $2016^{2}$ & MV patients with subsyndromal delirium & $\begin{array}{l}\text { Haloperidol (Planned } \\
\text { dose: } 1 \mathrm{mg} \text { ), } 34\end{array}$ & $\begin{array}{l}\text { Placebo (Planned } \\
\text { dose: } 5 \% \\
\text { dextroseml), } 34\end{array}$ & Intravenous & $\begin{array}{l}\text { During the ICU } \\
\text { admission }\end{array}$ & $12 / 34(35.3 \%)$ & $9 / 34(26.5 \%)$ & $\begin{array}{l}1.33(0.65 \text { to } \\
2.74)\end{array}$ \\
\hline Fukata, $2014^{3}$ & $\begin{array}{l}\text { Delirium after abdominal or orthopedic } \\
\text { surgery in elderly patients }\end{array}$ & $\begin{array}{l}\text { Haloperidol (Planned } \\
\text { dose: } 2.5 \mathrm{mg} \text { ), } 59\end{array}$ & Placebo (), 60 & Intravenous & $\begin{array}{l}\text { Patients were } \\
\text { limited to those } \\
\text { with a } \\
\text { preoperative } \\
\text { MMSE <25 }\end{array}$ & $31 / 59(52.8 \%)$ & $\begin{array}{l}26 / 60 \\
(43.52 \%)\end{array}$ & $\begin{array}{l}1.21(0.83 \text { to } \\
1.77)\end{array}$ \\
\hline Fukata, $2014^{3}$ & $\begin{array}{l}\text { Delirium after abdominal or orthopedic } \\
\text { surgery in elderly patients }\end{array}$ & $\begin{array}{l}\text { Haloperidol (Planned } \\
\text { dose: } 2.5 \mathrm{mg} \text { ), } 59\end{array}$ & Placebo (), 60 & Intravenous & $\begin{array}{l}\text { NEECHAM } \\
\text { score }<20\end{array}$ & 25 / 59 (42.4\%) & $20 / 60(33.3 \%)$ & $\begin{array}{l}1.27(0.80 \text { to } \\
2.02)\end{array}$ \\
\hline
\end{tabular}




\begin{tabular}{|c|c|c|c|c|c|c|c|c|}
\hline Author, year & Population & Intervention group, $\mathbf{n}$ & Control group, $\mathrm{n}$ & $\begin{array}{c}\text { Route of } \\
\text { administration }\end{array}$ & Outcome definition & $\begin{array}{c}n / N(\%) \\
\text { intervention } \\
\text { group }\end{array}$ & $\begin{array}{c}n / N(\%), \\
\text { control group }\end{array}$ & $\begin{array}{l}\text { Relative risk } \\
(95 \% \mathrm{Cl})\end{array}$ \\
\hline Kalisvaart, $2005^{6}$ & $\begin{array}{l}\text { Acute or elective hip surgery patients } \\
\geq 70 \text { years is at risk of delirium: } \\
\text { MMSE between less than or equal to } \\
24 ; \text { dehydration=BUN/creatinine }>\text { or }= \\
\text { to } 18 \text {, low visual acuity and/or } \\
\text { increased severity of illness on } \\
\text { APACHE II of } 16 \text { or greater. }\end{array}$ & $\begin{array}{l}\text { Haloperidol (Planned dose: } \\
0.5 \mathrm{mg}), 212\end{array}$ & $\begin{array}{l}\text { Placebo (Not } \\
\text { applicable), } 218\end{array}$ & Oral & Not applicable & $\begin{array}{l}32 / 212 \\
(15.1 \%)\end{array}$ & $\begin{array}{l}36 / 218 \\
(16.5 \%)\end{array}$ & $\begin{array}{l}0.91(0.59 \text { to } \\
1.42)\end{array}$ \\
\hline Kaneko, $1999^{7}$ & $\begin{array}{l}\text { Scheduled for elective gastrointestinal } \\
\text { surgery and admitted to ICU }\end{array}$ & $\begin{array}{l}\text { Haloperidol (Planned dose: } \\
\text { 5mg), } 38\end{array}$ & $\begin{array}{l}\text { Placebo (Planned } \\
\text { dose: } 1 \mathrm{ml}(0.9 \% \\
\text { normal saline)ml), } \\
40\end{array}$ & Intravenous & $\begin{array}{l}\text { based on DSM } \\
\text { criteria (not } \\
\text { specified) }\end{array}$ & $4 / 38(\%)$ & $13 / 40(\%)$ & $\begin{array}{l}0.32(0.12 \text { to } \\
0.91)\end{array}$ \\
\hline Khan, $2018^{8}$ & $\begin{array}{l}\text { English speaking individuals } \\
\text { undergoing thoracic surgery }\end{array}$ & $\begin{array}{l}\text { Haloperidol (Planned dose: } \\
0.5 \mathrm{mg} \text {, Planned duration: } 4 \\
\text { days (11 doses total)), } 68\end{array}$ & $\begin{array}{l}\text { Placebo (Planned } \\
\text { dose: NR, } \\
\text { Planned duration: } \\
4 \text { days (11 doses } \\
\text { total)), } 67\end{array}$ & Intravenous & $\begin{array}{l}\text { Incidence of delirium, } \\
\text { based on CAM-ICU }\end{array}$ & $\begin{array}{l}15 / 68 \\
(22.1 \%)\end{array}$ & $19 / 67(28.4 \%)$ & $\begin{array}{l}0.78(0.43 \text { to } \\
1.40)\end{array}$ \\
\hline Schrijver, $2018^{12}$ & Medical and surgical patients & $\begin{array}{l}\text { Haloperidol (Planned dose: } \\
1 \mathrm{mg}), 118\end{array}$ & Placebo, 124 & Oral & $\begin{array}{l}\text { DOSS score } \geq 3, \\
\text { confirmed by DSM-IV } \\
\text { criteria }\end{array}$ & $\begin{array}{l}23 / 118 \\
(19.5 \%)\end{array}$ & $\begin{array}{l}18 / 124 \\
(14.5 \%)\end{array}$ & $\begin{array}{l}1.34(0.76 \text { to } \\
2.36)\end{array}$ \\
\hline $\begin{array}{l}\text { van den Boogaard, } \\
2018^{15}\end{array}$ & ICU patients at high risk of delirium & $\begin{array}{l}\text { Haloperidol (Planned dose: } \\
2 \mathrm{mg}), 732\end{array}$ & $\begin{array}{l}\text { Placebo (Planned } \\
\text { dose: } 0.9 \% \\
\text { NaCl), } 707\end{array}$ & Intravenous & $\begin{array}{l}\text { Number of patients } \\
\text { who scored positive } \\
\text { at-least once for } \\
\text { CAM-ICU or ICDSC } \\
\text { during } 28 \text { days } \\
\text { assessment }\end{array}$ & $\begin{array}{l}244 / 732 \\
(33.3 \%)\end{array}$ & $\begin{array}{l}233 / 707 \\
(33 \%)\end{array}$ & $\begin{array}{l}1.01(0.87 \text { to } \\
1.17)\end{array}$ \\
\hline $\begin{array}{l}\text { van den Boogaard, } \\
2018^{15}\end{array}$ & ICU patients at high risk of delirium & $\begin{array}{l}\text { Haloperidol (Planned dose: } \\
1 \mathrm{mg}), 350\end{array}$ & $\begin{array}{l}\text { Placebo (Planned } \\
\text { dose: } 0.9 \% \\
\text { NaCl), } 707\end{array}$ & Intravenous & $\begin{array}{l}\text { Number of patients } \\
\text { who scored positive } \\
\text { at-least once for } \\
\text { CAM-ICU or ICDSC } \\
\text { during } 28 \text { days } \\
\text { assessment }\end{array}$ & $\begin{array}{l}139 / 350 \\
(39.7 \%)\end{array}$ & $\begin{array}{l}233 / 707 \\
(33 \%)\end{array}$ & $\begin{array}{l}1.21(1.02 \text { to } \\
1.42)\end{array}$ \\
\hline Wang, $2012^{16}$ & $\begin{array}{l}\text { Patients } 65 \text { or older admitted to the } \\
\text { ICU after noncardiac surgery }\end{array}$ & $\begin{array}{l}\text { Haloperidol (Planned dose: } 0.5 \\
\text { mg followed by continuous } \\
\text { infusion at a rate of } 1 \mathrm{~mL} / \mathrm{hr} \\
(0.1 \mathrm{mg} / \mathrm{hr} \text { haloperidol)), } 229\end{array}$ & $\begin{array}{l}\text { Placebo (Planned } \\
\text { dose: Normal } \\
\text { saline), } 228\end{array}$ & Intravenous & $\begin{array}{l}\text { Occurrence of } \\
\text { Delirium, based on } \\
\text { CAM-ICU }\end{array}$ & $\begin{array}{l}35 / 229 \\
(15.3 \%)\end{array}$ & $\begin{array}{l}53 / 228 \\
(23.2 \%)\end{array}$ & $\begin{array}{l}0.66(0.45 \text { to } \\
0.97)\end{array}$ \\
\hline
\end{tabular}




\begin{tabular}{|c|c|c|c|c|c|c|c|c|}
\hline Author, year & Population & Intervention group, $\mathbf{n}$ & Control group, $n$ & $\begin{array}{c}\text { Route of } \\
\text { administration }\end{array}$ & Outcome definition & $\begin{array}{l}\mathrm{n} / \mathrm{N}(\%) \\
\text { intervention } \\
\text { group }\end{array}$ & $\begin{array}{c}\mathrm{n} / \mathrm{N} \text { (\%), } \\
\text { control group }\end{array}$ & $\begin{array}{l}\text { Relative risk } \\
\quad(95 \% \mathrm{Cl})\end{array}$ \\
\hline \multicolumn{9}{|l|}{$\begin{array}{l}\text { Second-generation } \\
\text { antipsychotic vs. } \\
\text { placebo }\end{array}$} \\
\hline Hakim, $2012^{5}$ & $\begin{array}{l}\text { Patients } 65 \text { years or older } \\
\text { experiencing subsyndromal delirium } \\
\text { after on-pump cardiac surgery }\end{array}$ & $\begin{array}{l}\text { Risperidone (Planned dose: } \\
0.5 \mathrm{mg} \text { ), } 51\end{array}$ & $\begin{array}{l}\text { Placebo (Planned } \\
\text { dose: given every } \\
12 \text { hours), } 50\end{array}$ & Oral & DSM criteria & $\begin{array}{l}7 / 51 \\
(13.7 \%)\end{array}$ & 17 / 50 (34\%) & $\begin{array}{l}0.40(0.18 \text { to } \\
0.89)\end{array}$ \\
\hline Hakim, $2012^{5}$ & $\begin{array}{l}\text { Patients } 65 \text { years or older } \\
\text { experiencing subsyndromal delirium } \\
\text { after on-pump cardiac surgery }\end{array}$ & $\begin{array}{l}\text { Risperidone (Planned dose: } \\
0.5 \mathrm{mg} \text { ), }\end{array}$ & $\begin{array}{l}\text { Placebo (Planned } \\
\text { dose: given every } \\
12 \text { hours), }\end{array}$ & Oral & $\begin{array}{l}\text { Possible delirium: } \\
\text { ICDSC } \geq 4\end{array}$ & $\begin{array}{l}8 / 51 \\
(15.7 \%)\end{array}$ & $19 / 50(38 \%)$ & $\begin{array}{l}0.41(0.20 \text { to } \\
0.86)\end{array}$ \\
\hline Larsen, $2010^{9}$ & $\begin{array}{l}\text { Post-operative elderly joint } \\
\text { replacement surgery patients }\end{array}$ & $\begin{array}{l}\text { Olanzapine (Planned dose: } \\
5 \mathrm{mg}), 196\end{array}$ & $\begin{array}{l}\text { Placebo (Planned } \\
\text { dose: } 5 \mathrm{mg} \text { ), } 204\end{array}$ & Oral & DSM-IIIR & $\begin{array}{l}28 / 196 \\
(14.3 \%)\end{array}$ & $\begin{array}{l}82 / 204 \\
(40.2 \%)\end{array}$ & $\begin{array}{l}0.36(0.24 \text { to } \\
0.52)\end{array}$ \\
\hline $\begin{array}{l}\text { Prakanrattana, } \\
2007^{11}\end{array}$ & $\begin{array}{l}\text { Elective Cardiac Surgery with } \\
\text { cardiopulmonary bypass }\end{array}$ & $\begin{array}{l}\text { Risperidone (Planned dose: } \\
\text { 1mg), } 63\end{array}$ & $\begin{array}{l}\text { Placebo (Not } \\
\text { applicable), } 63\end{array}$ & $\begin{array}{l}\text { Orally } \\
\text { disintegrating } \\
\text { tablet }\end{array}$ & $\begin{array}{l}\text { The presence of } \\
\text { features } 1 \text { and } 2 \text { and } \\
\text { either feature } 3 \text { or } 4 \text {, } \\
\text { of CAM-ICU }\end{array}$ & $3 / 63(\%)$ & 15 / $63(\%)$ & $\begin{array}{l}0.20(0.06 \text { to } \\
0.66)\end{array}$ \\
\hline $\begin{array}{l}\text { Prakanrattana, } \\
2007^{11}\end{array}$ & $\begin{array}{l}\text { Elective Cardiac Surgery with } \\
\text { cardiopulmonary bypass }\end{array}$ & $\begin{array}{l}\text { Risperidone (Planned dose: } \\
\text { 1mg), } 63\end{array}$ & $\begin{array}{l}\text { Placebo (Not } \\
\text { applicable), } 63\end{array}$ & $\begin{array}{l}\text { Orally } \\
\text { disintegrating } \\
\text { tablet }\end{array}$ & $\begin{array}{l}\text { The presence of } \\
\text { features } 1 \text { and } 2 \text { and } \\
\text { either feature } 3 \text { or } 4 \text {, } \\
\text { of CAM-ICU }\end{array}$ & $\begin{array}{l}7 / 63 \\
(11.1 \%)\end{array}$ & $20 / 63(31.7 \%)$ & $\begin{array}{l}0.35(0.16 \text { to } \\
0.77)\end{array}$ \\
\hline
\end{tabular}




\begin{tabular}{|c|c|c|c|c|c|c|c|c|}
\hline Author, year & Population & Intervention group, $\mathbf{n}$ & Control group, $\mathrm{n}$ & $\begin{array}{c}\text { Route of } \\
\text { administration }\end{array}$ & $\begin{array}{l}\text { Outcome } \\
\text { definition }\end{array}$ & $\begin{array}{l}\mathrm{n} / \mathrm{N}(\%) \\
\text { intervention } \\
\text { group }\end{array}$ & $\begin{array}{c}\mathrm{n} / \mathrm{N} \text { (\%), } \\
\text { control group }\end{array}$ & $\begin{array}{c}\text { Relative risk } \\
(95 \% \mathrm{Cl})\end{array}$ \\
\hline \multicolumn{9}{|l|}{$\begin{array}{l}\text { First-generation } \\
\text { antipsychotic vs. } \\
\text { other }\end{array}$} \\
\hline Abdelgalel, $2016^{1}$ & $\begin{array}{l}\text { Adult intensive care patients of ASA } \\
\text { physical status III and IV aged } \\
\text { between } 26 \text { and } 70 \text { years }\end{array}$ & $\begin{array}{l}\text { Haloperidol (Planned dose: } \\
0.5-2 \mathrm{mg} / \mathrm{h} \text { preceded by a } \\
\text { loading dose of } 2.5 \mathrm{mg} \\
\text { intravenously over } 10 \mathrm{~min} \text { if } \\
\text { needed (if RASS }>2+\text { ), } 30\end{array}$ & $\begin{array}{l}\text { Dexmedetomidine } \\
\text { (Planned dose: } \\
0.2-0.7 \mathrm{mcg} / \mathrm{kg} / \mathrm{h} \\
\text { preceded by a } \\
\text { loading dose of } \\
1.0 \mathrm{ug} / \mathrm{kg} \\
\text { intravenously } \\
\text { over } 10 \mathrm{~min} \text { if } \\
\text { needed (if RASS } \\
>2+) \text { ), } 30\end{array}$ & Intravenous & $\begin{array}{l}\text { incidence of delirium } \\
\text { based on CAM-ICU } \\
\text { threshold }\end{array}$ & $\begin{array}{l}10 / 30 \\
(33.3 \%)\end{array}$ & $3 / 30(10 \%)$ & $\begin{array}{l}3.33(1.02 \text { to } \\
10.92)\end{array}$ \\
\hline
\end{tabular}

APACHE II= Acute Physiology and Chronic Health Evaluation 2; BUN=blood urea nitrogen ratio; CAM-ICU=Confusion Assessment Method for the ICU; CI=confidence interval; DSM=Diagnostic and Statistical Manual of Mental Disorders; ICDSC=Intensive Care Delirium Screening Checklist; ICU=intensive care unit; ICU=intensive care unit; Mg/hr=milligram per hour; Ml/hr=milliliter per hour; MMSE=Mini-Mental State Examination; MV=mechanical ventilation; NaCl=sodium chloride; NEECHAM=Neelon and Champagne Confusion Scale; RASS=Richmond Agitation and Sedation Scale; SD=standard deviation; Ug/kg/h=microgram per kilogram per hour 


\begin{tabular}{|c|c|c|c|c|c|c|c|c|}
\hline Author, year & Population & $\begin{array}{c}\text { Intervention } \\
\text { group, } \mathbf{n}\end{array}$ & Control group, $n$ & $\begin{array}{c}\text { Route of } \\
\text { administration }\end{array}$ & $\begin{array}{l}\text { Outcome } \\
\text { definition }\end{array}$ & $\begin{array}{c}\text { n / N (\%), intervention } \\
\text { group }\end{array}$ & $\begin{array}{c}\text { n I N (\%), control } \\
\text { group }\end{array}$ & $\begin{array}{l}\text { Relative risk (95\% } \\
\text { Cl) }\end{array}$ \\
\hline \multicolumn{9}{|l|}{$\begin{array}{l}\text { First-generation } \\
\text { antipsychotic } \\
\text { vs. placebo }\end{array}$} \\
\hline $\begin{array}{l}\text { Al-Qadheeb, } \\
2016^{2}\end{array}$ & $\begin{array}{l}\text { MV patients with } \\
\text { subsyndromal delirium }\end{array}$ & $\begin{array}{l}\text { Haloperidol } \\
\text { (Planned dose: } \\
\text { 1mg), } 34\end{array}$ & $\begin{array}{l}\text { Placebo (Planned } \\
\text { dose: } 5 \% \\
\text { dextroseml), } 34\end{array}$ & Intravenous & Excessive sedation & $1 / 34(2.9 \%)$ & $0 / 34(0 \%)$ & 3.00 (0.13 to 71.15$)$ \\
\hline $\begin{array}{l}\text { Kalisvaart, } \\
2005^{6}\end{array}$ & $\begin{array}{l}\text { Acute or elective hip surgery } \\
\text { patients } \geq 70 \text { years is at risk } \\
\text { of delirium: MMSE between } \\
\text { less than or equal to } 24 ; \\
\text { dehydration=BUN/creatinine } \\
>\text { or = to } 18 \text {, low visual acuity } \\
\text { and/or increased severity of } \\
\text { illness on APACHE II of } 16 \text { or } \\
\text { greater. }\end{array}$ & $\begin{array}{l}\text { Haloperidol } \\
\text { (Planned dose: } \\
0.5 \mathrm{mg}), 212\end{array}$ & $\begin{array}{l}\text { Placebo (Not } \\
\text { applicable), } 218\end{array}$ & Oral & Sedation & $0 / 212(0 \%)$ & $0 / 218(0 \%)$ & \\
\hline Page, $2013^{10}$ & $\begin{array}{l}\text { ICU patients needing MV } \\
\text { within } 72 \text { hours of admission } \\
\text { were enrolled }\end{array}$ & $\begin{array}{l}\text { Haloperidol } \\
\text { (Planned dose: } \\
2.5 \mathrm{mg}), 71\end{array}$ & $\begin{array}{l}\text { Placebo (Planned } \\
\text { dose: } 0.5 \mathrm{ml}), 70\end{array}$ & Intravenous & Oversedation & $11 / 71(15 \%)$ & $6 / 70(9 \%)$ & 1.81 (0.71 to 4.62$)$ \\
\hline $\begin{array}{l}\text { Schrijver, } \\
2018^{12}\end{array}$ & Medical and surgical patients & $\begin{array}{l}\text { Haloperidol } \\
\text { (Planned dose: } \\
\text { 1mg), } 118\end{array}$ & Placebo, 124 & Oral & Somnolence & $2 / 118$ (1.7\%) & $0 / 124(0 \%)$ & $\begin{array}{l}5.25(0.25 \text { to } \\
108.27)\end{array}$ \\
\hline
\end{tabular}

APACHE II= Acute Physiology and Chronic Health Evaluation 2; BUN=blood urea nitrogen ratio; CI=confidence interval; ICU=intensive care unit; Mg=milligram; MMSE=Mini-Mental State Examination; MV=mechanical ventilation; $\mathrm{N}=$ sample size 
Evidence Table D-25. Binary cardiac outcomes in studies comparing interventions to prevent development of delirium

\begin{tabular}{|c|c|c|c|c|c|c|c|c|}
\hline Author, year & Population & Intervention group, $\mathbf{n}$ & Control group, $n$ & $\begin{array}{c}\text { Route of } \\
\text { administration }\end{array}$ & Outcome definition & $\begin{array}{c}\mathrm{n} / \mathrm{N}(\%) \\
\text { intervention } \\
\text { group }\end{array}$ & $\begin{array}{l}\mathrm{n} / \mathrm{N}(\%) \\
\text { control } \\
\text { group }\end{array}$ & $\begin{array}{c}\text { Relative risk } \\
(95 \% \mathrm{Cl})\end{array}$ \\
\hline \multicolumn{9}{|l|}{$\begin{array}{l}\text { First-generation } \\
\text { antipsychotic vs. } \\
\text { placebo }\end{array}$} \\
\hline Abdelgalel, $2016^{1}$ & $\begin{array}{l}\text { Adult intensive care } \\
\text { patients of ASA } \\
\text { physical status III and } \\
\text { IV aged between } 26 \\
\text { and } 70 \text { years }\end{array}$ & $\begin{array}{l}\text { Haloperidol (Planned dose: } 0.5-2 \\
\mathrm{mg} / \mathrm{h} \text { preceded by a loading dose } \\
\text { of } 2.5 \mathrm{mg} \text { intravenously over } 10 \\
\text { min if needed (if RASS }>2+\text { ), } 30\end{array}$ & $\begin{array}{l}\text { Placebo (Planned dose: } 2-8 \mathrm{ml} \text {, loading } \\
\text { dose } 10 \mathrm{ml} \text { over } 10 \mathrm{~min} \text { if needed (if } \\
\text { RASS > 2+)), } 30\end{array}$ & Intravenous & Arrhythmia & $3 / 30(10 \%)$ & $2 / 30(7 \%)$ & $\begin{array}{l}1.50(0.27 \text { to } \\
8.34)\end{array}$ \\
\hline Abdelgalel, $2016^{1}$ & $\begin{array}{l}\text { Adult intensive care } \\
\text { patients of ASA } \\
\text { physical status III and } \\
\text { IV aged between } 26 \\
\text { and } 70 \text { years }\end{array}$ & $\begin{array}{l}\text { Haloperidol (Planned dose: } 0.5-2 \\
\mathrm{mg} / \mathrm{h} \text { preceded by a loading dose } \\
\text { of } 2.5 \mathrm{mg} \text { intravenously over } 10 \\
\mathrm{~min} \text { if needed (if RASS }>2+\text { ), } 30\end{array}$ & $\begin{array}{l}\text { Placebo (Planned dose: } 2-8 \mathrm{ml} \text {, loading } \\
\text { dose } 10 \mathrm{ml} \text { over } 10 \mathrm{~min} \text { if needed (if } \\
\text { RASS > 2+)), } 30\end{array}$ & Intravenous & $\begin{array}{l}\text { Bradycardia (Heart } \\
\text { rate decreased to } \leq 60 \\
\text { beats } / \text { min) }\end{array}$ & $2 / 30(7 \%)$ & $1 / 30(3 \%)$ & $\begin{array}{l}2.00(0.19 \text { to } \\
20.90)\end{array}$ \\
\hline Abdelgalel, $2016^{1}$ & $\begin{array}{l}\text { Adult intensive care } \\
\text { patients of ASA } \\
\text { physical status III and } \\
\text { IV aged between } 26 \\
\text { and } 70 \text { years }\end{array}$ & $\begin{array}{l}\text { Haloperidol (Planned dose: } 0.5-2 \\
\mathrm{mg} / \mathrm{h} \text { preceded by a loading dose } \\
\text { of } 2.5 \mathrm{mg} \text { intravenously over } 10 \\
\mathrm{~min} \text { if needed (if RASS }>2+\text { ), } 30\end{array}$ & $\begin{array}{l}\text { Placebo (Planned dose: } 2-8 \mathrm{ml} \text {, loading } \\
\text { dose } 10 \mathrm{ml} \text { over } 10 \mathrm{~min} \text { if needed (if } \\
\text { RASS > 2+)), } 30\end{array}$ & Intravenous & Hypotension & $3 / 30(10 \%)$ & $\begin{array}{l}3 / 30 \\
(10 \%)\end{array}$ & $\begin{array}{l}1.00(0.22 \text { to } \\
4.56)\end{array}$ \\
\hline Abdelgalel, $2016^{1}$ & $\begin{array}{l}\text { Adult intensive care } \\
\text { patients of ASA } \\
\text { physical status III and } \\
\text { IV aged between } 26 \\
\text { and } 70 \text { years }\end{array}$ & $\begin{array}{l}\text { Haloperidol (Planned dose: } 0.5-2 \\
\mathrm{mg} / \mathrm{h} \text { preceded by a loading dose } \\
\text { of } 2.5 \mathrm{mg} \text { intravenously over } 10 \\
\mathrm{~min} \text { if needed (if RASS }>2+\text { ), } 30\end{array}$ & $\begin{array}{l}\text { Placebo (Planned dose: } 2-8 \mathrm{ml} \text {, loading } \\
\text { dose } 10 \mathrm{ml} \text { over } 10 \mathrm{~min} \text { if needed (if } \\
\text { RASS > 2+)), } 30\end{array}$ & Intravenous & $\begin{array}{l}\text { Prolongation in the } \\
\text { QTc interval }>500 \mathrm{~ms}\end{array}$ & $2 / 30(7 \%)$ & $0 / 30(0 \%)$ & $\begin{array}{l}5.00(0.25 \text { to } \\
99.95)\end{array}$ \\
\hline Al-Qadheeb, $2016^{2}$ & $\begin{array}{l}\text { MV patients with } \\
\text { subsyndromal delirium }\end{array}$ & $\begin{array}{l}\text { Haloperidol (Planned dose: } 1 \mathrm{mg} \text { ), } \\
34\end{array}$ & $\begin{array}{l}\text { Placebo (Planned dose: } 5 \% \\
\text { dextroseml), } 34\end{array}$ & Intravenous & Hypotension & $1 / 34(2.9 \%)$ & $\begin{array}{l}1 / 34 \\
(2.9 \%)\end{array}$ & $\begin{array}{l}1.00(0.07 \text { to } \\
15.34)\end{array}$ \\
\hline Al-Qadheeb, $2016^{2}$ & $\begin{array}{l}\text { MV patients with } \\
\text { subsyndromal delirium }\end{array}$ & $\begin{array}{l}\text { Haloperidol (Planned dose: } 1 \mathrm{mg} \text { ), } \\
34\end{array}$ & $\begin{array}{l}\text { Placebo (Planned dose: } 5 \% \\
\text { dextroseml), } 34\end{array}$ & Intravenous & $\begin{array}{l}\text { Prolongation in the } \\
\text { QTc interval }\end{array}$ & $4 / 34(11.8 \%)$ & $\begin{array}{l}1 / 34 \\
(2.9 \%)\end{array}$ & $\begin{array}{l}4.00(0.47 \text { to } \\
33.97)\end{array}$ \\
\hline Girard, $2010^{4}$ & $\begin{array}{l}>18 \text { years MV medical } \\
\text { and surgical ICU } \\
\text { patients }\end{array}$ & $\begin{array}{l}\text { Haloperidol (Planned dose: } 5 \\
\text { Median dose: } 15 \text { (10.8-17), } 35\end{array}$ & Placebo (Planned dose: $5 \mathrm{ml}$ ), 36 & Oral & $\begin{array}{l}\text { Prolongation in the } \\
\text { QTc interval } \\
\text { prolongation of the } \\
\text { QTc }>500 \text { ms while } \\
\text { receiving study drug }\end{array}$ & $2 / 35(5.7 \%)$ & $\begin{array}{l}3 / 36 \\
(8.3 \%)\end{array}$ & $\begin{array}{l}0.69(0.12 \text { to } \\
3.86)\end{array}$ \\
\hline Girard, $2010^{4}$ & $\begin{array}{l}>18 \text { years MV medical } \\
\text { and surgical ICU } \\
\text { patients }\end{array}$ & $\begin{array}{l}\text { Haloperidol (Planned dose: } 5 \\
\text { Median dose: } 15 \text { (10.8-17), } 35\end{array}$ & Placebo (Planned dose: $5 \mathrm{ml}$ ), 36 & Oral & $\begin{array}{l}\text { Ventricular } \\
\text { arrhythmias }\end{array}$ & $0 / 35(\%)$ & $0 / 36(\%)$ & \\
\hline
\end{tabular}




\begin{tabular}{|c|c|c|c|c|c|c|c|c|}
\hline Author, year & Population & Intervention group, $\mathbf{n}$ & Control group, $\mathbf{n}$ & $\begin{array}{c}\text { Route of } \\
\text { administration }\end{array}$ & Outcome definition & $\begin{array}{c}\mathrm{n} / \mathrm{N}(\%) \\
\text { intervention } \\
\text { group }\end{array}$ & $\begin{array}{c}\text { n I N (\%), } \\
\text { control } \\
\text { group }\end{array}$ & $\begin{array}{l}\text { Relative risk } \\
(95 \% \mathrm{Cl})\end{array}$ \\
\hline Khan, $2018^{8}$ & $\begin{array}{l}\text { English speaking } \\
\text { individuals undergoing } \\
\text { thoracic surgery }\end{array}$ & $\begin{array}{l}\text { Haloperidol (Planned dose: } \\
0.5 \mathrm{mg} \text {, Planned duration: } 4 \text { days } \\
\text { (11 doses total)), } 68\end{array}$ & $\begin{array}{l}\text { Placebo (Planned dose: NR, Planned } \\
\text { duration: } 4 \text { days ( } 11 \text { doses total)), } 67\end{array}$ & Intravenous & Arrythmia & $9 / 68(13.2 \%)$ & $\begin{array}{l}10 / 67 \\
(14.9 \%)\end{array}$ & $\begin{array}{l}0.89(0.38 \text { to } \\
2.04)\end{array}$ \\
\hline Khan, $2018^{8}$ & $\begin{array}{l}\text { English speaking } \\
\text { individuals undergoing } \\
\text { thoracic surgery }\end{array}$ & $\begin{array}{l}\text { Haloperidol (Planned dose: } \\
0.5 \mathrm{mg} \text {, Planned duration: } 4 \text { days } \\
\text { (11 doses total)), } 68\end{array}$ & $\begin{array}{l}\text { Placebo (Planned dose: NR, Planned } \\
\text { duration: } 4 \text { days ( } 11 \text { doses total)), } 67\end{array}$ & Intravenous & QT prolongation & $3 / 68(4.4 \%)$ & $4 / 67(6 \%)$ & $\begin{array}{l}0.74 \text { (0.17 to } \\
3.18)\end{array}$ \\
\hline Khan, $2018^{8}$ & $\begin{array}{l}\text { English speaking } \\
\text { individuals undergoing } \\
\text { thoracic surgery }\end{array}$ & $\begin{array}{l}\text { Haloperidol (Planned dose: } \\
0.5 \mathrm{mg} \text {, Planned duration: } 4 \text { days } \\
\text { (11 doses total)), } 68\end{array}$ & $\begin{array}{l}\text { Placebo (Planned dose: NR, Planned } \\
\text { duration: } 4 \text { days (11 doses total)), } 67\end{array}$ & Intravenous & $\begin{array}{l}\text { Deep venous } \\
\text { thrombosis }\end{array}$ & $1 / 68(1.5 \%)$ & $\begin{array}{l}5 / 67 \\
(7.5 \%)\end{array}$ & $\begin{array}{l}0.2(0.02 \text { to } \\
1.64)\end{array}$ \\
\hline Page, $2013^{10}$ & $\begin{array}{l}\text { ICU patients needing } \\
\text { MV within } 72 \text { hours of } \\
\text { admission were } \\
\text { enrolled }\end{array}$ & $\begin{array}{l}\text { Haloperidol (Planned dose: } \\
2.5 \mathrm{mg}), 71\end{array}$ & Placebo (Planned dose: $0.5 \mathrm{ml}), 70$ & Intravenous & Atrial fibrillation & $7 / 71(10 \%)$ & $3 / 70(4 \%)$ & $\begin{array}{l}2.30(0.62 \text { to } \\
8.54)\end{array}$ \\
\hline Page, $2013^{10}$ & $\begin{array}{l}\text { ICU patients needing } \\
\text { MV within } 72 \text { hours of } \\
\text { admission were } \\
\text { enrolled }\end{array}$ & $\begin{array}{l}\text { Haloperidol (Planned dose: } \\
2.5 \mathrm{mg}), 71\end{array}$ & Placebo (Planned dose: $0.5 \mathrm{ml}), 70$ & Intravenous & Bradycardia & $2 / 71(3 \%)$ & $0 / 70(\%)$ & $\begin{array}{l}4.93(0.24 \text { to } \\
100.89)\end{array}$ \\
\hline Page, $2013^{10}$ & $\begin{array}{l}\text { ICU patients needing } \\
\text { MV within } 72 \text { hours of } \\
\text { admission were } \\
\text { enrolled }\end{array}$ & $\begin{array}{l}\text { Haloperidol (Planned dose: } \\
2.5 \mathrm{mg}), 71\end{array}$ & Placebo (Planned dose: $0.5 \mathrm{ml}), 70$ & Intravenous & Hypotension & $3 / 71(4 \%)$ & $2 / 70(3 \%)$ & $\begin{array}{l}1.48(0.25 \text { to } \\
8.58)\end{array}$ \\
\hline Page, $2013^{10}$ & $\begin{array}{l}\text { ICU patients needing } \\
\text { MV within } 72 \text { hours of } \\
\text { admission were } \\
\text { enrolled }\end{array}$ & $\begin{array}{l}\text { Haloperidol (Planned dose: } \\
2.5 \mathrm{mg}), 71\end{array}$ & Placebo (Planned dose: $0.5 \mathrm{ml}), 70$ & Intravenous & $\begin{array}{l}\text { Prolongation in the } \\
\text { QTc interval }>500 \mathrm{~ms}\end{array}$ & $7 / 71(10 \%)$ & $6 / 70(9 \%)$ & $\begin{array}{l}1.15(0.41 \text { to } \\
3.25)\end{array}$ \\
\hline Page, $2013^{10}$ & $\begin{array}{l}\text { ICU patients needing } \\
\text { MV within } 72 \text { hours of } \\
\text { admission were } \\
\text { enrolled }\end{array}$ & $\begin{array}{l}\text { Haloperidol (Planned dose: } \\
2.5 \mathrm{mg}), 71\end{array}$ & Placebo (Planned dose: $0.5 \mathrm{ml}), 70$ & Intravenous & $\begin{array}{l}\text { Supraventricular } \\
\text { Tachycardia }\end{array}$ & $4 / 71(6 \%)$ & $1 / 70(1 \%)$ & $\begin{array}{l}3.94(0.45 \text { to } \\
34.41)\end{array}$ \\
\hline
\end{tabular}




\begin{tabular}{|c|c|c|c|c|c|c|c|c|}
\hline Author, year & Population & Intervention group, $\mathbf{n}$ & Control group, $\mathbf{n}$ & $\begin{array}{c}\text { Route of } \\
\text { administration }\end{array}$ & Outcome definition & $\begin{array}{c}\mathrm{n} / \mathrm{N}(\%) \\
\text { intervention } \\
\text { group }\end{array}$ & $\begin{array}{c}\mathrm{n} / \mathrm{N}(\%) \\
\text { control } \\
\text { group }\end{array}$ & $\begin{array}{l}\text { Relative risk } \\
\quad(95 \% \mathrm{Cl})\end{array}$ \\
\hline Schrijver, $2018^{12}$ & $\begin{array}{l}\text { Medical and surgical } \\
\text { patients }\end{array}$ & $\begin{array}{l}\text { Haloperidol (Planned dose: } 1 \mathrm{mg} \text { ), } \\
118\end{array}$ & Placebo, 124 & Oral & $\begin{array}{l}\text { Acute coronary } \\
\text { syndrome }\end{array}$ & $2 / 118(1.7 \%)$ & $\begin{array}{l}2 / 124 \\
(1.6 \%)\end{array}$ & $\begin{array}{l}1.05(0.15 \text { to } \\
7.34)\end{array}$ \\
\hline Schrijver, $2018^{12}$ & $\begin{array}{l}\text { Medical and surgical } \\
\text { patients }\end{array}$ & Haloperidol (Planned dose: $1 \mathrm{mg}$ ), & Placebo, & Oral & $\begin{array}{l}\text { Prolongation in the } \\
\text { QTc interval } \\
\text { milliseconds }\end{array}$ & $-8.5(32.5 \%)$ & $-5.5(30 \%)$ & $\begin{array}{l}-2.92(-12.42 \\
\text { to } 6.59)\end{array}$ \\
\hline Schrijver, $2018^{12}$ & $\begin{array}{l}\text { Medical and surgical } \\
\text { patients }\end{array}$ & Haloperidol (Planned dose: $1 \mathrm{mg}$ ), & Placebo, & Oral & $\begin{array}{l}\text { Prolongation in the } \\
\text { QTc interval } \\
\text { milliseconds }\end{array}$ & $7.3(35 \%)$ & $\begin{array}{l}-5.1 \\
(45.5 \%)\end{array}$ & $\begin{array}{l}12.45(-12.26 \\
\text { to } 37.17)\end{array}$ \\
\hline Schrijver, $2018^{12}$ & $\begin{array}{l}\text { Medical and surgical } \\
\text { patients }\end{array}$ & Haloperidol (Planned dose: $1 \mathrm{mg}$ ), & Placebo, & Oral & $\begin{array}{l}\text { Prolongation in the } \\
\text { QTc interval } \\
\text { milliseconds }\end{array}$ & $-6.4(29.2)$ & $7.3(54.1)$ & $\begin{array}{l}-13.62(- \\
31.26 \text { to } \\
4.03)\end{array}$ \\
\hline $\begin{array}{l}\text { van den Boogaard, } \\
2018^{15}\end{array}$ & $\begin{array}{l}\text { ICU patients at high } \\
\text { risk of delirium }\end{array}$ & $\begin{array}{l}\text { Haloperidol (Planned dose: } 2 \mathrm{mg} \text { ), } \\
732\end{array}$ & $\begin{array}{l}\text { Placebo (Planned dose: } 0.9 \% \mathrm{NaCl} \text { ), } \\
707\end{array}$ & Intravenous & $\begin{array}{l}\text { Monomorphic } \\
\text { ventricular } \\
\text { tachycardia }\end{array}$ & $1 / 732(0.13 \%)$ & $\begin{array}{l}0 / 707 \\
(0 \%)\end{array}$ & $\begin{array}{l}2.90(0.12 \text { to } \\
71.01)\end{array}$ \\
\hline $\begin{array}{l}\text { van den Boogaard, } \\
2018^{15}\end{array}$ & $\begin{array}{l}\text { ICU patients at high } \\
\text { risk of delirium }\end{array}$ & $\begin{array}{l}\text { Haloperidol (Planned dose: } 1 \mathrm{mg} \text { ), } \\
350\end{array}$ & $\begin{array}{l}\text { Placebo (Planned dose: } 0.9 \% \mathrm{NaCl} \text { ), } \\
707\end{array}$ & Intravenous & $\begin{array}{l}\text { Monomorphic } \\
\text { ventricular } \\
\text { tachycardia }\end{array}$ & $2 / 350(0.57 \%)$ & $\begin{array}{l}0 / 707 \\
(0 \%)\end{array}$ & $\begin{array}{l}10.09(0.49 \\
\text { to } 209.51)\end{array}$ \\
\hline $\begin{array}{l}\text { van den Boogaard, } \\
2018^{15}\end{array}$ & $\begin{array}{l}\text { ICU patients at high } \\
\text { risk of delirium }\end{array}$ & Haloperidol (Planned dose: $2 \mathrm{mg}$ ), & Placebo (Planned dose: $0.9 \% \mathrm{NaCl}$ ), & Intravenous & $\begin{array}{l}\text { Prolongation in the } \\
\text { QTc interval } \\
\text { Maximum QTc } \\
\text { interval }\end{array}$ & $\begin{array}{l}\text { Median of the } \\
\text { maximum } \\
\text { times, } 465 \\
\text { (IQR, } 446 \text { to } \\
483 \text { ) }\end{array}$ & $\begin{array}{l}\text { Median of } \\
\text { the } \\
\text { maximum } \\
\text { times, } 463 \\
(\mathrm{IQR}, 440 \\
\text { to } 486)\end{array}$ & $1(-2$ to 5$)$ \\
\hline $\begin{array}{l}\text { van den Boogaard, } \\
2018^{15}\end{array}$ & $\begin{array}{l}\text { ICU patients at high } \\
\text { risk of delirium }\end{array}$ & Haloperidol (Planned dose: $1 \mathrm{mg}$ ), & Placebo (Planned dose: $0.9 \% \mathrm{NaCl}$ ), & Intravenous & $\begin{array}{l}\text { Prolongation in the } \\
\text { QTc interval } \\
\text { Maximum QTc } \\
\text { interval }\end{array}$ & $\begin{array}{l}\text { Median of the } \\
\text { maximum } \\
\text { times, } 465 \\
\text { (IQR, } 440 \text { to } \\
489 \text { ) }\end{array}$ & $\begin{array}{l}\text { Median of } \\
\text { the } \\
\text { maximum } \\
\text { times, } 463 \\
\text { (IQR, } 440 \\
\text { to } 486)\end{array}$ & \\
\hline $\begin{array}{l}\text { van den Boogaard, } \\
2018^{15}\end{array}$ & $\begin{array}{l}\text { ICU patients at high } \\
\text { risk of delirium }\end{array}$ & $\begin{array}{l}\text { Haloperidol (Planned dose: } 2 \mathrm{mg} \text { ), } \\
732\end{array}$ & $\begin{array}{l}\text { Placebo (Planned dose: } 0.9 \% \mathrm{NaCl} \text { ), } \\
707\end{array}$ & Intravenous & $\begin{array}{l}\text { Prolongation in the } \\
\text { QTc interval Number } \\
\text { of QTc time } \\
\text { prolongations }\end{array}$ & $33 / 732(4.5 \%)$ & $\begin{array}{l}36 / 707 \\
(5.1 \%)\end{array}$ & $\begin{array}{l}0.89(0.56 \text { to } \\
1.40)\end{array}$ \\
\hline $\begin{array}{l}\text { van den Boogaard, } \\
2018^{15}\end{array}$ & $\begin{array}{l}\text { ICU patients at high } \\
\text { risk of delirium }\end{array}$ & $\begin{array}{l}\text { Haloperidol (Planned dose: } 1 \mathrm{mg} \text { ), } \\
350\end{array}$ & $\begin{array}{l}\text { Placebo (Planned dose: } 0.9 \% \mathrm{NaCl} \text { ), } \\
707\end{array}$ & Intravenous & $\begin{array}{l}\text { Prolongation in the } \\
\text { QTc interval Number } \\
\text { of QTc time } \\
\text { prolongations }\end{array}$ & $31 / 350(8.9 \%)$ & $\begin{array}{l}36 / 707 \\
(5.1 \%)\end{array}$ & $\begin{array}{l}1.74(1.09 \text { to } \\
2.76)\end{array}$ \\
\hline
\end{tabular}




\begin{tabular}{|c|c|c|c|c|c|c|c|c|}
\hline Author, year & Population & Intervention group, $\mathbf{n}$ & Control group, $n$ & $\begin{array}{c}\text { Route of } \\
\text { administration }\end{array}$ & Outcome definition & $\begin{array}{c}\mathrm{n} / \mathrm{N}(\%) \\
\text { intervention } \\
\text { group }\end{array}$ & $\begin{array}{c}\text { n I N (\%), } \\
\text { control } \\
\text { group }\end{array}$ & $\begin{array}{c}\text { Relative risk } \\
(95 \% \mathrm{Cl})\end{array}$ \\
\hline Wang, $2012^{16}$ & $\begin{array}{l}\text { Patients } 65 \text { or older } \\
\text { admitted to the ICU } \\
\text { after noncardiac } \\
\text { surgery }\end{array}$ & $\begin{array}{l}\text { Haloperidol (Planned dose: } 0.5 \\
\mathrm{mg} \text { followed by continuous } \\
\text { infusion at a rate of } 1 \mathrm{~mL} / \mathrm{hr}(0.1 \\
\mathrm{mg} / \mathrm{hr} \text { haloperidol)), } 229\end{array}$ & $\begin{array}{l}\text { Placebo (Planned dose: Normal saline), } \\
228\end{array}$ & Intravenous & $\begin{array}{l}\text { Arrhythmia during } \\
\text { study drug infusion }\end{array}$ & $6 / 229(2.6 \%)$ & $\begin{array}{l}5 / 228 \\
(2.2 \%)\end{array}$ & $\begin{array}{l}1.19(0.37 \text { to } \\
3.86)\end{array}$ \\
\hline Wang, $2012^{16}$ & $\begin{array}{l}\text { Patients } 65 \text { or older } \\
\text { admitted to the ICU } \\
\text { after noncardiac } \\
\text { surgery }\end{array}$ & $\begin{array}{l}\text { Haloperidol (Planned dose: } 0.5 \\
\mathrm{mg} \text { followed by continuous } \\
\text { infusion at a rate of } 1 \mathrm{~mL} / \mathrm{hr}(0.1 \\
\mathrm{mg} / \mathrm{hr} \text { haloperidol)), }\end{array}$ & Placebo (Planned dose: Normal saline), & Intravenous & $\begin{array}{l}\text { Change of heart rate- } \\
\text { corrected QT interval } \\
\text { after study drug } \\
\text { infusion,, ms, mean } \\
+/- \text { SD }\end{array}$ & $1(26 \%)$ & $-1(31 \%)$ & $2(-3.2$ to 7.2$)$ \\
\hline Wang, 2012 & $\begin{array}{l}\text { Patients } 65 \text { or older } \\
\text { admitted to the ICU } \\
\text { after noncardiac } \\
\text { surgery }\end{array}$ & $\begin{array}{l}\text { Haloperidol (Planned dose: } 0.5 \\
\mathrm{mg} \text { followed by continuous } \\
\text { infusion at a rate of } 1 \mathrm{~mL} / \mathrm{hr}(0.1 \\
\mathrm{mg} / \mathrm{hr} \text { haloperidol })), 229\end{array}$ & $\begin{array}{l}\text { Placebo (Planned dose: Normal saline), } \\
228\end{array}$ & Intravenous & $\begin{array}{l}\text { Prolongation in the } \\
\text { QT interval Significant } \\
\text { heart rate-corrected } \\
\text { QT interval } \\
\text { prolongation after } \\
\text { study drug infusion }\end{array}$ & $4 / 229(1.7 \%)$ & $\begin{array}{l}5 / 228 \\
(2.2 \%)\end{array}$ & $\begin{array}{l}0.80(0.22 \text { to } \\
2.93)\end{array}$ \\
\hline
\end{tabular}




\begin{tabular}{|c|c|c|c|c|c|c|c|c|}
\hline Author, year & Population & Intervention group, $\mathbf{n}$ & Control group, $n$ & $\begin{array}{c}\text { Route of } \\
\text { administration }\end{array}$ & Outcome definition & $\begin{array}{c}\mathrm{n} / \mathrm{N}(\%), \\
\text { intervention } \\
\text { group }\end{array}$ & $\begin{array}{c}\mathrm{n} / \mathrm{N}(\%), \\
\text { control } \\
\text { group }\end{array}$ & $\begin{array}{c}\text { Relative risk } \\
(95 \% \mathrm{Cl})\end{array}$ \\
\hline \multicolumn{9}{|c|}{$\begin{array}{l}\text { Second-generation } \\
\text { antipsychotic vs. } \\
\text { placebo }\end{array}$} \\
\hline Girard, $2010^{4}$ & $\begin{array}{l}>18 \text { years MV medical } \\
\text { and surgical ICU } \\
\text { patients }\end{array}$ & $\begin{array}{l}\text { Ziprasidone (Planned dose: } 40 \\
\text { Median dose: } 113 \text { (81-140), } 30\end{array}$ & Placebo (Planned dose: $5 \mathrm{ml}$ ), 36 & Oral & $\begin{array}{l}\text { Prolongation in the } \\
\text { QTc interval } \\
\text { prolongation of the } \\
\text { QTc }>500 \text { ms while } \\
\text { receiving study drug }\end{array}$ & $5 / 30(\%)$ & $3 / 36(\%)$ & $\begin{array}{l}2.00(0.52 \text { to } \\
7.69)\end{array}$ \\
\hline Girard, $2010^{4}$ & $\begin{array}{l}>18 \text { years MV medical } \\
\text { and surgical ICU } \\
\text { patients }\end{array}$ & $\begin{array}{l}\text { Ziprasidone (Planned dose: } 40 \\
\text { Median dose: } 113 \text { (81-140), } 30\end{array}$ & Placebo (Planned dose: $5 \mathrm{ml}$ ), 36 & Oral & $\begin{array}{l}\text { Ventricular } \\
\text { arrhythmias }\end{array}$ & $0 / 30(\%)$ & $0 / 36(\%)$ & $\begin{array}{l}\text { Not } \\
\text { calculable }\end{array}$ \\
\hline Hakim, $2012^{5}$ & $\begin{array}{l}\text { Patients } 65 \text { years or } \\
\text { older experiencing } \\
\text { subsyndromal delirium } \\
\text { after on-pump cardiac } \\
\text { surgery }\end{array}$ & $\begin{array}{l}\text { Risperidone (Planned dose: } \\
0.5 \mathrm{mg}), 51\end{array}$ & $\begin{array}{l}\text { Placebo (Planned dose: given every } 12 \\
\text { hours), } 50\end{array}$ & Oral & $\begin{array}{l}\text { Prolongation in the } \\
\text { QT interval } \\
\text { Abnormality }\end{array}$ & $0 / 51(\%)$ & $0 / 50(\%)$ & $\begin{array}{l}\text { Not } \\
\text { calculable }\end{array}$ \\
\hline Larsen, $2010^{9}$ & $\begin{array}{l}\text { Post-operative elderly } \\
\text { joint replacement } \\
\text { surgery patients }\end{array}$ & $\begin{array}{l}\text { Olanzapine (Planned dose: } 5 \mathrm{mg} \text { ), } \\
196\end{array}$ & Placebo (Planned dose: 5mg), 204 & Oral & Arrhythmia & $2 / 196(1 \%)$ & $\begin{array}{l}1 / 204 \\
(0.5 \%)\end{array}$ & $\begin{array}{l}2.08(0.19 \text { to } \\
22.77)\end{array}$ \\
\hline Larsen, $2010^{9}$ & $\begin{array}{l}\text { Post-operative elderly } \\
\text { joint replacement } \\
\text { surgery patients }\end{array}$ & $\begin{array}{l}\text { Olanzapine (Planned dose: } 5 \mathrm{mg} \text { ), } \\
196\end{array}$ & Placebo (Planned dose: $5 \mathrm{mg}$ ), 204 & Oral & Atrial Fibrillation & $6 / 196(3.1 \%)$ & $\begin{array}{l}3 / 204 \\
(1.5 \%)\end{array}$ & $\begin{array}{l}2.08(0.53 \text { to } \\
8.21)\end{array}$ \\
\hline Larsen, $2010^{9}$ & $\begin{array}{l}\text { Post-operative elderly } \\
\text { joint replacement } \\
\text { surgery patients }\end{array}$ & $\begin{array}{l}\text { Olanzapine (Planned dose: } 5 \mathrm{mg} \text { ), } \\
196\end{array}$ & Placebo (Planned dose: $5 \mathrm{mg}$ ), 204 & Oral & $\mathrm{CHF}$ & $1 / 196(0.5 \%)$ & $\begin{array}{l}1 / 204 \\
(0.5 \%)\end{array}$ & $\begin{array}{l}1.04(0.07 \text { to } \\
16.53)\end{array}$ \\
\hline $\begin{array}{l}\text { Prakanrattana, } \\
2007^{11}\end{array}$ & $\begin{array}{l}\text { Elective Cardiac } \\
\text { Surgery with } \\
\text { cardiopulmonary } \\
\text { bypass }\end{array}$ & $\begin{array}{l}\text { Risperidone (Planned dose: } 1 \mathrm{mg} \text { ), } \\
63\end{array}$ & Placebo (Not applicable), 63 & $\begin{array}{l}\text { Orally } \\
\text { disintegrating } \\
\text { tablet }\end{array}$ & Arrhythmia & $6 / 63(9.5 \%)$ & $\begin{array}{l}6 / 63 \\
(9.5 \%)\end{array}$ & $\begin{array}{l}1.00(0.34 \text { to } \\
2.93)\end{array}$ \\
\hline
\end{tabular}




\begin{tabular}{|c|c|c|c|c|c|c|c|c|}
\hline Author, year & Population & Intervention group, $\mathbf{n}$ & Control group, $\mathrm{n}$ & $\begin{array}{c}\text { Route of } \\
\text { administration }\end{array}$ & Outcome definition & $\begin{array}{c}\mathrm{n} / \mathrm{N}(\%) \\
\text { intervention } \\
\text { group }\end{array}$ & $\begin{array}{c}\mathrm{n} / \mathrm{N}(\%) \\
\text { control } \\
\text { group }\end{array}$ & $\begin{array}{l}\text { Relative risk } \\
\text { (95\% Cl) }\end{array}$ \\
\hline \multicolumn{9}{|l|}{$\begin{array}{l}\text { First-generation } \\
\text { antipsychotic vs. } \\
\text { second-generation } \\
\text { antipsychotic } \\
\end{array}$} \\
\hline Girard, $2010^{4}$ & $\begin{array}{l}>18 \text { years MV medical } \\
\text { and surgical ICU } \\
\text { patients }\end{array}$ & $\begin{array}{l}\text { Ziprasidone (Planned dose: } 40 \\
\text { Median dose: } 113 \text { (81-140), } 30\end{array}$ & $\begin{array}{l}\text { Haloperidol (Planned dose: } 5 \text { Median } \\
\text { dose: } 15 \text { (10.8-17), } 35\end{array}$ & Oral & $\begin{array}{l}\text { Prolongation in the } \\
\text { QTc interval } \\
\text { prolongation of the } \\
\text { QTc }>500 \text { ms while } \\
\text { receiving study drug }\end{array}$ & $5 / 30(\%)$ & $2 / 35(\%)$ & $\begin{array}{l}2.92(0.61 \text { to } \\
13.96)\end{array}$ \\
\hline Girard, $2010^{4}$ & $\begin{array}{l}>18 \text { years MV medical } \\
\text { and surgical ICU } \\
\text { patients }\end{array}$ & $\begin{array}{l}\text { Ziprasidone (Planned dose: } 40 \\
\text { Median dose: } 113 \text { (81-140), } 30\end{array}$ & $\begin{array}{l}\text { Haloperidol (Planned dose: } 5 \text { Median } \\
\text { dose: } 15 \text { (10.8-17), } 35\end{array}$ & Oral & $\begin{array}{l}\text { Ventricular } \\
\text { arrhythmias }\end{array}$ & $0 / 30(\%)$ & $0 / 35(\%)$ & $\begin{array}{l}\text { Not } \\
\text { calculable }\end{array}$ \\
\hline \multicolumn{9}{|l|}{$\begin{array}{l}\text { First-generation } \\
\text { antipsychotic vs. } \\
\text { other }\end{array}$} \\
\hline Abdelgalel, $2016^{1}$ & $\begin{array}{l}\text { Adult intensive care } \\
\text { patients of ASA } \\
\text { physical status III and } \\
\text { IV aged between } 26 \\
\text { and } 70 \text { years }\end{array}$ & $\begin{array}{l}\text { Haloperidol (Planned dose: } 0.5-2 \\
\mathrm{mg} / \mathrm{h} \text { preceded by a loading dose } \\
\text { of } 2.5 \mathrm{mg} \text { intravenously over } 10 \\
\mathrm{~min} \text { if needed (if RASS }>2+\text { ), } 30\end{array}$ & $\begin{array}{l}\text { Dexmedetomidine (Planned dose: } 0.2- \\
0.7 \mathrm{mcg} / \mathrm{kg} / \mathrm{h} \text { preceded by a loading } \\
\text { dose of } 1.0 \mathrm{ug} / \mathrm{kg} \text { intravenously over } 10 \\
\mathrm{~min} \text { if needed (if RASS }>2+\text { )), } 30\end{array}$ & Intravenous & Arrhythmia & $3 / 30(10 \%)$ & $2 / 30(7 \%)$ & $\begin{array}{l}1.50(0.27 \text { to } \\
8.34)\end{array}$ \\
\hline Abdelgalel, $2016^{1}$ & $\begin{array}{l}\text { Adult intensive care } \\
\text { patients of ASA } \\
\text { physical status III and } \\
\text { IV aged between } 26 \\
\text { and } 70 \text { years }\end{array}$ & $\begin{array}{l}\text { Haloperidol (Planned dose: } 0.5-2 \\
\mathrm{mg} / \mathrm{h} \text { preceded by a loading dose } \\
\text { of } 2.5 \mathrm{mg} \text { intravenously over } 10 \\
\mathrm{~min} \text { if needed (if RASS }>2+\text { ), } 30\end{array}$ & $\begin{array}{l}\text { Dexmedetomidine (Planned dose: } 0.2- \\
0.7 \mathrm{mcg} / \mathrm{kg} / \mathrm{h} \text { preceded by a loading } \\
\text { dose of } 1.0 \mathrm{ug} / \mathrm{kg} \text { intravenously over } 10 \\
\mathrm{~min} \text { if needed (if RASS }>2+\text { )), } 30\end{array}$ & Intravenous & $\begin{array}{l}\text { Bradycardia (Heart } \\
\text { rate decreased to <= } \\
60 \text { beats } / \mathrm{min} \text { ) }\end{array}$ & $2 / 30(7 \%)$ & $\begin{array}{l}8 / 30 \\
(27 \%)\end{array}$ & $\begin{array}{l}0.25 \text { (0.06 to } \\
1.08)\end{array}$ \\
\hline Abdelgalel, $2016^{1}$ & $\begin{array}{l}\text { Adult intensive care } \\
\text { patients of ASA } \\
\text { physical status III and } \\
\text { IV aged between } 26 \\
\text { and } 70 \text { years }\end{array}$ & $\begin{array}{l}\text { Haloperidol (Planned dose: } 0.5-2 \\
\mathrm{mg} / \mathrm{h} \text { preceded by a loading dose } \\
\text { of } 2.5 \mathrm{mg} \text { intravenously over } 10 \\
\mathrm{~min} \text { if needed (if RASS }>2+\text { ), } 30\end{array}$ & $\begin{array}{l}\text { Dexmedetomidine (Planned dose: } 0.2- \\
0.7 \mathrm{mcg} / \mathrm{kg} / \mathrm{h} \text { preceded by a loading } \\
\text { dose of } 1.0 \mathrm{ug} / \mathrm{kg} \text { intravenously over } 10 \\
\mathrm{~min} \text { if needed (if RASS }>2+\text { )), } 30\end{array}$ & Intravenous & Hypotension & $3 / 30(10 \%)$ & $\begin{array}{l}4 / 30 \\
(13 \%)\end{array}$ & $\begin{array}{l}0.75(0.18 \text { to } \\
3.07)\end{array}$ \\
\hline Abdelgalel, $2016^{1}$ & $\begin{array}{l}\text { Adult intensive care } \\
\text { patients of ASA } \\
\text { physical status III and } \\
\text { IV aged between } 26 \\
\text { and } 70 \text { years }\end{array}$ & $\begin{array}{l}\text { Haloperidol (Planned dose: } 0.5-2 \\
\mathrm{mg} / \mathrm{h} \text { preceded by a loading dose } \\
\text { of } 2.5 \mathrm{mg} \text { intravenously over } 10 \\
\mathrm{~min} \text { if needed (if RASS }>2+\text { ), } 30\end{array}$ & $\begin{array}{l}\text { Dexmedetomidine (Planned dose: } 0.2- \\
0.7 \mathrm{mcg} / \mathrm{kg} / \mathrm{h} \mathrm{preceded} \mathrm{by} \mathrm{a} \mathrm{loading} \\
\text { dose of } 1.0 \mathrm{ug} / \mathrm{kg} \text { intravenously over } 10 \\
\mathrm{~min} \text { if needed (if RASS }>2+\text { )), } 30\end{array}$ & Intravenous & $\begin{array}{l}\text { Prolongation in the } \\
\text { QTc interval }>500 \mathrm{~ms}\end{array}$ & $2 / 30(7 \%)$ & $0 / 30(0 \%)$ & $\begin{array}{l}5.00(0.25 \text { to } \\
99.95)\end{array}$ \\
\hline
\end{tabular}

ventilation; N=sample size; QT=Q and T wave interval; QTc=corrected QT interval; RASS=Richmond Agitation and Sedation Scale; $\mathrm{SD}=$ standard deviation; Ug/kg=microgram per kilogram 


\begin{tabular}{|c|c|c|c|c|c|c|c|c|}
\hline Author, year & Population & Intervention group, $\mathbf{n}$ & Control group, $\mathbf{n}$ & $\begin{array}{c}\text { Route of } \\
\text { administration }\end{array}$ & Outcome definition & $\begin{array}{l}\text { Mean change } \\
\text { from baseline } \\
\text { (SD) in ms, } \\
\text { intervention } \\
\text { group }\end{array}$ & 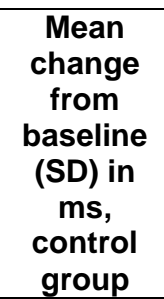 & $\begin{array}{c}\text { Mean } \\
\text { between- } \\
\text { group } \\
\text { difference } \\
(95 \% \mathrm{Cl}) \text { in } \\
\mathrm{ms}\end{array}$ \\
\hline \multicolumn{9}{|l|}{$\begin{array}{l}\text { First-generation } \\
\text { antipsychotic vs. } \\
\text { palcebo }\end{array}$} \\
\hline Schrijver, $2018^{13}$ & $\begin{array}{l}\text { Medical and surgical } \\
\text { patients age } 70 \text { and } \\
\text { above, with at least } \\
\text { three ECGs }\end{array}$ & $\begin{array}{l}\text { Haloperidol (Planned dose: } 1 \mathrm{mg} \text {, } \\
\text { bid, mean } 7 \text { days), } 38\end{array}$ & $\begin{array}{l}\text { Placebo (Planned dose: dose NR, bid, } \\
\text { mean } 7 \text { days), } 34\end{array}$ & Oral & QTc-automatic, ms & $\begin{array}{l}\text { Baseline: } \\
\text { Mean 435.4 } \\
\text { (SD 25) } \\
\text { Final: Mean } \\
437.8 \text { (SD 20) }\end{array}$ & $\begin{array}{l}\text { Baseline: } \\
\text { Mean } \\
431.3 \text { (SD } \\
24.7) \\
\text { Final: } \\
\text { Mean } \\
437.8 \text { (SD } \\
32.9)\end{array}$ & $\begin{array}{l}-4.10(95 \% \\
\mathrm{Cl}:-131.27 \\
\text { to } 123.07)\end{array}$ \\
\hline Schrijver, $2018^{13}$ & $\begin{array}{l}\text { Medical and surgical } \\
\text { patients age } 70 \text { and } \\
\text { above, with at least } \\
\text { three ECGs }\end{array}$ & $\begin{array}{l}\text { Haloperidol (Planned dose: } 1 \mathrm{mg} \text {, } \\
\text { bid, mean } 7 \text { days), } 38\end{array}$ & $\begin{array}{l}\text { Placebo (Planned dose: dose NR, bid, } \\
\text { mean } 7 \text { days), } 34\end{array}$ & Oral & QTc-Bazett, ms & $\begin{array}{l}\text { Baseline: } \\
\text { Mean 422.2 } \\
\text { (SD 33.6) } \\
\text { Final: Mean } \\
424.7 \text { (SD 53) }\end{array}$ & $\begin{array}{l}\text { Baseline: } \\
\text { Mean } \\
430.8 \text { (SD } \\
33.4) \\
\text { Final: } \\
\text { Mean } \\
411.1 \text { (SD } \\
27.3) \\
\end{array}$ & $\begin{array}{l}22.20(95 \% \\
\mathrm{Cl}:-165.43 \\
\text { to } 209.83)\end{array}$ \\
\hline Schrijver, $2018^{13}$ & $\begin{array}{l}\text { Medical and surgical } \\
\text { patients age } 70 \text { and } \\
\text { above, with at least } \\
\text { three ECGs }\end{array}$ & $\begin{array}{l}\text { Haloperidol (Planned dose: } 1 \mathrm{mg} \text {, } \\
\text { bid, mean } 7 \text { days), } 38\end{array}$ & $\begin{array}{l}\text { Placebo (Planned dose: dose NR, bid, } \\
\text { mean } 7 \text { days), } 34\end{array}$ & Oral & QTc-Framingham, ms & $\begin{array}{l}\text { Baseline: } \\
\text { Mean 403.1 } \\
\text { (SD 25.4) } \\
\text { Final: Mean } \\
400 \text { (SD 43.5) }\end{array}$ & $\begin{array}{l}\text { Baseline: } \\
\text { Mean } \\
414.9 \text { (SD } \\
29.3) \\
\text { Final: } \\
\text { Mean } \\
399.7 \text { (SD } \\
19.7)\end{array}$ & $\begin{array}{l}12.10(95 \% \\
\mathrm{Cl}:-138.38 \\
\text { to } 162.58)\end{array}$ \\
\hline
\end{tabular}




\begin{tabular}{|c|c|c|c|c|c|c|c|c|}
\hline Author, year & Population & Intervention group, $\mathbf{n}$ & Control group, $\mathbf{n}$ & $\begin{array}{c}\text { Route of } \\
\text { administration }\end{array}$ & Outcome definition & $\begin{array}{c}\text { Mean change } \\
\text { from baseline } \\
\text { (SD) in ms, } \\
\text { intervention } \\
\text { group }\end{array}$ & $\begin{array}{c}\text { Mean } \\
\text { change } \\
\text { from } \\
\text { baseline } \\
\text { (SD) in } \\
\text { ms, } \\
\text { control } \\
\text { group }\end{array}$ & $\begin{array}{c}\text { Mean } \\
\text { between- } \\
\text { group } \\
\text { difference } \\
(95 \% \mathrm{Cl}) \text { in } \\
\text { ms }\end{array}$ \\
\hline Schrijver, $2018^{13}$ & $\begin{array}{l}\text { Medical and surgical } \\
\text { patients age } 70 \text { and } \\
\text { above, with at least } \\
\text { three ECGs }\end{array}$ & $\begin{array}{l}\text { Haloperidol (Planned dose: } 1 \mathrm{mg} \text {, } \\
\text { bid, mean } 7 \text { days), } 38\end{array}$ & $\begin{array}{l}\text { Placebo (Planned dose: dose NR, bid, } \\
\text { mean } 7 \text { days), } 34\end{array}$ & Oral & QTc-Fridericia, ms & $\begin{array}{l}\text { Baseline: } \\
\text { Mean 402.9 } \\
\text { (SD 28.9) } \\
\text { Final: Mean } \\
400 \text { (SD 49) }\end{array}$ & $\begin{array}{l}\text { Baseline: } \\
\text { Mean } \\
414.8 \text { (SD } \\
30.7) \\
\text { Final: } \\
\text { Mean } 398 \\
\text { (SD 21.6) }\end{array}$ & $\begin{array}{l}13.90(95 \% \\
\mathrm{Cl}:-153.24 \\
\text { to } 181.04)\end{array}$ \\
\hline Schrijver, $2018^{13}$ & $\begin{array}{l}\text { Medical and surgical } \\
\text { patients age } 70 \text { and } \\
\text { above, with at least } \\
\text { three ECGs }\end{array}$ & $\begin{array}{l}\text { Haloperidol (Planned dose: } 1 \mathrm{mg} \text {, } \\
\text { bid, mean } 7 \text { days), } 38\end{array}$ & $\begin{array}{l}\text { Placebo (Planned dose: dose NR, bid, } \\
\text { mean } 7 \text { days), } 34\end{array}$ & Oral & QTc-Hodges, ms & $\begin{array}{l}\text { Baseline: } \\
\text { Mean 405.4 } \\
\text { (SD 25.9) } \\
\text { Final: Mean } \\
402 \text { (SD 58.6) }\end{array}$ & $\begin{array}{l}\text { Baseline: } \\
\text { Mean } \\
413.5 \text { (SD } \\
29.1) \\
\text { Final: } \\
\text { Mean } \\
388.5 \text { (SD } \\
\text { 13.5) }\end{array}$ & $\begin{array}{l}21.60(95 \% \\
\mathrm{Cl}:-150.46 \\
\text { to } 193.66)\end{array}$ \\
\hline
\end{tabular}


Evidence Table D-27. Cardiac outcomes in studies comparing interventions to prevent development of delirium: single arm studies

\begin{tabular}{|c|c|c|c|c|c|c|}
\hline Author, year & Study design & Population & Intervention group, $\mathbf{n}$ & $\begin{array}{c}\text { Route of } \\
\text { administration }\end{array}$ & Outcome definition & $\begin{array}{l}\mathrm{n} / \mathrm{N}(\%) \\
\text { intervention } \\
\text { group }\end{array}$ \\
\hline $\begin{array}{l}\text { van den } \\
\text { Boogaard, } \\
2013^{14}\end{array}$ & $\begin{array}{l}\text { Prospective cohort with a } \\
\text { comparison group, } \\
\text { analyzed as a single } \\
\text { group }\end{array}$ & $\begin{array}{l}\text { Patients in the ICU who are at a high } \\
\text { risk of delirium }\end{array}$ & $\begin{array}{l}\text { Haloperidol (Planned dose: } 1 \text { Median dose: } \\
2 \mathrm{mg} \text { ), }\end{array}$ & Intravenous & $\begin{array}{l}\text { Prolongation in the QTc } \\
\text { interval stopped medication } \\
\text { due to prolonged QTc-time }\end{array}$ & $9 / 177$ (5\%) \\
\hline
\end{tabular}

Mg=milligram; N=sample size; QTc=corrected QT interval 
Evidence Table D-28. Binary neurological outcomes in studies comparing interventions to prevent development of delirium

\begin{tabular}{|c|c|c|c|c|c|c|c|c|}
\hline Author, year & $\begin{array}{l}\text { Population } \\
\end{array}$ & $\begin{array}{c}\text { Intervention } \\
\text { group, } \mathbf{n}\end{array}$ & Control group, $n$ & $\begin{array}{c}\text { Route of } \\
\text { administration } \\
\end{array}$ & $\begin{array}{l}\text { Outcome } \\
\text { definition }\end{array}$ & $\begin{array}{c}\text { n I N (\%), intervention } \\
\text { group }\end{array}$ & $\begin{array}{c}\mathrm{n} / \mathrm{N} \text { (\%), control } \\
\text { group }\end{array}$ & $\begin{array}{c}\text { Relative risk (95\% } \\
\mathrm{Cl})\end{array}$ \\
\hline \multicolumn{9}{|l|}{$\begin{array}{l}\text { First-generation } \\
\text { antipsychotic vs. } \\
\text { placebo }\end{array}$} \\
\hline Al-Qadheeb, $2016^{2}$ & $\begin{array}{l}\text { MV patients with } \\
\text { subsyndromal delirium }\end{array}$ & $\begin{array}{l}\text { Haloperidol } \\
\text { (Planned dose: } \\
\text { 1mg), } 34\end{array}$ & $\begin{array}{l}\text { Placebo (Planned } \\
\text { dose: } 5 \% \\
\text { dextroseml), } 34\end{array}$ & Intravenous & $\begin{array}{l}\text { Extrapyramidal } \\
\text { symptoms }\end{array}$ & $1 / 34(2.9 \%)$ & $0 / 34(0 \%)$ & 3.00 (0.13 to 71.15$)$ \\
\hline Fukata, $2014^{3}$ & $\begin{array}{l}\text { Delirium after } \\
\text { abdominal or } \\
\text { orthopedic surgery in } \\
\text { elderly patients }\end{array}$ & $\begin{array}{l}\text { Haloperidol } \\
\text { (Planned dose: } \\
\text { 2.5mg), } 59\end{array}$ & Placebo (), 60 & Intravenous & $\begin{array}{l}\text { Convulsions and } \\
\text { temporary loss of } \\
\text { consciousness } 2 \\
\text { days after } \\
\text { completing the } \\
\text { preventive } \\
\text { administration of } \\
\text { haloperidol and T- } \\
\text { tube removal }\end{array}$ & $1 / 59(2 \%)$ & $0 / 60(0 \%)$ & 3.05 (0.13 to 73.39$)$ \\
\hline Girard, $2010^{4}$ & $\begin{array}{l}>18 \text { years MV medical } \\
\text { and surgical ICU } \\
\text { patients }\end{array}$ & $\begin{array}{l}\text { Haloperidol } \\
\text { (Planned dose: } 5 \\
\text { Median dose: } 15 \\
(10.8-17), 35\end{array}$ & $\begin{array}{l}\text { Placebo (Planned } \\
\text { dose: } 5 \mathrm{ml}), 36\end{array}$ & Oral & Akathisia & $10 / 35(29 \%)$ & $7 / 36(19 \%)$ & 1.47 (0.63 to 3.43$)$ \\
\hline Girard, $2010^{4}$ & $\begin{array}{l}>18 \text { years MV medical } \\
\text { and surgical ICU } \\
\text { patients }\end{array}$ & $\begin{array}{l}\text { Haloperidol } \\
\text { (Planned dose: } 5 \\
\text { Median dose: } 15 \\
(10.8-17), 35\end{array}$ & $\begin{array}{l}\text { Placebo (Planned } \\
\text { dose: } 5 \mathrm{ml}), 36\end{array}$ & Oral & $\begin{array}{l}\text { Extrapyramidal } \\
\text { symptoms }\end{array}$ & $4 / 35$ (11\%) & $6 / 36(17 \%)$ & $0.69(0.21$ to 2.22$)$ \\
\hline Girard, $2010^{4}$ & $\begin{array}{l}>18 \text { years MV medical } \\
\text { and surgical ICU } \\
\text { patients }\end{array}$ & $\begin{array}{l}\text { Haloperidol } \\
\text { (Planned dose: } 5 \\
\text { Median dose: } 15 \\
(10.8-17), 35\end{array}$ & $\begin{array}{l}\text { Placebo (Planned } \\
\text { dose: } 5 \mathrm{ml}), 36\end{array}$ & Oral & $\begin{array}{l}\text { Neuroleptic } \\
\text { malignant } \\
\text { syndrome }\end{array}$ & $0 / 35$ (\%) & $0 / 36(\%)$ & Not calculable \\
\hline
\end{tabular}




\begin{tabular}{|c|c|c|c|c|c|c|c|c|}
\hline Author, year & Population & $\begin{array}{c}\text { Intervention } \\
\text { group, } \mathrm{n}\end{array}$ & Control group, $\mathbf{n}$ & $\begin{array}{c}\text { Route of } \\
\text { administration }\end{array}$ & $\begin{array}{l}\text { Outcome } \\
\text { definition }\end{array}$ & $\begin{array}{c}\mathrm{n} / \mathrm{N}(\%), \text { intervention } \\
\text { group }\end{array}$ & $\begin{array}{c}\mathrm{n} / \mathrm{N} \text { (\%), control } \\
\text { group }\end{array}$ & $\begin{array}{l}\text { Relative risk (95\% } \\
\mathrm{Cl})\end{array}$ \\
\hline Kalisvaart, $2005^{6}$ & $\begin{array}{l}\text { Acute or elective hip } \\
\text { surgery patients } \geq 70 \\
\text { years is at risk of } \\
\text { delirium: MMSE } \\
\text { between less than or } \\
\text { equal to } 24 ; \\
\text { dehydration=BUN/creat } \\
\text { inine }>\text { or }=\text { to } 18 \text {, low } \\
\text { visual acuity and/or } \\
\text { increased severity of } \\
\text { illness on APACHE II of } \\
16 \text { or greater. }\end{array}$ & $\begin{array}{l}\text { Haloperidol } \\
\text { (Planned dose: } \\
0.5 \mathrm{mg}), 212\end{array}$ & $\begin{array}{l}\text { Placebo (Not } \\
\text { applicable), } 218\end{array}$ & Oral & $\begin{array}{l}\text { Extrapyramidal } \\
\text { symptoms }\end{array}$ & $0 / 212(\%)$ & $0 / 218$ (\%) & Not calculable \\
\hline Kaneko, $1999^{7}$ & $\begin{array}{l}\text { Scheduled for elective } \\
\text { gastrointestinal surgery } \\
\text { and admitted to ICU }\end{array}$ & $\begin{array}{l}\text { Haloperidol } \\
\text { (Planned dose: } \\
\text { 5mg), } 38\end{array}$ & $\begin{array}{l}\text { Placebo (Planned } \\
\text { dose: } 1 \mathrm{ml}(0.9 \% \\
\text { normal saline)ml), } \\
40\end{array}$ & Intravenous & $\begin{array}{l}\text { Extrapyramidal } \\
\text { symptoms }\end{array}$ & $0 / 38$ (\%) & $0 / 40$ (\%) & Not calculable \\
\hline Khan, $2018^{8}$ & $\begin{array}{l}\text { English speaking } \\
\text { individuals undergoing } \\
\text { thoracic surgery }\end{array}$ & $\begin{array}{l}\text { Haloperidol } \\
\text { (Planned dose: } \\
\text { 0.5mg, Planned } \\
\text { duration: } 4 \text { days (11 } \\
\text { doses total)), } 68\end{array}$ & $\begin{array}{l}\text { Placebo (Planned } \\
\text { dose: NR, Planned } \\
\text { duration: } 4 \text { days (11 } \\
\text { doses total)), } 67\end{array}$ & Intravenous & Elbow Rigidity & $0 / 68(0 \%)$ & $2 / 67(3 \%)$ & $0.2(0.01$ to 4.03$)$ \\
\hline Khan, $2018^{8}$ & $\begin{array}{l}\text { English speaking } \\
\text { individuals undergoing } \\
\text { thoracic surgery }\end{array}$ & $\begin{array}{l}\text { Haloperidol } \\
\text { (Planned dose: } \\
0.5 \mathrm{mg}, \text { Planned } \\
\text { duration: } 4 \text { days (11 } \\
\text { doses total)), } 68\end{array}$ & $\begin{array}{l}\text { Placebo (Planned } \\
\text { dose: NR, Planned } \\
\text { duration: } 4 \text { days (11 } \\
\text { doses total)), } 67\end{array}$ & Intravenous & Wrist Ridigidity & $1 / 68(1.5 \%)$ & $2 / 67(3 \%)$ & 0.49 (0.05 to 5.31$)$ \\
\hline
\end{tabular}




\begin{tabular}{|c|c|c|c|c|c|c|c|c|}
\hline Author, year & Population & $\begin{array}{c}\text { Intervention } \\
\text { group, } \mathbf{n}\end{array}$ & Control group, $\mathbf{n}$ & $\begin{array}{c}\text { Route of } \\
\text { administration }\end{array}$ & $\begin{array}{l}\text { Outcome } \\
\text { definition }\end{array}$ & $\begin{array}{c}\mathrm{n} / \mathrm{N} \text { (\%), intervention } \\
\text { group }\end{array}$ & $\begin{array}{c}\mathrm{n} / \mathrm{N}(\%), \text { control } \\
\text { group }\end{array}$ & $\begin{array}{l}\text { Relative risk (95\% } \\
\mathrm{Cl})\end{array}$ \\
\hline Khan, $2018^{8}$ & $\begin{array}{l}\text { English speaking } \\
\text { individuals undergoing } \\
\text { thoracic surgery }\end{array}$ & $\begin{array}{l}\text { Haloperidol } \\
\text { (Planned dose: } \\
0.5 \mathrm{mg}, \text { Planned } \\
\text { duration: } 4 \text { days (11 } \\
\text { doses total)), } 68\end{array}$ & $\begin{array}{l}\text { Placebo (Planned } \\
\text { dose: NR, Planned } \\
\text { duration: } 4 \text { days (11 } \\
\text { doses total)), } 67\end{array}$ & Intravenous & Resting Tremor & $1 / 68(1.5 \%)$ & $4 / 67$ (6\%) & 0.25 (0.03 to 2.15$)$ \\
\hline Khan, $2018^{8}$ & $\begin{array}{l}\text { English speaking } \\
\text { individuals undergoing } \\
\text { thoracic surgery }\end{array}$ & $\begin{array}{l}\text { Haloperidol } \\
\text { (Planned dose: } \\
0.5 \mathrm{mg}, \text { Planned } \\
\text { duration: } 4 \text { days (11 } \\
\text { doses total)), } 68\end{array}$ & $\begin{array}{l}\text { Placebo (Planned } \\
\text { dose: NR, Planned } \\
\text { duration: } 4 \text { days (11 } \\
\text { doses total)), } 67\end{array}$ & Intravenous & Dystonia & $0 / 68(0 \%)$ & $0 / 67(0 \%)$ & \\
\hline Khan, $2018^{8}$ & $\begin{array}{l}\text { English speaking } \\
\text { individuals undergoing } \\
\text { thoracic surgery }\end{array}$ & $\begin{array}{l}\text { Haloperidol } \\
\text { (Planned dose: } \\
0.5 \mathrm{mg}, \text { Planned } \\
\text { duration: } 4 \text { days (11 } \\
\text { doses total)), } 68\end{array}$ & $\begin{array}{l}\text { Placebo (Planned } \\
\text { dose: NR, Planned } \\
\text { duration: } 4 \text { days (11 } \\
\text { doses total)), } 67\end{array}$ & Intravenous & $\begin{array}{l}\text { Neuroleptic } \\
\text { Malignant } \\
\text { Syndrome }\end{array}$ & $0 / 68(0 \%)$ & $0 / 67(0 \%)$ & \\
\hline Khan, $2018^{8}$ & $\begin{array}{l}\text { English speaking } \\
\text { individuals undergoing } \\
\text { thoracic surgery }\end{array}$ & $\begin{array}{l}\text { Haloperidol } \\
\text { (Planned dose: } \\
\text { 0.5mg, Planned } \\
\text { duration: } 4 \text { days (11 } \\
\text { doses total)), } 68\end{array}$ & $\begin{array}{l}\text { Placebo (Planned } \\
\text { dose: NR, Planned } \\
\text { duration: } 4 \text { days (11 } \\
\text { doses total)), } 67\end{array}$ & Intravenous & Tardive Dyskinesia & 0 / 68 (0\%) & $0 / 67(0 \%)$ & \\
\hline Khan, $2018^{8}$ & $\begin{array}{l}\text { English speaking } \\
\text { individuals undergoing } \\
\text { thoracic surgery }\end{array}$ & $\begin{array}{l}\text { Haloperidol } \\
\text { (Planned dose: } \\
0.5 \mathrm{mg}, \text { Planned } \\
\text { duration: } 4 \text { days (11 } \\
\text { doses total)), } 68\end{array}$ & $\begin{array}{l}\text { Placebo (Planned } \\
\text { dose: NR, Planned } \\
\text { duration: } 4 \text { days (11 } \\
\text { doses total)), } 67\end{array}$ & Intravenous & $\begin{array}{l}\text { Pseudoparkinsonis } \\
\mathrm{m}\end{array}$ & $0 / 68(0 \%)$ & $0 / 67(0 \%)$ & \\
\hline
\end{tabular}




\begin{tabular}{|c|c|c|c|c|c|c|c|c|}
\hline Author, year & Population & $\begin{array}{c}\text { Intervention } \\
\text { group, } \mathrm{n}\end{array}$ & Control group, $\mathbf{n}$ & $\begin{array}{c}\text { Route of } \\
\text { administration }\end{array}$ & $\begin{array}{l}\text { Outcome } \\
\text { definition }\end{array}$ & $\begin{array}{l}\text { n I N (\%), intervention } \\
\text { group }\end{array}$ & $\begin{array}{c}\mathrm{n} / \mathrm{N}(\%), \text { control } \\
\text { group }\end{array}$ & $\begin{array}{l}\text { Relative risk (95\% } \\
\mathrm{Cl})\end{array}$ \\
\hline Page, $2013^{10}$ & $\begin{array}{l}\text { ICU patients needing } \\
\text { MV within } 72 \text { hours of } \\
\text { admission were } \\
\text { enrolled }\end{array}$ & $\begin{array}{l}\text { Haloperidol } \\
\text { (Planned dose: } \\
2.5 \mathrm{mg}), 71\end{array}$ & $\begin{array}{l}\text { Placebo (Planned } \\
\text { dose: } 0.5 \mathrm{ml}), 70\end{array}$ & Intravenous & Akathisia & $1 / 71(1 \%)$ & $2 / 70(3 \%)$ & 0.49 (0.05 to 5.31$)$ \\
\hline Page, $2013^{10}$ & $\begin{array}{l}\text { ICU patients needing } \\
\text { MV within } 72 \text { hours of } \\
\text { admission were } \\
\text { enrolled }\end{array}$ & $\begin{array}{l}\text { Haloperidol } \\
\text { (Planned dose: } \\
2.5 \mathrm{mg}), 71\end{array}$ & $\begin{array}{l}\text { Placebo (Planned } \\
\text { dose: } 0.5 \mathrm{ml}), 70\end{array}$ & Intravenous & Muscle Stiffness & $1 / 71(1 \%)$ & $1 / 70(3 \%)$ & 0.99 (0.06 to 15.45$)$ \\
\hline Page, $2013^{10}$ & $\begin{array}{l}\text { ICU patients needing } \\
\text { MV within } 72 \text { hours of } \\
\text { admission were } \\
\text { enrolled }\end{array}$ & $\begin{array}{l}\text { Haloperidol } \\
\text { (Planned dose: } \\
2.5 \mathrm{mg}), 71\end{array}$ & $\begin{array}{l}\text { Placebo (Planned } \\
\text { dose: } 0.5 \mathrm{ml}), 70\end{array}$ & Intravenous & Torticollis & $0 / 71(\%)$ & $1 / 70(3 \%)$ & 0.33 (0.01 to 7.93$)$ \\
\hline
\end{tabular}




\begin{tabular}{|c|c|c|c|c|c|c|c|c|}
\hline Author, year & Population & $\begin{array}{l}\text { Intervention } \\
\text { group, } \mathrm{n}\end{array}$ & Control group, $n$ & $\begin{array}{c}\text { Route of } \\
\text { administration }\end{array}$ & $\begin{array}{l}\text { Outcome } \\
\text { definition }\end{array}$ & $\begin{array}{c}\text { n I N (\%), intervention } \\
\text { group }\end{array}$ & $\begin{array}{l}\text { n I N (\%), control } \\
\text { group }\end{array}$ & $\begin{array}{c}\text { Relative risk (95\% } \\
\text { Cl) }\end{array}$ \\
\hline Schrijver, $2018^{12}$ & $\begin{array}{l}\text { Medical and surgical } \\
\text { patients }\end{array}$ & $\begin{array}{l}\text { Haloperidol } \\
\text { (Planned dose: } \\
\text { 1mg), } 118\end{array}$ & Placebo, 124 & Oral & $\begin{array}{l}\text { Extrapyramidal } \\
\text { symptoms }\end{array}$ & $2 / 118(1.7 \%)$ & $2 / 124(1.6 \%)$ & 1.05 (0.15 to 7.34$)$ \\
\hline Schrijver, 2018 & $\begin{array}{l}\text { Medical and surgical } \\
\text { patients }\end{array}$ & $\begin{array}{l}\text { Haloperidol } \\
\text { (Planned dose: } \\
\text { 1mg), } 118\end{array}$ & Placebo, 124 & Oral & $\begin{array}{l}\text { Transient ischemic } \\
\text { attack }\end{array}$ & $2 / 118(1.7 \%)$ & $0 / 124(0 \%)$ & $\begin{array}{l}5.25(0.25 \text { to } \\
108.27)\end{array}$ \\
\hline $\begin{array}{l}\text { van den Boogaard, } \\
2018^{15}\end{array}$ & $\begin{array}{l}\text { ICU patients at high } \\
\text { risk of delirium }\end{array}$ & $\begin{array}{l}\text { Haloperidol } \\
\text { (Planned dose: } \\
\text { 1mg), } 350\end{array}$ & $\begin{array}{l}\text { Placebo (Planned } \\
\text { dose: } 0.9 \% \mathrm{NaCl} \text { ), } \\
707\end{array}$ & Intravenous & $\begin{array}{l}\text { Extrapyramidal } \\
\text { symptoms }\end{array}$ & $9 / 350(2.6 \%)$ & $11 / 707$ (1.6\%) & 1.65 (0.69 to 3.95$)$ \\
\hline $\begin{array}{l}\text { van den Boogaard, } \\
2018^{15}\end{array}$ & $\begin{array}{l}\text { ICU patients at high } \\
\text { risk of delirium }\end{array}$ & $\begin{array}{l}\text { Haloperidol } \\
\text { (Planned dose: } \\
\text { 2mg), } 732\end{array}$ & $\begin{array}{l}\text { Placebo (Planned } \\
\text { dose: } 0.9 \% \mathrm{NaCl} \text {, } \\
707\end{array}$ & Intravenous & $\begin{array}{l}\text { Extrapyramidal } \\
\text { symptoms }\end{array}$ & 11 / 732 (1.5\%) & $11 / 707$ (1.6\%) & 0.97 (0.42 to 2.21$)$ \\
\hline $\begin{array}{l}\text { van den Boogaard, } \\
2018^{15}\end{array}$ & $\begin{array}{l}\text { ICU patients at high } \\
\text { risk of delirium }\end{array}$ & $\begin{array}{l}\text { Haloperidol } \\
\text { (Planned dose: } \\
1 \mathrm{mg}), 350\end{array}$ & $\begin{array}{l}\text { Placebo (Planned } \\
\text { dose: } 0.9 \% \mathrm{NaCl} \text { ), } \\
707\end{array}$ & Intravenous & $\begin{array}{l}\text { Suspected } \\
\text { malignant } \\
\text { neuroleptic } \\
\text { syndrome }\end{array}$ & 0 / 350 (\%) & $1 / 707(\%)$ & 0.67 (0.03 to 16.46$)$ \\
\hline $\begin{array}{l}\text { van den Boogaard, } \\
2018^{15}\end{array}$ & $\begin{array}{l}\text { ICU patients at high } \\
\text { risk of delirium }\end{array}$ & $\begin{array}{l}\text { Haloperidol } \\
\text { (Planned dose: } \\
2 \mathrm{mg}), 732\end{array}$ & $\begin{array}{l}\text { Placebo (Planned } \\
\text { dose: } 0.9 \% \mathrm{NaCl} \text { ), } \\
707\end{array}$ & Intravenous & $\begin{array}{l}\text { Suspected } \\
\text { malignant } \\
\text { neuroleptic } \\
\text { syndrome }\end{array}$ & $0 / 732(\%)$ & $1 / 707(\%)$ & 0.32 (0.01 to 7.89$)$ \\
\hline Wang, $2012^{16}$ & $\begin{array}{l}\text { Patients } 65 \text { or older } \\
\text { admitted to the ICU } \\
\text { after noncardiac } \\
\text { surgery }\end{array}$ & $\begin{array}{l}\text { Haloperidol } \\
\text { (Planned dose: } 0.5 \\
\mathrm{mg} \text { followed by } \\
\text { continuous infusion } \\
\text { at a rate of } 1 \mathrm{~mL} / \mathrm{hr} \\
(0.1 \mathrm{mg} / \mathrm{hr} \\
\text { haloperidol)), } 229\end{array}$ & $\begin{array}{l}\text { Placebo (Planned } \\
\text { dose: Normal } \\
\text { saline), } 228\end{array}$ & Intravenous & $\begin{array}{l}\text { Extrapyramidal } \\
\text { symptoms }\end{array}$ & 0 / 229 (0\%) & $0 / 228$ (0\%) & Not calculable \\
\hline
\end{tabular}




\begin{tabular}{|c|c|c|c|c|c|c|c|c|}
\hline Author, year & Population & $\begin{array}{l}\text { Intervention } \\
\text { group, } n\end{array}$ & Control group, $n$ & $\begin{array}{c}\text { Route of } \\
\text { administration }\end{array}$ & $\begin{array}{l}\text { Outcome } \\
\text { definition }\end{array}$ & $\begin{array}{c}\mathrm{n} / \mathrm{N}(\%), \text { intervention } \\
\text { group }\end{array}$ & $\begin{array}{c}\mathrm{n} / \mathrm{N} \text { (\%), control } \\
\text { group }\end{array}$ & $\begin{array}{l}\text { Relative risk (95\% } \\
\text { Cl) }\end{array}$ \\
\hline \multicolumn{9}{|l|}{$\begin{array}{l}\text { Second-generation } \\
\text { antipsychotic vs. } \\
\text { placebo }\end{array}$} \\
\hline Girard, $2010^{4}$ & $\begin{array}{l}>18 \text { years MV medical } \\
\text { and surgical ICU } \\
\text { patients }\end{array}$ & $\begin{array}{l}\text { Ziprasidone } \\
\text { (Planned dose: } 40 \\
\text { Median dose: } 113 \\
\text { (81-140), } 30\end{array}$ & $\begin{array}{l}\text { Placebo (Planned } \\
\text { dose: } 5 \mathrm{ml}), 36\end{array}$ & Oral & Akathisia & $6 / 30(20 \%)$ & $7 / 36(19 \%)$ & 1.03 (0.39 to 2.73$)$ \\
\hline Girard, $2010^{4}$ & $\begin{array}{l}>18 \text { years MV medical } \\
\text { and surgical ICU } \\
\text { patients }\end{array}$ & $\begin{array}{l}\text { Ziprasidone } \\
\text { (Planned dose: } 40 \\
\text { Median dose: } 113 \\
\text { (81-140), } 30\end{array}$ & $\begin{array}{l}\text { Placebo (Planned } \\
\text { dose: } 5 \mathrm{ml}), 36\end{array}$ & Oral & $\begin{array}{l}\text { Extrapyramidal } \\
\text { symptoms }\end{array}$ & $2 / 30(7 \%)$ & $6 / 36(17 \%)$ & 0.40 (0.09 to 1.84$)$ \\
\hline Girard, $2010^{4}$ & $\begin{array}{l}>18 \text { years MV medical } \\
\text { and surgical ICU } \\
\text { patients }\end{array}$ & $\begin{array}{l}\text { Ziprasidone } \\
\text { (Planned dose: } 40 \\
\text { Median dose: } 113 \\
\text { (81-140), } 30\end{array}$ & $\begin{array}{l}\text { Placebo (Planned } \\
\text { dose: } 5 \mathrm{ml}), 36\end{array}$ & Oral & $\begin{array}{l}\text { Neuroleptic } \\
\text { malignant } \\
\text { syndrome }\end{array}$ & $0 / 30(0 \%)$ & $0 / 36(0 \%)$ & Not calculable \\
\hline Hakim, $2012^{5}$ & $\begin{array}{l}\text { Patients } 65 \text { years or } \\
\text { older experiencing } \\
\text { subsyndromal delirium } \\
\text { after on-pump cardiac } \\
\text { surgery }\end{array}$ & $\begin{array}{l}\text { Risperidone } \\
\text { (Planned dose: } \\
0.5 \mathrm{mg}), 51\end{array}$ & $\begin{array}{l}\text { Placebo (Planned } \\
\text { dose: given every } \\
12 \text { hours), } 50\end{array}$ & Oral & $\begin{array}{l}\text { Extrapyramidal } \\
\text { symptoms }\end{array}$ & $2 / 51(3.9 \%)$ & $0 / 50(0 \%)$ & 4.90 (0.24 to 99.66$)$ \\
\hline
\end{tabular}




\begin{tabular}{|c|c|c|c|c|c|c|c|c|}
\hline Author, year & Population & $\begin{array}{l}\text { Intervention } \\
\text { group, } \mathrm{n}\end{array}$ & Control group, $n$ & $\begin{array}{c}\text { Route of } \\
\text { administration }\end{array}$ & $\begin{array}{l}\text { Outcome } \\
\text { definition }\end{array}$ & $\begin{array}{l}\text { n / N (\%), intervention } \\
\text { group }\end{array}$ & $\begin{array}{c}\text { n I N (\%), control } \\
\text { group }\end{array}$ & $\begin{array}{l}\text { Relative risk (95\% } \\
\text { Cl) }\end{array}$ \\
\hline \multicolumn{9}{|l|}{$\begin{array}{l}\text { First-generation } \\
\text { antipsychotic vs. } \\
\text { second-generation } \\
\text { antipsychotic }\end{array}$} \\
\hline Girard, $2010^{4}$ & $\begin{array}{l}>18 \text { years MV medical } \\
\text { and surgical ICU } \\
\text { patients }\end{array}$ & $\begin{array}{l}\text { Ziprasidone } \\
\text { (Planned dose: } 40 \\
\text { Median dose: } 113 \\
\text { (81-140), } 30\end{array}$ & $\begin{array}{l}\text { Haloperidol } \\
\text { (Planned dose: } 5 \\
\text { Median dose: } 15 \\
(10.8-17), 35\end{array}$ & Oral & Akathisia & $6 / 30(20 \%)$ & $10 / 35(29 \%)$ & $0.70(0.29$ to 1.70$)$ \\
\hline Girard, $2010^{4}$ & $\begin{array}{l}>18 \text { years MV medical } \\
\text { and surgical ICU } \\
\text { patients }\end{array}$ & $\begin{array}{l}\text { Ziprasidone } \\
\text { (Planned dose: } 40 \\
\text { Median dose: } 113 \\
\text { (81-140), } 30\end{array}$ & $\begin{array}{l}\text { Haloperidol } \\
\text { (Planned dose: } 5 \\
\text { Median dose: } 15 \\
(10.8-17), 35\end{array}$ & Oral & $\begin{array}{l}\text { Extrapyramidal } \\
\text { symptoms }\end{array}$ & $2 / 30(7 \%)$ & $4 / 35$ (11\%) & 0.58 (0.11 to 2.96$)$ \\
\hline Girard, $2010^{4}$ & $\begin{array}{l}>18 \text { years MV medical } \\
\text { and surgical ICU } \\
\text { patients }\end{array}$ & $\begin{array}{l}\text { Ziprasidone } \\
\text { (Planned dose: } 40 \\
\text { Median dose: } 113 \\
\text { (81-140), } 30\end{array}$ & $\begin{array}{l}\text { Haloperidol } \\
\text { (Planned dose: } 5 \\
\text { Median dose: } 15 \\
\text { (10.8-17), } 35\end{array}$ & Oral & $\begin{array}{l}\text { Neuroleptic } \\
\text { malignant } \\
\text { syndrome }\end{array}$ & $0 / 30(0 \%)$ & $0 / 35(0 \%)$ & Not calculable \\
\hline
\end{tabular}

APACHE II= Acute Physiology and Chronic Health Evaluation 2; CI=confidence interval; ICU=intensive care unit; Mg/hr=milligram per hour; mg=milligram; ml/hr=milliliter per hour; ml=milliliter; MMSE=Mini-Mental State Examination; $\mathrm{MV}=$ mechanical ventilation; $\mathrm{n}=$ sample size; $\mathrm{NaCl}=$ sodium chloride; $\mathrm{SD}=$ standard deviation 
Evidence Table D-29. Continuous neurological outcomes in studies comparing interventions to prevent development of delirium

Author, year $\mid$ Study design

Population

Intervention

Control group, $\mathbf{n}$

Route of

Outcome definition

Mean score

Mean score,

First-generation

antipsychotic vs

placebo

Khan, $2018^{8}$

\begin{tabular}{l|l} 
RCT & $\begin{array}{l}\text { English speaking } \\
\text { individuals }\end{array}$
\end{tabular}

\begin{tabular}{l|l} 
individuals & (Planned dose: \\
undergoing thoracic & $0.5 \mathrm{mg}$, Planned
\end{tabular}

surgery

Haloperido

$0.5 \mathrm{mg}$, Planned

duration: 4 days
doses total)), 68

\begin{tabular}{|l|l|l|l|l|}
$\begin{array}{l}\text { Placebo (Planned } \\
\text { dose: NR, Planned } \\
\text { duration: } 4 \text { days }(11 \\
\text { doses total)), } 67\end{array}$ & Intravenous & Akathisia, mean score & Mean: 1.2 (SD 1.4) & Mean: 1.2 (SD 1.6) \\
\hline
\end{tabular}

$\mathrm{mg}=$ =milligram; NR=not reported; RCT=randomized controlled trial; $\mathrm{SD}=$ =standard deviation 
Evidence Table D-30. Neurological outcomes in studies comparing interventions to prevent development of delirium: single arm studies

\begin{tabular}{|c|c|c|c|c|c|c|}
\hline Author, year & Study design & Population & Intervention group, $\mathbf{n}$ & Route of administration & Outcome definition & $\mathrm{n} / \mathrm{N}(\%)$, intervention group \\
\hline $\begin{array}{l}\text { van den } \\
\text { Boogaard, } \\
2013^{14}\end{array}$ & $\begin{array}{l}\text { Prospective cohort with a } \\
\text { comparison group, analyzed } \\
\text { as a single group }\end{array}$ & $\begin{array}{l}\text { Patients in the ICU } \\
\text { who are at a high risk } \\
\text { of delirium }\end{array}$ & $\begin{array}{l}\text { Haloperidol (Planned dose: } 1 \\
\text { Median dose: } 2 \mathrm{mg} \text { ), }\end{array}$ & Intravenous & $\begin{array}{l}\text { Medication stopped due to suspected } \\
\text { malignant neuroleptic syndrome (later } \\
\text { not confirmed) }\end{array}$ & $1 / 177$ (1\%) \\
\hline $\begin{array}{l}\text { van den } \\
\text { Boogaard, } \\
2013^{14}\end{array}$ & $\begin{array}{l}\text { Prospective cohort with a } \\
\text { comparison group, analyzed } \\
\text { as a single group }\end{array}$ & $\begin{array}{l}\text { Patients in the ICU } \\
\text { who are at a high risk } \\
\text { of delirium }\end{array}$ & $\begin{array}{l}\text { Haloperidol (Planned dose: } 1 \\
\text { Median dose: } 2 \mathrm{mg} \text { ), }\end{array}$ & Intravenous & $\begin{array}{l}\text { Medication stopped due to signs of } \\
\text { Parkinsonism }\end{array}$ & $1 / 177(1 \%)$ \\
\hline
\end{tabular}

CU=intensive care unit; $\mathrm{mg}=$ =milligram; $\mathrm{N}=$ sample size 
Evidence Table D-31. Short-term continuous delirium symptom outcomes for studies comparing interventions to treat delirium

\begin{tabular}{|c|c|c|c|c|c|c|c|c|}
\hline Author, year & Population & Intervention group, $\mathrm{n}$ & Control group, $\mathrm{n}$ & $\begin{array}{c}\text { Route of } \\
\text { administration }\end{array}$ & $\begin{array}{l}\text { Outcome } \\
\text { definition }\end{array}$ & $\begin{array}{l}\text { Median (IQR) } \\
\text { hours, } \\
\text { intervention } \\
\text { group } \\
\end{array}$ & $\begin{array}{l}\text { Median } \\
\text { (IQR) hours, } \\
\text { control } \\
\text { group } \\
\end{array}$ & $\begin{array}{c}\text { Measure of association } \\
(95 \% \mathrm{Cl})\end{array}$ \\
\hline \multicolumn{9}{|l|}{$\begin{array}{l}\text { Second-generation } \\
\text { antipsychotic vs. } \\
\text { placebo }\end{array}$} \\
\hline Devlin, 201026 & $\begin{array}{l}\text { Patients admitted to Medical \& } \\
\text { Surgical ICU with delirium. }\end{array}$ & $\begin{array}{l}\text { Quetiapine (Mean dose: } \\
\text { 50 Median dose: } 110 \text { (88- } \\
\text { 191 IQR) Max dose: } 200 \\
\text { Dose range: } 50 \text { to } 200 \mathrm{mg} \text { ), } \\
18\end{array}$ & $\begin{array}{l}\text { Placebo (Mean } \\
\text { dose: } 50 \text { Median } \\
\text { dose: } 210 \text { (116 - } \\
\text { 293) Max dose: } \\
\text { 200mg), } 18\end{array}$ & $\begin{array}{l}\text { Either orally or } \\
\text { NG tube }\end{array}$ & $\begin{array}{l}\text { Sedation } \\
\text { Agitated } \\
\text { Scale (SAS) } \\
\text { score } \geq 5 \text {, } \\
\text { hours }\end{array}$ & 6 (0 to 38$)$ & 36 (11 to 66$)$ & $p=0.02$ \\
\hline Tahir, $2010^{59}$ & $\begin{array}{l}\text { Patients in medical, surgical and } \\
\text { orthopedic wards, who met the } \\
\text { DSM-IV criteria for delirium on the } \\
\text { same day if they had a DRS-R-98 } \\
\text { total score of } 15 \text { or more }\end{array}$ & $\begin{array}{l}\text { Quetiapine (Mean dose: } \\
\text { 40 Dose range: } 25 \text { - } \\
\text { 175mg), } 21\end{array}$ & $\begin{array}{l}\text { Placebo (Mean } \\
\text { dose: } 25 \text { Max } \\
\text { dose: } 175 \mathrm{mg}), 21\end{array}$ & Oral & $\begin{array}{l}\text { Brief } \\
\text { Psychiatric } \\
\text { Rating Scale }\end{array}$ & Not reported & Not reported & $\begin{array}{l}\text { There were no significant } \\
\text { differences found between } \\
\text { treatment groups for MMSE, } \\
\text { BPRS, or CGI Global } \\
\text { scores. }\end{array}$ \\
\hline \multicolumn{9}{|l|}{$\begin{array}{l}\text { First-generation } \\
\text { antipsychotic vs. } \\
\text { second-generation } \\
\text { antipsychotic }\end{array}$} \\
\hline Lim, $2007^{46}$ & $\begin{array}{l}\text { Patients from internal medicine, } \\
\text { PMR, neurology, neurosurgery, } \\
\text { orthopedic surgery, or general } \\
\text { surgery that were referred to the } \\
\text { psychiatric department }\end{array}$ & $\begin{array}{l}\text { Olanzapine (Titrated (5 to } \\
20 \mathrm{mg} \text { ) }\end{array}$ & $\begin{array}{l}\text { Haloperidol } \\
\text { (Titrated (5 to } 20 \\
\text { mg) }\end{array}$ & Intramuscular & $\begin{array}{l}\text { Abnormal } \\
\text { Involuntary } \\
\text { Movement } \\
\text { Scale }\end{array}$ & Not reported & Not reported & $\mathrm{p}=\mathrm{NS}$ \\
\hline Maneeton, $2013^{49}$ & $\begin{array}{l}\text { General adult population (age 18- } \\
\text { 75) with hyperactive delirium, } \\
\text { referred to psychiatry consult liaison } \\
\text { service at tertiary care hospital in } \\
\text { Thailand }\end{array}$ & $\begin{array}{l}\text { Quetiapine (Mean dose: } \\
\text { 67.6 (SD 9.7) Dose range: } \\
\text { 25-100mg), }\end{array}$ & $\begin{array}{l}\text { Haloperidol (Mean } \\
\text { dose: } 0.8 \text { (SD 0.3) } \\
\text { Dose range: } 0.5- \\
\text { 2.0mg), }\end{array}$ & Oral & $\begin{array}{l}\text { Total sleep } \\
\text { times (hours) }\end{array}$ & $\begin{array}{l}\text { Change from } \\
\text { baseline: } \\
\text { Mean: } 6.5 \text { (SD: } \\
\text { 3) }\end{array}$ & $\begin{array}{l}\text { Change from } \\
\text { baseline: } \\
\text { Mean: } 6.1 \\
\text { (SD: } 3.4 \text { ) }\end{array}$ & $\begin{array}{l}-0.40(95 \% \mathrm{Cl}:-2.14 \text { to } \\
1.34)\end{array}$ \\
\hline
\end{tabular}

BPRS= Brief Psychiatric Rating Scale; CGI=Clinical Global Impressions Scale; CI=confidence interval; DRS-R-98= Delirium Rating Scale Revised-98; DSM-IV=Diagnostic and Statistical Manual of Mental Disorders, $4^{\text {th }}$ ed;

ICU=intensive care unit; IQR=interquartile range; mg=milligram; MMSE=Mini-Mental State Examination; NG=nadogastric tube; p=p-value; SAS=Sedation Agitated Scale; SD=standard deviation 


\begin{tabular}{|c|c|c|c|c|c|c|c|c|}
\hline Author, year & Population & $\begin{array}{c}\text { Intervention group, } \\
\mathbf{n}\end{array}$ & Control group, $\mathbf{n}$ & $\begin{array}{c}\text { Route of } \\
\text { administration }\end{array}$ & Outcome definition & $\begin{array}{c}n / \mathrm{N}(\%) \\
\text { intervention group }\end{array}$ & $\begin{array}{l}\text { n I N (\%), control } \\
\text { group }\end{array}$ & $\begin{array}{c}\text { Relative risk (95\% } \\
\text { CI) }\end{array}$ \\
\hline \multicolumn{9}{|l|}{$\begin{array}{l}\text { First-generation } \\
\text { antipsychotic vs. } \\
\text { placebo }\end{array}$} \\
\hline Page, $2013^{10}$ & $\begin{array}{l}\text { ICU patients } \\
\text { needing MV within } \\
72 \text { hours of } \\
\text { admission were } \\
\text { enrolled }\end{array}$ & $\begin{array}{l}\text { Haloperidol (Planned } \\
\text { dose: } 2.5 \mathrm{mg}), 71\end{array}$ & $\begin{array}{l}\text { Placebo (Planned } \\
\text { dose: } 0.5 \mathrm{ml}), 70\end{array}$ & Intravenous & RASS +2 & $9 / 71(13 \%)$ & $14 / 70(20 \%)$ & $0.63(0.29$ to 1.37$)$ \\
\hline \multicolumn{9}{|l|}{$\begin{array}{l}\text { First-generation } \\
\text { antipsychotic vs. } \\
\text { second-generation } \\
\text { antipsychotic } \\
\end{array}$} \\
\hline Grover, $2011^{30}$ & $\begin{array}{l}\text { Consecutive } \\
\text { patients }>-18 \text { years } \\
\text { old referred to } \\
\text { psychiatry and } \\
\text { diagnosed with } \\
\text { delirium }\end{array}$ & $\begin{array}{l}\text { Olanzapine (Mean } \\
\text { dose: } 3.05 \mathrm{mg} \text { Dose } \\
\text { range: } 1.25-10 \mathrm{mg} \\
\text { per day), } 23\end{array}$ & $\begin{array}{l}\text { Haloperidol (Mean } \\
\text { dose: } 0.88 \text { Dose } \\
\text { range: } 0.25-5 \mathrm{mg} \text { per } \\
\text { day), } 21\end{array}$ & Oral & $\begin{array}{l}\text { Increased duration of } \\
\text { sleep }\end{array}$ & $0 / 23(0 \%)$ & $1 / 21(5 \%)$ & 0.31 (0.01 to 7.12$)$ \\
\hline Grover, $2011^{30}$ & $\begin{array}{l}\text { Consecutive } \\
\text { patients }>-18 \text { years } \\
\text { old referred to } \\
\text { psychiatry and } \\
\text { diagnosed with } \\
\text { delirium }\end{array}$ & $\begin{array}{l}\text { Risperidone (Mean } \\
\text { dose: } 0.95 \mathrm{mg} \text { Dose } \\
\text { range: } 0.5-2.0 \mathrm{mg} \text { per } \\
\text { day), } 20\end{array}$ & $\begin{array}{l}\text { Haloperidol (Mean } \\
\text { dose: } 0.88 \text { Dose } \\
\text { range: } 0.25-5 \mathrm{mg} \text { per } \\
\text { day), } 21\end{array}$ & Oral & $\begin{array}{l}\text { Increased duration of } \\
\text { sleep }\end{array}$ & $1 / 20(5 \%)$ & $1 / 21(5 \%)$ & 1.05 (0.07 to 15.68$)$ \\
\hline \multicolumn{9}{|l|}{$\begin{array}{l}\text { Second-generation } \\
\text { antipsychotic vs. } \\
\text { second-generation } \\
\text { antipsychotic }\end{array}$} \\
\hline Grover, $2011^{30}$ & $\begin{array}{l}\text { Consecutive } \\
\text { patients }>-18 \text { years } \\
\text { old referred to } \\
\text { psychiatry and } \\
\text { diagnosed with } \\
\text { delirium }\end{array}$ & $\begin{array}{l}\text { Risperidone (Mean } \\
\text { dose: } 0.95 \mathrm{mg} \text { Dose } \\
\text { range: } 0.5-2.0 \mathrm{mg} \text { per } \\
\text { day), } 20\end{array}$ & $\begin{array}{l}\text { Olanzapine (Mean } \\
\text { dose: } 3.05 \mathrm{mg} \text { Dose } \\
\text { range: } 1.25-10 \mathrm{mg} \\
\text { per day), } 23\end{array}$ & Oral & $\begin{array}{l}\text { Increased duration of } \\
\text { sleep }\end{array}$ & $1 / 20(5 \%)$ & $0 / 23(0 \%)$ & $3.43(0.15$ to 79.74$)$ \\
\hline
\end{tabular}


Evidence Table D-33. Continuous delirium severity outcomes for studies comparing interventions to treat delirium

\begin{tabular}{|c|c|c|c|c|c|c|c|c|}
\hline Author, year & Population & Intervention group, $\mathbf{n}$ & Control group, $\mathbf{n}$ & $\begin{array}{c}\text { Route of } \\
\text { administration }\end{array}$ & $\begin{array}{l}\text { Outcome } \\
\text { definition }\end{array}$ & $\begin{array}{c}\text { Severity score, } \\
\text { intervention group }\end{array}$ & $\begin{array}{l}\text { Severity score, } \\
\text { control group }\end{array}$ & $\begin{array}{c}\text { Mean between- } \\
\text { group difference } \\
(95 \% \mathrm{Cl})\end{array}$ \\
\hline \multicolumn{9}{|l|}{$\begin{array}{l}\text { First-generation } \\
\text { antipsychotic vs. placebo }\end{array}$} \\
\hline Agar, $2017^{17}$ & $\begin{array}{l}\text { Patients in } \\
\text { Hospice and } \\
\text { palliative care with } \\
\text { delirium }\end{array}$ & $\begin{array}{l}\text { Haloperidol (Max dose: } 4 \\
\text { mg/day), } 59\end{array}$ & $\begin{array}{l}\text { Placebo (Max dose: } \\
4 \text { mg/day), } 64\end{array}$ & Oral & MDAS Scores & $\begin{array}{l}\text { Baseline: Mean } 15.99 \\
\text { (95\% Cl: } 13.19 \text { to } \\
18.74) \\
\text { Final: Mean } 13.61 \\
\text { (95\% Cl: } 10.86 \text { to } \\
16.46)\end{array}$ & $\begin{array}{l}\text { Baseline: Mean } 15.01 \\
\text { (95\% Cl: } 12.21 \text { to } \\
17.81) \\
\text { Final: Mean } 10.26 \\
\text { (95\% Cl: } 7.41 \text { to } \\
13.05)\end{array}$ & $\begin{array}{l}0.75 \text { (95\% Cl: - } \\
0.03 \text { to } 1.51) \\
p=0.06\end{array}$ \\
\hline Agar, $2017^{17}$ & $\begin{array}{l}\text { Patients in } \\
\text { Hospice and } \\
\text { palliative care with } \\
\text { delirium }\end{array}$ & $\begin{array}{l}\text { Haloperidol (Max dose: } 4 \\
\text { mg/day), }\end{array}$ & $\begin{array}{l}\text { Placebo (Max dose: } \\
4 \text { mg/day), }\end{array}$ & Oral & $\begin{array}{l}\text { NuDESC (Scores } \\
2,3,4)\end{array}$ & $\begin{array}{l}\text { Baseline: Mean } 2.6 \\
\text { (SD 1.48) } \\
\text { Final: Not reported }\end{array}$ & $\begin{array}{l}\text { Baseline: Mean } 2.54 \\
\text { (SD 1.43) } \\
\text { Final: Not reported }\end{array}$ & $\begin{array}{l}0.24 \text { (95\% Cl: } \\
0.06 \text { to } 0.42) \\
p=0.009\end{array}$ \\
\hline $\mathrm{Hu}, 2006^{35}$ & $\begin{array}{l}\text { Inpatients with } \\
\text { senile delirium } \\
\text { aged 65-99 }\end{array}$ & $\begin{array}{l}\text { Haloperidol (Dose range: } \\
2.5-10 \mathrm{mg}), 72\end{array}$ & Not reported29 & Intramuscular & $\begin{array}{l}\text { CGI-GI (global } \\
\text { improvement item } \\
\text { of the clinical global } \\
\text { impression scale) }\end{array}$ & $\begin{array}{l}\text { Baseline: Mean } 4.94 \\
\text { (SD 0.69) } \\
\text { Final: Mean } 1.79 \text { (SD } \\
\text { 1.12) }\end{array}$ & $\begin{array}{l}\text { Baseline: Mean } 5.21 \\
\text { (SD 0.77) } \\
\text { Final: Mean } 3.97 \text { (SD } \\
\text { 1.76) }\end{array}$ & $\begin{array}{l}-1.91(95 \% \mathrm{Cl}:- \\
2.85 \text { to }-0.97)\end{array}$ \\
\hline $\mathrm{Hu}, 2006^{35}$ & $\begin{array}{l}\text { Inpatients with } \\
\text { senile delirium } \\
\text { aged 65-99 }\end{array}$ & $\begin{array}{l}\text { Haloperidol (Dose range: } \\
2.5 \text {-10mg), } 72\end{array}$ & Not reported29 & Intramuscular & DRS score & $\begin{array}{l}\text { Baseline: Mean } 24.3 \\
\text { (SD 2.5) } \\
\text { Final: Mean } 7.2 \text { (SD } \\
\text { 4.6) }\end{array}$ & $\begin{array}{l}\text { Baseline: Mean } 24.7 \\
\text { (SD 3.5) } \\
\text { Final: Mean } 17.6 \text { (SD } \\
\text { 9.3) }\end{array}$ & $\begin{array}{l}-10.00(95 \% \mathrm{Cl}:- \\
14.58 \text { to }-5.42)\end{array}$ \\
\hline \multicolumn{9}{|l|}{$\begin{array}{l}\text { Second-generation } \\
\text { antipsychotic vs. placebo }\end{array}$} \\
\hline Agar, $2017^{17}$ & $\begin{array}{l}\text { Patients in } \\
\text { Hospice and } \\
\text { palliative care with } \\
\text { delirium }\end{array}$ & $\begin{array}{l}\text { Risperidone (Max dose: } 4 \\
\mathrm{mg} / \mathrm{d}), 46\end{array}$ & $\begin{array}{l}\text { Placebo (Max dose: } \\
4 \text { mg/day), } 64\end{array}$ & Oral & MDAS Scores & $\begin{array}{l}\text { Baseline: Mean } 16.97 \\
\text { (95\% Cl: } 14.08 \text { to } \\
\text { 19.81) } \\
\text { Final: Mean } 14.69 \\
\text { (95\% Cl: } 11.7 \text { to } \\
17.62 \text { ) }\end{array}$ & $\begin{array}{l}\text { Baseline: Mean } 15.01 \\
\text { (95\% Cl: } 12.21 \text { to } \\
17.81) \\
\text { Final: Mean } 10.26 \\
\text { (95\% Cl: } 7.41 \text { to } \\
13.05 \text { ) }\end{array}$ & $\begin{array}{l}0.96(95 \% \mathrm{Cl}: \\
0.16 \text { to } 1.77) \\
p=0.001\end{array}$ \\
\hline Agar, $2017^{17}$ & $\begin{array}{l}\text { Patients in } \\
\text { Hospice and } \\
\text { palliative care with } \\
\text { delirium }\end{array}$ & $\begin{array}{l}\text { Risperidone (Max dose: } 4 \\
\mathrm{mg} / \mathrm{d} \text { ), }\end{array}$ & $\begin{array}{l}\text { Placebo (Max dose: } \\
4 \text { mg/day), }\end{array}$ & Oral & $\begin{array}{l}\text { NuDESC (Scores } \\
2,3,4)\end{array}$ & $\begin{array}{l}\text { Baseline: Mean } 2.54 \\
\text { (SD 1.23) } \\
\text { Final: Not reported }\end{array}$ & $\begin{array}{l}\text { Baseline: Mean } 2.54 \\
\text { (SD 1.43) } \\
\text { Final: Not reported }\end{array}$ & $\begin{array}{l}0.48(95 \% \mathrm{Cl}: \\
0.09 \text { to } 0.86) \\
p=0.02\end{array}$ \\
\hline
\end{tabular}




\begin{tabular}{|c|c|c|c|c|c|c|c|c|}
\hline Author, year & Population & Intervention group, $\mathbf{n}$ & Control group, $n$ & $\begin{array}{c}\text { Route of } \\
\text { administration }\end{array}$ & $\begin{array}{l}\text { Outcome } \\
\text { definition }\end{array}$ & $\begin{array}{c}\text { Severity score, } \\
\text { intervention group }\end{array}$ & $\begin{array}{l}\text { Severity score, } \\
\text { control group }\end{array}$ & $\begin{array}{c}\text { Mean between- } \\
\text { group difference } \\
(95 \% \mathrm{Cl})\end{array}$ \\
\hline $\mathrm{Hu}, 2006^{35}$ & $\begin{array}{l}\text { Inpatients with } \\
\text { senile delirium } \\
\text { aged 65-99 }\end{array}$ & $\begin{array}{l}\text { Olanzapine (Dose range: } \\
1.25-20 \mathrm{mg}), 74\end{array}$ & Not reported29 & $\begin{array}{l}\text { Oral or } \\
\text { sublingually }\end{array}$ & $\begin{array}{l}\text { CGI-GI (global } \\
\text { improvement item } \\
\text { of the clinical global } \\
\text { impression scale) }\end{array}$ & $\begin{array}{l}\text { Baseline: Mean } 4.85 \\
\text { (SD 0.95) } \\
\text { Final: Mean } 2.05 \text { (SD } \\
0.99 \text { ) }\end{array}$ & $\begin{array}{l}\text { Baseline: Mean } 5.21 \\
\text { (SD 0.77) } \\
\text { Final: Mean } 3.97 \text { (SD } \\
1.76 \text { ) }\end{array}$ & $\begin{array}{l}-1.56(95 \% \mathrm{Cl}:- \\
2.54 \text { to }-0.58)\end{array}$ \\
\hline $\mathrm{Hu}, 2006^{35}$ & $\begin{array}{l}\text { Inpatients with } \\
\text { senile delirium } \\
\text { aged 65-99 }\end{array}$ & $\begin{array}{l}\text { Olanzapine (Dose range: } \\
1.25-20 \mathrm{mg}), 74\end{array}$ & Not reported29 & $\begin{array}{l}\text { Oral or } \\
\text { sublingually }\end{array}$ & DRS score & $\begin{array}{l}\text { Change from } \\
\text { baseline: Mean - } 17.2 \\
\text { (Not reported) }\end{array}$ & $\begin{array}{l}\text { Change from } \\
\text { baseline: Mean }-7.1 \\
\text { (Not reported) }\end{array}$ & $\begin{array}{l}-10.10(95 \% \mathrm{Cl}:- \\
14.71 \text { to }-5.49)\end{array}$ \\
\hline Tahir, $2010^{59}$ & $\begin{array}{l}\text { Patients in } \\
\text { medical, surgical } \\
\text { and orthopedic } \\
\text { wards, who met } \\
\text { the DSM-IV } \\
\text { criteria for delirium } \\
\text { on the same day if } \\
\text { they had a DRS- } \\
\text { R-98 total score of } \\
15 \text { or more }\end{array}$ & $\begin{array}{l}\text { Quetiapine (Mean dose: } \\
\text { 40 Dose range: } 25 \text { - } \\
\text { 175mg), } 21\end{array}$ & $\begin{array}{l}\text { Placebo (Mean } \\
\text { dose: } 25 \text { Max dose: } \\
\text { 175mg), } 21\end{array}$ & Oral & DRS-R-98 & $\begin{array}{l}\text { Baseline: Mean } 19.07 \\
\text { (SE 2.921) } \\
\text { Final: Mean } 9.673 \\
\text { (SE 2.647) }\end{array}$ & $\begin{array}{l}\text { Baseline: Mean } 19.07 \\
\text { (SE 2.921) } \\
\text { Final: Mean } 12.255 \\
\text { (SE 2.204) }\end{array}$ & $\begin{array}{l}-2.58(95 \% \mathrm{Cl}:- \\
15.45 \text { to } 10.29)\end{array}$ \\
\hline Tahir, $2010^{59}$ & $\begin{array}{l}\text { Patients in } \\
\text { medical, surgical } \\
\text { and orthopedic } \\
\text { wards, who met } \\
\text { the DSM-IV } \\
\text { criteria for delirium } \\
\text { on the same day if } \\
\text { they had a DRS- } \\
\text { R-98 total score of } \\
15 \text { or more }\end{array}$ & $\begin{array}{l}\text { Quetiapine (Mean dose: } \\
\text { 40mg Dose range: } 25 \text { - } \\
\text { 175mg), } 21\end{array}$ & $\begin{array}{l}\text { Placebo (Mean } \\
\text { dose: } 25 \mathrm{mg} \mathrm{Max} \\
\text { dose: } 175 \mathrm{mg} \text { ), } 21\end{array}$ & Oral & DRS-R-98 & $\begin{array}{l}\text { Baseline: Mean } 19.07 \\
\text { (SE 2.921) } \\
\text { Final: Mean } 7.132 \\
\text { (SE 3.347) }\end{array}$ & $\begin{array}{l}\text { Baseline: Mean } 19.07 \\
\text { (SE 2.921) } \\
\text { Final: Mean } 7.387 \\
\text { (SE 3.264) }\end{array}$ & $\begin{array}{l}-0.26(95 \% \mathrm{Cl}:- \\
15.21 \text { to } 14.70)\end{array}$ \\
\hline \multicolumn{9}{|c|}{$\begin{array}{l}\text { First-generation } \\
\text { antipsychotic vs. first- } \\
\text { generation antipsychotic }\end{array}$} \\
\hline Breitbart, $1996^{22}$ & $\begin{array}{l}\text { Medically } \\
\text { hospitalized adult } \\
\text { patients with AIDS } \\
\text { and a DRS score } \\
\text { of } 13 \text { or greater } \\
\text { (delirium) }\end{array}$ & $\begin{array}{l}\text { Haloperidol (Planned } \\
\text { dose: oral: } 0.25-5.0 \mathrm{mg} \text {, } \\
\text { Intramuscular: } 0.125- \\
\text { 3.0mg Mean dose: } 1.4 \\
\text { Dose range: } 0.4-3.6 \mathrm{mg} \text { ), } \\
11\end{array}$ & $\begin{array}{l}\text { Chlorpromazine } \\
\text { (Planned dose: Oral: } \\
\text { 10-200mg, } \\
\text { Intramuscular: 5- } \\
\text { 100mg Mean dose: } \\
\text { 36 Dose range: 10- } \\
\text { 80mg), } 13\end{array}$ & $\begin{array}{l}\text { Oral or } \\
\text { intramuscular }\end{array}$ & $\begin{array}{l}\text { Delirium Rating } \\
\text { Scale (older } \\
\text { version) score }\end{array}$ & $\begin{array}{l}\text { Baseline: Mean } 20.45 \\
\text { (SD 3.45) } \\
\text { Final: Mean } 11.64 \\
\text { (SD 6.1) }\end{array}$ & $\begin{array}{l}\text { Baseline: Mean } 20.62 \\
\text { (SD 3.88) } \\
\text { Final: Mean } 11.85 \\
\text { (SD 6.74) }\end{array}$ & $\begin{array}{l}-0.04(95 \% \mathrm{Cl}:- \\
6.17 \text { to } 6.09)\end{array}$ \\
\hline
\end{tabular}




\begin{tabular}{|c|c|c|c|c|c|c|c|c|}
\hline Author, year & Population & Intervention group, $\mathbf{n}$ & Control group, $\mathbf{n}$ & $\begin{array}{c}\text { Route of } \\
\text { administration }\end{array}$ & $\begin{array}{l}\text { Outcome } \\
\text { definition }\end{array}$ & $\begin{array}{c}\text { Severity score, } \\
\text { intervention group }\end{array}$ & $\begin{array}{l}\text { Severity score, } \\
\text { control group }\end{array}$ & $\begin{array}{l}\text { Mean between- } \\
\text { group difference } \\
(95 \% \mathrm{Cl})\end{array}$ \\
\hline \multicolumn{9}{|c|}{$\begin{array}{l}\text { First-generation } \\
\text { antipsychotic vs. second- } \\
\text { generation antipsychotic }\end{array}$} \\
\hline Grover, $2011^{30}$ & $\begin{array}{l}\text { Consecutive } \\
\text { patients }>-18 \\
\text { years old referred } \\
\text { to psychiatry and } \\
\text { diagnosed with } \\
\text { delirium }\end{array}$ & $\begin{array}{l}\text { Risperidone (Mean dose: } \\
0.95 \mathrm{mg} \text { Dose range: } 0.5- \\
2.0 \mathrm{mg} \text { per day), } 20\end{array}$ & $\begin{array}{l}\text { Haloperidol (Mean } \\
\text { dose: } 0.88 \text { Dose } \\
\text { range: } 0.25-5 \mathrm{mg} \text { per } \\
\text { day), } 21\end{array}$ & Oral & DRS-R-98 & $\begin{array}{l}\text { Baseline: Mean } 23.8 \\
\text { (SD 5.16) } \\
\text { Final: Mean } 8 \text { (SD } \\
7.27)\end{array}$ & $\begin{array}{l}\text { Baseline: Mean } 21.85 \\
\text { (SD 4.77) } \\
\text { Final: Mean } 6.09 \text { (SD } \\
7.19)\end{array}$ & $\begin{array}{l}-0.04(95 \% \mathrm{Cl}:- \\
5.82 \text { to } 5.74)\end{array}$ \\
\hline Grover, $2011^{30}$ & $\begin{array}{l}\text { Consecutive } \\
\text { patients }>-18 \\
\text { years old referred } \\
\text { to psychiatry and } \\
\text { diagnosed with } \\
\text { delirium }\end{array}$ & $\begin{array}{l}\text { Olanzapine (Mean dose: } \\
3.05 \mathrm{mg} \text { Dose range: } 1.25- \\
10 \mathrm{mg} \text { per day), } 23\end{array}$ & $\begin{array}{l}\text { Haloperidol (Mean } \\
\text { dose: } 0.88 \text { Dose } \\
\text { range: } 0.25-5 \mathrm{mg} \text { per } \\
\text { day), } 21\end{array}$ & Oral & DRS-R-98 & $\begin{array}{l}\text { Baseline: Mean } 22.56 \\
\text { (SD 4.49) } \\
\text { Final: Mean } 9.17 \text { (SD } \\
\text { 8.65) }\end{array}$ & $\begin{array}{l}\text { Baseline: Mean } 21.85 \\
\text { (SD 4.77) } \\
\text { Final: Mean } 6.09 \text { (SD } \\
\text { 7.19) }\end{array}$ & $\begin{array}{l}2.37 \text { (95\% Cl: - } \\
3.26 \text { to } 8.00)\end{array}$ \\
\hline Grover, $2016^{31}$ & $\begin{array}{l}\text { Patients }>18 \\
\text { years old referred } \\
\text { to Psychiatry who } \\
\text { met DSM-IV } \\
\text { criteria for delirium }\end{array}$ & $\begin{array}{l}\text { Quetiapine (Mean dose: } \\
26.63 \pm 15.61 \text { Dose } \\
\text { range: } 12.5-75 \mathrm{mg}), 31\end{array}$ & $\begin{array}{l}\text { Haloperidol (Mean } \\
\text { dose: } 0.67 \pm 0.35 \\
\text { Dose range: } 0.25- \\
1.25 \mathrm{mg} \text { ), } 32\end{array}$ & Oral & DRS-R-98 & $\begin{array}{l}\text { Change from } \\
\text { baseline: Mean } 4.86 \\
\text { (Not reported) }\end{array}$ & $\begin{array}{l}\text { Change from } \\
\text { baseline: Mean } 4.94 \\
\text { (Not reported) }\end{array}$ & $\begin{array}{l}-0.52(95 \% \mathrm{Cl}:- \\
3.57 \text { to } 2.53)\end{array}$ \\
\hline Han, $2004^{32}$ & $\begin{array}{l}\text { All patients } \\
\text { presenting with } \\
\text { altered mental } \\
\text { status (from both } \\
\text { ICU and non-ICU } \\
\text { units) who were } \\
\text { referred to the } \\
\text { consulting } \\
\text { psychiatry division } \\
\text { were evaluated. }\end{array}$ & $\begin{array}{l}\text { Risperidone (Mean dose: } \\
1.02 \text { Dose range: } 0.5 \text { to } \\
2.0 \mathrm{mg}), 12\end{array}$ & $\begin{array}{l}\text { Haloperidol (Mean } \\
\text { dose: } 1.7 \text { Dose } \\
\text { range: } 1.0 \text { to } 3.0 \mathrm{mg} \text { ), } \\
12\end{array}$ & Oral & DRS-R-98 (MDAS) & $\begin{array}{l}\text { Baseline: Mean } 33.8 \\
\text { (SD 6.8) } \\
\text { Final: Mean } 20.94 \\
\text { (SD 8.9) }\end{array}$ & $\begin{array}{l}\text { Baseline: Mean } 32.82 \\
\text { (SD 6.66) } \\
\text { Final: Mean } 15.18 \\
\text { (SD 10.48) }\end{array}$ & $\begin{array}{l}4.78(95 \% \mathrm{Cl}:- \\
5.95 \text { to } 15.51)\end{array}$ \\
\hline $\mathrm{Hu}, 2006^{35}$ & $\begin{array}{l}\text { Inpatients with } \\
\text { senile delirium } \\
\text { aged 65-99 }\end{array}$ & $\begin{array}{l}\text { Olanzapine (Dose range: } \\
1.25-20 \mathrm{mg}), 74\end{array}$ & $\begin{array}{l}\text { Haloperidol (Dose } \\
\text { range: } 2.5-10 \mathrm{mg}) \text {, } \\
72\end{array}$ & $\begin{array}{l}\text { Oral or } \\
\text { sublingually }\end{array}$ & $\begin{array}{l}\text { CGI-GI (global } \\
\text { improvement item } \\
\text { of the clinical global } \\
\text { impression scale) }\end{array}$ & $\begin{array}{l}\text { Baseline: Mean } 4.85 \\
\text { (SD 0.95) } \\
\text { Final: Mean } 2.05 \text { (SD } \\
0.99 \text { ) }\end{array}$ & $\begin{array}{l}\text { Baseline: Mean } 4.94 \\
\text { (SD 0.69) } \\
\text { Final: Mean } 1.79 \text { (SD } \\
\text { 1.12) }\end{array}$ & $\begin{array}{l}0.35(95 \% \mathrm{Cl}:- \\
0.14 \text { to } 0.84)\end{array}$ \\
\hline $\mathrm{Hu}, 2006^{35}$ & $\begin{array}{l}\text { Inpatients with } \\
\text { senile delirium } \\
\text { aged 65-99 }\end{array}$ & $\begin{array}{l}\text { Olanzapine (Dose range: } \\
1.25-20 \mathrm{mg}), 74\end{array}$ & $\begin{array}{l}\text { Haloperidol (Dose } \\
\text { range: } 2.5-10 \mathrm{mg} \text { ), } \\
72\end{array}$ & $\begin{array}{l}\text { Oral or } \\
\text { sublingually }\end{array}$ & DRS score & $\begin{array}{l}\text { Change from } \\
\text { baseline: Mean - } 17.2 \\
\text { (Not reported ) }\end{array}$ & $\begin{array}{l}\text { Change from } \\
\text { baseline: Mean -17.1 } \\
\text { (Not reported) }\end{array}$ & $\begin{array}{l}0.00(95 \% \mathrm{Cl}:- \\
1.78 \text { to } 1.78)\end{array}$ \\
\hline
\end{tabular}




\begin{tabular}{|c|c|c|c|c|c|c|c|c|}
\hline Author, year & Population & Intervention group, $\mathbf{n}$ & Control group, $n$ & $\begin{array}{c}\text { Route of } \\
\text { administration }\end{array}$ & $\begin{array}{l}\text { Outcome } \\
\text { definition }\end{array}$ & $\begin{array}{c}\text { Severity score, } \\
\text { intervention group }\end{array}$ & $\begin{array}{l}\text { Severity score, } \\
\text { control group }\end{array}$ & $\begin{array}{c}\text { Mean between- } \\
\text { group difference } \\
(95 \% \mathrm{Cl})\end{array}$ \\
\hline Jain, $2017^{38}$ & $\begin{array}{l}\text { Medical and } \\
\text { surgical inpatients }\end{array}$ & $\begin{array}{l}\text { Olanzapine (Mean dose: } \\
5.49 \mathrm{mg}), 47\end{array}$ & $\begin{array}{l}\text { Haloperidol (Mean } \\
\text { dose: } 2.10 \mathrm{mg} \text { ), } 53\end{array}$ & Oral & MDAS & $\begin{array}{l}\text { Baseline: Mean } 18.49 \\
\text { (Not reported) } \\
\text { Final: Mean } 8.43 \text { (Not } \\
\text { reported) }\end{array}$ & $\begin{array}{l}\text { Baseline: Mean } 17.79 \\
\text { (Not reported) } \\
\text { Final: Mean } 8 \text { (Not } \\
\text { reported) }\end{array}$ & $\begin{array}{l}-0.27 \text { (95\% Cl: } \\
\text { Not reported) }\end{array}$ \\
\hline Lim, $2007^{46}$ & $\begin{array}{l}\text { Patients from } \\
\text { internal medicine, } \\
\text { PMR, neurology, } \\
\text { neurosurgery, } \\
\text { orthopedic } \\
\text { surgery, or } \\
\text { general surgery } \\
\text { that were referred } \\
\text { to the psychiatric } \\
\text { department }\end{array}$ & $\begin{array}{l}\text { Olanzapine (Titrated (5 to } \\
20 \mathrm{mg}) 31\end{array}$ & $\begin{array}{l}\text { Haloperidol (Titrated } \\
\text { (5 to } 20 \mathrm{mg} \text { ) } 31\end{array}$ & Intramuscular & DRS-R-98 & $\begin{array}{l}\text { Baseline: Mean } 27.2 \\
\text { (SD 4) } \\
\text { Final: Mean } 6.8 \text { (SD } \\
\text { 4) }\end{array}$ & $\begin{array}{l}\text { Baseline: Mean } 22.8 \\
\text { (SD 9.4) } \\
\text { Final: Mean } 8 \text { (SD } \\
\text { 4.4) }\end{array}$ & $\begin{array}{l}-5.60(95 \% \mathrm{Cl}:- \\
11.61 \text { to } 0.41)\end{array}$ \\
\hline Lin, $2008^{47}$ & $\begin{array}{l}\text { Patients receiving } \\
\text { hospice or } \\
\text { palliative care with } \\
\text { advanced cancer } \\
\text { and met DSM-IV } \\
\text { criteria for delirium }\end{array}$ & $\begin{array}{l}\text { Olanzapine (Planned } \\
\text { dose: start dose 5mg Max } \\
\text { dose: } 15 \mathrm{mg} \text { ), } 5\end{array}$ & $\begin{array}{l}\text { Haloperidol } \\
\text { (Planned dose: start } \\
\text { dose } 5 \mathrm{mg} \text { Max dose: } \\
\text { 15mg), } 7\end{array}$ & Oral & $\begin{array}{l}\text { Clinical Global } \\
\text { Impression - } \\
\text { Severity }\end{array}$ & $\begin{array}{l}\text { Baseline: Mean } 5 \text { (SD } \\
\text { 1.03) } \\
\text { Final: Mean } 3.6 \text { (SD } \\
0.55)\end{array}$ & $\begin{array}{l}\text { Baseline: Mean } 4.5 \\
\text { (SD 1.02) } \\
\text { Final: Mean } 3.57 \text { (SD } \\
0.98 \text { ) }\end{array}$ & $\begin{array}{l}-0.47 \text { (95\% Cl: }- \\
1.77 \text { to } 0.83)\end{array}$ \\
\hline Lin, $2008^{47}$ & $\begin{array}{l}\text { Patients receiving } \\
\text { hospice or } \\
\text { palliative care with } \\
\text { advanced cancer } \\
\text { and met DSM-IV } \\
\text { criteria for delirium }\end{array}$ & $\begin{array}{l}\text { Olanzapine (Planned } \\
\text { dose: start dose } 5 \mathrm{mg} \text { Max } \\
\text { dose: } 15 \mathrm{mg}), 10\end{array}$ & $\begin{array}{l}\text { Haloperidol } \\
\text { (Planned dose: start } \\
\text { dose } 5 \mathrm{mg} \text { Max dose: } \\
\text { 15mg), } 14\end{array}$ & Oral & $\begin{array}{l}\text { Clinical Global } \\
\text { Impression - } \\
\text { Severity }\end{array}$ & $\begin{array}{l}\text { Baseline: Mean } 5 \text { (SD } \\
1.03) \\
\text { Final: Mean } 4.1 \text { (SD } \\
1.2)\end{array}$ & $\begin{array}{l}\text { Baseline: Mean } 4.5 \\
\text { (SD 1.02) } \\
\text { Final: Mean } 3.79 \text { (SD } \\
0.89)\end{array}$ & $\begin{array}{l}-0.19 \text { (95\% Cl: }- \\
1.46 \text { to } 1.08)\end{array}$ \\
\hline Lin, $2008^{47}$ & $\begin{array}{l}\text { Patients receiving } \\
\text { hospice or } \\
\text { palliative care with } \\
\text { advanced cancer } \\
\text { and met DSM-IV } \\
\text { criteria for delirium }\end{array}$ & $\begin{array}{l}\text { Olanzapine (Planned } \\
\text { dose: start dose } 5 \mathrm{mg} \text { Max } \\
\text { dose: } 15 \mathrm{mg}), 14\end{array}$ & $\begin{array}{l}\text { Haloperidol } \\
\text { (Planned dose: start } \\
\text { dose } 5 \mathrm{mg} \text { Max dose: } \\
\text { 15mg), } 14\end{array}$ & Oral & $\begin{array}{l}\text { Clinical Global } \\
\text { Impression- } \\
\text { Severity }\end{array}$ & $\begin{array}{l}\text { Baseline: Mean } 5 \text { (SD } \\
\text { 1.03) } \\
\text { Final: Mean } 4.07 \text { (SD } \\
1.21)\end{array}$ & $\begin{array}{l}\text { Baseline: Mean } 4.5 \\
\text { (SD 1.02) } \\
\text { Final: Mean } 3.57 \text { (SD } \\
0.65)\end{array}$ & $\begin{array}{l}0.00(95 \% \mathrm{Cl}:- \\
1.20 \text { to } 1.20)\end{array}$ \\
\hline
\end{tabular}




\begin{tabular}{|c|c|c|c|c|c|c|c|c|}
\hline Author, year & Population & Intervention group, $\mathbf{n}$ & Control group, $\mathrm{n}$ & $\begin{array}{c}\text { Route of } \\
\text { administration }\end{array}$ & $\begin{array}{l}\text { Outcome } \\
\text { definition }\end{array}$ & $\begin{array}{c}\text { Severity score, } \\
\text { intervention group }\end{array}$ & $\begin{array}{l}\text { Severity score, } \\
\text { control group }\end{array}$ & $\begin{array}{l}\text { Mean between- } \\
\text { group difference } \\
(95 \% \mathrm{Cl})\end{array}$ \\
\hline Lin, $2008^{47}$ & $\begin{array}{l}\text { Patients receiving } \\
\text { hospice or } \\
\text { palliative care with } \\
\text { advanced cancer } \\
\text { and met DSM-IV } \\
\text { criteria for delirium }\end{array}$ & $\begin{array}{l}\text { Olanzapine (Planned } \\
\text { dose: start dose 5mg Max } \\
\text { dose: } 15 \mathrm{mg}), 14\end{array}$ & $\begin{array}{l}\text { Haloperidol } \\
\text { (Planned dose: start } \\
\text { dose } 5 \mathrm{mg} \text { Max dose: } \\
15 \mathrm{mg}), 14\end{array}$ & Oral & DRS-C & $\begin{array}{l}\text { Baseline: Mean } 17.56 \\
\text { (SD 5.18) } \\
\text { Final: Mean } 14.29 \\
\text { (SD 4.55) }\end{array}$ & $\begin{array}{l}\text { Baseline: Mean } 16.5 \\
\text { (SD 4.7) } \\
\text { Final: Mean } 11.93 \\
\text { (SD 3.81) }\end{array}$ & $\begin{array}{l}1.30(95 \% \mathrm{Cl}:- \\
4.62 \text { to } 7.22)\end{array}$ \\
\hline Lin, $2008^{47}$ & $\begin{array}{l}\text { Patients receiving } \\
\text { hospice or } \\
\text { palliative care with } \\
\text { advanced cancer } \\
\text { and met DSM-IV } \\
\text { criteria for delirium }\end{array}$ & $\begin{array}{l}\text { Olanzapine (Planned } \\
\text { dose: start dose 5mg Max } \\
\text { dose: } 15 \mathrm{mg}), 10\end{array}$ & $\begin{array}{l}\text { Haloperidol } \\
\text { (Planned dose: start } \\
\text { dose } 5 \mathrm{mg} \text { Max dose: } \\
15 \mathrm{mg}), 14\end{array}$ & Oral & DRS-C & $\begin{array}{l}\text { Baseline: Mean } 17.56 \\
\text { (SD 5.18) } \\
\text { Final: Mean } 14.9 \text { (SD } \\
\text { 3.48) }\end{array}$ & $\begin{array}{l}\text { Baseline: Mean } 16.5 \\
\text { (SD 4.7) } \\
\text { Final: Mean } 13 \text { (SD } \\
5.02 \text { ) }\end{array}$ & $\begin{array}{l}0.84 \text { (95\% Cl: - } \\
5.45 \text { to } 7.13)\end{array}$ \\
\hline Lin, $2008^{47}$ & $\begin{array}{l}\text { Patients receiving } \\
\text { hospice or } \\
\text { palliative care with } \\
\text { advanced cancer } \\
\text { and met DSM-IV } \\
\text { criteria for delirium }\end{array}$ & $\begin{array}{l}\text { Olanzapine (Planned } \\
\text { dose: start dose 5mg Max } \\
\text { dose: } 15 \mathrm{mg} \text { ), } 5\end{array}$ & $\begin{array}{l}\text { Haloperidol } \\
\text { (Planned dose: start } \\
\text { dose } 5 \mathrm{mg} \text { Max dose: } \\
15 \mathrm{mg}), 7\end{array}$ & Oral & DRS-C & $\begin{array}{l}\text { Baseline: Mean } 17.56 \\
\text { (SD 5.18) } \\
\text { Final: Mean } 10.6 \text { (SD } \\
\text { 3.65) }\end{array}$ & $\begin{array}{l}\text { Baseline: Mean } 16.5 \\
\text { (SD 4.7) } \\
\text { Final: Mean } 12.29 \\
\text { (SD 5.59) }\end{array}$ & $\begin{array}{l}-2.75(95 \% \mathrm{Cl}:- \\
9.23 \text { to } 3.73)\end{array}$ \\
\hline Maneeton, $2013^{49}$ & $\begin{array}{l}\text { General adult } \\
\text { population (age } \\
\text { 18-75) with } \\
\text { hyperactive } \\
\text { delirium, referred } \\
\text { to psychiatry } \\
\text { consult liaison } \\
\text { service at tertiary } \\
\text { care hospital in } \\
\text { Thailand }\end{array}$ & $\begin{array}{l}\text { Quetiapine (Mean dose: } \\
\text { 67.6 (SD 9.7) Dose range: } \\
\text { 25-100mg), }\end{array}$ & $\begin{array}{l}\text { Haloperidol (Mean } \\
\text { dose: } 0.8 \text { (SD 0.3) } \\
\text { Dose range: 0.5- } \\
\text { 2.0mg), }\end{array}$ & Oral & $\begin{array}{l}\text { Clinical global } \\
\text { impression }\end{array}$ & $\begin{array}{l}\text { Change from } \\
\text { baseline: mean } \\
\text { difference -1.1 (SD 1) }\end{array}$ & $\begin{array}{l}\text { Change from } \\
\text { baseline: mean } \\
\text { difference -1.2 (SD } \\
1.4)\end{array}$ & $\begin{array}{l}-0.10(95 \% \mathrm{Cl}:- \\
0.75 \text { to } 0.55)\end{array}$ \\
\hline
\end{tabular}




\begin{tabular}{|c|c|c|c|c|c|c|c|c|}
\hline Author, year & Population & Intervention group, $\mathbf{n}$ & Control group, $\mathbf{n}$ & $\begin{array}{c}\text { Route of } \\
\text { administration }\end{array}$ & $\begin{array}{l}\text { Outcome } \\
\text { definition }\end{array}$ & $\begin{array}{c}\text { Severity score, } \\
\text { intervention group }\end{array}$ & $\begin{array}{l}\text { Severity score, } \\
\text { control group }\end{array}$ & $\begin{array}{c}\text { Mean between- } \\
\text { group difference } \\
(95 \% \mathrm{Cl})\end{array}$ \\
\hline Maneeton, $2013^{49}$ & $\begin{array}{l}\text { General adult } \\
\text { population (age } \\
\text { 18-75) with } \\
\text { hyperactive } \\
\text { delirium, referred } \\
\text { to psychiatry } \\
\text { consult liaison } \\
\text { service at tertiary } \\
\text { care hospital in } \\
\text { Thailand }\end{array}$ & $\begin{array}{l}\text { Quetiapine (Mean dose: } \\
67.6 \text { (SD 9.7) Dose range: } \\
25-100 \mathrm{mg}), 24\end{array}$ & $\begin{array}{l}\text { Haloperidol (Mean } \\
\text { dose: } 0.8 \text { (SD 0.3) } \\
\text { Dose range: } 0.5- \\
\text { 2.0mg), } 28\end{array}$ & Oral & DRS-R-98 & $\begin{array}{l}\text { Change from } \\
\text { baseline: Mean -22.9 } \\
\text { (SD 6.9) }\end{array}$ & $\begin{array}{l}\text { Change from } \\
\text { baseline: Mean -21.7 } \\
\text { (SD 6.7) }\end{array}$ & $\begin{array}{l}1.20(95 \% \mathrm{Cl}:- \\
2.51 \text { to } 4.91)\end{array}$ \\
\hline Skrobik, $2004^{57}$ & $\begin{array}{l}\text { Medical-surgical } \\
\text { ICU }\end{array}$ & $\begin{array}{l}\text { Olanzapine (Mean dose: } \\
\text { 4.54 Dose range: } 2.5 \text { to } \\
\text { 13.5mg), } 28\end{array}$ & $\begin{array}{l}\text { Haloperidol (Mean } \\
\text { dose: } 6.5 \text { Dose } \\
\text { range: } 1 \text { to } 28 \mathrm{mg} \text { ), } \\
45\end{array}$ & Oral & Delirium Index & $\begin{array}{l}\text { Baseline: Mean } 6.7 \\
\text { (Not reported) } \\
\text { Final: Mean } 5.4 \text { (Not } \\
\text { reported) }\end{array}$ & $\begin{array}{l}\text { Baseline: Mean } 7.4 \\
\text { (Not reported) } \\
\text { Final: Mean } 4.8 \text { (Not } \\
\text { reported) }\end{array}$ & $\begin{array}{l}1.30 \text { (95\% Cl: Not } \\
\text { reported) } p=0.64\end{array}$ \\
\hline \multicolumn{9}{|c|}{$\begin{array}{l}\text { Second-generation } \\
\text { antipsychotic vs. second- } \\
\text { generation antipsychotic }\end{array}$} \\
\hline Grover, 201130 & $\begin{array}{l}\text { Consecutive } \\
\text { patients }>-18 \\
\text { years old referred } \\
\text { to psychiatry and } \\
\text { diagnosed with } \\
\text { delirium }\end{array}$ & $\begin{array}{l}\text { Risperidone (Mean dose: } \\
0.95 \mathrm{mg} \text { Dose range: } 0.5- \\
2.0 \mathrm{mg} \text { per day), } 20\end{array}$ & $\begin{array}{l}\text { Olanzapine (Mean } \\
\text { dose: } 3.05 \mathrm{mg} \text { Dose } \\
\text { range: } 1.25-10 \mathrm{mg} \\
\text { per day), } 23\end{array}$ & Oral & DRS-R-98 & $\begin{array}{l}\text { Baseline: Mean } 23.8 \\
\text { (SD 5.16) } \\
\text { Final: Mean } 8 \text { (SD } \\
7.27)\end{array}$ & $\begin{array}{l}\text { Baseline: Mean } 22.56 \\
\text { (SD 4.49) } \\
\text { Final: Mean } 9.17 \text { (SD } \\
\text { 8.65) }\end{array}$ & $\begin{array}{l}-2.41(95 \% \mathrm{Cl}:- \\
8.24 \text { to } 3.42)\end{array}$ \\
\hline Kim, $2010^{42}$ & $\begin{array}{l}\text { General hospital } \\
\text { inpatients who } \\
\text { met DSM-IV } \\
\text { diagnostic criteria } \\
\text { for delirium }\end{array}$ & $\begin{array}{l}\text { Risperidone (Mean dose: } \\
0.9 \pm 0.6 \text { Dose range: } \\
0.25-2 \mathrm{mg}), 17\end{array}$ & $\begin{array}{l}\text { Olanzapine (Mean } \\
\text { dose: } 2.4 \pm 1.7 \text { Dose } \\
\text { range: } 1.25 \text { - } \\
7.5 \mathrm{mg}), 15\end{array}$ & Not reported & DRS-R-98 & $\begin{array}{l}\text { Baseline: Mean } 25.8 \\
\text { (SD 5.2) } \\
\text { Final: Mean } 7.62 \text { (Not } \\
\text { reported) }\end{array}$ & $\begin{array}{l}\text { Baseline: Mean } 23.5 \\
\text { (SD 5.1) } \\
\text { Final: Mean } 7.52 \text { (Not } \\
\text { reported) }\end{array}$ & $\begin{array}{l}-2.2 \text { (95\% Cl: Not } \\
\text { reported) }\end{array}$ \\
\hline Lee, $2005^{45}$ & $\begin{array}{l}\text { Patients referred } \\
\text { to the Psychiatric } \\
\text { Consultation that } \\
\text { met DSM-IV } \\
\text { criteria for delirium }\end{array}$ & 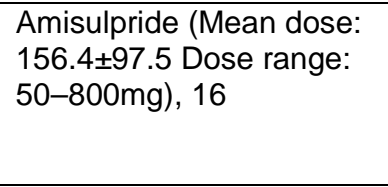 & $\begin{array}{l}\text { Quetiapine (Mean } \\
\text { dose: } 113 \pm 85.5 \\
\text { Dose range: } 50- \\
\text { 300mg), } 15\end{array}$ & Not reported & DRS-R-98 & $\begin{array}{l}\text { Baseline: Mean } 10.5 \\
\text { (SD 4.1) } \\
\text { Final: Mean } 3.5 \text { ( } 1.4)\end{array}$ & $\begin{array}{l}\text { Baseline: Mean } 10.1 \\
\text { (SD 4.1) } \\
\text { Final: Mean } 3.5 \text { ( 2.6) }\end{array}$ & $\begin{array}{l}-0.40(95 \% \mathrm{Cl}:- \\
5.06 \text { to } 4.26)\end{array}$ \\
\hline
\end{tabular}




\begin{tabular}{|c|c|c|c|c|c|c|c|c|}
\hline Author, year & Population & Intervention group, $\mathbf{n}$ & Control group, $n$ & $\begin{array}{c}\text { Route of } \\
\text { administration }\end{array}$ & $\begin{array}{l}\text { Outcome } \\
\text { definition }\end{array}$ & $\begin{array}{c}\text { Severity score, } \\
\text { intervention group }\end{array}$ & $\begin{array}{l}\text { Severity score, } \\
\text { control group }\end{array}$ & $\begin{array}{c}\text { Mean between- } \\
\text { group difference } \\
(95 \% \mathrm{Cl})\end{array}$ \\
\hline \multicolumn{9}{|l|}{$\begin{array}{l}\text { First-generation } \\
\text { antipsychotic vs. other }\end{array}$} \\
\hline Bakri, 2015 ${ }^{19}$ & $\begin{array}{l}\text { ICU trauma } \\
\text { patients without } \\
\text { severe injury, } \\
\text { comatose, or } \\
\text { moribund patients } \\
\text { were excluded } \\
\end{array}$ & $\begin{array}{l}\text { Haloperidol (Planned } \\
\text { dose: } 5 \mathrm{mg} \text { ), } 32\end{array}$ & $\begin{array}{l}\text { Ondansetron } \\
\text { (Planned dose: } 8 \\
\text { mg), } 32\end{array}$ & Intravenous & ICDSC score & $\begin{array}{l}\text { Baseline: Mean } 6.5 \\
\text { (SD 1.6) } \\
\text { Final: Mean } 3.4 \text { (SD } \\
1.1 \text { ) }\end{array}$ & $\begin{array}{l}\text { Baseline: Mean } 6.4 \\
\text { (SD 1.5) } \\
\text { Final: Mean } 3.5 \text { (SD } \\
1.3 \text { ) }\end{array}$ & $\begin{array}{l}-0.20(95 \% \mathrm{Cl}:- \\
1.44 \text { to } 1.04)\end{array}$ \\
\hline Bakri, $2015^{19}$ & $\begin{array}{l}\text { ICU trauma } \\
\text { patients without } \\
\text { severe injury, } \\
\text { comatose, or } \\
\text { moribund patients } \\
\text { were excluded }\end{array}$ & $\begin{array}{l}\text { Haloperidol (Planned } \\
\text { dose: } 5 \mathrm{mg} \text { ), } 32\end{array}$ & $\begin{array}{l}\text { Dexmedetomidine } \\
\text { (Planned dose: } 1 \\
\text { ug } / \mathrm{kg} \text { ), } 32\end{array}$ & Intravenous & ICDSC score & $\begin{array}{l}\text { Baseline: Mean } 6.5 \\
\text { (SD 1.6) } \\
\text { Final: Mean } 3.4 \text { (SD } \\
1.1 \text { ) }\end{array}$ & $\begin{array}{l}\text { Baseline: Mean } 6.7 \\
\text { (SD 1.3) } \\
\text { Final: Mean } 2.9 \text { (SD } \\
1.2)\end{array}$ & $\begin{array}{l}0.70(95 \% \mathrm{Cl}:- \\
0.45 \text { to } 1.85)\end{array}$ \\
\hline Breitbart, $1996^{22}$ & $\begin{array}{l}\text { Medically } \\
\text { hospitalized adult } \\
\text { patients with AIDS } \\
\text { and a DRS score } \\
\text { of } 13 \text { or greater } \\
\text { (delirium) }\end{array}$ & $\begin{array}{l}\text { Lorazepam (Planned } \\
\text { dose: Oral: } 0.50-4.0 \mathrm{mg} \text {, } \\
\text { Intramuscular: } 0.2-2.0 \mathrm{mg} \\
\text { Mean dose: } 4.6 \text { Dose } \\
\text { range: } 1.3-7.9 \mathrm{mg}), 6\end{array}$ & $\begin{array}{l}\text { Chlorpromazine } \\
\text { (Planned dose: Oral: } \\
\text { 10-200mg, } \\
\text { Intramuscular: 5- } \\
\text { 100mg Mean dose: } \\
\text { 36 Dose range: 10- } \\
\text { 80mg), 13 }\end{array}$ & $\begin{array}{l}\text { Oral or } \\
\text { intramuscular }\end{array}$ & $\begin{array}{l}\text { Delirium Rating } \\
\text { Scale (older } \\
\text { version) score }\end{array}$ & $\begin{array}{l}\text { Baseline: Mean } 18.33 \\
\text { (SD 2.58) } \\
\text { Final: Mean } 17 \text { (SD } \\
\text { 4.98) }\end{array}$ & $\begin{array}{l}\text { Baseline: Mean } 20.62 \\
\text { (SD 3.88) } \\
\text { Final: Mean } 11.85 \\
\text { (SD 6.74) }\end{array}$ & $\begin{array}{l}7.44 \text { (95\% Cl: } \\
1.53 \text { to } 13.35)\end{array}$ \\
\hline Breitbart, $1996^{22}$ & $\begin{array}{l}\text { Medically } \\
\text { hospitalized adult } \\
\text { patients with AIDS } \\
\text { and a DRS score } \\
\text { of } 13 \text { or greater } \\
\text { (delirium) }\end{array}$ & $\begin{array}{l}\text { Haloperidol (Planned } \\
\text { dose: oral: } 0.25-5.0 \mathrm{mg} \text {, } \\
\text { Intramuscular: } 0.125- \\
\text { 3.0mg Mean dose: } 1.4 \\
\text { Dose range: } 0.4-3.6 \mathrm{mg} \text {, } \\
11\end{array}$ & $\begin{array}{l}\text { Lorazepam (Planned } \\
\text { dose: Oral: 0.50- } \\
\text { 4.0mg, } \\
\text { Intramuscular: } 0.2- \\
\text { 2.0mg Mean dose: } \\
\text { 4.6 Dose range: } 1.3 \\
-7.9 \mathrm{mg}), 6\end{array}$ & $\begin{array}{l}\text { Oral or } \\
\text { intramuscular }\end{array}$ & $\begin{array}{l}\text { Delirium Rating } \\
\text { Scale (older } \\
\text { version) score }\end{array}$ & $\begin{array}{l}\text { Baseline: Mean } 20.45 \\
\text { (SD 3.45) } \\
\text { Final: Mean } 11.64 \\
\text { (SD 6.1) }\end{array}$ & $\begin{array}{l}\text { Baseline: Mean } 18.33 \\
\text { (SD 2.58) } \\
\text { Final: Mean } 17 \text { (SD } \\
\text { 4.98) }\end{array}$ & $\begin{array}{l}-7.48(95 \% \mathrm{Cl}:- \\
13.32 \text { to }-1.64)\end{array}$ \\
\hline
\end{tabular}

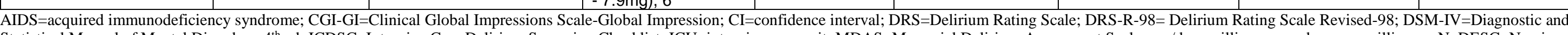

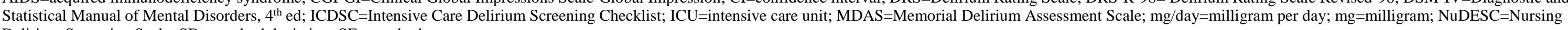
Delirium Screening Scale; SD=standard deviation; $\mathrm{SE}=$ standard error 
Evidence Table D-34. Binary delirium severity outcomes for studies comparing interventions to treat delirium

\begin{tabular}{|c|c|c|c|c|c|c|c|c|}
\hline Author, year & Population & Intervention group, $\mathbf{n}$ & Control group, $\mathbf{n}$ & $\begin{array}{c}\text { Route of } \\
\text { administration }\end{array}$ & Outcome definition & $\begin{array}{l}\mathrm{n} / \mathrm{N}(\%) \\
\text { intervention } \\
\text { group }\end{array}$ & $\begin{array}{c}\mathrm{n} / \mathrm{N}(\%) \\
\text { control } \\
\text { group }\end{array}$ & $\begin{array}{l}\text { Relative risk } \\
(95 \% \mathrm{Cl})\end{array}$ \\
\hline \multicolumn{9}{|c|}{$\begin{array}{l}\text { First-generation } \\
\text { antipsychotic vs. placebo }\end{array}$} \\
\hline $\mathrm{Hu}, 2006^{35}$ & $\begin{array}{l}\text { Inpatients with senile } \\
\text { delirium aged 65-99 }\end{array}$ & $\begin{array}{l}\text { Haloperidol (Dose range: } \\
2.5-10 \mathrm{mg}), 72\end{array}$ & Not reported & Intramuscular & $\begin{array}{l}\text { CGI-GI score of } \\
\text { "significantly improved" or } \\
\text { "completely alleviated" }\end{array}$ & $63 / 72(87.5 \%)$ & $\begin{array}{l}9 / 29 \\
(31 \%)\end{array}$ & $\begin{array}{l}2.82(1.62 \text { to } \\
4.88)\end{array}$ \\
\hline \multicolumn{9}{|c|}{$\begin{array}{l}\text { Second-generation } \\
\text { antipsychotic vs. placebo }\end{array}$} \\
\hline $\mathrm{Hu}, 2006^{35}$ & $\begin{array}{l}\text { Inpatients with senile } \\
\text { delirium aged 65-99 }\end{array}$ & $\begin{array}{l}\text { Olanzapine (Dose range: } \\
1.25-20 \mathrm{mg}), 74\end{array}$ & Not reported & $\begin{array}{l}\text { Oral or } \\
\text { sublingually }\end{array}$ & $\begin{array}{l}\text { CGI-GI score of } \\
\text { "significantly improved" or } \\
\text { "completely alleviated" }\end{array}$ & $61 / 74$ (82.4\%) & $\begin{array}{l}9 / 29 \\
(31 \%)\end{array}$ & $\begin{array}{l}2.66(1.53 \text { to } \\
4.62)\end{array}$ \\
\hline Tahir, $2010^{59}$ & $\begin{array}{l}\text { Patients in medical, } \\
\text { surgical and orthopedic } \\
\text { wards, who met the } \\
\text { DSM-IV criteria for } \\
\text { delirium on the same day } \\
\text { if they had a DRS-R-98 } \\
\text { total score of } 15 \text { or more }\end{array}$ & $\begin{array}{l}\text { Quetiapine (Mean dose: } \\
\text { 40 Dose range: } 25 \text { - } \\
\text { 175mg), } 21\end{array}$ & $\begin{array}{l}\text { Placebo (Mean dose: } \\
25 \mathrm{Max} \text { dose: } \\
\text { 175mg), } 21\end{array}$ & Oral & Clinical Global Improvement & & & $\begin{array}{l}\text { There were no } \\
\text { significant } \\
\text { differences found } \\
\text { between } \\
\text { treatment groups } \\
\text { for MMSE, } \\
\text { BPRS, or CGI } \\
\text { Global scores. }\end{array}$ \\
\hline \multicolumn{9}{|c|}{$\begin{array}{l}\text { First-generation } \\
\text { antipsychotic vs. second- } \\
\text { generation antipsychotic }\end{array}$} \\
\hline Grover, $2011^{30}$ & $\begin{array}{l}\text { Consecutive patients >- } \\
18 \text { years old referred to } \\
\text { psychiatry and } \\
\text { diagnosed with delirium }\end{array}$ & $\begin{array}{l}\text { Risperidone (Mean dose: } \\
\text { 0.95mg Dose range: } 0.5- \\
2.0 \mathrm{mg} \text { per day), } 20\end{array}$ & $\begin{array}{l}\text { Haloperidol (Mean } \\
\text { dose: } 0.88 \text { Dose } \\
\text { range: } 0.25-5 \mathrm{mg} \text { per } \\
\text { day), } 21\end{array}$ & Oral & $\begin{array}{l}\text { DRS-R-98 score }<10 \text { on day } \\
6\end{array}$ & 14 / $20(70 \%)$ & $\begin{array}{l}17 / 21 \\
(81 \%)\end{array}$ & $\begin{array}{l}0.86(0.61 \text { to } \\
1.23)\end{array}$ \\
\hline Grover, 201130 & $\begin{array}{l}\text { Consecutive patients >- } \\
18 \text { years old referred to } \\
\text { psychiatry and } \\
\text { diagnosed with delirium }\end{array}$ & $\begin{array}{l}\text { Olanzapine (Mean dose: } \\
3.05 \mathrm{mg} \text { Dose range: } 1.25- \\
10 \mathrm{mg} \text { per day), } 23\end{array}$ & $\begin{array}{l}\text { Haloperidol (Mean } \\
\text { dose: } 0.88 \text { Dose } \\
\text { range: } 0.25-5 \mathrm{mg} \text { per } \\
\text { day), } 21\end{array}$ & Oral & $\begin{array}{l}\text { DRS-R-98 score }<10 \text { on day } \\
6\end{array}$ & $16 / 23(69.56 \%)$ & $\begin{array}{l}17 / 21 \\
(81 \%)\end{array}$ & $\begin{array}{l}0.86(0.61 \text { to } \\
1.21)\end{array}$ \\
\hline
\end{tabular}




\begin{tabular}{|c|c|c|c|c|c|c|c|c|}
\hline Author, year & Population & Intervention group, $\mathbf{n}$ & Control group, $\mathbf{n}$ & $\begin{array}{c}\text { Route of } \\
\text { administration }\end{array}$ & Outcome definition & $\begin{array}{c}\text { n I N (\%), } \\
\text { intervention } \\
\text { group }\end{array}$ & $\begin{array}{c}\mathrm{n} \text { I N (\%), } \\
\text { control } \\
\text { group }\end{array}$ & $\begin{array}{l}\text { Relative risk } \\
(95 \% \mathrm{Cl})\end{array}$ \\
\hline Grover, 2016 ${ }^{31}$ & $\begin{array}{l}\text { Patients }>18 \text { years old } \\
\text { referred to Psychiatry } \\
\text { who met DSM-IV criteria } \\
\text { for delirium }\end{array}$ & $\begin{array}{l}\text { Quetiapine (Mean dose: } \\
26.63 \pm 15.61 \text { Dose } \\
\text { range: } 12.5-75 \mathrm{mg}), 31\end{array}$ & $\begin{array}{l}\text { Haloperidol (Mean } \\
\text { dose: } 0.67 \pm 0.35 \\
\text { Dose range: } 0.25- \\
1.25 \mathrm{mg} \text { ), } 32\end{array}$ & Oral & DRS-R-98 $<10$ & $21 / 31$ (67.74\%) & $\begin{array}{l}22 / 32 \\
(68.75 \%)\end{array}$ & $\begin{array}{l}0.99(0.70 \text { to } \\
1.38)\end{array}$ \\
\hline $\mathrm{Hu}, 2006^{35}$ & $\begin{array}{l}\text { Inpatients with senile } \\
\text { delirium aged 65-99 }\end{array}$ & $\begin{array}{l}\text { Olanzapine (Dose range: } \\
1.25-20 \mathrm{mg}), 74\end{array}$ & $\begin{array}{l}\text { Haloperidol (Dose } \\
\text { range: } 2.5-10 \mathrm{mg}), 72\end{array}$ & $\begin{array}{l}\text { Oral or } \\
\text { sublingually }\end{array}$ & $\begin{array}{l}\text { CGI-GI score of } \\
\text { "significantly improved" or } \\
\text { "completely alleviated" }\end{array}$ & $61 / 74$ (82.4\%) & $\begin{array}{l}63 / 72 \\
(87.5 \%)\end{array}$ & $\begin{array}{l}0.94(0.82 \text { to } \\
1.08)\end{array}$ \\
\hline Maneeton, $2013^{49}$ & $\begin{array}{l}\text { General adult population } \\
\text { (age 18-75) with } \\
\text { hyperactive delirium, } \\
\text { referred to psychiatry } \\
\text { consult liaison service at } \\
\text { tertiary care hospital in } \\
\text { Thailand }\end{array}$ & $\begin{array}{l}\text { Quetiapine (Mean dose: } \\
67.6 \text { (SD 9.7) Dose range: } \\
\text { 25-100mg), } 24\end{array}$ & $\begin{array}{l}\text { Haloperidol (Mean } \\
\text { dose: } 0.8 \text { (SD 0.3) } \\
\text { Dose range: } 0.5- \\
\text { 2.0mg), } 28\end{array}$ & Oral & $\begin{array}{l}\text { Remission rate, a DRS-R-98 } \\
\text { severity score }=<12 \text { without } \\
\text { relapse }\end{array}$ & $18 / 24(75 \%)$ & $\begin{array}{l}19 / 28 \\
(67.9 \%)\end{array}$ & $\begin{array}{l}1.11(0.78 \text { to } \\
1.56)\end{array}$ \\
\hline Maneeton, $2013^{49}$ & $\begin{array}{l}\text { General adult population } \\
\text { (age 18-75) with } \\
\text { hyperactive delirium, } \\
\text { referred to psychiatry } \\
\text { consult liaison service at } \\
\text { tertiary care hospital in } \\
\text { Thailand }\end{array}$ & $\begin{array}{l}\text { Quetiapine (Mean dose: } \\
67.6 \text { (SD 9.7) Dose range: } \\
\text { 25-100mg), } 24\end{array}$ & $\begin{array}{l}\text { Haloperidol (Mean } \\
\text { dose: } 0.8 \text { (SD 0.3) } \\
\text { Dose range: } 0.5- \\
\text { 2.0mg), } 28\end{array}$ & Oral & $\begin{array}{l}\text { Response rate, reduction in } \\
\text { DRS-R-98 severity score } \\
\geq 50 \% \text { or more from baseline }\end{array}$ & $19 / 24(79.2 \%)$ & $\begin{array}{l}22 / 28 \\
(78.6 \%)\end{array}$ & $\begin{array}{l}1.01(0.76 \text { to } \\
1.34)\end{array}$ \\
\hline \multicolumn{9}{|c|}{$\begin{array}{l}\text { Second-generation } \\
\text { antipsychotic vs. second- } \\
\text { generation antipsychotic }\end{array}$} \\
\hline Grover, $2011^{30}$ & $\begin{array}{l}\text { Consecutive patients >- } \\
18 \text { years old referred to } \\
\text { psychiatry and } \\
\text { diagnosed with delirium }\end{array}$ & $\begin{array}{l}\text { Risperidone (Mean dose: } \\
0.95 \mathrm{mg} \text { Dose range: } 0.5- \\
2.0 \mathrm{mg} \text { per day), } 20\end{array}$ & $\begin{array}{l}\text { Olanzapine (Mean } \\
\text { dose: } 3.05 \mathrm{mg} \text { Dose } \\
\text { range: } 1.25-10 \mathrm{mg} \\
\text { per day), } 23\end{array}$ & Oral & $\begin{array}{l}\text { DRS-R-98 score }<10 \text { on day } \\
6\end{array}$ & 14 / $20(70 \%)$ & $\begin{array}{l}16 / 23 \\
(69.56 \%)\end{array}$ & $\begin{array}{l}1.01(0.68 \text { to } \\
1.49)\end{array}$ \\
\hline
\end{tabular}




\begin{tabular}{|c|c|c|c|c|c|c|c|c|}
\hline Author, year & Population & Intervention group, $\mathbf{n}$ & Control group, $\mathrm{n}$ & $\begin{array}{c}\text { Route of } \\
\text { administration }\end{array}$ & Outcome definition & $\begin{array}{c}\mathrm{n} / \mathrm{N}(\%) \\
\text { intervention } \\
\text { group }\end{array}$ & $\begin{array}{l}\text { n I N (\%), } \\
\text { control } \\
\text { group }\end{array}$ & $\begin{array}{c}\text { Relative risk } \\
(95 \% \mathrm{Cl})\end{array}$ \\
\hline \multicolumn{9}{|c|}{$\begin{array}{l}\text { First-generation } \\
\text { antipsychotic vs. other }\end{array}$} \\
\hline Bakri, $2015^{19}$ & $\begin{array}{l}\text { ICU trauma patients } \\
\text { without severe injury, } \\
\text { comatose, or moribund } \\
\text { patients were excluded }\end{array}$ & $\begin{array}{l}\text { Haloperidol (Planned } \\
\text { dose: } 5 \mathrm{mg} \text { ), } 32\end{array}$ & $\begin{array}{l}\text { Dexmedetomidine } \\
\text { (Planned dose: } 1 \\
\text { ug/kg), } 32\end{array}$ & Intravenous & $\begin{array}{l}\text { Patients who remained } \\
\text { delirious, according to } \\
\text { ICDSC score }\end{array}$ & $2 / 32(6 \%)$ & 3 / $32(9 \%)$ & $\begin{array}{l}0.67(0.12 \text { to } \\
3.73)\end{array}$ \\
\hline Bakri, $2015^{19}$ & $\begin{array}{l}\text { ICU trauma patients } \\
\text { without severe injury, } \\
\text { comatose, or moribund } \\
\text { patients were excluded }\end{array}$ & $\begin{array}{l}\text { Haloperidol (Planned } \\
\text { dose: } 5 \mathrm{mg} \text { ), } 32\end{array}$ & $\begin{array}{l}\text { Ondansetron } \\
\text { (Planned dose: } 8 \\
\text { mg), } 32\end{array}$ & Intravenous & $\begin{array}{l}\text { Patients who remained } \\
\text { delirious, according to } \\
\text { ICDSC score }\end{array}$ & 2 / $32(6 \%)$ & $\begin{array}{l}6 / 32 \\
(19 \%)\end{array}$ & $\begin{array}{l}0.33(0.07 \text { to } \\
1.53)\end{array}$ \\
\hline
\end{tabular}

BPRS= Brief Psychiatric Rating Scale; CGI=Clinical Global Impressions Scale; CGI-GI=Clinical Global Impressions Scale-Global Impression; CI=confidence interval; DRS-R-98= Delirium Rating Scale Revised-98; DSM-IV=Diagnostic and Statistical Manual of Mental Disorders, $4^{\text {th }}$ ed; ICDSC=Intensive Care Delirium Screening Checklist; ICU=intensive care unit; mg=milligram; MMSE=Mini-Mental State Examination; N=sample size; SD=standard deviation 
Evidence Table D-35. Delirium free days outcomes for studies comparing interventions to treat delirium

\begin{tabular}{|c|c|c|c|c|c|c|c|c|}
\hline Author, year & Population & Intervention group, $\mathbf{n}$ & $\underset{n}{\text { Control group, }}$ & $\begin{array}{l}\text { Route of } \\
\text { administr } \\
\text { ation }\end{array}$ & Outcome definition & $\begin{array}{l}\text { Median (IQR) } \\
\text { days, } \\
\text { intervention } \\
\text { group }\end{array}$ & $\begin{array}{l}\text { Median (IQR), } \\
\text { days, control } \\
\text { group }\end{array}$ & $\begin{array}{c}\text { Measure of } \\
\text { association }(95 \% \mathrm{Cl})\end{array}$ \\
\hline \multicolumn{9}{|l|}{$\begin{array}{l}\text { First-generation } \\
\text { antipsychotic vs. } \\
\text { placebo }\end{array}$} \\
\hline Girard, $2010^{4}$ & $\begin{array}{l}>18 \text { years MV medical and } \\
\text { surgical ICU patients }\end{array}$ & $\begin{array}{l}\text { Haloperidol (Planned } \\
\text { dose: } 5 \text { Median dose: } 15 \\
\text { (10.8-17), } 35\end{array}$ & $\begin{array}{l}\text { Placebo } \\
\text { (Planned dose: } \\
5 \mathrm{ml}), 36\end{array}$ & Oral & $\begin{array}{l}\text { delirium/coma-free days } \\
\text { during the 21-day study } \\
\text { period. }\end{array}$ & 14 (6 to 18$)$ & 12.5 (1.2 to 17.2$)$ & \\
\hline Girard, $2018^{29}$ & $\begin{array}{l}\text { Patients over } 18 \text { years of age } \\
\text { in the medical or surgical ICU, } \\
\text { with delirium }\end{array}$ & $\begin{array}{l}\text { Haloperidol (Mean dose: } \\
11 \mathrm{mg} \text { Dose range: } 2.5- \\
\text { 20mg), } 192\end{array}$ & $\begin{array}{l}\text { Placebo (Mean } \\
\text { dose: Not } \\
\text { reported), } 184\end{array}$ & $\begin{array}{l}\text { Intravenou } \\
\mathrm{s}\end{array}$ & $\begin{array}{l}\text { Days alive without delirium or } \\
\text { coma }\end{array}$ & 8 (0 to 11$)$ & 7 (0 to 11$)$ & $\begin{array}{l}\text { OR } 0.88(95 \% \mathrm{Cl}: \\
0.64 \text { to } 1.21), p=0.26\end{array}$ \\
\hline Girard, $2018^{29}$ & $\begin{array}{l}\text { Patients over } 18 \text { years of age } \\
\text { in the medical or surgical ICU, } \\
\text { with delirium }\end{array}$ & $\begin{array}{l}\text { Haloperidol (Mean dose: } \\
11 \mathrm{mg} \text { Dose range: } 2.5- \\
\text { 20mg), } 192\end{array}$ & $\begin{array}{l}\text { Placebo (Mean } \\
\text { dose: Not } \\
\text { reported), } 184\end{array}$ & $\begin{array}{l}\text { Intravenou } \\
\mathrm{s}\end{array}$ & $\begin{array}{l}\text { Days alive without delirium or } \\
\text { coma }\end{array}$ & $\begin{array}{l}\text { Adjusted median: } \\
7.9(95 \% \mathrm{Cl}: 4.4 \\
\text { to } 9.6)\end{array}$ & $\begin{array}{l}\text { Adjusted median: } \\
8.5(95 \% \mathrm{Cl}: 5.6 \\
\text { to } 9.9)\end{array}$ & $\begin{array}{l}\text { OR } 0.88 \text { (95\% Cl: } \\
0.64 \text { to } 1.21), p=0.26\end{array}$ \\
\hline Page, $2013^{10,27}$ & $\begin{array}{l}\text { ICU patients needing MV } \\
\text { within } 72 \text { hours of admission } \\
\text { were enrolled }\end{array}$ & $\begin{array}{l}\text { Haloperidol (Planned } \\
\text { dose: } 2.5 \mathrm{mg}), 71\end{array}$ & $\begin{array}{l}\text { Placebo } \\
\text { (Planned dose: } \\
0.5 \mathrm{ml}), 70\end{array}$ & $\begin{array}{l}\text { Intravenou } \\
\mathrm{s}\end{array}$ & $\begin{array}{l}\text { Alive, delirium free, and coma } \\
\text { free days in first } 14 \text { days, } \\
\text { median (IQR) }\end{array}$ & 5 (0 to 10$)$ & $6(0$ to 11$)$ & $\begin{array}{l}\text { Mean difference }-0.48 \\
(95 \% \mathrm{Cl}:-2.08 \text { to } \\
1.21), p=0.53\end{array}$ \\
\hline Page, $2013^{10,27}$ & $\begin{array}{l}\text { ICU patients needing MV } \\
\text { within } 72 \text { hours of admission } \\
\text { were enrolled }\end{array}$ & $\begin{array}{l}\text { Haloperidol (Planned } \\
\text { dose: } 2.5 \mathrm{mg} \text { ), } 71\end{array}$ & $\begin{array}{l}\text { Placebo } \\
\text { (Planned dose: } \\
0.5 \mathrm{ml}), 70\end{array}$ & $\begin{array}{l}\text { Intravenou } \\
\mathrm{s}\end{array}$ & $\begin{array}{l}\text { Alive, delirium free, and coma } \\
\text { free days in first } 28 \text { days }\end{array}$ & 19 (0 to 24$)$ & 19.5 (0 to 25$)$ & $\begin{array}{l}\text { Mean difference }-0.26 \\
\text { (95\% Cl: }-3.72 \text { to } \\
3.46), p=0.57\end{array}$ \\
\hline \multicolumn{9}{|l|}{$\begin{array}{l}\text { Second- } \\
\text { generation } \\
\text { antipsychotic vs. } \\
\text { placebo }\end{array}$} \\
\hline Girard, $2010^{4}$ & $\begin{array}{l}>18 \text { years MV medical and } \\
\text { surgical ICU patients }\end{array}$ & $\begin{array}{l}\text { Ziprasidone (Planned } \\
\text { dose: } 40 \text { Median dose: } \\
113(81-140), 30\end{array}$ & $\begin{array}{l}\text { Placebo } \\
\text { (Planned dose: } \\
\text { 5ml), } 36\end{array}$ & Oral & $\begin{array}{l}\text { Delirium/coma-free days } \\
\text { during the } 21 \text {-day study } \\
\text { period. }\end{array}$ & 15 (9.1 to 18$)$ & $12.5(1.2$ to 17.2$)$ & \\
\hline Girard, $2018^{29}$ & $\begin{array}{l}\text { Patients over } 18 \text { years of age } \\
\text { in the medical or surgical ICU, } \\
\text { with delirium }\end{array}$ & $\begin{array}{l}\text { Ziprasidone (Mean dose: } \\
\text { 20mg Dose range: } 5.0- \\
\text { 40mg), } 190\end{array}$ & $\begin{array}{l}\text { Placebo (Mean } \\
\text { dose: Not } \\
\text { reported), } 184\end{array}$ & $\begin{array}{l}\text { Intravenou } \\
\mathrm{s}\end{array}$ & $\begin{array}{l}\text { Days alive without delirium or } \\
\text { coma }\end{array}$ & 8 (2 to 11$)$ & 7 (0 to 11$)$ & $\begin{array}{l}\text { OR 1.04 (95\% Cl: } \\
0.73-1.48), p=0.26\end{array}$ \\
\hline Girard, 2018 29 & $\begin{array}{l}\text { Patients over } 18 \text { years of age } \\
\text { in the medical or surgical ICU, } \\
\text { with delirium }\end{array}$ & $\begin{array}{l}\text { Ziprasidone (Mean dose: } \\
\text { 20mg Dose range: } 5.0- \\
\text { 40mg), } 190\end{array}$ & $\begin{array}{l}\text { Placebo (Mean } \\
\text { dose: Not } \\
\text { reported), } 184\end{array}$ & $\begin{array}{l}\text { Intravenou } \\
\mathrm{s}\end{array}$ & $\begin{array}{l}\text { Days alive without delirium or } \\
\text { coma }\end{array}$ & $\begin{array}{l}\text { Adjusted median: } \\
8.7(95 \% \mathrm{Cl}: 5.9 \\
\text { to } 10)\end{array}$ & $\begin{array}{l}\text { Adjusted median: } \\
8.5(95 \% \mathrm{Cl}: 5.6 \\
\text { to } 9.9)\end{array}$ & $\begin{array}{l}\text { OR 1.04 (95\% Cl: } \\
0.73-1.48), p=0.26\end{array}$ \\
\hline
\end{tabular}




\begin{tabular}{|c|c|c|c|c|c|c|c|c|}
\hline Author, year & Population & Intervention group, $\mathbf{n}$ & Control group, $n$ & $\begin{array}{l}\text { Route of } \\
\text { administr } \\
\text { ation }\end{array}$ & Outcome definition & $\begin{array}{c}\text { Median (IQR) } \\
\text { days, } \\
\text { intervention } \\
\text { group }\end{array}$ & $\begin{array}{l}\text { Median (IQR), } \\
\text { days, control } \\
\text { group }\end{array}$ & $\begin{array}{l}\text { Measure of } \\
\text { association } \\
(95 \% \mathrm{Cl})\end{array}$ \\
\hline \multicolumn{9}{|c|}{$\begin{array}{l}\text { First-generation } \\
\text { antipsychotic vs. second- } \\
\text { generation antipsychotic }\end{array}$} \\
\hline Girard, $2010^{4}$ & $\begin{array}{l}>18 \text { years MV medical and } \\
\text { surgical ICU patients }\end{array}$ & $\begin{array}{l}\text { Ziprasidone (Planned } \\
\text { dose: } 40 \text { Median dose: } \\
113 \text { (81-140), } 30\end{array}$ & $\begin{array}{l}\text { Haloperidol (Planned } \\
\text { dose: } 5 \text { Median dose: } 15 \\
\text { (10.8-17), } 35\end{array}$ & Oral & $\begin{array}{l}\text { Delirium/coma-free } \\
\text { days during the } 21 \text {-day } \\
\text { study period. }\end{array}$ & $15(9.1$ to 18$)$ & 14 (6 to 18$)$ & \\
\hline Girard, $2018^{29}$ & $\begin{array}{l}\text { Patients over } 18 \text { years of age } \\
\text { in the medical or surgical ICU, } \\
\text { with delirium }\end{array}$ & $\begin{array}{l}\text { Ziprasidone (Mean dose: } \\
\text { 20mg Dose range: } 5.0- \\
\text { 40mg), } 190\end{array}$ & $\begin{array}{l}\text { Haloperidol (Mean dose: } \\
\text { 11mg Dose range: } 2.5- \\
\text { 20mg), } 192\end{array}$ & $\begin{array}{l}\text { Intraveno } \\
\text { us }\end{array}$ & $\begin{array}{l}\text { Days alive without } \\
\text { delirium or coma }\end{array}$ & 8 (2 to 11$)$ & 8 (0 to 11$)$ & \\
\hline Girard, $2018^{29}$ & $\begin{array}{l}\text { Patients over } 18 \text { years of age } \\
\text { in the medical or surgical ICU, } \\
\text { with delirium }\end{array}$ & $\begin{array}{l}\text { Ziprasidone (Mean dose: } \\
\text { 20mg Dose range: } 5.0- \\
\text { 40mg), } 190\end{array}$ & $\begin{array}{l}\text { Haloperidol (Mean dose: } \\
\text { 11mg Dose range: 2.5- } \\
\text { 20mg), } 192\end{array}$ & $\begin{array}{l}\text { Intraveno } \\
\text { us }\end{array}$ & $\begin{array}{l}\text { Days alive without } \\
\text { delirium or coma }\end{array}$ & $\begin{array}{l}\text { Adjusted } \\
\text { median: } 8.7 \\
\text { (95\% Cl: } 5.9 \text { to } \\
10)\end{array}$ & $\begin{array}{l}\text { Adjusted } \\
\text { median: } 7.9 \\
\text { (95\% Cl: } 4.4 \text { to } \\
9.6 \text { ) }\end{array}$ & \\
\hline
\end{tabular}

$\mathrm{CI}=$ confidence interval; $\mathrm{ICU}=$ intensive care unit; $\mathrm{IQR}=$ interquartile range; $\mathrm{MV}=$ =mechanical ventilatio 
Evidence Table D-36. Duration of delirium outcomes for studies comparing interventions to treat delirium

\begin{tabular}{|c|c|c|c|c|c|c|c|c|}
\hline Author, year & Population & Intervention group, $\mathrm{n}$ & Control group, $n$ & $\begin{array}{l}\text { Route of } \\
\text { administration }\end{array}$ & Outcome definition & $\begin{array}{c}\text { Median } \\
\text { (IQR) days, } \\
\text { intervention } \\
\text { group } \\
\end{array}$ & $\begin{array}{l}\text { Median } \\
\text { (IQR), days, } \\
\text { control } \\
\text { group } \\
\end{array}$ & $\begin{array}{c}\text { Measure of } \\
\text { association }(95 \% \\
\mathrm{Cl})\end{array}$ \\
\hline \multicolumn{9}{|l|}{$\begin{array}{l}\text { First-generation } \\
\text { antipsychotic vs. } \\
\text { placebo }\end{array}$} \\
\hline Girard, $2010^{4}$ & $\begin{array}{l}>18 \text { years MV medical and } \\
\text { surgical ICU patients }\end{array}$ & $\begin{array}{l}\text { Haloperidol (Planned dose: } 5 \\
\text { Median dose: } 15 \text { (10.8-17), } \\
35\end{array}$ & $\begin{array}{l}\text { Placebo (Planned } \\
\text { dose: } 5 \mathrm{ml}), 36\end{array}$ & Oral & Delirium days & 4 (2 to 7$)$ & $4(2$ to 6$)$ & \\
\hline Girard, $2018^{29}$ & $\begin{array}{l}\text { Patients over } 18 \text { years of age } \\
\text { in the medical or surgical ICU, } \\
\text { with delirium }\end{array}$ & $\begin{array}{l}\text { Haloperidol (Mean dose: } \\
\text { 11mg Dose range: } 2.5- \\
\text { 20mg), } 192\end{array}$ & $\begin{array}{l}\text { Placebo (Not } \\
\text { reported), } 184\end{array}$ & Intravenous & Days with delirium & $4(2$ to 7$)$ & 4 (2 to 8$)$ & $\begin{array}{l}\text { OR } 1.12(95 \% \mathrm{Cl}: \\
0.86 \text { to } 1.46) \\
\mathrm{p}=\text { Not reported }\end{array}$ \\
\hline Girard, $2018^{29}$ & $\begin{array}{l}\text { Patients over } 18 \text { years of age } \\
\text { in the medical or surgical ICU, } \\
\text { with delirium }\end{array}$ & $\begin{array}{l}\text { Haloperidol (Mean dose: } \\
11 \mathrm{mg} \text { Dose range: } 2.5- \\
\text { 20mg), } 192\end{array}$ & $\begin{array}{l}\text { Placebo (Not } \\
\text { reported), } 184\end{array}$ & Intravenous & $\begin{array}{l}\text { Days with hyperactive } \\
\text { delirium (RASS >0) }\end{array}$ & 0 (0 to 1$)$ & 0 (0 to 1$)$ & $\begin{array}{l}\text { OR } 1.18(95 \% \mathrm{Cl}: \\
0.86 \text { to } 1.61) \\
\text { p=Not reported }\end{array}$ \\
\hline Girard, $2018^{29}$ & $\begin{array}{l}\text { Patients over } 18 \text { years of age } \\
\text { in the medical or surgical ICU, } \\
\text { with delirium }\end{array}$ & $\begin{array}{l}\text { Haloperidol (Mean dose: } \\
11 \mathrm{mg} \text { Dose range: } 2.5- \\
20 \mathrm{mg}), 192\end{array}$ & $\begin{array}{l}\text { Placebo (Not } \\
\text { reported), } 184\end{array}$ & Intravenous & $\begin{array}{l}\text { Days with hypoactive } \\
\text { delirium (RASS } \leq 0 \text { ) }\end{array}$ & 4 (2 to 6$)$ & 3 (2 to 8$)$ & $\begin{array}{l}\text { OR } 1.1(95 \% \mathrm{Cl}: \\
0.81 \text { to } 1.48), \\
\text { p=Not reported }\end{array}$ \\
\hline Page, $2013^{10,27}$ & $\begin{array}{l}\text { ICU patients needing MV } \\
\text { within } 72 \text { hours of admission } \\
\text { were enrolled }\end{array}$ & $\begin{array}{l}\text { Haloperidol (Planned dose: } \\
2.5 \mathrm{mg}), 71\end{array}$ & $\begin{array}{l}\text { Placebo (Planned } \\
\text { dose: } 0.5 \mathrm{ml}), 70\end{array}$ & Intravenous & Days in delirium & 5 (2 to 8$)$ & 5 (1 to 8$)$ & $\begin{array}{l}\text { Mean difference } \\
0.01(95 \% \mathrm{Cl}:-1.31 \\
\text { to } 1.33), \mathrm{p}=0.99\end{array}$ \\
\hline Page, $2013^{10,27}$ & $\begin{array}{l}\text { ICU patients needing MV } \\
\text { within } 72 \text { hours of admission } \\
\text { were enrolled }\end{array}$ & $\begin{array}{l}\text { Haloperidol (Planned dose: } \\
\text { 2.5mg), } 71\end{array}$ & $\begin{array}{l}\text { Placebo (Planned } \\
\text { dose: } 0.5 \mathrm{ml}), 70\end{array}$ & Intravenous & Days in delirium & 5 (2 to 10$)$ & 5 (1 to 9$)$ & $\begin{array}{l}\text { Mean difference - } \\
0.38(95 \% \mathrm{Cl}:-2.37 \\
\text { to } 1.62), \mathrm{p}=0.71\end{array}$ \\
\hline \multicolumn{9}{|l|}{$\begin{array}{l}\text { Second-generation } \\
\text { antipsychotic vs. } \\
\text { placebo }\end{array}$} \\
\hline Devlin, $2010^{26}$ & $\begin{array}{l}\text { Patients admitted to Medical \& } \\
\text { Surgical ICU with delirium. }\end{array}$ & $\begin{array}{l}\text { Quetiapine (Mean dose: } 50 \\
\text { Median dose: } 110 \text { (88-191 } \\
\text { IQR) Max dose: } 200 \text { Dose } \\
\text { range: } 50 \text { to 200mg), } 18\end{array}$ & $\begin{array}{l}\text { Placebo (Mean } \\
\text { dose: } 50 \text { Median } \\
\text { dose: } 210 \text { (116 - } \\
\text { 293) Max dose: } \\
\text { 200mg), } 18\end{array}$ & $\begin{array}{l}\text { Either orally or } \\
\text { NG tube }\end{array}$ & $\begin{array}{l}\text { Percent of time in } \\
\text { delirium, as the } \\
\text { percentage of time } \\
\text { subject was } \\
\text { administered study } \\
\text { drug }\end{array}$ & 53 (16 to 67$)$ & $\begin{array}{l}69(58 \text { to } \\
100)\end{array}$ & \\
\hline Devlin, $2010^{26}$ & $\begin{array}{l}\text { Patients admitted to Medical \& } \\
\text { Surgical ICU with delirium. }\end{array}$ & $\begin{array}{l}\text { Quetiapine (Mean dose: } 50 \\
\text { Median dose: } 110 \text { (88-191 } \\
\text { IQR) Max dose: } 200 \text { Dose } \\
\text { range: } 50 \text { to 200mg), } 18\end{array}$ & $\begin{array}{l}\text { Placebo (Mean } \\
\text { dose: } 50 \text { Median } \\
\text { dose: } 210 \text { (116 - } \\
\text { 293) Max dose: } \\
\text { 200mg), } 18 \\
\end{array}$ & $\begin{array}{l}\text { Either orally or } \\
\text { NG tube }\end{array}$ & $\begin{array}{l}\text { Time in delirium in } \\
\text { hours }\end{array}$ & 36 (12 to 87 ) & $\begin{array}{l}120(60 \text { to } \\
195)\end{array}$ & \\
\hline
\end{tabular}




\begin{tabular}{|c|c|c|c|c|c|c|c|c|}
\hline Author, year & Population & Intervention group, $\mathbf{n}$ & Control group, $n$ & $\begin{array}{c}\text { Route of } \\
\text { administration }\end{array}$ & Outcome definition & $\begin{array}{c}\text { Median } \\
\text { (IQR) days, } \\
\text { intervention } \\
\text { group }\end{array}$ & $\begin{array}{c}\text { Median } \\
\text { (IQR), days, } \\
\text { control } \\
\text { group } \\
\end{array}$ & $\begin{array}{c}\text { Measure of } \\
\text { association (95\% } \\
\text { Cl) }\end{array}$ \\
\hline Girard, $2010^{4}$ & $\begin{array}{l}>18 \text { years MV medical and } \\
\text { surgical ICU patients }\end{array}$ & $\begin{array}{l}\text { Ziprasidone (Planned dose: } \\
40 \text { Median dose: } 113 \text { (81- } \\
140), 30\end{array}$ & $\begin{array}{l}\text { Placebo (Planned } \\
\text { dose: } 5 \mathrm{ml}), 36\end{array}$ & Oral & Delirium days & $4(2$ to 8$)$ & $4(2$ to 6$)$ & \\
\hline Girard, $2018^{29}$ & $\begin{array}{l}\text { Patients over } 18 \text { years of age } \\
\text { in the medical or surgical ICU, } \\
\text { with delirium }\end{array}$ & $\begin{array}{l}\text { Ziprasidone (Mean dose: } \\
\text { 20mg Dose range: } 5.0- \\
\text { 40mg), } 190\end{array}$ & $\begin{array}{l}\text { Placebo (Not } \\
\text { reported), } 184\end{array}$ & Intravenous & Days with delirium & 4 (2 to 6$)$ & 4 (2 to 8$)$ & $\begin{array}{l}\text { OR } 1.02(95 \% \mathrm{Cl}: \\
0.69 \text { to } 1.51) \\
\text { p=Not reported }\end{array}$ \\
\hline Girard, $2018^{29}$ & $\begin{array}{l}\text { Patients over } 18 \text { years of age } \\
\text { in the medical or surgical ICU, } \\
\text { with delirium }\end{array}$ & $\begin{array}{l}\text { Ziprasidone (Mean dose: } \\
\text { 20mg Dose range: } 5.0- \\
40 \mathrm{mg} \text { ), } 190\end{array}$ & $\begin{array}{l}\text { Placebo (Not } \\
\text { reported), } 184\end{array}$ & Intravenous & $\begin{array}{l}\text { Days with hyperactive } \\
\text { delirium (RASS }>0 \text { ) }\end{array}$ & 0 (0 to 1$)$ & $0(0$ to 1$)$ & $\begin{array}{l}\text { OR } 1.09(95 \% \mathrm{Cl}: \\
0.7 \text { to } 1.7), p=\text { Not } \\
\text { reported }\end{array}$ \\
\hline Girard, $2018^{29}$ & $\begin{array}{l}\text { Patients over } 18 \text { years of age } \\
\text { in the medical or surgical ICU, } \\
\text { with delirium }\end{array}$ & $\begin{array}{l}\text { Ziprasidone (Mean dose: } \\
\text { 20mg Dose range: } 5.0- \\
40 \mathrm{mg} \text { ), } 190\end{array}$ & $\begin{array}{l}\text { Placebo (Not } \\
\text { reported), } 184\end{array}$ & Intravenous & $\begin{array}{l}\text { Days with hypoactive } \\
\text { delirium (RASS } \leq 0 \text { ) }\end{array}$ & 3 (2 to 6$)$ & 3 (2 to 8$)$ & $\begin{array}{l}\text { OR } 1(95 \% \text { Cl: } 0.68 \\
\text { to } 1.47), p=\text { Not } \\
\text { reported }\end{array}$ \\
\hline \multicolumn{9}{|l|}{$\begin{array}{l}\text { First-generation } \\
\text { antipsychotic vs. } \\
\text { second-generation } \\
\text { antipsychotic }\end{array}$} \\
\hline Girard, $2010^{4}$ & $\begin{array}{l}>18 \text { years MV medical and } \\
\text { surgical ICU patients }\end{array}$ & $\begin{array}{l}\text { Ziprasidone (Planned dose: } \\
40 \text { Median dose: } 113 \text { (81- } \\
140), 30\end{array}$ & $\begin{array}{l}\text { Haloperidol } \\
\text { (Planned dose: } 5 \\
\text { Median dose: } 15 \\
\text { (10.8-17), } 35\end{array}$ & Oral & Delirium days & $\begin{array}{l}\text { Median: } 4 \\
\text { (IQR: } 2 \text { to } 8 \text { ) }\end{array}$ & $\begin{array}{l}\text { Median: } 4 \\
\text { (IQR: } 2 \text { to } 7 \text { ) }\end{array}$ & \\
\hline Girard, $2018^{29}$ & $\begin{array}{l}\text { Patients over } 18 \text { years of age } \\
\text { in the medical or surgical ICU, } \\
\text { with delirium }\end{array}$ & $\begin{array}{l}\text { Ziprasidone (Mean dose: } \\
\text { 20mg Dose range: } 5.0- \\
\text { 40mg), } 190\end{array}$ & $\begin{array}{l}\text { Haloperidol (Mean } \\
\text { dose: } 11 \mathrm{mg} \text { Dose } \\
\text { range: } 2.5-20 \mathrm{mg} \text { ), } \\
192\end{array}$ & Intravenous & Days with delirium & 4 (2 to 6$)$ & $4(2$ to 7$)$ & $\begin{array}{l}\text { OR } 1.02(95 \% \mathrm{Cl}: \\
0.69 \text { to } 1.51) \\
p=\text { Not reported }\end{array}$ \\
\hline Girard, $2018^{29}$ & $\begin{array}{l}\text { Patients over } 18 \text { years of age } \\
\text { in the medical or surgical ICU, } \\
\text { with delirium }\end{array}$ & $\begin{array}{l}\text { Ziprasidone (Mean dose: } \\
\text { 20mg Dose range: } 5.0- \\
\text { 40mg), } 190\end{array}$ & $\begin{array}{l}\text { Haloperidol (Mean } \\
\text { dose: } 11 \mathrm{mg} \text { Dose } \\
\text { range: } 2.5-20 \mathrm{mg} \text { ), } \\
192\end{array}$ & Intravenous & $\begin{array}{l}\text { Days with hyperactive } \\
\text { delirium (RASS }>0 \text { ) }\end{array}$ & 0 (0 to 1$)$ & $0(0$ to 1$)$ & $\begin{array}{l}\text { OR } 1.09(95 \% \mathrm{Cl}: \\
0.7 \text { to } 1.7), p=\mathrm{Not} \\
\text { reported }\end{array}$ \\
\hline Girard, $2018^{29}$ & $\begin{array}{l}\text { Patients over } 18 \text { years of age } \\
\text { in the medical or surgical ICU, } \\
\text { with delirium }\end{array}$ & $\begin{array}{l}\text { Ziprasidone (Mean dose: } \\
\text { 20mg Dose range: } 5.0- \\
\text { 40mg), } 190\end{array}$ & $\begin{array}{l}\text { Haloperidol (Mean } \\
\text { dose: } 11 \mathrm{mg} \text { Dose } \\
\text { range: } 2.5-20 \mathrm{mg} \text { ), } \\
192\end{array}$ & Intravenous & $\begin{array}{l}\text { Days with hypoactive } \\
\text { delirium (RASS } \leq 0 \text { ) }\end{array}$ & 3 (2 to 6$)$ & 4 (2 to 6$)$ & $\begin{array}{l}\text { OR } 1(95 \% \text { Cl: } 0.68 \\
\text { to } 1.47), p=\text { Not } \\
\text { reported }\end{array}$ \\
\hline
\end{tabular}




\begin{tabular}{|c|c|c|c|c|c|c|c|c|}
\hline Author, year & Population & Intervention group, $\mathbf{n}$ & Control group, $\mathrm{n}$ & $\begin{array}{c}\text { Route of } \\
\text { administration }\end{array}$ & Outcome definition & $\begin{array}{l}\text { Median } \\
\text { (IQR) days, } \\
\text { intervention } \\
\text { group }\end{array}$ & $\begin{array}{l}\text { Median } \\
\text { (IQR), days, } \\
\text { control } \\
\text { group }\end{array}$ & $\begin{array}{c}\text { Measure of } \\
\text { association (95\% } \\
\mathrm{Cl})\end{array}$ \\
\hline Han, 2004 ${ }^{32}$ & $\begin{array}{l}\text { All patients presenting with } \\
\text { altered mental status (from } \\
\text { both ICU and non-ICU units) } \\
\text { who were referred to the } \\
\text { consulting psychiatry division } \\
\text { were evaluated. }\end{array}$ & $\begin{array}{l}\text { Risperidone (Mean dose: } \\
1.02 \text { Dose range: } 0.5 \text { to } \\
2.0 \mathrm{mg}), 12\end{array}$ & $\begin{array}{l}\text { Haloperidol (Mean } \\
\text { dose: } 1.7 \text { Dose } \\
\text { range: } 1.0 \text { to } 3.0 \mathrm{mg} \text { ), } \\
12\end{array}$ & Oral & $\begin{array}{l}\text { Period of time until } \\
\text { MDAS }<13\end{array}$ & $\begin{array}{l}\text { Mean: } 4.17 \\
\text { (SD: } 2.14)\end{array}$ & $\begin{array}{l}\text { Mean: } 4.22 \\
\text { (SD: } 2.48)\end{array}$ & $\begin{array}{l}\text { Difference between } \\
\text { means: }-0.05(95 \% \\
\text { Cl: }-1.45 \text { to } 1.35)\end{array}$ \\
\hline Jain, $2017^{38}$ & Medical and surgical inpatients & $\begin{array}{l}\text { Olanzapine (Mean dose: } \\
5.49 \mathrm{mg}), 47\end{array}$ & $\begin{array}{l}\text { Haloperidol (Mean } \\
\text { dose: } 2.10 \mathrm{mg}), 53\end{array}$ & Oral & MDAS score $<10$ & $\begin{array}{l}\text { Mean: } 3.57 \\
\text { (SD: } 0.71 \text { ) }\end{array}$ & $\begin{array}{l}\text { Mean: } 3.37 \\
\text { (SD: } 0.92 \text { ) }\end{array}$ & $\begin{array}{l}\text { Difference between } \\
\text { means: } 0.20(95 \% \\
\text { Cl: }-0.05 \text { to } 0.45)\end{array}$ \\
\hline Lim, $2007^{46}$ & $\begin{array}{l}\text { patients from internal } \\
\text { medicine, PMR, neurology, } \\
\text { neurosurgery, orthopedic } \\
\text { surgery, or general surgery } \\
\text { that were referred to the } \\
\text { psychiatric department }\end{array}$ & $\begin{array}{l}\text { Olanzapine (Titrated (5 to } 20 \\
\text { mg) } 31\end{array}$ & $\begin{array}{l}\text { Haloperidol (Titrated } \\
\text { (5 to } 20 \mathrm{mg}) 31\end{array}$ & Intramuscular & Time to recovery & $\begin{array}{l}\text { Mean: } 3.22 \\
\text { (SD: } 1.2)\end{array}$ & $\begin{array}{l}\text { Mean: } 3.14 \\
\text { (SD: } 1.1)\end{array}$ & $\begin{array}{l}\text { Difference between } \\
\text { means: } 0.08(95 \% \\
\text { Cl: }-0.31 \text { to } 0.47)\end{array}$ \\
\hline Maneeton, $2013^{49}$ & $\begin{array}{l}\text { General adult population (age } \\
\text { 18-75) with hyperactive } \\
\text { delirium, referred to psychiatry } \\
\text { consult liaison service at } \\
\text { tertiary care hospital in } \\
\text { Thailand }\end{array}$ & $\begin{array}{l}\text { Quetiapine (Mean dose: } 67.6 \\
\text { (SD 9.7) Dose range: } 25- \\
\text { 100mg), } 24\end{array}$ & $\begin{array}{l}\text { Haloperidol (Mean } \\
\text { dose: } 0.8 \text { (SD 0.3) } \\
\text { Dose range: } 0.5- \\
\text { 2.0mg), } 28\end{array}$ & Oral & $\begin{array}{l}\text { Time to first remission } \\
\text { (days) }\end{array}$ & $\begin{array}{l}\text { Mean: } 2.6 \\
\text { (SD: } 1.9)\end{array}$ & $\begin{array}{l}\text { Mean: } 1.8 \\
\text { (SD: } 1.5)\end{array}$ & $\begin{array}{l}\text { Hazard ratio } 1.15 \\
(95 \% \mathrm{Cl}: 0.6 \text { to } \\
2.19), p=0.68\end{array}$ \\
\hline \multicolumn{9}{|c|}{$\begin{array}{l}\text { Second-generation } \\
\text { antipsychotic vs. other }\end{array}$} \\
\hline Lee, $2005^{45}$ & $\begin{array}{l}\text { Patients referred to the } \\
\text { Psychiatric Consultation that } \\
\text { met DSM-IV criteria for } \\
\text { delirium }\end{array}$ & $\begin{array}{l}\text { Amisulpride (Mean dose: } \\
\text { 156.4 } \pm 97.5 \text { Dose range: } 50- \\
800 \mathrm{mg}), 16\end{array}$ & $\begin{array}{l}\text { Quetiapine (Mean } \\
\text { dose: } 113 \pm 85.5 \\
\text { Dose range: } 50- \\
\text { 300mg), } 15 \\
\end{array}$ & Not reported & $\begin{array}{l}\text { The mean duration of } \\
\text { stabilization }\end{array}$ & $\begin{array}{l}\text { Mean: } 6.3 \\
\text { (SD: } 4.4 \text { ) }\end{array}$ & $\begin{array}{l}\text { Mean: } 7.4 \\
\text { (SD: 4.1) }\end{array}$ & $\begin{array}{l}\text { Difference between } \\
\text { means: }-1.10 \text { (95\% } \\
\text { Cl: }-3.17 \text { to } 0.97) \\
p=0.4\end{array}$ \\
\hline \multicolumn{9}{|c|}{$\begin{array}{l}\text { First-generation } \\
\text { antipsychotic vs. other }\end{array}$} \\
\hline Atalan, $2013^{18}$ & $\begin{array}{l}\text { Post cardiac surgery patients } \\
\text { with hyperactive type delirium }\end{array}$ & $\begin{array}{l}\text { Morphine sulfate (Max dose: } \\
20 \mathrm{mg}), 27\end{array}$ & $\begin{array}{l}\text { Haloperidol (Max } \\
\text { dose: } 20 \mathrm{mg}), 26\end{array}$ & Intramuscular & Delirium duration & $\begin{array}{l}\text { Mean: } 31.56 \\
\text { (SD: 16.6) }\end{array}$ & $\begin{array}{l}\text { Mean: } 33.92 \\
\text { (SD: } 16.7 \text { ) }\end{array}$ & $\begin{array}{l}\text { Difference between } \\
\text { means: }-2.36 \text { (95\% } \\
\text { Cl: }-8.78 \text { to } 4.06) \\
p=0.607\end{array}$ \\
\hline
\end{tabular}

ratio; $\mathrm{p}=\mathrm{p}$-value; RASS=Richmond Agitation and Sedation Scale; $\mathrm{SD}=$ standard deviation 


\begin{tabular}{|c|c|c|c|c|c|c|c|c|}
\hline Author, year & Population & $\begin{array}{c}\text { Intervention group, } \\
\mathrm{n}\end{array}$ & Control group, $\mathbf{n}$ & $\begin{array}{c}\text { Route of } \\
\text { administration }\end{array}$ & Outcome definition & $\begin{array}{c}\mathrm{n} / \mathrm{N}(\%) \\
\text { intervention } \\
\text { group }\end{array}$ & $\begin{array}{c}\mathrm{n} / \mathrm{N}(\%), \\
\text { control } \\
\text { group }\end{array}$ & $\begin{array}{l}\text { Relative risk } \\
(95 \% \mathrm{Cl})\end{array}$ \\
\hline \multicolumn{9}{|l|}{$\begin{array}{l}\text { First-generation } \\
\text { antipsychotic vs. placebo }\end{array}$} \\
\hline Agar, $2017^{17}$ & $\begin{array}{l}\text { Patients in hospice and } \\
\text { palliative care with } \\
\text { delirium }\end{array}$ & $\begin{array}{l}\text { Haloperidol (Max } \\
\text { dose: } 4 \mathrm{mg} / \mathrm{d} \text { ) }\end{array}$ & $\begin{array}{l}\text { Placebo (Max dose: } 4 \\
\text { mg/day) }\end{array}$ & Oral & $\begin{array}{l}\text { Midazolam } 2.5 \mathrm{mg} \text { for } \\
\text { both placebo and } \\
\text { risperidone. } 4 \mathrm{mg} \text { for } \\
\text { haloperidol group }\end{array}$ & & & $\begin{array}{l}\text { Midazolam use } \\
\text { was significantly } \\
\text { lower among } \\
\text { those in the } \\
\text { placebo arm } \\
\text { compared with } \\
\text { the risperidone } \\
\text { and haloperidol } \\
\text { arms combined } \\
\text { on each study } \\
\text { day (13 of } 75 \\
{[17.3 \%] \text { vs } 50 \text { of }} \\
144[34.7 \%] \text { on } \\
\text { day } 1 ; \mathrm{P}=.007 ; \\
11 \text { of } 68[16.8 \%] \\
\text { vs } 40 \text { of } 121 \\
{[33.1 \%] \text { on day }} \\
2 ; \mathrm{P}=.01 ; \text { and } 9 \\
\text { of } 66[13.6 \%] \text { vs } \\
32 \text { of } 108 \\
{[29.6 \%] \text { on day }} \\
3 ; \mathrm{P}=.02) \text {. }\end{array}$ \\
\hline Girard, $2010^{4}$ & $\begin{array}{l}>18 \text { years MV medical } \\
\text { and surgical ICU } \\
\text { patients }\end{array}$ & $\begin{array}{l}\text { Haloperidol (Planned } \\
\text { dose: } 5 \text { Median dose: } \\
15 \text { (10.8-17), } 35\end{array}$ & $\begin{array}{l}\text { Placebo (Planned dose: } \\
5 \mathrm{ml}), 36\end{array}$ & Oral & $\begin{array}{l}\text { Haloperidol varies for } \\
\text { each group }\end{array}$ & $6 / 35(17 \%)$ & $\begin{array}{l}14 / 36 \\
(39 \%)\end{array}$ & $\begin{array}{l}0.44(0.19 \text { to } \\
1.02)\end{array}$ \\
\hline Girard, $2018^{29}$ & $\begin{array}{l}\text { Patients over } 18 \text { years } \\
\text { of age in the medical or } \\
\text { surgical ICU, with } \\
\text { delirium }\end{array}$ & $\begin{array}{l}\text { Haloperidol (Mean } \\
\text { dose: } 11 \mathrm{mg} \text { Dose } \\
\text { range: } 2.5-20 \mathrm{mg} \text { ), } \\
192\end{array}$ & $\begin{array}{l}\text { Placebo (Not reported), } \\
184\end{array}$ & Intravenous & $\begin{array}{l}\text { Open label antipsychotic } \\
\text { exposure }\end{array}$ & 39 / $192(20 \%)$ & $\begin{array}{l}38 / 184 \\
(22 \%)\end{array}$ & $\begin{array}{l}0.98 \text { (0.66 to } \\
1.47)\end{array}$ \\
\hline Page, $2013^{10}$ & $\begin{array}{l}\text { ICU patients needing } \\
\text { MV within } 72 \text { hours of } \\
\text { admission were enrolled }\end{array}$ & $\begin{array}{l}\text { Haloperidol (Planned } \\
\text { dose: } 2.5 \mathrm{mg}), 71\end{array}$ & $\begin{array}{l}\text { Placebo (Planned dose: } \\
0.5 \mathrm{ml}), 70\end{array}$ & Intravenous & Any anti-psychotic & $8 / 71(11 \%)$ & $\begin{array}{l}18 / 70 \\
(26 \%)\end{array}$ & $\begin{array}{l}0.44(0.20 \text { to } \\
0.94)\end{array}$ \\
\hline Page, $2013^{10}$ & $\begin{array}{l}\text { ICU patients needing } \\
\text { MV within } 72 \text { hours of } \\
\text { admission were enrolled }\end{array}$ & $\begin{array}{l}\text { Haloperidol (Planned } \\
\text { dose: } 2.5 \mathrm{mg} \text { ), } 71\end{array}$ & $\begin{array}{l}\text { Placebo (Planned dose: } \\
0.5 \mathrm{ml}), 70\end{array}$ & Intravenous & Open Label Haloperidol & $6 / 71(8 \%)$ & $\begin{array}{l}15 / 70 \\
(21 \%)\end{array}$ & $\begin{array}{l}0.39(0.16 \text { to } \\
0.96)\end{array}$ \\
\hline
\end{tabular}




\begin{tabular}{|c|c|c|c|c|c|c|c|c|}
\hline Author, year & Population & $\begin{array}{c}\text { Intervention group, } \\
\mathrm{n}\end{array}$ & Control group, $\mathbf{n}$ & $\begin{array}{c}\text { Route of } \\
\text { administration }\end{array}$ & Outcome definition & $\begin{array}{c}\mathrm{n} / \mathrm{N}(\%) \\
\text { intervention } \\
\text { group }\end{array}$ & $\begin{array}{c}\mathrm{n} / \mathrm{N}(\%), \\
\text { control } \\
\text { group }\end{array}$ & $\begin{array}{l}\text { Relative risk } \\
(95 \% \mathrm{Cl})\end{array}$ \\
\hline \multicolumn{9}{|c|}{$\begin{array}{l}\text { Second-generation } \\
\text { antipsychotic vs. placebo }\end{array}$} \\
\hline Agar, $2017^{17}$ & $\begin{array}{l}\text { Patients in Hospice and } \\
\text { palliative care with } \\
\text { delirium }\end{array}$ & $\begin{array}{l}\text { Risperidone (Max } \\
\text { dose: } 4 \mathrm{mg} / \mathrm{d} \text { ) }\end{array}$ & $\begin{array}{l}\text { Placebo (Max dose: } 4 \\
\text { mg/day) }\end{array}$ & Oral & $\begin{array}{l}\text { Midazolam } 2.5 \mathrm{mg} \text { for } \\
\text { both placebo and } \\
\text { risperidone. } 4 \mathrm{mg} \text { for } \\
\text { haloperidol group }\end{array}$ & & & $\begin{array}{l}\text { Midazolam use } \\
\text { was significantly } \\
\text { lower among } \\
\text { those in the } \\
\text { placebo arm } \\
\text { compared with } \\
\text { the risperidone } \\
\text { and haloperidol } \\
\text { arms combined } \\
\text { on each study } \\
\text { day (13 of } 75 \\
{[17.3 \%] \text { vs } 50 \text { of }} \\
144[34.7 \%] \text { on } \\
\text { day } 1 ; \mathrm{P}=.007 ; \\
11 \text { of } 68[16.8 \%] \\
\text { vs } 40 \text { of } 121 \\
{[33.1 \%] \text { on day }} \\
2 ; \mathrm{P}=.01 ; \text { and } 9 \\
\text { of } 66[13.6 \%] \text { vs } \\
32 \text { of } 108 \\
{[29.6 \%] \text { on day }} \\
3 ; \mathrm{P}=.02) \text {. }\end{array}$ \\
\hline Girard, $2010^{4}$ & $\begin{array}{l}>18 \text { years MV medical } \\
\text { and surgical ICU } \\
\text { patients }\end{array}$ & $\begin{array}{l}\text { Ziprasidone (Planned } \\
\text { dose: } 40 \text { Median } \\
\text { dose: } 113 \text { (81-140), } \\
30\end{array}$ & $\begin{array}{l}\text { Placebo (Planned dose: } \\
5 \mathrm{ml}), 36\end{array}$ & Oral & $\begin{array}{l}\text { Haloperidol varies for } \\
\text { each group }\end{array}$ & $9 / 30(30 \%)$ & $\begin{array}{l}14 / 36 \\
(39 \%)\end{array}$ & $\begin{array}{l}0.77 \text { (0.39 to } \\
1.53)\end{array}$ \\
\hline Girard, $2018^{29}$ & $\begin{array}{l}\text { Patients over } 18 \text { years } \\
\text { of age in the medical or } \\
\text { surgical ICU, with } \\
\text { delirium }\end{array}$ & $\begin{array}{l}\text { Ziprasidone (Mean } \\
\text { dose: } 20 \mathrm{mg} \text { Dose } \\
\text { range: } 5.0-40 \mathrm{mg} \text { ), } \\
190\end{array}$ & $\begin{array}{l}\text { Placebo (Not reported), } \\
184\end{array}$ & Intravenous & $\begin{array}{l}\text { Open label antipsychotic } \\
\text { exposure }\end{array}$ & $41 / 190$ (22\%) & $\begin{array}{l}38 / 184 \\
(22 \%)\end{array}$ & $\begin{array}{l}1.05(0.71 \text { to } \\
1.55)\end{array}$ \\
\hline
\end{tabular}




\begin{tabular}{|c|c|c|c|c|c|c|c|c|}
\hline Author, year & Population & $\begin{array}{c}\text { Intervention group, } \\
\mathrm{n}\end{array}$ & Control group, $\mathbf{n}$ & $\begin{array}{c}\text { Route of } \\
\text { administration }\end{array}$ & Outcome definition & $\begin{array}{c}\mathrm{n} / \mathrm{N}(\%) \\
\text { intervention } \\
\text { group }\end{array}$ & $\begin{array}{c}\mathrm{n} / \mathrm{N}(\%) \\
\text { control } \\
\text { group }\end{array}$ & $\begin{array}{l}\text { Relative risk } \\
(95 \% \mathrm{Cl})\end{array}$ \\
\hline \multicolumn{9}{|c|}{$\begin{array}{l}\text { First-generation } \\
\text { antipsychotic vs. second- } \\
\text { generation antipsychotic }\end{array}$} \\
\hline Agar, $2017^{17}$ & $\begin{array}{l}\text { Patients in hospice and } \\
\text { palliative care with } \\
\text { delirium }\end{array}$ & $\begin{array}{l}\text { Risperidone (Max } \\
\text { dose: } 4 \mathrm{mg} / \mathrm{d} \text { ), }\end{array}$ & $\begin{array}{l}\text { Haloperidol (Max dose: } 4 \\
\text { mg/d), }\end{array}$ & Oral & $\begin{array}{l}\text { Midazolam } 2.5 \mathrm{mg} \text { for } \\
\text { both placebo and } \\
\text { risperidone. } 4 \mathrm{mg} \text { for } \\
\text { haloperidol group }\end{array}$ & & & $\begin{array}{l}\text { Midazolam use } \\
\text { was significantly } \\
\text { lower among } \\
\text { those in the } \\
\text { placebo arm } \\
\text { compared with } \\
\text { the risperidone } \\
\text { and haloperidol } \\
\text { arms combined } \\
\text { on each study } \\
\text { day (13 of } 75 \\
{[17.3 \%] \text { vs } 50 \text { of }} \\
144[34.7 \%] \text { on } \\
\text { day } 1 ; \mathrm{P}=.007 ; \\
11 \text { of } 68[16.8 \%] \\
\text { vs } 40 \text { of } 121 \\
{[33.1 \%] \text { on day }} \\
2 ; P=.01 ; \text { and } 9 \\
\text { of } 66[13.6 \%] \text { vs } \\
32 \text { of } 108 \\
{[29.6 \%] \text { on day }} \\
3 ; P=.02) \text {. }\end{array}$ \\
\hline Girard, $2010^{4}$ & $\begin{array}{l}>18 \text { years MV medical } \\
\text { and surgical ICU } \\
\text { patients }\end{array}$ & $\begin{array}{l}\text { Ziprasidone (Planned } \\
\text { dose: } 40 \text { Median } \\
\text { dose: } 113 \text { (81-140), } \\
30\end{array}$ & $\begin{array}{l}\text { Haloperidol (Planned } \\
\text { dose: } 5 \text { Median dose: } 15 \\
\text { (10.8-17), } 35\end{array}$ & Oral & $\begin{array}{l}\text { Haloperidol varies for } \\
\text { each group }\end{array}$ & $9 / 30(30 \%)$ & $6 / 35(17 \%)$ & $\begin{array}{l}1.75(0.70 \text { to } \\
4.35)\end{array}$ \\
\hline Girard, 2018 29 & $\begin{array}{l}\text { Patients over } 18 \text { years } \\
\text { of age in the medical or } \\
\text { surgical ICU, with } \\
\text { delirium }\end{array}$ & $\begin{array}{l}\text { Ziprasidone (Mean } \\
\text { dose: } 20 \mathrm{mg} \text { Dose } \\
\text { range: } 5.0-40 \mathrm{mg} \text { ), } \\
190\end{array}$ & $\begin{array}{l}\text { Haloperidol (Mean dose: } \\
\text { 11mg Dose range: } 2.5- \\
\text { 20mg), } 192\end{array}$ & Intravenous & $\begin{array}{l}\text { Open label antipsychotic } \\
\text { exposure }\end{array}$ & $41 / 190(22 \%)$ & $\begin{array}{l}39 / 192 \\
(20 \%)\end{array}$ & $\begin{array}{l}1.06(0.72 \text { to } \\
1.57)\end{array}$ \\
\hline
\end{tabular}




\begin{tabular}{|c|c|c|c|c|c|c|c|c|}
\hline Author, year & Population & $\begin{array}{c}\text { Intervention group, } \\
\mathrm{n}\end{array}$ & Control group, $\mathbf{n}$ & $\begin{array}{c}\text { Route of } \\
\text { administration }\end{array}$ & Outcome definition & $\begin{array}{c}\mathrm{n} / \mathrm{N}(\%) \\
\text { intervention } \\
\text { group }\end{array}$ & $\begin{array}{c}\mathrm{n} / \mathrm{N}(\%) \\
\text { control } \\
\text { group }\end{array}$ & $\begin{array}{l}\text { Relative risk } \\
(95 \% \mathrm{Cl})\end{array}$ \\
\hline Lin, $2008^{47}$ & $\begin{array}{l}\text { Patients receiving } \\
\text { hospice or palliative } \\
\text { care with advanced } \\
\text { cancer and met DSM-IV } \\
\text { criteria for delirium }\end{array}$ & $\begin{array}{l}\text { Olanzapine (Planned } \\
\text { dose: start dose } 5 \mathrm{mg} \\
\text { Max dose: } 15 \mathrm{mg}), 5\end{array}$ & $\begin{array}{l}\text { Haloperidol (Planned } \\
\text { dose: start dose } 5 \mathrm{mg} \text { Max } \\
\text { dose: } 15 \mathrm{mg}), 7\end{array}$ & Oral & $\begin{array}{l}\text { Midazolam dose not } \\
\text { reported, as needed }\end{array}$ & & & $\begin{array}{l}\text { Only states } \\
\text { midozolam } \\
\text { intramuscular } \\
\text { injection across } \\
\text { time periods } \\
\text { were found to be } \\
\text { not significantly } \\
\text { different } \\
\text { statistically. }\end{array}$ \\
\hline Skrobik, $2004^{57}$ & Medical-surgical ICU & $\begin{array}{l}\text { Olanzapine (Mean } \\
\text { dose: } 4.54 \text { Dose } \\
\text { range: } 2.5 \text { to } \\
13.5 \mathrm{mg}), 28\end{array}$ & $\begin{array}{l}\text { Haloperidol (Mean dose: } \\
6.5 \text { Dose range: } 1 \text { to } \\
28 \mathrm{mg}), 45\end{array}$ & Oral & IV Haloperidol 1-5 mg & $10 / 28(\%)$ & 19 / $45(\%)$ & $\begin{array}{l}0.85(0.46 \text { to } \\
1.55)\end{array}$ \\
\hline \multicolumn{9}{|c|}{$\begin{array}{l}\text { Second-generation } \\
\text { antipsychotic vs. second- } \\
\text { generation antipsychotic }\end{array}$} \\
\hline Kim, $2010^{42}$ & $\begin{array}{l}\text { General hospital } \\
\text { inpatients who met } \\
\text { DSM-IV diagnostic } \\
\text { criteria for delirium }\end{array}$ & $\begin{array}{l}\text { Risperidone (Mean } \\
\text { dose: } 0.9 \pm 0.6 \text { Dose } \\
\text { range: } 0.25-2 \mathrm{mg} \text { ), } \\
17\end{array}$ & $\begin{array}{l}\text { Olanzapine (Mean dose: } \\
2.4 \pm 1.7 \text { Dose range: } 1.25 \\
-7.5 \mathrm{mg}), 15\end{array}$ & Not reported & Haloperidol IM & $3 / 17(18 \%)$ & $0 / 15(0 \%)$ & $\begin{array}{l}6.22(0.35 \text { to } \\
111.47)\end{array}$ \\
\hline Kim, $2010^{42}$ & $\begin{array}{l}\text { General hospital } \\
\text { inpatients who met } \\
\text { DSM-IV diagnostic } \\
\text { criteria for delirium } \\
\end{array}$ & $\begin{array}{l}\text { Risperidone (Mean } \\
\text { dose: } 0.9 \pm 0.6 \text { Dose } \\
\text { range: } 0.25-2 \mathrm{mg} \text { ), } \\
17\end{array}$ & $\begin{array}{l}\text { Olanzapine (Mean dose: } \\
2.4 \pm 1.7 \text { Dose range: } 1.25 \\
-7.5 \mathrm{mg}), 15\end{array}$ & Not reported & Benzodiazepine IM & $7 / 17(41.2 \%)$ & $\begin{array}{l}4 / 15 \\
(26.7 \%)\end{array}$ & $\begin{array}{l}1.54(0.56 \text { to } \\
4.25)\end{array}$ \\
\hline \multicolumn{9}{|l|}{$\begin{array}{l}\text { First-generation } \\
\text { antipsychotic vs. other }\end{array}$} \\
\hline Bakri, $2015^{19}$ & $\begin{array}{l}\text { ICU trauma patients } \\
\text { without severe injury, } \\
\text { comatose, or moribund } \\
\text { patients were excluded }\end{array}$ & $\begin{array}{l}\text { Haloperidol (Planned } \\
\text { dose: } 5 \mathrm{mg} \text { ), } 32\end{array}$ & $\begin{array}{l}\text { Ondansetron (Planned } \\
\text { dose: } 8 \mathrm{mg} \text { ), } 32\end{array}$ & Intravenous & Haloperidol & $3 / 32(9 \%)$ & $\begin{array}{l}11 / 32 \\
(34 \%)\end{array}$ & $\begin{array}{l}0.27(0.08 \text { to } \\
0.89)\end{array}$ \\
\hline Bakri, $2015^{19}$ & $\begin{array}{l}\text { ICU trauma patients } \\
\text { without severe injury, } \\
\text { comatose, or moribund } \\
\text { patients were excluded }\end{array}$ & $\begin{array}{l}\text { Haloperidol (Planned } \\
\text { dose: } 5 \mathrm{mg} \text { ), } 32\end{array}$ & $\begin{array}{l}\text { Dexmedetomidine } \\
\text { (Planned dose: } 1 \text { ug } / \mathrm{kg} \text { ), } \\
32\end{array}$ & Intravenous & Haloperidol & $3 / 32(9 \%)$ & $5 / 32(16 \%)$ & $\begin{array}{l}0.60(0.16 \text { to } \\
2.30)\end{array}$ \\
\hline
\end{tabular}

CI=confidence interval; DSM-IV=Diagnostic and Statistical Manual of Mental Disorders, $4^{\text {th }}$ ed; ICU=intensive care unit; IQR=interquartile range;
ventilation; NG=nadogastric tube; OR=odds ratio; p=p-value; p=p-value; RASS=Richmond Agitation and Sedation Scale; SD=standard deviation 
Evidence Table D-38. Mortality outcomes for studies comparing interventions to treat delirium

\begin{tabular}{|c|c|c|c|c|c|c|c|c|}
\hline Author, year & $\begin{array}{l}\text { Population } \\
\end{array}$ & Intervention group, $\mathbf{n}$ & Control group, $\mathrm{n}$ & $\begin{array}{c}\text { Route of } \\
\text { administration }\end{array}$ & $\begin{array}{l}\text { Outcome } \\
\text { definition }\end{array}$ & $\begin{array}{l}\mathrm{n} / \mathrm{N}(\%) \\
\text { intervention } \\
\text { group }\end{array}$ & $\begin{array}{l}\mathrm{n} / \mathrm{N} \\
(\%), \\
\text { control } \\
\text { group }\end{array}$ & $\begin{array}{l}\text { Relative } \\
\text { risk } \\
(95 \% \mathrm{Cl})\end{array}$ \\
\hline \multicolumn{9}{|l|}{$\begin{array}{l}\text { First-generation } \\
\text { antipsychotic vs. placebo }\end{array}$} \\
\hline Agar, $2017^{17}$ & $\begin{array}{l}\text { Patients in Hospice and palliative care } \\
\text { with delirium }\end{array}$ & $\begin{array}{l}\text { Haloperidol (Max dose: } 4 \mathrm{mg} / \mathrm{d} \text { ), } \\
81\end{array}$ & $\begin{array}{l}\text { Placebo (Max dose: } 4 \text { mg/day), } \\
84\end{array}$ & Oral & $\begin{array}{l}\text { Died } \\
\text { during } \\
\text { study } \\
\text { period }\end{array}$ & $\begin{array}{l}9 / 81 \\
(11.1 \%)\end{array}$ & $\begin{array}{l}9 / 84 \\
(10.7 \%)\end{array}$ & $\begin{array}{l}1.17 \\
(0.47 \text { to } \\
2.88)\end{array}$ \\
\hline Girard, $2010^{4}$ & $\begin{array}{l}>18 \text { years MV medical and surgical } \\
\text { ICU patients }\end{array}$ & $\begin{array}{l}\text { Haloperidol (Planned dose: } 5 \\
\text { Median dose: } 15 \text { (10.8-17), } 35\end{array}$ & Placebo (Planned dose: $5 \mathrm{ml}$ ), 36 & Oral & $\begin{array}{l}\text { 21-day } \\
\text { mortality, n } \\
(\%)\end{array}$ & $4 / 35(11 \%)$ & $\begin{array}{l}6 / 36 \\
(17 \%)\end{array}$ & $\begin{array}{l}0.69 \\
(0.21 \text { to } \\
2.22)\end{array}$ \\
\hline Girard, $2018^{29}$ & $\begin{array}{l}\text { Patients over } 18 \text { years of age in the } \\
\text { medical or surgical ICU, with delirium }\end{array}$ & $\begin{array}{l}\text { Haloperidol (Mean dose: } 11 \mathrm{mg} \\
\text { Dose range: } 2.5-20 \mathrm{mg}), 192\end{array}$ & Placebo (Not reported), 184 & Intravenous & $\begin{array}{l}\text { Death at } \\
30 \text { days }\end{array}$ & $\begin{array}{l}50 / 192 \\
(26 \%)\end{array}$ & $\begin{array}{l}50 / \\
184 \\
(27 \%)\end{array}$ & $\begin{array}{l}0.96 \\
(0.69 \text { to } \\
1.34)\end{array}$ \\
\hline Girard, $2018^{29}$ & $\begin{array}{l}\text { Patients over } 18 \text { years of age in the } \\
\text { medical or surgical ICU, with delirium }\end{array}$ & $\begin{array}{l}\text { Haloperidol (Mean dose: } 11 \mathrm{mg} \\
\text { Dose range: } 2.5-20 \mathrm{mg}), 192\end{array}$ & Placebo (Not reported), 184 & Intravenous & $\begin{array}{l}\text { Death at } \\
90 \text { days }\end{array}$ & $\begin{array}{l}73 / 192 \\
(38 \%)\end{array}$ & $\begin{array}{l}63 / \\
184 \\
(34 \%) \\
\end{array}$ & $\begin{array}{l}1.11 \\
(0.85 \text { to } \\
1.45)\end{array}$ \\
\hline $\begin{array}{l}\text { Page, } 2013^{10} \text { Drinkwater, } \\
2014^{27}\end{array}$ & $\begin{array}{l}\text { ICU patients needing MV within } 72 \\
\text { hours of admission were enrolled }\end{array}$ & $\begin{array}{l}\text { Haloperidol (Planned dose: } \\
2.5 \mathrm{mg}), 71\end{array}$ & $\begin{array}{l}\text { Placebo (Planned dose: } 0.5 \mathrm{ml} \text { ), } \\
70\end{array}$ & Intravenous & & $\begin{array}{l}20 / 71 \\
(28.2 \%)\end{array}$ & $\begin{array}{l}19 / 70 \\
(27.1 \%)\end{array}$ & $\begin{array}{l}1.04 \\
(0.61 \text { to } \\
1.77)\end{array}$ \\
\hline \multicolumn{9}{|l|}{$\begin{array}{l}\text { Second-generation } \\
\text { antipsychotic vs. placebo }\end{array}$} \\
\hline Agar, $2017^{17}$ & $\begin{array}{l}\text { Patients in Hospice and palliative care } \\
\text { with delirium }\end{array}$ & $\begin{array}{l}\text { Risperidone (Max dose: } 4 \text { mg/d), } \\
82\end{array}$ & $\begin{array}{l}\text { Placebo (Max dose: } 4 \text { mg/day), } \\
84\end{array}$ & Oral & $\begin{array}{l}\text { died during } \\
\text { study } \\
\text { period }\end{array}$ & $\begin{array}{l}16 / 82 \\
(19.5 \%)\end{array}$ & $\begin{array}{l}9 / 84 \\
(10.7 \%)\end{array}$ & $\begin{array}{l}1.82 \\
(0.85 \text { to } \\
3.89) \\
\end{array}$ \\
\hline Devlin, $2010^{26}$ & $\begin{array}{l}\text { Patients admitted to Medical \& } \\
\text { Surgical ICU with delirium. }\end{array}$ & $\begin{array}{l}\text { Quetiapine (Mean dose: } 50 \\
\text { Median dose: } 110 \text { ( } 88-191 \text { IQR) } \\
\text { Max dose: } 200 \text { Dose range: } 50 \text { to } \\
\text { 200mg), } 18\end{array}$ & $\begin{array}{l}\text { Placebo (Mean dose: } 50 \text { Median } \\
\text { dose: } 210 \text { (116 - 293) Max dose: } \\
\text { 200mg), } 18\end{array}$ & $\begin{array}{l}\text { Either orally or } \\
\text { NG tube }\end{array}$ & $\begin{array}{l}\text { \%, Hospital } \\
\text { Mortality }\end{array}$ & $2 / 18(11 \%)$ & $\begin{array}{l}3 / 18 \\
(17 \%)\end{array}$ & $\begin{array}{l}0.67 \\
(0.13 \text { to } \\
3.53)\end{array}$ \\
\hline Girard, $2010^{4}$ & $\begin{array}{l}\text { > } 18 \text { years MV medical and surgical } \\
\text { ICU patients }\end{array}$ & $\begin{array}{l}\text { Ziprasidone (Planned dose: } 40 \\
\text { Median dose: } 113 \text { (81-140), } 30\end{array}$ & Placebo (Planned dose: $5 \mathrm{ml}$ ), 36 & Oral & $\begin{array}{l}\text { 21-day } \\
\text { mortality, } \mathrm{n} \\
(\%)\end{array}$ & $4 / 30(13 \%)$ & $\begin{array}{l}6 / 36 \\
(17 \%)\end{array}$ & $\begin{array}{l}0.80 \\
(0.25 \text { to } \\
2.57) \\
\end{array}$ \\
\hline Girard, $2018^{29}$ & $\begin{array}{l}\text { Patients over } 18 \text { years of age in the } \\
\text { medical or surgical ICU, with delirium }\end{array}$ & $\begin{array}{l}\text { Ziprasidone (Mean dose: } 20 \mathrm{mg} \\
\text { Dose range: } 5.0-40 \mathrm{mg}), 190\end{array}$ & Placebo (Not reported), 184 & Intravenous & $\begin{array}{l}\text { Death at } \\
30 \text { days }\end{array}$ & $\begin{array}{l}53 / 190 \\
(28 \%)\end{array}$ & $\begin{array}{l}50 / \\
184 \\
(27 \%)\end{array}$ & $\begin{array}{l}1.03 \\
(0.74 \text { to } \\
1.43)\end{array}$ \\
\hline
\end{tabular}




\begin{tabular}{|c|c|c|c|c|c|c|c|c|}
\hline Author, year & Population & Intervention group, $\mathbf{n}$ & Control group, $n$ & $\begin{array}{c}\text { Route of } \\
\text { administration }\end{array}$ & $\begin{array}{l}\text { Outcome } \\
\text { definition }\end{array}$ & $\begin{array}{l}\mathrm{n} / \mathrm{N}(\%) \\
\text { intervention } \\
\text { group }\end{array}$ & $\begin{array}{l}\mathrm{n} / \mathrm{N} \\
(\%), \\
\text { control } \\
\text { group }\end{array}$ & $\begin{array}{l}\text { Relative } \\
\text { risk } \\
(95 \% \mathrm{Cl})\end{array}$ \\
\hline Girard, $2018^{29}$ & $\begin{array}{l}\text { Patients over } 18 \text { years of age in the } \\
\text { medical or surgical ICU, with delirium }\end{array}$ & $\begin{array}{l}\text { Ziprasidone (Mean dose: } 20 \mathrm{mg} \\
\text { Dose range: } 5.0-40 \mathrm{mg}), 190\end{array}$ & Placebo (Not reported), 184 & Intravenous & $\begin{array}{l}\text { Death at } \\
90 \text { days }\end{array}$ & $\begin{array}{l}65 / 190 \\
(34 \%)\end{array}$ & $\begin{array}{l}63 / \\
184 \\
(34 \%) \\
\end{array}$ & $\begin{array}{l}1.00 \\
(0.75 \text { to } \\
1.32)\end{array}$ \\
\hline Tahir, $2010^{59}$ & $\begin{array}{l}\text { Patients in medical, surgical and } \\
\text { orthopedic wards, who met the DSM- } \\
\text { IV criteria for delirium on the same day } \\
\text { if they had a DRS-R-98 total score of } \\
15 \text { or more }\end{array}$ & $\begin{array}{l}\text { Quetiapine (Mean dose: } 40 \text { Dose } \\
\text { range: } 25 \text { - 175mg), } 21\end{array}$ & $\begin{array}{l}\text { Placebo (Mean dose: } 25 \text { Max } \\
\text { dose: } 175 \mathrm{mg}), 21\end{array}$ & Oral & $\begin{array}{l}\text { Death } \\
\text { within } 30 \\
\text { days of } \\
\text { entering } \\
\text { the study } \\
\end{array}$ & $4 / 21(19 \%)$ & $\begin{array}{l}3 / 21 \\
(\%)\end{array}$ & $\begin{array}{l}1.33 \\
(0.34 \text { to } \\
5.24)\end{array}$ \\
\hline Tahir, $2010^{59}$ & $\begin{array}{l}\text { Patients in medical, surgical and } \\
\text { orthopedic wards, who met the DSM- } \\
\text { IV criteria for delirium on the same day } \\
\text { if they had a DRS-R-98 total score of } \\
15 \text { or more }\end{array}$ & $\begin{array}{l}\text { Quetiapine (Mean dose: } 40 \text { Dose } \\
\text { range: } 25 \text { - 175mg), } 21\end{array}$ & $\begin{array}{l}\text { Placebo (Mean dose: } 25 \text { Max } \\
\text { dose: } 175 \mathrm{mg}), 21\end{array}$ & Oral & $\begin{array}{l}\text { Deaths } \\
\text { before the } \\
\text { completion } \\
\text { of study }\end{array}$ & $\begin{array}{l}3 / 21 \\
(14.3 \%)\end{array}$ & $\begin{array}{l}1 / 21 \\
(4.8 \%)\end{array}$ & $\begin{array}{l}3.00 \\
(0.34 \text { to } \\
26.56)\end{array}$ \\
\hline \multicolumn{9}{|c|}{$\begin{array}{l}\text { First-generation } \\
\text { antipsychotic vs. first- } \\
\text { generation antipsychotic }\end{array}$} \\
\hline Breitbart, $1996^{22}$ & $\begin{array}{l}\text { Medically hospitalized adult patients } \\
\text { with AIDS and a DRS score of } 13 \text { or } \\
\text { greater (delirium) }\end{array}$ & $\begin{array}{l}\text { Haloperidol (Planned dose: oral: } \\
0.25-5.0 \mathrm{mg} \text {, Intramuscular: } \\
0.125-3.0 \mathrm{mg} \text { Mean dose: } 1.4 \\
\text { Dose range: } 0.4-3.6 \mathrm{mg}), 11 \\
\end{array}$ & $\begin{array}{l}\text { Chlorpromazine (Planned dose: } \\
\text { Oral: } 10-200 \mathrm{mg} \text {, Intramuscular: } \\
\text { 5-100mg Mean dose: } 36 \text { Dose } \\
\text { range: } 10-80 \mathrm{mg}), 13\end{array}$ & $\begin{array}{l}\text { Oral or } \\
\text { intramuscular }\end{array}$ & & $\begin{array}{l}2 / 11 \\
(18.2 \%)\end{array}$ & $\begin{array}{l}2 / 13 \\
(15.4 \%)\end{array}$ & $\begin{array}{l}1.18 \\
(0.20 \text { to } \\
7.06)\end{array}$ \\
\hline \multicolumn{9}{|c|}{$\begin{array}{l}\text { First-generation } \\
\text { antipsychotic vs. second- } \\
\text { generation antipsychotic }\end{array}$} \\
\hline Agar, $2017^{17}$ & $\begin{array}{l}\text { Patients in Hospice and palliative care } \\
\text { with delirium }\end{array}$ & $\begin{array}{l}\text { Risperidone (Max dose: } 4 \mathrm{mg} / \mathrm{d} \text { ), } \\
82\end{array}$ & $\begin{array}{l}\text { Haloperidol (Max dose: } 4 \mathrm{mg} / \mathrm{d} \text { ), } \\
81\end{array}$ & Oral & $\begin{array}{l}\text { died during } \\
\text { study } \\
\text { period }\end{array}$ & $\begin{array}{l}16 / 82 \\
(19.5 \%)\end{array}$ & $\begin{array}{l}9 / 81 \\
(11.1 \%)\end{array}$ & $\begin{array}{l}1.76 \\
(0.82 \text { to } \\
3.74)\end{array}$ \\
\hline Girard, $2010^{4}$ & $\begin{array}{l}>18 \text { years MV medical and surgical } \\
\text { ICU patients }\end{array}$ & $\begin{array}{l}\text { Ziprasidone (Planned dose: } 40 \\
\text { Median dose: } 113 \text { (81-140), } 30\end{array}$ & $\begin{array}{l}\text { Haloperidol (Planned dose: } 5 \\
\text { Median dose: } 15 \text { (10.8-17), } 35\end{array}$ & Oral & $\begin{array}{l}\text { 21-day } \\
\text { mortality, } \mathrm{n} \\
(\%)\end{array}$ & $4 / 30$ (13\%) & $\begin{array}{l}4 / 35 \\
(11 \%)\end{array}$ & $\begin{array}{l}1.17(0.32 \\
\text { to } 4.27)\end{array}$ \\
\hline Girard, $2018^{29}$ & $\begin{array}{l}\text { Patients over } 18 \text { years of age in the } \\
\text { medical or surgical ICU, with delirium }\end{array}$ & $\begin{array}{l}\text { Ziprasidone (Mean dose: } 20 \mathrm{mg} \\
\text { Dose range: } 5.0-40 \mathrm{mg} \text { ), } 190\end{array}$ & $\begin{array}{l}\text { Haloperidol (Mean dose: } 11 \mathrm{mg} \\
\text { Dose range: } 2.5-20 \mathrm{mg}), 192\end{array}$ & Intravenous & $\begin{array}{l}\text { Death at } \\
30 \text { days }\end{array}$ & $\begin{array}{l}53 / 190 \\
(28 \%)\end{array}$ & $\begin{array}{l}50 / \\
192 \\
(26 \%)\end{array}$ & $\begin{array}{l}1.07 \\
(0.77 \text { to } \\
1.49)\end{array}$ \\
\hline Girard, $2018^{29}$ & $\begin{array}{l}\text { Patients over } 18 \text { years of age in the } \\
\text { medical or surgical ICU, with delirium }\end{array}$ & $\begin{array}{l}\text { Ziprasidone (Mean dose: } 20 \mathrm{mg} \\
\text { Dose range: } 5.0-40 \mathrm{mg}), 190\end{array}$ & $\begin{array}{l}\text { Haloperidol (Mean dose: } 11 \mathrm{mg} \\
\text { Dose range: } 2.5-20 \mathrm{mg}), 192\end{array}$ & Intravenous & $\begin{array}{l}\text { Death at } \\
90 \text { days }\end{array}$ & $\begin{array}{l}65 / 190 \\
(34 \%)\end{array}$ & $\begin{array}{l}73 / \\
192 \\
(38 \%) \\
\end{array}$ & $\begin{array}{l}0.90 \\
(0.69 \text { to } \\
1.18)\end{array}$ \\
\hline
\end{tabular}




\begin{tabular}{|c|c|c|c|c|c|c|c|c|}
\hline Author, year & Population & Intervention group, $\mathbf{n}$ & Control group, $\mathrm{n}$ & $\begin{array}{c}\text { Route of } \\
\text { administration }\end{array}$ & $\begin{array}{l}\text { Outcome } \\
\text { definition }\end{array}$ & $\begin{array}{l}\mathrm{n} / \mathrm{N}(\%) \\
\text { intervention } \\
\text { group }\end{array}$ & $\begin{array}{l}\mathrm{n} / \mathrm{N} \\
(\%), \\
\text { control } \\
\text { group }\end{array}$ & $\begin{array}{l}\text { Relative } \\
\text { risk } \\
(95 \% \mathrm{Cl})\end{array}$ \\
\hline Grover, 201130 & $\begin{array}{l}\text { Consecutive patients }>-18 \text { years old } \\
\text { referred to psychiatry and diagnosed } \\
\text { with delirium }\end{array}$ & $\begin{array}{l}\text { Risperidone (Mean dose: } 0.95 \mathrm{mg} \\
\text { Dose range: } 0.5-2.0 \mathrm{mg} \text { per day), } \\
22\end{array}$ & $\begin{array}{l}\text { Haloperidol (Mean dose: } 0.88 \\
\text { Dose range: } 0.25-5 \mathrm{mg} \text { per day), } \\
26\end{array}$ & Oral & & $0 / 22(0 \%)$ & $\begin{array}{l}0 / 26 \\
(0 \%)\end{array}$ & \\
\hline Grover, $2011^{30}$ & $\begin{array}{l}\text { Consecutive patients }>-18 \text { years old } \\
\text { referred to psychiatry and diagnosed } \\
\text { with delirium }\end{array}$ & $\begin{array}{l}\text { Olanzapine (Mean dose: } 3.05 \mathrm{mg} \\
\text { Dose range: } 1.25-10 \mathrm{mg} \text { per } \\
\text { day), } 26\end{array}$ & $\begin{array}{l}\text { Haloperidol (Mean dose: } 0.88 \\
\text { Dose range: } 0.25-5 \mathrm{mg} \text { per day), } \\
26\end{array}$ & Oral & & $0 / 26(0 \%)$ & $\begin{array}{l}0 / 26 \\
(\% 0)\end{array}$ & \\
\hline Jain, $2017^{38}$ & Medical and surgical inpatients & $\begin{array}{l}\text { Olanzapine (Mean dose: } \\
5.49 \mathrm{mg}), 66\end{array}$ & $\begin{array}{l}\text { Haloperidol (Mean dose: } \\
2.10 \mathrm{mg}), 66\end{array}$ & Oral & $\begin{array}{l}\text { Not } \\
\text { applicable }\end{array}$ & $\begin{array}{l}9 / 66 \\
(13.6 \%)\end{array}$ & $\begin{array}{l}7 / 66 \\
(10.6 \%)\end{array}$ & $\begin{array}{l}1.29 \\
(0.51 \text { to } \\
3.25)\end{array}$ \\
\hline Maneeton, $2013^{49}$ & $\begin{array}{l}\text { General adult population (age 18-75) } \\
\text { with hyperactive delirium, referred to } \\
\text { psychiatry consult liaison service at } \\
\text { tertiary care hospital in Thailand }\end{array}$ & $\begin{array}{l}\text { Quetiapine (Mean dose: } 67.6 \text { (SD } \\
\text { 9.7) Dose range: } 25-100 \mathrm{mg}), 24\end{array}$ & $\begin{array}{l}\text { Haloperidol (Mean dose: } 0.8 \text { (SD } \\
0.3 \text { ) Dose range: } 0.5-2.0 \mathrm{mg}), 28\end{array}$ & Oral & $\begin{array}{l}\text { Not } \\
\text { applicable }\end{array}$ & $1 / 24(4.2 \%)$ & $\begin{array}{l}1 / 28 \\
(3.6 \%)\end{array}$ & $\begin{array}{l}1.17(0.08 \\
\text { to } 17.67)\end{array}$ \\
\hline \multicolumn{9}{|c|}{$\begin{array}{l}\text { Second-generation } \\
\text { antipsychotic vs. second- } \\
\text { generation antipsychotic }\end{array}$} \\
\hline Grover, $2011^{30}$ & $\begin{array}{l}\text { Consecutive patients }>-18 \text { years old } \\
\text { referred to psychiatry and diagnosed } \\
\text { with delirium }\end{array}$ & $\begin{array}{l}\text { Risperidone (Mean dose: } 0.95 \mathrm{mg} \\
\text { Dose range: } 0.5-2.0 \mathrm{mg} \text { per day), } \\
22\end{array}$ & $\begin{array}{l}\text { Olanzapine (Mean dose: } 3.05 \mathrm{mg} \\
\text { Dose range: } 1.25-10 \mathrm{mg} \text { per } \\
\text { day), } 26\end{array}$ & Oral & & $0 / 22(0 \%)$ & $\begin{array}{l}0 / 26 \\
(0 \%)\end{array}$ & \\
\hline \multicolumn{9}{|l|}{$\begin{array}{l}\text { First-generation } \\
\text { antipsychotic vs. other }\end{array}$} \\
\hline Atalan, $2013^{18}$ & $\begin{array}{l}\text { Post cardiac surgery patients with } \\
\text { hyperactive type delirium }\end{array}$ & $\begin{array}{l}\text { Haloperidol (Max dose: } 20 \mathrm{mg} \text { ), } \\
26\end{array}$ & $\begin{array}{l}\text { Morphine sulfate (Max dose: } \\
20 \mathrm{mg}), 27\end{array}$ & Intramuscular & $\begin{array}{l}\text { Hospital } \\
\text { mortality }\end{array}$ & $2 / 26(7.7 \%)$ & $\begin{array}{l}1 / 27 \\
(3.7 \%)\end{array}$ & $\begin{array}{l}2.08 \\
(0.20 \text { to } \\
21.55)\end{array}$ \\
\hline Breitbart, $1996^{22}$ & $\begin{array}{l}\text { Medically hospitalized adult patients } \\
\text { with AIDS and a DRS score of } 13 \text { or } \\
\text { greater (delirium) }\end{array}$ & $\begin{array}{l}\text { Chlorpromazine (Planned dose: } \\
\text { Oral: } 10-200 \mathrm{mg} \text {, Intramuscular: } \\
\text { 5-100mg Mean dose: } 36 \text { Dose } \\
\text { range: } 10-80 \mathrm{mg} \text { ), } 13\end{array}$ & $\begin{array}{l}\text { Lorazepam (Planned dose: Oral: } \\
0.50-4.0 \mathrm{mg} \text {, Intramuscular: 0.2- } \\
\text { 2.0mg Mean dose: } 4.6 \text { Dose } \\
\text { range: } 1.3-7.9 \mathrm{mg}), 6\end{array}$ & $\begin{array}{l}\text { Oral or } \\
\text { intramuscular }\end{array}$ & & $2 / 13(\%)$ & $\begin{array}{l}1 / 6 \\
(16.7 \%)\end{array}$ & $\begin{array}{l}0.92 \\
(0.10 \text { to } \\
8.31)\end{array}$ \\
\hline Breitbart, $1996^{22}$ & $\begin{array}{l}\text { Medically hospitalized adult patients } \\
\text { with AIDS and a DRS score of } 13 \text { or } \\
\text { greater (delirium) }\end{array}$ & $\begin{array}{l}\text { Haloperidol (Planned dose: oral: } \\
0.25-5.0 \mathrm{mg} \text {, Intramuscular: } \\
0.125-3.0 \mathrm{mg} \text { Mean dose: } 1.4 \\
\text { Dose range: } 0.4-3.6 \mathrm{mg}), 11\end{array}$ & $\begin{array}{l}\text { Lorazepam (Planned dose: Oral: } \\
0.50-4.0 \mathrm{mg} \text {, Intramuscular: 0.2- } \\
\text { 2.0mg Mean dose: } 4.6 \text { Dose } \\
\text { range: } 1.3-7.9 \mathrm{mg}), 6\end{array}$ & $\begin{array}{l}\text { Oral or } \\
\text { intramuscular }\end{array}$ & & $2 / 11(\%)$ & $\begin{array}{l}1 / 6 \\
(16.7 \%)\end{array}$ & $\begin{array}{l}1.09 \\
(0.12 \text { to } \\
9.70)\end{array}$ \\
\hline
\end{tabular}

AIDS=acquired immunodeficiency syndrome; CI=confidence interval; DRS-R-98= Delirium Rating Scale Revised-98; DSM-IV=Diagnostic and Statistical Manual of Mental Disorders, $4^{\text {th }}$ ed; ICU=intensive care unit; mg=milligram; $\mathrm{N}=$ sample size 
Evidence Table D-39. Survival outcomes for studies comparing interventions to treat delirium

\begin{tabular}{|c|c|c|c|c|c|c|c|c|}
\hline Author, year & Population & Intervention group, $\mathrm{n}$ & Control group, $\mathbf{n}$ & $\begin{array}{c}\text { Route of } \\
\text { administration }\end{array}$ & $\begin{array}{l}\text { Outcome } \\
\text { definition }\end{array}$ & $\begin{array}{l}\text { Median (IQR) days, } \\
\text { intervention group }\end{array}$ & $\begin{array}{l}\text { Median (IQR) } \\
\text { days, control } \\
\text { group }\end{array}$ & $\begin{array}{c}\text { Measure of } \\
\text { association (95\% } \\
\mathrm{Cl})\end{array}$ \\
\hline \multicolumn{9}{|l|}{$\begin{array}{l}\text { First-generation } \\
\text { antipsychotic vs. placebo }\end{array}$} \\
\hline Agar, $2017^{17}$ & $\begin{array}{l}\text { Patients in } \\
\text { Hospice and } \\
\text { palliative care } \\
\text { with delirium }\end{array}$ & $\begin{array}{l}\text { Haloperidol (Max dose: } \\
4 \mathrm{mg} / \mathrm{d} \text { ), } 81\end{array}$ & $\begin{array}{l}\text { Placebo (Max dose: } 4 \\
\text { mg/day), } 84\end{array}$ & Oral & $\begin{array}{l}\text { Median survival, } \\
\text { days }\end{array}$ & 16 & 26 & \\
\hline \multicolumn{9}{|l|}{$\begin{array}{l}\text { Second-generation } \\
\text { antipsychotic vs. placebo }\end{array}$} \\
\hline Agar, $2017^{17}$ & $\begin{array}{l}\text { Patients in } \\
\text { Hospice and } \\
\text { palliative care } \\
\text { with delirium }\end{array}$ & $\begin{array}{l}\text { Risperidone (Max } \\
\text { dose: } 4 \mathrm{mg} / \mathrm{d}), 82\end{array}$ & $\begin{array}{l}\text { Placebo (Max dose: } 4 \\
\text { mg/day), } 84\end{array}$ & Oral & $\begin{array}{l}\text { Median survival, } \\
\text { days }\end{array}$ & 17 & 26 & \\
\hline \multicolumn{9}{|l|}{$\begin{array}{l}\text { First-generation } \\
\text { antipsychotic vs. second- } \\
\text { generation antipsychotic }\end{array}$} \\
\hline Agar, $2017^{17}$ & $\begin{array}{l}\text { Patients in } \\
\text { Hospice and } \\
\text { palliative care } \\
\text { with delirium }\end{array}$ & $\begin{array}{l}\text { Risperidone (Max } \\
\text { dose: } 4 \mathrm{mg} / \mathrm{d}), 82\end{array}$ & $\begin{array}{l}\text { Haloperidol (Max } \\
\text { dose: } 4 \mathrm{mg} / \mathrm{d}), 81\end{array}$ & Oral & $\begin{array}{l}\text { Median survival, } \\
\text { days }\end{array}$ & 17 & 16 & \\
\hline
\end{tabular}

CI=confidence interval; IQR=interquartile range; $\mathrm{mg} / \mathrm{d}=$ =milligram per day; $\mathrm{N}=$ sample size 
Evidence Table D-40. Cognitive outcomes for studies comparing interventions to treat delirium

\begin{tabular}{|c|c|c|c|c|c|c|c|c|}
\hline Author, year & Population & Intervention group, $\mathbf{n}$ & Control group, $\mathrm{n}$ & $\begin{array}{c}\text { Route of } \\
\text { administration }\end{array}$ & Outcome definition & $\begin{array}{l}\text { MMSE, } \\
\text { intervention } \\
\text { group }\end{array}$ & $\begin{array}{l}\text { MMSE, } \\
\text { control } \\
\text { group }\end{array}$ & $\begin{array}{c}\text { Mean between } \\
\text { group } \\
\text { difference } \\
(95 \% \mathrm{Cl})\end{array}$ \\
\hline \multicolumn{9}{|c|}{$\begin{array}{l}\text { Second-generation } \\
\text { antipsychotic vs. placebo }\end{array}$} \\
\hline Tahir, $2010^{59}$ & $\begin{array}{l}\text { Patients in medical, surgical } \\
\text { and orthopedic wards, who } \\
\text { met the DSM-IV criteria for } \\
\text { delirium on the same day if } \\
\text { they had a DRS-R-98 total } \\
\text { score of } 15 \text { or more }\end{array}$ & $\begin{array}{l}\text { Quetiapine (Mean dose: } \\
\text { 40 Dose range: } 25 \text { - } \\
\text { 175mg), } 21\end{array}$ & $\begin{array}{l}\text { Placebo (Mean dose: } \\
25 \text { Max dose: } \\
\text { 175mg), } 21\end{array}$ & Oral & Mini Mental Status: & $\begin{array}{l}\text { Baseline: Mean } \\
\text { 11.829 (SE 4.08) } \\
\text { Final: Mean } \\
\text { 16.317 (SE } \\
\text { 3.689) }\end{array}$ & $\begin{array}{l}\text { Baseline: } \\
\text { Mean } 11.829 \\
\text { (SE 4.08) } \\
\text { Final: Mean } \\
16.773 \text { (SE } \\
3.838 \text { ) }\end{array}$ & $\begin{array}{l}-0.46(95 \% \mathrm{Cl}:- \\
19.29 \text { to } 18.38)\end{array}$ \\
\hline Tahir, $2010^{59}$ & $\begin{array}{l}\text { Patients in medical, surgical } \\
\text { and orthopedic wards, who } \\
\text { met the DSM-IV criteria for } \\
\text { delirium on the same day if } \\
\text { they had a DRS-R-98 total } \\
\text { score of } 15 \text { or more }\end{array}$ & $\begin{array}{l}\text { Quetiapine (Mean dose: } \\
\text { 40 Dose range: } 25 \text { - } \\
\text { 175mg), } 21\end{array}$ & $\begin{array}{l}\text { Placebo (Mean dose: } \\
25 \text { Max dose: } \\
\text { 175mg), } 21\end{array}$ & Oral & Mini Mental Status: & $\begin{array}{l}\text { Baseline: Mean } \\
\text { 11.829 (SE 4.08) } \\
\text { Final: Mean } \\
\text { 18.504 (SE } \\
4.739)\end{array}$ & $\begin{array}{l}\text { Baseline: } \\
\text { Mean 11.829 } \\
\text { (SE 4.08) } \\
\text { Final: Mean } \\
\text { 18.534 (SE } \\
\text { 4.757) }\end{array}$ & $\begin{array}{l}-0.03(95 \% \mathrm{Cl}:- \\
21.24 \text { to } 21.18)\end{array}$ \\
\hline \multicolumn{9}{|c|}{$\begin{array}{l}\text { First-generation } \\
\text { antipsychotic vs. first- } \\
\text { generation antipsychotic }\end{array}$} \\
\hline Breitbart, $1996^{22}$ & $\begin{array}{l}\text { Medically hospitalized adult } \\
\text { patients with AIDS and a } \\
\text { DRS score of } 13 \text { or greater } \\
\text { (delirium) }\end{array}$ & $\begin{array}{l}\text { Haloperidol (Planned } \\
\text { dose: oral: } 0.25-5.0 \mathrm{mg} \text {, } \\
\text { Intramuscular: } 0.125- \\
\text { 3.Omg Mean dose: } 1.4 \\
\text { Dose range: } 0.4 \text { - } \\
\text { 3.6mg), } 11\end{array}$ & $\begin{array}{l}\text { Chlorpromazine } \\
\text { (Planned dose: Oral: } \\
\text { 10-200mg, } \\
\text { Intramuscular: } 5- \\
\text { 100mg Mean dose: } \\
\text { 36 Dose range: } 10- \\
\text { 80mg), } 13\end{array}$ & $\begin{array}{l}\text { Oral or } \\
\text { intramuscular }\end{array}$ & Mini Mental Status: & $\begin{array}{l}\text { Baseline: Mean } \\
\text { 13.45 (SD 6.95) } \\
\text { Final: Mean } \\
17.18 \text { (SD 12.12) }\end{array}$ & $\begin{array}{l}\text { Baseline: } \\
\text { Mean 10.92 } \\
\text { (SD 8.87) } \\
\text { Final: Mean } \\
15.08 \text { (SD } \\
10.43 \text { ) }\end{array}$ & $\begin{array}{l}-0.43(95 \% \mathrm{Cl}:- \\
12.20 \text { to } 11.34)\end{array}$ \\
\hline \multicolumn{9}{|c|}{$\begin{array}{l}\text { First-generation } \\
\text { antipsychotic vs. second- } \\
\text { generation antipsychotic }\end{array}$} \\
\hline Grover, $2011^{30}$ & $\begin{array}{l}\text { Consecutive patients }>-18 \\
\text { years old referred to } \\
\text { psychiatry and diagnosed } \\
\text { with delirium }\end{array}$ & $\begin{array}{l}\text { Risperidone (Mean dose: } \\
\text { 0.95mg Dose range: } 0.5- \\
\text { 2.0mg per day), } 20\end{array}$ & $\begin{array}{l}\text { Haloperidol (Mean } \\
\text { dose: } 0.88 \text { Dose } \\
\text { range: } 0.25-5 \mathrm{mg} \text { per } \\
\text { day), } 21\end{array}$ & Oral & Mini Mental Status: & $\begin{array}{l}\text { Baseline: Mean } \\
6.84 \text { (SD 5.33) } \\
\text { Final: Mean } \\
22.31 \text { (SD 6.63) }\end{array}$ & $\begin{array}{l}\text { Baseline: } \\
\text { Mean 6.38 } \\
\text { (SD 5.02) } \\
\text { Final: Mean } \\
\text { 21.71 (SD } \\
7.66)\end{array}$ & $\begin{array}{l}0.14 \text { (95\% } \mathrm{Cl}:- \\
5.95 \text { to } 6.23)\end{array}$ \\
\hline
\end{tabular}




\begin{tabular}{|c|c|c|c|c|c|c|c|c|}
\hline Author, year & Population & Intervention group, $\mathbf{n}$ & Control group, $\mathrm{n}$ & $\begin{array}{c}\text { Route of } \\
\text { administration }\end{array}$ & $\begin{array}{l}\text { Outcome } \\
\text { definition }\end{array}$ & $\begin{array}{l}\text { MMSE, intervention } \\
\text { group }\end{array}$ & $\begin{array}{l}\text { MMSE, control } \\
\text { group }\end{array}$ & $\begin{array}{l}\text { Mean between- } \\
\text { group } \\
\text { difference } \\
(95 \% \mathrm{Cl})\end{array}$ \\
\hline Grover, $2011^{30}$ & $\begin{array}{l}\text { Consecutive patients >- } \\
18 \text { years old referred to } \\
\text { psychiatry and } \\
\text { diagnosed with delirium }\end{array}$ & $\begin{array}{l}\text { Olanzapine (Mean dose: } \\
3.05 \mathrm{mg} \text { Dose range: } 1.25- \\
10 \mathrm{mg} \text { per day), } 23\end{array}$ & $\begin{array}{l}\text { Haloperidol (Mean } \\
\text { dose: } 0.88 \text { Dose } \\
\text { range: } 0.25-5 \mathrm{mg} \text { per } \\
\text { day), } 21\end{array}$ & Oral & $\begin{array}{l}\text { Mini Mental } \\
\text { Status: }\end{array}$ & $\begin{array}{l}\text { Baseline: Mean } 9.72 \\
\text { (SD 6.3) } \\
\text { Final: Mean } 20.77 \text { (SD } \\
\text { 8.14) }\end{array}$ & $\begin{array}{l}\text { Baseline: Mean } \\
6.38 \text { (SD 5.02) } \\
\text { Final: Mean 21.71 } \\
\text { (SD 7.66) }\end{array}$ & $\begin{array}{l}-4.28(95 \% \mathrm{Cl}:- \\
10.61 \text { to } 2.05)\end{array}$ \\
\hline Grover, $2016^{31}$ & $\begin{array}{l}\text { Patients }>18 \text { years old } \\
\text { referred to Psychiatry } \\
\text { who met DSM-IV } \\
\text { criteria for delirium }\end{array}$ & $\begin{array}{l}\text { Quetiapine (Mean dose: } \\
26.63 \pm 15.61 \text { Dose range: } \\
12.5-75 \mathrm{mg}), 31\end{array}$ & $\begin{array}{l}\text { Haloperidol (Mean } \\
\text { dose: } 0.67 \pm 0.35 \\
\text { Dose range: } 0.25- \\
1.25 \mathrm{mg}), 32\end{array}$ & Oral & $\begin{array}{l}\text { Mini Mental } \\
\text { Status: }\end{array}$ & $\begin{array}{l}\text { Change from baseline: } \\
\text { Mean 12.23 (Not } \\
\text { reported) } \\
\text { Baseline: Mean } 6.83 \\
\text { (SD 4.45) } \\
\text { Final: Mean } 22.54 \text { (SD } \\
\text { 5.34) }\end{array}$ & $\begin{array}{l}\text { Change from } \\
\text { baseline: Mean } \\
12.38 \text { (Not } \\
\text { reported) } \\
\text { Baseline: } 7.5 \text { SD } \\
\text { (3.83 Not } \\
\text { reported) } \\
\text { Final: } 23 \text { SD (4.75 } \\
\text { Not reported) }\end{array}$ & $\begin{array}{l}0.15 \text { (95\% Cl: }- \\
3.43 \text { to } 3.73)\end{array}$ \\
\hline \multicolumn{9}{|c|}{$\begin{array}{l}\text { Second-generation } \\
\text { antipsychotic vs. second- } \\
\text { generation antipsychotic }\end{array}$} \\
\hline Grover, $2011^{30}$ & $\begin{array}{l}\text { Consecutive patients >- } \\
18 \text { years old referred to } \\
\text { psychiatry and } \\
\text { diagnosed with delirium }\end{array}$ & $\begin{array}{l}\text { Risperidone (Mean dose: } \\
\text { 0.95mg Dose range: } 0.5- \\
2.0 \mathrm{mg} \text { per day), } 20\end{array}$ & $\begin{array}{l}\text { Olanzapine (Mean } \\
\text { dose: } 3.05 \mathrm{mg} \text { Dose } \\
\text { range: } 1.25-10 \mathrm{mg} \\
\text { per day), } 23\end{array}$ & Oral & $\begin{array}{l}\text { Mini Mental } \\
\text { Status: }\end{array}$ & $\begin{array}{l}\text { Baseline: Mean } 6.84 \\
\text { (SD 5.33) } \\
\text { Final: Mean } 22.31 \text { (SD } \\
6.63) \\
\end{array}$ & $\begin{array}{l}\text { Baseline: Mean } \\
9.72 \text { (SD 6.3) } \\
\text { Final: Mean 20.77 } \\
\text { (SD 8.14) } \\
\end{array}$ & $\begin{array}{l}4.42(95 \% \mathrm{Cl}:- \\
2.16 \text { to } 11.00)\end{array}$ \\
\hline \multicolumn{9}{|l|}{$\begin{array}{l}\text { First-generation } \\
\text { antipsychotic vs. other }\end{array}$} \\
\hline Breitbart, $1996^{22}$ & $\begin{array}{l}\text { Medically hospitalized } \\
\text { adult patients with AIDS } \\
\text { and a DRS score of } 13 \\
\text { or greater (delirium) }\end{array}$ & $\begin{array}{l}\text { Lorazepam (Planned dose: } \\
\text { Oral: } 0.50-4.0 \mathrm{mg} \text {, } \\
\text { Intramuscular: } 0.2-2.0 \mathrm{mg} \\
\text { Mean dose: } 4.6 \text { Dose } \\
\text { range: } 1.3-7.9 \mathrm{mg}), 6\end{array}$ & $\begin{array}{l}\text { Chlorpromazine } \\
\text { (Planned dose: Oral: } \\
\text { 10-200mg, } \\
\text { Intramuscular: 5- } \\
\text { 100mg Mean dose: } \\
\text { 36 Dose range: } 10- \\
80 \mathrm{mg} \text { ), } 13\end{array}$ & $\begin{array}{l}\text { Oral or } \\
\text { intramuscular }\end{array}$ & $\begin{array}{l}\text { Mini Mental } \\
\text { Status: }\end{array}$ & $\begin{array}{l}\text { Baseline: Mean } 15.17 \\
\text { (SD 5.31) } \\
\text { Final: Mean } 11.5 \text { (SD } \\
\text { 8.69) }\end{array}$ & $\begin{array}{l}\text { Baseline: Mean } \\
10.92 \text { (SD 8.87) } \\
\text { Final: Mean 15.08 } \\
\text { (SD 10.43) }\end{array}$ & $\begin{array}{l}-7.83(95 \% \mathrm{Cl}:- \\
19.15 \text { to } 3.49)\end{array}$ \\
\hline Breitbart, $1996^{22}$ & $\begin{array}{l}\text { Medically hospitalized } \\
\text { adult patients with AIDS } \\
\text { and a DRS score of } 13 \\
\text { or greater (delirium) }\end{array}$ & $\begin{array}{l}\text { Lorazepam (Planned dose: } \\
\text { Oral: } 0.50-4.0 \mathrm{mg} \text {, } \\
\text { Intramuscular: } 0.2-2.0 \mathrm{mg} \\
\text { Mean dose: } 4.6 \text { Dose } \\
\text { range: } 1.3-7.9 \mathrm{mg}), 6\end{array}$ & $\begin{array}{l}\text { Haloperidol (Planned } \\
\text { dose: oral: } 0.25- \\
\text { 5.0mg, Intramuscular: } \\
0.125-3.0 \mathrm{mg} \text { Mean } \\
\text { dose: 1.4 Dose } \\
\text { range: } 0.4 \text { - 3.6mg), } \\
11\end{array}$ & $\begin{array}{l}\text { Oral or } \\
\text { intramuscular }\end{array}$ & $\begin{array}{l}\text { Mini Mental } \\
\text { Status: }\end{array}$ & $\begin{array}{l}\text { Baseline: Mean } 15.17 \\
\text { (SD 5.31) } \\
\text { Final: Mean } 11.5 \text { (SD } \\
\text { 8.69) }\end{array}$ & $\begin{array}{l}\text { Baseline: Mean } \\
\text { 13.45 (SD 6.95) } \\
\text { Final: Mean 17.18 } \\
\text { (SD 12.12) }\end{array}$ & $\begin{array}{l}-7.40(95 \% \mathrm{Cl}:- \\
19.11 \text { to } 4.31)\end{array}$ \\
\hline
\end{tabular}

Examination; SD=standard deviation; SE=standard error 
Evidence Table D-41. Intensive care unit readmission outcomes for studies comparing interventions to treat delirium

\begin{tabular}{|c|c|c|c|c|c|c|c|c|}
\hline Author, year & Population & $\begin{array}{c}\text { Intervention group, } \\
\mathbf{n}\end{array}$ & Control group, $n$ & $\begin{array}{c}\text { Route of } \\
\text { administration }\end{array}$ & Outcome definition & $\begin{array}{c}\text { n I N (\%), intervention } \\
\text { group }\end{array}$ & $\begin{array}{l}\text { n I N (\%), control } \\
\text { group }\end{array}$ & $\begin{array}{c}\text { Relative risk (95\% } \\
\mathrm{Cl})\end{array}$ \\
\hline \multicolumn{9}{|l|}{$\begin{array}{l}\text { First-generation } \\
\text { antipsychotic vs. } \\
\text { placebo }\end{array}$} \\
\hline Girard, $2018^{29}$ & $\begin{array}{l}\text { Patients over } 18 \\
\text { years of age in the } \\
\text { medical or surgical } \\
\text { ICU, with delirium }\end{array}$ & $\begin{array}{l}\text { Haloperidol (Mean } \\
\text { dose: } 11 \mathrm{mg} \text { Dose } \\
\text { range: } 2.5-20 \mathrm{mg} \text { ), } \\
192\end{array}$ & $\begin{array}{l}\text { Placebo (Not } \\
\text { reported), } 184\end{array}$ & Intravenous & $\begin{array}{l}\text { ICU Readmission, \% } \\
\text { patients }\end{array}$ & 27 / 192 (14\%) & $23 / 184(12 \%)$ & 1.13 (0.67 to 1.89$)$ \\
\hline Page, $2013^{10}$ & $\begin{array}{l}\text { ICU patients } \\
\text { needing MV within } \\
72 \text { hours of } \\
\text { admission were } \\
\text { enrolled }\end{array}$ & $\begin{array}{l}\text { Haloperidol } \\
\text { (Planned dose: } \\
2.5 \mathrm{mg}), 71\end{array}$ & $\begin{array}{l}\text { Placebo (Planned } \\
\text { dose: } 0.5 \mathrm{ml}), 70\end{array}$ & Intravenous & $\begin{array}{l}\text { Readmission to ICU } \\
\text { with sepsis }\end{array}$ & $1 / 71(3 \%)$ & $1 / 70(1 \%)$ & 0.99 (0.06 to 15.45$)$ \\
\hline \multicolumn{9}{|l|}{$\begin{array}{l}\text { Second-generation } \\
\text { antipsychotic vs. } \\
\text { placebo }\end{array}$} \\
\hline Girard, $2018^{29}$ & $\begin{array}{l}\text { Patients over } 18 \\
\text { years of age in the } \\
\text { medical or surgical } \\
\text { ICU, with delirium }\end{array}$ & $\begin{array}{l}\text { Ziprasidone (Mean } \\
\text { dose: } 20 \mathrm{mg} \text { Dose } \\
\text { range: } 5.0-40 \mathrm{mg} \text { ), } \\
190\end{array}$ & $\begin{array}{l}\text { Placebo (Not } \\
\text { reported), } 184\end{array}$ & Intravenous & $\begin{array}{l}\text { ICU Readmission, \% } \\
\text { patients }\end{array}$ & $18 / 190(9 \%)$ & $23 / 184(12 \%)$ & 0.76 (0.42 to 1.36$)$ \\
\hline \multicolumn{9}{|l|}{$\begin{array}{l}\text { First-generation } \\
\text { antipsychotic vs. } \\
\text { Second-generation } \\
\text { antipsychotic }\end{array}$} \\
\hline Girard, $2018^{29}$ & $\begin{array}{l}\text { Patients over } 18 \\
\text { years of age in the } \\
\text { medical or surgical } \\
\text { ICU, with delirium }\end{array}$ & $\begin{array}{l}\text { Ziprasidone (Mean } \\
\text { dose: } 20 \mathrm{mg} \text { Dose } \\
\text { range: } 5.0-40 \mathrm{mg} \text { ), } \\
190\end{array}$ & $\begin{array}{l}\text { Haloperidol (Mean } \\
\text { dose: } 11 \mathrm{mg} \text { Dose } \\
\text { range: } 2.5-20 \mathrm{mg} \text { ), } \\
192\end{array}$ & Intravenous & $\begin{array}{l}\text { ICU Readmission, \% } \\
\text { patients }\end{array}$ & 18 / $190(9 \%)$ & 27 / $192(14 \%)$ & 0.67 (0.38 to 1.18$)$ \\
\hline
\end{tabular}




\begin{tabular}{|c|c|c|c|c|c|c|c|c|}
\hline Author, year & Population & $\begin{array}{c}\text { Intervention group, } \\
\mathrm{n}\end{array}$ & Control group, $n$ & $\begin{array}{c}\text { Route of } \\
\text { administration }\end{array}$ & Outcome definition & $\begin{array}{l}\text { n I N (\%), intervention } \\
\text { group }\end{array}$ & $\begin{array}{l}\text { n I N (\%), control } \\
\text { group }\end{array}$ & $\begin{array}{l}\text { Relative risk (95\% } \\
\mathrm{Cl})\end{array}$ \\
\hline \multicolumn{9}{|l|}{$\begin{array}{l}\text { First-generation } \\
\text { antipsychotic vs. } \\
\text { other }\end{array}$} \\
\hline Atalan, $2013^{18}$ & $\begin{array}{l}\text { Post cardiac } \\
\text { surgery patients } \\
\text { with hyperactive } \\
\text { type delirium }\end{array}$ & $\begin{array}{l}\text { Haloperidol (Max } \\
\text { dose: } 20 \mathrm{mg}), 26\end{array}$ & $\begin{array}{l}\text { Morphine sulfate } \\
\text { (Max dose: } 20 \mathrm{mg} \text { ), } \\
27\end{array}$ & Intramuscular & Duration of ICU stay & $8 / 26(30.8 \%)$ & $7 / 27(25.9 \%)$ & 1.19 (0.50 to 2.80$)$ \\
\hline
\end{tabular}

CI=confidence interval; ICU=intensive care unit; $\mathrm{mg}=$ =milligram; $\mathrm{ml}=$ =milliliter; $\mathrm{n}=$ =sample size 
Evidence Table D-42. Length of hospital stay outcomes for studies comparing interventions to treat delirium

\begin{tabular}{|c|c|c|c|c|c|c|c|c|}
\hline Author, year & Population & Intervention group, $\mathbf{n}$ & Control group, $\mathrm{n}$ & $\begin{array}{c}\text { Route of } \\
\text { administration }\end{array}$ & Outcome definition & $\begin{array}{c}\text { Median } \\
\text { (IQR) days, } \\
\text { intervention } \\
\text { group }\end{array}$ & $\begin{array}{l}\text { Median } \\
\text { (IQR), } \\
\text { days, } \\
\text { control } \\
\text { group }\end{array}$ & $\begin{array}{c}\text { Measure of } \\
\text { association (95\% } \\
\text { Cl) }\end{array}$ \\
\hline \multicolumn{9}{|l|}{$\begin{array}{l}\text { First-generation } \\
\text { antipsychotic vs. } \\
\text { placebo }\end{array}$} \\
\hline Girard, $2010^{4}$ & $\begin{array}{l}>18 \text { years MV medical } \\
\text { and surgical ICU patients }\end{array}$ & $\begin{array}{l}\text { Haloperidol (Planned dose: } 5 \text { Median } \\
\text { dose: } 15 \text { (10.8-17), } 35\end{array}$ & $\begin{array}{l}\text { Placebo (Planned dose: } \\
5 \mathrm{ml}), 36\end{array}$ & Oral & $\begin{array}{l}\text { Length of stay, days } \\
\text { in Hospital }\end{array}$ & $\begin{array}{l}13.8(9.4 \text { to } \\
\text { Not reported) }\end{array}$ & $\begin{array}{l}15.4(8.9 \text { to } \\
\text { Not } \\
\text { reported })\end{array}$ & \\
\hline Girard, $2018^{29}$ & $\begin{array}{l}\text { Patients over } 18 \text { years of } \\
\text { age in the medical or } \\
\text { surgical ICU, with } \\
\text { delirium }\end{array}$ & $\begin{array}{l}\text { Haloperidol (Mean dose: } 11 \mathrm{mg} \text { Dose } \\
\text { range: } 2.5-20 \mathrm{mg}), 192\end{array}$ & $\begin{array}{l}\text { Placebo (Not reported), } \\
184\end{array}$ & Intravenous & $\begin{array}{l}\text { Days to hospital } \\
\text { discharge }\end{array}$ & 13 (8 to 22$)$ & 13 (8 to 23$)$ & $\begin{array}{l}\text { HR } 1.03(95 \% \mathrm{Cl}: \\
0.85 \text { to } 1.23), p=\end{array}$ \\
\hline Page, $2013^{10,27}$ & $\begin{array}{l}\text { ICU patients needing MV } \\
\text { within } 72 \text { hours of } \\
\text { admission were enrolled }\end{array}$ & $\begin{array}{l}\text { Haloperidol (Planned dose: } 2.5 \mathrm{mg} \text { ), } \\
42\end{array}$ & $\begin{array}{l}\text { Placebo (Planned dose: } \\
0.5 \mathrm{ml}), 47\end{array}$ & Intravenous & $\begin{array}{l}\text { Length of hospital } \\
\text { stay, Excluding } \\
\text { patients who died in } \\
\text { hospital }\end{array}$ & $\begin{array}{l}18.5(12 \text { to } \\
31)\end{array}$ & $\begin{array}{l}26(15 \text { to } \\
40)\end{array}$ & $\begin{array}{l}\text { Mean difference - } \\
5.13 \text { ( } 95 \% \mathrm{Cl}:- \\
21.75 \text { to } 11.48) \\
\mathrm{p}=0.54\end{array}$ \\
\hline \multicolumn{9}{|l|}{$\begin{array}{l}\text { Second- } \\
\text { generation } \\
\text { antipsychotic vs. } \\
\text { placebo }\end{array}$} \\
\hline Devlin, $2010^{26}$ & $\begin{array}{l}\text { Patients admitted to } \\
\text { Medical \& Surgical ICU } \\
\text { with delirium. }\end{array}$ & $\begin{array}{l}\text { Quetiapine (Mean dose: } 50 \text { Median } \\
\text { dose: } 110 \text { (88-191 IQR) Max dose: } \\
200 \text { Dose range: } 50 \text { to 200mg), } 18\end{array}$ & $\begin{array}{l}\text { Placebo (Mean dose: } 50 \\
\text { Median dose: } 210 \text { (116 - } \\
\text { 293) Max dose: } 200 \mathrm{mg} \text { ), } \\
\text { 18) }\end{array}$ & $\begin{array}{l}\text { Either orally or } \\
\text { NG tube }\end{array}$ & $\begin{array}{l}\text { Duration of } \\
\text { hospitalization, days }\end{array}$ & 24 (11 to 33 ) & $\begin{array}{l}26 \text { (17 to } \\
49)\end{array}$ & \\
\hline Girard, $2010^{4}$ & $\begin{array}{l}>18 \text { years MV medical } \\
\text { and surgical ICU patients }\end{array}$ & $\begin{array}{l}\text { Ziprasidone (Planned dose: } 40 \\
\text { Median dose: } 113 \text { (81-140), } 30\end{array}$ & $\begin{array}{l}\text { Placebo (Planned dose: } \\
5 \mathrm{ml}), 36\end{array}$ & Oral & $\begin{array}{l}\text { Length of stay, days } \\
\text { in Hospital }\end{array}$ & $\begin{array}{l}13.5(9.3 \text { to } \\
\text { Not reported) }\end{array}$ & $\begin{array}{l}15.4(8.9 \text { to } \\
\text { Not } \\
\text { reported) }\end{array}$ & \\
\hline Girard, $2018^{29}$ & $\begin{array}{l}\text { Patients over } 18 \text { years of } \\
\text { age in the medical or } \\
\text { surgical ICU, with } \\
\text { delirium }\end{array}$ & $\begin{array}{l}\text { Ziprasidone (Mean dose: } 20 \mathrm{mg} \text { Dose } \\
\text { range: } 5.0-40 \mathrm{mg}), 190\end{array}$ & $\begin{array}{l}\text { Placebo (Not reported), } \\
184\end{array}$ & Intravenous & $\begin{array}{l}\text { Days to hospital } \\
\text { discharge }\end{array}$ & 12 (8 to 21 ) & 13 (8 to 23$)$ & $\begin{array}{l}\text { HR } 1.05(95 \% \mathrm{Cl}: \\
0.88 \text { to } 1.25) \\
\mathrm{p}=\text { Not reported }\end{array}$ \\
\hline
\end{tabular}




\begin{tabular}{|c|c|c|c|c|c|c|c|c|}
\hline Author, year & Population & Intervention group, $\mathbf{n}$ & Control group, $\mathbf{n}$ & $\begin{array}{c}\text { Route of } \\
\text { administration }\end{array}$ & Outcome definition & $\begin{array}{l}\text { Median } \\
\text { (IQR) days, } \\
\text { intervention } \\
\text { group }\end{array}$ & $\begin{array}{l}\text { Median } \\
\text { (IQR), } \\
\text { days, } \\
\text { control } \\
\text { group } \\
\end{array}$ & $\begin{array}{c}\text { Measure of } \\
\text { association (95\% } \\
\mathrm{Cl})\end{array}$ \\
\hline \multicolumn{9}{|c|}{$\begin{array}{l}\text { First-generation } \\
\text { antipsychotic vs. } \\
\text { second-generation } \\
\text { antipsychotic }\end{array}$} \\
\hline Girard, $2010^{4}$ & $\begin{array}{l}>18 \text { years MV medical } \\
\text { and surgical ICU patients }\end{array}$ & $\begin{array}{l}\text { Ziprasidone (Planned dose: } 40 \\
\text { Median dose: } 113 \text { (81-140), } 30\end{array}$ & $\begin{array}{l}\text { Haloperidol (Planned dose: } \\
5 \text { Median dose: } 15 \text { (10.8- } \\
\text { 17), } 35\end{array}$ & Oral & $\begin{array}{l}\text { Length of stay, days } \\
\text { in Hospital }\end{array}$ & $\begin{array}{l}13.5 \text { ( } 9.3 \text { to } \\
\text { Not reported) }\end{array}$ & $\begin{array}{l}13.8(9.4 \text { to } \\
\text { Not } \\
\text { reported })\end{array}$ & \\
\hline Girard, $2018^{29}$ & $\begin{array}{l}\text { Patients over } 18 \text { years of } \\
\text { age in the medical or } \\
\text { surgical ICU, with } \\
\text { delirium }\end{array}$ & $\begin{array}{l}\text { Ziprasidone (Mean dose: } 20 \mathrm{mg} \text { Dose } \\
\text { range: } 5.0-40 \mathrm{mg}), 190\end{array}$ & $\begin{array}{l}\text { Haloperidol (Mean dose: } \\
\text { 11mg Dose range: } 2.5- \\
\text { 20mg), } 192\end{array}$ & Intravenous & $\begin{array}{l}\text { Days to hospital } \\
\text { discharge }\end{array}$ & 12 (8 to 21 ) & 13 (8 to 22 ) & $\begin{array}{l}\text { HR } 1.05(95 \% \mathrm{Cl}: \\
0.88 \text { to } 1.25) \\
p=\text { Not reported }\end{array}$ \\
\hline \multicolumn{9}{|l|}{$\begin{array}{l}\text { First-generation } \\
\text { antipsychotic vs. } \\
\text { other }\end{array}$} \\
\hline Atalan, $2013^{18}$ & $\begin{array}{l}\text { Post cardiac surgery } \\
\text { patients with hyperactive } \\
\text { type delirium }\end{array}$ & $\begin{array}{l}\text { Morphine sulfate (Max dose: } 20 \mathrm{mg} \text { ), } \\
27\end{array}$ & $\begin{array}{l}\text { Haloperidol (Max dose: } \\
\text { 20mg), } 26\end{array}$ & Intramuscular & $\begin{array}{l}\text { length of hospital } \\
\text { stay, in days }\end{array}$ & $\begin{array}{l}\text { Mean: } 8.93 \\
\text { (SD: } 3.11 \text { ) }\end{array}$ & $\begin{array}{l}\text { Mean: } 8.54 \\
\text { (SD: } 3.44)\end{array}$ & \\
\hline
\end{tabular}


Evidence Table D-43. Length of intensive care unit stay outcomes for studies comparing interventions to treat delirium

\begin{tabular}{|c|c|c|c|c|c|c|c|c|}
\hline Author, year & Population & Intervention group, $\mathbf{n}$ & Control group, $n$ & $\begin{array}{c}\text { Route of } \\
\text { administration }\end{array}$ & Outcome definition & $\begin{array}{l}\text { Median (IQR) } \\
\text { days, } \\
\text { intervention } \\
\text { group }\end{array}$ & $\begin{array}{l}\text { Median (IQR), } \\
\text { days, control } \\
\text { group }\end{array}$ & $\begin{array}{c}\text { Measure of } \\
\text { association }(95 \% \mathrm{Cl})\end{array}$ \\
\hline \multicolumn{9}{|l|}{$\begin{array}{l}\text { First-generation } \\
\text { antipsychotic vs. } \\
\text { placebo }\end{array}$} \\
\hline Girard, $2010^{4}$ & $\begin{array}{l}>18 \text { years MV medical } \\
\text { and surgical ICU patients }\end{array}$ & $\begin{array}{l}\text { Haloperidol (Planned } \\
\text { dose: } 5 \text { Median dose: } 15 \\
\text { (10.8-17), } 35\end{array}$ & $\begin{array}{l}\text { Placebo (Planned } \\
\text { dose: } 5 \mathrm{ml}), 36\end{array}$ & Oral & $\begin{array}{l}\text { Length of stay, days } \\
\text { in ICU }\end{array}$ & 11.7 (4.6 to 15.7$)$ & $\begin{array}{l}7.3(4.7 \text { to } \\
12.3)\end{array}$ & \\
\hline Girard, $2018^{29}$ & $\begin{array}{l}\text { Patients over } 18 \text { years of } \\
\text { age in the medical or } \\
\text { surgical ICU, with } \\
\text { delirium }\end{array}$ & $\begin{array}{l}\text { Haloperidol (Mean dose: } \\
\text { 11mg Dose range: 2.5- } \\
\text { 20mg), } 192\end{array}$ & $\begin{array}{l}\text { Placebo (Not } \\
\text { reported), } 184\end{array}$ & Intravenous & $\begin{array}{l}\text { Days to ICU } \\
\text { discharge }\end{array}$ & 5 (3 to 13$)$ & 5 (3 to 14$)$ & $\begin{array}{l}\text { Hazard ratio } 0.95 \\
\text { (95\% Cl: } 0.81 \text { to } 1.12) \\
p=\text { Not reported }\end{array}$ \\
\hline Page, $2013^{10,27}$ & $\begin{array}{l}\text { ICU patients needing MV } \\
\text { within } 72 \text { hours of } \\
\text { admission were enrolled }\end{array}$ & $\begin{array}{l}\text { Haloperidol (Planned } \\
\text { dose: } 2.5 \mathrm{mg} \text { ), } 50\end{array}$ & $\begin{array}{l}\text { Placebo (Planned } \\
\text { dose: } 0.5 \mathrm{ml}), 52\end{array}$ & Intravenous & $\begin{array}{l}\text { Length of critical } \\
\text { care stay, excluding } \\
\text { patients who died in } \\
\text { ICU }\end{array}$ & 9.5 (5 to 14$)$ & 9 (5 to 18$)$ & $\begin{array}{l}\text { Mean difference }-1.45 \\
\text { (95\% Cl: }-5.42 \text { to } \\
2.52), p=0.47\end{array}$ \\
\hline \multicolumn{9}{|l|}{$\begin{array}{l}\text { Second-generation } \\
\text { antipsychotic vs. } \\
\text { placebo }\end{array}$} \\
\hline Devlin, $2010^{26}$ & $\begin{array}{l}\text { Patients admitted to } \\
\text { Medical \& Surgical ICU } \\
\text { with delirium. }\end{array}$ & $\begin{array}{l}\text { Quetiapine (Mean dose: } \\
\text { 50 Median dose: } 110 \\
\text { (88-191 IQR) Max dose: } \\
\text { 200 Dose range: } 50 \text { to } \\
\text { 200mg), } 18\end{array}$ & $\begin{array}{l}\text { Placebo (Mean dose: } \\
50 \text { Median dose: } 210 \\
(116-293) \text { Max dose: } \\
200 \mathrm{mg}), 18\end{array}$ & $\begin{array}{l}\text { Either orally or } \\
\text { NG tube }\end{array}$ & $\begin{array}{l}\text { Duration of intensive } \\
\text { care unit stay, days }\end{array}$ & 16 (10 to 22$)$ & 16 (13 to 32$)$ & \\
\hline Girard, $2010^{4}$ & $\begin{array}{l}>18 \text { years MV medical } \\
\text { and surgical ICU patients }\end{array}$ & $\begin{array}{l}\text { Ziprasidone (Planned } \\
\text { dose: } 40 \text { Median dose: } \\
113 \text { (81-140), } 30\end{array}$ & $\begin{array}{l}\text { Placebo (Planned } \\
\text { dose: } 5 \mathrm{ml}), 36\end{array}$ & Oral & $\begin{array}{l}\text { Length of stay, days } \\
\text { in ICU }\end{array}$ & 9.6 (3.8 to 14.5 ) & $\begin{array}{l}7.3(4.7 \text { to } \\
12.3)\end{array}$ & \\
\hline Girard, $2018^{29}$ & $\begin{array}{l}\text { Patients over } 18 \text { years of } \\
\text { age in the medical or } \\
\text { surgical ICU, with } \\
\text { delirium }\end{array}$ & $\begin{array}{l}\text { Ziprasidone (Mean dose: } \\
\text { 20mg Dose range: } 5.0- \\
\text { 40mg), } 190\end{array}$ & $\begin{array}{l}\text { Placebo (Not } \\
\text { reported), } 184\end{array}$ & Intravenous & $\begin{array}{l}\text { Days to ICU } \\
\text { discharge }\end{array}$ & 6 (3 to 10$)$ & 5 (3 to 14$)$ & $\begin{array}{l}\text { Hazard ratio } 1.02 \\
\text { (95\% Cl: } 0.88 \text { to } 1.17 \text { ) } \\
\text { p=Not reported }\end{array}$ \\
\hline
\end{tabular}




\begin{tabular}{|c|c|c|c|c|c|c|c|c|}
\hline Author, year & Population & Intervention group, $\mathbf{n}$ & Control group, $\mathbf{n}$ & $\begin{array}{c}\text { Route of } \\
\text { administration }\end{array}$ & Outcome definition & $\begin{array}{c}\text { Median (IQR) } \\
\text { days, } \\
\text { intervention } \\
\text { group } \\
\end{array}$ & $\begin{array}{l}\text { Median (IQR), } \\
\text { days, control } \\
\text { group }\end{array}$ & $\begin{array}{c}\text { Measure of } \\
\text { association }(95 \% \mathrm{Cl})\end{array}$ \\
\hline \multicolumn{9}{|l|}{$\begin{array}{l}\text { First-generation } \\
\text { antipsychotic vs. } \\
\text { second-generation } \\
\text { antipsychotic } \\
\end{array}$} \\
\hline Girard, $2010^{4}$ & $\begin{array}{l}>18 \text { years MV medical } \\
\text { and surgical ICU patients }\end{array}$ & $\begin{array}{l}\text { Ziprasidone (Planned } \\
\text { dose: } 40 \text { Median dose: } \\
113 \text { (81-140), } 30\end{array}$ & $\begin{array}{l}\text { Haloperidol (Planned } \\
\text { dose: } 5 \text { Median dose: } \\
15 \text { (10.8-17), } 35\end{array}$ & Oral & $\begin{array}{l}\text { Length of stay, days } \\
\text { in ICU }\end{array}$ & 9.6 (3.8 to 14.5$)$ & $\begin{array}{l}11.7(4.6 \text { to } \\
15.7)\end{array}$ & \\
\hline Girard, $2018^{29}$ & $\begin{array}{l}\text { Patients over } 18 \text { years of } \\
\text { age in the medical or } \\
\text { surgical ICU, with } \\
\text { delirium }\end{array}$ & $\begin{array}{l}\text { Ziprasidone (Mean dose: } \\
\text { 20mg Dose range: } 5.0- \\
40 \mathrm{mg} \text { ), } 190\end{array}$ & $\begin{array}{l}\text { Haloperidol (Mean } \\
\text { dose: } 11 \mathrm{mg} \text { Dose } \\
\text { range: } 2.5-20 \mathrm{mg}), 192\end{array}$ & Intravenous & $\begin{array}{l}\text { Days to ICU } \\
\text { discharge }\end{array}$ & 6 (3 to 10 ) & 5 (3 to 13 ) & \\
\hline \multicolumn{9}{|c|}{$\begin{array}{l}\text { First-generation } \\
\text { antipsychotic vs. other }\end{array}$} \\
\hline Atalan, $2013^{18}$ & $\begin{array}{l}\text { Post cardiac surgery } \\
\text { patients with hyperactive } \\
\text { type delirium }\end{array}$ & $\begin{array}{l}\text { Morphine sulfate (Max } \\
\text { dose: } 20 \mathrm{mg}), 27\end{array}$ & $\begin{array}{l}\text { Haloperidol (Max } \\
\text { dose: } 20 \mathrm{mg} \text { ), } 26\end{array}$ & Intramuscular & ICU time (d) & $\begin{array}{l}\text { Mean: } 2.85 \text { (SD: } \\
\text { 1.48) }\end{array}$ & $\begin{array}{l}\text { Mean: } 3.31 \\
\text { (SD: } 2.32 \text { ) }\end{array}$ & \\
\hline
\end{tabular}


Evidence Table D-44. Incidence of delirium outcomes for studies comparing interventions to treat delirium

\begin{tabular}{|c|c|c|c|c|c|c|c|c|}
\hline Author, year & Population & Intervention group, $\mathbf{n}$ & Control group, $n$ & $\begin{array}{c}\text { Route of } \\
\text { administration }\end{array}$ & Outcome definition & $\begin{array}{l}\text { n / N (\%), intervention } \\
\text { group }\end{array}$ & $\begin{array}{l}\text { n I N (\%), control } \\
\text { group }\end{array}$ & $\begin{array}{l}\text { Relative risk (95\% } \\
\mathrm{Cl})\end{array}$ \\
\hline \multicolumn{9}{|c|}{$\begin{array}{l}\text { Second-generation } \\
\text { antipsychotic vs. } \\
\text { placebo }\end{array}$} \\
\hline Devlin, $2010^{26}$ & $\begin{array}{l}\text { Patients admitted } \\
\text { to Medical \& } \\
\text { Surgical ICU with } \\
\text { delirium. }\end{array}$ & $\begin{array}{l}\text { Quetiapine (Mean dose: } \\
\text { 50 Median dose: } 110 \\
\text { (88-191 IQR) Max dose: } \\
\text { 200 Dose range: } 50 \text { to } \\
\text { 200mg), } 18\end{array}$ & $\begin{array}{l}\text { Placebo (Mean } \\
\text { dose: } 50 \text { Median } \\
\text { dose: } 210 \text { (116 - } \\
\text { 293) Max dose: } \\
\text { 200mg), } 18\end{array}$ & $\begin{array}{l}\text { Either orally or NG } \\
\text { tube }\end{array}$ & $\begin{array}{l}\text { Delirium in the 14-day } \\
\text { period after study } \\
\text { drug discontinued, or } \\
\text { until subject } \\
\text { discharged/transferred } \\
\text { from hospital. }\end{array}$ & $4 / 18(20 \%)$ & 10 / $18(56 \%)$ & $0.40(0.15$ to 1.04$)$ \\
\hline
\end{tabular}

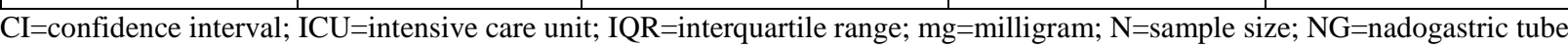


Evidence Table D-45. Sedation outcomes for studies comparing interventions to treat delirium

\begin{tabular}{|c|c|c|c|c|c|c|c|c|c|}
\hline Author, year & $\begin{array}{l}\text { Study } \\
\text { design }\end{array}$ & Population & Intervention group, $\mathbf{n}$ & Control group, $\mathbf{n}$ & $\begin{array}{c}\text { Route of } \\
\text { administration }\end{array}$ & $\begin{array}{l}\text { Outcome } \\
\text { definition }\end{array}$ & $\begin{array}{l}\mathrm{n} / \mathrm{N}(\%) \\
\text { intervention } \\
\text { group }\end{array}$ & $\begin{array}{l}\mathrm{n} / \mathrm{N} \\
(\%), \\
\text { control } \\
\text { group }\end{array}$ & $\begin{array}{c}\text { Relative risk } \\
(95 \% \mathrm{Cl})\end{array}$ \\
\hline \multicolumn{10}{|l|}{$\begin{array}{l}\text { First-generation } \\
\text { antipsychotic vs. } \\
\text { placebo }\end{array}$} \\
\hline Girard, $2018^{29}$ & RCT & $\begin{array}{l}\text { Patients over } 18 \text { years of age in the } \\
\text { medical or surgical ICU, with } \\
\text { delirium }\end{array}$ & $\begin{array}{l}\text { Haloperidol (Mean dose: } 11 \mathrm{mg} \\
\text { Dose range: } 2.5-20 \mathrm{mg}), 192\end{array}$ & $\begin{array}{l}\text { Placebo (Not } \\
\text { reported), } 184\end{array}$ & Intravenous & $\begin{array}{l}\text { Reasons for } \\
\text { temporary holds: } \\
\text { Oversedation }\end{array}$ & $\begin{array}{l}42 / 192 \\
(22 \%)\end{array}$ & $\begin{array}{l}46 / 184 \\
(25 \%)\end{array}$ & $\begin{array}{l}\text { RR } 0.88 \\
(95 \% \text { Cl: } 0.61 \\
\text { to } 1.26)\end{array}$ \\
\hline Page, $2013^{10}$ & RCT & $\begin{array}{l}\text { ICU patients needing MV within } 72 \\
\text { hours of admission were enrolled }\end{array}$ & $\begin{array}{l}\text { Haloperidol (Planned dose: } \\
2.5 \mathrm{mg}), 71\end{array}$ & $\begin{array}{l}\text { Placebo (Planned } \\
\text { dose: } 0.5 \mathrm{ml}), 70\end{array}$ & Intravenous & Oversedation & $\begin{array}{l}11 / 71 \\
(15 \%)\end{array}$ & $\begin{array}{l}6 / 70 \\
(9 \%)\end{array}$ & $\begin{array}{l}\text { RR 1.81 } \\
\text { (95\% Cl: } 0.71 \\
\text { to 4.62) }\end{array}$ \\
\hline \multicolumn{10}{|c|}{$\begin{array}{l}\text { Second-generation } \\
\text { antipsychotic vs. } \\
\text { placebo }\end{array}$} \\
\hline Devlin, $2010^{26}$ & RCT & $\begin{array}{l}\text { Patients admitted to Medical \& } \\
\text { Surgical ICU with delirium. }\end{array}$ & $\begin{array}{l}\text { Quetiapine (Mean dose: } 50 \\
\text { Median dose: } 110 \text { (88-191 } \\
\text { IQR) Max dose: } 200 \text { Dose } \\
\text { range: } 50 \text { to 200mg), }\end{array}$ & $\begin{array}{l}\text { Placebo (Mean } \\
\text { dose: } 50 \text { Median } \\
\text { dose: } 210 \text { (116 - } \\
\text { 293) Max dose: } \\
\text { 200mg), }\end{array}$ & $\begin{array}{l}\text { Either orally or } \\
\text { NG tube }\end{array}$ & $\begin{array}{l}\text { Episodes of } \\
\text { somnolence }\end{array}$ & $\begin{array}{l}\text { Number of } \\
\text { Events: } 5\end{array}$ & $\begin{array}{l}\text { Number } \\
\text { of } \\
\text { Events: } \\
2\end{array}$ & \\
\hline Devlin, $2010^{26}$ & RCT & $\begin{array}{l}\text { Patients admitted to Medical \& } \\
\text { Surgical ICU with delirium. }\end{array}$ & $\begin{array}{l}\text { Quetiapine (Mean dose: } 50 \\
\text { Median dose: } 110 \text { (88-191 } \\
\text { IQR) Max dose: } 200 \text { Dose } \\
\text { range: } 50 \text { to 200mg), } 18\end{array}$ & $\begin{array}{l}\text { Placebo (Mean } \\
\text { dose: } 50 \text { Median } \\
\text { dose: } 210 \text { (116 - } \\
\text { 293) Max dose: } \\
\text { 200mg), } 18\end{array}$ & $\begin{array}{l}\text { Either orally or } \\
\text { NG tube }\end{array}$ & $\begin{array}{l}\text { Subjects } \\
\text { experiencing } \\
\text { somnolence, \% }\end{array}$ & $4 / 18(22 \%)$ & $\begin{array}{l}2 / 18 \\
(11 \%)\end{array}$ & $\begin{array}{l}\text { RR 2.00 } \\
\text { (95\% Cl: } 0.42 \\
\text { to } 9.58)\end{array}$ \\
\hline Girard, $2018^{29}$ & RCT & $\begin{array}{l}\text { Patients over } 18 \text { years of age in the } \\
\text { medical or surgical ICU, with } \\
\text { delirium }\end{array}$ & $\begin{array}{l}\text { Ziprasidone (Mean dose: } \\
20 \mathrm{mg} \text { Dose range: 5.0-40mg), } \\
190\end{array}$ & $\begin{array}{l}\text { Placebo (Not } \\
\text { reported), } 184\end{array}$ & Intravenous & $\begin{array}{l}\text { Reasons for } \\
\text { temporary holds: } \\
\text { Oversedation }\end{array}$ & $\begin{array}{l}50 / 190 \\
(26 \%)\end{array}$ & $\begin{array}{l}46 / 184 \\
(25 \%)\end{array}$ & $\begin{array}{l}\text { RR 1.05 } \\
\text { (95\% Cl: } 0.75 \\
\text { to 1.49) }\end{array}$ \\
\hline Tahir, $2010^{59}$ & RCT & $\begin{array}{l}\text { Patients in medical, surgical and } \\
\text { orthopedic wards, who met the } \\
\text { DSM-IV criteria for delirium on the } \\
\text { same day if they had a DRS-R-98 } \\
\text { total score of } 15 \text { or more }\end{array}$ & $\begin{array}{l}\text { Quetiapine (Mean dose: } 40 \\
\text { Dose range: } 25 \text { - 175mg), } 21\end{array}$ & $\begin{array}{l}\text { Placebo (Mean } \\
\text { dose: } 25 \text { Max } \\
\text { dose: } 175 \mathrm{mg}), 21\end{array}$ & Oral & $\begin{array}{l}\text { Complained of } \\
\text { sedation }\end{array}$ & $1 / 21(4.8 \%)$ & $\begin{array}{l}0 / 21 \\
(\%)\end{array}$ & $\begin{array}{l}\text { RR 3.00 } \\
\text { (95\% Cl: } 0.13 \\
\text { to } 69.70)\end{array}$ \\
\hline
\end{tabular}




\begin{tabular}{|c|c|c|c|c|c|c|c|c|c|}
\hline Author, year & $\begin{array}{l}\text { Study } \\
\text { design }\end{array}$ & Population & Intervention group, $\mathbf{n}$ & Control group, $\mathrm{n}$ & $\begin{array}{c}\text { Route of } \\
\text { administration }\end{array}$ & $\begin{array}{l}\text { Outcome } \\
\text { definition }\end{array}$ & $\begin{array}{l}\mathrm{n} / \mathrm{N}(\%) \\
\text { intervention } \\
\text { group }\end{array}$ & $\begin{array}{l}n / N \\
(\%), \\
\text { control } \\
\text { group }\end{array}$ & $\begin{array}{c}\text { Relative risk } \\
(95 \% \mathrm{Cl})\end{array}$ \\
\hline \multicolumn{10}{|l|}{$\begin{array}{l}\text { First-generation } \\
\text { antipsychotic vs. } \\
\text { second-generation } \\
\text { antipsychotic }\end{array}$} \\
\hline Boettger, 2015 & $\begin{array}{l}\text { Cohort w/ } \\
\text { comparison } \\
\text { group }\end{array}$ & Cancer patients & $\begin{array}{l}\text { Olanzapine (Mean dose: } 3.5 \\
\text { Dose range: } 2.5 \text { to } 10 \mathrm{mg}), 21\end{array}$ & $\begin{array}{l}\text { Haloperidol (Mean } \\
\text { dose: } 4.9 \text { Dose } \\
\text { range: } 1.5 \text { to } \\
\text { 16mg), } 21\end{array}$ & Not reported & Not applicable & $\begin{array}{l}6 / 21 \\
(28.6 \%)\end{array}$ & $\begin{array}{l}0 / 21 \\
(0 \%)\end{array}$ & $\begin{array}{l}\text { RR 13.00 } \\
\text { (95\% Cl: } 0.78 \\
\text { to } 217.03)\end{array}$ \\
\hline Boettger, 201521 & $\begin{array}{l}\text { Cohort w/ } \\
\text { comparison } \\
\text { group }\end{array}$ & Cancer patients & $\begin{array}{l}\text { Risperidone (Mean dose: } 0.9 \\
\text { Dose range: } 0.5 \text { to } 2 \mathrm{mg} \text { ), } 21\end{array}$ & $\begin{array}{l}\text { Haloperidol (Mean } \\
\text { dose: } 4.9 \text { Dose } \\
\text { range: } 1.5 \text { to } \\
\text { 16mg), } 21\end{array}$ & Not reported & Not applicable & $0 / 21(0 \%)$ & $\begin{array}{l}0 / 21 \\
(0 \%)\end{array}$ & \\
\hline Boettger, $2015^{21}$ & $\begin{array}{l}\text { Cohort w/ } \\
\text { comparison } \\
\text { group }\end{array}$ & Cancer patients & $\begin{array}{l}\text { Aripiprazole (Mean dose: } 15.2 \\
\text { Dose range: } 5 \text { to } 30 \mathrm{mg} \text { ), } 21\end{array}$ & $\begin{array}{l}\text { Haloperidol (Mean } \\
\text { dose: } 4.9 \text { Dose } \\
\text { range: } 1.5 \text { to } \\
\text { 16mg), } 21\end{array}$ & Not reported & Not applicable & $0 / 21(0 \%)$ & $\begin{array}{l}0 / 21 \\
(0 \%)\end{array}$ & \\
\hline $\begin{array}{l}\text { Charoenporn, } \\
2018^{25}\end{array}$ & $\begin{array}{l}\text { Cohort w/ } \\
\text { comparison } \\
\text { group }\end{array}$ & Physically ill patients & $\begin{array}{l}\text { Quetiapine (Mean dose: } 26.7 \\
\text { Median dose: } 25), 31\end{array}$ & $\begin{array}{l}\text { Haloperidol (Mean } \\
\text { dose: } 0.9 \text { Median } \\
\text { dose: } 0.5 \mathrm{mg}), 11 \\
\end{array}$ & Oral & Not applicable & $3 / 31(9.7 \%)$ & $\begin{array}{l}1 / 11 \\
(9.1 \%)\end{array}$ & $\begin{array}{l}\text { RR } 1.06 \\
\text { (95\% Cl: } 0.12 \\
\text { to } 9.19)\end{array}$ \\
\hline $\begin{array}{l}\text { Charoenporn, } \\
2018^{25}\end{array}$ & $\begin{array}{l}\text { Cohort w/ } \\
\text { comparison } \\
\text { group }\end{array}$ & Physically ill patients & $\begin{array}{l}\text { Risperidone (Mean dose: } 0.6 \\
\text { Median dose: } 0.5 \mathrm{mg} \text { ), } 14\end{array}$ & $\begin{array}{l}\text { Haloperidol (Mean } \\
\text { dose: } 0.9 \text { Median } \\
\text { dose: } 0.5 \mathrm{mg} \text { ), } 11 \\
\end{array}$ & Oral & Not applicable & $\begin{array}{l}4 / 14 \\
(28.6 \%)\end{array}$ & $\begin{array}{l}1 / 11 \\
(9.1 \%)\end{array}$ & $\begin{array}{l}\text { RR 3.14 } \\
\text { (95\% Cl: } 0.41 \\
\text { to } 24.27)\end{array}$ \\
\hline Girard, $2018^{29}$ & RCT & $\begin{array}{l}\text { Patients over } 18 \text { years of age in the } \\
\text { medical or surgical ICU, with } \\
\text { delirium }\end{array}$ & $\begin{array}{l}\text { Ziprasidone (Mean dose: } \\
\text { 20mg Dose range: } 5.0-40 \mathrm{mg} \text { ), } \\
190\end{array}$ & $\begin{array}{l}\text { Haloperidol (Mean } \\
\text { dose: } 11 \mathrm{mg} \text { Dose } \\
\text { range: } 2.5-20 \mathrm{mg} \text { ), } \\
192\end{array}$ & Intravenous & $\begin{array}{l}\text { Reasons for } \\
\text { temporary holds: } \\
\text { Oversedation }\end{array}$ & $\begin{array}{l}50 / 190 \\
(26 \%)\end{array}$ & $\begin{array}{l}42 / 192 \\
(22 \%)\end{array}$ & $\begin{array}{l}\text { RR 1.20 } \\
\text { (95\% Cl: } 0.84 \\
\text { to } 1.72 \text { ) }\end{array}$ \\
\hline Grover, $2011^{30}$ & RCT & $\begin{array}{l}\text { Consecutive patients }>-18 \text { years old } \\
\text { referred to psychiatry and } \\
\text { diagnosed with delirium }\end{array}$ & $\begin{array}{l}\text { Olanzapine (Mean dose: } \\
\text { 3.05mg Dose range: } 1.25-10 \\
\text { mg per day), } 23\end{array}$ & $\begin{array}{l}\text { Haloperidol (Mean } \\
\text { dose: } 0.88 \text { Dose } \\
\text { range: } 0.25-5 \mathrm{mg} \\
\text { per day), } 21\end{array}$ & Oral & Sleepiness/sedation & $0 / 23(0 \%)$ & $\begin{array}{l}1 / 21 \\
(4.8 \%)\end{array}$ & $\begin{array}{l}\text { RR } 0.31 \\
\text { (95\% Cl: } 0.01 \\
\text { to } 7.12)\end{array}$ \\
\hline Grover, $2011^{30}$ & RCT & $\begin{array}{l}\text { Consecutive patients }>-18 \text { years old } \\
\text { referred to psychiatry and } \\
\text { diagnosed with delirium }\end{array}$ & $\begin{array}{l}\text { Risperidone (Mean dose: } \\
\text { 0.95mg Dose range: } 0.5- \\
\text { 2.0mg per day), } 20\end{array}$ & $\begin{array}{l}\text { Haloperidol (Mean } \\
\text { dose: } 0.88 \text { Dose } \\
\text { range: } 0.25-5 \mathrm{mg} \\
\text { per day), } 21\end{array}$ & Oral & Sleepiness/sedation & $3 / 20$ (15\%) & $\begin{array}{l}1 / 21 \\
(4.8 \%)\end{array}$ & $\begin{array}{l}\text { RR 3.15 } \\
\text { (95\% Cl: } 0.36 \\
\text { to } 27.83 \text { ) }\end{array}$ \\
\hline
\end{tabular}




\begin{tabular}{|c|c|c|c|c|c|c|c|c|c|}
\hline Author, year & $\begin{array}{l}\text { Study } \\
\text { design }\end{array}$ & Population & Intervention group, $\mathbf{n}$ & Control group, $\mathbf{n}$ & $\begin{array}{c}\text { Route of } \\
\text { administration }\end{array}$ & $\begin{array}{l}\text { Outcome } \\
\text { definition }\end{array}$ & $\begin{array}{l}\mathrm{n} / \mathrm{N}(\%) \\
\text { intervention } \\
\text { group }\end{array}$ & $\begin{array}{c}\mathrm{n} / \mathrm{N} \\
(\%), \\
\text { control } \\
\text { group } \\
\end{array}$ & $\begin{array}{c}\text { Relative risk } \\
(95 \% \mathrm{Cl})\end{array}$ \\
\hline Han, $2004^{32}$ & RCT & $\begin{array}{l}\text { All patients presenting with altered } \\
\text { mental status (from both ICU and } \\
\text { non-ICU units) who were referred to } \\
\text { the consulting psychiatry division } \\
\text { were evaluated. }\end{array}$ & $\begin{array}{l}\text { Risperidone (Mean dose: } 1.02 \\
\text { Dose range: } 0.5 \text { to } 2.0 \mathrm{mg} \text { ), } 14\end{array}$ & $\begin{array}{l}\text { Haloperidol (Mean } \\
\text { dose: } 1.7 \text { Dose } \\
\text { range: } 1.0 \text { to } \\
3.0 \mathrm{mg}), 14\end{array}$ & Oral & $\begin{array}{l}\text { Withdrawal due to } \\
\text { severe sedation }\end{array}$ & $0 / 14(0 \%)$ & $\begin{array}{l}1 / 14 \\
(7.1 \%)\end{array}$ & $\begin{array}{l}\text { RR } 0.33 \\
\text { (95\% Cl: } 0.01 \\
\text { to } 7.55)\end{array}$ \\
\hline Jain, $2017^{38}$ & RCT & Medical and surgical inpatients & $\begin{array}{l}\text { Olanzapine (Mean dose: } \\
5.49 \mathrm{mg}), 47\end{array}$ & $\begin{array}{l}\text { Haloperidol (Mean } \\
\text { dose: } 2.10 \mathrm{mg}), 53\end{array}$ & Oral & Excessive sedation & $1 / 47(2.1 \%)$ & $\begin{array}{l}0 / 53 \\
(0 \%)\end{array}$ & $\begin{array}{l}\text { RR 3.38 } \\
\text { (95\% Cl: } 0.14 \\
\text { to } 80.91)\end{array}$ \\
\hline Kim, $2005^{41}$ & $\begin{array}{l}\text { Cohort w/ } \\
\text { comparison } \\
\text { group }\end{array}$ & $\begin{array}{l}\text { Hospital patients referred to the } \\
\text { psychiatry division }\end{array}$ & Risperidone (Not reported), 18 & $\begin{array}{l}\text { Haloperidol (Not } \\
\text { reported), } 24\end{array}$ & Not reported & Drowsiness & $0 / 18(0 \%)$ & $\begin{array}{l}1 / 24 \\
(4.2 \%)\end{array}$ & $\begin{array}{l}\text { RR 0.44 } \\
\text { (95\% Cl: } 0.02 \\
\text { to } 10.18) \\
\end{array}$ \\
\hline Lim, $2007^{46}$ & $\mathrm{RCT}$ & $\begin{array}{l}\text { Patients from internal medicine, } \\
\text { PMR, neurology, neurosurgery, } \\
\text { orthopedic surgery, or general } \\
\text { surgery that were referred to the } \\
\text { psychiatric department }\end{array}$ & $\begin{array}{l}\text { Olanzapine (Titrated (5 to } 20 \\
\mathrm{mg} \text { ) }\end{array}$ & $\begin{array}{l}\text { Haloperidol } \\
\text { (Titrated (5 to } 20 \\
\mathrm{mg} \text { ) }\end{array}$ & Intramuscular & Somnolence & $2 / 31(6.5 \%)$ & $\begin{array}{l}1 / 31 \\
(3.2 \%)\end{array}$ & $\begin{array}{l}\text { RR 2.00 } \\
\text { (95\% Cl: } 0.19 \\
\text { to 20.93) }\end{array}$ \\
\hline Maneeton, $2013^{49}$ & RCT & $\begin{array}{l}\text { General adult population (age 18- } \\
\text { 75) with hyperactive delirium, } \\
\text { referred to psychiatry consult } \\
\text { liaison service at tertiary care } \\
\text { hospital in Thailand }\end{array}$ & $\begin{array}{l}\text { Quetiapine (Mean dose: } 67.6 \\
\text { (SD 9.7) Dose range: } 25- \\
\text { 100mg), } 24\end{array}$ & $\begin{array}{l}\text { Haloperidol (Mean } \\
\text { dose: } 0.8 \text { (SD 0.3) } \\
\text { Dose range: } 0.5- \\
\text { 2.0mg), } 28\end{array}$ & Oral & Hypersomnia & $\begin{array}{l}10 / 24 \\
(41.7 \%)\end{array}$ & $\begin{array}{l}8 / 28 \\
(28.6 \%)\end{array}$ & $\begin{array}{l}\text { RR 1.46 } \\
\text { (95\% Cl: } 0.69 \\
\text { to } 3.10)\end{array}$ \\
\hline $\begin{array}{l}\text { Sipahimalani, } \\
1998^{56}\end{array}$ & $\begin{array}{l}\text { Cohort w/ } \\
\text { comparison } \\
\text { group }\end{array}$ & Hospital patients & $\begin{array}{l}\text { Olanzapine (Mean dose: } 8.2 \\
\text { Max dose: } 15 \mathrm{mg}), 11\end{array}$ & $\begin{array}{l}\text { Haloperidol (Dose } \\
\text { range: } 1.5 \text { to } \\
\text { 10mg), } 11\end{array}$ & Not reported & $\begin{array}{l}\text { Excessively } \\
\text { sedated }\end{array}$ & $0 / 11(0 \%)$ & $\begin{array}{l}2 / 11 \\
(18.2 \%)\end{array}$ & $\begin{array}{l}\text { RR } 0.20 \\
\text { (95\% Cl: } 0.01 \\
\text { to 3.74) }\end{array}$ \\
\hline Yoon, $2013^{62}$ & $\begin{array}{l}\text { Cohort w/ } \\
\text { comparison } \\
\text { group }\end{array}$ & $\begin{array}{l}\text { Referred to consultation-liaison } \\
\text { psychiatric service for a mental } \\
\text { status change }\end{array}$ & $\begin{array}{l}\text { Quetiapine (Mean dose: } 47.9 \\
\text { Dose range: } 25-200 \mathrm{mg} \text { ), } 18\end{array}$ & $\begin{array}{l}\text { Haloperidol (Mean } \\
\text { dose: } 1.2 \text { Dose } \\
\text { range: } 0.5-10 \mathrm{mg}) \\
23\end{array}$ & Not reported & $\begin{array}{l}\text { Exacerbation of } \\
\text { sedation or } \\
\text { sleepiness }\end{array}$ & $\begin{array}{l}2 / 18 \\
(11.1 \%)\end{array}$ & $\begin{array}{l}4 / 23 \\
(17.4 \%)\end{array}$ & $\begin{array}{l}\text { RR } 0.64 \\
\text { (95\% Cl: } 0.13 \\
\text { to 3.11) }\end{array}$ \\
\hline Yoon, $2013^{62}$ & $\begin{array}{l}\text { Cohort w/ } \\
\text { comparison } \\
\text { group }\end{array}$ & $\begin{array}{l}\text { Referred to consultation-liaison } \\
\text { psychiatric service for a mental } \\
\text { status change }\end{array}$ & $\begin{array}{l}\text { Olanzapine (Mean dose: } 2.9 \\
\text { Dose range: } 1-20 \mathrm{mg}), 18\end{array}$ & $\begin{array}{l}\text { Haloperidol (Mean } \\
\text { dose: } 1.2 \text { Dose } \\
\text { range: } 0.5-10 \mathrm{mg}) \\
23\end{array}$ & Not reported & $\begin{array}{l}\text { Exacerbation of } \\
\text { sedation or } \\
\text { sleepiness }\end{array}$ & $\begin{array}{l}4 / 18 \\
(22.2 \%)\end{array}$ & $\begin{array}{l}4 / 23 \\
(17.4 \%)\end{array}$ & $\begin{array}{l}\text { RR } 1.28 \\
\text { (95\% Cl: } 0.37 \\
\text { to } 4.42 \text { ) }\end{array}$ \\
\hline Yoon, $2013^{62}$ & $\begin{array}{l}\text { Cohort wl } \\
\text { comparison } \\
\text { group }\end{array}$ & $\begin{array}{l}\text { Referred to consultation-liaison } \\
\text { psychiatric service for a mental } \\
\text { status change }\end{array}$ & $\begin{array}{l}\text { Risperidone (Mean dose: } 1.1 \\
\text { Dose range: } 0.25-4 \mathrm{mg}), 21\end{array}$ & $\begin{array}{l}\text { Haloperidol (Mean } \\
\text { dose: } 1.2 \text { Dose } \\
\text { range: } 0.5-10 \mathrm{mg}) \\
23\end{array}$ & Not reported & $\begin{array}{l}\text { Exacerbation of } \\
\text { sedation or } \\
\text { sleepiness }\end{array}$ & $\begin{array}{l}3 / 21 \\
(14.3 \%)\end{array}$ & $\begin{array}{l}4 / 23 \\
(17.4 \%)\end{array}$ & $\begin{array}{l}\text { RR 0.82 } \\
\text { (95\% Cl: } 0.21 \\
\text { to 3.25) }\end{array}$ \\
\hline
\end{tabular}




\begin{tabular}{|c|c|c|c|c|c|c|c|c|c|}
\hline Author, year & $\begin{array}{l}\text { Study } \\
\text { design }\end{array}$ & Population & Intervention group, $\mathbf{n}$ & Control group, $\mathbf{n}$ & $\begin{array}{c}\text { Route of } \\
\text { administration }\end{array}$ & $\begin{array}{l}\text { Outcome } \\
\text { definition }\end{array}$ & $\begin{array}{l}\mathrm{n} / \mathrm{N}(\%) \\
\text { intervention } \\
\text { group }\end{array}$ & $\begin{array}{c}\mathrm{n} / \mathrm{N} \\
(\%), \\
\text { control } \\
\text { group }\end{array}$ & $\begin{array}{l}\text { Relative risk } \\
\quad(95 \% \mathrm{Cl})\end{array}$ \\
\hline \multicolumn{10}{|l|}{$\begin{array}{l}\text { Second-generation } \\
\text { antipsychotic vs. } \\
\text { second-generation } \\
\text { antipsychotic }\end{array}$} \\
\hline Boettger, 2015 & $\begin{array}{l}\text { Cohort w/ } \\
\text { comparison } \\
\text { group }\end{array}$ & Cancer patients & $\begin{array}{l}\text { Olanzapine (Mean dose: } 3.5 \\
\text { Dose range: } 2.5 \text { to } 10 \mathrm{mg}), 21\end{array}$ & $\begin{array}{l}\text { Aripiprazole (Mean } \\
\text { dose: } 15.2 \text { Dose } \\
\text { range: } 5 \text { to } 30 \mathrm{mg} \text { ), } \\
21\end{array}$ & Not reported & Not applicable & $\begin{array}{l}6 / 21 \\
(28.6 \%)\end{array}$ & $\begin{array}{l}0 / 21 \\
(0 \%)\end{array}$ & $\begin{array}{l}\text { RR } 13.00 \\
\text { (95\% Cl: } 0.78 \\
\text { to } 217.03)\end{array}$ \\
\hline Boettger, $2015^{21}$ & $\begin{array}{l}\text { Cohort w/ } \\
\text { comparison } \\
\text { group }\end{array}$ & Cancer patients & $\begin{array}{l}\text { Risperidone (Mean dose: } 0.9 \\
\text { Dose range: } 0.5 \text { to } 2 \mathrm{mg} \text { ), } 21\end{array}$ & $\begin{array}{l}\text { Aripiprazole (Mean } \\
\text { dose: } 15.2 \text { Dose } \\
\text { range: } 5 \text { to } 30 \mathrm{mg} \text { ), } \\
21\end{array}$ & Not reported & Not applicable & $0 / 21(0 \%)$ & $\begin{array}{l}0 / 21 \\
(0 \%)\end{array}$ & \\
\hline Boettger, $2015^{21}$ & $\begin{array}{l}\text { Cohort w/ } \\
\text { comparison } \\
\text { group }\end{array}$ & Cancer patients & $\begin{array}{l}\text { Risperidone (Mean dose: } 0.9 \\
\text { Dose range: } 0.5 \text { to } 2 \mathrm{mg} \text { ), } 21\end{array}$ & $\begin{array}{l}\text { Olanzapine (Mean } \\
\text { dose: } 3.5 \text { Dose } \\
\text { range: } 2.5 \text { to } \\
\text { 10mg), } 21\end{array}$ & Not reported & Not applicable & $0 / 21(0 \%)$ & $\begin{array}{l}6 / 21 \\
(28.6 \%)\end{array}$ & $\begin{array}{l}\text { RR } 0.08 \\
\text { (95\% Cl: } 0.00 \\
\text { to } 1.28)\end{array}$ \\
\hline $\begin{array}{l}\text { Charoenporn, } \\
2018^{25}\end{array}$ & $\begin{array}{l}\text { Cohort w/ } \\
\text { comparison } \\
\text { group }\end{array}$ & Physically ill patients & $\begin{array}{l}\text { Risperidone (Mean dose: } 0.6 \\
\text { Median dose: } 0.5 \mathrm{mg} \text { ), } 14\end{array}$ & $\begin{array}{l}\text { Quetiapine (Mean } \\
\text { dose: } 26.7 \text { Median } \\
\text { dose: } 25), 31 \\
\end{array}$ & Oral & Not applicable & $\begin{array}{l}4 / 14 \\
(28.6 \%)\end{array}$ & $\begin{array}{l}3 / 31 \\
(9.7 \%)\end{array}$ & $\begin{array}{l}\text { RR 2.95 } \\
\text { (95\% Cl: } 0.76 \\
\text { to 11.47) }\end{array}$ \\
\hline Fox, $2018^{28}$ & $\begin{array}{l}\text { Cohort w/ } \\
\text { comparison } \\
\text { group }\end{array}$ & $\begin{array}{l}\text { Critically ill patients in the medical } \\
\text { and cardiovascular ICU }\end{array}$ & $\begin{array}{l}\text { Quetiapine (Mean dose: } 153.8 \\
\text { Max dose: } 800 \mathrm{mg} \text { ), } 20\end{array}$ & $\begin{array}{l}\text { Lurasidone (Mean } \\
\text { dose: } 42.6 \mathrm{Max} \\
\text { dose: } 120 \mathrm{mg}), 20\end{array}$ & Not reported & $\begin{array}{l}\text { Discontinuation of } \\
\text { study drug due to } \\
\text { increased sedation }\end{array}$ & $4 / 20(20 \%)$ & $\begin{array}{l}2 / 20 \\
(10 \%)\end{array}$ & $\begin{array}{l}\text { RR 2.00 } \\
\text { (95\% Cl: } 0.41 \\
\text { to } 9.71 \text { ) }\end{array}$ \\
\hline Fox, $2018^{28}$ & $\begin{array}{l}\text { Cohort w/ } \\
\text { comparison } \\
\text { group }\end{array}$ & $\begin{array}{l}\text { Critically ill patients in the medical } \\
\text { and cardiovascular ICU }\end{array}$ & $\begin{array}{l}\text { Quetiapine (Mean dose: } 153.8 \\
\text { Max dose: } 800 \mathrm{mg} \text { ), } 20\end{array}$ & $\begin{array}{l}\text { Lurasidone (Mean } \\
\text { dose: } 42.6 \mathrm{Max} \\
\text { dose: } 120 \mathrm{mg}), 20\end{array}$ & Not reported & $\begin{array}{l}\text { Percentage of time } \\
\text { the patient was } \\
\text { oversedated (RASS } \\
<=-3 \text { ) }\end{array}$ & $\begin{array}{l}\text { Median } 2.7 \\
\text { (IQR } 0.9 \text { to } \\
11.7 \text { ) }\end{array}$ & $\begin{array}{l}\text { Median } \\
2.8(\text { IQR } \\
0 \text { to } \\
29.8)\end{array}$ & \\
\hline Grover, $2011^{30}$ & RCT & $\begin{array}{l}\text { Consecutive patients }>-18 \text { years old } \\
\text { referred to psychiatry and } \\
\text { diagnosed with delirium }\end{array}$ & $\begin{array}{l}\text { Risperidone (Mean dose: } \\
\text { 0.95mg Dose range: } 0.5- \\
2.0 \mathrm{mg} \text { per day), } 20\end{array}$ & $\begin{array}{l}\text { Olanzapine (Mean } \\
\text { dose: } 3.05 \mathrm{mg} \\
\text { Dose range: } 1.25- \\
10 \mathrm{mg} \text { per day), } 23\end{array}$ & Oral & Sleepiness/sedation & $3 / 20(15 \%)$ & $\begin{array}{l}0 / 23 \\
(0 \%)\end{array}$ & $\begin{array}{l}\text { RR } 8.00 \\
\text { (95\% Cl: } 0.44 \\
\text { to } 146.08)\end{array}$ \\
\hline
\end{tabular}




\begin{tabular}{|c|c|c|c|c|c|c|c|c|c|}
\hline Author, year & $\begin{array}{l}\text { Study } \\
\text { design }\end{array}$ & Population & Intervention group, $\mathbf{n}$ & Control group, $\mathbf{n}$ & $\begin{array}{c}\text { Route of } \\
\text { administration }\end{array}$ & $\begin{array}{l}\text { Outcome } \\
\text { definition }\end{array}$ & $\begin{array}{l}\mathrm{n} / \mathrm{N}(\%) \\
\text { intervention } \\
\text { group }\end{array}$ & $\begin{array}{l}\mathbf{n} / \mathbf{N} \\
(\%), \\
\text { control } \\
\text { group }\end{array}$ & $\begin{array}{c}\text { Relative risk } \\
(95 \% \mathrm{Cl})\end{array}$ \\
\hline Kim, $2010^{42}$ & RCT & $\begin{array}{l}\text { General hospital inpatients who met } \\
\text { DSM-IV diagnostic criteria for } \\
\text { delirium }\end{array}$ & $\begin{array}{l}\text { Risperidone (Mean dose: } 0.9 \pm \\
0.6 \text { Dose range: } 0.25-2 \mathrm{mg} \text { ), } \\
17\end{array}$ & $\begin{array}{l}\text { Olanzapine (Mean } \\
\text { dose: } 2.4 \pm 1.7 \\
\text { Dose range: } 1.25 \text { - } \\
7.5 \mathrm{mg}), 15\end{array}$ & Not reported & $\begin{array}{l}\text { Exacerbation of } \\
\text { daytime } \\
\text { somnolence or } \\
\text { increased duration } \\
\text { of sleep }\end{array}$ & $\begin{array}{l}5 / 17 \\
(29.4 \%)\end{array}$ & $\begin{array}{l}5 / 15 \\
(33.3 \%)\end{array}$ & $\begin{array}{l}\text { RR } 0.88 \\
\text { (95\% Cl: } 0.32 \\
\text { to 2.46) }\end{array}$ \\
\hline Lee, $2005^{45}$ & RCT & $\begin{array}{l}\text { Patients referred to the Psychiatric } \\
\text { Consultation that met DSM-IV } \\
\text { criteria for delirium }\end{array}$ & 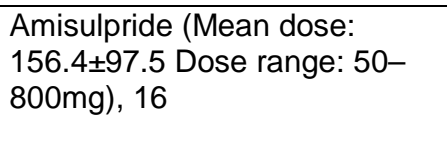 & $\begin{array}{l}\text { Quetiapine (Mean } \\
\text { dose: } 113 \pm 85.5 \\
\text { Dose range: } 50- \\
\text { 300mg), } 15\end{array}$ & Not reported & Oversedation & $0 / 16(0 \%)$ & $\begin{array}{l}0 / 15 \\
(0 \%)\end{array}$ & \\
\hline Yoon, $2013^{62}$ & $\begin{array}{l}\text { Cohort w/ } \\
\text { comparison } \\
\text { group }\end{array}$ & $\begin{array}{l}\text { Referred to consultation-liaison } \\
\text { psychiatric service for a mental } \\
\text { status change }\end{array}$ & $\begin{array}{l}\text { Quetiapine (Mean dose: } 47.9 \\
\text { Dose range: } 25-200 \mathrm{mg} \text { ), } 18\end{array}$ & $\begin{array}{l}\text { Olanzapine (Mean } \\
\text { dose: } 2.9 \text { Dose } \\
\text { range: } 1-20 \mathrm{mg} \text { ), } \\
18\end{array}$ & Not reported & $\begin{array}{l}\text { Exacerbation of } \\
\text { sedation or } \\
\text { sleepiness }\end{array}$ & $\begin{array}{l}2 / 18 \\
(11.1 \%)\end{array}$ & $\begin{array}{l}4 / 18 \\
(22.2 \%)\end{array}$ & $\begin{array}{l}\text { RR } 0.50 \\
\text { (95\% Cl: } 0.10 \\
\text { to } 2.40)\end{array}$ \\
\hline Yoon, $2013^{62}$ & $\begin{array}{l}\text { Cohort w/ } \\
\text { comparison } \\
\text { group }\end{array}$ & $\begin{array}{l}\text { Referred to consultation-liaison } \\
\text { psychiatric service for a mental } \\
\text { status change }\end{array}$ & $\begin{array}{l}\text { Risperidone (Mean dose: } 1.1 \\
\text { Dose range: } 0.25-4 \mathrm{mg} \text { ), } 21\end{array}$ & $\begin{array}{l}\text { Olanzapine (Mean } \\
\text { dose: } 2.9 \text { Dose } \\
\text { range: } 1-20 \mathrm{mg} \text { ), } \\
18\end{array}$ & Not reported & $\begin{array}{l}\text { Exacerbation of } \\
\text { sedation or } \\
\text { sleepiness }\end{array}$ & $\begin{array}{l}3 / 21 \\
(14.3 \%)\end{array}$ & $\begin{array}{l}4 / 18 \\
(22.2 \%)\end{array}$ & $\begin{array}{l}\text { RR } 0.64 \\
\text { (95\% Cl: } 0.17 \\
\text { to } 2.50)\end{array}$ \\
\hline Yoon, $2013^{62}$ & $\begin{array}{l}\text { Cohort w/ } \\
\text { comparison } \\
\text { group }\end{array}$ & $\begin{array}{l}\text { Referred to consultation-liaison } \\
\text { psychiatric service for a mental } \\
\text { status change }\end{array}$ & $\begin{array}{l}\text { Risperidone (Mean dose: } 1.1 \\
\text { Dose range: } 0.25-4 \mathrm{mg} \text { ), } 21\end{array}$ & $\begin{array}{l}\text { Quetiapine (Mean } \\
\text { dose: } 47.9 \text { Dose } \\
\text { range: } 25- \\
\text { 200mg),18 }\end{array}$ & Not reported & $\begin{array}{l}\text { Exacerbation of } \\
\text { sedation or } \\
\text { sleepiness }\end{array}$ & $\begin{array}{l}3 / 21 \\
(14.3 \%)\end{array}$ & $\begin{array}{l}2 / 18 \\
(11.1 \%)\end{array}$ & $\begin{array}{l}\text { RR 1.29 } \\
\text { (95\% Cl: } 0.24 \\
\text { to 6.86) }\end{array}$ \\
\hline
\end{tabular}

CI=confidence interval; DRS-R-98= Delirium Rating Scale Revised-98; DSM-IV=Diagnostic and Statistical Manual of Mental Disorders, $4^{\text {th }}$ ed; ICU=intensive care unit; IQR=interquartile range; mg=milligram; MV=mechanical ventilation; N=sample size; RCT=randomized controlled trial; RR=risk ratio 


\begin{tabular}{|c|c|c|c|c|c|c|}
\hline Author, year & $\begin{array}{l}\text { Study } \\
\text { design }\end{array}$ & Population & Intervention group, $\mathbf{n}$ & $\begin{array}{c}\text { Route of } \\
\text { administration }\end{array}$ & Outcome definition & $\begin{array}{c}\mathrm{n} / \mathrm{N}(\%) \\
\text { intervention } \\
\text { group }\end{array}$ \\
\hline $\begin{array}{l}\text { Breitbart, } \\
2002^{23}\end{array}$ & $\begin{array}{l}\text { Cohort } \\
\text { w/o } \\
\text { compariso } \\
\text { n group }\end{array}$ & Cancer patients & $\begin{array}{l}\text { Olanzapine (Mean dose: } 3.0 \text { Dose range: } 2.5 \text { to } \\
\text { 10mg) }\end{array}$ & Oral & Not applicable & $(30 \%)$ \\
\hline $\begin{array}{l}\text { Breitbart, } \\
2002^{23}\end{array}$ & $\begin{array}{l}\text { Cohort } \\
\text { w/o } \\
\text { compariso } \\
\text { n group }\end{array}$ & Cancer patients & $\begin{array}{l}\text { Olanzapine (Mean dose: } 3.0 \text { Dose range: } 2.5 \text { to } \\
\text { 10mg) }\end{array}$ & Oral & Not applicable & $(30 \%)$ \\
\hline $\begin{array}{l}\text { Breitbart, } \\
2002^{23}\end{array}$ & $\begin{array}{l}\text { Cohort } \\
\text { w/o } \\
\text { compariso } \\
\text { n group }\end{array}$ & Cancer patients & $\begin{array}{l}\text { Olanzapine (Mean dose: } 3.0 \text { Dose range: } 2.5 \text { to } \\
\text { 10mg), } 79\end{array}$ & Oral & Dosage reduced due to sedation & $8 / 79(10 \%)$ \\
\hline $\begin{array}{l}\text { Horikawa, } \\
2003^{34}\end{array}$ & $\begin{array}{l}\text { Cohort } \\
\text { w/o } \\
\text { compariso } \\
\text { n group }\end{array}$ & Medical and surgical inpatients & $\begin{array}{l}\text { Risperidone (Planned dose: } 0.5 \text { Max dose: } \\
1.7 \mathrm{mg} \text { ), } 10\end{array}$ & Oral & Daytime sleepiness & $3 / 10(30 \%)$ \\
\hline Ikezawa, $2008^{37}$ & $\begin{array}{l}\text { Open- } \\
\text { label trial }\end{array}$ & Elderly patients with hyperactive-hypervalent delirium & $\begin{array}{l}\text { Risperidone (Mean dose: } 1.5 \text { (0.7) Dose range: } \\
0.5-3 \mathrm{mg}), 22\end{array}$ & Oral & Somnolence & $4 / 22(18 \%)$ \\
\hline Kim, $2001^{39}$ & $\begin{array}{l}\text { Open } \\
\text { label study }\end{array}$ & $\begin{array}{l}\text { Medico-surgical patients from Neurology, } \\
\text { Neurosurgery and BMT unit }\end{array}$ & $\begin{array}{l}\text { Olanzapine (Mean dose: } 5.9 \text { (1.5) Max dose: } \\
8.8(2.2), 20\end{array}$ & Oral & Mild sedation & $2 / 20(10 \%)$ \\
\hline Kim, $2003^{40}$ & $\begin{array}{l}\text { Cohort } \\
\text { w/o } \\
\text { compariso } \\
\text { n group }\end{array}$ & Patients in the acute medical units & $\begin{array}{l}\text { Quetiapine (Planned dose: } 25 \text { Mean dose: } \\
93.75 \mathrm{mg}), 12\end{array}$ & Not reported & Not applicable & $2 / 12(17 \%)$ \\
\hline Kishi, $2012^{43}$ & $\begin{array}{l}\text { Cohort } \\
\text { w/o } \\
\text { compariso } \\
\text { n group }\end{array}$ & Delirious cancer patients & Risperidone (Mean dose: 1.4mg), 29 & Oral & Mild sedation & $0 / 29(0 \%)$ \\
\hline $\begin{array}{l}\text { Maneeton, } \\
2007^{48}\end{array}$ & $\begin{array}{l}\text { Open- } \\
\text { label study }\end{array}$ & $\begin{array}{l}\text { All physically ill in-patients whose pcp consulted } \\
\text { psychiatrists }\end{array}$ & $\begin{array}{l}\text { Quetiapine (Mean dose: } 45.7 \text { (28.7) Max dose: } \\
100 \text { Dose range: } 25-100 \mathrm{mg}) .17\end{array}$ & Oral & Daytime sleepiness & $13 / 17(76 \%)$ \\
\hline Mittal, $2004^{50}$ & $\begin{array}{l}\text { Cohort } \\
\text { w/o } \\
\text { compariso } \\
\text { n group }\end{array}$ & Hospitalized patients & Risperidone (Mean dose: $0.75 \mathrm{mg}$ ), 10 & Not reported & Mild sedation & $2 / 10(20 \%)$ \\
\hline
\end{tabular}




\begin{tabular}{|c|c|c|c|c|c|c|}
\hline Author, year & $\begin{array}{l}\text { Study } \\
\text { design }\end{array}$ & Population & Intervention group, $\mathbf{n}$ & $\begin{array}{c}\text { Route of } \\
\text { administration }\end{array}$ & Outcome definition & $\begin{array}{c}\mathbf{n} / \mathbf{N}(\%) \\
\text { intervention } \\
\text { group }\end{array}$ \\
\hline Omura, $2003^{52}$ & $\begin{array}{l}\text { Cohort } \\
\text { w/o } \\
\text { compariso } \\
\text { n group }\end{array}$ & Hospitalized patients & $\begin{array}{l}\text { Quetiapine (Mean dose: } 54.7 \text { Dose range: } 25 \text { to } \\
\text { 125mg), } 24\end{array}$ & Not reported & Somnolence & $3 / 24(13 \%)$ \\
\hline Omura, 200352 & $\begin{array}{l}\text { Cohort } \\
\text { w/o } \\
\text { compariso } \\
\text { n group }\end{array}$ & Hospitalized patients & $\begin{array}{l}\text { Quetiapine (Mean dose: } 54.7 \text { Dose range: } 25 \text { to } \\
\text { 125mg), } 24\end{array}$ & Not reported & Oversedation & $0 / 24(0 \%)$ \\
\hline Pae, $2004^{53}$ & $\begin{array}{l}\text { Cohort } \\
\text { w/o } \\
\text { compariso } \\
\text { n group }\end{array}$ & $\begin{array}{l}\text { Delirious patients recruited from the departments of } \\
\text { neurosurgery, orthopedic surgery, and oncology }\end{array}$ & Quetiapine (Mean dose: $127.1 \mathrm{mg}), 22$ & Oral & $\begin{array}{l}\text { Stopped medication due to } \\
\text { sedation }\end{array}$ & $2 / 22(9 \%)$ \\
\hline Pae, $2004^{53}$ & $\begin{array}{l}\text { Cohort } \\
\text { w/o } \\
\text { compariso } \\
\text { n group }\end{array}$ & $\begin{array}{l}\text { Delirious patients recruited from the departments of } \\
\text { neurosurgery, orthopedic surgery, and oncology }\end{array}$ & Quetiapine (Mean dose: $127.1 \mathrm{mg}), 22$ & Oral & Mild sedation & $3 / 22(14 \%)$ \\
\hline $\begin{array}{l}\text { Parellada, } \\
2004^{54}\end{array}$ & $\begin{array}{l}\text { Cohort } \\
\text { w/o } \\
\text { compariso } \\
\text { n group }\end{array}$ & General hospital patients & $\begin{array}{l}\text { Risperidone (Mean dose: day 1, 2.6; day 3, 2.6; } \\
\text { day } 7,1.5 \mathrm{mg}), 64\end{array}$ & Oral & Drowsiness & $2 / 64(3 \%)$ \\
\hline Sasaki, $2003^{55}$ & $\begin{array}{l}\text { Cohort } \\
\text { w/o } \\
\text { compariso } \\
\text { n group }\end{array}$ & Inpatients and outpatients & $\begin{array}{l}\text { Quetiapine (Mean dose: } 44.9 \text { Max dose: } \\
63.5 \mathrm{mg}), 12\end{array}$ & Oral & $\begin{array}{l}\text { Excessive sedation or } \\
\text { somnolence in the daytime }\end{array}$ & $0 / 12(0 \%)$ \\
\hline Toda, $2005^{60}$ & $\begin{array}{l}\text { Cohort } \\
\text { w/o } \\
\text { compariso } \\
\text { n group }\end{array}$ & Hospital patients with delirium & $\begin{array}{l}\text { Risperidone (Mean dose: } 0.92 \text { Max dose: 1.38), } \\
10\end{array}$ & Oral & Somnolence & $2 / 10(20 \%)$ \\
\hline
\end{tabular}


Evidence Table D-47. Binary cardiac outcomes for studies comparing interventions to treat delirium

\begin{tabular}{|c|c|c|c|c|c|c|c|c|c|}
\hline Author, year & $\begin{array}{l}\text { Study } \\
\text { design }\end{array}$ & Population & Intervention group, $\mathbf{n}$ & Control group, $n$ & $\begin{array}{c}\text { Route of } \\
\text { administration }\end{array}$ & Outcome definition & $\begin{array}{c}\mathrm{n} / \mathrm{N}(\%), \\
\text { intervention } \\
\text { group }\end{array}$ & $\begin{array}{l}\mathrm{n} \text { I N (\%), } \\
\text { control } \\
\text { group }\end{array}$ & $\begin{array}{l}\text { Relative } \\
\text { risk (95\% } \\
\text { Cl) }\end{array}$ \\
\hline \multicolumn{10}{|l|}{$\begin{array}{l}\text { First-generation } \\
\text { antipsychotic vs. } \\
\text { placebo }\end{array}$} \\
\hline Girard, $2010^{4}$ & RCT & $\begin{array}{l}>18 \text { years MV medical and } \\
\text { surgical ICU patients }\end{array}$ & $\begin{array}{l}\text { Haloperidol (Planned } \\
\text { dose: } 5 \text { Median dose: } \\
15 \text { (10.8-17),35 }\end{array}$ & $\begin{array}{l}\text { Placebo (Planned } \\
\text { dose: } 5 \mathrm{ml}), 36\end{array}$ & Oral & $\begin{array}{l}\text { Prolongation in the QTc } \\
\text { interval prolongation of the } \\
\text { QTc }>500 \mathrm{~ms} \text { while receiving } \\
\text { study drug }\end{array}$ & $2 / 35(5.7 \%)$ & $3 / 36(8.3 \%)$ & $\begin{array}{l}\text { RR } 0.69 \\
\text { (95\% Cl: } \\
0.12 \text { to } \\
3.86)\end{array}$ \\
\hline Girard, $2010^{4}$ & RCT & $\begin{array}{l}>18 \text { years MV medical and } \\
\text { surgical ICU patients }\end{array}$ & $\begin{array}{l}\text { Haloperidol (Planned } \\
\text { dose: } 5 \text { Median dose: } \\
15 \text { (10.8-17), } 35\end{array}$ & $\begin{array}{l}\text { Placebo (Planned } \\
\text { dose: } 5 \mathrm{ml}), 36\end{array}$ & Oral & Ventricular arrhythmias & $0 / 35(0 \%)$ & $0 / 36(0 \%)$ & \\
\hline Girard, $2018^{29}$ & RCT & $\begin{array}{l}\text { Patients over } 18 \text { years of age in } \\
\text { the medical or surgical ICU, with } \\
\text { delirium }\end{array}$ & $\begin{array}{l}\text { Haloperidol (Mean } \\
\text { dose: } 11 \mathrm{mg} \text { Dose } \\
\text { range: } 2.5-20 \mathrm{mg} \text { ), } 192\end{array}$ & $\begin{array}{l}\text { Placebo (Not } \\
\text { reported), } 184\end{array}$ & Intravenous & $\begin{array}{l}\text { Reasons for temporary holds: } \\
\text { Prolonged QTc }\end{array}$ & $\begin{array}{l}13 / 192 \\
(7 \%)\end{array}$ & $\begin{array}{l}10 / 184 \\
(5 \%)\end{array}$ & $\begin{array}{l}\text { RR } 1.25 \\
(95 \% \mathrm{Cl} \text { : } \\
0.56 \text { to } \\
2.77)\end{array}$ \\
\hline Girard, $2018^{29}$ & $\mathrm{RCT}$ & $\begin{array}{l}\text { Patients over } 18 \text { years of age in } \\
\text { the medical or surgical ICU, with } \\
\text { delirium }\end{array}$ & $\begin{array}{l}\text { Haloperidol (Mean } \\
\text { dose: } 11 \mathrm{mg} \text { Dose } \\
\text { range: } 2.5-20 \mathrm{mg}), 192\end{array}$ & $\begin{array}{l}\text { Placebo (Not } \\
\text { reported), } 184\end{array}$ & Intravenous & $\begin{array}{l}\text { Reasons for permanent } \\
\text { discontinuation: Suspected } \\
\text { torsades des pointes }\end{array}$ & $0 / 192(0 \%)$ & $0 / 184(0 \%)$ & \\
\hline Page, $2013^{10}$ & RCT & $\begin{array}{l}\text { ICU patients needing MV within } \\
72 \text { hours of admission were } \\
\text { enrolled }\end{array}$ & $\begin{array}{l}\text { Haloperidol (Planned } \\
\text { dose: } 2.5 \mathrm{mg}), 71\end{array}$ & $\begin{array}{l}\text { Placebo (Planned } \\
\text { dose: } 0.5 \mathrm{ml}), 70\end{array}$ & Intravenous & Atrial fibrillation & $7 / 71(10 \%)$ & $3 / 70(4 \%)$ & $\begin{array}{l}\text { RR 2.30 } \\
(95 \% \mathrm{Cl} \text { : } \\
0.62 \text { to } \\
8.54)\end{array}$ \\
\hline Page, $2013^{10}$ & RCT & $\begin{array}{l}\text { ICU patients needing MV within } \\
72 \text { hours of admission were } \\
\text { enrolled }\end{array}$ & $\begin{array}{l}\text { Haloperidol (Planned } \\
\text { dose: } 2.5 \mathrm{mg}), 71\end{array}$ & $\begin{array}{l}\text { Placebo (Planned } \\
\text { dose: } 0.5 \mathrm{ml}), 70\end{array}$ & Intravenous & Bradycardia & $2 / 71(2.8 \%)$ & $0 / 70(0 \%)$ & $\begin{array}{l}\text { RR 4.93 } \\
\text { (95\% Cl: } \\
0.24 \text { to } \\
100.89) \\
\end{array}$ \\
\hline Page, $2013^{10}$ & RCT & $\begin{array}{l}\text { ICU patients needing MV within } \\
72 \text { hours of admission were } \\
\text { enrolled }\end{array}$ & $\begin{array}{l}\text { Haloperidol (Planned } \\
\text { dose: } 2.5 \mathrm{mg} \text { ), } 71\end{array}$ & $\begin{array}{l}\text { Placebo (Planned } \\
\text { dose: } 0.5 \mathrm{ml}), 70\end{array}$ & Intravenous & Hypotension & $3 / 71(4 \%)$ & $2 / 70(3 \%)$ & $\begin{array}{l}\text { RR } 1.48 \\
\text { (95\% Cl: } \\
0.25 \text { to } \\
8.58)\end{array}$ \\
\hline Page, $2013^{10}$ & RCT & $\begin{array}{l}\text { ICU patients needing MV within } \\
72 \text { hours of admission were } \\
\text { enrolled }\end{array}$ & $\begin{array}{l}\text { Haloperidol (Planned } \\
\text { dose: } 2.5 \mathrm{mg}), 71\end{array}$ & $\begin{array}{l}\text { Placebo (Planned } \\
\text { dose: } 0.5 \mathrm{ml}), 70\end{array}$ & Intravenous & $\begin{array}{l}\text { Prolongation in the QTC } \\
\text { interval > 500 ms }\end{array}$ & $7 / 71(10 \%)$ & $6 / 70(9 \%)$ & $\begin{array}{l}\text { RR 1.15 } \\
\text { (95\% Cl: } \\
0.41 \text { to } \\
3.25)\end{array}$ \\
\hline Page, $2013^{10}$ & RCT & $\begin{array}{l}\text { ICU patients needing MV within } \\
72 \text { hours of admission were } \\
\text { enrolled }\end{array}$ & $\begin{array}{l}\text { Haloperidol (Planned } \\
\text { dose: } 2.5 \mathrm{mg} \text { ), } 71\end{array}$ & $\begin{array}{l}\text { Placebo (Planned } \\
\text { dose: } 0.5 \mathrm{ml}), 70\end{array}$ & Intravenous & Supraventricular Tachycardia & $4 / 71(6 \%)$ & $1 / 70(1 \%)$ & $\begin{array}{l}\text { RR 3.94 } \\
(95 \% \mathrm{Cl}: \\
0.45 \text { to } \\
34.41)\end{array}$ \\
\hline
\end{tabular}




\begin{tabular}{|c|c|c|c|c|c|c|c|c|c|}
\hline Author, year & $\begin{array}{l}\text { Study } \\
\text { design }\end{array}$ & Population & Intervention group, $\mathbf{n}$ & Control group, $\mathbf{n}$ & $\begin{array}{c}\text { Route of } \\
\text { administration }\end{array}$ & Outcome definition & $\begin{array}{l}\mathrm{n} / \mathrm{N}(\%) \\
\text { intervention } \\
\text { group }\end{array}$ & $\begin{array}{l}\mathrm{n} / \mathrm{N}(\%) \\
\text { control } \\
\text { group }\end{array}$ & $\begin{array}{l}\text { Relative } \\
\text { risk }(95 \% \\
\text { Cl) }\end{array}$ \\
\hline \multicolumn{10}{|c|}{$\begin{array}{l}\text { Second-generation } \\
\text { antipsychotic vs. } \\
\text { placebo }\end{array}$} \\
\hline Devlin, $2010^{26}$ & RCT & $\begin{array}{l}\text { Patients admitted to Medical \& } \\
\text { Surgical ICU with delirium. }\end{array}$ & $\begin{array}{l}\text { Quetiapine (Mean } \\
\text { dose: } 50 \text { Median dose: } \\
110 \text { (88-191 IQR) Max } \\
\text { dose: } 200 \text { Dose range: } \\
50 \text { to } 200 \mathrm{mg}), 18\end{array}$ & $\begin{array}{l}\text { Placebo (Mean } \\
\text { dose: } 50 \text { Median } \\
\text { dose: } 210 \text { (116 - } \\
\text { 293) Max dose: } \\
\text { 200mg), } 18\end{array}$ & $\begin{array}{l}\text { Either orally or } \\
\text { NG tube }\end{array}$ & Hypotension & $1 / 18(6 \%)$ & $0 / 18(0 \%)$ & $\begin{array}{l}\text { RR 3.00 } \\
\text { (95\% Cl: } \\
0.13 \text { to } \\
69.09 \text { ) }\end{array}$ \\
\hline Devlin, $2010^{26}$ & RCT & $\begin{array}{l}\text { Patients admitted to Medical \& } \\
\text { Surgical ICU with delirium. }\end{array}$ & $\begin{array}{l}\text { Quetiapine (Mean } \\
\text { dose: } 50 \text { Median dose: } \\
110 \text { (88-191 IQR) Max } \\
\text { dose: } 200 \text { Dose range: } \\
50 \text { to } 200 \mathrm{mg} \text { ) }\end{array}$ & $\begin{array}{l}\text { Placebo (Mean } \\
\text { dose: } 50 \text { Median } \\
\text { dose: } 210 \text { (116 - } \\
\text { 293) Max dose: } \\
\text { 200mg) }\end{array}$ & $\begin{array}{l}\text { Either orally or } \\
\text { NG tube }\end{array}$ & $\begin{array}{l}\text { Prolongation in the QTc } \\
\text { interval Episodes of QTC } \\
\text { interval > } 60 \mathrm{~m} / \mathrm{sec} \text { above } \\
\text { baseline }\end{array}$ & $\begin{array}{l}\text { Number of } \\
\text { events: } 20\end{array}$ & $\begin{array}{l}\text { Number of } \\
\text { events: } 34\end{array}$ & $\mathrm{p}=0.7$ \\
\hline Devlin, $2010^{26}$ & RCT & $\begin{array}{l}\text { Patients admitted to Medical \& } \\
\text { Surgical ICU with delirium. }\end{array}$ & $\begin{array}{l}\text { Quetiapine (Mean } \\
\text { dose: } 50 \text { Median dose: } \\
110 \text { (88-191 IQR) Max } \\
\text { dose: } 200 \text { Dose range: } \\
50 \text { to } 200 \mathrm{mg} \text { ) }\end{array}$ & $\begin{array}{l}\text { Placebo (Mean } \\
\text { dose: } 50 \text { Median } \\
\text { dose: } 210 \text { (116 - } \\
\text { 293) Max dose: } \\
\text { 200mg) }\end{array}$ & $\begin{array}{l}\text { Either orally or } \\
\text { NG tube }\end{array}$ & $\begin{array}{l}\text { Prolongation in the QTC } \\
\text { interval Episodes of QTC } \\
\text { interval > 500 m/sec }\end{array}$ & $\begin{array}{l}\text { Number of } \\
\text { events: } 8\end{array}$ & $\begin{array}{l}\text { Number of } \\
\text { events: } 8\end{array}$ & $\mathrm{p}=1$ \\
\hline Devlin, $2010^{26}$ & RCT & $\begin{array}{l}\text { Patients admitted to Medical \& } \\
\text { Surgical ICU with delirium. }\end{array}$ & $\begin{array}{l}\text { Quetiapine (Mean } \\
\text { dose: } 50 \text { Median dose: } \\
110 \text { (88-191 IQR) Max } \\
\text { dose: } 200 \text { Dose range: } \\
50 \text { to } 200 \mathrm{mg} \text { ) }\end{array}$ & $\begin{array}{l}\text { Placebo (Mean } \\
\text { dose: } 50 \text { Median } \\
\text { dose: } 210 \text { (116 - } \\
\text { 293) Max dose: } \\
\text { 200mg) }\end{array}$ & $\begin{array}{l}\text { Either orally or } \\
\text { NG tube }\end{array}$ & $\begin{array}{l}\text { Prolongation in the QTC } \\
\text { interval Episodes of QTC } \\
\text { interval prolongation }\end{array}$ & $\begin{array}{l}\text { Number of } \\
\text { events: } 30\end{array}$ & $\begin{array}{l}\text { Number of } \\
\text { events: } 41\end{array}$ & $\mathrm{p}=0.32$ \\
\hline Devlin, $2010^{26}$ & RCT & $\begin{array}{l}\text { Patients admitted to Medical \& } \\
\text { Surgical ICU with delirium. }\end{array}$ & $\begin{array}{l}\text { Quetiapine (Mean } \\
\text { dose: } 50 \text { Median dose: } \\
110 \text { (88-191 IQR) Max } \\
\text { dose: } 200 \text { Dose range: } \\
50 \text { to } 200 \mathrm{mg}), 18\end{array}$ & $\begin{array}{l}\text { Placebo (Mean } \\
\text { dose: } 50 \text { Median } \\
\text { dose: } 210 \text { (116 - } \\
\text { 293) Max dose: } \\
\text { 200mg), } 18\end{array}$ & $\begin{array}{l}\text { Either orally or } \\
\text { NG tube }\end{array}$ & $\begin{array}{l}\text { Prolongation in the QTC } \\
\text { interval Subjects experiencing } \\
\text { QTc interval > } 60 \text { msec above } \\
\text { baseline, \% }\end{array}$ & $7 / 18$ (39\%) & $8 / 18$ (44\%) & $\begin{array}{l}\text { RR } 0.88 \\
\text { (95\% Cl: } \\
0.40 \text { to } \\
1.90)\end{array}$ \\
\hline Devlin, $2010^{26}$ & RCT & $\begin{array}{l}\text { Patients admitted to Medical \& } \\
\text { Surgical ICU with delirium. }\end{array}$ & $\begin{array}{l}\text { Quetiapine (Mean } \\
\text { dose: } 50 \text { Median dose: } \\
110 \text { (88-191 IQR) Max } \\
\text { dose: } 200 \text { Dose range: } \\
50 \text { to } 200 \mathrm{mg} \text { ), } 18\end{array}$ & $\begin{array}{l}\text { Placebo (Mean } \\
\text { dose: } 50 \text { Median } \\
\text { dose: } 210 \text { (116 - } \\
\text { 293) Max dose: } \\
\text { 200mg), } 18\end{array}$ & $\begin{array}{l}\text { Either orally or } \\
\text { NG tube }\end{array}$ & $\begin{array}{l}\text { Prolongation in the QTC } \\
\text { interval Subjects experiencing } \\
\text { QTc interval > } 500 \mathrm{~m} / \mathrm{sec}, \%\end{array}$ & $4 / 18$ (22\%) & $5 / 18$ (28\%) & $\begin{array}{l}\text { RR 0.80 } \\
\text { (95\% Cl: } \\
0.26 \text { to } \\
2.50)\end{array}$ \\
\hline
\end{tabular}




\begin{tabular}{|c|c|c|c|c|c|c|c|c|c|}
\hline Author, year & $\begin{array}{l}\text { Study } \\
\text { design }\end{array}$ & Population & Intervention group, $\mathbf{n}$ & Control group, $\mathbf{n}$ & $\begin{array}{c}\text { Route of } \\
\text { administration }\end{array}$ & Outcome definition & $\begin{array}{l}\mathrm{n} / \mathrm{N}(\%) \\
\text { intervention } \\
\text { group }\end{array}$ & $\begin{array}{c}\mathrm{n} / \mathrm{N}(\%) \\
\text { control } \\
\text { group }\end{array}$ & $\begin{array}{l}\text { Relative } \\
\text { risk (95\% } \\
\mathrm{Cl})\end{array}$ \\
\hline Devlin, $2010^{26}$ & $\mathrm{RCT}$ & $\begin{array}{l}\text { Patients admitted to Medical \& } \\
\text { Surgical ICU with delirium. }\end{array}$ & $\begin{array}{l}\text { Quetiapine (Mean } \\
\text { dose: } 50 \text { Median dose: } \\
110 \text { ( } 88-191 \text { IQR) Max } \\
\text { dose: } 200 \text { Dose range: } \\
50 \text { to } 200 \mathrm{mg}), 18\end{array}$ & $\begin{array}{l}\text { Placebo (Mean } \\
\text { dose: } 50 \text { Median } \\
\text { dose: } 210 \text { (116 - } \\
\text { 293) Max dose: } \\
\text { 200mg), } 18\end{array}$ & $\begin{array}{l}\text { Either orally or } \\
\text { NG tube }\end{array}$ & $\begin{array}{l}\text { Prolongation in the QTC } \\
\text { interval Subjects experiencing } \\
\text { QTc prolongation, \% }\end{array}$ & $9 / 18(50 \%)$ & $\begin{array}{l}13 / 18 \\
(72 \%)\end{array}$ & $\begin{array}{l}\text { RR } 0.69 \\
\text { (95\% Cl: } \\
0.40 \text { to } \\
1.19)\end{array}$ \\
\hline Devlin, $2010^{26}$ & $\mathrm{RCT}$ & $\begin{array}{l}\text { Patients admitted to Medical \& } \\
\text { Surgical ICU with delirium. }\end{array}$ & $\begin{array}{l}\text { Quetiapine (Mean } \\
\text { dose: } 50 \text { Median dose: } \\
110 \text { (88-191 IQR) Max } \\
\text { dose: } 200 \text { Dose range: } \\
50 \text { to } 200 \mathrm{mg} \text { ), } 18\end{array}$ & $\begin{array}{l}\text { Placebo (Mean } \\
\text { dose: } 50 \text { Median } \\
\text { dose: } 210 \text { (116 - } \\
\text { 293) Max dose: } \\
\text { 200mg), } 18\end{array}$ & $\begin{array}{l}\text { Either orally or } \\
\text { NG tube }\end{array}$ & Torsades de pointes & $0 / 18(0 \%)$ & $0 / 18(0 \%)$ & \\
\hline Girard, $2010^{4}$ & $\mathrm{RCT}$ & $\begin{array}{l}>18 \text { years MV medical and } \\
\text { surgical ICU patients }\end{array}$ & $\begin{array}{l}\text { Ziprasidone (Planned } \\
\text { dose: } 40 \text { Median dose: } \\
113(81-140) 30\end{array}$ & $\begin{array}{l}\text { Placebo (Planned } \\
\text { dose: } 5 \mathrm{ml}), 36\end{array}$ & Oral & $\begin{array}{l}\text { Prolongation in the QTc } \\
\text { interval prolongation of the } \\
\text { QTc }>500 \mathrm{~ms} \text { while receiving } \\
\text { study drug }\end{array}$ & $\begin{array}{l}5 / 30 \\
(16.7 \%)\end{array}$ & $3 / 36(8.3 \%)$ & $\begin{array}{l}\text { RR 2.00 } \\
\text { (95\% Cl: } \\
0.52 \text { to } \\
7.69)\end{array}$ \\
\hline Girard, $2010^{4}$ & $\mathrm{RCT}$ & $\begin{array}{l}>18 \text { years MV medical and } \\
\text { surgical ICU patients }\end{array}$ & $\begin{array}{l}\text { Ziprasidone (Planned } \\
\text { dose: } 40 \text { Median dose: } \\
113 \text { (81-140), } 30\end{array}$ & $\begin{array}{l}\text { Placebo (Planned } \\
\text { dose: } 5 \mathrm{ml}), 36\end{array}$ & Oral & Ventricular arrhythmias & $0 / 30(0 \%)$ & $0 / 36(0 \%)$ & \\
\hline Girard, $2018^{29}$ & $\mathrm{RCT}$ & $\begin{array}{l}\text { Patients over } 18 \text { years of age in } \\
\text { the medical or surgical ICU, with } \\
\text { delirium }\end{array}$ & $\begin{array}{l}\text { Ziprasidone (Mean } \\
\text { dose: } 20 \mathrm{mg} \text { Dose } \\
\text { range: } 5.0-40 \mathrm{mg}), 190\end{array}$ & $\begin{array}{l}\text { Placebo (Not } \\
\text { reported), } 184\end{array}$ & Intravenous & $\begin{array}{l}\text { Reasons for temporary holds: } \\
\text { Prolonged QTC }\end{array}$ & $\begin{array}{l}20 / 190 \\
(11 \%)\end{array}$ & $\begin{array}{l}10 / 184 \\
(5 \%)\end{array}$ & $\begin{array}{l}\text { RR } 1.94 \\
\text { (95\% Cl: } \\
0.93 \text { to } \\
4.03)\end{array}$ \\
\hline Girard, $2018^{29}$ & $\mathrm{RCT}$ & $\begin{array}{l}\text { Patients over } 18 \text { years of age in } \\
\text { the medical or surgical ICU, with } \\
\text { delirium }\end{array}$ & $\begin{array}{l}\text { Ziprasidone (Mean } \\
\text { dose: } 20 \mathrm{mg} \text { Dose } \\
\text { range: } 5.0-40 \mathrm{mg}), 192\end{array}$ & $\begin{array}{l}\text { Placebo (Not } \\
\text { reported), } 184\end{array}$ & Intravenous & $\begin{array}{l}\text { Reasons for permanent } \\
\text { discontinuation: Suspected } \\
\text { torsades des pointes }\end{array}$ & $0 / 192(0 \%)$ & $0 / 184(0 \%)$ & \\
\hline \multicolumn{10}{|c|}{$\begin{array}{l}\text { First-generation } \\
\text { antipsychotic vs. } \\
\text { second-generation } \\
\text { antipsychotic }\end{array}$} \\
\hline $\begin{array}{l}\text { Charoenporn, } \\
2018^{25}\end{array}$ & $\begin{array}{l}\text { Cohort w/ } \\
\text { comparison } \\
\text { group }\end{array}$ & Physically ill patients & $\begin{array}{l}\text { Quetiapine (Mean } \\
\text { dose: } 26.7 \text { Median } \\
\text { dose: } 25), 31\end{array}$ & $\begin{array}{l}\text { Haloperidol } \\
\text { (Mean dose: } 0.9 \\
\text { Median dose: } \\
0.5 \mathrm{mg} \text { ), } 11\end{array}$ & Oral & Arrhythmia & $0 / 31(0 \%)$ & $1 / 11(9.1 \%)$ & $\begin{array}{l}\mathrm{RR} 0.13 \\
\text { (95\% Cl: } \\
0.01 \text { to } \\
2.86 \text { ) }\end{array}$ \\
\hline $\begin{array}{l}\text { Charoenporn, } \\
2018^{25}\end{array}$ & $\begin{array}{l}\text { Cohort w/ } \\
\text { comparison } \\
\text { group }\end{array}$ & Physically ill patients & $\begin{array}{l}\text { Risperidone (Mean } \\
\text { dose: } 0.6 \text { Median } \\
\text { dose: } 0.5 \mathrm{mg}), 14\end{array}$ & $\begin{array}{l}\text { Haloperidol } \\
\text { (Mean dose: } 0.9 \\
\text { Median dose: } \\
0.5 \mathrm{mg} \text { ), } 11 \\
\end{array}$ & Oral & Arrhythmia & $1 / 14(7.1 \%)$ & $1 / 11(9.1 \%)$ & $\begin{array}{l}\text { RR 0.79 } \\
\text { (95\% Cl: } \\
0.06 \text { to } \\
11.20)\end{array}$ \\
\hline
\end{tabular}




\begin{tabular}{|c|c|c|c|c|c|c|c|c|c|}
\hline Author, year & $\begin{array}{l}\text { Study } \\
\text { design }\end{array}$ & Population & Intervention group, $\mathbf{n}$ & Control group, $\mathrm{n}$ & $\begin{array}{c}\text { Route of } \\
\text { administration }\end{array}$ & Outcome definition & $\begin{array}{l}\mathrm{n} / \mathrm{N}(\%) \\
\text { intervention } \\
\text { group }\end{array}$ & $\begin{array}{c}\mathrm{n} / \mathrm{N}(\%) \\
\text { control } \\
\text { group }\end{array}$ & $\begin{array}{c}\text { Relative } \\
\text { risk (95\% } \\
\text { Cl) }\end{array}$ \\
\hline $\begin{array}{l}\text { Charoenporn, } \\
2018^{25}\end{array}$ & $\begin{array}{l}\text { Cohort w/ } \\
\text { comparison } \\
\text { group }\end{array}$ & Physically ill patients & $\begin{array}{l}\text { Quetiapine (Mean } \\
\text { dose: } 26.7 \text { Median } \\
\text { dose: } 25), 31\end{array}$ & $\begin{array}{l}\text { Haloperidol } \\
\text { (Mean dose: } 0.9 \\
\text { Median dose: } \\
0.5 \mathrm{mg} \text { ), } 11 \\
\end{array}$ & Oral & Orthostatic hypotension & $1 / 31(3.3 \%)$ & $0 / 11(0 \%)$ & $\begin{array}{l}\text { RR } 1.13 \\
\text { (95\% Cl: } \\
0.05 \text { to } \\
25.76)\end{array}$ \\
\hline $\begin{array}{l}\text { Charoenporn, } \\
2018^{25}\end{array}$ & $\begin{array}{l}\text { Cohort w/ } \\
\text { comparison } \\
\text { group }\end{array}$ & Physically ill patients & $\begin{array}{l}\text { Risperidone (Mean } \\
\text { dose: } 0.6 \text { Median } \\
\text { dose: } 0.5 \mathrm{mg}), 14\end{array}$ & $\begin{array}{l}\text { Haloperidol } \\
\text { (Mean dose: } 0.9 \\
\text { Median dose: } \\
0.5 \mathrm{mg} \text { ), } 11 \\
\end{array}$ & Oral & Orthostatic hypotension & $0 / 14(0 \%)$ & $0 / 11(0 \%)$ & \\
\hline Girard, $2010^{4}$ & RCT & $\begin{array}{l}>18 \text { years MV medical and } \\
\text { surgical ICU patients }\end{array}$ & $\begin{array}{l}\text { Ziprasidone (Planned } \\
\text { dose: } 40 \text { Median dose: } \\
113 \text { (81-140), } 30\end{array}$ & $\begin{array}{l}\text { Haloperidol } \\
\text { (Planned dose: } 5 \\
\text { Median dose: } 15 \\
(10.8-17), 35\end{array}$ & Oral & $\begin{array}{l}\text { Prolongation in the QTC } \\
\text { interval prolongation of the } \\
\text { QTc }>500 \text { ms while receiving } \\
\text { study drug }\end{array}$ & $\begin{array}{l}5 / 30 \\
(16.7 \%)\end{array}$ & $2 / 35(5.7 \%)$ & $\begin{array}{l}\text { RR 2.92 } \\
(95 \% \mathrm{Cl}: \\
0.61 \text { to } \\
13.96)\end{array}$ \\
\hline Girard, $2010^{4}$ & RCT & $\begin{array}{l}>18 \text { years MV medical and } \\
\text { surgical ICU patients }\end{array}$ & $\begin{array}{l}\text { Ziprasidone (Planned } \\
\text { dose: } 40 \text { Median dose: } \\
113 \text { (81-140), } 30\end{array}$ & $\begin{array}{l}\text { Haloperidol } \\
\text { (Planned dose: } 5 \\
\text { Median dose: } 15 \\
(10.8-17), 35\end{array}$ & Oral & Ventricular arrhythmias & $0 / 30(0 \%)$ & $0 / 35(0 \%)$ & \\
\hline Girard, $2018^{29}$ & RCT & $\begin{array}{l}\text { Patients over } 18 \text { years of age in } \\
\text { the medical or surgical ICU, with } \\
\text { delirium }\end{array}$ & $\begin{array}{l}\text { Ziprasidone (Mean } \\
\text { dose: } 20 \mathrm{mg} \text { Dose } \\
\text { range: } 5.0-40 \mathrm{mg} \text { ), } 190\end{array}$ & $\begin{array}{l}\text { Haloperidol } \\
\text { (Mean dose: } \\
\text { 11mg Dose } \\
\text { range: } 2.5-20 \mathrm{mg} \text { ), } \\
192\end{array}$ & Intravenous & $\begin{array}{l}\text { Reasons for temporary holds: } \\
\text { Prolonged QTc }\end{array}$ & $\begin{array}{l}20 / 190 \\
(11 \%)\end{array}$ & $\begin{array}{l}13 / 192 \\
(7 \%)\end{array}$ & $\begin{array}{l}\text { RR 1.55 } \\
\text { (95\% Cl: } \\
0.80 \text { to } \\
3.03)\end{array}$ \\
\hline Girard, 2018 29 & RCT & $\begin{array}{l}\text { Patients over } 18 \text { years of age in } \\
\text { the medical or surgical ICU, with } \\
\text { delirium }\end{array}$ & $\begin{array}{l}\text { Ziprasidone (Mean } \\
\text { dose: } 20 \mathrm{mg} \text { Dose } \\
\text { range: } 5.0-40 \mathrm{mg}), 192\end{array}$ & $\begin{array}{l}\text { Haloperidol } \\
\text { (Mean dose: } \\
\text { 11mg Dose } \\
\text { range: } 2.5-20 \mathrm{mg} \text { ), } \\
184\end{array}$ & Intravenous & $\begin{array}{l}\text { Reasons for permanent } \\
\text { discontinuation: Suspected } \\
\text { torsades des pointes }\end{array}$ & $0 / 192(0 \%)$ & $0 / 184(0 \%)$ & \\
\hline Hatta, $2014^{33}$ & $\begin{array}{l}\text { Cohort w/ } \\
\text { comparison } \\
\text { group }\end{array}$ & $\begin{array}{l}\text { Patients in a general hospital } \\
\text { who developed delirium and } \\
\text { were managed by a psychiatrist }\end{array}$ & $\begin{array}{l}\text { Olanzapine (Max } \\
\text { dose: } 10.2 \mathrm{mg}), 87\end{array}$ & $\begin{array}{l}\text { Haloperidol (Max } \\
\text { dose: } 6.40 \mathrm{mg}) \\
480\end{array}$ & Not reported & $\begin{array}{l}\text { Cardiovascular events (see } \\
\text { below for details) }\end{array}$ & $0 / 87(0 \%)$ & $0 / 480(0 \%)$ & \\
\hline Hatta, $2014^{33}$ & $\begin{array}{l}\text { Cohort w/ } \\
\text { comparison } \\
\text { group }\end{array}$ & $\begin{array}{l}\text { Patients in a general hospital } \\
\text { who developed delirium and } \\
\text { were managed by a psychiatrist }\end{array}$ & $\begin{array}{l}\text { Quetiapine (Max dose: } \\
71.8 \mathrm{mg}), 779\end{array}$ & $\begin{array}{l}\text { Haloperidol (Max } \\
\text { dose: } 6.40 \mathrm{mg}) \\
480\end{array}$ & Not applicable & $\begin{array}{l}\text { Cardiovascular events (see } \\
\text { below for details) }\end{array}$ & $\begin{array}{l}1 / 779 \\
(0.1 \%)\end{array}$ & $0 / 480(0 \%)$ & $\begin{array}{l}\text { RR 1.85 } \\
\text { (95\% Cl: } \\
0.08 \text { to } \\
45.32) \\
\end{array}$ \\
\hline Hatta, $2014^{33}$ & $\begin{array}{l}\text { Cohort w/ } \\
\text { comparison } \\
\text { group }\end{array}$ & $\begin{array}{l}\text { Patients in a general hospital } \\
\text { who developed delirium and } \\
\text { were managed by a psychiatrist }\end{array}$ & $\begin{array}{l}\text { Risperidone (Max } \\
\text { dose: } 1.35 \mathrm{mg}), 835\end{array}$ & $\begin{array}{l}\text { Haloperidol (Max } \\
\text { dose: } 6.40 \mathrm{mg}) \\
480\end{array}$ & Not applicable & $\begin{array}{l}\text { Cardiovascular events (see } \\
\text { below for details) }\end{array}$ & $\begin{array}{l}1 / 835 \\
(0.1 \%)\end{array}$ & $0 / 480(0 \%)$ & $\begin{array}{l}\text { RR } 1.73 \\
\text { (95\% Cl: } \\
0.07 \text { to } \\
42.29)\end{array}$ \\
\hline
\end{tabular}




\begin{tabular}{|c|c|c|c|c|c|c|c|c|c|}
\hline Author, year & $\begin{array}{l}\text { Study } \\
\text { design }\end{array}$ & Population & Intervention group, $\mathbf{n}$ & Control group, $\mathbf{n}$ & $\begin{array}{c}\text { Route of } \\
\text { administration }\end{array}$ & Outcome definition & $\begin{array}{l}\mathrm{n} / \mathrm{N}(\%) \\
\text { intervention } \\
\text { group }\end{array}$ & $\begin{array}{c}\mathrm{n} / \mathrm{N}(\%) \\
\text { control } \\
\text { group }\end{array}$ & $\begin{array}{l}\text { Relative } \\
\text { risk (95\% } \\
\mathrm{Cl})\end{array}$ \\
\hline Hatta, $2014^{33}$ & $\begin{array}{l}\text { Cohort w/ } \\
\text { comparison } \\
\text { group }\end{array}$ & $\begin{array}{l}\text { Patients in a general hospital } \\
\text { who developed delirium and } \\
\text { were managed by a psychiatrist }\end{array}$ & $\begin{array}{l}\text { Risperidone (Max } \\
\text { dose: } 1.35 \mathrm{mg}), 835\end{array}$ & $\begin{array}{l}\text { Haloperidol (Max } \\
\text { dose: } 6.40 \mathrm{mg}) \\
480\end{array}$ & Not applicable & Venous thromboembolism & $0 / 835(0 \%)$ & $\begin{array}{l}1 / 480 \\
(0.2 \%)\end{array}$ & $\begin{array}{l}\text { RR } 0.19 \\
\text { (95\% Cl: } \\
0.01 \text { to } \\
4.70)\end{array}$ \\
\hline Hatta, 201433 & $\begin{array}{l}\text { Cohort wl } \\
\text { comparison } \\
\text { group }\end{array}$ & $\begin{array}{l}\text { Patients in a general hospital } \\
\text { who developed delirium and } \\
\text { were managed by a psychiatrist }\end{array}$ & $\begin{array}{l}\text { Olanzapine (Max } \\
\text { dose: } 10.2 \mathrm{mg}), 87\end{array}$ & $\begin{array}{l}\text { Haloperidol (Max } \\
\text { dose: } 6.40 \mathrm{mg}) \\
480\end{array}$ & Not reported & Venous thromboembolism & $0 / 87(0 \%)$ & $\begin{array}{l}1 / 480 \\
(0.2 \%)\end{array}$ & $\begin{array}{l}\text { RR } 1.82 \\
\text { (95\% Cl: } \\
0.07 \text { to } \\
44.36)\end{array}$ \\
\hline Hatta, $2014^{33}$ & $\begin{array}{l}\text { Cohort w/ } \\
\text { comparison } \\
\text { group }\end{array}$ & $\begin{array}{l}\text { Patients in a general hospital } \\
\text { who developed delirium and } \\
\text { were managed by a psychiatrist }\end{array}$ & $\begin{array}{l}\text { Quetiapine (Max dose: } \\
71.8 \mathrm{mg}), 779\end{array}$ & $\begin{array}{l}\text { Haloperidol (Max } \\
\text { dose: } \\
6.40 \mathrm{mg}), 480\end{array}$ & Not applicable & Venous thromboembolism & $0 / 779(0 \%)$ & $\begin{array}{l}1 / 480 \\
(0.2 \%)\end{array}$ & $\begin{array}{l}\text { RR 0.21 } \\
\text { (95\% Cl: } \\
0.01 \text { to } \\
5.04)\end{array}$ \\
\hline Hatta, 201433 & $\begin{array}{l}\text { Cohort w/ } \\
\text { comparison } \\
\text { group }\end{array}$ & $\begin{array}{l}\text { Patients in a general hospital } \\
\text { who developed delirium and } \\
\text { were managed by a psychiatrist }\end{array}$ & $\begin{array}{l}\text { Aripiprazole (Max } \\
\text { dose: } 7.23 \mathrm{mg}), 61\end{array}$ & $\begin{array}{l}\text { Haloperidol (Max } \\
\text { dose: } 6.40 \mathrm{mg}) \\
480\end{array}$ & Not reported & $\begin{array}{l}\text { Cardiovascular events (see } \\
\text { below for details) }\end{array}$ & $0 / 61(0 \%)$ & $0 / 480(0 \%)$ & \\
\hline Hatta, $2014^{33}$ & $\begin{array}{l}\text { Cohort wl } \\
\text { comparison } \\
\text { group }\end{array}$ & $\begin{array}{l}\text { Patients in a general hospital } \\
\text { who developed delirium and } \\
\text { were managed by a psychiatrist }\end{array}$ & $\begin{array}{l}\text { Aripiprazole (Max } \\
\text { dose: } 7.23 \mathrm{mg}), 61\end{array}$ & $\begin{array}{l}\text { Haloperidol (Max } \\
\text { dose: } 6.40 \mathrm{mg}) \\
480\end{array}$ & Not reported & Venous thromboembolism & $0 / 61(0 \%)$ & $\begin{array}{l}1 / 480 \\
(0.2 \%)\end{array}$ & $\begin{array}{l}\text { RR 2.59 } \\
\text { (95\% Cl: } \\
0.11 \text { to } \\
62.79) \\
\end{array}$ \\
\hline Lim, $2007^{46}$ & $\mathrm{RCT}$ & $\begin{array}{l}\text { Patients from internal medicine, } \\
\text { PMR, neurology, neurosurgery, } \\
\text { orthopedic surgery, or general } \\
\text { surgery that were referred to the } \\
\text { psychiatric department }\end{array}$ & $\begin{array}{l}\text { Olanzapine (Titrated } \\
\text { (5 to } 20 \mathrm{mg} \text { ) }\end{array}$ & $\begin{array}{l}\text { Haloperidol } \\
\text { (Titrated (5 to } 20 \\
\mathrm{mg} \text { ) }\end{array}$ & Intramuscular & Ventricular tachycardia & $0 / 31(0 \%)$ & $0 / 31(0 \%)$ & $\mathrm{p}=\mathrm{NS}$ \\
\hline Lim, $2007^{46}$ & RCT & $\begin{array}{l}\text { Patients from internal medicine, } \\
\text { PMR, neurology, neurosurgery, } \\
\text { orthopedic surgery, or general } \\
\text { surgery that were referred to the } \\
\text { psychiatric department }\end{array}$ & $\begin{array}{l}\text { Olanzapine (Titrated } \\
\text { (5 to } 20 \mathrm{mg} \text { ) }\end{array}$ & $\begin{array}{l}\text { Haloperidol } \\
\text { (Titrated (5 to } 20 \\
\mathrm{mg} \text { ) }\end{array}$ & Intramuscular & QT prolongation & 0 / $31(0 \%)$ & $0 / 31(0 \%)$ & $\mathrm{p}=\mathrm{NS}$ \\
\hline Lim, $2007^{46}$ & RCT & $\begin{array}{l}\text { Patients from internal medicine, } \\
\text { PMR, neurology, neurosurgery, } \\
\text { orthopedic surgery, or general } \\
\text { surgery that were referred to the } \\
\text { psychiatric department }\end{array}$ & $\begin{array}{l}\text { Olanzapine (Titrated } \\
\text { (5 to } 20 \mathrm{mg} \text { ) }\end{array}$ & $\begin{array}{l}\text { Haloperidol } \\
\text { (Titrated (5 to } 20 \\
\text { mg) }\end{array}$ & Intramuscular & $\begin{array}{l}\text { Other serious cardiovascular } \\
\text { complications }\end{array}$ & $0 / 31(0 \%)$ & 0 / $31(0 \%)$ & $p=N S$ \\
\hline
\end{tabular}




\begin{tabular}{|c|c|c|c|c|c|c|c|c|c|}
\hline Author, year & $\begin{array}{l}\text { Study } \\
\text { design }\end{array}$ & Population & Intervention group, $\mathbf{n}$ & Control group, $\mathbf{n}$ & $\begin{array}{c}\text { Route of } \\
\text { administration }\end{array}$ & Outcome definition & $\begin{array}{c}\mathrm{n} / \mathrm{N}(\%) \\
\text { intervention } \\
\text { group }\end{array}$ & $\begin{array}{c}\text { n I N (\%), } \\
\text { control } \\
\text { group }\end{array}$ & $\begin{array}{c}\text { Relative } \\
\text { risk (95\% } \\
\mathrm{Cl})\end{array}$ \\
\hline Maneeton, $2013^{49}$ & RCT & $\begin{array}{l}\text { General adult population (age } \\
\text { 18-75) with hyperactive delirium, } \\
\text { referred to psychiatry consult } \\
\text { liaison service at tertiary care } \\
\text { hospital in Thailand }\end{array}$ & $\begin{array}{l}\text { Quetiapine (Mean } \\
\text { dose: } 67.6 \text { (SD 9.7) } \\
\text { Dose range: } 25- \\
\text { 100mg), } 24\end{array}$ & $\begin{array}{l}\text { Haloperidol } \\
\text { (Mean dose: } 0.8 \\
\text { (SD 0.3) Dose } \\
\text { range: } 0.5- \\
2.0 \mathrm{mg} \text { ), } 28\end{array}$ & Oral & Withdrawal due to AV block & $0 / 24(0 \%)$ & $1 / 28(3.6 \%)$ & $\begin{array}{l}\text { RR } 0.39 \\
(95 \% \mathrm{Cl}: \\
0.02 \text { to } \\
9.07)\end{array}$ \\
\hline \multicolumn{10}{|l|}{$\begin{array}{l}\text { Second-generation } \\
\text { antipsychotic vs. } \\
\text { second-generation } \\
\text { antipsychotic } \\
\end{array}$} \\
\hline $\begin{array}{l}\text { Charoenporn, } \\
2018^{25}\end{array}$ & $\begin{array}{l}\text { Cohort w/ } \\
\text { comparison } \\
\text { group }\end{array}$ & Physically ill patients & $\begin{array}{l}\text { Risperidone (Mean } \\
\text { dose: } 0.6 \text { Median } \\
\text { dose: } 0.5 \mathrm{mg}), 14\end{array}$ & $\begin{array}{l}\text { Quetiapine (Mean } \\
\text { dose: } 26.7 \\
\text { Median dose: } 25) \text {, } \\
31\end{array}$ & Oral & Arrhythmia & $1 / 14(7.1 \%)$ & $0 / 31(0 \%)$ & $\begin{array}{l}\text { RR 6.40 } \\
\text { (95\% Cl: } \\
0.28 \text { to } \\
148.06) \\
\end{array}$ \\
\hline $\begin{array}{l}\text { Charoenporn, } \\
2018^{25}\end{array}$ & $\begin{array}{l}\text { Cohort w/ } \\
\text { comparison } \\
\text { group }\end{array}$ & Physically ill patients & $\begin{array}{l}\text { Risperidone (Mean } \\
\text { dose: } 0.6 \text { Median } \\
\text { dose: } 0.5 \mathrm{mg}), 14\end{array}$ & $\begin{array}{l}\text { Quetiapine (Mean } \\
\text { dose: } 26.7 \\
\text { Median dose: } 25) \text {, } \\
31\end{array}$ & Oral & Orthostatic hypotension & $0 / 14(0 \%)$ & $1 / 31(3.3 \%)$ & $\begin{array}{l}\text { RR 0.71 } \\
\text { (95\% Cl: } \\
0.03 \text { to } \\
16.45)\end{array}$ \\
\hline Fox, $2018^{28}$ & $\begin{array}{l}\text { Cohort w/ } \\
\text { comparison } \\
\text { group }\end{array}$ & $\begin{array}{l}\text { Critically ill patients in the } \\
\text { medical and cardiovascular ICU }\end{array}$ & $\begin{array}{l}\text { Quetiapine (Mean } \\
\text { dose: } 153.8 \mathrm{Max} \\
\text { dose: } 800 \mathrm{mg} \text { ), } 20\end{array}$ & $\begin{array}{l}\text { Lurasidone } \\
\text { (Mean dose: } 42.6 \\
\text { Max dose: } \\
\text { 120mg), } 20\end{array}$ & Not reported & $\begin{array}{l}\text { Life-threatening arrhythmia or } \\
\text { torsades de pointes }\end{array}$ & $0 / 20(0 \%)$ & $0 / 20(0 \%)$ & \\
\hline Fox, $2018^{28}$ & $\begin{array}{l}\text { Cohort w/ } \\
\text { comparison } \\
\text { group }\end{array}$ & $\begin{array}{l}\text { Critically ill patients in the } \\
\text { medical and cardiovascular ICU }\end{array}$ & $\begin{array}{l}\text { Quetiapine (Mean } \\
\text { dose: } 153.8 \mathrm{Max} \\
\text { dose: } 800 \mathrm{mg} \text { ), } 20\end{array}$ & $\begin{array}{l}\text { Lurasidone } \\
\text { (Mean dose: } 42.6 \\
\text { Max dose: } \\
\text { 120mg), } 20\end{array}$ & Not reported & $\begin{array}{l}\text { Prolongation in the QTc } \\
\text { interval change from baseline } \\
\text { QTc interval of }>60 \\
\text { milliseconds }\end{array}$ & $2 / 20(10 \%)$ & $2 / 20(10 \%)$ & $\begin{array}{l}\text { RR } 1.00 \\
\text { (95\% Cl: } \\
0.16 \text { to } \\
6.42)\end{array}$ \\
\hline Fox, $2018^{28}$ & $\begin{array}{l}\text { Cohort w/ } \\
\text { comparison } \\
\text { group }\end{array}$ & $\begin{array}{l}\text { Critically ill patients in the } \\
\text { medical and cardiovascular ICU }\end{array}$ & $\begin{array}{l}\text { Quetiapine (Mean } \\
\text { dose: } 153.8 \mathrm{Max} \\
\text { dose: } 800 \mathrm{mg} \text { ), } 20\end{array}$ & $\begin{array}{l}\text { Lurasidone } \\
\text { (Mean dose: } 42.6 \\
\text { Max dose: } \\
\text { 120mg), } 20\end{array}$ & Not reported & $\begin{array}{l}\text { Prolongation in the QTc } \\
\text { interval discontinuation of } \\
\text { study drug due to an increase } \\
\text { in the QTc interval }\end{array}$ & $1 / 20(5 \%)$ & $0 / 20(0 \%)$ & $\begin{array}{l}\text { RR } 3.00 \\
(95 \% \mathrm{Cl}: \\
0.13 \text { to } \\
69.52)\end{array}$ \\
\hline
\end{tabular}




\begin{tabular}{|c|c|c|c|c|c|c|c|c|c|}
\hline Author, year & $\begin{array}{l}\text { Study } \\
\text { design }\end{array}$ & Population & Intervention group, $\mathbf{n}$ & Control group, $n$ & $\begin{array}{c}\text { Route of } \\
\text { administration }\end{array}$ & Outcome definition & $\begin{array}{c}\mathrm{n} / \mathrm{N}(\%), \\
\text { intervention } \\
\text { group }\end{array}$ & $\begin{array}{c}\mathrm{n} / \mathrm{N}(\%), \\
\text { control } \\
\text { group }\end{array}$ & $\begin{array}{l}\text { Relative } \\
\text { risk (95\% } \\
\text { Cl) }\end{array}$ \\
\hline Hatta, 201433 & $\begin{array}{l}\text { Cohort w/ } \\
\text { comparison } \\
\text { group }\end{array}$ & $\begin{array}{l}\text { Patients in a general hospital } \\
\text { who developed delirium and } \\
\text { were managed by a psychiatrist }\end{array}$ & $\begin{array}{l}\text { Olanzapine (Max } \\
\text { dose: } 10.2 \mathrm{mg}), 87\end{array}$ & $\begin{array}{l}\text { Aripiprazole (Max } \\
\text { dose: } 7.23 \mathrm{mg}) \\
61\end{array}$ & Not reported & $\begin{array}{l}\text { Cardiovascular events (see } \\
\text { below for details) }\end{array}$ & $0 / 87(0 \%)$ & $0 / 61(0 \%)$ & \\
\hline Hatta, 2014 33 & $\begin{array}{l}\text { Cohort w/ } \\
\text { comparison } \\
\text { group }\end{array}$ & $\begin{array}{l}\text { Patients in a general hospital } \\
\text { who developed delirium and } \\
\text { were managed by a psychiatrist }\end{array}$ & $\begin{array}{l}\text { Quetiapine (Max dose: } \\
71.8 \mathrm{mg}), 779\end{array}$ & $\begin{array}{l}\text { Aripiprazole (Max } \\
\text { dose: } 7.23 \mathrm{mg}) \\
61\end{array}$ & Not applicable & $\begin{array}{l}\text { Cardiovascular events (see } \\
\text { below for details) }\end{array}$ & $\begin{array}{l}1 / 779 \\
(0.1 \%)\end{array}$ & $0 / 61(0 \%)$ & $\begin{array}{l}\text { RR } 0.24 \\
\text { (95\% Cl: } \\
0.01 \text { to } \\
5.79 \text { ) }\end{array}$ \\
\hline Hatta, $2014^{33}$ & $\begin{array}{l}\text { Cohort w/ } \\
\text { comparison } \\
\text { group }\end{array}$ & $\begin{array}{l}\text { Patients in a general hospital } \\
\text { who developed delirium and } \\
\text { were managed by a psychiatrist }\end{array}$ & $\begin{array}{l}\text { Quetiapine (Max dose: } \\
71.8 \mathrm{mg}), 779\end{array}$ & $\begin{array}{l}\text { Olanzapine (Max } \\
\text { dose: } 10.2 \mathrm{mg}) \\
87\end{array}$ & Not applicable & $\begin{array}{l}\text { Cardiovascular events (see } \\
\text { below for details) }\end{array}$ & $\begin{array}{l}1 / 779 \\
(0.1 \%)\end{array}$ & $0 / 87(0 \%)$ & $\begin{array}{l}\text { RR } 0.34 \\
\text { (95\% Cl: } \\
0.01 \text { to } \\
8.25 \text { ) }\end{array}$ \\
\hline Hatta, 201433 & $\begin{array}{l}\text { Cohort w/ } \\
\text { comparison } \\
\text { group }\end{array}$ & $\begin{array}{l}\text { Patients in a general hospital } \\
\text { who developed delirium and } \\
\text { were managed by a psychiatrist }\end{array}$ & $\begin{array}{l}\text { Risperidone (Max } \\
\text { dose: } 1.35 \mathrm{mg}), 835\end{array}$ & $\begin{array}{l}\text { Aripiprazole (Max } \\
\text { dose: } 7.23 \mathrm{mg}) \\
61\end{array}$ & Not applicable & $\begin{array}{l}\text { Cardiovascular events (see } \\
\text { below for details) }\end{array}$ & $\begin{array}{l}1 / 835 \\
(0.1 \%)\end{array}$ & $0 / 61(0 \%)$ & $\begin{array}{l}\text { RR } 0.22 \\
\text { (95\% Cl: } \\
0.01 \text { to } \\
5.40 \text { ) }\end{array}$ \\
\hline Hatta, 2014 33 & $\begin{array}{l}\text { Cohort w/ } \\
\text { comparison } \\
\text { group }\end{array}$ & $\begin{array}{l}\text { Patients in a general hospital } \\
\text { who developed delirium and } \\
\text { were managed by a psychiatrist }\end{array}$ & $\begin{array}{l}\text { Risperidone (Max } \\
\text { dose: } 1.35 \mathrm{mg}), 835\end{array}$ & $\begin{array}{l}\text { Olanzapine (Max } \\
\text { dose: } 10.2 \mathrm{mg}) \\
87\end{array}$ & Not applicable & $\begin{array}{l}\text { Cardiovascular events (see } \\
\text { below for details) }\end{array}$ & $\begin{array}{l}1 / 835 \\
(0.1 \%)\end{array}$ & $0 / 87(0 \%)$ & $\begin{array}{l}\text { RR } 0.32 \\
\text { (95\% Cl: } \\
0.01 \text { to } \\
7.69 \text { ) }\end{array}$ \\
\hline
\end{tabular}




\begin{tabular}{|c|c|c|c|c|c|c|c|c|c|}
\hline Author, year & $\begin{array}{l}\text { Study } \\
\text { design }\end{array}$ & Population & Intervention group, $\mathbf{n}$ & Control group, $\mathbf{n}$ & $\begin{array}{c}\text { Route of } \\
\text { administration }\end{array}$ & Outcome definition & $\begin{array}{l}\mathrm{n} / \mathrm{N}(\%) \\
\text { intervention } \\
\text { group }\end{array}$ & $\begin{array}{c}\text { n I N (\%), } \\
\text { control } \\
\text { group }\end{array}$ & $\begin{array}{c}\text { Relative } \\
\text { risk (95\% } \\
\text { Cl) }\end{array}$ \\
\hline Hatta, $2014^{33}$ & $\begin{array}{l}\text { Cohort w/ } \\
\text { comparison } \\
\text { group }\end{array}$ & $\begin{array}{l}\text { Patients in a general hospital } \\
\text { who developed delirium and } \\
\text { were managed by a psychiatrist }\end{array}$ & $\begin{array}{l}\text { Risperidone (Max } \\
\text { dose: } 1.35 \mathrm{mg}), 835\end{array}$ & $\begin{array}{l}\text { Quetiapine (Max } \\
\text { dose: } 71.8 \mathrm{mg}) \\
779\end{array}$ & Not applicable & $\begin{array}{l}\text { Cardiovascular events (see } \\
\text { below for details) }\end{array}$ & $\begin{array}{l}1 / 835 \\
(0.1 \%)\end{array}$ & $\begin{array}{l}1 / 779 \\
(0.1 \%)\end{array}$ & $\begin{array}{l}\text { RR } 0.93 \\
\text { (95\% Cl: } \\
0.06 \text { to } \\
14.89)\end{array}$ \\
\hline Hatta, 201433 & $\begin{array}{l}\text { Cohort w/ } \\
\text { comparison } \\
\text { group }\end{array}$ & $\begin{array}{l}\text { Patients in a general hospital } \\
\text { who developed delirium and } \\
\text { were managed by a psychiatrist }\end{array}$ & $\begin{array}{l}\text { Risperidone (Max } \\
\text { dose: } 1.35 \mathrm{mg}), 835\end{array}$ & $\begin{array}{l}\text { Quetiapine (Max } \\
\text { dose: } 71.8 \mathrm{mg}) \\
779\end{array}$ & Not applicable & Venous thromboembolism & $0 / 835(0 \%)$ & $0 / 779(0 \%)$ & \\
\hline Hatta, $2014^{33}$ & $\begin{array}{l}\text { Cohort w/ } \\
\text { comparison } \\
\text { group }\end{array}$ & $\begin{array}{l}\text { Patients in a general hospital } \\
\text { who developed delirium and } \\
\text { were managed by a psychiatrist }\end{array}$ & $\begin{array}{l}\text { Quetiapine (Max dose: } \\
71.8 \mathrm{mg}), 779\end{array}$ & $\begin{array}{l}\text { Olanzapine (Max } \\
\text { dose: } 10.2 \mathrm{mg}) \\
87\end{array}$ & Not applicable & Venous thromboembolism & $0 / 779(0 \%)$ & $0 / 87(0 \%)$ & \\
\hline Hatta, $2014^{33}$ & $\begin{array}{l}\text { Cohort w/ } \\
\text { comparison } \\
\text { group }\end{array}$ & $\begin{array}{l}\text { Patients in a general hospital } \\
\text { who developed delirium and } \\
\text { were managed by a psychiatrist }\end{array}$ & $\begin{array}{l}\text { Olanzapine (Max } \\
\text { dose: } 10.2 \mathrm{mg}), 87\end{array}$ & $\begin{array}{l}\text { Aripiprazole (Max } \\
\text { dose: } 7.23 \mathrm{mg}) \\
61\end{array}$ & Not reported & Venous thromboembolism & $0 / 87(0 \%)$ & $0 / 61(0 \%)$ & \\
\hline Hatta, $2014^{33}$ & $\begin{array}{l}\text { Cohort w/ } \\
\text { comparison } \\
\text { group }\end{array}$ & $\begin{array}{l}\text { Patients in a general hospital } \\
\text { who developed delirium and } \\
\text { were managed by a psychiatrist }\end{array}$ & $\begin{array}{l}\text { Quetiapine (Max dose: } \\
71.8 \mathrm{mg}), 779\end{array}$ & $\begin{array}{l}\text { Aripiprazole (Max } \\
\text { dose: } 7.23 \mathrm{mg}) \\
61\end{array}$ & Not applicable & Venous thromboembolism & $0 / 779(0 \%)$ & $0 / 61(0 \%)$ & \\
\hline Hatta, $2014^{33}$ & $\begin{array}{l}\text { Cohort wl } \\
\text { comparison } \\
\text { group }\end{array}$ & $\begin{array}{l}\text { Patients in a general hospital } \\
\text { who developed delirium and } \\
\text { were managed by a psychiatrist }\end{array}$ & $\begin{array}{l}\text { Risperidone (Max } \\
\text { dose: } 1.35 \mathrm{mg}), 835\end{array}$ & $\begin{array}{l}\text { Aripiprazole (Max } \\
\text { dose: } 7.23 \mathrm{mg}) \\
61\end{array}$ & Not applicable & Venous thromboembolism & $0 / 835(0 \%)$ & $0 / 61(0 \%)$ & \\
\hline Hatta, $2014^{33}$ & $\begin{array}{l}\text { Cohort w/ } \\
\text { comparison } \\
\text { group }\end{array}$ & $\begin{array}{l}\text { Patients in a general hospital } \\
\text { who developed delirium and } \\
\text { were managed by a psychiatrist }\end{array}$ & $\begin{array}{l}\text { Risperidone (Max } \\
\text { dose: } 1.35 \mathrm{mg}), 835\end{array}$ & $\begin{array}{l}\text { Olanzapine (Max } \\
\text { dose: } 10.2 \mathrm{mg}) \\
87\end{array}$ & Not applicable & Venous thromboembolism & $0 / 835(0 \%)$ & $0 / 87(0 \%)$ & \\
\hline
\end{tabular}




\begin{tabular}{|c|c|c|c|c|c|c|c|c|c|}
\hline Author, year & $\begin{array}{l}\text { Study } \\
\text { design }\end{array}$ & Population & Intervention group, $\mathbf{n}$ & Control group, $\mathbf{n}$ & $\begin{array}{c}\text { Route of } \\
\text { administratio } \\
n\end{array}$ & Outcome definition & $\begin{array}{c}\mathrm{n} / \mathrm{N}(\%) \\
\text { intervention } \\
\text { group }\end{array}$ & $\begin{array}{c}\text { n I N (\%), } \\
\text { control } \\
\text { group }\end{array}$ & $\begin{array}{c}\text { Relative } \\
\text { risk (95\% } \\
\text { Cl) }\end{array}$ \\
\hline \multicolumn{10}{|l|}{$\begin{array}{l}\text { First-generation } \\
\text { antipsychotic vs. } \\
\text { other }\end{array}$} \\
\hline Bakri, 2015 & RCT & $\begin{array}{l}\text { ICU trauma patients without } \\
\text { severe injury, comatose, or } \\
\text { moribund patients were } \\
\text { excluded }\end{array}$ & $\begin{array}{l}\text { Haloperidol (Planned } \\
\text { dose: } 5 \mathrm{mg} \text { ), } 32\end{array}$ & $\begin{array}{l}\text { Dexmedetomidin } \\
\text { e (Planned dose: } \\
1 \text { ug } / \mathrm{kg}), 32\end{array}$ & Intravenous & Hypotension & $0 / 32(0 \%)$ & $0 / 32(0 \%)$ & \\
\hline Bakri, $2015^{19}$ & RCT & $\begin{array}{l}\text { ICU trauma patients without } \\
\text { severe injury, comatose, or } \\
\text { moribund patients were } \\
\text { excluded }\end{array}$ & $\begin{array}{l}\text { Haloperidol (Planned } \\
\text { dose: } 5 \mathrm{mg} \text { ), } 32\end{array}$ & $\begin{array}{l}\text { Dexmedetomidin } \\
\text { e (Planned dose: } \\
1 \text { ug } / \mathrm{kg}), 32\end{array}$ & Intravenous & Incidence of bradycardia & $0 / 32(0 \%)$ & $0 / 32(0 \%)$ & \\
\hline Bakri, $2015^{19}$ & RCT & $\begin{array}{l}\text { ICU trauma patients without } \\
\text { severe injury, comatose, or } \\
\text { moribund patients were } \\
\text { excluded }\end{array}$ & $\begin{array}{l}\text { Haloperidol (Planned } \\
\text { dose: } 5 \mathrm{mg} \text { ), } 32\end{array}$ & $\begin{array}{l}\text { Dexmedetomidin } \\
\text { e (Planned dose: } \\
1 \text { ug } / \mathrm{kg}), 32\end{array}$ & Intravenous & $\begin{array}{l}\text { Prolongation in the QTc } \\
\text { interval prolonged QTc } \\
\text { interval }\end{array}$ & $0 / 32(0 \%)$ & $0 / 32(0 \%)$ & \\
\hline Bakri, $2015^{19}$ & RCT & $\begin{array}{l}\text { ICU trauma patients without } \\
\text { severe injury, comatose, or } \\
\text { moribund patients were } \\
\text { excluded }\end{array}$ & $\begin{array}{l}\text { Ondansetron (Planned } \\
\text { dose: } 8 \mathrm{mg} \text { ), } 32\end{array}$ & $\begin{array}{l}\text { Haloperidol } \\
\text { (Planned dose: } 5 \\
\text { mg), } 32\end{array}$ & Intravenous & Hypotension & $0 / 32(0 \%)$ & $0 / 32(0 \%)$ & \\
\hline Bakri, $2015^{19}$ & RCT & $\begin{array}{l}\text { ICU trauma patients without } \\
\text { severe injury, comatose, or } \\
\text { moribund patients were } \\
\text { excluded }\end{array}$ & $\begin{array}{l}\text { Ondansetron (Planned } \\
\text { dose: } 8 \mathrm{mg} \text { ), } 32\end{array}$ & $\begin{array}{l}\text { Haloperidol } \\
\text { (Planned dose: } 5 \\
\text { mg), } 32\end{array}$ & Intravenous & Incidence of bradycardia & $0 / 32(0 \%)$ & 0 / $32(0 \%)$ & \\
\hline Bakri, $2015^{19}$ & RCT & $\begin{array}{l}\text { ICU trauma patients without } \\
\text { severe injury, comatose, or } \\
\text { moribund patients were } \\
\text { excluded }\end{array}$ & $\begin{array}{l}\text { Ondansetron (Planned } \\
\text { dose: } 8 \mathrm{mg} \text { ), }\end{array}$ & $\begin{array}{l}\text { Haloperidol } \\
\text { (Planned dose: } 5 \\
\text { mg), }\end{array}$ & Intravenous & $\begin{array}{l}\text { Prolongation in the QTC } \\
\text { interval prolonged QTc } \\
\text { interval }\end{array}$ & $0 / 32(0 \%)$ & $0 / 32(0 \%)$ & \\
\hline
\end{tabular}

CI=confidence interval; ICU=intensive care unit; Mg=milligram; Ml=milliliter; MV=mechanical ventilation; $\mathrm{N}=$ sample size; NG=nadogastric tube; QTc=corrected QT interval; RCT=randomized controlled trial; RR=risk ratio 
Evidence Table D-48. Binary cardiac outcomes for studies comparing interventions to treat delirium: single arm studies

\begin{tabular}{|c|c|c|c|c|c|c|c|}
\hline Author, year & $\begin{array}{c}\text { Study } \\
\text { design }\end{array}$ & Population & Control group, $\mathbf{n}$ & $\begin{array}{l}\text { Route of } \\
\text { administra } \\
\text { tion }\end{array}$ & Outcome definition & $\begin{array}{l}\text { n I N (\%), control } \\
\text { group }\end{array}$ & $\begin{array}{l}\text { Measure of } \\
\text { association }\end{array}$ \\
\hline Kim, $2003^{40}$ & $\begin{array}{l}\text { Cohort w/o } \\
\text { comparison } \\
\text { group }\end{array}$ & $\begin{array}{l}\text { Patients in the acute } \\
\text { medical units }\end{array}$ & $\begin{array}{l}\text { Quetiapine (Planned dose: } 25 \text { Mean } \\
\text { dose: } 93.75 \mathrm{mg} \text { ), } 12\end{array}$ & $\begin{array}{l}\text { Not } \\
\text { reported }\end{array}$ & Fatal acute myocardial infarction & $1 / 12(0.00 \%)$ & \\
\hline Maneeton, $2007^{48}$ & $\begin{array}{l}\text { Open-label } \\
\text { study }\end{array}$ & $\begin{array}{l}\text { All physically ill in-patients } \\
\text { whose pcp consulted } \\
\text { psychiatrists }\end{array}$ & $\begin{array}{l}\text { Quetiapine (Mean dose: } 45.7(28.7) \\
\text { Max dose: } 100 \text { Dose range: } 25- \\
\text { 100mg), } 17\end{array}$ & Oral & Hypotension & 2/17(11.76\%) & \\
\hline Mittal, $2004^{50}$ & $\begin{array}{l}\text { Cohort w/o } \\
\text { comparison } \\
\text { group }\end{array}$ & Hospitalized patients & Risperidone (Mean dose: $0.75 \mathrm{mg}$ ) & $\begin{array}{l}\text { Not } \\
\text { reported }\end{array}$ & QTc interval, ms & $\begin{array}{l}\text { Baseline: Mean } \\
\text { 436 (SE 10) } \\
\text { Final: } 432 \text { (SE 15) } \\
\end{array}$ & $\begin{array}{l}\text { Change from } \\
\text { baseline: }-4\end{array}$ \\
\hline Mittal, $2004^{50}$ & $\begin{array}{l}\text { Cohort w/o } \\
\text { comparison } \\
\text { group }\end{array}$ & Hospitalized patients & $\begin{array}{l}\text { Risperidone (Mean dose: } 0.75 \mathrm{mg} \text { ), } \\
10\end{array}$ & $\begin{array}{l}\text { Not } \\
\text { reported }\end{array}$ & $\begin{array}{l}\text { Treatment discontinued because of severe, } \\
\text { aggressively treated congestive heart failure, } \\
\text { bradycardia, and worsening of hypotension }\end{array}$ & $1 / 10(8.33 \%)$ & \\
\hline Omura, $2003^{52}$ & $\begin{array}{l}\text { Cohort w/o } \\
\text { comparison } \\
\text { group }\end{array}$ & Hospitalized patients & $\begin{array}{l}\text { Quetiapine (Mean dose: } 54.7 \text { Dose } \\
\text { range: } 25 \text { to } 125 \mathrm{mg} \text { ), } 24\end{array}$ & $\begin{array}{l}\text { Not } \\
\text { reported }\end{array}$ & Hypotension & 0/24(0.00\%) & \\
\hline Straker, $2006^{58}$ & $\begin{array}{l}\text { Cohort w/o } \\
\text { comparison } \\
\text { group }\end{array}$ & $\begin{array}{l}\text { Medically ill delirium } \\
\text { patients }\end{array}$ & $\begin{array}{l}\text { Aripiprazole (Mean dose: 8.9 Dose } \\
\text { range: } 5 \text { to } 15 \mathrm{mg}), 14\end{array}$ & $\begin{array}{l}\text { Not } \\
\text { reported }\end{array}$ & Cardiac arrest & 1/14(7.14\%) & \\
\hline Straker, $2006^{58}$ & $\begin{array}{l}\text { Cohort w/o } \\
\text { comparison } \\
\text { group }\end{array}$ & $\begin{array}{l}\text { Medically ill delirium } \\
\text { patients }\end{array}$ & $\begin{array}{l}\text { Aripiprazole (Mean dose: 8.9 Dose } \\
\text { range: } 5 \text { to } 15 \mathrm{mg} \text { ), } 14\end{array}$ & $\begin{array}{l}\text { Not } \\
\text { reported }\end{array}$ & Ventricular arrhythmias & 0/14(0.00\%) & \\
\hline Straker, $2006^{58}$ & $\begin{array}{l}\text { Cohort w/o } \\
\text { comparison } \\
\text { group }\end{array}$ & $\begin{array}{l}\text { Medically ill delirium } \\
\text { patients }\end{array}$ & $\begin{array}{l}\text { Aripiprazole (Mean dose: } 8.9 \text { Dose } \\
\text { range: } 5 \text { to } 15 \mathrm{mg} \text { ) }\end{array}$ & $\begin{array}{l}\text { Not } \\
\text { reported }\end{array}$ & Prolongation in the qtc interval milliseconds & $\begin{array}{l}\text { Baseline: Mean } \\
442 \text { (SD 44) } \\
\text { Final: } 434 \text { (SD 22) }\end{array}$ & $\begin{array}{l}\text { Change from } \\
\text { baseline: }-8\end{array}$ \\
\hline Straker, $2006^{58}$ & $\begin{array}{l}\text { Cohort w/o } \\
\text { comparison } \\
\text { group }\end{array}$ & $\begin{array}{l}\text { Medically ill delirium } \\
\text { patients }\end{array}$ & $\begin{array}{l}\text { Aripiprazole (Mean dose: } 8.9 \text { Dose } \\
\text { range: } 5 \text { to } 15 \mathrm{mg} \text { ), } 14\end{array}$ & $\begin{array}{l}\text { Not } \\
\text { reported }\end{array}$ & Prolongation in the qtc interval increase & $3 / 14(0.00 \%)$ & \\
\hline
\end{tabular}

Mg=milligram; N=sample size; QTc=corrected QT interval; SD=standard deviation; SE=standard error 
Evidence Table D-49. Continuous cardiac outcomes for studies comparing interventions to treat delirium

\begin{tabular}{|c|c|c|c|c|c|c|c|c|c|}
\hline Author, year & Study design & Population & $\begin{array}{c}\text { Intervention group, } \\
\mathrm{n}\end{array}$ & Control group, $\mathrm{n}$ & $\begin{array}{c}\text { Route of } \\
\text { administration }\end{array}$ & $\begin{array}{l}\text { Outcome } \\
\text { definition }\end{array}$ & $\begin{array}{c}\text { MMSE, } \\
\text { intervention } \\
\text { group }\end{array}$ & $\begin{array}{l}\text { MMSE, control } \\
\text { group }\end{array}$ & $\begin{array}{l}\text { Mean } \\
\text { between- } \\
\text { group } \\
\text { difference } \\
(95 \% \mathrm{Cl}) \\
\end{array}$ \\
\hline \multicolumn{10}{|l|}{$\begin{array}{l}\text { First-generation } \\
\text { antipsychotic vs. } \\
\text { placebo }\end{array}$} \\
\hline Naksuk, $2017^{51}$ & $\begin{array}{l}\text { Case control } \\
\text { analyzed as cohort } \\
\text { with comparison } \\
\text { group }\end{array}$ & $\begin{array}{l}\text { Patients } \\
\text { admitted to the } \\
\text { coronary care } \\
\text { unit }\end{array}$ & $\begin{array}{l}\text { Haloperidol (Median } \\
\text { dose: } 5.0 \text { Dose } \\
\text { range: } 1.0 \text { to } \\
57.5 \mathrm{mg}), 124\end{array}$ & Not Reported, 681 & Not reported & $\begin{array}{l}\text { Prolongation } \\
\text { in the QTc } \\
\text { interval } \\
\text { milliseconds }\end{array}$ & $\begin{array}{l}\text { Baseline: Mean } \\
448 \text { (SD 56) } \\
\text { Final: Mean } 471 \\
\text { (SD 52) }\end{array}$ & $\begin{array}{l}\text { Baseline: Mean } \\
459 \text { (SD 60) } \\
\text { Final: Mean (SD ) }\end{array}$ & \\
\hline Naksuk, $2017^{51}$ & $\begin{array}{l}\text { Case control } \\
\text { analyzed as cohort } \\
\text { with comparison } \\
\text { group }\end{array}$ & $\begin{array}{l}\text { Patients } \\
\text { admitted to the } \\
\text { coronary care } \\
\text { unit }\end{array}$ & $\begin{array}{l}\text { Haloperidol (Median } \\
\text { dose: } 5.0 \text { Dose } \\
\text { range: } 1.0 \text { to } \\
57.5 \mathrm{mg}), 124\end{array}$ & Not Reported, 681 & Not reported & $\begin{array}{l}\text { Prolongation } \\
\text { in the QTC } \\
\text { interval } \\
\text { milliseconds }\end{array}$ & $\begin{array}{l}\text { Baseline: Mean } \\
448 \text { (SD 56) } \\
\text { Final: Mean } 426 \\
\text { (SD 64) }\end{array}$ & $\begin{array}{l}\text { Baseline: Mean } \\
459 \text { (SD 60) } \\
\text { Final: Mean (SD ) }\end{array}$ & \\
\hline Naksuk, $2017^{51}$ & $\begin{array}{l}\text { Case control } \\
\text { analyzed as cohort } \\
\text { with comparison } \\
\text { group }\end{array}$ & $\begin{array}{l}\text { Patients } \\
\text { admitted to the } \\
\text { coronary care } \\
\text { unit }\end{array}$ & $\begin{array}{l}\text { Haloperidol (Median } \\
\text { dose: } 5.0 \text { Dose } \\
\text { range: } 1.0 \text { to } \\
57.5 \mathrm{mg}), 124\end{array}$ & Not Reported, 681 & Not reported & $\begin{array}{l}\text { Prolongation } \\
\text { in the QTC } \\
\text { interval } \\
\text { milliseconds }\end{array}$ & $\begin{array}{l}\text { Baseline: Mean } \\
448 \text { (SD 56) } \\
\text { Final: Mean } 442 \\
\text { (SD 34) }\end{array}$ & $\begin{array}{l}\text { Baseline: Mean } \\
459 \text { (SD 60) } \\
\text { Final: Mean } 458 \\
\text { (SD 44) }\end{array}$ & $\begin{array}{l}-5.00(95 \% \\
\text { Cl: }-194.06 \text { to } \\
184.06)\end{array}$ \\
\hline Naksuk, $2017^{51}$ & $\begin{array}{l}\text { Case control } \\
\text { analyzed as cohort } \\
\text { with comparison } \\
\text { group }\end{array}$ & $\begin{array}{l}\text { Patients } \\
\text { admitted to the } \\
\text { coronary care } \\
\text { unit }\end{array}$ & $\begin{array}{l}\text { Haloperidol (Median } \\
\text { dose: } 5.0 \text { Dose } \\
\text { range: } 1.0 \text { to } \\
57.5 \mathrm{mg}), 124\end{array}$ & Not Reported, 681 & Not reported & $\begin{array}{l}\text { Prolongation } \\
\text { in the QTC } \\
\text { interval } \\
\text { milliseconds }\end{array}$ & $\begin{array}{l}\text { Baseline: Mean } \\
448 \text { (SD 56) } \\
\text { Final: Mean } 458 \\
\text { (SD 57) }\end{array}$ & $\begin{array}{l}\text { Baseline: Mean } \\
459 \text { (SD 60) } \\
\text { Final: Mean (SD ) }\end{array}$ & \\
\hline Naksuk, $2017^{51}$ & $\begin{array}{l}\text { Case control } \\
\text { analyzed as cohort } \\
\text { with comparison } \\
\text { group }\end{array}$ & $\begin{array}{l}\text { Patients } \\
\text { admitted to the } \\
\text { coronary care } \\
\text { unit }\end{array}$ & $\begin{array}{l}\text { Haloperidol (Median } \\
\text { dose: } 5.0 \text { Dose } \\
\text { range: } 1.0 \text { to } \\
57.5 \mathrm{mg}), 124\end{array}$ & Not Reported, 681 & Not reported & $\begin{array}{l}\text { Prolongation } \\
\text { in the QTc } \\
\text { interval } \\
\text { milliseconds }\end{array}$ & $\begin{array}{l}\text { Baseline: Mean } \\
448 \text { (SD 56) } \\
\text { Final: Mean } 454 \\
\text { (SD 49) }\end{array}$ & $\begin{array}{l}\text { Baseline: Mean } \\
459 \text { (SD 60) } \\
\text { Final: Mean (SD ) }\end{array}$ & \\
\hline
\end{tabular}




\begin{tabular}{|c|c|c|c|c|c|c|c|c|c|}
\hline Author, year & Study design & Population & $\begin{array}{c}\text { Intervention group, } \\
\mathrm{n}\end{array}$ & Control group, $\mathbf{n}$ & $\begin{array}{c}\text { Route of } \\
\text { administration }\end{array}$ & $\begin{array}{l}\text { Outcome } \\
\text { definition }\end{array}$ & $\begin{array}{c}\text { MMSE, } \\
\text { intervention } \\
\text { group }\end{array}$ & $\begin{array}{l}\text { MMSE, control } \\
\text { group }\end{array}$ & $\begin{array}{c}\text { Mean } \\
\text { between- } \\
\text { group } \\
\text { difference } \\
(95 \% \mathrm{Cl}) \\
\end{array}$ \\
\hline \multicolumn{10}{|l|}{$\begin{array}{l}\text { Second-generation } \\
\text { antipsychotic vs. } \\
\text { placebo }\end{array}$} \\
\hline Naksuk, $2017^{51}$ & $\begin{array}{l}\text { Case control } \\
\text { analyzed as cohort } \\
\text { with comparison } \\
\text { group }\end{array}$ & $\begin{array}{l}\text { Patients } \\
\text { admitted to the } \\
\text { coronary care } \\
\text { unit }\end{array}$ & $\begin{array}{l}\text { Quetiapine (Median } \\
\text { dose: } 25.0 \text { Dose } \\
\text { range: } 4.2 \text { to } \\
\text { 150.0mg), } 59\end{array}$ & Not Reported, 681 & Not reported & $\begin{array}{l}\text { Prolongation } \\
\text { in the QTC } \\
\text { interval } \\
\text { milliseconds }\end{array}$ & $\begin{array}{l}\text { Baseline: Mean } \\
470 \text { (SD 66) } \\
\text { Final: Mean } 455 \\
\text { (SD 53) }\end{array}$ & $\begin{array}{l}\text { Baseline: Mean } \\
459 \text { (SD 60) } \\
\text { Final: Mean (SD ) }\end{array}$ & \\
\hline Naksuk, $2017^{51}$ & $\begin{array}{l}\text { Case control } \\
\text { analyzed as cohort } \\
\text { with comparison } \\
\text { group }\end{array}$ & $\begin{array}{l}\text { Patients } \\
\text { admitted to the } \\
\text { coronary care } \\
\text { unit } \\
\end{array}$ & $\begin{array}{l}\text { Quetiapine (Median } \\
\text { dose: } 25.0 \text { Dose } \\
\text { range: } 4.2 \text { to } \\
150.0 \mathrm{mg}), 59\end{array}$ & Not Reported, 681 & Not reported & $\begin{array}{l}\text { Prolongation } \\
\text { in the QTC } \\
\text { interval } \\
\text { milliseconds }\end{array}$ & $\begin{array}{l}\text { Baseline: Mean } \\
470 \text { (SD 66) } \\
\text { Final: Mean } 451 \\
\text { (SD 51) } \\
\end{array}$ & $\begin{array}{l}\text { Baseline: Mean } \\
459 \text { (SD 60) } \\
\text { Final: Mean (SD ) }\end{array}$ & \\
\hline Naksuk, $2017^{51}$ & $\begin{array}{l}\text { Case control } \\
\text { analyzed as cohort } \\
\text { with comparison } \\
\text { group }\end{array}$ & $\begin{array}{l}\text { Patients } \\
\text { admitted to the } \\
\text { coronary care } \\
\text { unit }\end{array}$ & $\begin{array}{l}\text { Quetiapine (Median } \\
\text { dose: } 25.0 \text { Dose } \\
\text { range: } 4.2 \text { to } \\
\text { 150.0mg), } 59\end{array}$ & Not Reported, 681 & Not reported & $\begin{array}{l}\text { Prolongation } \\
\text { in the QTc } \\
\text { interval } \\
\text { milliseconds }\end{array}$ & $\begin{array}{l}\text { Baseline: Mean } \\
470 \text { (SD 66) } \\
\text { Final: Mean } 454 \\
\text { (SD 51) }\end{array}$ & $\begin{array}{l}\text { Baseline: Mean } \\
459 \text { (SD 60) } \\
\text { Final: Mean } 458 \\
\text { (SD 44) }\end{array}$ & $\begin{array}{l}-15.00(95 \% \\
\mathrm{Cl}:-201.67 \text { to } \\
171.67)\end{array}$ \\
\hline Naksuk, $2017^{51}$ & $\begin{array}{l}\text { Case control } \\
\text { analyzed as cohort } \\
\text { with comparison } \\
\text { group }\end{array}$ & $\begin{array}{l}\text { Patients } \\
\text { admitted to the } \\
\text { coronary care } \\
\text { unit }\end{array}$ & $\begin{array}{l}\text { Quetiapine (Median } \\
\text { dose: } 25.0 \text { Dose } \\
\text { range: } 4.2 \text { to } \\
\text { 150.0mg), } 59\end{array}$ & Not Reported, 681 & Not reported & $\begin{array}{l}\text { Prolongation } \\
\text { in the QTC } \\
\text { interval } \\
\text { milliseconds }\end{array}$ & $\begin{array}{l}\text { Baseline: Mean } \\
470 \text { (SD 66) } \\
\text { Final: Mean } 467 \\
\text { (SD 68) } \\
\end{array}$ & $\begin{array}{l}\text { Baseline: Mean } \\
459 \text { (SD 60) } \\
\text { Final: Mean (SD ) }\end{array}$ & \\
\hline Naksuk, $2017^{51}$ & $\begin{array}{l}\text { Case control } \\
\text { analyzed as cohort } \\
\text { with comparison } \\
\text { group }\end{array}$ & $\begin{array}{l}\text { Patients } \\
\text { admitted to the } \\
\text { coronary care } \\
\text { unit } \\
\end{array}$ & $\begin{array}{l}\text { Quetiapine (Median } \\
\text { dose: } 25.0 \text { Dose } \\
\text { range: } 4.2 \text { to } \\
150.0 \mathrm{mg}), 59\end{array}$ & Not Reported, 681 & Not reported & $\begin{array}{l}\text { Prolongation } \\
\text { in the QTC } \\
\text { interval } \\
\text { milliseconds }\end{array}$ & $\begin{array}{l}\text { Baseline: Mean } \\
470 \text { (SD } 66) \\
\text { Final: } 457 \text { ( } 41)\end{array}$ & $\begin{array}{l}\text { Baseline: Mean } \\
459 \text { (SD 60) } \\
\text { Final: ( ) }\end{array}$ & \\
\hline
\end{tabular}




\begin{tabular}{|c|c|c|c|c|c|c|c|c|c|}
\hline Author, year & Study design & Population & $\begin{array}{c}\text { Intervention group, } \\
\mathrm{n}\end{array}$ & Control group, $n$ & $\begin{array}{c}\text { Route of } \\
\text { administration }\end{array}$ & $\begin{array}{l}\text { Outcome } \\
\text { definition }\end{array}$ & $\begin{array}{c}\text { MMSE, } \\
\text { intervention } \\
\text { group }\end{array}$ & $\begin{array}{l}\text { MMSE, control } \\
\text { group }\end{array}$ & $\begin{array}{c}\text { Mean } \\
\text { between- } \\
\text { group } \\
\text { difference } \\
(95 \% \mathrm{Cl}) \\
\end{array}$ \\
\hline \multicolumn{10}{|l|}{$\begin{array}{l}\text { First-generation } \\
\text { antipsychotic vs. } \\
\text { second-generation } \\
\text { antipsychotic }\end{array}$} \\
\hline Naksuk, $2017^{51}$ & $\begin{array}{l}\text { Case control } \\
\text { analyzed as cohort } \\
\text { with comparison } \\
\text { group }\end{array}$ & $\begin{array}{l}\text { Patients } \\
\text { admitted to the } \\
\text { coronary care } \\
\text { unit }\end{array}$ & $\begin{array}{l}\text { Quetiapine (Median } \\
\text { dose: } 25.0 \text { Dose } \\
\text { range: } 4.2 \text { to } \\
\text { 150.0mg) } 59\end{array}$ & $\begin{array}{l}\text { Haloperidol (Median } \\
\text { dose: } 5.0 \text { Dose } \\
\text { range: } 1.0 \text { to } \\
57.5 \mathrm{mg}) .124\end{array}$ & $\begin{array}{l}\text { Median dose: } 25.0 \\
\text { Dose range: } 4.2 \text { to } \\
\text { 150.0mg }\end{array}$ & $\begin{array}{l}24 \text { hours after } \\
\text { last dose }\end{array}$ & $\begin{array}{l}\text { Baseline: Mean } \\
470 \text { (SD 66) } \\
\text { Final: Mean } 455 \\
\text { (SD 53) }\end{array}$ & $\begin{array}{l}\text { Baseline: Mean } \\
448 \text { (SD 56) } \\
\text { Final: Mean } 471 \\
\text { (SD 52) }\end{array}$ & $\begin{array}{l}-38.00(95 \% \\
\mathrm{Cl}:-268.46 \text { to } \\
192.46)\end{array}$ \\
\hline Naksuk, $2017^{51}$ & $\begin{array}{l}\text { Case control } \\
\text { analyzed as cohort } \\
\text { with comparison } \\
\text { group }\end{array}$ & $\begin{array}{l}\text { Patients } \\
\text { admitted to the } \\
\text { coronary care } \\
\text { unit }\end{array}$ & $\begin{array}{l}\text { Quetiapine (Median } \\
\text { dose: } 25.0 \text { Dose } \\
\text { range: } 4.2 \text { to } \\
\text { 150.0mg), } 59\end{array}$ & $\begin{array}{l}\text { Haloperidol (Median } \\
\text { dose: } 5.0 \text { Dose } \\
\text { range: } 1.0 \text { to } \\
57.5 \mathrm{mg}), 124\end{array}$ & $\begin{array}{l}\text { Median dose: } 25.0 \\
\text { Dose range: } 4.2 \text { to } \\
\text { 150.0mg }\end{array}$ & $\begin{array}{l}48 \text { hours after } \\
\text { last dose }\end{array}$ & $\begin{array}{l}\text { Baseline: Mean } \\
470 \text { (SD 66) } \\
\text { Final: Mean } 451 \\
\text { (SD 51) }\end{array}$ & $\begin{array}{l}\text { Baseline: Mean } \\
448 \text { (SD 56) } \\
\text { Final: Mean } 426 \\
\text { (SD 64) }\end{array}$ & $\begin{array}{l}3.00(95 \% \mathrm{Cl}: \\
-242.80 \text { to } \\
248.80)\end{array}$ \\
\hline Naksuk, $2017^{51}$ & $\begin{array}{l}\text { Case control } \\
\text { analyzed as cohort } \\
\text { with comparison } \\
\text { group }\end{array}$ & $\begin{array}{l}\text { Patients } \\
\text { admitted to the } \\
\text { coronary care } \\
\text { unit }\end{array}$ & $\begin{array}{l}\text { Quetiapine (Median } \\
\text { dose: } 25.0 \text { Dose } \\
\text { range: } 4.2 \text { to } \\
150.0 \mathrm{mg}), 59\end{array}$ & $\begin{array}{l}\text { Haloperidol (Median } \\
\text { dose: } 5.0 \text { Dose } \\
\text { range: } 1.0 \text { to } \\
57.5 \mathrm{mg}), 124\end{array}$ & $\begin{array}{l}\text { Median dose: } 25.0 \\
\text { Dose range: } 4.2 \text { to } \\
\text { 150.0mg }\end{array}$ & $\begin{array}{l}\text { During } \\
\text { admission } \\
\text { (mean QTC } \\
\text { intervals } \\
\text { during CCU } \\
\text { admission, } \\
\text { excluding } \\
\text { baseline) }\end{array}$ & $\begin{array}{l}\text { Baseline: Mean } \\
470 \text { (SD 66) } \\
\text { Final: Mean } 454 \\
\text { (SD 51) }\end{array}$ & $\begin{array}{l}\text { Baseline: Mean } \\
448 \text { (SD 56) } \\
\text { Final: Mean } 442 \\
\text { (SD 34) }\end{array}$ & $\begin{array}{l}-10.00(95 \% \\
\text { Cl: }-216.57 \text { to } \\
196.57)\end{array}$ \\
\hline Naksuk, $2017^{51}$ & $\begin{array}{l}\text { Case control } \\
\text { analyzed as cohort } \\
\text { with comparison } \\
\text { group }\end{array}$ & $\begin{array}{l}\text { Patients } \\
\text { admitted to the } \\
\text { coronary care } \\
\text { unit }\end{array}$ & $\begin{array}{l}\text { Quetiapine (Median } \\
\text { dose: } 25.0 \text { Dose } \\
\text { range: } 4.2 \text { to } \\
\text { 150.0mg), } 59\end{array}$ & $\begin{array}{l}\text { Haloperidol (Median } \\
\text { dose: } 5.0 \text { Dose } \\
\text { range: } 1.0 \text { to } \\
57.5 \mathrm{mg} \text { ), } 124\end{array}$ & $\begin{array}{l}\text { Median dose: } 25.0 \\
\text { Dose range: } 4.2 \text { to } \\
\text { 150.0mg }\end{array}$ & $\begin{array}{l}\text { During therapy } \\
\text { within } 6 \text { hours } \\
\text { after each } \\
\text { dose }\end{array}$ & $\begin{array}{l}\text { Baseline: Mean } \\
470 \text { (SD 66) } \\
\text { Final: Mean } 467 \\
\text { (SD 68) }\end{array}$ & $\begin{array}{l}\text { Baseline: Mean } \\
448 \text { (SD 56) } \\
\text { Final: Mean } 458 \\
\text { (SD 57) }\end{array}$ & $\begin{array}{l}-13.00(95 \% \\
\mathrm{Cl}:-260.81 \text { to } \\
234.81)\end{array}$ \\
\hline Naksuk, $2017^{51}$ & $\begin{array}{l}\text { Case control } \\
\text { analyzed as cohort } \\
\text { with comparison } \\
\text { group }\end{array}$ & $\begin{array}{l}\text { Patients } \\
\text { admitted to the } \\
\text { coronary care } \\
\text { unit }\end{array}$ & $\begin{array}{l}\text { Quetiapine (Median } \\
\text { dose: } 25.0 \text { Dose } \\
\text { range: } 4.2 \text { to } \\
\text { 150.0mg), } 59\end{array}$ & $\begin{array}{l}\text { Haloperidol (Median } \\
\text { dose: } 5.0 \text { Dose } \\
\text { range: } 1.0 \text { to } \\
57.5 \mathrm{mg}), 124\end{array}$ & $\begin{array}{l}\text { Median dose: } 25.0 \\
\text { Dose range: } 4.2 \text { to } \\
\text { 150.0mg }\end{array}$ & $\begin{array}{l}\text { During therapy } \\
\text { within } 6 \text { hours } \\
\text { after third } \\
\text { dose }\end{array}$ & $\begin{array}{l}\text { Baseline: Mean } \\
470 \text { (SD 66) } \\
\text { Final: Mean } 457 \\
\text { (SD 41) }\end{array}$ & $\begin{array}{l}\text { Baseline: Mean } \\
448 \text { (SD 56) } \\
\text { Final: Mean } 454 \\
\text { (SD 49) }\end{array}$ & $\begin{array}{l}-19.00(95 \% \\
\text { Cl: }-237.62 \text { to } \\
199.62)\end{array}$ \\
\hline
\end{tabular}


Evidence Table D-50. Binary neurological outcomes for studies comparing interventions to treat delirium

\begin{tabular}{|c|c|c|c|c|c|c|c|c|c|}
\hline Author, year & $\begin{array}{l}\text { Study } \\
\text { design }\end{array}$ & Population & Intervention group, $\mathbf{n}$ & Control group, $\mathrm{n}$ & $\begin{array}{c}\text { Route of } \\
\text { administration }\end{array}$ & Outcome definition & $\begin{array}{c}\mathrm{n} / \mathrm{N}(\%) \\
\text { intervention } \\
\text { group }\end{array}$ & $\begin{array}{c}\text { n I N (\%), } \\
\text { control } \\
\text { group }\end{array}$ & $\begin{array}{l}\text { Relative risk } \\
(95 \% \mathrm{Cl})\end{array}$ \\
\hline \multicolumn{10}{|l|}{$\begin{array}{l}\text { First-generation } \\
\text { antipsychotic vs. } \\
\text { placebo }\end{array}$} \\
\hline Girard, $2010^{4}$ & RCT & $\begin{array}{l}>18 \text { years MV medical and surgical ICU } \\
\text { patients }\end{array}$ & $\begin{array}{l}\text { Haloperidol (Planned } \\
\text { dose: } 5 \text { Median dose: } 15 \\
\text { (10.8-17), } 35\end{array}$ & $\begin{array}{l}\text { Placebo (Planned } \\
\text { dose: } 5 \mathrm{ml}), 36\end{array}$ & Oral & Akathisia & $\begin{array}{l}10 / 35 \\
(29 \%)\end{array}$ & $\begin{array}{l}7 / 36 \\
(19 \%)\end{array}$ & $\begin{array}{l}\text { RR 1.47 } \\
\text { (95\% Cl: } 0.63 \\
\text { to 3.43) }\end{array}$ \\
\hline Girard, $2010^{4}$ & $\mathrm{RCT}$ & $\begin{array}{l}>18 \text { years MV medical and surgical ICU } \\
\text { patients }\end{array}$ & $\begin{array}{l}\text { Haloperidol (Planned } \\
\text { dose: } 5 \text { Median dose: } 15 \\
\text { (10.8-17), } 35\end{array}$ & $\begin{array}{l}\text { Placebo (Planned } \\
\text { dose: } 5 \mathrm{ml}), 36\end{array}$ & Oral & $\begin{array}{l}\text { Extrapyramidal } \\
\text { symptoms }\end{array}$ & $4 / 35(11 \%)$ & $\begin{array}{l}6 / 36 \\
(17 \%)\end{array}$ & $\begin{array}{l}\text { RR 0.69 } \\
\text { (95\% Cl: } 0.21 \\
\text { to 2.22) }\end{array}$ \\
\hline Girard, $2010^{4}$ & RCT & $\begin{array}{l}>18 \text { years MV medical and surgical ICU } \\
\text { patients }\end{array}$ & $\begin{array}{l}\text { Haloperidol (Planned } \\
\text { dose: } 5 \text { Median dose: } 15 \\
\text { (10.8-17), } 35\end{array}$ & $\begin{array}{l}\text { Placebo (Planned } \\
\text { dose: } 5 \mathrm{ml}), 36\end{array}$ & Oral & $\begin{array}{l}\text { Neuroleptic } \\
\text { malignant syndrome }\end{array}$ & $0 / 35(0 \%)$ & $\begin{array}{l}0 / 36 \\
(0 \%)\end{array}$ & \\
\hline Girard, $2018^{29}$ & $\mathrm{RCT}$ & $\begin{array}{l}\text { Patients over } 18 \text { years of age in the } \\
\text { medical or surgical ICU, with delirium }\end{array}$ & $\begin{array}{l}\text { Haloperidol (Mean dose: } \\
11 \mathrm{mg} \text { Dose range: } 2.5- \\
20 \mathrm{mg}), 192\end{array}$ & $\begin{array}{l}\text { Placebo (Not } \\
\text { reported), } 184\end{array}$ & Intravenous & $\begin{array}{l}\text { Extrapyramidal } \\
\text { symptoms or } \\
\text { Dystonia }\end{array}$ & $2 / 192(1 \%)$ & $\begin{array}{l}1 / 184 \\
(1 \%)\end{array}$ & $\begin{array}{l}\text { RR 1.92 } \\
\text { (95\% Cl: } 0.18 \\
\text { to 20.96) }\end{array}$ \\
\hline Girard, $2018^{29}$ & $\mathrm{RCT}$ & $\begin{array}{l}\text { Patients over } 18 \text { years of age in the } \\
\text { medical or surgical ICU, with delirium }\end{array}$ & $\begin{array}{l}\text { Haloperidol (Mean dose: } \\
11 \mathrm{mg} \text { Dose range: } 2.5- \\
\text { 20mg), } 192\end{array}$ & $\begin{array}{l}\text { Placebo (Not } \\
\text { reported), } 184\end{array}$ & Intravenous & $\begin{array}{l}\text { Reasons for } \\
\text { temporary holds: } \\
\text { Extrapyramidal } \\
\text { symptoms }\end{array}$ & $0 / 192(0 \%)$ & $\begin{array}{l}0 / 184 \\
(0 \%)\end{array}$ & \\
\hline Girard, $2018^{29}$ & RCT & $\begin{array}{l}\text { Patients over } 18 \text { years of age in the } \\
\text { medical or surgical ICU, with delirium }\end{array}$ & $\begin{array}{l}\text { Haloperidol (Mean dose: } \\
11 \mathrm{mg} \text { Dose range: } 2.5- \\
\text { 20mg), } 192\end{array}$ & $\begin{array}{l}\text { Placebo (Not } \\
\text { reported), } 184\end{array}$ & Intravenous & $\begin{array}{l}\text { Reasons for } \\
\text { temporary holds: } \\
\text { Dystonia }\end{array}$ & $0 / 192(0 \%)$ & $\begin{array}{l}0 / 184 \\
(0 \%)\end{array}$ & \\
\hline Girard, $2018^{29}$ & RCT & $\begin{array}{l}\text { Patients over } 18 \text { years of age in the } \\
\text { medical or surgical ICU, with delirium }\end{array}$ & $\begin{array}{l}\text { Haloperidol (Mean dose: } \\
11 \mathrm{mg} \text { Dose range: } 2.5- \\
20 \mathrm{mg}), 192\end{array}$ & $\begin{array}{l}\text { Placebo (Not } \\
\text { reported), } 184\end{array}$ & Intravenous & $\begin{array}{l}\text { Reasons for } \\
\text { permanent } \\
\text { discontinuation: } \\
\text { Suspected } \\
\text { neuroleptic } \\
\text { malignant syndrome }\end{array}$ & $0 / 192(0 \%)$ & $\begin{array}{l}0 / 184 \\
(0 \%)\end{array}$ & \\
\hline Page, $2013^{10}$ & RCT & $\begin{array}{l}\text { ICU patients needing MV within } 72 \\
\text { hours of admission were enrolled }\end{array}$ & $\begin{array}{l}\text { Haloperidol (Planned } \\
\text { dose: } 2.5 \mathrm{mg} \text { ), } 71\end{array}$ & $\begin{array}{l}\text { Placebo (Planned } \\
\text { dose: } 0.5 \mathrm{ml}), 70\end{array}$ & Intravenous & Akathisia & $1 / 71(1 \%)$ & $\begin{array}{l}2 / 70 \\
(3 \%)\end{array}$ & $\begin{array}{l}\text { RR } 0.49 \\
\text { (95\% Cl: } 0.05 \\
\text { to 5.31) }\end{array}$ \\
\hline Page, $2013^{10}$ & $\mathrm{RCT}$ & $\begin{array}{l}\text { ICU patients needing MV within } 72 \\
\text { hours of admission were enrolled }\end{array}$ & $\begin{array}{l}\text { Haloperidol (Planned } \\
\text { dose: } 2.5 \mathrm{mg} \text { ), } 71\end{array}$ & $\begin{array}{l}\text { Placebo (Planned } \\
\text { dose: } 0.5 \mathrm{ml}), 70\end{array}$ & Intravenous & Muscle Stiffness & $1 / 71(1 \%)$ & $\begin{array}{l}1 / 70 \\
(1.4 \%)\end{array}$ & $\begin{array}{l}\text { RR } 0.99 \\
\text { (95\% Cl: } 0.06 \\
\text { to 15.45) }\end{array}$ \\
\hline Page, $2013^{10}$ & $\mathrm{RCT}$ & $\begin{array}{l}\text { ICU patients needing MV within } 72 \\
\text { hours of admission were enrolled }\end{array}$ & $\begin{array}{l}\text { Haloperidol (Planned } \\
\text { dose: } 2.5 \mathrm{mg}), 71\end{array}$ & $\begin{array}{l}\text { Placebo (Planned } \\
\text { dose: } 0.5 \mathrm{ml}), 70\end{array}$ & Intravenous & Torticollis & $0 / 71(0 \%)$ & $\begin{array}{l}1 / 70 \\
(1.4 \%)\end{array}$ & $\begin{array}{l}\text { RR } 0.33 \\
\text { (95\% Cl: } 0.01 \\
\text { to } 7.93 \text { ) }\end{array}$ \\
\hline
\end{tabular}




\begin{tabular}{|c|c|c|c|c|c|c|c|c|c|}
\hline Author, year & $\begin{array}{l}\text { Study } \\
\text { design }\end{array}$ & Population & Intervention group, $\mathbf{n}$ & Control group, $\mathbf{n}$ & $\begin{array}{c}\text { Route of } \\
\text { administration }\end{array}$ & Outcome definition & $\begin{array}{l}\mathrm{n} / \mathrm{N}(\%) \\
\text { intervention } \\
\text { group }\end{array}$ & $\begin{array}{c}\text { n I N (\%), } \\
\text { control } \\
\text { group }\end{array}$ & $\begin{array}{l}\text { Relative risk } \\
\quad(95 \% \mathrm{Cl})\end{array}$ \\
\hline \multicolumn{10}{|c|}{$\begin{array}{l}\text { Second-generation } \\
\text { antipsychotic vs. } \\
\text { placebo }\end{array}$} \\
\hline Devlin, $2010^{26}$ & RCT & $\begin{array}{l}\text { Patients admitted to Medical \& Surgical } \\
\text { ICU with delirium. }\end{array}$ & $\begin{array}{l}\text { Quetiapine (Mean dose: } \\
\text { 50 Median dose: } 110 \\
\text { (88-191 IQR) Max dose: } \\
\text { 200 Dose range: } 50 \text { to } \\
\text { 200mg) }\end{array}$ & $\begin{array}{l}\text { Placebo (Mean } \\
\text { dose: } 50 \text { Median } \\
\text { dose: } 210 \text { (116 - } \\
\text { 293) Max dose: } \\
\text { 200mg) }\end{array}$ & $\begin{array}{l}\text { Either orally or } \\
\text { NG tube }\end{array}$ & $\begin{array}{l}\text { Extrapyramidal } \\
\text { symptoms }\end{array}$ & $\begin{array}{l}\text { Number of } \\
\text { events: } 0\end{array}$ & $\begin{array}{l}\text { Number } \\
\text { of events: } \\
0\end{array}$ & $p=1$ \\
\hline Girard, $2010^{4}$ & RCT & $\begin{array}{l}>18 \text { years MV medical and surgical ICU } \\
\text { patients }\end{array}$ & $\begin{array}{l}\text { Ziprasidone (Planned } \\
\text { dose: } 40 \text { Median dose: } \\
113 \text { (81-140), } 30\end{array}$ & $\begin{array}{l}\text { Placebo (Planned } \\
\text { dose: } 5 \mathrm{ml}), 36\end{array}$ & Oral & Akathisia & $6 / 30(20 \%)$ & $\begin{array}{l}7 / 36 \\
(19 \%)\end{array}$ & $\begin{array}{l}\text { RR 1.03 } \\
\text { (95\% Cl: } 0.39 \\
\text { to 2.73) } \\
\end{array}$ \\
\hline Girard, $2010^{4}$ & RCT & $\begin{array}{l}>18 \text { years MV medical and surgical ICU } \\
\text { patients }\end{array}$ & $\begin{array}{l}\text { Ziprasidone (Planned } \\
\text { dose: } 40 \text { Median dose: } \\
113 \text { (81-140), } 30\end{array}$ & $\begin{array}{l}\text { Placebo (Planned } \\
\text { dose: } 5 \mathrm{ml}), 36\end{array}$ & Oral & $\begin{array}{l}\text { Extrapyramidal } \\
\text { symptoms }\end{array}$ & $2 / 30(7 \%)$ & $\begin{array}{l}6 / 36 \\
(17 \%)\end{array}$ & $\begin{array}{l}\text { RR } 0.40 \\
\text { (95\% Cl: } 0.09 \\
\text { to } 1.84)\end{array}$ \\
\hline Girard, $2010^{4}$ & RCT & $\begin{array}{l}>18 \text { years MV medical and surgical ICU } \\
\text { patients }\end{array}$ & $\begin{array}{l}\text { Ziprasidone (Planned } \\
\text { dose: } 40 \text { Median dose: } \\
113 \text { (81-140), } 30\end{array}$ & $\begin{array}{l}\text { Placebo (Planned } \\
\text { dose: } 5 \mathrm{ml}), 36\end{array}$ & Oral & $\begin{array}{l}\text { Neuroleptic } \\
\text { malignant syndrome }\end{array}$ & $0 / 30(0 \%)$ & $\begin{array}{l}0 / 36 \\
(0 \%)\end{array}$ & \\
\hline Girard, $2018^{29}$ & RCT & $\begin{array}{l}\text { Patients over } 18 \text { years of age in the } \\
\text { medical or surgical ICU, with delirium }\end{array}$ & $\begin{array}{l}\text { Ziprasidone (Mean dose: } \\
\text { 20mg Dose range: } 5.0- \\
\text { 40mg), } 190\end{array}$ & $\begin{array}{l}\text { Placebo (Not } \\
\text { reported), } 184\end{array}$ & Intravenous & $\begin{array}{l}\text { Extrapyramidal } \\
\text { symptoms or } \\
\text { Dystonia }\end{array}$ & $1 / 190(1 \%)$ & $\begin{array}{l}1 / 184 \\
(1 \%)\end{array}$ & $\begin{array}{l}\text { RR } 0.97 \\
\text { (95\% Cl: } 0.06 \\
\text { to } 15.37 \text { ) }\end{array}$ \\
\hline Girard, $2018^{29}$ & RCT & $\begin{array}{l}\text { Patients over } 18 \text { years of age in the } \\
\text { medical or surgical ICU, with delirium }\end{array}$ & $\begin{array}{l}\text { Ziprasidone (Mean dose: } \\
\text { 20mg Dose range: } 5.0- \\
\text { 40mg), } 190\end{array}$ & $\begin{array}{l}\text { Placebo (Not } \\
\text { reported), } 184\end{array}$ & Intravenous & $\begin{array}{l}\text { Reasons for } \\
\text { temporary holds: } \\
\text { Extrapyramidal } \\
\text { symptoms } \\
\end{array}$ & $0 / 190(0 \%)$ & $\begin{array}{l}0 / 184 \\
(0 \%)\end{array}$ & \\
\hline Girard, $2018^{29}$ & RCT & $\begin{array}{l}\text { Patients over } 18 \text { years of age in the } \\
\text { medical or surgical ICU, with delirium }\end{array}$ & $\begin{array}{l}\text { Ziprasidone (Mean dose: } \\
\text { 20mg Dose range: } 5.0- \\
\text { 40mg), } 190\end{array}$ & $\begin{array}{l}\text { Placebo (Not } \\
\text { reported), } 184\end{array}$ & Intravenous & $\begin{array}{l}\text { Reasons for } \\
\text { temporary holds: } \\
\text { Dystonia }\end{array}$ & $0 / 190(0 \%)$ & $\begin{array}{l}0 / 184 \\
(0 \%)\end{array}$ & \\
\hline
\end{tabular}




\begin{tabular}{|c|c|c|c|c|c|c|c|c|c|}
\hline Author, year & $\begin{array}{l}\text { Study } \\
\text { design }\end{array}$ & Population & Intervention group, $\mathbf{n}$ & Control group, $\mathrm{n}$ & $\begin{array}{c}\text { Route of } \\
\text { administration }\end{array}$ & Outcome definition & $\begin{array}{l}\mathrm{n} / \mathrm{N}(\%) \\
\text { intervention } \\
\text { group }\end{array}$ & $\begin{array}{c}\mathrm{n} / \mathrm{N}(\%) \\
\text { control } \\
\text { group }\end{array}$ & $\begin{array}{c}\text { Relative risk } \\
(95 \% \mathrm{Cl})\end{array}$ \\
\hline Girard, $2018^{29}$ & $\mathrm{RCT}$ & $\begin{array}{l}\text { Patients over } 18 \text { years of age in the } \\
\text { medical or surgical ICU, with delirium }\end{array}$ & $\begin{array}{l}\text { Ziprasidone (Mean dose: } \\
\text { 20mg Dose range: } 5.0- \\
\text { 40mg), } 192\end{array}$ & $\begin{array}{l}\text { Placebo (Not } \\
\text { reported), } 184\end{array}$ & Intravenous & $\begin{array}{l}\text { Reasons for } \\
\text { permanent } \\
\text { discontinuation: } \\
\text { Suspected } \\
\text { neuroleptic } \\
\text { malignant syndrome }\end{array}$ & $0 / 192(0 \%)$ & $\begin{array}{l}0 / 184 \\
(0 \%)\end{array}$ & \\
\hline Tahir, $2010^{59}$ & RCT & $\begin{array}{l}\text { Patients in medical, surgical and } \\
\text { orthopedic wards, who met the DSM-IV } \\
\text { criteria for delirium on the same day if } \\
\text { they had a DRS-R-98 total score of } 15 \\
\text { or more }\end{array}$ & $\begin{array}{l}\text { Quetiapine (Mean dose: } \\
\text { 40 Dose range: } 25 \text { - } \\
\text { 175mg), } 21\end{array}$ & $\begin{array}{l}\text { Placebo (Mean } \\
\text { dose: } 25 \text { Max } \\
\text { dose: } 175 \mathrm{mg} \text { ), } 21\end{array}$ & Oral & $\begin{array}{l}\text { Abnormal } \\
\text { involuntary } \\
\text { movements }\end{array}$ & $1 / 21(4.8 \%)$ & $\begin{array}{l}3 / 21 \\
(14.3 \%)\end{array}$ & $\begin{array}{l}\text { RR } 0.33 \\
\text { (95\% Cl: } 0.04 \\
\text { to } 2.95)\end{array}$ \\
\hline \multicolumn{10}{|l|}{$\begin{array}{l}\text { First-generation } \\
\text { antipsychotic vs. } \\
\text { second-generation } \\
\text { antipsychotic }\end{array}$} \\
\hline Boettger, 201120 & $\begin{array}{l}\text { Cohort w/ } \\
\text { comparison } \\
\text { group }\end{array}$ & Cancer patients & $\begin{array}{l}\text { Risperidone (Mean dose: } \\
\text { 1.0 Dose range: } 0.25 \text { to } \\
\text { 2), } 32\end{array}$ & $\begin{array}{l}\text { Haloperidol } \\
\text { (Mean dose: } 4.6 \\
\text { Dose range: } 1 \text { to } \\
\text { 10mg), } 32 \\
\end{array}$ & Not reported & Dystonia & $1 / 32(3.1 \%)$ & $\begin{array}{l}3 / 32 \\
(9.4 \%)\end{array}$ & $\begin{array}{l}\text { RR } 0.33 \\
\text { (95\% Cl: } 0.04 \\
\text { to 3.04) }\end{array}$ \\
\hline Boettger, $2011^{20}$ & $\begin{array}{l}\text { Cohort w/ } \\
\text { comparison } \\
\text { group }\end{array}$ & Cancer patients & $\begin{array}{l}\text { Risperidone (Mean dose: } \\
\text { 1.0 Dose range: } 0.25 \text { to } \\
\text { 2), } 32\end{array}$ & $\begin{array}{l}\text { Haloperidol } \\
\text { (Mean dose: } 4.6 \\
\text { Dose range: } 1 \text { to } \\
\text { 10mg), } 32\end{array}$ & Not reported & Parkinsonism & $1 / 32(3.1 \%)$ & $\begin{array}{l}7 / 32 \\
(21.9 \%)\end{array}$ & $\begin{array}{l}\text { RR } 0.14 \\
\text { (95\% Cl: } 0.02 \\
\text { to } 1.10)\end{array}$ \\
\hline Boettger, $2015^{21}$ & $\begin{array}{l}\text { Cohort w/ } \\
\text { comparison } \\
\text { group }\end{array}$ & Cancer patients & $\begin{array}{l}\text { Risperidone (Mean dose: } \\
\text { 0.9 Dose range: } 0.5 \text { to } \\
2 \mathrm{mg}), 21\end{array}$ & $\begin{array}{l}\text { Haloperidol } \\
\text { (Mean dose: } 4.9 \\
\text { Dose range: } 1.5 \\
\text { to } 16 \mathrm{mg} \text { ), } 21 \\
\end{array}$ & Not reported & EPS - dystonia & $0 / 21(0 \%)$ & $\begin{array}{l}2 / 21 \\
(9.5 \%)\end{array}$ & $\begin{array}{l}\text { RR } 0.20 \\
\text { (95\% Cl: } 0.01 \\
\text { to 3.93) }\end{array}$ \\
\hline Boettger, $2015^{21}$ & $\begin{array}{l}\text { Cohort w/ } \\
\text { comparison } \\
\text { group }\end{array}$ & Cancer patients & $\begin{array}{l}\text { Olanzapine (Mean dose: } \\
\text { 3.5 Dose range: } 2.5 \text { to } \\
\text { 10mg), } 21\end{array}$ & $\begin{array}{l}\text { Haloperidol } \\
\text { (Mean dose: } 4.9 \\
\text { Dose range: } 1.5 \\
\text { to } 16 \mathrm{mg} \text { ), } 21 \\
\end{array}$ & Not reported & EPS - dystonia & $0 / 21(0 \%)$ & $\begin{array}{l}2 / 21 \\
(9.5 \%)\end{array}$ & $\begin{array}{l}\text { RR } 0.20 \\
\text { (95\% Cl: } 0.01 \\
\text { to 3.93) }\end{array}$ \\
\hline Boettger, $2015^{21}$ & $\begin{array}{l}\text { Cohort w/ } \\
\text { comparison } \\
\text { group }\end{array}$ & Cancer patients & $\begin{array}{l}\text { Aripiprazole (Mean dose: } \\
\text { 15.2 Dose range: } 5 \text { to } \\
\text { 30mg), } 21\end{array}$ & $\begin{array}{l}\text { Haloperidol } \\
\text { (Mean dose: } 4.9 \\
\text { Dose range: } 1.5 \\
\text { to } 16 \mathrm{mg} \text { ), } 21 \\
\end{array}$ & Not reported & EPS - dystonia & $0 / 21(0 \%)$ & $\begin{array}{l}2 / 21 \\
(9.5 \%)\end{array}$ & $\begin{array}{l}\text { RR } 0.20 \\
\text { (95\% Cl: } 0.01 \\
\text { to 3.93) }\end{array}$ \\
\hline
\end{tabular}




\begin{tabular}{|c|c|c|c|c|c|c|c|c|c|}
\hline Author, year & $\begin{array}{l}\text { Study } \\
\text { design }\end{array}$ & Population & Intervention group, $\mathbf{n}$ & Control group, $n$ & $\begin{array}{c}\text { Route of } \\
\text { administration }\end{array}$ & Outcome definition & $\begin{array}{c}\mathrm{n} / \mathrm{N}(\%) \\
\text { intervention } \\
\text { group }\end{array}$ & $\begin{array}{c}\mathrm{n} / \mathrm{N}(\%) \\
\text { control } \\
\text { group }\end{array}$ & $\begin{array}{l}\text { Relative risk } \\
(95 \% \mathrm{Cl})\end{array}$ \\
\hline Boettger, $2015^{21}$ & $\begin{array}{l}\text { Cohort w/ } \\
\text { comparison } \\
\text { group }\end{array}$ & Cancer patients & $\begin{array}{l}\text { Olanzapine (Mean dose: } \\
3.5 \text { Dose range: } 2.5 \text { to } \\
\text { 10mg), } 21\end{array}$ & $\begin{array}{l}\text { Haloperidol } \\
\text { (Mean dose: } 4.9 \\
\text { Dose range: } 1.5 \\
\text { to } 16 \mathrm{mg} \text { ), } 21\end{array}$ & Not reported & EPS - parkinsonism & $0 / 21(0 \%)$ & $\begin{array}{l}4 / 21 \\
(19 \%)\end{array}$ & $\begin{array}{l}\text { RR } 0.11 \\
\text { (95\% Cl: } 0.01 \\
\text { to } 1.94)\end{array}$ \\
\hline Boettger, $2015^{21}$ & $\begin{array}{l}\text { Cohort w/ } \\
\text { comparison } \\
\text { group }\end{array}$ & Cancer patients & $\begin{array}{l}\text { Risperidone (Mean dose: } \\
0.9 \text { Dose range: } 0.5 \text { to } \\
\text { 2mg), } 21\end{array}$ & $\begin{array}{l}\text { Haloperidol } \\
\text { (Mean dose: } 4.9 \\
\text { Dose range: } 1.5 \\
\text { to } 16 \mathrm{mg} \text { ), } 21\end{array}$ & Not reported & EPS - parkinsonism & $1 / 21(4.8 \%)$ & $\begin{array}{l}4 / 21 \\
(19 \%)\end{array}$ & $\begin{array}{l}\text { RR } 0.25 \\
\text { (95\% Cl: } 0.03 \\
\text { to } 2.05 \text { ) }\end{array}$ \\
\hline Boettger, $2015^{21}$ & $\begin{array}{l}\text { Cohort w/ } \\
\text { comparison } \\
\text { group }\end{array}$ & Cancer patients & $\begin{array}{l}\text { Aripiprazole (Mean dose: } \\
\text { 15.2 Dose range: } 5 \text { to } \\
30 \mathrm{mg}), 21\end{array}$ & $\begin{array}{l}\text { Haloperidol } \\
\text { (Mean dose: } 4.9 \\
\text { Dose range: } 1.5 \\
\text { to } 16 \mathrm{mg} \text { ), } 21\end{array}$ & Not reported & EPS - parkinsonism & $0 / 21(0 \%)$ & $\begin{array}{l}4 / 21 \\
(19 \%)\end{array}$ & $\begin{array}{l}\text { RR } 0.11 \\
\text { (95\% Cl: } 0.01 \\
\text { to } 1.94)\end{array}$ \\
\hline Boettger, $2015^{21}$ & $\begin{array}{l}\text { Cohort w/ } \\
\text { comparison } \\
\text { group }\end{array}$ & Cancer patients & $\begin{array}{l}\text { Olanzapine (Mean dose: } \\
3.5 \text { Dose range: } 2.5 \text { to } \\
\text { 10mg), } 21\end{array}$ & $\begin{array}{l}\text { Haloperidol } \\
\text { (Mean dose: } 4.9 \\
\text { Dose range: } 1.5 \\
\text { to } 16 \mathrm{mg} \text { ), } 21\end{array}$ & Not reported & $\begin{array}{l}\text { Extrapyramidal } \\
\text { symptoms }\end{array}$ & $0 / 21(0 \%)$ & $\begin{array}{l}4 / 21 \\
(19 \%)\end{array}$ & $\begin{array}{l}\text { RR } 0.11 \\
\text { (95\% Cl: } 0.01 \\
\text { to } 1.94)\end{array}$ \\
\hline Boettger, $2015^{21}$ & $\begin{array}{l}\text { Cohort w/ } \\
\text { comparison } \\
\text { group }\end{array}$ & Cancer patients & $\begin{array}{l}\text { Risperidone (Mean dose: } \\
0.9 \text { Dose range: } 0.5 \text { to } \\
2 \mathrm{mg}), 21\end{array}$ & $\begin{array}{l}\text { Haloperidol } \\
\text { (Mean dose: } 4.9 \\
\text { Dose range: } 1.5 \\
\text { to } 16 \mathrm{mg} \text { ), } 21\end{array}$ & Not reported & $\begin{array}{l}\text { Extrapyramidal } \\
\text { symptoms }\end{array}$ & $1 / 21(4.8 \%)$ & $\begin{array}{l}4 / 21 \\
(19 \%)\end{array}$ & $\begin{array}{l}\text { RR } 0.25 \\
\text { (95\% Cl: } 0.03 \\
\text { to } 2.05 \text { ) }\end{array}$ \\
\hline Boettger, 2015 & $\begin{array}{l}\text { Cohort w/ } \\
\text { comparison } \\
\text { group }\end{array}$ & Cancer patients & $\begin{array}{l}\text { Aripiprazole (Mean dose: } \\
\text { 15.2 Dose range: } 5 \text { to } \\
30 \mathrm{mg} \text { ), } 21\end{array}$ & $\begin{array}{l}\text { Haloperidol } \\
\text { (Mean dose: } 4.9 \\
\text { Dose range: } 1.5 \\
\text { to } 16 \mathrm{mg} \text { ), } 21\end{array}$ & Not reported & $\begin{array}{l}\text { Extrapyramidal } \\
\text { symptoms }\end{array}$ & $0 / 21(0 \%)$ & $\begin{array}{l}4 / 21 \\
(19 \%)\end{array}$ & $\begin{array}{l}\text { RR } 0.11 \\
\text { (95\% Cl: } 0.01 \\
\text { to } 1.94)\end{array}$ \\
\hline $\begin{array}{l}\text { Charoenporn, } \\
2018^{25}\end{array}$ & $\begin{array}{l}\text { Cohort w/ } \\
\text { comparison } \\
\text { group }\end{array}$ & Physically ill patients & $\begin{array}{l}\text { Quetiapine (Mean dose: } \\
26.7 \text { Median dose: } 25) \text {, } \\
31\end{array}$ & $\begin{array}{l}\text { Haloperidol } \\
\text { (Mean dose: } 0.9 \\
\text { Median dose: } \\
0.5 \mathrm{mg} \text { ), } 11\end{array}$ & Oral & $\begin{array}{l}\text { Extrapyramidal } \\
\text { symptoms }\end{array}$ & $3 / 31(9.7 \%)$ & $\begin{array}{l}1 / 11 \\
(9.1 \%)\end{array}$ & $\begin{array}{l}\text { RR 1.06 } \\
\text { (95\% Cl: } 0.12 \\
\text { to 9.19) }\end{array}$ \\
\hline $\begin{array}{l}\text { Charoenporn, } \\
2018^{25}\end{array}$ & $\begin{array}{l}\text { Cohort w/ } \\
\text { comparison } \\
\text { group }\end{array}$ & Physically ill patients & $\begin{array}{l}\text { Risperidone (Mean dose: } \\
0.6 \text { Median dose: } \\
0.5 \mathrm{mg}), 14\end{array}$ & $\begin{array}{l}\text { Haloperidol } \\
\text { (Mean dose: } 0.9 \\
\text { Median dose: } \\
0.5 \mathrm{mg} \text { ), } 11\end{array}$ & Oral & $\begin{array}{l}\text { Extrapyramidal } \\
\text { symptoms }\end{array}$ & $\begin{array}{l}2 / 14 \\
(14.2 \%)\end{array}$ & $\begin{array}{l}1 / 11 \\
(9.1 \%)\end{array}$ & $\begin{array}{l}\text { RR } 1.57 \\
\text { (95\% Cl: } 0.16 \\
\text { to } 15.16)\end{array}$ \\
\hline Girard, $2010^{4}$ & RCT & $\begin{array}{l}>18 \text { years MV medical and surgical ICU } \\
\text { patients }\end{array}$ & $\begin{array}{l}\text { Ziprasidone (Planned } \\
\text { dose: } 40 \text { Median dose: } \\
113 \text { (81-140), } 30\end{array}$ & $\begin{array}{l}\text { Haloperidol } \\
\text { (Planned dose: } 5 \\
\text { Median dose: } 15 \\
(10.8-17), 35\end{array}$ & Oral & Akathisia & $6 / 30(20 \%)$ & $\begin{array}{l}10 / 35 \\
(29 \%)\end{array}$ & $\begin{array}{l}\text { RR } 0.70 \\
(95 \% \text { Cl: } 0.29 \\
\text { to } 1.70)\end{array}$ \\
\hline
\end{tabular}




\begin{tabular}{|c|c|c|c|c|c|c|c|c|c|}
\hline Author, year & $\begin{array}{l}\text { Study } \\
\text { design }\end{array}$ & Population & Intervention group, $\mathbf{n}$ & Control group, $n$ & $\begin{array}{c}\text { Route of } \\
\text { administration }\end{array}$ & Outcome definition & $\begin{array}{c}\mathrm{n} / \mathrm{N}(\%) \\
\text { intervention } \\
\text { group }\end{array}$ & $\begin{array}{l}\mathrm{n} / \mathrm{N}(\%) \\
\text { control } \\
\text { group }\end{array}$ & $\begin{array}{c}\text { Relative risk } \\
(95 \% \mathrm{Cl})\end{array}$ \\
\hline Girard, $2010^{4}$ & RCT & $\begin{array}{l}>18 \text { years MV medical and surgical ICU } \\
\text { patients }\end{array}$ & $\begin{array}{l}\text { Ziprasidone (Planned } \\
\text { dose: } 40 \text { Median dose: } \\
113 \text { (81-140), } 30\end{array}$ & $\begin{array}{l}\text { Haloperidol } \\
\text { (Planned dose: } 5 \\
\text { Median dose: } 15 \\
(10.8-17), 35\end{array}$ & Oral & $\begin{array}{l}\text { Extrapyramidal } \\
\text { symptoms }\end{array}$ & $2 / 30(7 \%)$ & $\begin{array}{l}4 / 35 \\
(11 \%)\end{array}$ & $\begin{array}{l}\text { RR } 0.58 \\
\text { (95\% Cl: } 0.11 \\
\text { to } 2.96)\end{array}$ \\
\hline Girard, $2010^{4}$ & $\mathrm{RCT}$ & $\begin{array}{l}>18 \text { years MV medical and surgical ICU } \\
\text { patients }\end{array}$ & $\begin{array}{l}\text { Ziprasidone (Planned } \\
\text { dose: } 40 \text { Median dose: } \\
113 \text { (81-140), } 30\end{array}$ & $\begin{array}{l}\text { Haloperidol } \\
\text { (Planned dose: } 5 \\
\text { Median dose: } 15 \\
(10.8-17), 35\end{array}$ & Oral & $\begin{array}{l}\text { Neuroleptic } \\
\text { malignant syndrome }\end{array}$ & $0 / 30(0 \%)$ & $\begin{array}{l}0 / 35 \\
(0 \%)\end{array}$ & \\
\hline Girard, $2018^{29}$ & RCT & $\begin{array}{l}\text { Patients over } 18 \text { years of age in the } \\
\text { medical or surgical ICU, with delirium }\end{array}$ & $\begin{array}{l}\text { Ziprasidone (Mean dose: } \\
\text { 20mg Dose range: } 5.0- \\
\text { 40mg), } 190\end{array}$ & $\begin{array}{l}\text { Haloperidol } \\
\text { (Mean dose: } \\
\text { 11mg Dose } \\
\text { range: } 2.5-20 \mathrm{mg} \text { ), } \\
192\end{array}$ & Intravenous & $\begin{array}{l}\text { Extrapyramidal } \\
\text { symptoms or } \\
\text { Dystonia }\end{array}$ & $1 / 190(1 \%)$ & $\begin{array}{l}2 / 192 \\
(1 \%)\end{array}$ & $\begin{array}{l}\text { RR 0.51 } \\
\text { (95\% Cl: } 0.05 \\
\text { to 5.53) }\end{array}$ \\
\hline Girard, $2018^{29}$ & RCT & $\begin{array}{l}\text { Patients over } 18 \text { years of age in the } \\
\text { medical or surgical ICU, with delirium }\end{array}$ & $\begin{array}{l}\text { Ziprasidone (Mean dose: } \\
\text { 20mg Dose range: } 5.0- \\
\text { 40mg), } 190\end{array}$ & $\begin{array}{l}\text { Haloperidol } \\
\text { (Mean dose: } \\
\text { 11mg Dose } \\
\text { range: } 2.5-20 \mathrm{mg} \text { ), } \\
192\end{array}$ & Intravenous & $\begin{array}{l}\text { Reasons for } \\
\text { temporary holds: } \\
\text { Extrapyramidal } \\
\text { symptoms }\end{array}$ & $0 / 190(0 \%)$ & $\begin{array}{l}0 / 192 \\
(0 \%)\end{array}$ & \\
\hline Girard, $2018^{29}$ & RCT & $\begin{array}{l}\text { Patients over } 18 \text { years of age in the } \\
\text { medical or surgical ICU, with delirium }\end{array}$ & $\begin{array}{l}\text { Ziprasidone (Mean dose: } \\
\text { 20mg Dose range: } 5.0- \\
\text { 40mg), } 190\end{array}$ & $\begin{array}{l}\text { Haloperidol } \\
\text { (Mean dose: } \\
\text { 11mg Dose } \\
\text { range: } 2.5-20 \mathrm{mg} \text { ), } \\
192\end{array}$ & Intravenous & $\begin{array}{l}\text { Reasons for } \\
\text { temporary holds: } \\
\text { Dystonia }\end{array}$ & $0 / 190(0 \%)$ & $\begin{array}{l}0 / 192 \\
(0 \%)\end{array}$ & \\
\hline Girard, $2018^{29}$ & RCT & $\begin{array}{l}\text { Patients over } 18 \text { years of age in the } \\
\text { medical or surgical ICU, with delirium }\end{array}$ & $\begin{array}{l}\text { Ziprasidone (Mean dose: } \\
\text { 20mg Dose range: } 5.0- \\
\text { 40mg), } 192\end{array}$ & $\begin{array}{l}\text { Haloperidol } \\
\text { (Mean dose: } \\
\text { 11mg Dose } \\
\text { range: } 2.5-20 \mathrm{mg} \text { ), } \\
184\end{array}$ & Intravenous & $\begin{array}{l}\text { Reasons for } \\
\text { permanent } \\
\text { discontinuation: } \\
\text { Suspected } \\
\text { neuroleptic } \\
\text { malignant syndrome }\end{array}$ & $0 / 192(0 \%)$ & $\begin{array}{l}0 / 184 \\
(0 \%)\end{array}$ & \\
\hline Grover, $2011^{30}$ & RCT & $\begin{array}{l}\text { Consecutive patients }>-18 \text { years old } \\
\text { referred to psychiatry and diagnosed } \\
\text { with delirium }\end{array}$ & $\begin{array}{l}\text { Olanzapine (Mean dose: } \\
\text { 3.05mg Dose range: } \\
1.25-10 \mathrm{mg} \text { per day), } 23\end{array}$ & $\begin{array}{l}\text { Haloperidol } \\
\text { (Mean dose: } 0.88 \\
\text { Dose range: } 0.25- \\
\text { 5mg per day), } 21\end{array}$ & Oral & Jaw movement & $0 / 23(0 \%)$ & $\begin{array}{l}0 / 21 \\
(0 \%)\end{array}$ & \\
\hline
\end{tabular}




\begin{tabular}{|c|c|c|c|c|c|c|c|c|c|}
\hline Author, year & $\begin{array}{l}\text { Study } \\
\text { design }\end{array}$ & Population & Intervention group, $\mathbf{n}$ & Control group, $n$ & $\begin{array}{c}\text { Route of } \\
\text { administration }\end{array}$ & Outcome definition & $\begin{array}{l}\mathrm{n} / \mathrm{N}(\%) \\
\text { intervention } \\
\text { group }\end{array}$ & $\begin{array}{c}\mathrm{n} / \mathrm{N}(\%) \\
\text { control } \\
\text { group }\end{array}$ & $\begin{array}{c}\text { Relative risk } \\
(95 \% \mathrm{Cl})\end{array}$ \\
\hline Grover, $2011^{30}$ & RCT & $\begin{array}{l}\text { Consecutive patients }>-18 \text { years old } \\
\text { referred to psychiatry and diagnosed } \\
\text { with delirium }\end{array}$ & $\begin{array}{l}\text { Risperidone (Mean dose: } \\
0.95 \mathrm{mg} \text { Dose range: } 0.5- \\
2.0 \mathrm{mg} \text { per day), } 20\end{array}$ & $\begin{array}{l}\text { Haloperidol } \\
\text { (Mean dose: } 0.88 \\
\text { Dose range: } 0.25- \\
\text { 5mg per day), } 21\end{array}$ & Oral & Jaw movement & $1 / 20(5 \%)$ & $\begin{array}{l}0 / 21 \\
(0 \%)\end{array}$ & $\begin{array}{l}\text { RR } 3.14 \\
\text { (95\% Cl: } 0.14 \\
\text { to } 72.92)\end{array}$ \\
\hline Grover, $2011^{30}$ & $\mathrm{RCT}$ & $\begin{array}{l}\text { Consecutive patients }>-18 \text { years old } \\
\text { referred to psychiatry and diagnosed } \\
\text { with delirium }\end{array}$ & $\begin{array}{l}\text { Risperidone (Mean dose: } \\
\text { 0.95mg Dose range: } 0.5- \\
\text { 2.0mg per day), } 20\end{array}$ & $\begin{array}{l}\text { Haloperidol } \\
\text { (Mean dose: } 0.88 \\
\text { Dose range: } 0.25- \\
\text { 5mg per day), } 21\end{array}$ & Oral & $\begin{array}{l}\text { Lips and perioral } \\
\text { movements }\end{array}$ & $1 / 20(5 \%)$ & $\begin{array}{l}0 / 21 \\
(0 \%)\end{array}$ & $\begin{array}{l}\text { RR 3.14 } \\
\text { (95\% Cl: } 0.14 \\
\text { to } 72.92)\end{array}$ \\
\hline Grover, $2011^{30}$ & RCT & $\begin{array}{l}\text { Consecutive patients }>-18 \text { years old } \\
\text { referred to psychiatry and diagnosed } \\
\text { with delirium }\end{array}$ & $\begin{array}{l}\text { Olanzapine (Mean dose: } \\
\text { 3.05mg Dose range: } \\
\text { 1.25-10 mg per day), } 23\end{array}$ & $\begin{array}{l}\text { Haloperidol } \\
\text { (Mean dose: } 0.88 \\
\text { Dose range: } 0.25- \\
\text { 5mg per day), } 21\end{array}$ & Oral & $\begin{array}{l}\text { Lips and perioral } \\
\text { movements }\end{array}$ & $0 / 23(0 \%)$ & $\begin{array}{l}0 / 21 \\
(0 \%)\end{array}$ & \\
\hline Grover, $2011^{30}$ & RCT & $\begin{array}{l}\text { Consecutive patients }>-18 \text { years old } \\
\text { referred to psychiatry and diagnosed } \\
\text { with delirium }\end{array}$ & $\begin{array}{l}\text { Olanzapine (Mean dose: } \\
\text { 3.05mg Dose range: } \\
\text { 1.25-10 mg per day), } 23\end{array}$ & $\begin{array}{l}\text { Haloperidol } \\
\text { (Mean dose: } 0.88 \\
\text { Dose range: } 0.25- \\
5 \mathrm{mg} \text { per day), } 21\end{array}$ & Oral & $\begin{array}{l}\text { Movements of the } \\
\text { tongue }\end{array}$ & $0 / 23(0 \%)$ & $\begin{array}{l}0 / 21 \\
(0 \%)\end{array}$ & \\
\hline Grover, $2011^{30}$ & RCT & $\begin{array}{l}\text { Consecutive patients }>-18 \text { years old } \\
\text { referred to psychiatry and diagnosed } \\
\text { with delirium }\end{array}$ & $\begin{array}{l}\text { Risperidone (Mean dose: } \\
\text { 0.95mg Dose range: } 0.5- \\
\text { 2.0mg per day), } 20\end{array}$ & $\begin{array}{l}\text { Haloperidol } \\
\text { (Mean dose: } 0.88 \\
\text { Dose range: } 0.25- \\
\text { 5mg per day), } 21\end{array}$ & Oral & $\begin{array}{l}\text { Movements of the } \\
\text { tongue }\end{array}$ & $2 / 20(10 \%)$ & $\begin{array}{l}0 / 21 \\
(0 \%)\end{array}$ & $\begin{array}{l}\text { RR } 5.24 \\
\text { (95\% Cl: } 0.27 \\
\text { to } 102.81)\end{array}$ \\
\hline Grover, $2011^{30}$ & RCT & $\begin{array}{l}\text { Consecutive patients }>-18 \text { years old } \\
\text { referred to psychiatry and diagnosed } \\
\text { with delirium }\end{array}$ & $\begin{array}{l}\text { Olanzapine (Mean dose: } \\
\text { 3.05mg Dose range: } \\
1.25-10 \mathrm{mg} \text { per day), } 23\end{array}$ & $\begin{array}{l}\text { Haloperidol } \\
\text { (Mean dose: } 0.88 \\
\text { Dose range: } 0.25- \\
\text { 5mg per day), } 21\end{array}$ & Oral & Rigidity & $0 / 23(0 \%)$ & $\begin{array}{l}3 / 21 \\
(14.3 \%)\end{array}$ & $\begin{array}{l}\text { RR } 0.13 \\
\text { (95\% Cl: } 0.01 \\
\text { to } 2.39 \text { ) }\end{array}$ \\
\hline Grover, $2011^{30}$ & RCT & $\begin{array}{l}\text { Consecutive patients }>-18 \text { years old } \\
\text { referred to psychiatry and diagnosed } \\
\text { with delirium }\end{array}$ & $\begin{array}{l}\text { Risperidone (Mean dose: } \\
0.95 \mathrm{mg} \text { Dose range: } 0.5- \\
\text { 2.0mg per day), } 20\end{array}$ & $\begin{array}{l}\text { Haloperidol } \\
\text { (Mean dose: } 0.88 \\
\text { Dose range: } 0.25- \\
5 \mathrm{mg} \text { per day), } 21\end{array}$ & Oral & Rigidity & $2 / 20(10 \%)$ & $\begin{array}{l}3 / 21 \\
(14.3 \%)\end{array}$ & $\begin{array}{l}\text { RR } 0.70 \\
\text { (95\% Cl: } 0.13 \\
\text { to } 3.76 \text { ) }\end{array}$ \\
\hline Grover, $2011^{30}$ & RCT & $\begin{array}{l}\text { Consecutive patients }>-18 \text { years old } \\
\text { referred to psychiatry and diagnosed } \\
\text { with delirium }\end{array}$ & $\begin{array}{l}\text { Risperidone (Mean dose: } \\
0.95 \mathrm{mg} \text { Dose range: } 0.5- \\
\text { 2.0mg per day), } 20\end{array}$ & $\begin{array}{l}\text { Haloperidol } \\
\text { (Mean dose: } 0.88 \\
\text { Dose range: } 0.25- \\
5 \mathrm{mg} \text { per day), } 21\end{array}$ & Oral & Tremors & $2 / 20(10 \%)$ & $\begin{array}{l}4 / 21 \\
(19 \%)\end{array}$ & $\begin{array}{l}\text { RR } 0.53 \\
\text { (95\% Cl: } 0.11 \\
\text { to } 2.56)\end{array}$ \\
\hline Grover, $2011^{30}$ & RCT & $\begin{array}{l}\text { Consecutive patients }>-18 \text { years old } \\
\text { referred to psychiatry and diagnosed } \\
\text { with delirium }\end{array}$ & $\begin{array}{l}\text { Olanzapine (Mean dose: } \\
\text { 3.05mg Dose range: } \\
1.25-10 \mathrm{mg} \text { per day), } 23\end{array}$ & $\begin{array}{l}\text { Haloperidol } \\
\text { (Mean dose: } 0.88 \\
\text { Dose range: } 0.25- \\
\text { 5mg per day), } 21\end{array}$ & Oral & Tremors & $2 / 23(8.7 \%)$ & $\begin{array}{l}4 / 21 \\
(19 \%)\end{array}$ & $\begin{array}{l}\text { RR } 0.46 \\
\text { (95\% Cl: } 0.09 \\
\text { to } 2.24)\end{array}$ \\
\hline
\end{tabular}




\begin{tabular}{|c|c|c|c|c|c|c|c|c|c|}
\hline Author, year & $\begin{array}{c}\text { Study } \\
\text { design }\end{array}$ & Population & Intervention group, $\mathbf{n}$ & Control group, $\mathrm{n}$ & $\begin{array}{c}\text { Route of } \\
\text { administration }\end{array}$ & Outcome definition & $\begin{array}{l}\mathrm{n} / \mathrm{N}(\%) \\
\text { intervention } \\
\text { group }\end{array}$ & $\begin{array}{c}\mathrm{n} / \mathrm{N}(\%) \\
\text { control } \\
\text { group }\end{array}$ & $\begin{array}{l}\text { Relative risk } \\
(95 \% \mathrm{Cl})\end{array}$ \\
\hline Han, 200432 & RCT & $\begin{array}{l}\text { All patients presenting with altered } \\
\text { mental status (from both ICU and non- } \\
\text { ICU units) who were referred to the } \\
\text { consulting psychiatry division were } \\
\text { evaluated. }\end{array}$ & $\begin{array}{l}\text { Risperidone (Mean dose: } \\
\text { 1.02 Dose range: } 0.5 \text { to } \\
2.0 \mathrm{mg}), 12\end{array}$ & $\begin{array}{l}\text { Haloperidol } \\
\text { (Mean dose: } 1.7 \\
\text { Dose range: } 1.0 \\
\text { to } 3.0 \mathrm{mg} \text { ), } 12\end{array}$ & Oral & $\begin{array}{l}\text { Extrapyramidal } \\
\text { symptoms }\end{array}$ & $0 / 12(0 \%)$ & $\begin{array}{l}1 / 12 \\
(8.3 \%)\end{array}$ & $\begin{array}{l}\text { RR } 0.33 \\
\text { (95\% Cl: } 0.01 \\
\text { to } 7.45)\end{array}$ \\
\hline Hatta, 2014 33 & $\begin{array}{l}\text { Cohort w/ } \\
\text { comparison } \\
\text { group }\end{array}$ & $\begin{array}{l}\text { Patients in a general hospital who } \\
\text { developed delirium and were managed } \\
\text { by a psychiatrist }\end{array}$ & $\begin{array}{l}\text { Quetiapine (Max dose: } \\
71.8 \mathrm{mg}), 779\end{array}$ & $\begin{array}{l}\text { Haloperidol (Max } \\
\text { dose: } 6.40 \mathrm{mg}) \\
480\end{array}$ & Not applicable & $\begin{array}{l}\text { Extrapyramidal } \\
\text { symptoms }\end{array}$ & $\begin{array}{l}34 / 779 \\
(4.4 \%)\end{array}$ & $\begin{array}{l}30 / 480 \\
(6.3 \%)\end{array}$ & $\begin{array}{l}\text { RR } 0.70 \\
\text { (95\% Cl: } 0.43 \\
\text { to } 1.13)\end{array}$ \\
\hline Hatta, 2014 33 & $\begin{array}{l}\text { Cohort w/ } \\
\text { comparison } \\
\text { group }\end{array}$ & $\begin{array}{l}\text { Patients in a general hospital who } \\
\text { developed delirium and were managed } \\
\text { by a psychiatrist }\end{array}$ & $\begin{array}{l}\text { Olanzapine (Max dose: } \\
\text { 10.2mg), } 87\end{array}$ & $\begin{array}{l}\text { Haloperidol (Max } \\
\text { dose: } \\
6.40 \mathrm{mg}), 480 \\
\end{array}$ & Not reported & $\begin{array}{l}\text { Extrapyramidal } \\
\text { symptoms }\end{array}$ & $4 / 87(4.6 \%)$ & $\begin{array}{l}30 / 480 \\
(6.3 \%)\end{array}$ & $\begin{array}{l}\text { RR } 0.74 \\
\text { (95\% Cl: } 0.27 \\
\text { to } 2.04)\end{array}$ \\
\hline Hatta, 201433 & $\begin{array}{l}\text { Cohort w/ } \\
\text { comparison } \\
\text { group }\end{array}$ & $\begin{array}{l}\text { Patients in a general hospital who } \\
\text { developed delirium and were managed } \\
\text { by a psychiatrist }\end{array}$ & $\begin{array}{l}\text { Risperidone (Max dose: } \\
1.35 \mathrm{mg}), 835\end{array}$ & $\begin{array}{l}\text { Haloperidol (Max } \\
\text { dose: } 6.40 \mathrm{mg}) \\
480\end{array}$ & Not applicable & $\begin{array}{l}\text { Extrapyramidal } \\
\text { symptoms }\end{array}$ & $\begin{array}{l}53 / 835 \\
(6.3 \%)\end{array}$ & $\begin{array}{l}30 / 480 \\
(6.3 \%)\end{array}$ & $\begin{array}{l}\text { RR 1.02 } \\
\text { (95\% Cl: } 0.66 \\
\text { to } 1.57)\end{array}$ \\
\hline Hatta, 2014 33 & $\begin{array}{l}\text { Cohort w/ } \\
\text { comparison } \\
\text { group }\end{array}$ & $\begin{array}{l}\text { Patients in a general hospital who } \\
\text { developed delirium and were managed } \\
\text { by a psychiatrist }\end{array}$ & $\begin{array}{l}\text { Aripiprazole (Max dose: } \\
7.23 \mathrm{mg} \text { ), } 61\end{array}$ & $\begin{array}{l}\text { Haloperidol (Max } \\
\text { dose: } 6.40 \mathrm{mg}) \\
480\end{array}$ & Not reported & $\begin{array}{l}\text { Extrapyramidal } \\
\text { symptoms }\end{array}$ & $2 / 61(3.3 \%)$ & $\begin{array}{l}30 / 480 \\
(6.3 \%)\end{array}$ & $\begin{array}{l}\text { RR } 0.52 \\
\text { (95\% Cl: } 0.13 \\
\text { to } 2.14)\end{array}$ \\
\hline Jain, $2017^{38}$ & RCT & Medical and surgical inpatients & $\begin{array}{l}\text { Olanzapine (Mean dose: } \\
5.49 \mathrm{mg}), 47\end{array}$ & $\begin{array}{l}\text { Haloperidol } \\
\text { (Mean dose: } \\
2.10 \mathrm{mg} \text { ), } 53\end{array}$ & Oral & Akathisia & $1 / 47(2.1 \%)$ & $\begin{array}{l}0 / 53 \\
(0 \%)\end{array}$ & $\begin{array}{l}\text { RR 3.38 } \\
\text { (95\% Cl: } 0.14 \\
\text { to } 80.91)\end{array}$ \\
\hline Jain, $2017^{38}$ & RCT & Medical and surgical inpatients & $\begin{array}{l}\text { Olanzapine (Mean dose: } \\
5.49 \mathrm{mg}), 47\end{array}$ & $\begin{array}{l}\text { Haloperidol } \\
\text { (Mean dose: } \\
2.10 \mathrm{mg} \text { ), } 53\end{array}$ & Oral & $\begin{array}{l}\text { Drug-induced } \\
\text { parkinsonism }\end{array}$ & $0 / 47(0 \%)$ & $\begin{array}{l}3 / 53 \\
(5.7 \%)\end{array}$ & $\begin{array}{l}\text { RR 0.16 } \\
\text { (95\% Cl: } 0.01 \\
\text { to 3.03) }\end{array}$ \\
\hline Kim, $2005^{41}$ & $\begin{array}{l}\text { Cohort w/ } \\
\text { comparison } \\
\text { group }\end{array}$ & $\begin{array}{l}\text { Hospital patients referred to the } \\
\text { psychiatry division }\end{array}$ & $\begin{array}{l}\text { Risperidone (Not } \\
\text { reported), } 18\end{array}$ & $\begin{array}{l}\text { Haloperidol (Not } \\
\text { reported), } 24\end{array}$ & Not reported & $\begin{array}{l}\text { Extrapyramidal } \\
\text { symptoms }\end{array}$ & $0 / 18(0 \%)$ & $\begin{array}{l}0 / 24 \\
(0 \%)\end{array}$ & \\
\hline
\end{tabular}




\begin{tabular}{|c|c|c|c|c|c|c|c|c|c|}
\hline Author, year & $\begin{array}{l}\text { Study } \\
\text { design }\end{array}$ & Population & Intervention group, $\mathrm{n}$ & Control group, $n$ & $\begin{array}{c}\text { Route of } \\
\text { administration }\end{array}$ & Outcome definition & $\begin{array}{l}\mathrm{n} / \mathrm{N}(\%) \\
\text { intervention } \\
\text { group }\end{array}$ & $\begin{array}{c}\text { n I N (\%), } \\
\text { control } \\
\text { group }\end{array}$ & $\begin{array}{c}\text { Relative risk } \\
(95 \% \mathrm{Cl})\end{array}$ \\
\hline Lim, $2007^{46}$ & RCT & $\begin{array}{l}\text { Patients from internal medicine, PMR, } \\
\text { neurology, neurosurgery, orthopedic } \\
\text { surgery, or general surgery that were } \\
\text { referred to the psychiatric department }\end{array}$ & $\begin{array}{l}\text { Olanzapine (Titrated (5 } \\
\text { to } 20 \mathrm{mg} \text { ) }\end{array}$ & $\begin{array}{l}\text { Haloperidol } \\
\text { (Titrated (5 to } 20 \\
\mathrm{mg} \text { ) }\end{array}$ & Intramuscular & EPS & $0 / 31(0 \%)$ & $\begin{array}{l}3 / 31 \\
(9.6 \%)\end{array}$ & $\begin{array}{l}\text { RR } 0.14 \\
\text { (95\% Cl: } 0.01 \\
\text { to } 2.66)\end{array}$ \\
\hline Lim, $2007^{46}$ & RCT & $\begin{array}{l}\text { Patients from internal medicine, PMR, } \\
\text { neurology, neurosurgery, orthopedic } \\
\text { surgery, or general surgery that were } \\
\text { referred to the psychiatric department }\end{array}$ & $\begin{array}{l}\text { Olanzapine (Titrated (5 } \\
\text { to } 20 \mathrm{mg} \text { ) }\end{array}$ & $\begin{array}{l}\text { Haloperidol } \\
\text { (Titrated (5 to } 20 \\
\mathrm{mg} \text { ) }\end{array}$ & Intramuscular & Akathisia & $0 / 31(0 \%)$ & $\begin{array}{l}2 / 31 \\
(6.4 \%)\end{array}$ & $\begin{array}{l}\text { RR } 0.20 \\
\text { (95\% Cl: } 0.01 \\
\text { to } 4.00)\end{array}$ \\
\hline Lin, $2008^{47}$ & RCT & $\begin{array}{l}\text { Patients receiving hospice or palliative } \\
\text { care with advanced cancer and met } \\
\text { DSM-IV criteria for delirium }\end{array}$ & $\begin{array}{l}\text { Olanzapine (Planned } \\
\text { dose: start dose } 5 \mathrm{mg} \\
\text { Max dose: } 15 \mathrm{mg} \text { ) }\end{array}$ & $\begin{array}{l}\text { Haloperidol } \\
\text { (Planned dose: } \\
\text { start dose } 5 \mathrm{mg} \\
\text { Max dose: } 15 \mathrm{mg} \text { ) }\end{array}$ & Oral & $\begin{array}{l}\text { Extrapyramidal } \\
\text { symptoms }\end{array}$ & & & $\begin{array}{l}\mathrm{p}=\mathrm{no} \\
\text { significant } \\
\text { findings }\end{array}$ \\
\hline Maneeton, $2013^{49}$ & RCT & $\begin{array}{l}\text { General adult population (age 18-75) } \\
\text { with hyperactive delirium, referred to } \\
\text { psychiatry consult liaison service at } \\
\text { tertiary care hospital in Thailand }\end{array}$ & $\begin{array}{l}\text { Quetiapine (Mean dose: } \\
67.6 \text { (SD 9.7) Dose } \\
\text { range: } 25-100 \mathrm{mg}), 24\end{array}$ & $\begin{array}{l}\text { Haloperidol } \\
\text { (Mean dose: } 0.8 \\
\text { (SD 0.3) Dose } \\
\text { range: } 0.5- \\
2.0 \mathrm{mg} \text { ), } 28\end{array}$ & Oral & Akathisia & $0 / 24(0 \%)$ & $\begin{array}{l}1 / 28 \\
(3.6 \%)\end{array}$ & $\begin{array}{l}\text { RR } 0.39 \\
(95 \% \text { Cl: } 0.02 \\
\text { to } 9.07)\end{array}$ \\
\hline Maneeton, $2013^{49}$ & RCT & $\begin{array}{l}\text { General adult population (age 18-75) } \\
\text { with hyperactive delirium, referred to } \\
\text { psychiatry consult liaison service at } \\
\text { tertiary care hospital in Thailand }\end{array}$ & $\begin{array}{l}\text { Quetiapine (Mean dose: } \\
67.6 \text { (SD 9.7) Dose } \\
\text { range: } 25-100 \mathrm{mg}), 24\end{array}$ & $\begin{array}{l}\text { Haloperidol } \\
\text { (Mean dose: } 0.8 \\
\text { (SD 0.3) Dose } \\
\text { range: } 0.5- \\
\text { 2.0ma). } 28\end{array}$ & Oral & Tics & $0 / 24(0 \%)$ & $\begin{array}{l}1 / 28 \\
(3.6 \%)\end{array}$ & $\begin{array}{l}\text { RR } 0.39 \\
\text { (95\% Cl: } 0.02 \\
\text { to } 9.07)\end{array}$ \\
\hline Maneeton, $2013^{49}$ & RCT & $\begin{array}{l}\text { General adult population (age 18-75) } \\
\text { with hyperactive delirium, referred to } \\
\text { psychiatry consult liaison service at } \\
\text { tertiary care hospital in Thailand }\end{array}$ & $\begin{array}{l}\text { Quetiapine (Mean dose: } \\
67.6 \text { (SD 9.7) Dose } \\
\text { range: } 25-100 \mathrm{mg}), 24\end{array}$ & $\begin{array}{l}\text { Haloperidol } \\
\text { (Mean dose: } 0.8 \\
\text { (SD 0.3) Dose } \\
\text { range: } 0.5- \\
\text { 2.0mg), } 28\end{array}$ & Oral & Tremor & $0 / 24(0 \%)$ & $\begin{array}{l}1 / 28 \\
(3.6 \%)\end{array}$ & $\begin{array}{l}\text { RR } 0.39 \\
\text { (95\% Cl: } 0.02 \\
\text { to } 9.07)\end{array}$ \\
\hline Maneeton, $2013^{49}$ & RCT & $\begin{array}{l}\text { General adult population (age 18-75) } \\
\text { with hyperactive delirium, referred to } \\
\text { psychiatry consult liaison service at } \\
\text { tertiary care hospital in Thailand }\end{array}$ & $\begin{array}{l}\text { Quetiapine (Mean dose: } \\
67.6 \text { (SD 9.7) Dose } \\
\text { range: } 25-100 \mathrm{mg}), 24\end{array}$ & $\begin{array}{l}\text { Haloperidol } \\
\text { (Mean dose: } 0.8 \\
\text { (SD 0.3) Dose } \\
\text { range: } 0.5- \\
\text { 2.0mg), } 28\end{array}$ & Oral & $\begin{array}{l}\text { Withdrawal due to } \\
\text { seizures }\end{array}$ & $1 / 24(4.2 \%)$ & $\begin{array}{l}0 / 28 \\
(0 \%)\end{array}$ & $\begin{array}{l}\text { RR 3.48 } \\
\text { (95\% Cl: } 0.15 \\
\text { to 81.66) }\end{array}$ \\
\hline
\end{tabular}




\begin{tabular}{|c|c|c|c|c|c|c|c|c|c|}
\hline Author, year & $\begin{array}{l}\text { Study } \\
\text { design }\end{array}$ & Population & Intervention group, $\mathbf{n}$ & Control group, $\mathrm{n}$ & $\begin{array}{c}\text { Route of } \\
\text { administration }\end{array}$ & Outcome definition & $\begin{array}{l}\mathrm{n} / \mathrm{N}(\%) \\
\text { intervention } \\
\text { group }\end{array}$ & $\begin{array}{c}\text { n I N (\%), } \\
\text { control } \\
\text { group }\end{array}$ & $\begin{array}{c}\text { Relative risk } \\
(95 \% \mathrm{Cl})\end{array}$ \\
\hline $\begin{array}{l}\text { Sipahimalani, } \\
1998^{56}\end{array}$ & $\begin{array}{l}\text { Cohort w/ } \\
\text { comparison } \\
\text { group }\end{array}$ & Hospital patients & $\begin{array}{l}\text { Olanzapine (Mean dose: } \\
8.2 \text { Max dose: } 15 \mathrm{mg}), 11\end{array}$ & $\begin{array}{l}\text { Haloperidol (Dose } \\
\text { range: } 1.5 \text { to } \\
\text { 10mg), } 11\end{array}$ & Not reported & $\begin{array}{l}\text { Extrapyramidal } \\
\text { symptoms }\end{array}$ & $0 / 11(0 \%)$ & $\begin{array}{l}3 / 11 \\
(27.3 \%)\end{array}$ & $\begin{array}{l}\text { RR } 0.14 \\
(95 \% \mathrm{Cl}: 0.01 \\
\text { to } 2.48)\end{array}$ \\
\hline Skrobik, $2004^{57}$ & $\begin{array}{l}\text { Cohort w/ } \\
\text { comparison } \\
\text { group }\end{array}$ & Medical-surgical ICU & $\begin{array}{l}\text { Olanzapine (Mean dose: } \\
4.54 \text { Dose range: } 2.5 \text { to } \\
13.5 \mathrm{mg}), 28\end{array}$ & $\begin{array}{l}\text { Haloperidol } \\
\text { (Mean dose: } 6.5 \\
\text { Dose range: } 1 \text { to } \\
\text { 28mg), } 45\end{array}$ & Oral & $\begin{array}{l}\text { Extrapyramidal } \\
\text { symptoms }\end{array}$ & $0 / 28(0 \%)$ & $\begin{array}{l}6 / 45 \\
(13.3 \%)\end{array}$ & $\begin{array}{l}\text { RR } 0.12 \\
\text { (95\% Cl: } 0.01 \\
\text { to 2.09) }\end{array}$ \\
\hline Yoon, $2013^{62}$ & $\begin{array}{l}\text { Cohort w/ } \\
\text { comparison } \\
\text { group }\end{array}$ & $\begin{array}{l}\text { Referred to consultation-liaison } \\
\text { psychiatric service for a mental status } \\
\text { change }\end{array}$ & $\begin{array}{l}\text { Olanzapine (Mean dose: } \\
\text { 2.9 Dose range: } 1- \\
\text { 20mg), } 18\end{array}$ & $\begin{array}{l}\text { Haloperidol } \\
\text { (Mean dose: } 1.2 \\
\text { Dose range: } 0.5- \\
\text { 10mg), } 23\end{array}$ & Not reported & Akathisia & $0 / 18(0 \%)$ & $\begin{array}{l}1 / 23 \\
(4.3 \%)\end{array}$ & $\begin{array}{l}\text { RR } 0.42 \\
(95 \% \text { Cl: } 0.02 \\
\text { to } 9.76)\end{array}$ \\
\hline Yoon, $2013^{62}$ & $\begin{array}{l}\text { Cohort w/ } \\
\text { comparison } \\
\text { group }\end{array}$ & $\begin{array}{l}\text { Referred to consultation-liaison } \\
\text { psychiatric service for a mental status } \\
\text { change }\end{array}$ & $\begin{array}{l}\text { Quetiapine (Mean dose: } \\
\text { 47.9 Dose range: } 25- \\
\text { 200mg), } 18\end{array}$ & $\begin{array}{l}\text { Haloperidol } \\
\text { (Mean dose: } 1.2 \\
\text { Dose range: } 0.5- \\
\text { 10mg), } 23\end{array}$ & Not reported & Akathisia & $0 / 18(0 \%)$ & $\begin{array}{l}1 / 23 \\
(4.3 \%)\end{array}$ & $\begin{array}{l}\text { RR } 0.42 \\
(95 \% \text { Cl: } 0.02 \\
\text { to } 9.76)\end{array}$ \\
\hline Yoon, $2013^{62}$ & $\begin{array}{l}\text { Cohort w/ } \\
\text { comparison } \\
\text { group }\end{array}$ & $\begin{array}{l}\text { Referred to consultation-liaison } \\
\text { psychiatric service for a mental status } \\
\text { change }\end{array}$ & $\begin{array}{l}\text { Risperidone (Mean dose: } \\
\text { 1.1 Dose range: } 0.25- \\
4 \mathrm{mg}), 21\end{array}$ & $\begin{array}{l}\text { Haloperidol } \\
\text { (Mean dose: } 1.2 \\
\text { Dose range: } 0.5- \\
\text { 10mg), } 23\end{array}$ & Not reported & Akathisia & $0 / 21(0 \%)$ & $\begin{array}{l}1 / 23 \\
(4.3 \%)\end{array}$ & $\begin{array}{l}\text { RR } 0.36 \\
\text { (95\% Cl: } 0.02 \\
\text { to 8.47) }\end{array}$ \\
\hline
\end{tabular}




\begin{tabular}{|c|c|c|c|c|c|c|c|c|c|}
\hline Author, year & $\begin{array}{l}\text { Study } \\
\text { design }\end{array}$ & Population & Intervention group, $\mathbf{n}$ & Control group, $n$ & $\begin{array}{c}\text { Route of } \\
\text { administration }\end{array}$ & Outcome definition & $\begin{array}{l}\mathrm{n} / \mathrm{N}(\%) \\
\text { intervention } \\
\text { group }\end{array}$ & $\begin{array}{l}\mathrm{n} / \mathrm{N}(\%) \\
\text { control } \\
\text { group }\end{array}$ & $\begin{array}{c}\text { Relative risk } \\
(95 \% \mathrm{Cl})\end{array}$ \\
\hline Yoon, $2013^{62}$ & $\begin{array}{l}\text { Cohort w/ } \\
\text { comparison } \\
\text { group }\end{array}$ & $\begin{array}{l}\text { Referred to consultation-liaison } \\
\text { psychiatric service for a mental status } \\
\text { change }\end{array}$ & $\begin{array}{l}\text { Quetiapine (Mean dose: } \\
\text { 47.9 Dose range: } 25- \\
\text { 200mg), } 18\end{array}$ & $\begin{array}{l}\text { Haloperidol } \\
\text { (Mean dose: } 1.2 \\
\text { Dose range: } 0.5- \\
\text { 10mg), } 23\end{array}$ & Not reported & Bradykinesia & $0 / 18(0 \%)$ & $\begin{array}{l}1 / 23 \\
(4.3 \%)\end{array}$ & $\begin{array}{l}\text { RR } 0.42 \\
\text { (95\% Cl: } 0.02 \\
\text { to } 9.76 \text { ) }\end{array}$ \\
\hline Yoon, $2013^{62}$ & $\begin{array}{l}\text { Cohort w/ } \\
\text { comparison } \\
\text { group }\end{array}$ & $\begin{array}{l}\text { Referred to consultation-liaison } \\
\text { psychiatric service for a mental status } \\
\text { change }\end{array}$ & $\begin{array}{l}\text { Risperidone (Mean dose: } \\
1.1 \text { Dose range: } 0.25- \\
4 \mathrm{mg}), 21\end{array}$ & $\begin{array}{l}\text { Haloperidol } \\
\text { (Mean dose: } 1.2 \\
\text { Dose range: } 0.5- \\
\text { 10mg), } 23\end{array}$ & Not reported & Bradykinesia & $1 / 21(4.8 \%)$ & $\begin{array}{l}1 / 23 \\
(4.3 \%)\end{array}$ & $\begin{array}{l}\text { RR 1.10 } \\
\text { (95\% Cl: } 0.07 \\
\text { to } 16.43)\end{array}$ \\
\hline Yoon, $2013^{62}$ & $\begin{array}{l}\text { Cohort w/ } \\
\text { comparison } \\
\text { group }\end{array}$ & $\begin{array}{l}\text { Referred to consultation-liaison } \\
\text { psychiatric service for a mental status } \\
\text { change }\end{array}$ & $\begin{array}{l}\text { Olanzapine (Mean dose: } \\
\text { 2.9 Dose range: } 1- \\
\text { 20mg), } 18\end{array}$ & $\begin{array}{l}\text { Haloperidol } \\
\text { (Mean dose: } 1.2 \\
\text { Dose range: } 0.5- \\
\text { 10mg), } 23\end{array}$ & Not reported & Bradykinesia & $1 / 18(5.6 \%)$ & $\begin{array}{l}1 / 23 \\
(4.3 \%)\end{array}$ & $\begin{array}{l}\text { RR 1.28 } \\
\text { (95\% Cl: } 0.09 \\
\text { to } 19.06)\end{array}$ \\
\hline Yoon, $2013^{62}$ & $\begin{array}{l}\text { Cohort w/ } \\
\text { comparison } \\
\text { group }\end{array}$ & $\begin{array}{l}\text { Referred to consultation-liaison } \\
\text { psychiatric service for a mental status } \\
\text { change }\end{array}$ & $\begin{array}{l}\text { Olanzapine (Mean dose: } \\
\text { 2.9 Dose range: } 1- \\
\text { 20mg), } 18\end{array}$ & $\begin{array}{l}\text { Haloperidol } \\
\text { (Mean dose: } 1.2 \\
\text { Dose range: } 0.5- \\
\text { 10mg), } 23\end{array}$ & Not reported & Dystonia & $0 / 18(0 \%)$ & $\begin{array}{l}0 / 23 \\
(0 \%)\end{array}$ & \\
\hline Yoon, $2013^{62}$ & $\begin{array}{l}\text { Cohort w/ } \\
\text { comparison } \\
\text { group }\end{array}$ & $\begin{array}{l}\text { Referred to consultation-liaison } \\
\text { psychiatric service for a mental status } \\
\text { change }\end{array}$ & $\begin{array}{l}\text { Quetiapine (Mean dose: } \\
\text { 47.9 Dose range: } 25- \\
\text { 200mg), } 18\end{array}$ & $\begin{array}{l}\text { Haloperidol } \\
\text { (Mean dose: } 1.2 \\
\text { Dose range: } 0.5- \\
\text { 10mg), } 23\end{array}$ & Not reported & Dystonia & $0 / 18(0 \%)$ & $\begin{array}{l}0 / 23 \\
(0 \%)\end{array}$ & \\
\hline Yoon, $2013^{62}$ & $\begin{array}{l}\text { Cohort w/ } \\
\text { comparison } \\
\text { group }\end{array}$ & $\begin{array}{l}\text { Referred to consultation-liaison } \\
\text { psychiatric service for a mental status } \\
\text { change }\end{array}$ & $\begin{array}{l}\text { Risperidone (Mean dose: } \\
1.1 \text { Dose range: } 0.25- \\
4 \mathrm{mg}), 21\end{array}$ & $\begin{array}{l}\text { Haloperidol } \\
\text { (Mean dose: } 1.2 \\
\text { Dose range: } 0.5- \\
\text { 10mg), } 23\end{array}$ & Not reported & Dystonia & $0 / 21(0 \%)$ & $\begin{array}{l}0 / 23 \\
(0 \%)\end{array}$ & \\
\hline Yoon, $2013^{62}$ & $\begin{array}{l}\text { Cohort w/ } \\
\text { comparison } \\
\text { group }\end{array}$ & $\begin{array}{l}\text { Referred to consultation-liaison } \\
\text { psychiatric service for a mental status } \\
\text { change }\end{array}$ & $\begin{array}{l}\text { Quetiapine (Mean dose: } \\
47.9 \text { Dose range: } 25- \\
\text { 200mg), } 18\end{array}$ & $\begin{array}{l}\text { Haloperidol } \\
\text { (Mean dose: } 1.2 \\
\text { Dose range: } 0.5- \\
\text { 10mg), } 23\end{array}$ & Not reported & Rigidity & $1 / 18(5.6 \%)$ & $\begin{array}{l}2 / 23 \\
(8.7 \%)\end{array}$ & $\begin{array}{l}\text { RR } 0.64 \\
\text { (95\% Cl: } 0.06 \\
\text { to } 6.50)\end{array}$ \\
\hline Yoon, $2013^{62}$ & $\begin{array}{l}\text { Cohort w/ } \\
\text { comparison } \\
\text { group }\end{array}$ & $\begin{array}{l}\text { Referred to consultation-liaison } \\
\text { psychiatric service for a mental status } \\
\text { change }\end{array}$ & $\begin{array}{l}\text { Olanzapine (Mean dose: } \\
\text { 2.9 Dose range: } 1- \\
\text { 20mg), } 18\end{array}$ & $\begin{array}{l}\text { Haloperidol } \\
\text { (Mean dose: } 1.2 \\
\text { Dose range: } 0.5- \\
\text { 10mg), } 23\end{array}$ & Not reported & Rigidity & $1 / 18(5.6 \%)$ & $\begin{array}{l}2 / 23 \\
(8.7 \%)\end{array}$ & $\begin{array}{l}\text { RR } 0.64 \\
\text { (95\% Cl: } 0.06 \\
\text { to } 6.50)\end{array}$ \\
\hline Yoon, $2013^{62}$ & $\begin{array}{l}\text { Cohort w/ } \\
\text { comparison } \\
\text { group }\end{array}$ & $\begin{array}{l}\text { Referred to consultation-liaison } \\
\text { psychiatric service for a mental status } \\
\text { change }\end{array}$ & $\begin{array}{l}\text { Risperidone (Mean dose: } \\
1.1 \text { Dose range: } 0.25- \\
4 \mathrm{mg}), 21\end{array}$ & $\begin{array}{l}\text { Haloperidol } \\
\text { (Mean dose: } 1.2 \\
\text { Dose range: } 0.5- \\
\text { 10mg), } 23\end{array}$ & Not reported & Rigidity & $1 / 21(4.8 \%)$ & $\begin{array}{l}2 / 23 \\
(8.7 \%)\end{array}$ & $\begin{array}{l}\text { RR } 0.55 \\
\text { (95\% Cl: } 0.05 \\
\text { to 5.61) }\end{array}$ \\
\hline
\end{tabular}




\begin{tabular}{|c|c|c|c|c|c|c|c|c|c|}
\hline Author, year & $\begin{array}{l}\text { Study } \\
\text { design }\end{array}$ & Population & Intervention group, $\mathbf{n}$ & Control group, $n$ & $\begin{array}{c}\text { Route of } \\
\text { administration }\end{array}$ & Outcome definition & $\begin{array}{c}\mathrm{n} / \mathrm{N}(\%) \\
\text { intervention } \\
\text { group }\end{array}$ & $\begin{array}{c}\mathrm{n} / \mathrm{N}(\%) \\
\text { control } \\
\text { group }\end{array}$ & $\begin{array}{c}\text { Relative risk } \\
(95 \% \mathrm{Cl})\end{array}$ \\
\hline Yoon, $2013^{62}$ & $\begin{array}{l}\text { Cohort w/ } \\
\text { comparison } \\
\text { group }\end{array}$ & $\begin{array}{l}\text { Referred to consultation-liaison } \\
\text { psychiatric service for a mental status } \\
\text { change }\end{array}$ & $\begin{array}{l}\text { Olanzapine (Mean dose: } \\
\text { 2.9 Dose range: } 1- \\
\text { 20mg), } 18\end{array}$ & $\begin{array}{l}\text { Haloperidol } \\
\text { (Mean dose: } 1.2 \\
\text { Dose range: } 0.5- \\
\text { 10mg), } 23\end{array}$ & Not reported & Tremors & $1 / 18(5.6 \%)$ & $\begin{array}{l}3 / 23 \\
(13 \%)\end{array}$ & $\begin{array}{l}\text { RR } 0.43 \\
\text { (95\% Cl: } 0.05 \\
\text { to 3.76) }\end{array}$ \\
\hline Yoon, $2013^{62}$ & $\begin{array}{l}\text { Cohort w/ } \\
\text { comparison } \\
\text { group }\end{array}$ & $\begin{array}{l}\text { Referred to consultation-liaison } \\
\text { psychiatric service for a mental status } \\
\text { change }\end{array}$ & $\begin{array}{l}\text { Quetiapine (Mean dose: } \\
\text { 47.9 Dose range: } 25- \\
\text { 200mg), } 18\end{array}$ & $\begin{array}{l}\text { Haloperidol } \\
\text { (Mean dose: } 1.2 \\
\text { Dose range: } 0.5- \\
\text { 10mg), } 23\end{array}$ & Not reported & Tremors & $1 / 18(5.6 \%)$ & $\begin{array}{l}3 / 23 \\
(13 \%)\end{array}$ & $\begin{array}{l}\text { RR 0.43 } \\
\text { (95\% Cl: } 0.05 \\
\text { to 3.76) }\end{array}$ \\
\hline Yoon, $2013^{62}$ & $\begin{array}{l}\text { Cohort w/ } \\
\text { comparison } \\
\text { group }\end{array}$ & $\begin{array}{l}\text { Referred to consultation-liaison } \\
\text { psychiatric service for a mental status } \\
\text { change }\end{array}$ & $\begin{array}{l}\text { Risperidone (Mean dose: } \\
1.1 \text { Dose range: } 0.25- \\
4 \mathrm{mg}), 21\end{array}$ & $\begin{array}{l}\text { Haloperidol } \\
\text { (Mean dose: } 1.2 \\
\text { Dose range: } 0.5- \\
\text { 10mg), } 23\end{array}$ & Not reported & Tremors & $2 / 21(9.5 \%)$ & $\begin{array}{l}3 / 23 \\
(13 \%)\end{array}$ & $\begin{array}{l}\text { RR } 0.73 \\
\text { (95\% Cl: } 0.13 \\
\text { to 3.95) }\end{array}$ \\
\hline \multicolumn{10}{|c|}{$\begin{array}{l}\text { Second-generation } \\
\text { antipsychotic vs. } \\
\text { second-generation } \\
\text { antipsychotic }\end{array}$} \\
\hline Boettger, $2015^{21}$ & $\begin{array}{l}\text { Cohort w/ } \\
\text { comparison } \\
\text { group }\end{array}$ & Cancer patients & $\begin{array}{l}\text { Risperidone (Mean dose: } \\
0.9 \text { Dose range: } 0.5 \text { to } \\
2 \mathrm{mg}), 21\end{array}$ & $\begin{array}{l}\text { Olanzapine } \\
\text { (Mean dose: } 3.5 \\
\text { Dose range: } 2.5 \\
\text { to } 10 \mathrm{mg} \text { ), } 21\end{array}$ & Not reported & EPS - dystonia & $0 / 21(0 \%)$ & $\begin{array}{l}0 / 21 \\
(0 \%)\end{array}$ & \\
\hline Boettger, $2015^{21}$ & $\begin{array}{l}\text { Cohort w/ } \\
\text { comparison } \\
\text { group }\end{array}$ & Cancer patients & $\begin{array}{l}\text { Risperidone (Mean dose: } \\
0.9 \text { Dose range: } 0.5 \text { to } \\
2 \mathrm{mg}), 21\end{array}$ & $\begin{array}{l}\text { Aripiprazole } \\
\text { (Mean dose: } 15.2 \\
\text { Dose range: } 5 \text { to } \\
\text { 30mg), } 21\end{array}$ & Not reported & EPS - dystonia & $0 / 21(0 \%)$ & $\begin{array}{l}0 / 21 \\
(0 \%)\end{array}$ & \\
\hline Boettger, $2015^{21}$ & $\begin{array}{l}\text { Cohort w/ } \\
\text { comparison } \\
\text { group }\end{array}$ & Cancer patients & $\begin{array}{l}\text { Olanzapine (Mean dose: } \\
3.5 \text { Dose range: } 2.5 \text { to } \\
\text { 10mg), } 21\end{array}$ & $\begin{array}{l}\text { Aripiprazole } \\
\text { (Mean dose: } 15.2 \\
\text { Dose range: } 5 \text { to } \\
\text { 30mg), } 21\end{array}$ & Not reported & EPS - dystonia & $0 / 21(0 \%)$ & $\begin{array}{l}0 / 21 \\
(0 \%)\end{array}$ & \\
\hline Boettger, $2015^{21}$ & $\begin{array}{l}\text { Cohort w/ } \\
\text { comparison } \\
\text { group }\end{array}$ & Cancer patients & $\begin{array}{l}\text { Olanzapine (Mean dose: } \\
3.5 \text { Dose range: } 2.5 \text { to } \\
\text { 10mg), } 21\end{array}$ & $\begin{array}{l}\text { Aripiprazole } \\
\text { (Mean dose: } 15.2 \\
\text { Dose range: } 5 \text { to } \\
\text { 30mg), } 21\end{array}$ & Not reported & EPS - parkinsonism & $0 / 21(0 \%)$ & $\begin{array}{l}0 / 21 \\
(0 \%)\end{array}$ & \\
\hline
\end{tabular}




\begin{tabular}{|c|c|c|c|c|c|c|c|c|c|}
\hline Author, year & $\begin{array}{c}\text { Study } \\
\text { design }\end{array}$ & Population & Intervention group, $\mathbf{n}$ & Control group, $n$ & $\begin{array}{c}\text { Route of } \\
\text { administration }\end{array}$ & Outcome definition & $\begin{array}{l}\mathrm{n} / \mathrm{N}(\%) \\
\text { intervention } \\
\text { group }\end{array}$ & $\begin{array}{c}\mathrm{n} / \mathrm{N}(\%) \\
\text { control } \\
\text { group }\end{array}$ & $\begin{array}{c}\text { Relative risk } \\
(95 \% \mathrm{Cl})\end{array}$ \\
\hline Boettger, $2015^{21}$ & $\begin{array}{l}\text { Cohort w/ } \\
\text { comparison } \\
\text { group }\end{array}$ & Cancer patients & $\begin{array}{l}\text { Risperidone (Mean dose: } \\
0.9 \text { Dose range: } 0.5 \text { to } \\
2 \mathrm{mg}), 21\end{array}$ & $\begin{array}{l}\text { Aripiprazole } \\
\text { (Mean dose: } 15.2 \\
\text { Dose range: } 5 \text { to } \\
\text { 30mg), } 21\end{array}$ & Not reported & EPS - parkinsonism & $1 / 21(4.8 \%)$ & $\begin{array}{l}0 / 21 \\
(0 \%)\end{array}$ & $\begin{array}{l}\text { RR } 3.00 \\
\text { (95\% Cl: } 0.13 \\
\text { to } 69.70)\end{array}$ \\
\hline Boettger, $2015^{21}$ & $\begin{array}{l}\text { Cohort w/ } \\
\text { comparison } \\
\text { group }\end{array}$ & Cancer patients & $\begin{array}{l}\text { Risperidone (Mean dose: } \\
0.9 \text { Dose range: } 0.5 \text { to } \\
2 \mathrm{mg}), 21\end{array}$ & $\begin{array}{l}\text { Olanzapine } \\
\text { (Mean dose: } 3.5 \\
\text { Dose range: } 2.5 \\
\text { to } 10 \mathrm{mg} \text { ), } 21\end{array}$ & Not reported & EPS - parkinsonism & $1 / 21(4.8 \%)$ & $\begin{array}{l}0 / 21 \\
(0 \%)\end{array}$ & $\begin{array}{l}\text { RR } 3.00 \\
\text { (95\% Cl: } 0.13 \\
\text { to } 69.70)\end{array}$ \\
\hline Boettger, $2015^{21}$ & $\begin{array}{l}\text { Cohort w/ } \\
\text { comparison } \\
\text { group }\end{array}$ & Cancer patients & $\begin{array}{l}\text { Olanzapine (Mean dose: } \\
\text { 3.5 Dose range: } 2.5 \text { to } \\
\text { 10mg), } 21\end{array}$ & $\begin{array}{l}\text { Aripiprazole } \\
\text { (Mean dose: } 15.2 \\
\text { Dose range: } 5 \text { to } \\
\text { 30mg), } 21\end{array}$ & Not reported & $\begin{array}{l}\text { Extrapyramidal } \\
\text { symptoms }\end{array}$ & $0 / 21(0 \%)$ & $\begin{array}{l}0 / 21 \\
(0 \%)\end{array}$ & \\
\hline Boettger, $2015^{21}$ & $\begin{array}{l}\text { Cohort w/ } \\
\text { comparison } \\
\text { group }\end{array}$ & Cancer patients & $\begin{array}{l}\text { Risperidone (Mean dose: } \\
0.9 \text { Dose range: } 0.5 \text { to } \\
2 \mathrm{mg}), 21\end{array}$ & $\begin{array}{l}\text { Aripiprazole } \\
\text { (Mean dose: } 15.2 \\
\text { Dose range: } 5 \text { to } \\
\text { 30mg), } 21\end{array}$ & Not reported & $\begin{array}{l}\text { Extrapyramidal } \\
\text { symptoms }\end{array}$ & $1 / 21(4.8 \%)$ & $\begin{array}{l}0 / 21 \\
(0 \%)\end{array}$ & $\begin{array}{l}\text { RR } 3.00 \\
\text { (95\% Cl: } 0.13 \\
\text { to } 69.70)\end{array}$ \\
\hline Boettger, $2015^{21}$ & $\begin{array}{l}\text { Cohort w/ } \\
\text { comparison } \\
\text { group }\end{array}$ & Cancer patients & $\begin{array}{l}\text { Risperidone (Mean dose: } \\
0.9 \text { Dose range: } 0.5 \text { to } \\
2 \mathrm{mg}), 21\end{array}$ & $\begin{array}{l}\text { Olanzapine } \\
\text { (Mean dose: } 3.5 \\
\text { Dose range: } 2.5 \\
\text { to } 10 \mathrm{mg} \text { ), } 21\end{array}$ & Not reported & $\begin{array}{l}\text { Extrapyramidal } \\
\text { symptoms }\end{array}$ & $1 / 21(4.8 \%)$ & $\begin{array}{l}0 / 21 \\
(0 \%)\end{array}$ & $\begin{array}{l}\text { RR } 3.00 \\
\text { (95\% Cl: } 0.13 \\
\text { to } 69.70)\end{array}$ \\
\hline $\begin{array}{l}\text { Charoenporn, } \\
2018^{25}\end{array}$ & $\begin{array}{l}\text { Cohort w/ } \\
\text { comparison } \\
\text { group }\end{array}$ & Physically ill patients & $\begin{array}{l}\text { Risperidone (Mean dose: } \\
0.6 \text { Median dose: } \\
0.5 \mathrm{mg}), 14\end{array}$ & $\begin{array}{l}\text { Quetiapine (Mean } \\
\text { dose: } 26.7 \\
\text { Median dose: } 25) \text {, } \\
31\end{array}$ & Oral & $\begin{array}{l}\text { Extrapyramidal } \\
\text { symptoms }\end{array}$ & $\begin{array}{l}2 / 14 \\
(14.2 \%)\end{array}$ & $\begin{array}{l}3 / 31 \\
(9.7 \%)\end{array}$ & $\begin{array}{l}\text { RR 1.48 } \\
\text { (95\% Cl: } 0.28 \\
\text { to } 7.87)\end{array}$ \\
\hline Grover, $2011^{30}$ & RCT & $\begin{array}{l}\text { Consecutive patients }>-18 \text { years old } \\
\text { referred to psychiatry and diagnosed } \\
\text { with delirium }\end{array}$ & $\begin{array}{l}\text { Risperidone (Mean dose: } \\
\text { 0.95mg Dose range: } 0.5- \\
\text { 2.0mg per day), } 20\end{array}$ & $\begin{array}{l}\text { Olanzapine } \\
\text { (Mean dose: } \\
\text { 3.05mg Dose } \\
\text { range: } 1.25-10 \\
\text { mg per day), } 23\end{array}$ & Oral & Jaw movement & $1 / 20(5 \%)$ & $\begin{array}{l}0 / 23 \\
(0 \%)\end{array}$ & $\begin{array}{l}\text { RR } 3.43 \\
\text { (95\% Cl: } 0.15 \\
\text { to } 79.74)\end{array}$ \\
\hline Grover, $2011^{30}$ & RCT & $\begin{array}{l}\text { Consecutive patients }>-18 \text { years old } \\
\text { referred to psychiatry and diagnosed } \\
\text { with delirium }\end{array}$ & $\begin{array}{l}\text { Risperidone (Mean dose: } \\
\text { 0.95mg Dose range: } 0.5- \\
\text { 2.0mg per day), } 20\end{array}$ & $\begin{array}{l}\text { Olanzapine } \\
\text { (Mean dose: } \\
3.05 \mathrm{mg} \text { Dose } \\
\text { range: } 1.25-10 \\
\text { mg per day), } 23\end{array}$ & Oral & $\begin{array}{l}\text { Lips and perioral } \\
\text { movements }\end{array}$ & $1 / 20(5 \%)$ & $\begin{array}{l}0 / 23 \\
(0 \%)\end{array}$ & $\begin{array}{l}\text { RR } 3.43 \\
\text { (95\% Cl: } 0.15 \\
\text { to } 79.74)\end{array}$ \\
\hline
\end{tabular}




\begin{tabular}{|c|c|c|c|c|c|c|c|c|c|}
\hline Author, year & $\begin{array}{c}\text { Study } \\
\text { design }\end{array}$ & Population & Intervention group, $\mathbf{n}$ & Control group, $\mathbf{n}$ & $\begin{array}{c}\text { Route of } \\
\text { administration }\end{array}$ & Outcome definition & $\begin{array}{l}\mathrm{n} / \mathrm{N}(\%) \\
\text { intervention } \\
\text { group }\end{array}$ & $\begin{array}{l}\mathrm{n} / \mathrm{N}(\%) \\
\text { control } \\
\text { group }\end{array}$ & $\begin{array}{c}\text { Relative risk } \\
(95 \% \mathrm{Cl})\end{array}$ \\
\hline Grover, $2011^{30}$ & RCT & $\begin{array}{l}\text { Consecutive patients }>-18 \text { years old } \\
\text { referred to psychiatry and diagnosed } \\
\text { with delirium }\end{array}$ & $\begin{array}{l}\text { Risperidone (Mean dose: } \\
\text { 0.95mg Dose range: } 0.5- \\
2.0 \mathrm{mg} \text { per day), } 20\end{array}$ & $\begin{array}{l}\text { Olanzapine } \\
\text { (Mean dose: } \\
3.05 \mathrm{mg} \text { Dose } \\
\text { range: } 1.25-10 \\
\text { mg per day), } 23\end{array}$ & Oral & $\begin{array}{l}\text { Movements of the } \\
\text { tongue }\end{array}$ & $2 / 20(10 \%)$ & $\begin{array}{l}0 / 23 \\
(0 \%)\end{array}$ & $\begin{array}{l}\text { RR } 5.71 \\
\text { (95\% Cl: } 0.29 \\
\text { to } 112.43)\end{array}$ \\
\hline Grover, $2011^{30}$ & RCT & $\begin{array}{l}\text { Consecutive patients }>-18 \text { years old } \\
\text { referred to psychiatry and diagnosed } \\
\text { with delirium }\end{array}$ & $\begin{array}{l}\text { Risperidone (Mean dose: } \\
\text { 0.95mg Dose range: } 0.5- \\
\text { 2.0mg per day), } 20\end{array}$ & $\begin{array}{l}\text { Olanzapine } \\
\text { (Mean dose: } \\
\text { 3.05mg Dose } \\
\text { range: } 1.25-10 \\
\text { mg per day), } 23\end{array}$ & Oral & Rigidity & $2 / 20(10 \%)$ & $\begin{array}{l}0 / 23 \\
(0 \%)\end{array}$ & $\begin{array}{l}\text { RR 5.71 } \\
\text { (95\% Cl: } 0.29 \\
\text { to } 112.43)\end{array}$ \\
\hline Grover, $2011^{30}$ & RCT & $\begin{array}{l}\text { Consecutive patients }>-18 \text { years old } \\
\text { referred to psychiatry and diagnosed } \\
\text { with delirium }\end{array}$ & $\begin{array}{l}\text { Risperidone (Mean dose: } \\
\text { 0.95mg Dose range: } 0.5- \\
2.0 \mathrm{mg} \text { per day), } 20\end{array}$ & $\begin{array}{l}\text { Olanzapine } \\
\text { (Mean dose: } \\
\text { 3.05mg Dose } \\
\text { range: } 1.25-10 \\
\text { mg per day), } 23\end{array}$ & Oral & Tremors & $2 / 20(10 \%)$ & $\begin{array}{l}2 / 23 \\
(8.7 \%)\end{array}$ & $\begin{array}{l}\text { RR 1.15 } \\
\text { (95\% Cl: } 0.18 \\
\text { to } 7.43)\end{array}$ \\
\hline Hatta, 2014 33 & $\begin{array}{l}\text { Cohort wl } \\
\text { comparison } \\
\text { group }\end{array}$ & $\begin{array}{l}\text { Patients in a general hospital who } \\
\text { developed delirium and were managed } \\
\text { by a psychiatrist }\end{array}$ & $\begin{array}{l}\text { Olanzapine (Max dose: } \\
\text { 10.2mg), } 87\end{array}$ & $\begin{array}{l}\text { Aripiprazole (Max } \\
\text { dose: } 7.23 \mathrm{mg}), 61\end{array}$ & Not reported & $\begin{array}{l}\text { Extrapyramidal } \\
\text { symptoms }\end{array}$ & $4 / 87$ (4.6\%) & $\begin{array}{l}2 / 61 \\
(3.3 \%)\end{array}$ & $\begin{array}{l}\text { RR 1.40 } \\
\text { (95\% Cl: } 0.27 \\
\text { to } 7.42)\end{array}$ \\
\hline Hatta, $2014^{33}$ & $\begin{array}{l}\text { Cohort w/ } \\
\text { comparison } \\
\text { group }\end{array}$ & $\begin{array}{l}\text { Patients in a general hospital who } \\
\text { developed delirium and were managed } \\
\text { by a psychiatrist }\end{array}$ & $\begin{array}{l}\text { Quetiapine (Max dose: } \\
71.8 \mathrm{mg}), 779\end{array}$ & $\begin{array}{l}\text { Aripiprazole (Max } \\
\text { dose: } 7.23 \mathrm{mg}), 61\end{array}$ & Not applicable & $\begin{array}{l}\text { Extrapyramidal } \\
\text { symptoms }\end{array}$ & $\begin{array}{l}34 / 779 \\
(4.4 \%)\end{array}$ & $\begin{array}{l}2 / 61 \\
(3.3 \%)\end{array}$ & $\begin{array}{l}\text { RR } 1.33 \\
\text { (95\% Cl: } 0.33 \\
\text { to } 5.41)\end{array}$ \\
\hline Hatta, $2014^{33}$ & $\begin{array}{l}\text { Cohort w/ } \\
\text { comparison } \\
\text { group }\end{array}$ & $\begin{array}{l}\text { Patients in a general hospital who } \\
\text { developed delirium and were managed } \\
\text { by a psychiatrist }\end{array}$ & $\begin{array}{l}\text { Risperidone (Max dose: } \\
1.35 \mathrm{mg}), 835\end{array}$ & $\begin{array}{l}\text { Quetiapine (Max } \\
\text { dose: } 71.8 \mathrm{mg}) \\
779\end{array}$ & Not applicable & $\begin{array}{l}\text { Extrapyramidal } \\
\text { symptoms }\end{array}$ & $\begin{array}{l}53 / 835 \\
(6.3 \%)\end{array}$ & $\begin{array}{l}34 / 779 \\
(4.4 \%)\end{array}$ & $\begin{array}{l}\text { RR 1.45 } \\
\text { (95\% Cl: } 0.96 \\
\text { to 2.21) }\end{array}$ \\
\hline Hatta, $2014^{33}$ & $\begin{array}{l}\text { Cohort w/ } \\
\text { comparison } \\
\text { group }\end{array}$ & $\begin{array}{l}\text { Patients in a general hospital who } \\
\text { developed delirium and were managed } \\
\text { by a psychiatrist }\end{array}$ & $\begin{array}{l}\text { Risperidone (Max dose: } \\
1.35 \mathrm{mg}), 835\end{array}$ & $\begin{array}{l}\text { Aripiprazole (Max } \\
\text { dose: } 7.23 \mathrm{mg}), 61\end{array}$ & Not applicable & $\begin{array}{l}\text { Extrapyramidal } \\
\text { symptoms }\end{array}$ & $\begin{array}{l}53 / 835 \\
(6.3 \%)\end{array}$ & $\begin{array}{l}2 / 61 \\
(3.3 \%)\end{array}$ & $\begin{array}{l}\text { RR 1.94 } \\
\text { (95\% Cl: } 0.48 \\
\text { to } 7.75)\end{array}$ \\
\hline Hatta, $2014^{33}$ & $\begin{array}{l}\text { Cohort w/ } \\
\text { comparison } \\
\text { group }\end{array}$ & $\begin{array}{l}\text { Patients in a general hospital who } \\
\text { developed delirium and were managed } \\
\text { by a psychiatrist }\end{array}$ & $\begin{array}{l}\text { Risperidone (Max dose: } \\
1.35 \mathrm{mg}), 835\end{array}$ & $\begin{array}{l}\text { Olanzapine (Max } \\
\text { dose: 10.2mg), } 87\end{array}$ & Not applicable & $\begin{array}{l}\text { Extrapyramidal } \\
\text { symptoms }\end{array}$ & $\begin{array}{l}53 / 835 \\
(6.3 \%)\end{array}$ & $\begin{array}{l}4 / 87 \\
(4.6 \%)\end{array}$ & $\begin{array}{l}\text { RR 1.38 } \\
\text { (95\% Cl: } 0.51 \\
\text { to 3.72) }\end{array}$ \\
\hline Hatta, $2014^{33}$ & $\begin{array}{l}\text { Cohort wl } \\
\text { comparison } \\
\text { group }\end{array}$ & $\begin{array}{l}\text { Patients in a general hospital who } \\
\text { developed delirium and were managed } \\
\text { by a psychiatrist }\end{array}$ & $\begin{array}{l}\text { Quetiapine (Max dose: } \\
71.8 \mathrm{mg}), 779\end{array}$ & $\begin{array}{l}\text { Olanzapine (Max } \\
\text { dose: } 10.2 \mathrm{mg}), 87\end{array}$ & Not applicable & $\begin{array}{l}\text { Extrapyramidal } \\
\text { symptoms }\end{array}$ & $\begin{array}{l}34 / 779 \\
(4.4 \%)\end{array}$ & $\begin{array}{l}4 / 87 \\
(4.6 \%)\end{array}$ & $\begin{array}{l}\text { RR } 0.95 \\
\text { (95\% Cl: } 0.35 \\
\text { to 2.61) }\end{array}$ \\
\hline
\end{tabular}




\begin{tabular}{|c|c|c|c|c|c|c|c|c|c|}
\hline Author, year & $\begin{array}{c}\text { Study } \\
\text { design }\end{array}$ & Population & Intervention group, $\mathbf{n}$ & Control group, $n$ & $\begin{array}{c}\text { Route of } \\
\text { administration }\end{array}$ & Outcome definition & $\begin{array}{l}\mathrm{n} / \mathrm{N}(\%) \\
\text { intervention } \\
\text { group }\end{array}$ & $\begin{array}{c}\mathrm{n} / \mathrm{N}(\%) \\
\text { control } \\
\text { group }\end{array}$ & $\begin{array}{l}\text { Relative risk } \\
(95 \% \mathrm{Cl})\end{array}$ \\
\hline Kim, $2010^{42}$ & RCT & $\begin{array}{l}\text { General hospital inpatients who met } \\
\text { DSM-IV diagnostic criteria for delirium }\end{array}$ & $\begin{array}{l}\text { Risperidone (Mean dose: } \\
0.9 \pm 0.6 \text { Dose range: } \\
0.25-2 \mathrm{mg}), 17\end{array}$ & $\begin{array}{l}\text { Olanzapine } \\
\text { (Mean dose: } 2.4 \pm \\
\text { 1.7 Dose range: } \\
1.25-7.5 \mathrm{mg} \text { ), } 15\end{array}$ & Not reported & Akathisia & $0 / 17(0 \%)$ & $\begin{array}{l}2 / 15 \\
(13.3 \%)\end{array}$ & $\begin{array}{l}\text { RR } 0.18 \\
\text { (95\% Cl: } 0.01 \\
\text { to } 3.43 \text { ) }\end{array}$ \\
\hline Kim, $2010^{42}$ & RCT & $\begin{array}{l}\text { General hospital inpatients who met } \\
\text { DSM-IV diagnostic criteria for delirium }\end{array}$ & $\begin{array}{l}\text { Risperidone (Mean dose: } \\
0.9 \pm 0.6 \text { Dose range: } \\
0.25-2 \mathrm{mg}), 17\end{array}$ & $\begin{array}{l}\text { Olanzapine } \\
\text { (Mean dose: } 2.4 \pm \\
\text { 1.7 Dose range: } \\
1.25-7.5 \mathrm{mg} \text { ), } 15\end{array}$ & Not reported & $\begin{array}{l}\text { Tremor and } \\
\text { bradykinesia }\end{array}$ & $\begin{array}{l}2 / 17 \\
(11.8 \%)\end{array}$ & $\begin{array}{l}1 / 15 \\
(6.7 \%)\end{array}$ & $\begin{array}{l}\text { RR } 1.76 \\
\text { (95\% Cl: } 0.18 \\
\text { to } 17.56)\end{array}$ \\
\hline Lee, $2005^{45}$ & RCT & $\begin{array}{l}\text { Patients referred to the Psychiatric } \\
\text { Consultation that met DSM-IV criteria } \\
\text { for delirium }\end{array}$ & 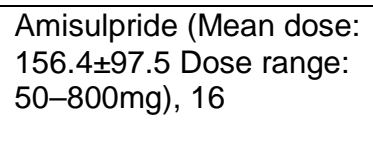 & $\begin{array}{l}\text { Quetiapine (Mean } \\
\text { dose: } 113 \pm 85.5 \\
\text { Dose range: } 50- \\
\text { 300mg), } 15\end{array}$ & Not reported & Acute dystonia & $0 / 16(0 \%)$ & $\begin{array}{l}0 / 15 \\
(0 \%)\end{array}$ & \\
\hline Lee, $2005^{45}$ & RCT & $\begin{array}{l}\text { Patients referred to the Psychiatric } \\
\text { Consultation that met DSM-IV criteria } \\
\text { for delirium }\end{array}$ & 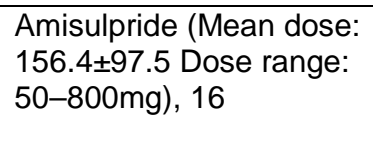 & $\begin{array}{l}\text { Quetiapine (Mean } \\
\text { dose: } 113 \pm 85.5 \\
\text { Dose range: } 50- \\
\text { 300mg), } 15\end{array}$ & Not reported & Dyskinesia & $0 / 16(0 \%)$ & $\begin{array}{l}0 / 15 \\
(0 \%)\end{array}$ & \\
\hline Yoon, $2013^{62}$ & $\begin{array}{l}\text { Cohort w/ } \\
\text { comparison } \\
\text { group }\end{array}$ & $\begin{array}{l}\text { Referred to consultation-liaison } \\
\text { psychiatric service for a mental status } \\
\text { change }\end{array}$ & $\begin{array}{l}\text { Quetiapine (Mean dose: } \\
47.9 \text { Dose range: } 25- \\
\text { 200mg), } 18\end{array}$ & $\begin{array}{l}\text { Olanzapine } \\
\text { (Mean dose: } 2.9 \\
\text { Dose range: } 1- \\
\text { 20mg), } 18\end{array}$ & Not reported & Akathisia & $0 / 18(0 \%)$ & $\begin{array}{l}0 / 18 \\
(0 \%)\end{array}$ & \\
\hline Yoon, $2013^{62}$ & $\begin{array}{l}\text { Cohort w/ } \\
\text { comparison } \\
\text { group }\end{array}$ & $\begin{array}{l}\text { Referred to consultation-liaison } \\
\text { psychiatric service for a mental status } \\
\text { change }\end{array}$ & $\begin{array}{l}\text { Risperidone (Mean dose: } \\
1.1 \text { Dose range: } 0.25- \\
4 \mathrm{mg}), 21\end{array}$ & $\begin{array}{l}\text { Olanzapine } \\
\text { (Mean dose: } 2.9 \\
\text { Dose range: } 1- \\
\text { 20mg), } 18\end{array}$ & Not reported & Akathisia & $0 / 21(0 \%)$ & $\begin{array}{l}0 / 18 \\
(0 \%)\end{array}$ & \\
\hline Yoon, $2013^{62}$ & $\begin{array}{l}\text { Cohort w/ } \\
\text { comparison } \\
\text { group }\end{array}$ & $\begin{array}{l}\text { Referred to consultation-liaison } \\
\text { psychiatric service for a mental status } \\
\text { change }\end{array}$ & $\begin{array}{l}\text { Risperidone (Mean dose: } \\
1.1 \text { Dose range: } 0.25- \\
4 \mathrm{mg}), 21\end{array}$ & $\begin{array}{l}\text { Quetiapine (Mean } \\
\text { dose: } 47.9 \text { Dose } \\
\text { range: } 25- \\
200 \mathrm{mg}), 18\end{array}$ & Not reported & Akathisia & $0 / 21(0 \%)$ & $\begin{array}{l}0 / 18 \\
(0 \%)\end{array}$ & \\
\hline Yoon, $2013^{62}$ & $\begin{array}{l}\text { Cohort w/ } \\
\text { comparison } \\
\text { group }\end{array}$ & $\begin{array}{l}\text { Referred to consultation-liaison } \\
\text { psychiatric service for a mental status } \\
\text { change }\end{array}$ & $\begin{array}{l}\text { Risperidone (Mean dose: } \\
1.1 \text { Dose range: } 0.25- \\
4 \mathrm{mg}), 21\end{array}$ & $\begin{array}{l}\text { Quetiapine (Mean } \\
\text { dose: } 47.9 \text { Dose } \\
\text { range: } 25- \\
200 \mathrm{mg} \text { ), } 18\end{array}$ & Not reported & Bradykinesia & $1 / 21(4.8 \%)$ & $\begin{array}{l}0 / 18 \\
(0 \%)\end{array}$ & $\begin{array}{l}\text { RR 2.59 } \\
\text { (95\% Cl: } 0.11 \\
\text { to } 59.93)\end{array}$ \\
\hline Yoon, $2013^{62}$ & $\begin{array}{l}\text { Cohort w/ } \\
\text { comparison } \\
\text { group }\end{array}$ & $\begin{array}{l}\text { Referred to consultation-liaison } \\
\text { psychiatric service for a mental status } \\
\text { change }\end{array}$ & $\begin{array}{l}\text { Quetiapine (Mean dose: } \\
\text { 47.9 Dose range: } 25- \\
\text { 200mg), } 18\end{array}$ & $\begin{array}{l}\text { Olanzapine } \\
\text { (Mean dose: } 2.9 \\
\text { Dose range: } 1- \\
\text { 20mg), } 18\end{array}$ & Not reported & Bradykinesia & $0 / 18(0 \%)$ & $\begin{array}{l}1 / 18 \\
(5.6 \%)\end{array}$ & $\begin{array}{l}\text { RR } 0.33 \\
\text { (95\% Cl: } 0.01 \\
\text { to } 7.68)\end{array}$ \\
\hline
\end{tabular}




\begin{tabular}{|c|c|c|c|c|c|c|c|c|c|}
\hline Author, year & $\begin{array}{c}\text { Study } \\
\text { design }\end{array}$ & Population & Intervention group, $\mathbf{n}$ & Control group, $n$ & $\begin{array}{c}\text { Route of } \\
\text { administratio } \\
n\end{array}$ & $\begin{array}{l}\text { Outcome } \\
\text { definition }\end{array}$ & $\begin{array}{c}\mathrm{n} / \mathrm{N}(\%) \\
\text { interventio } \\
\mathrm{n} \text { group }\end{array}$ & $\begin{array}{c}\mathrm{n} / \mathrm{N}(\%) \\
\text { control } \\
\text { group }\end{array}$ & $\begin{array}{c}\text { Relative risk } \\
(95 \% \mathrm{Cl})\end{array}$ \\
\hline Yoon, $2013^{62}$ & $\begin{array}{l}\text { Cohort w/ } \\
\text { compariso } \\
\text { n group }\end{array}$ & $\begin{array}{l}\text { Referred to consultation-liaison } \\
\text { psychiatric service for a mental status } \\
\text { change }\end{array}$ & $\begin{array}{l}\text { Risperidone (Mean dose: } \\
1.1 \text { Dose range: } 0.25- \\
4 \mathrm{mg}), 21\end{array}$ & $\begin{array}{l}\text { Olanzapine (Mean } \\
\text { dose: } 2.9 \text { Dose range: } \\
\text { 1-20mg), } 18\end{array}$ & Not reported & Bradykinesia & $\begin{array}{l}1 / 21 \\
(4.8 \%)\end{array}$ & $\begin{array}{l}1 / 18 \\
(5.6 \%)\end{array}$ & $\begin{array}{l}\text { RR } 0.86 \\
\text { (95\% Cl: } 0.06 \\
\text { to } 12.75)\end{array}$ \\
\hline Yoon, $2013^{62}$ & $\begin{array}{l}\text { Cohort w/ } \\
\text { compariso } \\
\text { n group }\end{array}$ & $\begin{array}{l}\text { Referred to consultation-liaison } \\
\text { psychiatric service for a mental status } \\
\text { change }\end{array}$ & $\begin{array}{l}\text { Quetiapine (Mean dose: } \\
\text { 47.9 Dose range: } 25- \\
\text { 200mg), } 18\end{array}$ & $\begin{array}{l}\text { Olanzapine (Mean } \\
\text { dose: } 2.9 \text { Dose range: } \\
\text { 1-20mg), } 18\end{array}$ & Not reported & Dystonia & $0 / 18(0 \%)$ & $\begin{array}{l}0 / 18 \\
(0 \%)\end{array}$ & \\
\hline Yoon, $2013^{62}$ & $\begin{array}{l}\text { Cohort w/ } \\
\text { compariso } \\
\text { n group }\end{array}$ & $\begin{array}{l}\text { Referred to consultation-liaison } \\
\text { psychiatric service for a mental status } \\
\text { change }\end{array}$ & $\begin{array}{l}\text { Risperidone (Mean dose: } \\
1.1 \text { Dose range: } 0.25- \\
4 \mathrm{mg}), 21\end{array}$ & $\begin{array}{l}\text { Quetiapine (Mean dose: } \\
\text { 47.9 Dose range: } 25- \\
\text { 200mg), } 18\end{array}$ & Not reported & Dystonia & $0 / 21(0 \%)$ & $\begin{array}{l}0 / 18 \\
(0 \%)\end{array}$ & \\
\hline Yoon, $2013^{62}$ & $\begin{array}{l}\text { Cohort w/ } \\
\text { compariso } \\
\text { n group }\end{array}$ & $\begin{array}{l}\text { Referred to consultation-liaison } \\
\text { psychiatric service for a mental status } \\
\text { change }\end{array}$ & $\begin{array}{l}\text { Risperidone (Mean dose: } \\
1.1 \text { Dose range: } 0.25- \\
4 \mathrm{mg}), 21\end{array}$ & $\begin{array}{l}\text { Olanzapine (Mean } \\
\text { dose: } 2.9 \text { Dose range: } \\
\text { 1-20mg), } 18\end{array}$ & Not reported & Dystonia & $0 / 21(0 \%)$ & $\begin{array}{l}0 / 18 \\
(0 \%)\end{array}$ & \\
\hline Yoon, $2013^{62}$ & $\begin{array}{l}\text { Cohort w/ } \\
\text { compariso } \\
\text { n group }\end{array}$ & $\begin{array}{l}\text { Referred to consultation-liaison } \\
\text { psychiatric service for a mental status } \\
\text { change }\end{array}$ & $\begin{array}{l}\text { Quetiapine (Mean dose: } \\
\text { 47.9 Dose range: } 25- \\
\text { 200mg), } 18\end{array}$ & $\begin{array}{l}\text { Olanzapine (Mean } \\
\text { dose: } 2.9 \text { Dose range: } \\
\text { 1-20mg), } 18\end{array}$ & Not reported & Rigidity & $\begin{array}{l}1 / 18 \\
(5.6 \%)\end{array}$ & $\begin{array}{l}1 / 18 \\
(5.6 \%)\end{array}$ & $\begin{array}{l}\text { RR 1.00 } \\
\text { (95\% Cl: } 0.07 \\
\text { to } 14.79)\end{array}$ \\
\hline Yoon, $2013^{62}$ & $\begin{array}{l}\text { Cohort w/ } \\
\text { compariso } \\
\text { n group }\end{array}$ & $\begin{array}{l}\text { Referred to consultation-liaison } \\
\text { psychiatric service for a mental status } \\
\text { change }\end{array}$ & $\begin{array}{l}\text { Risperidone (Mean dose: } \\
1.1 \text { Dose range: } 0.25- \\
4 \mathrm{mg}), 21\end{array}$ & $\begin{array}{l}\text { Olanzapine (Mean } \\
\text { dose: } 2.9 \text { Dose range: } \\
\text { 1-20mg), } 18\end{array}$ & Not reported & Rigidity & $\begin{array}{l}1 / 21 \\
(4.8 \%)\end{array}$ & $\begin{array}{l}1 / 18 \\
(5.6 \%)\end{array}$ & $\begin{array}{l}\text { RR 0.86 } \\
\text { (95\% Cl: } 0.06 \\
\text { to } 12.75)\end{array}$ \\
\hline Yoon, $2013^{62}$ & $\begin{array}{l}\text { Cohort w/ } \\
\text { compariso } \\
\text { n group }\end{array}$ & $\begin{array}{l}\text { Referred to consultation-liaison } \\
\text { psychiatric service for a mental status } \\
\text { change }\end{array}$ & $\begin{array}{l}\text { Risperidone (Mean dose: } \\
1.1 \text { Dose range: } 0.25- \\
4 \mathrm{mg}), 21\end{array}$ & $\begin{array}{l}\text { Quetiapine (Mean dose: } \\
\text { 47.9 Dose range: } 25- \\
\text { 200mg), } 18\end{array}$ & Not reported & Rigidity & $\begin{array}{l}1 / 21 \\
(4.8 \%)\end{array}$ & $\begin{array}{l}1 / 18 \\
(5.6 \%)\end{array}$ & $\begin{array}{l}\text { RR } 0.86 \\
\text { (95\% Cl: } 0.06 \\
\text { to } 12.75)\end{array}$ \\
\hline Yoon, $2013^{62}$ & $\begin{array}{l}\text { Cohort w/ } \\
\text { compariso } \\
\text { n group }\end{array}$ & $\begin{array}{l}\text { Referred to consultation-liaison } \\
\text { psychiatric service for a mental status } \\
\text { change }\end{array}$ & $\begin{array}{l}\text { Risperidone (Mean dose: } \\
1.1 \text { Dose range: } 0.25- \\
4 \mathrm{mg}), 21\end{array}$ & $\begin{array}{l}\text { Olanzapine (Mean } \\
\text { dose: } 2.9 \text { Dose range: } \\
\text { 1-20mg), } 18\end{array}$ & Not reported & Tremors & $\begin{array}{l}2 / 21 \\
(9.5 \%)\end{array}$ & $\begin{array}{l}1 / 18 \\
(5.6 \%)\end{array}$ & $\begin{array}{l}\text { RR 1.71 } \\
\text { (95\% Cl: } 0.17 \\
\text { to } 17.38) \\
\end{array}$ \\
\hline Yoon, $2013^{62}$ & $\begin{array}{l}\text { Cohort w/ } \\
\text { compariso } \\
\text { n group }\end{array}$ & $\begin{array}{l}\text { Referred to consultation-liaison } \\
\text { psychiatric service for a mental status } \\
\text { change }\end{array}$ & $\begin{array}{l}\text { Quetiapine (Mean dose: } \\
47.9 \text { Dose range: } 25- \\
\text { 200mg), } 18\end{array}$ & $\begin{array}{l}\text { Olanzapine (Mean } \\
\text { dose: } 2.9 \text { Dose range: } \\
\text { 1-20mg), } 18\end{array}$ & Not reported & Tremors & $\begin{array}{l}1 / 18 \\
(5.6 \%)\end{array}$ & $\begin{array}{l}1 / 18 \\
(5.6 \%)\end{array}$ & $\begin{array}{l}\text { RR 1.00 } \\
\text { (95\% Cl: } 0.07 \\
\text { to } 14.79)\end{array}$ \\
\hline Yoon, $2013^{62}$ & $\begin{array}{l}\text { Cohort w/ } \\
\text { compariso } \\
\text { n group }\end{array}$ & $\begin{array}{l}\text { Referred to consultation-liaison } \\
\text { psychiatric service for a mental status } \\
\text { change }\end{array}$ & $\begin{array}{l}\text { Risperidone (Mean dose: } \\
\text { 1.1 Dose range: } 0.25- \\
\text { 4mg), } 21\end{array}$ & $\begin{array}{l}\text { Quetiapine (Mean dose: } \\
\text { 47.9 Dose range: } 25- \\
\text { 200mg), } 18\end{array}$ & Not reported & Tremors & $\begin{array}{l}2 / 21 \\
(9.5 \%)\end{array}$ & $\begin{array}{l}1 / 18 \\
(5.6 \%)\end{array}$ & $\begin{array}{l}\text { RR 1.71 } \\
\text { (95\% Cl: } 0.17 \\
\text { to } 17.38)\end{array}$ \\
\hline
\end{tabular}

CI=confidence interval; DSM-IV=Diagnostic and Statistical Manual of Mental Disorders, $4^{\text {th }}$ ed; EPS=extrapyramidal symptoms; ICU=intensive care unit; mg=milligram; ml=milliliter; MV=mechanical ventilation; $\mathrm{n}=$ =sample size;

$\mathrm{RCT}=$ randomized controlled trial; $\mathrm{RR}=$ risk ratio; $\mathrm{SD}=$ standard deviation 
Evidence Table D-51. Binary neurological outcomes for studies comparing interventions to treat delirium: single arm studies

\begin{tabular}{|c|c|c|c|c|c|c|}
\hline $\begin{array}{c}\text { Author, } \\
\text { year }\end{array}$ & Study design & Population & $\begin{array}{l}\text { Intervention group, } \mathbf{n} \\
\end{array}$ & $\begin{array}{c}\text { Route of } \\
\text { administration }\end{array}$ & $\begin{array}{l}\text { Outcome } \\
\text { definition }\end{array}$ & $\begin{array}{c}\mathrm{n} / \mathrm{N}(\%) \\
\text { intervention group }\end{array}$ \\
\hline $\begin{array}{l}\text { Horikawa, } \\
2003^{34}\end{array}$ & $\begin{array}{l}\text { Cohort w/o } \\
\text { comparison group }\end{array}$ & Medical and surgical inpatients & $\begin{array}{l}\text { Risperidone (Planned dose: } 0.5 \text { Max dose: } \\
1.7 \mathrm{mg}), 10\end{array}$ & Oral & Mild parkinsonism & $1 / 10(10 \%)$ \\
\hline $\begin{array}{l}\text { Ikezawa, } \\
2008^{37}\end{array}$ & Open-label trial & Elderly patients with hyperactive-hypervalent delirium & $\begin{array}{l}\text { Risperidone (Mean dose: } 1.5 \text { (0.7) Dose range: } \\
0.5-3 \mathrm{mg}), 22\end{array}$ & Oral & $\begin{array}{l}\text { Extrapyramidal } \\
\text { symptoms }\end{array}$ & $1 / 22(5 \%)$ \\
\hline $\begin{array}{l}\text { Kim, } \\
2003^{40}\end{array}$ & $\begin{array}{l}\text { Cohort w/o } \\
\text { comparison group }\end{array}$ & Patients in the acute medical units & $\begin{array}{l}\text { Quetiapine (Planned dose: } 25 \text { Mean dose: } \\
93.75 \mathrm{mg}), 12\end{array}$ & Not reported & $\begin{array}{l}\text { Extrapyramidal } \\
\text { symptoms }\end{array}$ & $0 / 12(0 \%)$ \\
\hline $\begin{array}{l}\text { Kishi, } \\
2012^{43}\end{array}$ & $\begin{array}{l}\text { Cohort w/o } \\
\text { comparison group }\end{array}$ & Delirious cancer patients & Risperidone (Mean dose: $1.4 \mathrm{mg}), 29$ & Oral & $\begin{array}{l}\text { Extrapyramidal } \\
\text { symptoms }\end{array}$ & $0 / 29(0 \%)$ \\
\hline $\begin{array}{l}\text { Maneeton, } \\
2007^{48}\end{array}$ & Open-label study & All physically ill in-patients whose pcp consulted psychiatrists & $\begin{array}{l}\text { Quetiapine (Mean dose: } 45.7 \text { (28.7) Max dose: } \\
100 \text { Dose range: } 25-100 \mathrm{mg}), 17\end{array}$ & Oral & $\begin{array}{l}\text { Extrapyramidal } \\
\text { symptoms }\end{array}$ & $2 / 17(12 \%)$ \\
\hline $\begin{array}{l}\text { Mittal, } \\
2004^{50}\end{array}$ & $\begin{array}{l}\text { Cohort w/o } \\
\text { comparison group }\end{array}$ & Hospitalized patients & Risperidone (Mean dose: $0.75 \mathrm{mg}$ ), 10 & Not reported & $\begin{array}{l}\text { ESRS dyskinesia } \\
\text { scale }\end{array}$ & $\begin{array}{l}\text { Baseline: Mean } 0.2 \\
\text { (SE 0.6) } \\
\text { Final: Mean } 0 \text { (Not } \\
\text { reported) }\end{array}$ \\
\hline $\begin{array}{l}\text { Mittal, } \\
2004^{50}\end{array}$ & $\begin{array}{l}\text { Cohort w/o } \\
\text { comparison group }\end{array}$ & Hospitalized patients & Risperidone (Mean dose: $0.75 \mathrm{mg}$ ), 10 & Not reported & $\begin{array}{l}\text { ESRS dystonia } \\
\text { scores }\end{array}$ & $\begin{array}{l}\text { Baseline: Mean } 0 \\
\text { (Not reported) } \\
\text { Final: Mean } 0 \text { (Not } \\
\text { reported) }\end{array}$ \\
\hline $\begin{array}{l}\text { Mittal, } \\
2004^{50}\end{array}$ & $\begin{array}{l}\text { Cohort w/o } \\
\text { comparison group }\end{array}$ & Hospitalized patients & Risperidone (Mean dose: $0.75 \mathrm{mg}$ ), 10 & Not reported & $\begin{array}{l}\text { ESRS } \\
\text { Parkinsonism scale }\end{array}$ & $\begin{array}{l}\text { Baseline: Mean } 1.9 \\
\text { (SE 2.7) } \\
\text { Final: Mean } 0.6 \text { (SE } \\
0.4 \text { ) }\end{array}$ \\
\hline $\begin{array}{l}\text { Omura, } \\
2003^{52}\end{array}$ & $\begin{array}{l}\text { Cohort w/o } \\
\text { comparison group }\end{array}$ & Hospitalized patients & $\begin{array}{l}\text { Quetiapine (Mean dose: } 54.7 \text { Dose range: } 25 \text { to } \\
125 \mathrm{mg} \text { ), } 24\end{array}$ & Not reported & $\begin{array}{l}\text { Extrapyramidal } \\
\text { symptoms }\end{array}$ & $0 / 24(0 \%)$ \\
\hline $\begin{array}{l}\mathrm{Pae}, \\
2004^{53}\end{array}$ & $\begin{array}{l}\text { Cohort w/o } \\
\text { comparison group }\end{array}$ & $\begin{array}{l}\text { Delirious patients recruited from the departments of } \\
\text { neurosurgery, orthopedic surgery, and oncology }\end{array}$ & Quetiapine (Mean dose: $127.1 \mathrm{mg}$ ), 22 & Oral & $\begin{array}{l}\text { Extrapyramidal } \\
\text { symptoms }\end{array}$ & $0 / 22(0 \%)$ \\
\hline $\begin{array}{l}\text { Parellada, } \\
2004^{54}\end{array}$ & $\begin{array}{l}\text { Cohort w/o } \\
\text { comparison group }\end{array}$ & General hospital patients & $\begin{array}{l}\text { Risperidone (Mean dose: day 1, 2.6; day 3, 2.6; } \\
\text { day } 7,1.5 \mathrm{mg}), 64\end{array}$ & Oral & $\begin{array}{l}\text { Neurologic } \\
\text { subscale of the } \\
\text { UKU }\end{array}$ & $\begin{array}{l}\text { Baseline: Mean } 1.2 \\
\text { (SE 0.3) } \\
\text { Final: Mean } 1 \text { (SE } \\
0.1 \text { ) }\end{array}$ \\
\hline $\begin{array}{l}\text { Parellada, } \\
2004^{54}\end{array}$ & $\begin{array}{l}\text { Cohort w/o } \\
\text { comparison group }\end{array}$ & General hospital patients & $\begin{array}{l}\text { Risperidone (Mean dose: day 1, 2.6; day 3, 2.6; } \\
\text { day 7, 1.5mg), } 64\end{array}$ & Oral & $\begin{array}{l}\text { Neurologic } \\
\text { subscale of the } \\
\text { UKU }\end{array}$ & $\begin{array}{l}\text { Baseline: Mean } 1.2 \\
\text { (SE 0.3) } \\
\text { Final: Mean } 1.1 \text { (SE } \\
0.1 \text { ) }\end{array}$ \\
\hline $\begin{array}{l}\text { Parellada, } \\
2004^{54}\end{array}$ & $\begin{array}{l}\text { Cohort w/o } \\
\text { comparison group }\end{array}$ & General hospital patients & $\begin{array}{l}\text { Risperidone (Mean dose: day 1, 2.6; day 3, 2.6; } \\
\text { day } 7,1.5 \mathrm{mg} \text { ), } 64\end{array}$ & Oral & $\begin{array}{l}\text { Cerebrovascular } \\
\text { events }\end{array}$ & $0 / 64(0 \%)$ \\
\hline
\end{tabular}




\begin{tabular}{|c|c|c|c|c|c|c|}
\hline $\begin{array}{c}\text { Author, } \\
\text { year }\end{array}$ & Study design & Population & Intervention group, $\mathbf{n}$ & $\begin{array}{c}\text { Route of } \\
\text { administration }\end{array}$ & $\begin{array}{l}\text { Outcome } \\
\text { definition }\end{array}$ & $\begin{array}{c}\mathbf{n} / \mathbf{N}(\%) \\
\text { intervention group }\end{array}$ \\
\hline $\begin{array}{l}\text { Sasaki, } \\
2003^{55}\end{array}$ & $\begin{array}{l}\text { Cohort w/o } \\
\text { comparison group }\end{array}$ & Inpatients and outpatients & $\begin{array}{l}\text { Quetiapine (Mean dose: } 44.9 \text { Max dose: } \\
63.5 \mathrm{mg}), 10\end{array}$ & Oral & DIEPSS score & $\begin{array}{l}\text { Baseline: Mean } 1.5 \\
\text { (SD 1.7) } \\
\text { Final: Mean } 0.7 \text { (SD } \\
\text { 1.3) }\end{array}$ \\
\hline $\begin{array}{l}\text { Straker, } \\
2006^{58}\end{array}$ & $\begin{array}{l}\text { Cohort w/o } \\
\text { comparison group }\end{array}$ & Medically ill delirium patients & $\begin{array}{l}\text { Aripiprazole (Mean dose: } 8.9 \text { Dose range: } \\
5 \text { to } 15 \mathrm{mg} \text { ), } 14\end{array}$ & Not reported & $\begin{array}{l}\text { Cerebrovascular } \\
\text { accidents }\end{array}$ & $0 / 14(0 \%)$ \\
\hline $\begin{array}{l}\text { Yoon, } \\
2011^{61}\end{array}$ & Open-label pilot trial & $\begin{array}{l}\text { Patients who were referred to psychiatrists at Korea } \\
\text { University Ansan Hospital }\end{array}$ & $\begin{array}{l}\text { Paliperidone (Mean dose: } 3.75 \text { (1.06) Max } \\
\text { dose: } 9 \mathrm{mg}), 15\end{array}$ & Oral & Akathisia & $2 / 15(13 \%)$ \\
\hline
\end{tabular}

University Ansan Hospital

dose: 9mg), 15

Akathisia

$2 / 15(13 \%)$ 


\begin{tabular}{|c|c|c|c|c|c|c|c|c|c|}
\hline Author, year & $\begin{array}{c}\text { Study } \\
\text { design }\end{array}$ & $\begin{array}{l}\text { Population } \\
\end{array}$ & Intervention group, $\mathbf{n}$ & Control group, $\mathbf{n}$ & $\begin{array}{c}\text { Route of } \\
\text { administration }\end{array}$ & $\begin{array}{l}\text { Outcome } \\
\text { definition }\end{array}$ & $\begin{array}{l}\text { Mean score, } \\
\text { intervention }\end{array}$ & $\begin{array}{l}\text { Mean } \\
\text { score, } \\
\text { control } \\
\text { group }\end{array}$ & $\begin{array}{l}\text { Relative } \\
\text { risk (95\% } \\
\text { Cl) }\end{array}$ \\
\hline \multicolumn{10}{|l|}{$\begin{array}{l}\text { First-generation } \\
\text { antipsychotic vs. } \\
\text { placebo }\end{array}$} \\
\hline Agar, $2017^{17}$ & RCT & $\begin{array}{l}\text { Patients in hospice and palliative } \\
\text { care with delirium }\end{array}$ & $\begin{array}{l}\text { Haloperidol (Max dose: } 4 \\
\text { mg/d), }\end{array}$ & $\begin{array}{l}\text { Placebo (Max dose: } 4 \\
\text { mg/day), }\end{array}$ & Oral & $\begin{array}{l}\text { Mean of Extra } \\
\text { pyramidal } \\
\text { symptom rating } \\
\text { scale. }\end{array}$ & Not reported & $\begin{array}{l}\text { Not } \\
\text { reported }\end{array}$ & $\begin{array}{l}\text { Mean } \\
\text { difference } \\
0.79(95 \% \\
\text { Cl: } 0.17 \text { to } \\
1.41) \\
p=0.01\end{array}$ \\
\hline \multicolumn{10}{|l|}{$\begin{array}{l}\text { Second- } \\
\text { generation } \\
\text { antipsychotic vs. } \\
\text { placebo } \\
\end{array}$} \\
\hline Agar, $2017^{17}$ & RCT & $\begin{array}{l}\text { Patients in hospice and palliative } \\
\text { care with delirium }\end{array}$ & $\begin{array}{l}\text { Risperidone (Max dose: } 4 \\
\mathrm{mg} / \mathrm{d} \text { ), }\end{array}$ & $\begin{array}{l}\text { Placebo (Max dose: } 4 \\
\text { mg/day), }\end{array}$ & Oral & $\begin{array}{l}\text { Mean of Extra } \\
\text { pyramidal } \\
\text { symptom rating } \\
\text { scale. }\end{array}$ & Not reported & $\begin{array}{l}\text { Not } \\
\text { reported }\end{array}$ & $\begin{array}{l}\text { Mean } \\
\text { difference } \\
0.73(95 \% \\
\mathrm{Cl}: 0.09 \text { to } \\
1.37), \\
\mathrm{p}=0.03\end{array}$ \\
\hline \multicolumn{10}{|l|}{$\begin{array}{l}\text { First-generation } \\
\text { antipsychotic vs. } \\
\text { first-generation } \\
\text { antipsychotic }\end{array}$} \\
\hline Breitbart, $1996^{22}$ & RCT & $\begin{array}{l}\text { Medically hospitalized adult } \\
\text { patients with AIDS and a DRS } \\
\text { score of } 13 \text { or greater (delirium) }\end{array}$ & $\begin{array}{l}\text { Haloperidol (Planned dose: } \\
\text { oral: } 0.25-5.0 \mathrm{mg}, \\
\text { Intramuscular: } 0.125-3.0 \mathrm{mg} \\
\text { Mean dose: } 1.4 \text { Dose range: } \\
0.4 \text { - 3.6mg), } 11\end{array}$ & $\begin{array}{l}\text { Chlorpromazine (Planned } \\
\text { dose: Oral: } 10-200 \mathrm{mg}, \\
\text { Intramuscular: 5-100mg } \\
\text { Mean dose: } 36 \text { Dose range: } \\
\text { 10-80mg), } 13\end{array}$ & $\begin{array}{l}\text { Oral or } \\
\text { intramuscular }\end{array}$ & $\begin{array}{l}\text { Extrapyramidal } \\
\text { Symptom Rating } \\
\text { Scale: } \\
\text { Parkinson's } \\
\text { subscale }\end{array}$ & $\begin{array}{l}\text { Baseline: } \\
\text { Mean } 7 \text { (SD } \\
6.8) \\
\text { Final: Mean } \\
5.54 \text { (SD } \\
6.76)\end{array}$ & $\begin{array}{l}\text { Baseline: } \\
\text { Mean } \\
7.42 \text { (SD } \\
8.08 \text { ) } \\
\text { Final: } \\
\text { Mean } \\
5.08 \text { (SD } \\
4.48 \text { ) }\end{array}$ & $\begin{array}{l}0.88(95 \% \\
\text { Cl: }-29.37 \text { to } \\
31.13)\end{array}$ \\
\hline
\end{tabular}




\begin{tabular}{|c|c|c|c|c|c|c|c|c|c|}
\hline Author, year & $\begin{array}{l}\text { Study } \\
\text { design }\end{array}$ & Population & Intervention group, $\mathbf{n}$ & Control group, $\mathbf{n}$ & $\begin{array}{c}\text { Route of } \\
\text { administration }\end{array}$ & $\begin{array}{l}\text { Outcome } \\
\text { definition }\end{array}$ & $\begin{array}{l}\text { Mean score, } \\
\text { intervention }\end{array}$ & $\begin{array}{l}\text { Mean } \\
\text { score, } \\
\text { control } \\
\text { group }\end{array}$ & $\begin{array}{l}\text { Relative } \\
\text { risk (95\% } \\
\text { Cl) }\end{array}$ \\
\hline \multicolumn{10}{|l|}{$\begin{array}{l}\text { First-generation } \\
\text { antipsychotic vs. } \\
\text { second-generation } \\
\text { antipsychotic } \\
\end{array}$} \\
\hline Maneeton, $2013^{49}$ & $\mathrm{RCT}$ & $\begin{array}{l}\text { General adult population (age 18- } \\
\text { 75) with hyperactive delirium, } \\
\text { referred to psychiatry consult } \\
\text { liaison service at tertiary care } \\
\text { hospital in Thailand }\end{array}$ & $\begin{array}{l}\text { Quetiapine (Mean dose: } \\
67.6 \text { (SD 9.7) Dose range: } \\
\text { 25-100mg), } 24\end{array}$ & $\begin{array}{l}\text { Haloperidol (Mean dose: } 0.8 \\
\text { (SD 0.3) Dose range: } 0.5- \\
2.0 \mathrm{mg}), 28\end{array}$ & Oral & MSAS score & $\begin{array}{l}\text { Baseline: } \\
\text { Mean } 0.1 \text { (SD } \\
0.5) \\
\text { Final: Mean } \\
0.3 \text { (SD 0.7) }\end{array}$ & $\begin{array}{l}\text { Baseline: } \\
\text { Mean 0.2 } \\
\text { (SD 0.8) } \\
\text { Final: } \\
\text { Mean 0.3 } \\
\text { (SD 1.1) }\end{array}$ & $\begin{array}{l}0.10(95 \% \\
\text { Cl: }-3.65 \text { to } \\
3.85)\end{array}$ \\
\hline Maneeton, $2013^{49}$ & $\mathrm{RCT}$ & $\begin{array}{l}\text { General adult population (age 18- } \\
\text { 75) with hyperactive delirium, } \\
\text { referred to psychiatry consult } \\
\text { liaison service at tertiary care } \\
\text { hospital in Thailand }\end{array}$ & $\begin{array}{l}\text { Quetiapine (Mean dose: } \\
67.6 \text { (SD 9.7) Dose range: } \\
\text { 25-100mg), } 24\end{array}$ & $\begin{array}{l}\text { Haloperidol (Mean dose: } 0.8 \\
\text { (SD 0.3) Dose range: } 0.5- \\
2.0 \mathrm{mg}), 28\end{array}$ & Oral & MSAS score & $\begin{array}{l}\text { Baseline: } \\
\text { Mean } 0.1 \text { (SD } \\
0.5) \\
\text { Final: Mean } \\
0.3(\text { SD } 0.7)\end{array}$ & $\begin{array}{l}\text { Baseline: } \\
\text { Mean } 0.2 \\
\text { (SD 0.8) } \\
\text { Final: } \\
\text { Mean 0.3 } \\
(\text { SD 1.1) }\end{array}$ & $\begin{array}{l}0.10(95 \% \\
\text { Cl: }-3.65 \text { to } \\
3.85)\end{array}$ \\
\hline \multicolumn{10}{|l|}{$\begin{array}{l}\text { First-generation } \\
\text { antipsychotic vs. } \\
\text { other }\end{array}$} \\
\hline Breitbart, $1996^{22}$ & $\mathrm{RCT}$ & $\begin{array}{l}\text { Medically hospitalized adult } \\
\text { patients with AIDS and a DRS } \\
\text { score of } 13 \text { or greater (delirium) }\end{array}$ & $\begin{array}{l}\text { Lorazepam (Planned dose: } \\
\text { Oral: 0.50-4.0mg, } \\
\text { Intramuscular: 0.2-2.0mg } \\
\text { Mean dose: } 4.6 \text { Dose range: } \\
1.3-7.9 \mathrm{mg}), 6\end{array}$ & $\begin{array}{l}\text { Chlorpromazine (Planned } \\
\text { dose: Oral: } 10-200 \mathrm{mg}, \\
\text { Intramuscular: 5-100mg } \\
\text { Mean dose: } 36 \text { Dose range: } \\
\text { 10-80mg), } 13\end{array}$ & $\begin{array}{l}\text { Oral or } \\
\text { intramuscular }\end{array}$ & $\begin{array}{l}\text { Extrapyramidal } \\
\text { Symptom Rating } \\
\text { Scale: } \\
\text { Parkinson's } \\
\text { subscale }\end{array}$ & $\begin{array}{l}\text { Baseline: } \\
\text { Mean 7.6 (SD } \\
\text { 10.11) } \\
\text { Final: Mean } \\
\text { 12.2 (SD } \\
\text { 8.93) }\end{array}$ & $\begin{array}{l}\text { Baseline: } \\
\text { Mean } \\
7.42 \text { (SD } \\
8.08) \\
\text { Final: } \\
\text { Mean } \\
5.08 \text { (SD } \\
4.48) \\
\end{array}$ & $\begin{array}{l}6.94(95 \% \\
\text { Cl: }-23.88 \text { to } \\
37.76)\end{array}$ \\
\hline Breitbart, $1996^{22}$ & $\mathrm{RCT}$ & $\begin{array}{l}\text { Medically hospitalized adult } \\
\text { patients with AIDS and a DRS } \\
\text { score of } 13 \text { or greater (delirium) }\end{array}$ & $\begin{array}{l}\text { Lorazepam (Planned dose: } \\
\text { Oral: 0.50-4.Omg, } \\
\text { Intramuscular: 0.2-2.0mg } \\
\text { Mean dose: } 4.6 \text { Dose range: } \\
1.3-7.9 \mathrm{mg}), 6\end{array}$ & $\begin{array}{l}\text { Haloperidol (Planned dose: } \\
\text { oral: } 0.25-5.0 \mathrm{mg}, \\
\text { Intramuscular: } 0.125-3.0 \mathrm{mg} \\
\text { Mean dose: } 1.4 \text { Dose range: } \\
0.4 \text { - 3.6mg), } 11\end{array}$ & $\begin{array}{l}\text { Oral or } \\
\text { intramuscular }\end{array}$ & $\begin{array}{l}\text { Extrapyramidal } \\
\text { Symptom Rating } \\
\text { Scale: } \\
\text { Parkinson's } \\
\text { subscale }\end{array}$ & $\begin{array}{l}\text { Baseline: } \\
\text { Mean 7.6 (SD } \\
\text { 10.11) } \\
\text { Final: Mean } \\
\text { 12.2 (SD } \\
\text { 8.93) }\end{array}$ & $\begin{array}{l}\text { Baseline: } \\
\text { Mean } 7 \\
\text { (SD 6.8) } \\
\text { Final: } \\
\text { Mean } \\
5.54 \text { (SD } \\
6.76) \\
\end{array}$ & $\begin{array}{l}6.06(95 \% \\
\text { Cl: }-27.11 \text { to } \\
39.23)\end{array}$ \\
\hline
\end{tabular}

AIDS=acquired immunodeficiency syndrome; CI=confidence interval; DRS=Delirium Rating Scale; Mg/day=milligram per day; Mg=milligram; P=p-value; SD=standard deviation 
Evidence Table D-53. Aspiration pneumonia outcomes for studies comparing interventions to treat delirium

\begin{tabular}{|c|c|c|c|c|c|c|c|c|c|}
\hline Author, year & Study design & Population & Intervention group, $\mathbf{n}$ & $\underset{n}{\text { Control group, }}$ & $\begin{array}{c}\text { Route of } \\
\text { administration }\end{array}$ & $\begin{array}{l}\text { Outcome } \\
\text { definition }\end{array}$ & $\begin{array}{l}\mathrm{n} / \mathrm{N}(\%) \\
\text { intervention } \\
\text { group }\end{array}$ & $\begin{array}{l}\mathrm{n} \text { I N (\%), } \\
\text { control } \\
\text { group }\end{array}$ & $\begin{array}{l}\text { Relative } \\
\text { risk (95\% } \\
\mathrm{Cl})\end{array}$ \\
\hline \multicolumn{10}{|c|}{$\begin{array}{l}\text { First-generation } \\
\text { antipsychotic vs. second- } \\
\text { generation antipsychotic }\end{array}$} \\
\hline Hatta, 2014 & $\begin{array}{l}\text { Cohort w/ } \\
\text { comparison } \\
\text { group }\end{array}$ & $\begin{array}{l}\text { Patients in a general hospital who } \\
\text { developed delirium and were } \\
\text { managed by a psychiatrist }\end{array}$ & $\begin{array}{l}\text { Quetiapine (Max dose: } \\
71.8 \mathrm{mg} \text { ), }\end{array}$ & $\begin{array}{l}\text { Haloperidol } \\
\text { (Max dose: } \\
6.40 \mathrm{mg} \text { ), }\end{array}$ & Not applicable & serious & $4 / 779(0.5 \%)$ & $\begin{array}{l}3 / 480 \\
(0.6 \%)\end{array}$ & $\begin{array}{l}0.82 \\
(0.18 \text { to } \\
3.65)\end{array}$ \\
\hline Hatta, $2014^{33}$ & $\begin{array}{l}\text { Cohort w/ } \\
\text { comparison } \\
\text { group }\end{array}$ & $\begin{array}{l}\text { Patients in a general hospital who } \\
\text { developed delirium and were } \\
\text { managed by a psychiatrist }\end{array}$ & $\begin{array}{l}\text { Olanzapine (Max dose: } \\
\text { 10.2mg), }\end{array}$ & $\begin{array}{l}\text { Haloperidol } \\
\text { (Max dose: } \\
6.40 \mathrm{mg} \text { ), }\end{array}$ & Not reported & serious & $2 / 87(2.3 \%)$ & $\begin{array}{l}3 / 480 \\
(0.6 \%)\end{array}$ & $\begin{array}{l}3.68 \\
(0.62 \text { to } \\
21.69) \\
\end{array}$ \\
\hline Hatta, 2014 33 & $\begin{array}{l}\text { Cohort w/ } \\
\text { comparison } \\
\text { group }\end{array}$ & $\begin{array}{l}\text { Patients in a general hospital who } \\
\text { developed delirium and were } \\
\text { managed by a psychiatrist }\end{array}$ & $\begin{array}{l}\text { Risperidone (Max dose: } \\
1.35 \mathrm{mg} \text { ), }\end{array}$ & $\begin{array}{l}\text { Haloperidol } \\
\text { (Max dose: } \\
6.40 \mathrm{mg} \text { ) }\end{array}$ & Not applicable & serious & $7 / 835(0.8 \%)$ & $\begin{array}{l}3 / 480 \\
(0.6 \%)\end{array}$ & $\begin{array}{l}1.34 \\
(0.35 \text { to } \\
5.16)\end{array}$ \\
\hline Hatta, $2014^{33}$ & $\begin{array}{l}\text { Cohort w/ } \\
\text { comparison } \\
\text { group }\end{array}$ & $\begin{array}{l}\text { Patients in a general hospital who } \\
\text { developed delirium and were } \\
\text { managed by a psychiatrist }\end{array}$ & $\begin{array}{l}\text { Haloperidol (Max dose: } \\
6.40 \mathrm{mg} \text { ), }\end{array}$ & $\begin{array}{l}\text { Aripiprazole } \\
\text { (Max dose: } \\
7.23 \mathrm{mg} \text { ), }\end{array}$ & Intravenous & serious & $3 / 480(0.6 \%)$ & $0 / 61(0 \%)$ & $\begin{array}{l}0.90 \\
(0.05 \text { to } \\
17.26)\end{array}$ \\
\hline
\end{tabular}

\section{Second-generation}

antipsychotic vs. second-

generation antipsychotic

Hatta, 201433

\begin{tabular}{|c|c|c|c|c|}
\hline Hatta, 2014 & $\begin{array}{l}\text { Cohort wl } \\
\text { comparison } \\
\text { group }\end{array}$ & $\begin{array}{l}\text { Patients in a general hospital who } \\
\text { developed delirium and were } \\
\text { managed by a psychiatrist }\end{array}$ & $\begin{array}{l}\text { Risperidone (Max dose: } \\
1.35 \mathrm{mg} \text { ), }\end{array}$ & $\begin{array}{l}\text { Quetiapine (Max } \\
\text { dose: 71.8mg), }\end{array}$ \\
\hline Hatta, 2014 $4^{33}$ & $\begin{array}{l}\text { Cohort w/ } \\
\text { comparison } \\
\text { group }\end{array}$ & $\begin{array}{l}\text { Patients in a general hospital who } \\
\text { developed delirium and were } \\
\text { managed by a psychiatrist }\end{array}$ & $\begin{array}{l}\text { Quetiapine (Max dose: } \\
\text { 71.8mg), }\end{array}$ & $\begin{array}{l}\text { Olanzapine } \\
\text { (Max dose: } \\
\text { 10.2mg), }\end{array}$ \\
\hline Hatta, $2014^{33}$ & $\begin{array}{l}\text { Cohort w/ } \\
\text { comparison } \\
\text { group }\end{array}$ & $\begin{array}{l}\text { Patients in a general hospital who } \\
\text { developed delirium and were } \\
\text { managed by a psychiatrist }\end{array}$ & $\begin{array}{l}\text { Risperidone (Max dose: } \\
1.35 \mathrm{mg}),\end{array}$ & $\begin{array}{l}\text { Olanzapine } \\
\text { (Max dose: } \\
\text { 10.2mg), }\end{array}$ \\
\hline Hatta, 2014 $4^{33}$ & $\begin{array}{l}\text { Cohort w/ } \\
\text { comparison } \\
\text { group }\end{array}$ & $\begin{array}{l}\text { Patients in a general hospital who } \\
\text { developed delirium and were } \\
\text { managed by a psychiatrist }\end{array}$ & $\begin{array}{l}\text { Quetiapine (Max dose: } \\
71.8 \mathrm{mg} \text { ), }\end{array}$ & $\begin{array}{l}\text { Aripiprazole } \\
\text { (Max dose: } \\
7.23 \mathrm{mg} \text { ), }\end{array}$ \\
\hline Hatta, $2014^{33}$ & $\begin{array}{l}\text { Cohort w/ } \\
\text { comparison } \\
\text { group }\end{array}$ & $\begin{array}{l}\text { Patients in a general hospital who } \\
\text { developed delirium and were } \\
\text { managed by a psychiatrist }\end{array}$ & $\begin{array}{l}\text { Olanzapine (Max dose: } \\
\text { 10.2mg), }\end{array}$ & $\begin{array}{l}\text { Aripiprazole } \\
\text { (Max dose: } \\
\text { 7.23mg), }\end{array}$ \\
\hline Hatta, $2014^{33}$ & $\begin{array}{l}\text { Cohort w/ } \\
\text { comparison } \\
\text { group }\end{array}$ & $\begin{array}{l}\text { Patients in a general hospital who } \\
\text { developed delirium and were } \\
\text { managed by a psychiatrist }\end{array}$ & $\begin{array}{l}\text { Risperidone (Max dose: } \\
\text { 1.35mg), }\end{array}$ & $\begin{array}{l}\text { Aripiprazole } \\
\text { (Max dose: } \\
7.23 \mathrm{mg} \text { ), }\end{array}$ \\
\hline
\end{tabular}

\begin{tabular}{|c|c|c|c|c|}
\hline Not applicable & serious & $7 / 835$ (0.8\%) & $\begin{array}{l}4 / 779 \\
(0.5 \%)\end{array}$ & $\begin{array}{l}1.63 \\
(0.48 \text { to } \\
5.56)\end{array}$ \\
\hline Not applicable & serious & $4 / 779(0.5 \%)$ & $\begin{array}{l}2 / 87 \\
(2.3 \%)\end{array}$ & $\begin{array}{l}0.22 \\
(0.04 \text { to } \\
1.20)\end{array}$ \\
\hline Not applicable & serious & $7 / 835$ (0.8\%) & $\begin{array}{l}2 / 87 \\
(2.3 \%)\end{array}$ & $\begin{array}{l}0.36 \\
(0.08 \text { to } \\
1.73)\end{array}$ \\
\hline Not applicable & serious & $4 / 779(0.5 \%)$ & $0 / 61(0 \%)$ & $\begin{array}{l}0.72 \\
(0.04 \text { to } \\
13.14)\end{array}$ \\
\hline Not reported & serious & $2 / 87(2.3 \%)$ & $0 / 61(0 \%)$ & $\begin{array}{l}3.52 \\
(0.17 \text { to } \\
72.11)\end{array}$ \\
\hline Not applicable & serious & $7 / 835(0.8 \%)$ & $0 / 61(0 \%)$ & $\begin{array}{l}1.11 \\
(0.06 \text { to } \\
19.25)\end{array}$ \\
\hline
\end{tabular}

$\mathrm{CI}=$ confidence interval; $\mathrm{N}=$ sample size; $\mathrm{mg}=$ =milligram 
Evidence Table D-54. Aspiration pneumonia outcomes for studies comparing interventions to treat delirium: single arm studies

\begin{tabular}{c|c|c|l|c|c} 
Author, year & Study design & Population & Intervention group, $\mathbf{n}$ & $\begin{array}{c}\text { Route of } \\
\text { administration }\end{array}$ \\
\hline
\end{tabular}
administration

Outcome

definition

n I N (\%), intervention

Straker,

Cohort w/o comparison

Medically ill delirium

Aripiprazole (Mean dose: 8.9 Dose range: 5 to

Not reported

Not applicable

group

$2006^{58} \quad$ group

\begin{tabular}{l|l} 
patients & $15 \mathrm{mg}$ ) \\
\hline
\end{tabular}

$1 / 14(7 \%)$ 
Evidence Table D-55. Cochrane Risk of Bias assessment for randomized controlled trials comparing interventions to prevent development of delirium

\begin{tabular}{|c|c|c|c|c|c|c|c|}
\hline Author, year & $\begin{array}{c}\text { Risk of Bias in } \\
\text { Sequence Generation } \\
\text { is: }\end{array}$ & $\begin{array}{c}\text { Risk of Bias in } \\
\text { Allocation } \\
\text { Concealment is: }\end{array}$ & $\begin{array}{l}\text { Risk of Bias in binding of } \\
\text { participants, personnel and } \\
\text { outcomes assessors is: }\end{array}$ & $\begin{array}{c}\text { Risk of Bias from } \\
\text { missing outcome } \\
\text { data }\end{array}$ & $\begin{array}{c}\text { Risk of Bias from } \\
\text { Selective Outcome } \\
\text { Reporting }\end{array}$ & $\begin{array}{c}\text { The study appears to be } \\
\text { free of other sources of } \\
\text { bias. }\end{array}$ & $\begin{array}{c}\text { Overall risk } \\
\text { of bias }\end{array}$ \\
\hline Abdelgalel, $2016^{1}$ & Low & Low & Unclear & Unclear & Unclear & Unclear & Unclear \\
\hline Al-Qadheeb, 2016² & Low & Low & Low & Low & Low & Yes & Low \\
\hline Fukata, $2014^{3}$ & Low & High & High & Unclear & Unclear & Yes & High \\
\hline Girard, $2010^{4}$ & Low & Low & Low & Low & Low & Yes & Low \\
\hline Hakim, $2012^{5}$ & Low & Low & Low & Low & Unclear & Yes & Low \\
\hline Kalisvaart, $2005^{6}$ & Low & Low & Low & Low & Low & Yes & Low \\
\hline Kaneko, $1999^{7}$ & Unclear & Unclear & Unclear & Unclear & Unclear & Unclear & Unclear \\
\hline Khan, $2018^{8}$ & Low & Low & Low & Unclear & Low & Low & Low \\
\hline Larsen, $2010^{9}$ & Low & Low & Low & Unclear & Low & Yes & Low \\
\hline Page, $2013^{10}$ & Low & Low & Unclear & Low & Low & No & Low \\
\hline Prakanrattana, $2007^{11}$ & Low & Low & High & Low & Unclear & Yes & High \\
\hline Schrijver, $2018^{12,13}$ & Low & Low & Low & Low & Low & Yes & Low \\
\hline van den Boogaard, $2018^{15}$ & Low & Low & Low & Low & Low & Yes & Low \\
\hline Wang, $2012^{16}$ & Low & Unclear & Low & Low & Unclear & Unclear & Unclear \\
\hline
\end{tabular}

Wang $2012^{16}$

Unclear

Low

Low

Unclear

Unclear

Low 
Evidence Table D-56. Cochrane Risk of Bias assessment for randomized controlled trials comparing interventions to treat delirium

\begin{tabular}{|c|c|c|c|c|c|c|c|}
\hline Author, year & $\begin{array}{c}\text { Risk of Bias in } \\
\text { Sequence Generation } \\
\text { is: }\end{array}$ & $\begin{array}{l}\text { Risk of Bias in } \\
\text { Allocation } \\
\text { Concealment is: }\end{array}$ & $\begin{array}{l}\text { Risk of Bias in binding of } \\
\text { participants, personnel and } \\
\text { outcomes assessors is: }\end{array}$ & $\begin{array}{l}\text { Risk of bias from } \\
\text { missing outcome } \\
\text { data }\end{array}$ & $\begin{array}{c}\text { Risk of Bias from } \\
\text { Selective Outcome } \\
\text { Reporting }\end{array}$ & $\begin{array}{l}\text { The study appears to be } \\
\text { free of other sources of } \\
\text { bias. }\end{array}$ & $\begin{array}{c}\text { Overall risk } \\
\text { of bias }\end{array}$ \\
\hline Agar, $2017^{17}$ & Low & Low & Low & Low & Low & Yes & Low \\
\hline Atalan, $2013^{18}$ & Unclear & Unclear & Low & Unclear & Unclear & Unclear & Unclear \\
\hline Bakri, $2015^{19}$ & Low & Low & Low & Unclear & Unclear & Unclear & Low \\
\hline Breitbart, $1996^{22}$ & Unclear & Unclear & Unclear & Unclear & Unclear & Unclear & Unclear \\
\hline Devlin, $2010^{26}$ & Low & Low & Low & Low & Unclear & Unclear & Low \\
\hline Girard, $2010^{4}$ & Low & Low & Low & Low & Low & Yes & Low \\
\hline Girard, $2018^{29}$ & Low & Low & Low & Low & Low & Unclear & Low \\
\hline Grover, $2011^{30}$ & Low & Unclear & Low & Unclear & Unclear & Unclear & Unclear \\
\hline Grover, $2016^{31}$ & Low & Unclear & Low & Low & Unclear & Unclear & Low \\
\hline Han, $2004^{32}$ & Unclear & Unclear & Unclear & Unclear & Unclear & Unclear & Unclear \\
\hline $\mathrm{Hu}, 2006^{35}$ & Unclear & Unclear & Unclear & Unclear & Unclear & Unclear & Unclear \\
\hline Jain, $2017^{38}$ & Low & Unclear & High & High & Unclear & Unclear & High \\
\hline Kim, $2010^{42}$ & Unclear & Unclear & Low & High & Unclear & Unclear & High \\
\hline Lee, $2005^{45}$ & Unclear & Unclear & High & Unclear & Unclear & Unclear & High \\
\hline Lim, $2007^{46}$ & Low & Low & Unclear & Low & Low & Low & Low \\
\hline Lin, $2008^{47}$ & Unclear & Unclear & High & High & Unclear & Yes & High \\
\hline Maneeton, $2013^{49}$ & Low & Unclear & Low & Low & Low & Unclear & Low \\
\hline Page, $2013^{10}$ & Low & Low & Unclear & Low & Low & No & Low \\
\hline Tahir, $2010^{59}$ & Low & Low & Low & Unclear & Unclear & Unclear & Low \\
\hline
\end{tabular}

Tahir, $2010^{59}$ Ligh risk of bias; Low=low risk of bias; Unclear=unclear risk of bias 
Evidence Table D-57. Cochrane Risk of Bias in non-randomized studies assessing interventions to prevent development of delirium

\begin{tabular}{|c|c|c|c|c|c|c|c|c|}
\hline Author, year & $\begin{array}{l}\text { Domain 1: Bias } \\
\text { Due to } \\
\text { Confounding }\end{array}$ & $\begin{array}{l}\text { Domain 2: Bias in } \\
\text { Selection of } \\
\text { Participants into Study }\end{array}$ & $\begin{array}{l}\text { Domain 3: Bias in } \\
\text { Classification of } \\
\text { Interventions }\end{array}$ & $\begin{array}{c}\text { Domain 4: Bias Due to } \\
\text { Deviations from Intended } \\
\text { Interventions }\end{array}$ & $\begin{array}{c}\text { Domain 5: Bias } \\
\text { Due to Missing } \\
\text { Data }\end{array}$ & $\begin{array}{c}\text { Domain 6: Bias in } \\
\text { Measurement of } \\
\text { Outcomes }\end{array}$ & $\begin{array}{l}\text { Domain 7: Bias in } \\
\text { Selection of } \\
\text { Reported Results }\end{array}$ & $\begin{array}{l}\text { Overall Risk of } \\
\text { Bias Judgment }\end{array}$ \\
\hline $\begin{array}{l}\text { van den } \\
\text { Boogaaard, } \\
2013^{14}\end{array}$ & Low & Low & Low & Low & Low & Moderate & Low & Moderate \\
\hline
\end{tabular}

$2013^{14} \quad$ Low

Low

Low

main 3: Bias

4: Bias Due to

Moderate 
Evidence Table D-58. Cochrane Risk of Bias in non-randomized studies assessing interventions to treat delirium

\begin{tabular}{|c|c|c|c|c|c|c|c|c|}
\hline Author, year & $\begin{array}{l}\text { Domain 1: Bias } \\
\text { Due to } \\
\text { Confounding }\end{array}$ & $\begin{array}{l}\text { Domain 2: Bias in } \\
\text { Selection of } \\
\text { Participants into Study }\end{array}$ & $\begin{array}{c}\text { Domain 3: Bias in } \\
\text { Classification of } \\
\text { Interventions }\end{array}$ & $\begin{array}{l}\text { Domain 4: Bias Due to } \\
\text { Deviations from Intended } \\
\text { Interventions }\end{array}$ & $\begin{array}{c}\text { Domain 5: Bias } \\
\text { Due to Missing } \\
\text { Data }\end{array}$ & $\begin{array}{c}\text { Domain 6: Bias in } \\
\text { Measurement of } \\
\text { Outcomes }\end{array}$ & $\begin{array}{l}\text { Domain 7: Bias in } \\
\text { Selection of } \\
\text { Reported Results }\end{array}$ & $\begin{array}{l}\text { Overall Risk of } \\
\text { Bias Judgment }\end{array}$ \\
\hline Boettger, $2011^{20}$ & Serious & Serious & Serious & Moderate & Moderate & Serious & Moderate & Serious \\
\hline Boettger, $2015^{21}$ & Serious & Moderate & Moderate & Serious & Moderate & Serious & Moderate & Serious \\
\hline Breitbart, $2002^{23}$ & Moderate & Moderate & Serious & Moderate & Moderate & Serious & Moderate & Moderate \\
\hline Carrasco, $2016^{24}$ & Serious & Serious & Moderate & Serious & Moderate & Serious & Moderate & Serious \\
\hline $\begin{array}{l}\text { Charoenporn, } \\
2018^{25}\end{array}$ & Moderate & Moderate & Serious & Moderate & Moderate & Serious & Moderate & Moderate \\
\hline Fox, $2018^{28}$ & Serious & Low & Low & Moderate & Low & Moderate & Low & Moderate \\
\hline Hatta, 2014 ${ }^{33}$ & Serious & Low & Moderate & Moderate & Low & Serious & Low & Serious \\
\hline Horikawa, $2003^{34}$ & Serious & Moderate & Serious & Serious & Moderate & Serious & Moderate & Serious \\
\hline Hui, $2017^{36}$ & Low & Low & Low & Low & Low & Low & Low & Low \\
\hline Ikezawa, $2008^{37}$ & Serious & Serious & Serious & Moderate & Moderate & Serious & Moderate & Serious \\
\hline Kim, $2001^{39}$ & Serious & Serious & Moderate & Moderate & Moderate & Low & Moderate & Serious \\
\hline Kim, $2003^{40}$ & Serious & Moderate & Serious & Moderate & Moderate & Serious & Moderate & Moderate \\
\hline Kim, $2005^{41}$ & Serious & Serious & Moderate & Moderate & Low & Moderate & Moderate & Serious \\
\hline Kishi, $2012^{43}$ & Serious & Serious & Low & Low & Low & Moderate & Low & Serious \\
\hline Maneeton, $2007^{48}$ & Serious & Moderate & Low & Low & Moderate & Moderate & Low & Serious \\
\hline Mittal, $2004^{50}$ & Serious & Low & Low & Low & Low & Moderate & Low & Moderate \\
\hline Naksuk, $2017^{51}$ & Serious & Moderate & Serious & Moderate & Moderate & Serious & Low & Serious \\
\hline Omura, $2003^{52}$ & Serious & Serious & Serious & Serious & Moderate & Serious & Moderate & Serious \\
\hline Pae, $2004^{53}$ & Serious & Serious & Serious & Serious & Moderate & Serious & Moderate & Serious \\
\hline Parellada, $2004^{54}$ & Serious & Moderate & Moderate & Moderate & Moderate & Serious & Moderate & Moderate \\
\hline Sasaki, $2003^{55}$ & Serious & Serious & Serious & Serious & Moderate & Serious & Moderate & Serious \\
\hline $\begin{array}{l}\text { Sipahimalani, } \\
1998^{56}\end{array}$ & Serious & Serious & Serious & Moderate & Moderate & Serious & Moderate & Serious \\
\hline Skrobik, 200457 & Low & Low & Low & Low & Low & Low & Low & Low \\
\hline Straker, $2006^{58}$ & Serious & Serious & Low & Moderate & Low & Moderate & Low & Serious \\
\hline Toda, $2005^{60}$ & Serious & Serious & Moderate & Low & Low & Moderate & Low & Serious \\
\hline Yoon, 2011 ${ }^{61}$ & Serious & Low & Low & Low & Low & Moderate & Low & Serious \\
\hline Yoon, 201362 & Serious & Low & Serious & Low & Low & Serious & Low & Serious \\
\hline
\end{tabular}

Low=low risk of bias; Moderate=moderate risk of bias; Serious=serious risk of bias 


\section{References for Appendix D}

1. Abdelgalel EF. Dexmedetomidine versus haloperidol for prevention of delirium during non-invasive mechanical ventilation. Egyptian Journal of Anaesthesia. 2016;32(4):473-81. doi: 10.1016/j.egja.2016.05.008.

2. Al-Qadheeb NS, Skrobik Y, Schumaker G, et al. Preventing ICU Subsyndromal Delirium Conversion to Delirium With Low-Dose IV Haloperidol: A Double-Blind, Placebo-Controlled Pilot Study. Crit Care Med. 2016 Mar;44(3):583-91. doi: 10.1097/ccm.0000000000001411. PMID: 26540397.

3. Fukata S, Kawabata Y, Fujisiro K, et al. Haloperidol prophylaxis does not prevent postoperative delirium in elderly patients: a randomized, open-label prospective trial. Surg Today. 2014 Dec;44(12):2305-13. doi: 10.1007/s00595-014-0859-7. PMID: 24532143.

4. Girard TD, Pandharipande PP, Carson SS, et al. Feasibility, efficacy, and safety of antipsychotics for intensive care unit delirium: the MIND randomized, placebocontrolled trial. Crit Care Med. 2010 Feb;38(2):428-37. PMID: 20095068.

5. Hakim SM, Othman AI, Naoum DO. Early treatment with risperidone for subsyndromal delirium after on-pump cardiac surgery in the elderly: a randomized trial. Anesthesiology. 2012 May;116(5):987-97. doi: 10.1097/ALN.0b013e31825153cc. PMID: 22436797.

6. Kalisvaart KJ, de Jonghe JF, Bogaards MJ, et al. Haloperidol prophylaxis for elderly hip-surgery patients at risk for delirium: a randomized placebo-controlled study. J Am Geriatr Soc. 2005 Oct;53(10):1658-66. doi: 10.1111/j.1532-5415.2005.53503.x. PMID: 16181163.

7. Kaneko T, Cai J, Ishikura T, et al. Prophylactic consecutive administration of haloperidol can reduce the occurrence of postoperative delirium in gastrointestinal surgery. Yonago Acta Medica. 1999;42(3):179-84.

8. Khan BA, Perkins AJ, Campbell NL, et al. Preventing Postoperative Delirium After Major Noncardiac Thoracic Surgery-A Randomized Clinical Trial. J Am Geriatr Soc. 2018 Dec;66(12):2289-97. doi: 10.1111/jgs.15640. PMID: 30460981.

9. Larsen KA, Kelly SE, Stern TA, et al. Administration of olanzapine to prevent postoperative delirium in elderly jointreplacement patients: a randomized, controlled trial. Psychosomatics. 2010 SepOct;51(5):409-18. doi: 10.1176/appi.psy.51.5.409. PMID: 20833940.
10. Page VJ, Ely EW, Gates S, et al. Effect of intravenous haloperidol on the duration of delirium and coma in critically ill patients (Hope-ICU): a randomised, double-blind, placebo-controlled trial. Lancet Respir Med. 2013 Sep;1(7):515-23. doi: 10.1016/s22132600(13)70166-8. PMID: 24461612.

11. Prakanrattana U, Prapaitrakool S. Efficacy of risperidone for prevention of postoperative delirium in cardiac surgery. Anaesth Intensive Care. 2007 Oct;35(5):714-9. PMID: 17933157.

12. Schrijver EJM, de Vries OJ, van de Ven PM, et al. Haloperidol versus placebo for delirium prevention in acutely hospitalised older at risk patients: a multi-centre doubleblind randomised controlled clinical trial. Age Ageing. 2018 Jan 1;47(1):48-55. doi: 10.1093/ageing/afx124. PMID: 28985255.

13. Schrijver EJM, Verstraaten M, Van De Ven $\mathrm{PM}$, et al. Low dose oral haloperidol does not prolong QTc interval in older acutely hospitalised adults: Results fromA subanalysis of a randomised double-blind placebo-controlled study. Journal of Geriatric Cardiology. 2018;15(6):401-7.

14. van den Boogaard $M$, Schoonhoven $L$, van Achterberg T, et al. Haloperidol prophylaxis in critically ill patients with a high risk for delirium. Crit Care. 2013 Jan 17;17(1):R9. doi: 10.1186/cc11933. PMID: 23327295.

15. van den Boogaard M, Slooter AJC, Bruggemann RJM, et al. Effect of Haloperidol on Survival Among Critically Ill Adults With a High Risk of Delirium: The REDUCE Randomized Clinical Trial. JAMA. 2018 Feb 20;319(7):680-90. doi: 10.1001/jama.2018.0160. PMID: 29466591.

16. Wang W, Li HL, Wang DX, et al. Haloperidol prophylaxis decreases delirium incidence in elderly patients after noncardiac surgery: a randomized controlled trial*. Crit Care Med. 2012 Mar;40(3):731-9. doi: 10.1097/CCM.0b013e3182376e4f. PMID: 22067628.

17. Agar MR, Lawlor PG, Quinn S, et al. Efficacy of Oral Risperidone, Haloperidol, or Placebo for Symptoms of Delirium Among Patients in Palliative Care: A Randomized Clinical Trial. JAMA Intern Med. 2017 Jan 1;177(1):34-42. doi: 10.1001/jamainternmed.2016.7491. PMID: 27918778.

18. Atalan N, Efe Sevim M, Akgun S, et al. Morphine is a reasonable alternative to haloperidol in the treatment of postoperative hyperactive-type delirium after cardiac surgery. J Cardiothorac Vasc Anesth. 2013 Oct;27(5):933-8. doi: 10.1053/j.jvca.2013.01.017. PMID: 23791495. 

ondansetron with haloperidol for treatment of postoperative delirium in trauma patients admitted to intensive care unit: Randomized controlled trial. Anaesthesia, Pain and Intensive Care. 2015;19(2):118-23.

20. Boettger S, Breitbart W, Passik S. Haloperidol and risperidone in the treatment of delirium and its subtypes. European Journal of Psychiatry. 2011;25(2):59-67.

21. Boettger S, Jenewein J, Breitbart W Haloperidol, risperidone, olanzapine and aripiprazole in the management of delirium: A comparison of efficacy, safety, and side effects. Palliative \& supportive care. 2015;13(4):1079-85. doi: 10.1017/s1478951514001059.

22. Breitbart W, Marotta R, Platt MM, et al. A double-blind trial of haloperidol, chlorpromazine, and lorazepam in the treatment of delirium in hospitalized AIDS patients. Am J Psychiatry. 1996 Feb;153(2):231-7. doi: 10.1176/ajp.153.2.231. PMID: 8561204.

23. Breitbart W, Tremblay A, Gibson C. An open trial of olanzapine for the treatment of delirium in hospitalized cancer patients. Psychosomatics. 2002 May-Jun;43(3):17582. doi: 10.1176/appi.psy.43.3.175. PMID: 12075032

24. Carrasco G, Baeza N, Cabre L, et al Dexmedetomidine for the Treatment of Hyperactive Delirium Refractory to Haloperidol in Nonintubated ICU Patients: A Nonrandomized Controlled Trial. Crit Care Med. 2016 Jul;44(7):1295-306. doi: 10.1097/ccm.0000000000001622. PMID: 26925523.

25. Charoenporn V. Efficacy and safety of antipsychotic medications in the treatment of delirium. Journal of the Medical Association of Thailand. 2018;101(3):361-6.

26. Devlin JW, Roberts RJ, Fong JJ, et al. Efficacy and safety of quetiapine in critically ill patients with delirium: a prospective, multicenter, randomized, double-blind, placebo-controlled pilot study. Crit Care Med. 2010 Feb;38(2):419-27. doi: 10.1097/CCM.0b013e3181b9e302. PMID: 19915454.

27. Drinkwater B, Billyard T. Effect of intravenous haloperidol on the duration of delirium and coma in critically ill patients (hope-ICU). Journal of the Intensive Care Society. 2014;15(2):167-8. doi: 10.1177/175114371401500218.

28. Fox MA, Elefritz JL, Huang BM, et al. Comparison of Lurasidone Versus Quetiapine for the Treatment of Delirium in Critically Ill Patients. J Intensive Care Med. 2018 Jan 1:885066617754187. doi: 10.1177/0885066617754187. PMID: 29357776.
Girard TD, Exline MC, Carson SS, et al. Haloperidol and Ziprasidone for Treatment of Delirium in Critical Illness. N Engl J Med. 2018 Oct 22doi:

10.1056/NEJMoa1808217. PMID: 30346242.

30. Grover S, Kumar V, Chakrabarti S. Comparative efficacy study of haloperidol, olanzapine and risperidone in delirium. J Psychosom Res. 2011 Oct;71(4):277-81. doi: 10.1016/j.jpsychores.2011.01.019. PMID: 21911107.

31. Grover S, Mahajan S, Chakrabarti S, et al. Comparative effectiveness of quetiapine and haloperidol in delirium: A single blind randomized controlled study. World J Psychiatry. 2016 Sep 22;6(3):365-71. doi: 10.5498/wjp.v6.i3.365. PMID: 27679777.

32. Han CS, Kim YK. A double-blind trial of risperidone and haloperidol for the treatment of delirium. Psychosomatics. 2004 JulAug;45(4):297-301. doi: 10.1016/s00333182(04)70170-x. PMID: 15232043.

33. Hatta K, Kishi Y, Wada K, et al Antipsychotics for delirium in the general hospital setting in consecutive 2453 inpatients: a prospective observational study. Int J Geriatr Psychiatry. 2014 Mar;29(3):253-62. doi: 10.1002/gps.3999. PMID: 23801358.

34. Horikawa N, Yamazaki T, Miyamoto K, et al. Treatment for delirium with risperidone: results of a prospective open trial with 10 patients. Gen Hosp Psychiatry. 2003 JulAug;25(4):289-92. PMID: 12850662.

35. Hu H, Deng W, Yang H, et al. Olanzapine and haloperidol for senile delirium: A randomized controlled observation. Chinese Journal of Clinical Rehabilitation. 2006;10(42):188-90.

36. Hui D, Frisbee-Hume S, Wilson A, et al. Effect of Lorazepam With Haloperidol vs Haloperidol Alone on Agitated Delirium in Patients With Advanced Cancer Receiving Palliative Care: A Randomized Clinical Trial. JAMA. 2017 Sep 19;318(11):1047-56. doi: 10.1001/jama.2017.11468. PMID: 28975307.

37. Ikezawa K, Canuet L, Ishii R, et al. Efficacy of risperidone in the treatment of delirium in elderly patients. Psychogeriatrics. 2008;8(2):62-5. doi: 10.1111/j.14798301.2008.00227.x.

38. Jain R, Arun P, Sidana A, et al. Comparison of efficacy of haloperidol and olanzapine in the treatment of delirium. Indian J Psychiatry. 2017 Oct-Dec;59(4):451-6. doi: 10.4103/psychiatry.IndianJPsychiatry_59_1 7. PMID: 29497187.

39. Kim KS, Pae CU, Chae JH, et al. An open pilot trial of olanzapine for delirium in the Korean population. Psychiatry Clin Neurosci. 2001 Oct;55(5):515-9. doi: 10.1046/j.1440-1819.2001.00898.x. PMID: 11555348. 

Treatment of delirium in older adults with quetiapine. J Geriatr Psychiatry Neurol. 2003 Mar;16(1):29-31. doi:

10.1177/0891988702250533. PMID: 12641370 .

41. Kim JY, Jung IK, Han C, et al.

Antipsychotics and dopamine transporter gene polymorphisms in delirium patients. Psychiatry Clin Neurosci. 2005 Apr;59(2):183-8. doi: 10.1111/j.14401819.2005.01355.x. PMID: 15823165.

42. Kim SW, Yoo JA, Lee SY, et al. Risperidone versus olanzapine for the treatment of delirium. Hum Psychopharmacol. 2010 Jun-Jul;25(4):298302. doi: 10.1002/hup.1117. PMID: 20521319.

43. Kishi Y, Kato M, Okuyama T, et al. Treatment of delirium with risperidone in cancer patients. Psychiatry Clin Neurosci. 2012 Aug;66(5):411-7. doi: 10.1111/j.14401819.2012.02346.x. PMID: 22834659.

44. Konkayev AK, Bekmagambetova NV [Comparison of sedation with dexmedetomidine and haloperidol in patients with delirium after femoral neck fractures]. Anesteziol Reanimatol. 2015 JanFeb;60(1):8-11. PMID: 26027216.

45. Lee KU, Won WY, Lee HK, et al. Amisulpride versus quetiapine for the treatment of delirium: a randomized, open prospective study. Int Clin Psychopharmacol. 2005 Nov;20(6):311-4. PMID: 16192839.

46. Lim HK, Paik IH, Oh K, et al. Comparison of the Clinical Efficacy and Safety between Intramuscular Olanzapine and Intramuscular Haloperidol Injection in the Treatment of Delirium. Korean journal of psychopharmacology; 2007. p. 423-8.

47. Lin CJ, Sun FJ, Fang CK, et al. An open trial comparing haloperidol with olanzapine for the treatment of delirium in palliative and hospice center cancer patients. Journal of Internal Medicine of Taiwan. 2008;19(4):346-54.

48. Maneeton B, Maneeton N, Srisurapanont M An open-label study of quetiapine for delirium. J Med Assoc Thai. 2007 Oct;90(10):2158-63. PMID: 18041437.

49. Maneeton B, Maneeton N, Srisurapanont M, et al. Quetiapine versus haloperidol in the treatment of delirium: a double-blind, randomized, controlled trial. Drug Des Devel Ther. 2013;7:657-67. doi: 10.2147/dddt.s45575. PMID: 23926422.

50. Mittal D, Jimerson NA, Neely EP, et al. Risperidone in the treatment of delirium: results from a prospective open-label trial. J Clin Psychiatry. 2004 May;65(5):662-7. PMID: 15163252.
51. Naksuk N, Thongprayoon C, Park JY, et al. Editor's Choice-Clinical impact of delirium and antipsychotic therapy: 10-Year experience from a referral coronary care unit. Eur Heart J Acute Cardiovasc Care. 2017 Sep;6(6):560-8. doi: 10.1177/2048872615592232. PMID: 26124454

52. Omura K, Amano N. Clinical experience of quetiapine in 24 elderly patients with delirium. Psychogeriatrics. 2003;3(2):69-72. doi: 10.1046/j.1479-8301.2003.00012.x. PMID: 2004-19733-005.

53. Pae CU, Lee SJ, Lee CU, et al. A pilot trial of quetiapine for the treatment of patients with delirium. Hum Psychopharmacol. 2004 Mar;19(2):125-7. doi: 10.1002/hup.559. PMID: 14994323.

54. Parellada E, Baeza I, de Pablo J, et al. Risperidone in the treatment of patients with delirium. J Clin Psychiatry. 2004 Mar;65(3):348-53. PMID: 15096074.

55. Sasaki Y, Matsuyama T, Inoue S, et al. A prospective, open-label, flexible-dose study of quetiapine in the treatment of delirium. J Clin Psychiatry. 2003 Nov;64(11):1316-21. PMID: 14658945.

56. Sipahimalani A, Masand PS. Olanzapine in the treatment of delirium. Psychosomatics. 1998 Sep-Oct;39(5):422-30. doi: 10.1016/s0033-3182(98)71301-5. PMID: 9775699.

57. Skrobik YK, Bergeron N, Dumont M, et al. Olanzapine vs haloperidol: treating delirium in a critical care setting. Intensive Care Med. 2004 Mar;30(3):444-9. doi: 10.1007/s00134-003-2117-0. PMID: 14685663.

58. Straker DA, Shapiro PA, Muskin PR. Aripiprazole in the treatment of delirium. Psychosomatics. 2006 Sep-Oct;47(5):38591. doi: 10.1176/appi.psy.47.5.385. PMID: 16959926.

59. Tahir TA, Eeles E, Karapareddy V, et al. A randomized controlled trial of quetiapine versus placebo in the treatment of delirium. J Psychosom Res. 2010 Nov;69(5):485-90. doi: 10.1016/j.jpsychores.2010.05.006. PMID: 20955868.

60. Toda H, Kusumi I, Sasaki Y, et al. Relationship between plasma concentration levels of risperidone and clinical effects in the treatment of delirium. Int Clin Psychopharmacol. 2005 Nov;20(6):331-3. PMID: 16192843.

61. Yoon HK, Kim YK, Han C, et al. Paliperidone in the treatment of delirium: results of a prospective open-label pilot trial. Acta Neuropsychiatr. 2011 Aug;23(4):17983. doi: 10.1111/j.1601-5215.2011.00568.x. PMID: 25379796.

62. Yoon HJ, Park KM, Choi WJ, et al. Efficacy and safety of haloperidol versus atypical antipsychotic medications in the treatment of delirium. BMC Psychiatry. 2013 Sep 30;13:240. doi: 10.1186/1471-244x-13-240. PMID: 24074357. 
